Florida International University FIU Digital Commons

7-28-1994

\title{
A new parallel technique for the solution of sparse nonlinear equations
}

Maria Cereijo Martinez

Florida International University

DOI: $10.25148 /$ etd.FI14060129

Follow this and additional works at: https://digitalcommons.fiu.edu/etd

Part of the Computer Sciences Commons

\section{Recommended Citation}

Cereijo Martinez, Maria, "A new parallel technique for the solution of sparse nonlinear equations" (1994). FIU Electronic Theses and Dissertations. 2097.

https://digitalcommons.fiu.edu/etd/2097

This work is brought to you for free and open access by the University Graduate School at FIU Digital Commons. It has been accepted for inclusion in FIU Electronic Theses and Dissertations by an authorized administrator of FIU Digital Commons. For more information, please contact dcc@fiu.edu. 


\title{
FLORIDA INTERNATIONAL UNIVERSITY
}

\author{
Miami, Florida
}

\section{A NEW PARALLEL TECHNIQUE FOR THE SOLUTION OF SPARSE NONLINEAR EQUATIONS}

A dissertation submitted in partial satisfaction of the

requirements for the degree of

DOCTOR OF PHILOSOPHY

in

COMPUTER SCIENCE

by

Maria Cereijo Martinez

1994 
To: Dean Arthur W. Herriott

College of Arts and Sciences

This dissertation, written by Maria Cereijo Martinez, and titled A New Parallel Technique for the Solution of Sparse Nonlinear Equations, having been approved in respect to style and intellectual content, is referred to you for judgement.

We have read this dissertation and recommend that it be approved.

John Comfort

Nand Tripathi

Osama Mohammed

Gustavo Roig

Date of Defense: July 28th, 1994

David Barton, Major Professor

The dissertation of Maria Cereijo Martinez is approved.

Dean Arthur W. Herriott

College of Arts and Science

Dr. Richard L. Campbell

Dean of Graduate Studies

Florida International University, 1994 
CCOPYRIGHT 1994 by Maria Cereijo Martinez

All rights reserved 
I dedicate this dissertation to my children Daniel, Stephanie, and Elizabeth. 


\section{ACKNOWLEDGMENTS}

I would like to express my appreciation and gratitude to my committee for their help, guidance, and useful comments; To Dr. John Comfort for always being a part of my professional education, first as my mentor in Faculty Scholars and now participating in my guidance committee; To Dr. Osama Mohammed and Dr. Gustavo Roig for their participation and input to this project; To Dr. Nand Tripathi for teaching me about power systems and always willing to help and for providing the FPL data to test this project. A special thanks must go to my major professor, Dr. David Barton, for the advice, contribution, and dedication he has given me in this project.

I would like to give a special gratitude to my family for their love and support. To my parents I give my deepest appreciation; To my father for his confidence in me, his example, and his constant help; To my mother, whom I could not have done this without, I thank for always being there, and always willing to help especially with my children. To my husband I give a special thanks for without his encouragement, help and patience I would never haved finished. Finally I would like to thank my children, for whom I dedicate this dissertation, for their love. I hope this will be an encouragement to them to always try their best at what they do. 


\title{
A NEW PARALLEL TECHNIQUE FOR THE SOLUTION OF SPARSE NONLINEAR EQUATIONS
}

\author{
by \\ Maria Cereijo Martinez \\ Florida International University, 1994 \\ Professor David Barton, Major Professor
}

Solving nonlinear systems of equations is a central problem in numerical analysis, with enormous significance for science and engineering. A special case, sparse systems of equations, occurs frequently in various applications. Sparsity occurs in the analysis of many types of complex systems because of the local nature of the dependence or connectivity among system components.

One such system which may be modeled by a nonlinear sparse set of equations is the power system load flow analysis. This is a mathematical study performed by electrical utilities to monitor the electrical power system. The data from system components are used to create a set of nonlinear equations. These equations are then solved to find the voltage profile of the power network. With these data, control and security of the power system are achieved.

Solving problems of this type is very time consuming when the system is large. This dissertation proposes a highly parallel computer architecture for solving large sets of nonlinear sparse equations. The goal of this architecture is to reduce the processing time required to solve this type of problem. In particular, the load flow problem is analyzed and implemented on this architecture. For the FPL network, the speed is increased by a factor of about 2000 . 


\section{Contents}

1 INTRODUCTION 1

1.1 Numerical Solutions . . . . . . . . . . . . . . . 2

1.2 Previous Work Done . . . . . . . . . . . . . . 3

1.3 Application ................... 5

2 PARALLEL SOFTWARE SOLUTION

2.1 Statement of Problem ................. 7

2.1 .1 Nonlinear Equations . . . . . . . . . . . . . 8

2.1 .2 Sparsity ........................ 8

2.2 Numerical Solutions . . . . . . . . . . . . . . . . . . . . . 9

2.2 .1 Jacobi Method . . . . . . . . . . . . . . 9

2.2.2 Gauss-Seidel Method . . . . . . . . . . . . . . 9

2.2 .3 Newton-Raphson Iteration . . . . . . . . . . . . . . 10

2.2 .4 Other Methods . . . . . . . . . . . . . . . . 10

2.2.5 Application to Sparse Systerns . . . . . . . . . . . . . 11

2.2.6 Convergence of Algorithm . . . . . . . . . . . . 12

2.3 PLF Iterative Solution . . . . . . . . . . . . . . . . . 12

2.3.1 Graph Representation ................. 13

2.3.2 Description of Algorithm . . . . . . . . . . . . 14

2.3.3 Time Complexity . . . . . . . . . . . . . 16

2.3.4 Speedup and Efficiency . . . . . . . . . . . . 16

2.3.5 Synchronization . . . . . . . . . . . . . 17

2.3.6 Termination of Algorithm .............. 18

3 PARALLEL HARDWARE SOLUTION 20

3.1 General Design Considerations . . . . . . . . . . . . . . . 20

3.1 .1 Computing Power . . . . . . . . . . . . . . 20 
3.1.2 Loosely Coupled vs. Tightly Coupled Systems . . . . . . . . 21

3.1.3 Input/Output Considerations . . . . . . . . . . . 21

3.2 Communications Subsystem . . . . . . . . . . . . . . 22

3.2.1 Bus Connected Systems . . . . . . . . . . . . 22

3.2 .2 Switching Networks . . . . . . . . . . . . . . . 23

3.2.3 Network Control . . . . . . . . . . . . . . . 25

3.3 Review of Possible Parallel Architectures . . . . . . . . . . . . . 26

3.3.1 Pipeline Computers . . . . . . . . . . . . . . . . . 27

3.3 .2 SIMD Machines . . . . . . . . . . . . . . . . 27

3.3.3 MIMD Machines . . . . . . . . . . . . . . . 28

3.3.4 Dataflow Architecture . . . . . . . . . . . . . 30

3.4 PLF Architecture . . . . . . . . . . . . . . . . . . 30

3.4 .1 Control Unit. . . . . . . . . . . . . . . . . 32

3.4 .2 Processing Unit . . . . . . . . . . . . . . . . . 36

3.4 .3 Switching Network . . . . . . . . . . . . . 37

4 PERFORMANCE EVALUATION 43

4.1 Design Configuration . . . . . . . . . . . . . . . . . 43

4.2 Analysis and Experiments . . . . . . . . . . . . . . 44

4.2 .1 Simulations . . . . . . . . . . . . . . . 44

$4.2 .2 \quad$ Transfer Rate . . . . . . . . . . . . . . . . 46

4.3 Results . . . . . . . . . . . . . . . . . . . . 47

4.3 .1 Circuit Setup Time . . . . . . . . . . . . 47

4.3 .2 Communication Time . . . . . . . . . . . . . . . 49

5 POWER FLOW 50

5.1 Examination of the Problem . . . . . . . . . . . . . . 51

5.1 .1 Partition of the Power Network . . . . . . . . . . . 53 
5.1.2 Find the Parallelism in the Algorithms . . . . . . . . . 53

5.1 .3 Possible Architectures . . . . . . . . . . . . . . . 53

5.2 Mathematical Formulation of Load Flow . . . . . . . . . . . . . 54

5.2 .1 Formulation of the Problem . . . . . . . . . . 55

5.2 .2 Three Bus Example . . . . . . . . . . . . . . . . . 61

5.2.3 Jacobi and Gauss-Seidel Methods . . . . . . . . . . . . 62

5.2 .4 Jacobi Example . . . . . . . . . . . . . . . . 65

5.2 .5 Newton-Raphson Iteration . . . . . . . . . . . 65

5.2.6 Newton-Raphson Example . . . . . . . . . . . . . . 67

5.2 .7 Fast Decoupled Load Flow . . . . . . . . . . . . . 68

$5.2 .8 \quad$ Fast Decoupled Example . . . . . . . . . . . . . . 70

5.2 .9 Comparison of methods . . . . . . . . . . 70

5.2.10 Control of Load Flow Variables . . . . . . . . . . . 71

5.3 Review of Previous Research . . . . . . . . . . . . . . . . 72

5.3 .1 Solutions by Decomposition . . . . . . . . . . . 72

5.3 .2 Other Solutions . . . . . . . . . . . . . . 76

$5.3 .3 \quad$ EPRI Research $\ldots \ldots \ldots \ldots \ldots \ldots$

5.4 Load Flow Solution . . . . . . . . . . . . . . . . . . 81

5.4 .1 Parallel Machine Configuration ............ 81

5.4 .2 Convergence of the System . . . . . . . . . . . 82

6 LOADFLOW PERFORMANCE EVALUATION 84

6.1 Software Simulation. . . . . . . . . . . . . . 84

6.1 .1 Data Input ................... 84

6.1 .2 Data Types ...................... 86

6.1.3 Data Calculated . . . . . . . . . . . . 87

6.1 .4 Generators...................... 88

6.1 .5 Transformers $\ldots \ldots \ldots \ldots \ldots$ 
6.1 .6 Transmission Lines $\ldots \ldots \ldots \ldots$

6.1 .7 Load . . . . . . . . . . . . . . . . . . . 89

6.1 .8 Shunt Capacitors and Inductors . . . . . . . . . . . . . 89

6.2 Results . . . . . . . . . . . . . . . . . . . 90

7 CONCLUSION $\quad 94$

7.1 Future Research . . . . . . . . . . . . . . . . . 95

$\begin{array}{lr}\text { BIBLIOGRAPHY } & 96\end{array}$

$\begin{array}{ll}\text { A STOPPING ALGORITHM } & 101\end{array}$

$\begin{array}{lr}\text { B SWITCH SETUP } & 102\end{array}$

$\begin{array}{lr}\text { C ALGORITHMS } & 103\end{array}$

$\begin{array}{lr}\text { D OUTPUTS } & 146\end{array}$

\section{List of Figures}

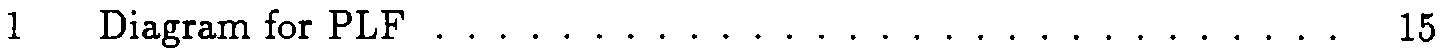

2 Single Stage Networks _ . . . . . . . . . . . . . . . . . . 24

3 PLF System Overview . . . . . . . . . . . . . . 32

4 Control Unit . . . . . . . . . . . . . . . . . . . 33

5 Processing Unit . . . . . . . . . . . . . . . . 36

6 Three Stage Clos Network . . . . . . . . . . . . . . . . . 39

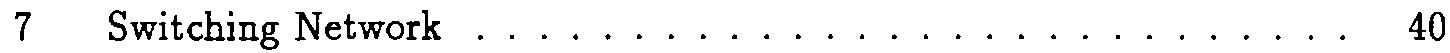

$8 \quad$ Middle Stage $512 \times 512 \ldots \ldots \ldots \ldots \ldots \ldots \ldots$

9 Connections of Transputers $\ldots \ldots \ldots \ldots \ldots \ldots \ldots$

10 Transfer Time . . . . . . . . . . . . . . . . 48

11 One-line diagram $\ldots \ldots \ldots \ldots \ldots \ldots \ldots \ldots$ 
12 Representation of network elements . . . . . . . . . . . . 58

13 Three bus systern . . . . . . . . . . . . . . . . 62

14 Rate of Convergence . . . . . . . . . . . . . . . . 83

\section{List of Tables}

$1 \quad$ Number of Buses . . . . . . . . . . . . . . . 46

2 Computation and Transfer Rates . . . . . . . . . . . . . 47

3 Summary of bus types $\ldots \ldots \ldots 61$

4 Comparison of methods ................ 71

$5 \quad$ Data Type Per Bus . . . . . . . . . . . . . . . . 88

6 Test Case ........................ 90

7 Time for Convergence $\ldots \ldots \ldots . \ldots \ldots$

830 Bus - Number of Iterations . . . . . . . . . . . . . 91

957 Bus - Number of Iterations . . . . . . . . . . . . . 92

10118 Bus - Number of Iterations . . . . . . . . . . . . . 92

11 FPL Test Case - Number of Iterations . . . . . . . . . . . 92 


\section{INTRODUCTION}

The problem of solving a system of nonlinear equations is one that has been often studied in numerical analysis. As the size of the systems increases the manipulations become more complex, storage requirements increase, and the total time for solving the equations becomes extended. The problem becomes more complex when the equations are sparse, meaning that it is thinly populated, or in a matrix formulation were the number of non-zero elements are small as compared to zero terms. Several numerical techniques have been developed to optimize the solution of sparse systems of equations. The problem addressed in this research is the development of an original architecture with maximum parallelism for any such sparse system and the development of appropriate numerical procedures in the parallel environment.

The general form of a system of nonlinear equations is

$$
\begin{array}{r}
f_{1}\left(x_{1}, x_{2}, \ldots, x_{n}\right)=0, \\
f_{2}\left(x_{1}, x_{2}, \ldots, x_{n}\right)=0, \\
\vdots \\
f_{n}\left(x_{1}, x_{2}, \ldots, x_{n}\right)=0,
\end{array}
$$

where each function $f_{i}$ maps $\mathrm{n}$-dimensional space, $R^{n}$, into the real line $R$. A function, F, maps $R^{n}$ into $R^{n}$ by

$$
F\left(x_{1}, x_{2}, \ldots, x_{n}\right)=\left(f_{1}\left(x_{1}, x_{2}, \ldots, x_{n}\right), f_{2}\left(x_{1}, x_{2}, \ldots, x_{n}\right), \ldots, f_{n}\left(x_{1}, x_{2}, \ldots, x_{n}\right)\right)
$$

Writing

$$
x=\left(x_{1}, x_{2}, \ldots, x_{n}\right),
$$

we have

$$
F(x)=0 .
$$


In sparse systems, the derivative $\partial f_{i} / \partial x_{j}$ is zero for many of the pairs $i, j,(i=$ $1 \ldots n, j=1 \ldots n)$. The connectivity, $c_{i}$, of each $f_{i}$ is the number of non-zero values $\partial f_{i} / \partial x_{j}, j=1 \ldots n$. For the systems under study the $c_{i}$ are small. Interaction between variables can be thought of as local in nature. Thus, for a parallel implementation, the processors will need to communicate only with their nearest connections.

The system can be rewritten in the following way:

$$
\begin{array}{r}
x_{1}=g_{1}\left(x_{1}, x_{2}, \ldots, x_{n}\right), \\
x_{2}=g_{2}\left(x_{1}, x_{2}, \ldots, x_{n}\right), \\
\vdots \\
x_{n}=g_{n}\left(x_{1}, x_{2}, \ldots, x_{n}\right),
\end{array}
$$

or

$$
x=g(x) .
$$

\subsection{Numerical Solutions}

Fixed point iterative solutions can now be obtained to this problem [56]. A GaussJacobi type solution is found by the iteration:

$$
x_{i}^{m+1}=g_{i}\left(x_{1}^{m}, x_{2}^{m}, \ldots, x_{n}^{m}\right)
$$

Alternatively the Gauss-Seidel iteration :

$$
x_{i}^{m+1}=g_{i}\left(x_{i}^{m+1}, \ldots, x_{i-1}^{m+1}, x_{i}^{m}, \ldots, x_{n}^{m}\right)
$$

may be used. A more commonly used method is the Newton-Raphson Iteration which assures quadratic convergence but involves computing the inverse of the Jacobian matrix of partial derivatives at every step, making it more computationally intensive [24]. Suppose vector $\theta$ is the exact solution to equation 1.8 , and the present approximation 
$x$ can be written $x=\theta+h$. Compute $f_{i}\left(x_{i}, \ldots, x_{n}\right)$. and call the known values $b_{i}$. Hence

$$
f_{i}\left(\theta_{1}+h_{1}, \theta_{2}+h_{2}, \ldots, \theta_{n}+h_{n}\right)=b_{i},
$$

Taylor expansion yields

$$
f_{i}\left(\theta_{1}, \theta_{2}, \ldots, \theta_{n}\right)+\sum_{k=1}^{n} h_{k}\left(\partial f_{i} / \partial \theta_{k}\right)+(\text { higher order terms })=b_{i} .
$$

By definition the first term is zero. Neglecting higher order terms we get, $J h=b$ and $h=J^{-1} b$, where $J_{i k}=\partial f_{i} / \partial x_{k}$ provided $\mathrm{J}$ is nonsingular. Thus an improved $x$ is given by the iterative formula

$$
x^{m+1}=x^{m}-J^{-1} f\left(x^{m}\right) .
$$

where $\mathrm{J}$ is the well known Jacobian matrix.

In Chapter 2 of this dissertation, a parallel software solution is designed that enables all of the $f_{i}$ terms to be computed simultaneously in parallel. A Jacobi type algorithm is used to control the numerical programs as it perserves the sparse nature of the equations.

A parallel hardware design is given in Chapter 3. This parallel architecture is called PLF. The PLF architecture is based on a Mutiple Instruction Multiple Data (MIMD) machine the INMOS Transputer.

In Chapter 4 a result of the simulations performed on the Transputer system is given.

\subsection{Previous Work Done}

Systems of sparse nonlinear equations have been analyzed by engineers and scientist for many years. Before computers were invented these problems had to be solved using primitive methods, such as pencil and paper. This limited the size of the problems 
being solved and took a significant amount of time with a high level of error. The techniques used were ones such as the Jacobi and Gauss-Seidel methods.

With the invention of computers the techniques for solving these equations changed. Now larger problems could be solved and with a better degree of accuracy. The Newton-Raphson method was now the method most commonly used. To improve on the speed of solving these problems many techniques have been developed such as sparse matrix techniques [22]. The speedup achieved by these methods using sequential machines has reached its peak.

The development of parallel computer architectures has brought a possibility of improving the solution times for these problems. This will allow for larger problems to be solved at much faster rates. New techniques are now being used to solve these problems in parallel. Some of the methods involve using the Newton-Raphson method in parallel. This divides the system of equations into groups and solves each group separately in a different computer using the Newton-Raphson method $[65,60,7]$. Another technique is to try to find the steps that can be done in parallel while inverting the Jacobian matrix $[73,53]$. Other approaches using relaxation techniques have been developed $[29,15,14]$. These techniques combine the Newton method with relaxation techniques, such as Jacobi, to increase the amount of parallelism exploited.

The aforementioned algorithms were tested mostly using vector computers, hypercube architectures [33], shared memory architectures, or on supercomputers. Some experiments have been done using transputers systems $[13,63]$. All these methods have used architectures that use few processors. Most developments in parallel processing have been application specific.

In this research a massivelly parallel architecture is being developed to solve the set of sparse nonlinear equations. The algorithm used is based on the Jacobi method. 


\subsection{Application}

The procedure, PLF, designed in the first three chapters can be applied to any system represented by sparse nonlinear equations and in this dissertation it is to the problem of power system load flow analysis. Chapter 5 examines the load flow analysis in detail.

The electrical power system is modeled in the computer using a technique called load flow analysis. This technique is also used to determine the security of the power network by studying the effects of outages or failures of system components. The load flow algorithm involves solving a large set of sparse nonlinear equations and is currently done on sequential computers using iterative methods. Conventional load flow analysis techniques employed to solve the power system model take too much time to run, thus the model employed does not adequately represent the power system network in fast enough time. Using a parallel algorithm and architecture, we are able to solve the power system load flow analysis in much shorter time, and thus obtain a more accurate representation of the power system.

An electrical power system consists of power stations, an electric power network, and distribution centers. The power stations are where the electricity is generated in bulk quantities. This electricity is transmitted over an electric power network to all the customers. The power network consists of transmission lines where the huge bulk of electric power moves on grids. This grid is divided into subnetworks to enable the electrical power to be delivered to the distribution network. The distribution network delivers the electric power to the customers.

An important requirement of the electrical utilities is the economic secure operation of its electrical power system. Such an objective requires the use of advanced systems analysis and control technologies, and uses very large complex hardwaresoftware systems. It is incorporated into the security and control functions of the Energy Control Center (ECC) [17,6]. 
A power system is in an emergency condition when its operational limits are violated [68]. The most severe and least predictable violations result from contingencies. Contingencies are disturbances in the power system such as transmission line critical outages, transformer outages, and generator outages. An important part of security revolves around the power systems ability to withstand the effects of contingencies. The probable results of certain outages are analyzed in real time using the current state of the power system by the ECC. To do this, the ECC calculates the current state of the system using a state estimation algorithm [64]. The effects of many contingencies are then tested using a power flow algorithm.

The load flow analysis is a technique that determines the state of the electric power network at a particular time. It uses real time information sent to the ECC and information stored in the database to build the network model. From this information, a set of nonlinear equations is developed and solved to determine the unknown information of the system. The most common numerical methods used are the Gauss-Seidel, the Newton-Raphson Iteration, and the Fast Decoupled Load Flow. [67].

As utilities expand, the number of power system components increases as well as the number of contingencies being tested. This creates a problem in terms of computing time. The latter part of this dissertation explains the power flow problem and its inherent parallelism and shows how the parallel architecture and algorithm developed here can be applied to speed up the solution of the problem.

In Chapter 6 of this dissertation a performance evaluation of the load flow analysis, as solved by the PLF system, is given. 


\section{PARALLEL SOFTWARE SOLUTION}

The need for fast solutions of very large computational problems has led to the study of parallel algorithms. Technological advances have raised the possibility of massively parallel computation. Parallel techniques make possible different numerical procedures for solutions of certain problems. Some issues that were of great importance in solving numerical algorithms sequentially become irrevelant when they are solved in parallel.

There are other issues relating to the parallelization of algorithms that must be resolved. There is the need for task allocation, for the breakdown of total workload into smaller tasks, and for the sequencing of the tasks that are interdependent. There are also communication issues such as the communication of interim computations, carrying out communication efficiently, and estimating the impact on performance. Lastly there is the issue of synchronization of computations on different processors, these can be implemented either synchronously or asynchronously.

This chapter is concerned with the software solution of the problem of solving sparse nonlinear equations. In Section 2.1 this statement of the problem is given mathematically. The different numerical solutions for this problem are discussed in Section 2.2. The design of the parallel solution for solving the sparse nonlinear equations is given in Section 2.3.

\subsection{Statement of Problem}

The general mathematical problems being studied here are those which are represented by sets of nonlinear algebraic equations. Many physical systems such as transportation and electrical machines can be modeled by nonlinear equations. In particular, a subclass of these problems is that of sparse systems. Sparse systems are very important in the study of many different areas of science and engineering. 


\subsubsection{Nonlinear Equations}

The general form of a system of nonlinear equations is:

$$
\begin{array}{r}
f_{1}\left(x_{1}, x_{2}, \ldots, x_{n}\right)=0, \\
f_{2}\left(x_{1}, x_{2}, \ldots, x_{n}\right)=0, \\
\vdots \\
f_{n}\left(x_{1}, x_{2}, \ldots, x_{n}\right)=0,
\end{array}
$$

where each function maps n-dimensional space $R^{n}$, into the real line $R$. The system can be represented by a function $\mathrm{F}$ mapping $R^{n}$ into $R^{n}$ by

$$
F\left(x_{1}, x_{2}, \ldots, x_{n}\right)=\left(f_{1}\left(x_{1}, \ldots, x_{n}\right), \ldots, f_{n}\left(x_{1}, \ldots, x_{n}\right)\right)
$$

where

$$
x=\left(x_{1}, x_{2}, \ldots, x_{n}\right)
$$

So the equation becomes $F(x)=0$.

A nonlinear system of the form $F(x)=0$ can be transformed into an equivalent fixed point problem $G(x)=x$. That is

$$
\begin{array}{r}
x_{1}=g_{1}\left(x_{1}, x_{2}, \ldots, x_{n}\right), \\
x_{2}=g_{2}\left(x_{1}, x_{2}, \ldots, x_{n}\right), \\
\vdots \\
x_{n}=g_{n}\left(x_{1}, x_{2}, \ldots, x_{n}\right) .
\end{array}
$$

\subsubsection{Sparsity}

For a system to be sparse, thinly populated, the interaction between variables must be small compared to the size of the system. Using the nonlinear equations of 2.12 , sparsity means that many of the $\partial f_{i} / \partial x_{j}$ are zero. Many types of systems are sparse, because of the local nature or connectivity that occurs among system components. 
Some important areas that involve the solution of sparse nonlinear equations are partial differential equations, vibration analysis of structures, and control theory. An application of which is the power system loadflow analysis that is used in this dissertation.

\subsection{Numerical Solutions}

\subsubsection{Jacobi Method}

To solve the above type of equation an iterative fixed point process is applied to $G$ [24]. To do this, we select $x^{(0)}=\left(x_{1}^{(0)}, \ldots, x_{n}^{(0)}\right)$, and generate the sequence of vectors $x^{(k)}=\left(x_{1}^{(k)}, \ldots, x_{n}^{(k)}\right)$ by

$$
x^{(k)}=G\left(x^{(k-1)}\right) \text { for each } k=1,2, \ldots, t
$$

Component wise:

$$
\begin{array}{r}
x_{1}^{(k)}=g_{1}\left(x_{1}^{(k-1)}, \ldots, x_{n}^{(k-1)}\right), \\
x_{2}^{(k)}=g_{2}\left(x_{1}^{(k-1)}, \ldots, x_{n}^{(k-1)}\right), \\
\vdots \\
x_{n}^{(k)}=g_{n}\left(x_{1}^{(k-1)}, \ldots, x_{n}^{(k-1)}\right) .
\end{array}
$$

If the sequence $x^{(k)}$ generated converges to $x^{*}$ and $G$ is continuous, then $x^{*}$ is a fixed point of $G$ and $x^{*}=G\left(x^{*}\right)$.

\subsubsection{Gauss-Seidel Method}

One possible way of improving the rate of convergence is to use the latest estimates $x_{1}^{(k)}, \ldots, x_{i-1}^{(k)}$ to compute $x_{i}^{(k)}$ and is called the Gauss-Seidel method [25]. The general formula then becomes:

$$
x_{i}^{(k+1)}=g_{i}\left(x_{1}^{(k+1)}, \ldots, x_{i-1}^{(k+1)}, x_{i}^{(k)}, \ldots, x_{n}^{(k)}\right)
$$


This generally works better than Jacobi for sequential systems because newer estimates are used. There are systems for which the Jacobi method converges better than the Gauss-Seidel [76].

\subsubsection{Newton-Raphson Iteration}

Another method for solving the non-linear sets of equations $f_{i}\left(x_{1}, x_{2}, \ldots, x_{n}\right)=0$ for $i=1, \ldots, n$ is Newton-Raphson Iteration [8]. Suppose that a vector $\alpha$ is the exact solution to the system and that the present approximation $x$ can be written $\alpha=x+h$. Taylor series expansion yields

$$
f_{i}\left(x_{1}, x_{2}, \ldots, x_{n}\right)+\sum h_{k}\left(\partial f_{i} / \partial x_{j}\right)+(\text { higher order terms })=0
$$

or

$$
f_{i}\left(\alpha_{1}, \alpha_{2}, \ldots, \alpha_{n}\right)=0
$$

Neglecting the higher order terms we get, $J h=-f$ and $h=-J^{-1} f$. J is the Jacobian which is obtained by taking the gradients of the different components, $J_{i j}=$ $\partial f_{i} / \partial x_{j}$ and we assume $\mathrm{J}$ is nonsingular. The exact solution is obtained with the iterative formula

$$
x^{k+1}=x^{k}-J^{-1} f\left(x^{k}\right)
$$

This method assures quadratic convergence and yields a smaller number of iterations. The drawback is in finding the derivatives and that it involves the inverse of a matrix at every iteration step, thus becoming computationally far more intensive than the Gauss-Seidel or the Jacobi methods.

\subsubsection{Other Methods}

There are modifications to the above methods that can be used to solve a set of nonlinear equations. The successive overrelaxation method, SOR, can be applied to the 
Jacobi or Gauss-Seidel to improve the convergence rate [79]. At each iteration of the solution of $x_{i}^{k+1}$ by the Jacobi or the Gauss-Seidel a new value is calculated by

$$
x_{i}^{k+1}=x_{i}^{k}+w\left(x_{i}^{k+1}-x_{i}^{k}\right)
$$

This $w$ is known as an acceleration factor. Studies show that $w$ must lie in the range $0<w<2$ for convergence [79]. The PLF algorithm developed here is based on a parallel Jacobi Successive Overrelaxation method.

The Newton-SOR method is another possibility [8]. It is Newton's method as primary iteration and the SOR as secondary iteration. This takes away the quadratic convergence of Newton-Raphson Iteration and is consequentialy numerically intensive.

An incomplete Choleski-conjugate gradient algorithm is given in [1] to solve large sparse sets of simultaneous equations. It is used in the Newton-Raphson method when the Jacobian is symmetric. This improves on the computing time of the classical Newton-Raphson method.

\subsubsection{Application to Sparse Systems}

Although Newton's method is theoretically attractive, it may be difficult to use in practice for this formulation. For problems arising from partial differential equations and in control theory, in which the dimensions of the system may be thousands, this is a difficult task. Moreover, at each step, not only the n components of $f\left(x^{k}\right)$ but also the $n^{2}$ entries of $\partial f_{i} / \partial x_{j}$ are needed. Unless the partial derivatives $\partial f_{i} / \partial x_{j}$ have a simple functional form, it may be desirable to avoid their computation altogether.

The Jacobi method involves simple operations at each iteration but is very slow on sequential computers. This is eliminated in a parallel computer owing to the fact that all equations can be solved simultaneously. By using an acceleration factor, the number of iterations can be reduced one or two orders of magnitude compared to the Gauss-Seidel method [25]. In our case it reduced the number of iterations by a factor of two. 


\subsubsection{Convergence of Algorithm}

Let the maximum norm, $\|A\|_{\infty}$, of a matrix $\mathrm{A}$, be defined as $\max _{i} \sum_{k}\left|a_{i k}\right|$ where $A=\left(a_{i k}\right)$. The Jacobi method converges for an initial sufficiently good approximation when $\|A\|_{\infty}<1$ where $a_{i j}=\sup _{x}\left|\partial g_{i} / \partial x_{k}\right|$. [24].

In [79] the Jacobi method is proved to converge if $A$ is irreducible and has weak diagonal dominance. Weak diagonal dominance is defined for $A=\left(a_{i k}\right)$ as

$$
\left|a_{i i}\right| \geq \sum_{j \neq i}\left|a_{i j}\right| \text { for all i, }
$$

and for at least one $i$

$$
\left|a_{i i}\right|>\sum_{j}\left|a_{i j}\right| \text {. }
$$

An irredducible matrix is a matrix $A=\left(a_{i k}\right)$ of order $\mathrm{N}$ where $N=1$ or if $N>1$ then, given any two nonempty disjoint subsets $\mathrm{S}$ and $\mathrm{T}$ of $\mathrm{W}$, where $\mathrm{W}=1,2, \ldots, \mathrm{N}$, such that $\mathrm{S}+\mathrm{T}=\mathrm{W}$, there exists $i \epsilon S$ and $j \epsilon T$ such that $a_{i k} \neq 0$.

The convergence of the Successive Overrelaxation method is proved in [79]. Writing the SOR method in matrix form

$$
\begin{array}{r}
u^{n+1}=w\left(L u^{n+1}+U u^{n}+c\right)+(1-w) u^{n} \\
u^{n+1}=B u^{n}+(I-w L)^{-1} w c
\end{array}
$$

where

$$
B=(I-w L)^{-1}(w U+(1-w) I)
$$

Let $S(B)$ be the spectral radius of $B . S(B)$ is $\left|\lambda_{1}\right|$, where $\lambda_{1}$ is an eigenvalue of $B$ with greatest absolute value. If $S(B) \leq 1$, then $0 \leq w \leq 2$. For convergence $w$ must lie between 0 and 2 .

\subsection{PLF Iterative Solution}

The solution of sets of sparse nonlinear equations will be performed on a specially designed parallel system PLF. A parallel Jacobi successive overrelaxation method 
is implemented to run on this parallel hardware. This is called the PLF software solution. The next sections discuss this algorithm.

\subsubsection{Graph Representation}

A Directed Acyclic Graph (DAG) can be used to represent a parallel algorithm. This is a graph that has no positive cycles, that is no cycle consisting of only forward arcs [59]. Let $G=(N, A)$ be a DAG where $\mathrm{N}$ is the set of $n$ nodes and $A$ is the set of directed arcs $\operatorname{arc}(i, j) \epsilon A$. An arc $(i, j)$ represents an operation corresponding to node $j$ which uses results of the operation corresponding to node $i$. $i \in N$ is said to be a predecessor of node $j \epsilon N$. The following are definitions used in creating a DAG:

- in-degree of $i$ is number of predecessors of $i$

- out-degree of $i$ is the number of nodes for which $i$ is the predecessor

- input nodes are those nodes with in-degree zero

- output nodes are those nodes with out-degree zero

- positive path sequence $i_{0}, \ldots, i_{K}$ of nodes such that $\left(i_{k}, i_{k+1}\right) \epsilon A$ for $k=0, \ldots, K-1$ and the length of the path is $K$

- depth, D, of a DAG is the largest length of the positive paths

Let the result of the operation at node $i$ be $x_{i}$. Functional dependencies are represented as follows:

$$
x_{i}=f_{i}\left(x_{j} \mid j \text { is a predecessor of } i\right)
$$

when $f_{i}$ is a function describing the operation of ith node. $P_{i}$ is the processor assigned the responsibility of performing the corresponding operation.

The time that the operation is completed at node $i$ is specified by a positive integer $t_{i}$. A processor can perform one operation at a time. That is if $i, j \in N, i \neq j$ 
and $t_{i}=t_{j}$ then $P_{i} \neq P_{j}$. Thus if two different nodes complete an operation at the same time then they are assigned different processors. If $(i, j) \epsilon A$ then $t_{j} \geq t_{i}+1$, that is an operation at $j$ can only start after node $i$ has finished. If the transmission of a message requires $\tau$ time then $t_{j} \geq t_{i}+\tau+1$. From this a scheduled DAG for parallel execution is defined as

$$
\left(i, P_{i}, t_{i}\right) \mid i \in N
$$

\subsubsection{Description of Algorithm}

The iterative algorithm $x=G(x)$ can be parallelized by letting each of $\mathrm{n}$ processors update a different component of $\mathrm{x}$. At the $k t h$ stage the $i t h$ processor knows the value of $x_{j}^{(k)}$ on which $g_{i}$ depends. It computes $x_{i}^{(k+1)}$ and communicates it to the other nodes.

A dependency graph is defined as a Directed Acyclic Graph, $G=(N, A)$, where a set of nodes $N$ correspond to components of $x$. For nodes $i$ and $j$ let $(i, j)$ be an arc of the dependency graph if and only if $g_{j}$ depends on $x_{i}$, that is processor i needs to communicate the values of $x_{i}^{(k)}$ to processor $\mathrm{j}$.

Assume iterations to be carried out for $t=1,2, \ldots, T$ where $\mathrm{T}$ is some positive integer. Let us assign a parallel DAG to be an unfolding in time. Nodes are renamed $[i, t]$ where $i \epsilon N$ and $t$ is the iteration count. Arcs are of the form $([i, t],[j, t+1]) \epsilon A$. The parallel DAG for this algorithm is given in the Figure 1.

One node of the above graph represents one equation of each iteration. The indegree of node $\mathrm{i}$ is the number of unknowns for that equation. The out-degree for a node is the number of nodes that have it as an unknown. Each out going arc represents the newly calculated value. 


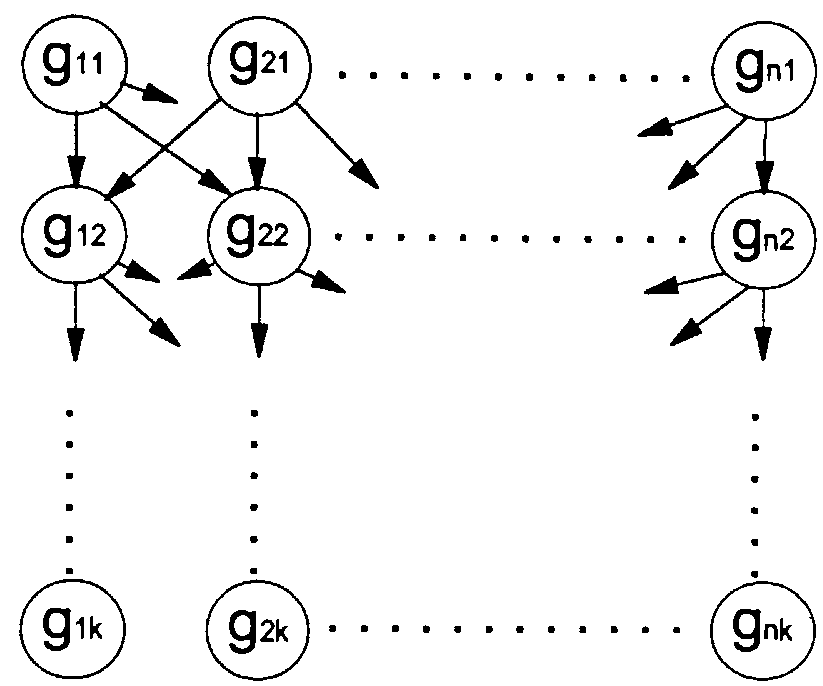

$$
\begin{aligned}
\text { gij } & =\text { equation set for node } i j \\
i & =\text { equation number } \\
j & =\text { iteration } \# \\
n & =\text { max number of equations } \\
k & =\text { max number of iterations }
\end{aligned}
$$

Figure 1: Diagram for PLF 


\subsubsection{Time Complexity}

Let $G=(N, A)$ be a DAG for a parallel algorithm. Let

$$
\left(i, P_{i}, t_{i}\right) \mid i \epsilon N
$$

be a schedule. The time spent by a schedule is $\max _{i \in N} t_{i}$. If $\mathrm{p}$ is the number of processors used, the minimum of $\max _{i \in N} t_{i}$ taken over all possible schedules that use $\mathrm{p}$ processors is called the time complexity of $\mathrm{G}$ and is written $T_{p}$.

$$
T_{\infty}=\min _{p \geq 1} T_{p}
$$

$T_{p}$ is a nonincreasing function of $\mathrm{p}$ bounded below by zero. The minimum number such that $T_{p}=T_{\infty}$ for all $p \geq p^{*}$ is $p^{*}$. Thus $T_{1}$ is the time needed for serial execution and $T_{\infty}$ is equal to the depth of the DAG.

For the system PLF the number of processors, $p$, is equal to a number of sets of equations, $\mathrm{n}$. The time spent on the schedule will depend on the speed of the slowest processor $p_{i}$. This is explained as follows. Each processor will do calculations and then output the results. It then reads in the values of the results of the processor it is dependent on. It will not proceed to evaluate its equations until it has received all the new values.

\subsubsection{Speedup and Efficiency}

Let $T_{p}(n)$ be termination time for problem size $n$ and p processors. Let $T^{*}(n)$ be the optimal serial time to solve the problem. The speedup or speed advantage of a parallel algorithm is given by

$$
S_{p}(n)=\frac{T^{*}(n)}{T_{p}(n)}
$$

The fraction of time a processor is employed or its efficiency is given by:

$$
E_{p}(n)=\frac{S_{p}(n)}{p}=\frac{T^{*}(n)}{p T_{p}(n)}
$$

Ideally $S_{p}(n)=p$ and $E_{p}(n)=1$. 


\subsubsection{Synchronization}

It is necessary to coordinate the activities of different processors in any parallel algorithm. This can be done in two ways, synchronously or asynchronously.

The algorithm is synchronous if it is mathematically equivalent to one for which the times of computation, message transmission, and message reception are fixed and given beforehand. Synchronization is performed by dividing the algorithm into phases. During each phase, every processor performs some computation that depends on the results of computations of other processors in previous phases. In order to implement a synchronous algorithm in an inherently asynchronous system, a synchronization mechanism is needed. An algorithm that is superimposed on the original and by which every processor can detect the end of the phase is called a synchronizer. Synchronizers can either be local or global.

In global synchronization, the idea is to let each processor detect when all messages sent during a phase have been received and then start doing the computation of the next phase. Since there is no global clock, this is done through the use of timeouts or phase termination messages. With timeouts, the processors measure the length of any time interval by using a local clock. A phase termination is indicated when a processor sends to every other processor a message signaling that it has received all of its messages.

The main concept in local synchronization is that once a processor knows which message to expect at the end of each phase, then it starts a new phase after having received all these messages.

For asynchronous algorithms, the concept of phases is not used, and the coordination of processors is less strict. An asynchronous algorithm is one in which the times for computation, message transmission, and message reception can vary widely in different executions of the algorithm. The local algorithms do not wait for predetermined data to become available, but keep on computing trying to solve the problem 
with whatever data are available.

In our problem, we have an n-processor system and an n-dimensional fixed point problem, that is, we want to find a vector $x=\left(x_{1}, x_{2}, \ldots, x_{n}\right)$. that satisfies:

$$
x_{i}=g_{i}\left(x_{1}, x_{2}, \ldots, x_{n}\right) \text { for } \mathrm{i}=1,2, \ldots, \mathrm{n}
$$

where $f_{i}$ are given functions of $\mathrm{n}$ variables. Each processor $P_{i}$ will update the variable $x_{i}$ according to the above equation, starting from a set of initial values for variables. A local synchronous implementation of this problem is one in which a processor, $P_{i}$, does not carry out its kth update without first receiving the results of the (k-1) updates from the processors whose variables appear in the function $f_{i}$. In an asynchronous implementation of this algorithm, what is required is that the kth update at processor $i$ is carried out with knowledge of the results of some past update of every other processor, not necessarily the most recent update.

The PLF software system that has been developed uses the local synchronous implementation.

\subsubsection{Termination of Algorithm}

To terminate the PLF system a stopping algorithm is needed. This algorithm is described as follows. All the nodes of the system are connected in a spanning tree. Each node knows its parent, its children, if it is leaf or if it is the root. If a node is a leaf it will check to see if it has converged and send the appropriate message to its parent. The message is a one if it has converged, and zero if it has not. When a parent has received a one bit from all its children it checks to see if it has converged. If it has converged then a one is sent up the tree to its parent. This process continues up the tree on every iteration. When the root receives a one from all its children, it continues to receive for the height of the spanning tree. If it has received a one for each iteration for the height of the spanning tree then the system has converged. It then proceeds to send a STOP message to all its children. When a node receives a 
STOP message it sends it down to its children and stops calculating. In Appendix A an algorthm is given to do this.

Each node in the system will run this algorithm. When the root node receives all ones from each of the children for the height of the tree then there is convergence. The root then initiates the STOP message down the tree. 


\section{PARALLEL HARDWARE SOLUTION}

A massively parallel architecture has been developed to reduce the solution time for solving large sets of nonlinear sparse equations. The system, called PLF, consist of many thousands of powerful processors. One or more processors are coupled to form a processor unit. These processor units are linked by a communications network. The systems that can be modeled by this architecture are systems of sparse nonlinear equations, e.g. power system load flow analysis, and the equations of computational fluid dynamics and weather predications. The main processor used for this architecture is the INMOS T800 Transputer. The transputer is a 32-bit processor with 7.5 to 10 million instructions per second (MIPS) processing speed. Each transputer has four bidirectional serial communication links which can be used to communicate with other processors or controllers. Each link has a speed of up to 20 Megabits/second.

\subsection{General Design Considerations}

\subsubsection{Computing Power}

Computing power can be crudely measured by million instructions per second (MIPS). The effective computing power when a parallel machine is used to solve a problem largely depends on the parallelism and granularity of the algorithm employed and how well that matches the parallel machine. There is a design criterion on how much computing power each individual processor of the parallel machine should have. When each individual processor has small computation power, efficient use will require that the system have a potentially large number of processors or high parallelism. If the processors are too small, and the problem to be solved has large sequential sub tasks, then the performance will be low. For our problems, the processors must be powerful enough to handle floating point arithmetic efficiently. As many processors are required as sets of equations (2048) in the sparse system. 


\subsubsection{Loosely Coupled vs. Tightly Coupled Systems}

Processes in loosely coupled multiprocessor systems communicate by exchanging messages. In tightly coupled systems, processors communicate through a shared main memory. In loosely coupled systems, processors communicate through message passing, and use their local memories to store results. If a processor is to access data in another processor's memory, it must send a message through the communication subsystem requesting the other processor to send these data.

Our design is a loosely coupled system. It consist of processors with large local memories in which they store their instructions and data. Processes that execute on different processor modules communicate by exchanging messages through a message transfer system. In our case, the message transfer system is a multistage network of switches. Loosely coupled systems are most efficient when interactions between tasks are minimal. In our application we have minimal interaction between processors.

\subsubsection{Input/Output Considerations}

When designing loosely coupled parallel systems, the input/output bandwidth for communication, internal and external, may become a bottleneck for the performance of the entire system.

Let $\mathrm{PE}$ be a processing element or processor. Let $\mathrm{C}$ be defined as computational bandwidth, the number of operations per second delivered by the PE. IO is input/output bandwidth, the number of words per second that the PE can communicate with the external environment. Let $M$ be size of local memory, the number of words of local memory. A PE is said to be balanced in carrying out a computation if the I/O time equals the computation time.

Let TC represent the number of operations a PE must perform for a given computation and TIO the number of words the PE must exchange with the outside world. Then the required computation and $\mathrm{I} / \mathrm{O}$ times are $T C / C$ and $T I O / I O$ respectively. 
Therefore the PE is balanced for the given computation if and only if:

$$
\frac{T C}{C}=\frac{T I O}{I O}
$$

For the application being studied less than 100 bytes of local memory is needed to keep intermediary results used in the computation.

\subsection{Communications Subsystem}

The communications subsytem linking processors, memories, and I/O controllers in a parallel processing system is one of its most important architectural features, and has a profound impact on system capabilities, performance, size, and cost. There are two basic architectural alternatives for the communications subsystem, the single bus and the network.

\subsubsection{Bus Connected Systems}

A high-bandwidth communications bus provides a satisfactory communication subsystem if each processor has its own cache memory and if the number of processors is no more than 50 [12], assuming present bus and memory technologies. For larger numbers of processors the bus bottleneck is eliminated by using a communications network instead of the bus to provide the desired connectivity and performance. In general, an interconnection network that will provide the desired connectivity and performance at minimum cost is required for communications in a parallel processing system with large numbers of processors.

The two basic types of interconnection networks for parallel processing systems are shared path networks and switching networks. The shared path network consists of a communication path that may be used by more than one pair of processors to exchange information. This shared path can be called a bus. The simplest such system is a single bus system. 
To improve the performance of single bus system, buses can be used hierarchically in a parallel arrangement as in the $C_{\boldsymbol{m}}^{*}[18]$ system. This is a Multiple Instruction Multiple Data (MIMD) system designed at Carnegie Mellon University that is composed of a large number of computer modules grouped into clusters and connected by a hierarchy of buses. Mathematical and simulation model analysis have shown that this system has the limitations inherent in shared communications path system which make them unsuitable for massive parallelism.

\subsubsection{Switching Networks}

A network providing a separate path between any two system components at all times is a crossbar network. The hardware required to implement the switch is complex. The crossbar switch provides the highest performance, but is not effective for a large multiprocessor system because of its cost and its complexity.

A single stage interconnection network can be used to solve the problem of providing fast, reliable and efficient communications at a reasonable cost in large parallel processing systems. In a single stage interconnection, network data may have to be passed through the switches several times before reaching its final destination. The data will travel through a few nodes before reaching the final one. The single stage network can be viewed as an interconnected set of $\mathrm{N}$ input units and $\mathrm{N}$ output units. The way the input units are connected to the output units determines the functional characteristics of the network. There are different ways to do this that form the basis of most interconnection networks that have been proposed. A few of these are the mesh, the cube, the shuffle-exchange networks. Information on these networks can be found in $[18,51]$. An example of these networks is shown in Figure 2.

The mesh network consist of $\mathrm{N}=n^{2}$ nodes, each connected to four neighbours. The input unit $i$ will be connected to output units $i+1, i-1, i+n, i-n,(\operatorname{modulo} N$ ).

The cube network is defined by $m=\log _{2} N$ interconnections. The connectivity of 


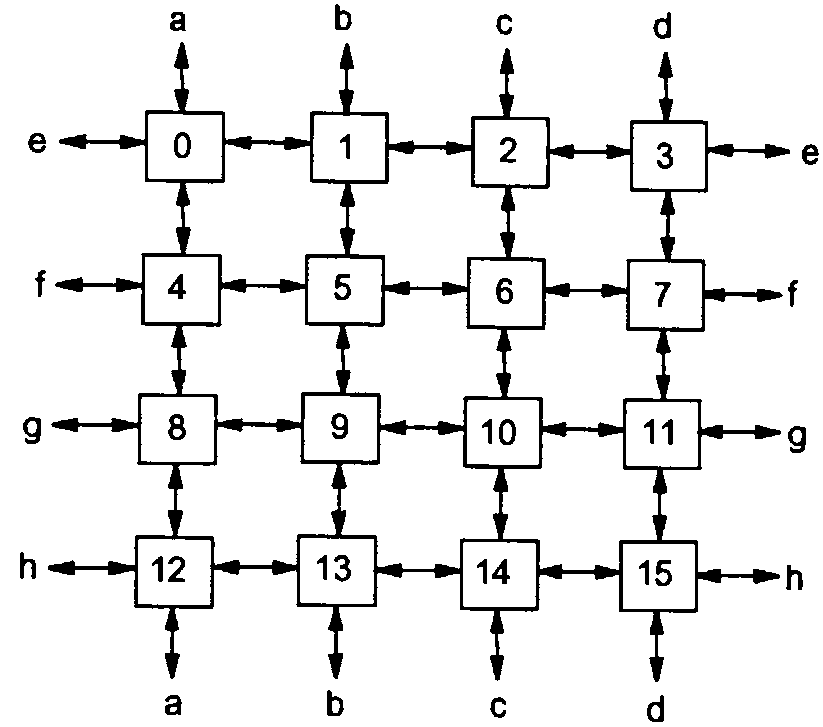

Mesh Network $N=16$

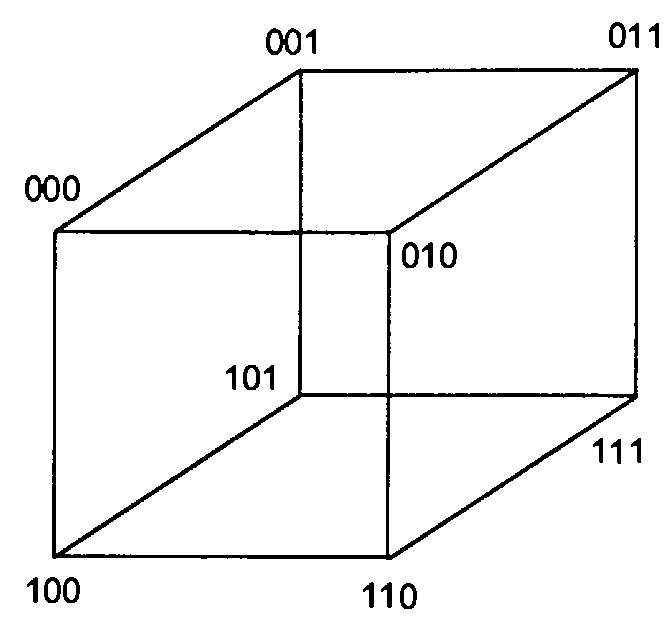

Three-Dimensional Binary Cube

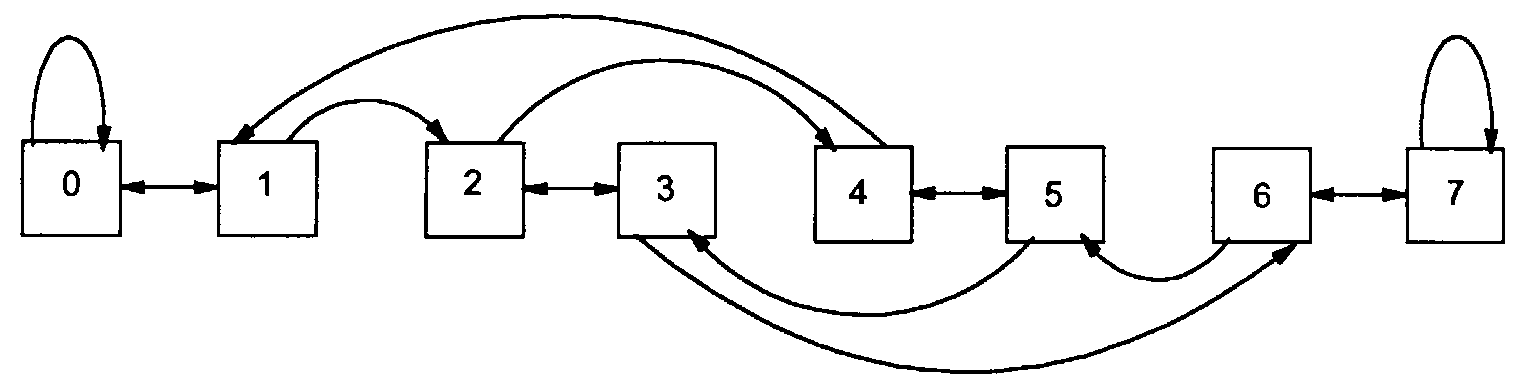

Shuffle-Exchange Network $\mathrm{N}=8$

Figure 2: Single Stage Networks 
this network can be represented by an $m$ dimensional cube whose corners represent the system component addresses. The interconnections can be written as a function of the binary representation of the system component addresses. Let $b^{\prime}$ be the complement of bit $b$.

$$
C_{i}\left(b_{m-1} b_{m-2} \ldots b_{i+1} b_{i} b_{i-1} \ldots b_{1} b_{0}\right)=b_{m-1} \ldots b_{i}^{\prime} \ldots b_{0}
$$

Neighbor addresses differ in only one bit position.

The shuffle-exchange network consist of a shuffle function and an exchange function. The shuffle function is equivalent to intermixing two halves of a deck of cards.

$$
S\left(b_{m-1} b_{m-2} \ldots b_{1} b_{0}\right)=b_{m-2} b_{m-3} \ldots b_{1} b_{0} b_{m-1}
$$

The exchange function connects each system component to the component whose address differs from it in the low order bit position.

$$
E\left(b_{m-1} b_{m-2} \ldots b_{1} b_{0}\right)=b_{m-1} b_{m-2} \ldots b_{1} b_{0}^{\prime}
$$

Another way to solve the problem of fast reliable communication at an acceptable cost is to use multistage networks. In a multistage network, one pass of the multiple stages of switches is usually enough. Multistage interconnection networks are built from stages of the basic single stage networks.

In the design of PLF, a multistage network is used. The stages consist of crossbar switch combinations. This switch network chosen is non-blocking, to allow all nodes to communicate simultaneously. It is built using the INMOS C004. The C004 is a programmable link switch designed to provide a full crossbar switch between 32 link inputs and 32 link outputs. The design of this network is discussed in another section.

\subsubsection{Network Control}

The network control structure is implemented by switching algorithms that generate the control settings on the switching elements to ensure reliable data routings from 
source to destination. It is also implemented by the communications protocols involved in the link control procedures that provide the handshaking process among switching points.

A network can be operated in circuit or packet switching mode. In circuit switching mode, once a path is established, the interchanges in the path remain in their specified states until the path is released. Thus a complete circuit is established from input to output for that path. Once a path is established, the only delay in the network is propagation delay. This is desirable when large blocks of data must be transferred, and when conflict-free connections that change infrequently can be set up. In the packet switching mode, a packet moves from stage to stage, releasing links and interchange paths immediately after using them. This involves overhead for routing, and setting switches, and waiting time delay in buffers at each stage. This is desirable when the communications among system components involve short messages, and when the paths through the network change frequently. Our network will use circuit switching, since, in our problem, the circuit does not change frequently once it is set up.

\subsection{Review of Possible Parallel Architectures}

Four types of parallel architectures where considered to be used for solving a set of sparse nonlinear equations in parallel.

- Pipeline processors,

- Single Instruction Multiple Data (SIMD) Machines,

- Multiple Instruction Multiple Data (MIMD) Machines,

- Dataflow Machines.

A pipelined processor is a processor with pipelined arithmetic units, whose operands can be input in a streamed fashion as into a pipe. Typical computers relying on 
pipelining for their speed are the C-MP CRAY and CDC CYBER. The Connection Machine [10], is a Single Instruction Multiple Data (SIMD) computers consist of many identical processors doing the same calculation on differents sets of data at the same time. The INMOS Transputer and Dataflow Machine are Multiple Data Multiple Instruction (MIMD) computers that perform separate calculations on different sets of data in parallel. Dataflow machines are a different form of parallel processing were data sets are processed in parallel if they are not dependent on each other .

\subsubsection{Pipeline Computers}

One such computer is the supercomputer (i.e. CDC Cyber). A few supercomputers can be connected in such a way that different equations can be run on each for different sections of the system $[65,78]$. These methods reduce the network of equations into many smaller decoupled subnetworks. Each computer would then solve a section of the problem separately and in parallel. Once each computer has finished solving the results are joined and the necessary adjustments made. Presently most numerical problems are solved using these supercomputers.

\subsubsection{SIMD Machines}

A class of computer considered were the Single Intruction Multiple Data (SIMD) machines. These computers perform an instruction on a set of data in parallel. The SIMD computer that was considered to be used was Thinking Machine Corporation's the Connection Machine.

The Connection Machine system from Thinking Machine Corporation implements data level parallelism. It combines a large number of processors with the communications capability necessary to match data topologies. The Connection Machine executes instructions that operate on a whole set of data elements at once [10].

The Connection Machine operates by receiving instructions from the front end 
computer. A microcontroller receives the instructions, expands them to machine instructions, and broadcasts them one at a time to all processors. It can include up to 65,536 physical processors and can be configured to an even larger number of logical processors. Each physical processor has 4096 bits of memory, which can total up to 32 megabytes for the entire machine.

The simplest form of communication between the processors is between nearest neighbors. Each processor is wired to the north, east, west, and south neighbors by a communications network called the NEWS grid. Information is passed one bit at a time. General intercommunication and dynamic reconfiguration is performed by the router. Full messages can be sent from any processor to any other and can be any length. Each of the 65,536 physical processors is connected to 16 other physical processors in a 16-dimension hypercube. This provides large numbers of direct paths to distant parts of the system.

The Connection Machine can have a floating point accelerator. It can operate on single precision (32 bit) or double precision(64 bit) floating point numbers. It supports IEEE standard floating-point formats and operations [11].

This machine is not suitable for our application because it performs the same operation on different data sets and our application requires different operations on data sets.

\subsubsection{MIMD Machines}

The computer used to develop the architecture for the present problem is the INMOS Transputer $[39,37,36]$. It is used to build an architecture that is Multiple Data Multiple Instruction (MIMD) type. This means that different instructions are working on different data at the same time independent of each other.

The transputer is a microcomputer with its own local memory and four links for connecting one transputer to another. The transputer is programmed to implement 
a set of concurrent processes. A resident operating system shares the processor time among the concurrent processes and performs interprocess communication.

The transputer can be programmed in most high level languages. To gain the most benefit from the transputer architecture, the whole system is programmed in the parallel language Occam. Occam provides all the advantages of a high level language, the maximum program efficiency and the ability to use the special features of the transputer.

The transputer system uses point to point communication links. Every transputer has four standard links that can be connected to a link of any other transputer. This allows for networks of almost any size and topology to be constructed. The links for the IMS T808 transputer are standard, full duplex, serial links that provide concurrent message passing capability to other transputer devices at up to $20 \mathrm{Mbits} / \mathrm{sec}$. The IMST808 processor does floating point arithmetic.

Each INMOS link provides two channels, one in each direction. Communication via any link may occur concurrently with that on other links and program execution. Synchronization of processes at each link is handled by the operating system. Each link consists of a serial input and serial output that can carry both data and link information. A message is transmitted as a series of bytes. The sending transputer waits for an acknowledgement, signifying that the receiving transputer is ready to receive another byte. The receiving transputer transmits an acknowledgement as soon as it starts to receive a data byte, so that transmission can be continuous. This protocol synchronizes the communication of each byte of data, ensuring that slow and fast transputers can communicate.

Each transputer in a system uses its own local memory. This memory consist of $2 \mathrm{~K}$ bytes and supports multiport access for the processor and each of the four links. Memory bandwidth is proportional to the number of transputers in the system. 


\subsubsection{Dataflow Architecture}

The concept of dataflow architectures come from dataflow graphs $[4,69,16]$. A dataflow graph is a directed graph in which the nodes represent primitive functions and the arcs represent data dependencies between functions. Dataflow architecures are language based architectures in which dataflow program graphs are the base language.

A dataflow program graph is made up of operators called actors connected by arcs that are used to show how data is moved. An actor has input and output arcs that move tokens from one actor to another. When a token is present on an input arc and there is no token on any of the output arcs the actor is enabled or fired. This means taking one token from each input arc, doing the specified operation and placing tokens labeled with the result value on the output arcs.

Two different approaches have been studied for the dataflow model: static dataflow and dynamic or tagged-token dataflow. The static dataflow provides a fixed amount of storage per arc. The dynamic dataflow provides dynamic allocation of token storage out of a common pool and requires that tokens carry tags to indicate their logical position in the arcs.

This system was not used in our implementation of the problem because of its lack of a dynamic switching network that needs to be updated with every new problem. The Dataflow Machine was not appropriate, to this problem, but the idea of token passing is very similar to the design of the PLF system.

\subsection{PLF Architecture}

PLF is the parallel architecture developed by the author for the solution of sparse nonlinear equations. PLF is a high level MIMD (Multiple Instruction Stream and Multiple Data Stream) system with distributed memory. It is an asynchronous multiprocessor system composed of $n$ nodes, each with its own memory, that communicate 
via a circuit switched communications network. It is divided into three parts (as seen in Figure 3). They are the Control Unit (CU), the Processing Units (PUs), and the Switching Network (SN). The Control Unit consist of a Sun Computer with a Transputer residing in it. The Control Unit performs the initialization of the Switching Network and the setting up of the switch. A Processing Unit consist of at most three Transputers that have up to eight connections to the Switching Network. This allows us to represent equations with eight unknowns or equations that have a connectivity of eight (i.e. eight receiving and eight sending messages). The Processing Unit does the processing for a small set of equations and connects to the Switching Network. The Switching Network handles communication with the other Processing Units. It is a circuit switching, multistage network.

The goal of this architecture is to model any set of sparse nonlinear equations with connectivity of eight or less. It provides a uniform parallel environment so that communication between any two processors is similar to that between any others. Therefore, the network topology is transparent to the application processes and can handle any set of such equations.

The communication links between any two processors will represent the connectivity of the equations in the system. This is accomplished by the Switching Network. This network will consist of thousands of switching units. It consists of a circuit switching network that, once it has been set up, is not changed for the duration of the calculations.

When in use, there is an initialization phase for the system, performed by the Control Unit, that consists of three steps. The first step is the configuration of the system or assigning to $\mathrm{PUj}$, a particular set of equations $s_{j}$. The second step is to load the code to the Processing Units for each equation set. Finally the last step is to establish connection through the switch to the other Processing Units.

The Processing Unit is responsible for solving the equations assigned to it, and 


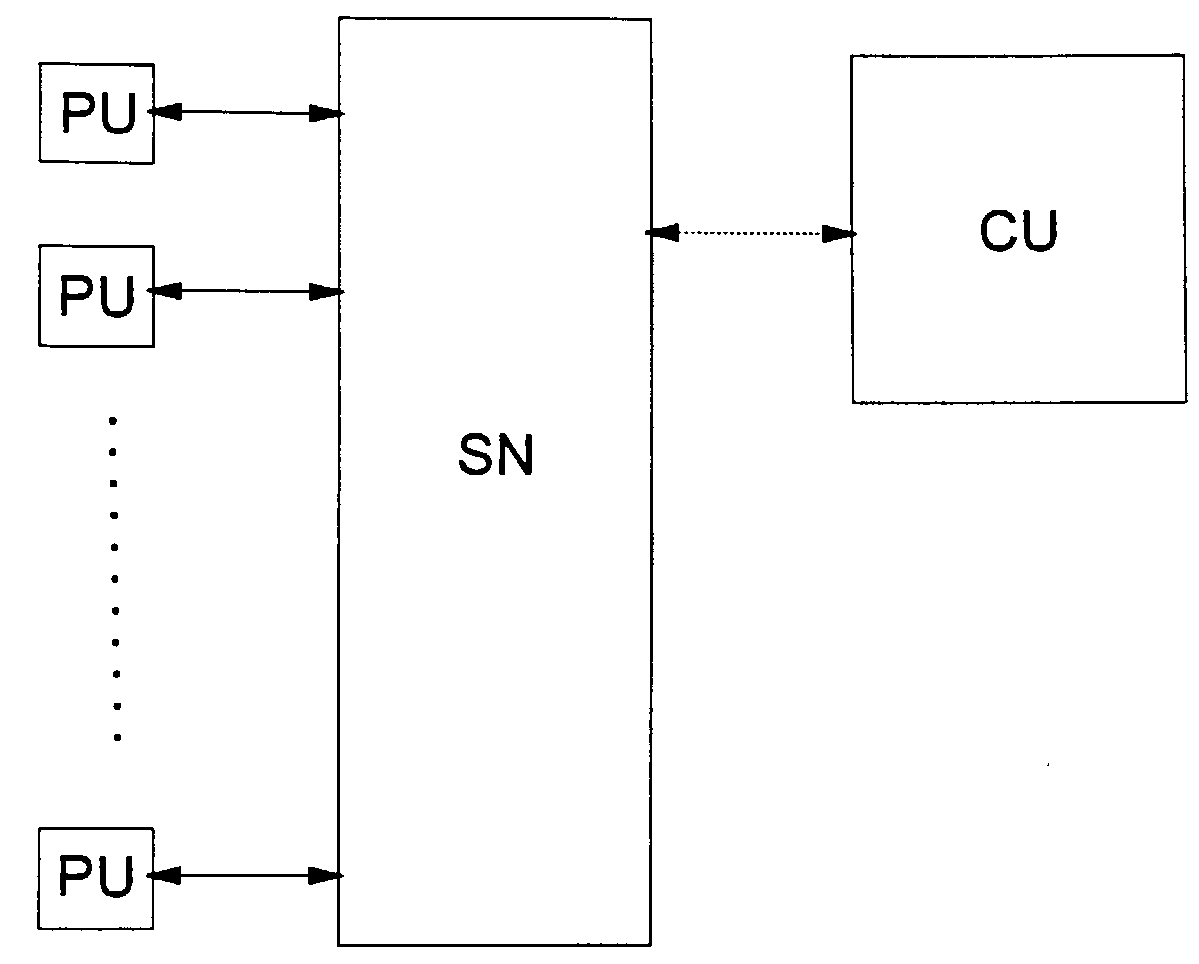

Figure 3: PLF System Overview

communicating its data and status via the Switching Network simultaneously with other Processing Units.

\subsubsection{Control Unit}

The Control Unit consist of an INMOS T800 Transputer connected to five INMOS C004s. The $\mathrm{C} 004$ is a programable crossbar switch with 32 inputs and 32 outputs. The Control Unit is shown in Figure 4. The Transputer resides inside a Sun Computer.

The Control Unit is responsible for the initialization of the Switching Network, that is, for setting up the circuits, and keeping track of the current status of the network. It sends the signals to set up the circuit. 


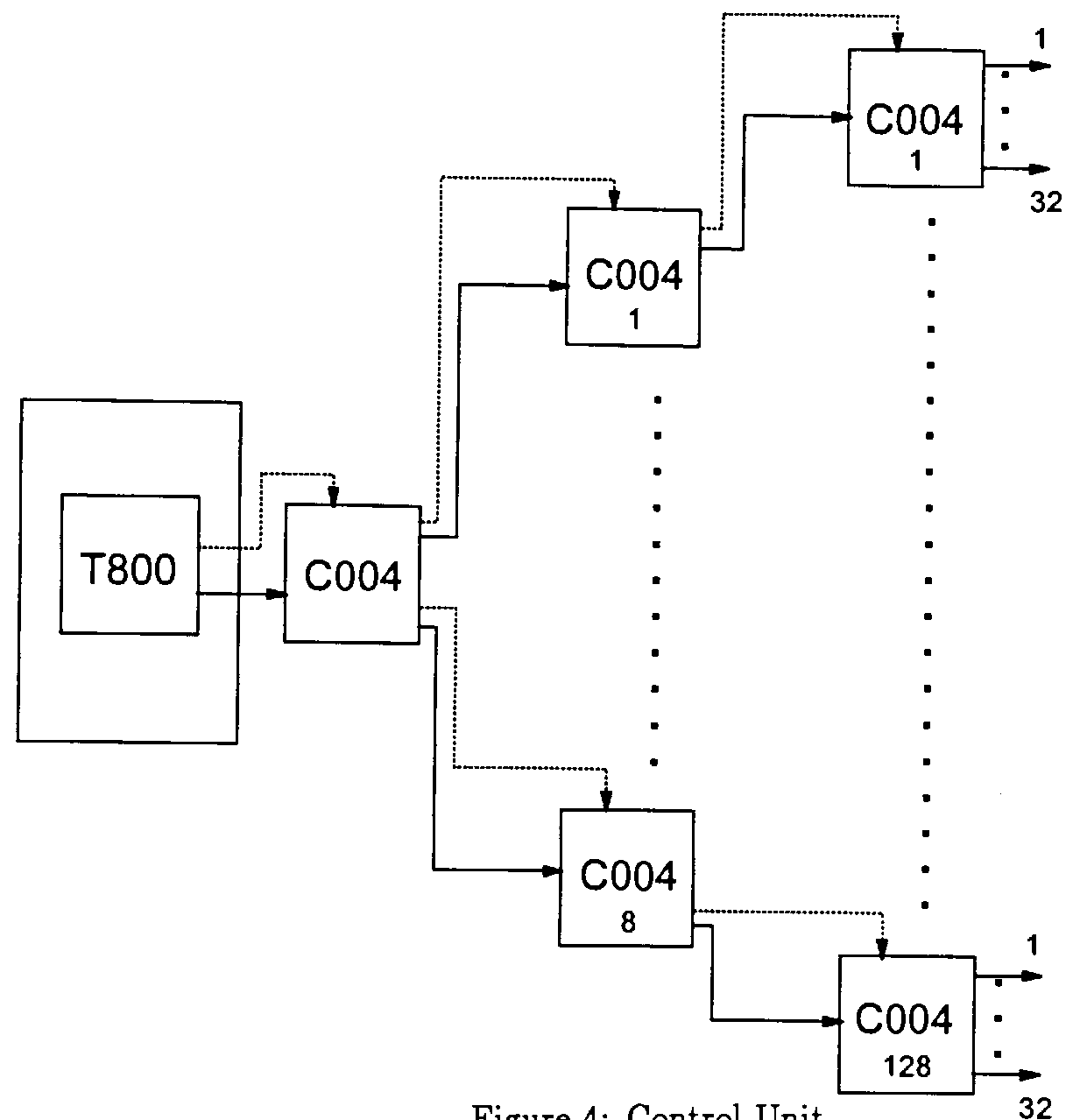

Figure 4: Control Unit

32

A table of the interconnections of the system being simulated is input to the Control Unit. This table has an entry for all connections of this system. An entry is an ordered pair $(\mathrm{i}, \mathrm{j})$ signifying there is to be a connection between PUi and PUj. Each Processing Unit has four input ports and four output ports.

An algorithm has been developed to set up these connections between the Processing Units. Each Processing Unit has four ports connected to the input side of the switch and four ports connected to the output side of the switch. As connections are made, there can occur conflicts where two Processing Units that are to communicate with each other but have only output ports available. The algorithm sets up the connections and resolves these conflicts. Conflicts are resolved by looking at the 
previously made connections until one is found that has available ports and then changing them.

The following algorithm sets up the connection between $P U_{i}$ and $P U_{j}$ that is given in a table as entry Connections $(i, j)$. This algorithm creates a table called tableofconnections for the system. The four input ports to the $P U_{i}$ is $i_{p o r t} t_{i, k}$ and the output ports are numbered oport $t_{i, k}$ for $k=1,2,3,4$. An algortihm for setting up the network is given in Appendix B.

The algortihm make - port - available $(X, T)$ is called when there is no available port to make a connection. $X$ is a Processing Unit and $T$ is input or output. This is called when a $X$ has no available $T$ ports, iport $t_{i, k}$ or oport $t_{j, k}$. If this is true, there have been four calls to $X$ on its $T$ ports. We have to make a call to $X$ from another Processing Unit and we can have at most eight calls to $X$, because of system design at most three of the opposite port (non $T$ ) from $X$ are in use. So upon entry to this algorithm there is an available non $T$ port on $X$.

A connection is defined as $T$ - stream in the direction $T$ port to non $T$ port . There are four $T$-stream connections from $X$ on entry. This algorithm constructs a set $S$ which is the set of processing Units reachable from $X$ by any finite sequence of $T$-stream connections from $X . S$ is not null since it will have at least five members, $X$ and its immediate friends. In constructing set $S$ loops such as $(A \rightarrow B \rightarrow C \rightarrow A)$ must be avoided. However the set of Processing Units is finite and so is the set of connections and a connection needs to be crossed only once.

Lemma 1: There are no $T$ - stream connections from the members of $S$ to Processing Units outside $S$.

Proof: (That is how $S$ is constructed).

Lemma 2: There is a member of $S($ called $Y$ ) with an available $T$ port. 
Proof: Suppose not- then all the $T$ ports on members

of $S$ are used. None are connected outside S.

(Lemma 1) - so all are connected inside $S$ - so all

non $-T$ ports on the members of $S$ are used since

there are four of each on every Processing Unit.

But $X$ is in $S$ and has an available non $-T$ port.

This contradiction proves Lemma 2.

Lemma 3: $X$ is not $Y$

Proof: $\quad$ All the $T$ ports on $X$ are used.

Lemma 4: There is at least one path along $T$-stream connection between $X$ and $Y$.

Proof: That is how $S$ was constructed and $X$ is not $Y$.

Choose one of the paths indicated in Lemma 4 and reverse it. This is possible using the available non $-T$ port on $X$ and the available $T$ port $Y$. All the Processing Units on the path have a $T$ and a non - $T$ port devoted to the path which can be used to reconstruct it in the opposite direction. Now $X$ has an available $T$ port, and this concludes make - port - available.

The Switch Setup algorithm is performed once at the initialization of the system. It will set the Switching Network and make it available for communication when the system starts solving the equations. It will need to be run again if there is a change in the communication links of the Processing Unit. 


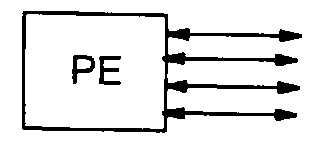

TO SN

Up to 4 connections

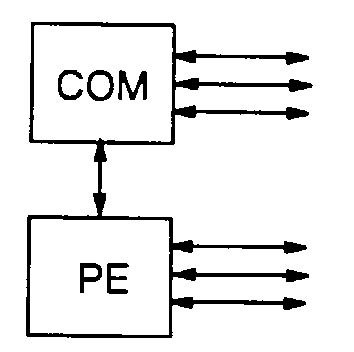

TO SN

5 and 6 connections

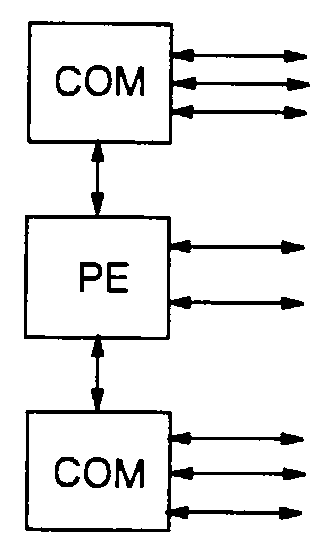

TO SN

7 and 8 connections

Figure 5: Processing Unit

\subsubsection{Processing Unit}

Several Processing Units are shown in Figure 5. The choice of unit is determined by the number of unknowns of the equation set $s_{j}$. This also determines the number of connections to the Switching Network.

These units are built from Transputers. Each Transputer has four bidirectional links. For a Processing Unit that has seven or eight connections three Transputers are required. Two are called Communicators(COM) and are used to shift data to the third which is the Processing Element(PE). This allows for each Processing Unit to have eight incoming or outgoing messages at the same time or to communicate simultaneously with eight other Processing Units. Each Processing Unit can receive 
and send data without having to wait for a channel to be available. This means that each equation set in the system can have eight unknowns.

For a Processing Unit that has five or six connections only two Transputers are needed one Communicator and Processing Element. Each Transputer has three links to the switch and one link to each other. For four or fewer connections on a Processing Unit only one Transputer is used, PE. Depending on the number of unknowns of an equation, the size of the Processing Unit will vary from worst case of three Transputers to best case of one Transputer.

Once each Processing Unit has its equations, it knows to which other Processing unit it needs to communicate. It was given data by the Control Unit which it stored in its local memory. As soon as the circuit switching network is set up, the Processing Unit can start calculations and transmissions of results. Each Processing Unit is responsible for the handling of the calculations for a certain equation set of the system. For each Processing Unit in the network, an iterative process is followed that, given a set of estimated values for the unknowns of the equations, calculates a new value for each unknown from the estimated values. A new set of values for the variables are then obtained for the Processing Unit, and used to calculate still another set of values. Each calculation of a new set of values is called an iteration. This process is repeated until the changes at the Processing Unit are less than a specified minimum value.

\subsubsection{Switching Network}

The Switching Network (SN) is a circuit-switching network. It is a three-stage Clos network $[45,46,9]$. The structure of the network is shown in the Figure 6. Each box in the figure is a $n \times m$ crossbar switching component. The switches have three columns: the input switches, the intermediary switches and the output switches. The links between the switches are bidirectional. The leftmost column will be the output 
side of the processors and the rightmost column is the input side of the processors. The links between the processors and the input switches are called the input c-links. The links between the processors and the output switches are called the output c-links. The links between the input switches and the intermediary switches are called the input s-links and the links between the intermediary switches and the ouput switches the output s-links.

The input switches are $n \times m$ switches, the output switches are $m \times n$ switches, and the intermediary switches are $r \times r$ switches. $N(m, n, r)$ is the notation used to denote such a network. Two important properties of a $N(m, n, r)$ network are proven in $[3,9]$.

1. If $m \geq n$, then for every partitioning of the set of all processors into pairs, there exist connection configurations where all the pairs talk simultaneously, i.e., there is no bottleneck in the switches.

2. When $m \geq 2 n-1$, the network is non-blocking, meaning that there is always a path available between any idle input c-link and any idle output c-link, independently of the connection sequence, i.e., there is no need to prearrange a special connection configuration to avoid bottleneck.

The connections between the processors and the input switches and the connections from the output switches to the processors are static. At the hardware level the switching components are normally passive, the connections have to be made by the Control Unit during the initialization phase. The circuits between the processors are bidirectional. Whenever a path from processor $i$ to $j$ is setup, a path from $j$ to $i$ is set up also.

In our system for a 2048 by 2048 equation set with connectivity of eight and one equation per Processing Unit, the Switching Network is represented in Figure 7. The input switches are $16 \times 32$. The output switches are $32 \times 16$. There are 512 input 


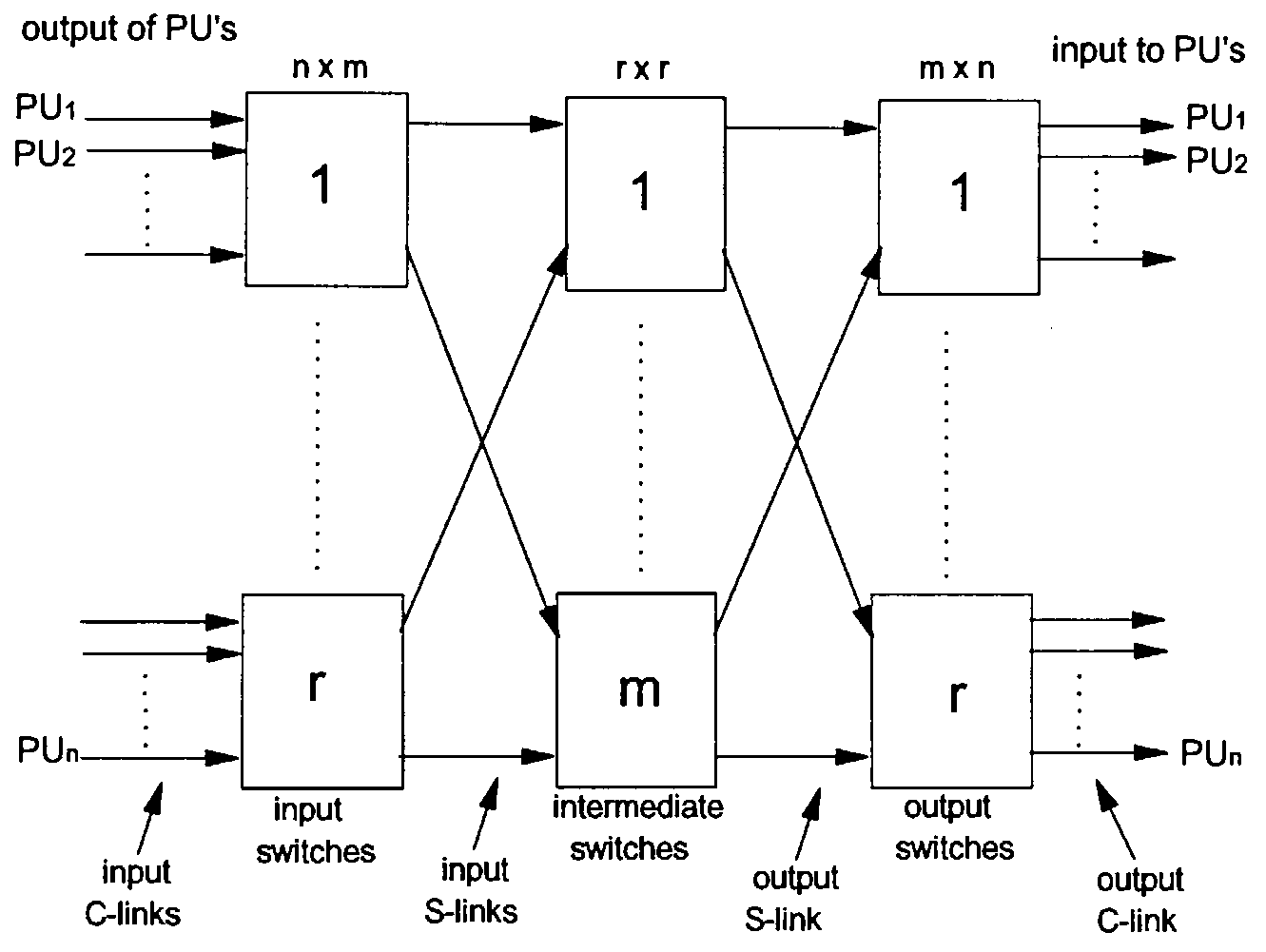

Figure 6: Three Stage Clos Network 


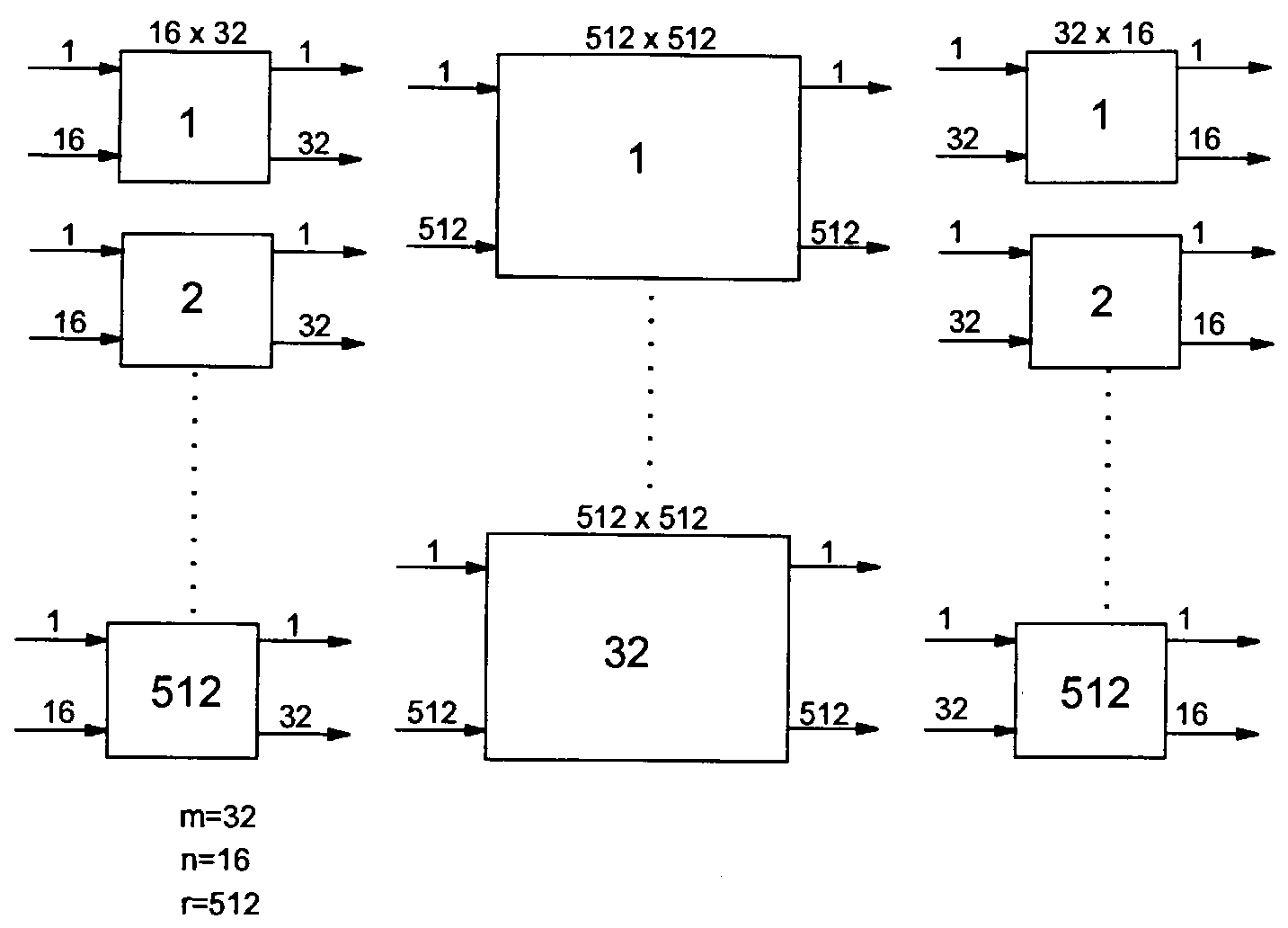

Figure 7: Switching Network

switches and the same number of output switches. The middle stage is $512 \times 512$ switches. There are 32 of these switches.

The switches are made using C004s. A C004 is a crossbar switch consisting of 32 inputs and 32 outputs. Therefore each input and output switch is made from one C004. The middle stage is made from a non-blocking network of C004s, as shown in Figure 8. Each input of this switch is made from a $16 \times 32$ switch. The output is a 32 x 16 switch. There are 32 input switches and 32 output switches. The intermediary switches are $32 \times 32$ switches and there are 32 of these.

In essence, the $\mathrm{SN}$ is a three stage nonblocking Clos network, where the middle stage is made up of another three stage nonblocking network. Our particular network 

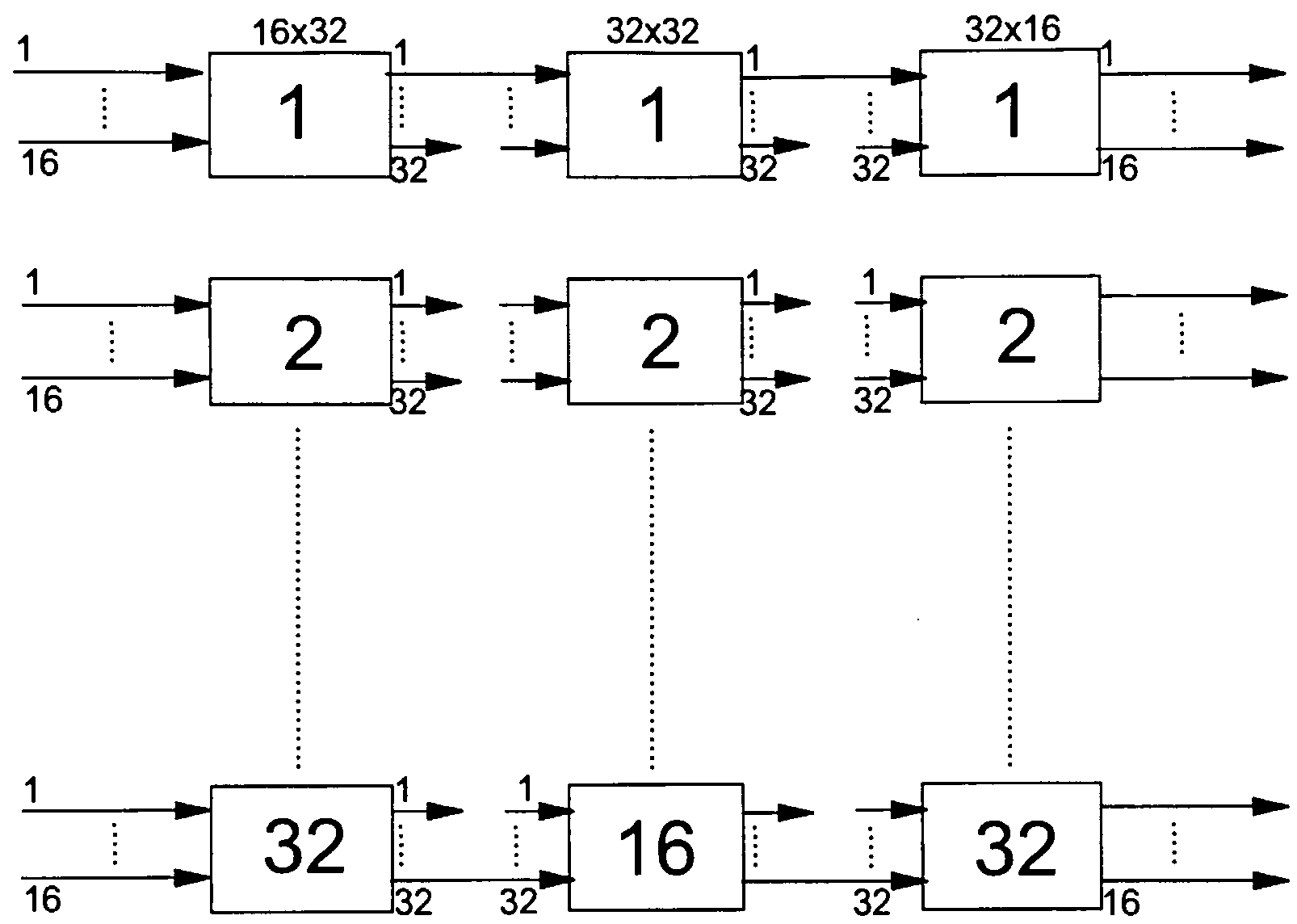

$$
\begin{aligned}
& m=32 \\
& n=16 \\
& r=16
\end{aligned}
$$

Figure 8: Middle Stage 512x512 
is implemented using 4608 C004s. By using Algorithm 1 to set up the switch, any Processing Unit can communicate with eight others. $32 \geq 2 * 16-1$ so the network is non-blocking. For the middle network, again $m=32$ and $n=16$ and therefore it is non-blocking. There will be no bottleneck in the system. 


\section{PERFORMANCE EVALUATION}

The operation of the PLF algorithm in parallel was simulated using the Transputer hardware currently available at the University. The purpose was to verify that interprocess communication time does not overwhelm the computation time and to demonstrate that the algorithm will perform at the speed of its slowest member and that the speed is independent of the number of nodes.

\subsection{Design Configuration}

The PLF system is designed to solve sparse non-linear sets of equations,

$$
x_{i}=g_{i}\left(x_{1}, x_{2}, \ldots, x_{n}\right) \text { for } i=1, \ldots, 2048
$$

The maximum number of equations was chosen as 2048 , because that is enough equations to model the Florida power network. The maximum number of unknowns per equation was chosen as eight, because this system can be modeled with eight connections per node. For each equation to have eight unknowns the PLF system needs three Transputers to model them. The network has a possible of 2048 equations, so the PLF system consists of 6152 INMOS Transputers [35].

A 3-stage Clos circuit switching network $\mathrm{N}(16,512,16)$, with the middle stage being another 3-stage Clos circuit switching network $\mathrm{N}(16,32,16)$ is used to interconnect the Processing Units. This network consist of 4608 INMOS COO4 dynamic reconfigureable switches $(32 \times 32)$ [38]. This network is non-blocking, so there are connections available for all of the eight lines. All communication links are serial links and have a speed of 20 Megabits/second. The links in the circuit switching network are also serial links with the same speed. Experiments have shown that the effective one-directional transfer rate is $8 \mathrm{Mega}$ bits/second. The circuit switching network can provide a link between any Processing Unit and eight other Processing Units simultaneously. It also allows for eight processors to receive data at the same time. 


\subsection{Analysis and Experiments}

Many experiments have been done to simulate the PLF system. These were programmed using Occam. Figure 9 shows the connections of the Transputer system that was constructed. There are 24 Transputers used in the simulation. Eight of them are T800 and 16 are T414. The connections between link 0 and link 3 of each machine go through a C004. Connections 1 and 2 form a hardwire chain. Two of the T800 reside in an INMOS B014 inside a SUN computer system.

\subsubsection{Simulations}

The Transputer network was connected so as to simulate different system scenarios. The three types of Processing Units were built. The first Processing Unit type is the one with one Processing Element and four input/output lines, that represents equations with up to four unknowns. The second type is one Processing Element with three input/output lines connected to a Communicator with three input/output lines. This represents equations with five or six unknowns. The third type is two Communicators connected to a Processing Element, each with three input/output lines and two input/output lines from the bus. Equations with seven or eight unknown can be represented by this configuration. One Transputer is used to simulate each Processing Element, and one to simulate the Communicator. Computational intensive arithmetic (i.e. sine, cosine,..) was performed on each Transputer to simulate computations at a node.

These test are summarized in Table 1. The amount of data transfered is two floating point numbers -64 bits output and 64 input after each calculation. There are 32 operations performed at each Processing Element. The calculations involved adding and multiplying sines and cosines. 


\section{Sun}

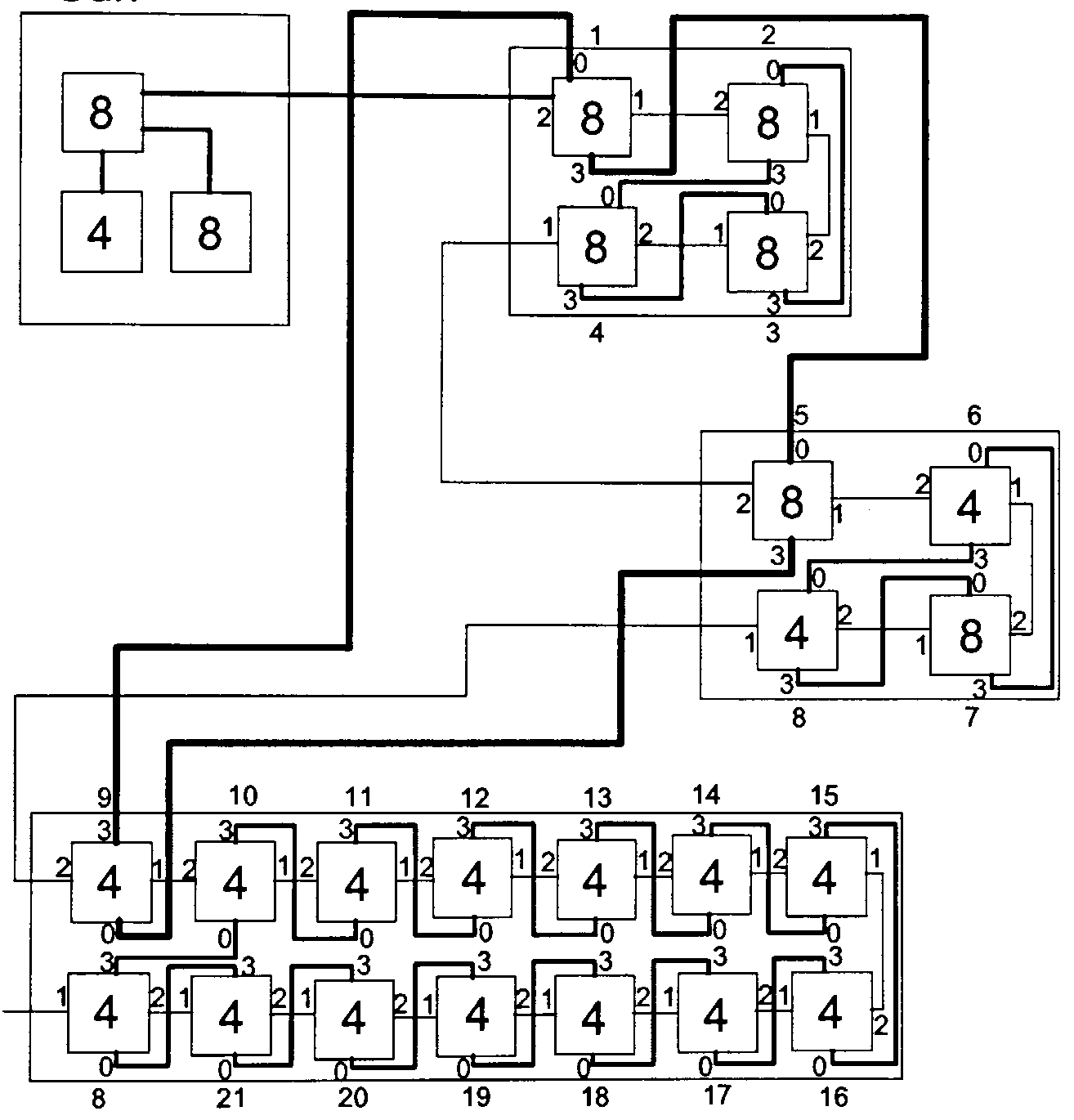

$\begin{array}{ll}\text { Hardwired } & \text { 8-T800 Transputer } \\ \text { Software } & \text { 4-T414 Transputer } \\ \text { Pipe 2-1 } & \text { C004-link 0 - 3 }\end{array}$

Figure 9: Connections of Transputers 


\begin{tabular}{||r|r|r||}
\hline buses & communicators & tics \\
\hline 12 & 10 & 67131 \\
\hline 18 & 4 & 67131 \\
\hline 21 & 1 & 64614 \\
\hline 22 & 0 & 61171 \\
\hline \multicolumn{2}{|r|}{ tic $=63 \times 10^{-6}$ secs } \\
\hline
\end{tabular}

Time for 10000 iterations

Table 1: Number of Buses

Table 1 shows that the number of Processing Units does not change the time of execution of the program. The program will run at approximate 67,000 tics for 10,000 iterations. A tic is $63 \times 10^{-6}$ secs. The amount of arithmetic done was kept the same for each test. A Communicator is a Transputer that is used to pass information forward but does no calculations on the data. This allowed for the simulation of a bus with six connections and eight connections. The data has to travel through two Transputers before going out to the switch when a Communicator is used. This explains the slow down in time.

Table 2 shows what happens when the calculation becomes more complex and when the amount of data transfered increases. These calculations were performed on a system with 22 PEs and no Communicators. It was done with 10,000 iterations. An observation made was that the time for computation was governed by the slowest Transputer. The Transputer with the most calculations set the time for computations.

\subsubsection{Transfer Rate}

Other tests done were to determine the rate of transfer of data from one Transputer to the next and the reduction of this rate when data went thru a C004. One circuit 


\begin{tabular}{||r|r|r|r|r||}
\hline transfers & calculations & transfer time & computation time & total time \\
\hline 2 & 2 & 13625 & 10235 & 23860 \\
\hline 2 & 16 & 31097 & 79829 & 110926 \\
\hline 2 & 8 & 21112 & 40059 & 61171 \\
\hline 4 & 8 & 27693 & 40059 & 67752 \\
\hline 16 & 8 & 67219 & 40059 & 107278 \\
\hline
\end{tabular}

Table 2: Computation and Transfer Rates

was built to find the time for two Transputers to communicate. This was found to be .25937 tics to transfer 128 bits (two floating point numbers) both ways. Another circuit was built to find the time for data to pass from one Transputer to the next through a C004. This time was .27656 tics to transfer 128 bits. These two circuits are shown in Figure 10. There is a slow down of $7 \%$ for data transfer thru a C004. This gives a transfer rate of 8 mega bits per second from one Transputer to the next.

The interprocess communication time is less by a factor of two than the computation time. The input/output at the Transputer uses significant amounts of CPU time when small amounts of data are transmitted, so the 20 megabits/ sec bandwidth is not achieved.

\subsection{Results}

\subsubsection{Circuit Setup Time}

The circuit setup for a set of nonlinear equations is done once for a problem. The time for circuit setup is dependent on the number of equations in the system. It does not effect the performance of the system during its normal operation. Given that the number of equations for a particular problem is $n$, then each node is numbered in sequence by the Control Unit from $P U_{1}$ to $P U_{n}$. The Control Unit is given the network setup. It then does the setting up of the Switching Network. 


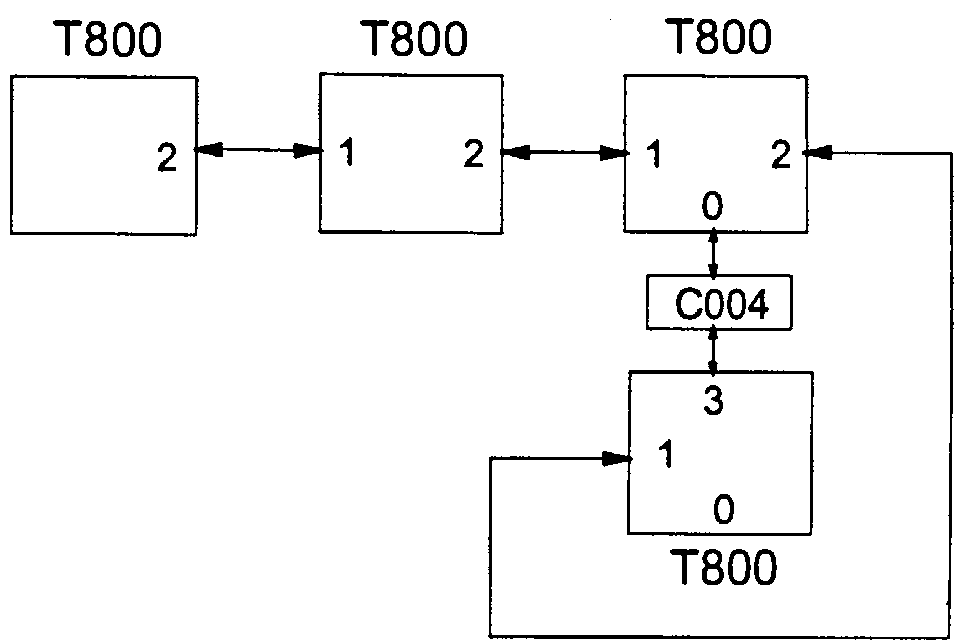

7800 Transfer - .25937 tics

C004 Transfer - .27656 tics

Figure 10: Transfer Time 


\subsubsection{Communication Time}

After the links are set up, the calculation begins. The Processing Units simultaneously run the PLF algorithm to solve for each pair of equations. When a particular Processing Unit has finished calculating its equation, it must send the newly calculated data to the nodes it has set up on its sending lines. This data must first travel through one or two levels of Transputers, then the Switching network and finally back up to a Communicator Transputer to the Processing Transputer.

In the worst case, the Processing Unit consist of two layers of Transputers. Let $\tau_{P U}$ be the transfer time through the Processing Unit. It was measured to be $16.34 \mathrm{x}$ $10^{-6}$ secs. Let $\tau_{S N}$ be the transfer time of the data through the Switching Network. The circuit switching Clos network or the Switching Network consist of five layers of $\mathrm{COO} 4 \mathrm{~s}$ so the transfer time of the data through this circuit is $5 \times 1.08 \times 10^{-6}$ secs. The data transfer time from sending to receiving is $2\left(\tau_{P U}\right)+\tau_{S N}=38.08 \times 10^{-6}$ secs.

After sending the data a Processing Unit can read its incoming lines to see if there is any new data. When data has arrived it starts calculating again. 


\section{POWER FLOW}

The electric utilities are responsible for systemwide coordination of the electrical power generation, transmission, distribution and interconnections. This is done at a central location, Energy Control Center (ECC). Power plants are responsible for generating the large amounts of electric power. They exchange data and control signals with the ECC, which is then responsible for the coordination of the overall interconnection levels. Interconnections consist of the transmission lines and transformers that transfer the electric power from power plants to distribution centers which provide it to the customers. Through continuous exchange of information, decision, and control signals, the entire control process is finely tuned.

A typical modern control center is computerized. Large digital computers in the ECC make it possible to track the changing conditions in the power system by representing the system by a mathematical model in the computer. This real time model attempts to portray the current system as accurately as possible. It is used to assess the security of the system by simulating outages or contingencies and to observe the consequences of possible correction measures. The contingencies are generator outages, transmission line outages, and transformer outages. The system model must be calibrated using real-time measurements before any contingencies can be analyzed.

As utilities expand, and the requirement for better performance increases, these programs take longer times to run, and thus do not represent the up to the minute state of the power system. Steady-state analysis for a large number of contingencies needs to be done quickly enough to provide timely alert messages. It becomes necessary to improve on the current technologies and algorithms. The development of computer systems made of many processing elements can make it feasible to design faster algorithms to use in the control sequence.

A parallel load flow algorithm has been developed using the PLF software de- 
scribed previously, to run on the PLF parallel computer architecture. The network will be partitioned into segments that will run in parallel, and take the fullest advantage of the parallelism of the hardware. The object of this dissertation is to reduce the processing time required to solve the power system load flow problem, and have it represent the real time power system accurately.

\subsection{Examination of the Problem}

The power system of an electrical utility company consists of generators, transmission lines, transformers, shunt capacitors, shunt admitances, and loads. Generators are located at power stations, where electricity is generated in bulk quantities. The substations are distribution centers where the electric power is distributed to customers or users. Shunt capacitors and shunt inductors are used to support the voltages in the system to maintain them at acceptable levels. Transformers are used to step up the voltages before sending power through the transmission lines, and to step down the voltages at the distribution centers. The transmission lines transfer the power from the power stations to the distribution centers, forming an electric power network. Nodes or buses are where these elements are modeled and connected.

For each bus, certain values are telemetered and sent to a central location, the ECC (Energy Control Center). At the ECC, a collection of computer aided analysis procedures determine the model of the power system network. These programs are supported by a database that contains the description of the network in terms of its parameters. The building of this model is done in three steps. The first step is the determination of the network topology and it is done by combining the database with the telemetered data. The next step in the process is the solution of the complex bus voltages and states of the network. This solution is done by a state estimator. Finally the load flow analysis then determines the actual power flow patterns in the

given power system. This is done by writting power flow equations for each bus in 
the system and solving the unknowns (i.e. voltages) in the power system.

Power companies use very elaborate computers and programs for making load flow studies. A typical system is capable of handling more than 2000 buses, 3000 lines, and 500 transformers. Programs can be expanded for larger systems provided the available computer facilities are adequate.

The model of the power system can be used to test the security of the system or its ability to withstand contingencies. Once the model of the system is built, contingency analysis can be performed. The core of this analysis is the load flow algorithm.

In most applications, the solution of the load flow problem is desired in as fast a time as possible. This means the solution of the power flow problem before the conditions of the analysis have changed. Digital computer methods have the disadvantage of lengthy solution time that increases very rapidly with system size. In recent years, there has been growing interest in using computer systems comprised of several processors for the solution of power system problems. Because of developments in computer technology, parallel processing can be used to aid in the solution of the load flow problem.

The application of parallel processing to power system analysis is motivated solely by the need for faster computation. There is no inherent parallelism in the mathematical structure of the power system problems. Therefore a parallel or near parallel formulation has to be found that is amenable to a particular algorithm. A parallel machine has to be used that is computationally efficient and suitable to the parallel algorithm.

Three areas have been analyzed in order to develop a parallel technique for solving the load flow for the power system. The first is how to partition the power network, the second is which algorithm has the most inherent parallelism, and the third is what type of computer to use. 


\subsubsection{Partition of the Power Network}

There are several possibilities to divide the power system network. One such way is for the network to be studied as one system and modeled mathematically as one entity. This is the way it is currently done with the algorithms used solving the network as one big unit sequentially.

Another possibility is to partition the network into several sections. Each network section can be modeled separately. The following questions would need to be answered if this approach were to be used.

- How many sections should the network be divided into?

- Where should the network be divided?

- What size should each network section be?

- How will the coordinating processor handle the tie line information?

Finally, the power network can be partitioned by its buses. Each bus can be modeled by its set of equations. Each bus knows the information of its neighbors. This is the way this research has handled the partioning of the network.

\subsubsection{Find the Parallelism in the Algorithms}

The next problem that has to be considered is what numerical procedure to use to solve the load flow analysis. The currently used algorithms are the Newton-Raphson, Gauss-Seidel, and Fast Decoupled. As described in Chapter 2 this present work uses the Jacobi type algorithm to solve the numerical equations.

\subsubsection{Possible Architectures}

Finally, the computer architecture that will be used has to be decided. Previously, different architectures have been examined to solve the load flow problem. These will be referred to in the next Section 5.3. 


\subsection{Mathematical Formulation of Load Flow}

Load Flow Analysis consists of calculating the unknown bus voltages, the unknown power generations and the power flows of a given power system for given bus voltages, generations and loads. The objectives are to determine the real and reactive power flows in the transmission lines of a system, based on certain assumptions regarding loads and generations, and to compute the voltages at all system buses. Thus the problem can be stated : given load demands at various load buses and assumed generation levels at specified supply voltage, find the overall voltage profile of the power network, the real and reactive flows on transmission lines and transformers, bus voltage magnitudes and phase angles, line currents, line losses and other related steady state variables.

The load flow problem is at the core of power system analysis $[17,26,52]$. It is used as an integral part of other activities such as:

- Keeping voltages and power flows within tolerances specified for the system. Check that no transmission lines are overloaded and there are no voltage problems.

- Testing for inter-utility power transfer limits. Planning to meet contracts for supplying power to neighboring power systems.

- Planning studies for additions or expansions. Check effect of new generation sites, projected load growth and new transmission line locations.

- Determining the specific load flow pattern that results in optimum dispatch. Selection of the most economic operation of the generators in the system.

- Contingency analysis of the system. Test for the effect of line and generator outages. Rerouting of power in case of emergencies. Analyze the effectiveness of corrective measures to alleviate emergencies. 
The power system cannot be modeled by an electrical network, because loads are complex powers not impedances, generators behave like power sources and not as voltage supplies and Kirshoff's Laws cannot be used. Therefore, the problem becomes a mathematical problem of solving a large number of nonlinear equations.

\subsubsection{Formulation of the Problem}

The components of the power system which are of interest are: power generators, transformers, transmission lines, shunt capacitors and inductors, and loads. Singleline diagrams are used to show the electrical interconnections and locations for generators, transformers, transmission lines, loads, circuit breakers, reactors, and some switches. Power system information is recorded on these diagrams. A bus in a singleline diagram is like a node in a circuit diagram. Points on a bus are separated by negligible impedance and therefore have the same voltage. Figure 11 is an example of a one line diagram showing buses, generators, loads, transmission lines and shunt capacitors [21]. Two equations can be written for every bus of the system. The system variables used in these equations are of the following type:

- Demand variables. Given values of real and reactive load demand.

- Control or Input variables. These are voltage magnitudes at generation buses, real power generation at all but one generation bus (the slack bus), switch status of capacitors and inductor banks, and tap settings of regulating transformers.

- State variables. Those whose knowledge will enable the computation of all other relevant quantities. These are the complex bus voltages.

- Output variables. Real and reactive line power flows, reactive generation, slack bus real generation, injected bus complex currents, and line current magnitudes. 


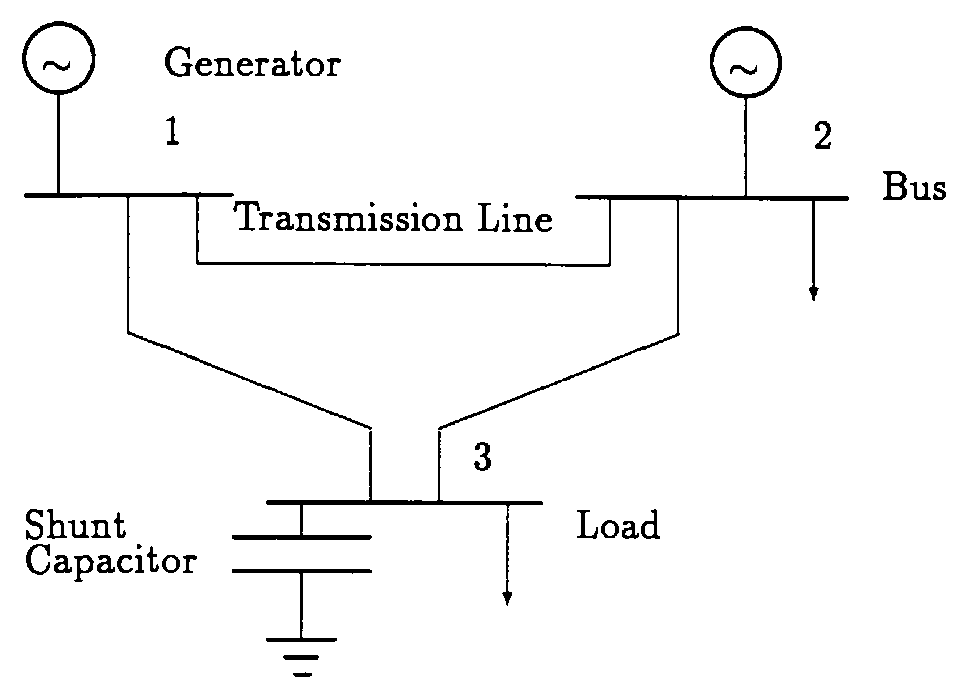

Figure 11: One-line diagram

Complex variables are used, and the engineering convention that $j$, rather than $i$, represents $\sqrt{-1}$. The following standard notation is used:

$$
\begin{aligned}
z & =x+j y \\
|z| & =\sqrt{x^{2}+y^{2}}
\end{aligned}
$$

The angular orientation or phase $\angle z$ of $z$ in reference to the real axis is

$$
\angle z=\arctan \frac{y}{x} .
$$

Let $\mathrm{V}$ (voltage) and $\mathrm{I}$ (current) correspond to the complex numbers $V e^{j \alpha}$ and $I e^{j \beta}$ and $*$ is the complex conjugate. The complex power flowing into the network is defined as

$$
S=\mathbf{V I}^{*}
$$

Therefore the following can be written:

$$
S=\left(V e^{j \alpha}\right)\left(I e^{j \beta}\right)^{*}
$$




$$
S=V I e^{j(\boldsymbol{\alpha}-\beta)}
$$

The complex power $S$ can be written as $S=P+j Q$, were $P$ is called the active power or real power and $Q$ is called the reactive power. The units of $S$ are Voltamperes written as VA, the units of $P$ are Watts written as W, and the units of $Q$ are Voltamperes-reactive written as Var.

In power system analysis, it is common to normalize or scale dimensioned quantities [30]. This is referred to as the per unit system. The per unit scaling equation is

$$
\text { Per unit value }=\frac{\text { Actual value }}{\text { Base value }}
$$

The base value and the actual value have the same units so the per unit value is dimensionless. The base value is real and the actual value is complex, so the phase of the per unit value is always the same as that of the actual value. The "pu" subscript is used to imply per unit. Two values are chosen for any of the following Vbase, Ibase, Zbase, or Sbase. Then the others are calculated using the following formulae

$$
\begin{array}{r}
\text { VbaseIbase }=\text { Sbase } \\
\text { Zbase }=\frac{\text { Vbase }}{\text { Ibase }}
\end{array}
$$

A transmission line is represented by its $\Pi$ equivalent model. Figure 12 is a representation of the network elements of a transmission line represented by its $\Pi$ equivalent. The following notations and definitions correspond to the above variables that make up the equations:

$$
\begin{aligned}
P_{i} & =\text { real power at bus } \mathrm{i} \\
& =\text { real power generated minus real power load at bus } \mathrm{i} \\
Q_{i} & =\text { reactive power at bus } \mathrm{i} \\
& =\text { reactive power generated minus reactive power load at bus } \mathrm{i}
\end{aligned}
$$




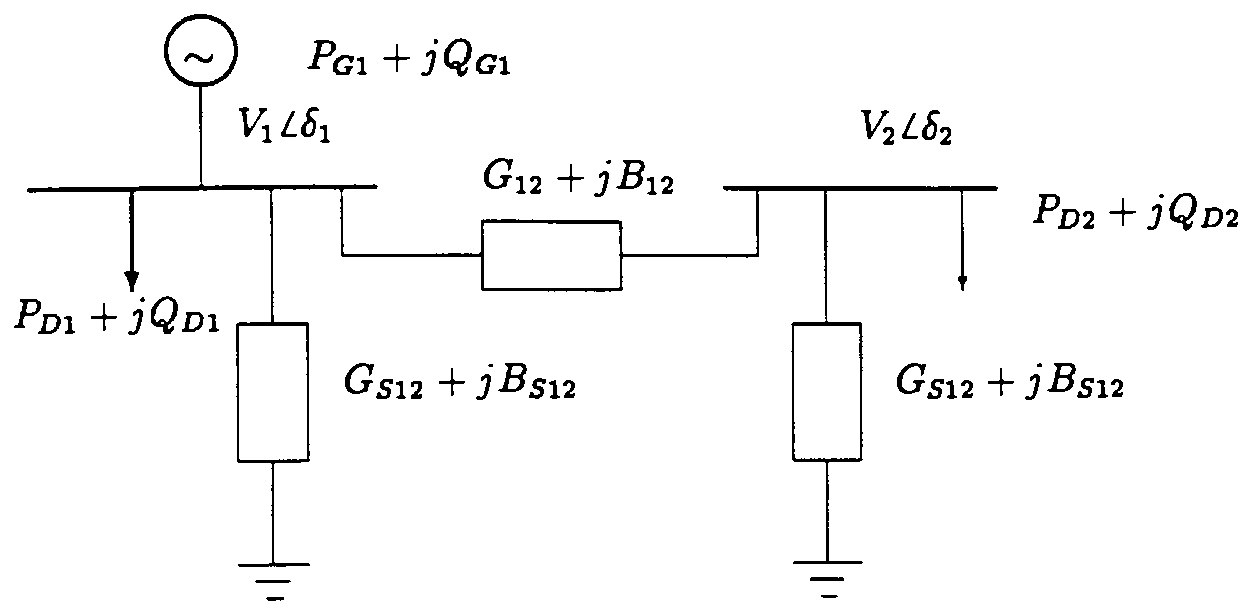

Figure 12: Representation of network elements

$$
\begin{aligned}
S_{i} & =P_{i}+j Q_{i} \\
& =\text { Complex power at bus } \mathrm{i} \\
V_{i} & =\text { voltage magnitude at bus } \mathrm{i} \\
\delta_{i} & =\text { voltage phase or angle at bus } \mathrm{i} \\
k_{i} & =\text { set of buses connected to bus } \mathrm{i} \\
R_{i j} & =\text { transmission line series resistance } \\
X_{i j} & =\text { transmission line series reactance } \\
G_{i j} & =\frac{R_{i j}}{R_{i j}^{2}+X_{i j}^{2}} \\
G_{i i} & =\sum_{j \in k(i)}\left(G_{S i j}+G_{i j}\right) \\
G_{S i j} & =\text { shunt capacitance } \\
B_{i j} & =\frac{-X_{i j}}{R_{i j}^{2}+X_{i j}^{2}} \\
B_{i i} & =B_{S i}+\sum_{j \in k(i)}\left(B_{S i j}+B_{i j}\right) \\
B_{S i} & =\text { net device shunt susceptance at bus } \mathrm{i} \\
Y_{i j} & =G_{i j}+j B_{i j}
\end{aligned}
$$




$$
\begin{aligned}
& =\text { inverse of line series impedance } \\
Y_{S i j} & =G_{S i j}+j B_{S i j} \\
& =\text { shunt capacitance in parallel with shunt conductance } \\
t & =\text { transformer tap ratio } \\
Y_{e i j} & =\text { Branch admitance for transformer branch } \\
Y_{T i j} & =t Y_{e i j} \\
Y_{T S i j} & =\left(t^{2}-t\right) Y_{e i j} \text { for } i<j \\
& =Y_{S i j} \text { for transformer branch } \\
Y_{T S j i} & =(1-t) Y_{e i j} \text { for } i<j \\
& =Y_{S j i} \text { for transformer branch }
\end{aligned}
$$

Given these definitions, the complex power at a bus can be written in terms of complex voltages[17] :

$$
\begin{aligned}
S_{i}= & V_{i} I_{i}^{*} \\
I_{i}= & V_{i} Y_{i i}-\sum_{j \in k(i)} V_{j} Y_{i j} \\
S_{i}= & V_{i}^{2} G_{i i}-V_{i} \sum_{j \in k(i)} V_{j}\left[G_{i j} \cos \left(\delta_{i}-\delta_{j}\right)+B_{i j} \sin \left(\delta_{i}-\delta_{j}\right)\right] \\
& j\left(-V_{i}^{2} B_{i i}-V_{i} \sum_{j \in k(i)} V_{j}\left[G_{i j} \sin \left(\delta_{i}-\delta_{j}\right)-B_{i j} \cos \left(\delta_{i}-\delta_{j}\right)\right]\right)
\end{aligned}
$$

This can be separated into real and imaginary components:

$$
\begin{aligned}
& P_{i}=V_{i}^{2} G_{i i}-V_{i} \sum_{j \in k(i)} V_{j}\left[G_{i j} \cos \left(\delta_{i}-\delta_{j}\right)+B_{i j} \sin \left(\delta_{i}-\delta_{j}\right)\right] \\
& Q_{i}=-V_{i}^{2} B_{i i}-V_{i} \sum_{j \in k(i)} V_{j}\left[G_{i j} \sin \left(\delta_{i}-\delta_{j}\right)-B_{i j} \cos \left(\delta_{i}-\delta_{j}\right)\right]
\end{aligned}
$$

The power flow equations are written in terms of phase differences between connecting buses. One of the buses, the slack bus, is normalized with phase zero and is numbered as bus number one. This bus is a generator bus and is the last quantity of 
power calculated since it depends on and must take up the yet unknown line losses. This is because the amount of generation is dependent on system losses which are functions of the problem solution.

The three types of buses in the system are as follows:

1. Slack bus. The one bus of the system on which the real and reactive power are allowed to swing and for which the voltage magnitude and angle are specified. This bus is numbered as one. For this bus the following can be written:

$$
\begin{aligned}
& \delta_{1}=0 \\
& V_{1}=1
\end{aligned}
$$

2. Load bus. Any bus where the injected real and reactive power are specified. $P_{i}$ is the negative of the real load at the bus and $Q_{i}$ is the negative of the reactive load at the bus. Most buses in a typical power flow program are of this type. The two equations are:

$$
\begin{aligned}
P_{i} & =V_{i}^{2} G_{i i}-V_{i} \sum_{j \in k(i)} V_{j}\left[G_{i j} \cos \left(\delta_{i}-\delta_{j}\right)+B_{i j} \sin \left(\delta_{i}-\delta_{j}\right)\right] \\
Q_{i} & =-V_{i}^{2} B_{i i}-V_{i} \sum_{j \in k(i)} V_{j}\left[G_{i j} \sin \left(\delta_{i}-\delta_{j}\right)-B_{i j} \cos \left(\delta_{i}-\delta_{j}\right)\right]
\end{aligned}
$$

3. Voltage controlled bus. Any bus where the voltage magnitude and the injected real power are specified. $V_{i}$ is the specified voltage value and $P_{i}$ is the real power generated at bus $i$ minus the real power load at that bus. It is the nonslack generation buses. Buses to which generators switched shunt capacitors are connected. It can also be a bus which has a tap changing transformer connected to it.

$$
P_{i}=V_{i}^{2} G_{i i}-V_{i} \sum_{j \in k(i)} V_{j}\left[G_{i j} \cos \left(\delta_{i}-\delta_{j}\right)+B_{i j} \sin \left(\delta_{i}-\delta_{j}\right)\right]
$$

Table 1 summarizes the bus types for a real power system such as FPL 


\begin{tabular}{|l|c|l|l|l|}
\hline \hline & bus type & knowns & unknowns & approximate number \\
\hline 1 & slack & $V_{i}=1.0$ & $P_{i}$ & 1 \\
& & $\delta_{i}=0$ & $Q_{i}$ & \\
\hline 2 & load & $P_{i}$ & $V_{i}$ & $85 \%$ \\
& & $Q_{i}$ & $\delta_{i}$ & \\
\hline 3 & voltage controlled-generator & $P_{i}$ & $\delta_{i}$ & $10 \%$ \\
& & $V_{i}$ & $Q_{i}$ & \\
\hline 3 & voltage controlled-transformer & $P_{i}=0$ & $\delta_{i}$ & $5 \%$ \\
& & $Q_{i}=0$ & $t$ & \\
& & $V_{i}$ & & \\
\hline
\end{tabular}

Table 3: Summary of bus types

\subsubsection{Three Bus Example}

For the three bus system described in Figure 13, the load flow equations will be written and examined in detail.

Bus 1 equations (slack bus)-

$$
\begin{gathered}
\delta_{1}=0 \\
V_{1}=1
\end{gathered}
$$

Bus 2 equations (generation bus)-

$$
\begin{aligned}
& V_{2}=.95 \\
& P_{2}=2.0 \\
& P_{2}=2(.95)^{2}-.95\left(\cos \left(\delta_{2}\right)-10 \sin \left(\delta_{2}\right)\right)-.95 V_{3}\left(\cos \left(\delta_{2}-\delta_{3}\right)-9 \sin \left(\delta_{2}-\delta_{3}\right)\right)
\end{aligned}
$$

Bus 3 equations (load bus)-

$$
\begin{aligned}
& P_{3}=-4.0 \\
& P_{3}=1.5 V_{3}^{2}-V_{3}\left(0.5 \cos \left(\delta_{3}\right)-7 \sin \left(\delta_{3}\right)\right)-.95 V_{3}\left(\cos \left(\delta_{3}-\delta_{2}\right)-9 \sin \left(\delta_{3}-\delta_{2}\right)\right)
\end{aligned}
$$




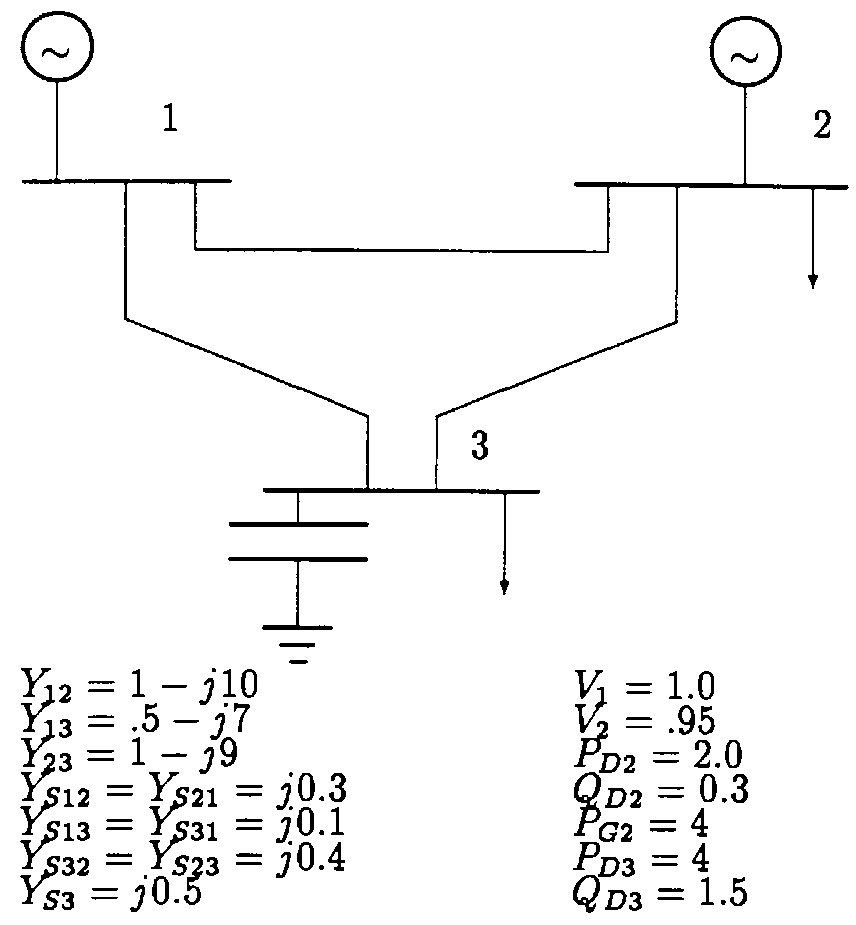

Figure 13: Three bus system

$$
\begin{aligned}
& Q_{3}=-1.5 \\
& Q_{3}=15 V_{3}^{2}-V_{3}\left(0.5 \sin \left(\delta_{3}\right)+7 \cos \left(\delta_{3}\right)\right)-.95 V_{3}\left(\sin \left(\delta_{3}-\delta_{2}\right)+9 \cos \left(\delta_{3}-\delta_{2}\right)\right)
\end{aligned}
$$

The procedure for solving the load flow problem is as follows. First, a set of $2 n+m-1$ equations that correspond to all voltage magnitudes and phases at all the $n$ load buses and $m$ voltage controlled buses of the system are written. Next, solve for the voltage magnitudes and phases of the state variables. Finally, solve for the output variables. The first step is to write two equations per load bus and one equation per voltage controlled bus. This was shown in the above formulation and example.

\subsubsection{Jacobi and Gauss-Seidel Methods}

The Jacobi and Gauss-Seidel methods can be applied to the equation $I_{k}=\frac{P_{h}-j Q_{h}}{V_{h}^{*}}$ to solve for $V_{k}^{i+1}$. 


$$
\frac{P_{k}-j Q_{k}}{\hat{V}_{k}^{*}}=\sum_{n=1}^{N} \hat{V}_{n} Y_{k n}
$$

For all buses, except the slack bus, the following equation is applied first for the Gauss-Seidel:

$$
V_{k}^{i+1} e^{j \delta_{k}^{i+1}}=\frac{1}{Y_{k k}}\left[\frac{P_{k}-j Q_{k}}{V_{k}^{i} e^{-j \delta_{k}^{i}}}+\sum_{n=1}^{k-1} Y_{k n} V_{n}^{i+1} e^{j \delta_{n}^{i+1}}+\sum_{n=k+1}^{N} Y_{k n} V_{n}^{i} e^{j \delta_{n}^{i}}\right]
$$

The equation is the same for the Jacobi except the $i$ iteration is used through out the formula and not $i+1$.

For a voltage controlled bus the following equation is then calculated.

$$
\begin{aligned}
Q_{k}^{i+1}= & -V_{k}^{2} B_{k k}-V_{k} \\
& {\left[\sum_{n=1}^{k-1} V_{n}^{i+1}\left[G_{k n} \sin \left(\delta_{k}^{i+1}-\delta_{n}^{i+1}\right)-B_{k n} \cos \left(\delta_{k}^{i+1}-\delta_{n}^{i+1}\right)\right]\right.} \\
& \left.+\sum_{n=k+1}^{N} V_{n}^{i}\left[G_{k n} \sin \left(\delta_{k}^{i}-\delta_{n}^{i}\right)-B_{k n} \cos \left(\delta_{k}^{i}-\delta_{n}^{i}\right)\right]\right]
\end{aligned}
$$

$Q_{k}$ is checked to see if it is within its limits, $Q_{k \max } \geq Q_{k} \geq Q_{k m i n}$. If it is within the limits then $Q_{k}$ is used to calculate $V_{k}^{i+1}$. If it is not within limits it is set to the appropriate limit and $V_{k}^{i+1}$ calculated using this value.

The calculated value for $V_{k}$ will not agree with the estimated value $V_{k-1}$. By substituting the conjugate of the calculated value of $V_{k}$ for $V_{k}^{*}$ to calculate another value for $V_{k}$, agreement would be reached to a good degree of accuracy after several iterations and would be the correct value for $V_{k}$ with the estimated voltages and without regard to power at the other buses. The process is repeated at each bus consecutively throughout the network, except at the slack bus, to complete the first iteration. Then the entire process is carried out repeatetly until the amount of correction in voltage at every bus is less than some predetermined precision. The algorithm starts at bus one, and calculates the unknowns for the other buses consecutively using the new values as it proceeds until the solution converges. 
Convergence to an erroneous solution may occur if the bus voltages are widely different from the correct values. Erroneous convergence is usually avoided if the original values are of reasonable magnitude and do not differ too widely in phase. Any unwanted solution is usually detected easily by inspection of the results, since the voltages of the system do not normally have a range in phase wider that $45^{\circ}$ and the difference between nearby buses is less than about $10^{\circ}$, and often very small.

Experience with the Gauss-Seidel method of solution of power-flow problems has shown that an excessive number of iterations are required before the voltage corrections are within an acceptable precision, if the corrected voltage at the bus merely replaces the best previous value as the computations proceed from bus to bus. The number of iterations required is reduced considerably if the correction in voltage at each bus is multiplied by some constant that increases the amount of correction to bring the voltage closer to the value it is approaching. The multipliers that accomplish this improved convergence are called the acceleration factors. The difference between the newly calculated voltage and the best previous voltage at the bus is multiplied by the appropriate acceleration factor to obtain a better correction to be added to the previour value. The acceleration factor for the real component of the correction may differ from that of the imaginary component. Poor choice of factors may result in less rapid convergence or make convergence impossible.

$$
\begin{array}{r}
V_{k}^{i+1}=V_{k}^{i}+\sigma \Delta V_{k}^{i+1} \\
\sigma \geq 1 \\
\Delta V_{k}^{i+1}=V_{k}^{i+1}-V_{k}^{i}
\end{array}
$$

The factor $\sigma$ is called the acceleration factor and for the power flow problem it is chosen to be 1.6 [30]. 


\subsubsection{Jacobi Example}

This example uses the Jacobi to solve Figure 13 of Example 1. For bus three we can solve for $V_{3}^{1} e^{j \delta_{3}^{1}}$ as follows by solving for the real part of the complex voltage $V_{3 r}$ and the imaginary part $V_{3 i}$.

$$
\begin{aligned}
V_{3 r}^{1} & =\frac{1}{(1.5)^{2}+(15)^{2}}[-4 * 1.5+1.5 *-15-.75-128] \\
V_{3 r}^{1} & =.6919 \\
V_{3 i}^{1} & =\frac{1}{(1.5)^{2}+(15)^{2}}[-4 * 15+1.5 * 1.5+23.325-21.75] \\
V_{3 i}^{1} & =-.2472
\end{aligned}
$$

For $V_{2}^{1}$ the real part is given, and the the reactive power must be found, $Q_{2}^{1}$.

$$
\begin{aligned}
Q_{2}^{1} & =-.95^{2} *(-18.3)-.95[10+9] \\
Q_{2}^{1} & =-1.5 \\
V_{2 i}^{1} & =\frac{1}{(2)^{2}+(18.3)^{2}}[(.95 *-18.3+1.5 * 2) / .95-18.3-20-18.3+18] \\
V_{2 i}^{1} & =-.1025
\end{aligned}
$$

This is the first iteration for the system. The equations are solved again with the new values.

\subsubsection{Newton-Raphson Iteration}

One method for solving the equations is by applying Newton-Raphson iteration. In the load flow problem, it is convenient to deal with $V, \delta, P$, and $Q$. Given that $n$ is the number of load buses and $m$ is the number of voltage controlled buses, the following vectors are defined. The slack bus is numbered one, the voltage controlled 
buses are numbered 2 to $m$, and the load buses are numbered $m+1$ to $m+n$.

$$
x=\left[\begin{array}{c}
\delta_{2} \\
\vdots \\
\delta_{m+n} \\
V_{m+1} \\
\vdots \\
V_{m+n}
\end{array}\right] ; b=\left[\begin{array}{c}
P_{2} \\
\vdots \\
P_{m+n} \\
Q_{m+1} \\
\vdots \\
Q_{m+n}
\end{array}\right]
$$

The Jacobian matrix now can be expressed in block form

$$
J=\left[\begin{array}{ll}
\frac{\partial P}{\partial \delta} & \frac{\partial P}{\partial V} \\
\frac{\partial Q}{\partial \delta} & \frac{\partial Q}{\partial V}
\end{array}\right]
$$

The individual terms in the four blocks are derived from the following

$$
\begin{aligned}
& \frac{\partial P_{i}}{\partial \delta_{i}}=V_{i} \sum_{j \in k(i)} V_{j}\left(G_{i j} \sin \left(\delta_{i}-\delta_{j}\right)-B_{i j} \cos \left(\delta_{i}-\delta_{j}\right)\right) \\
& \frac{\partial P_{i}}{\partial \delta_{j}}=-V_{i} V_{j}\left(G_{i j} \sin \left(\delta_{i}-\delta_{j}\right)-B_{i j} \cos \left(\delta_{i}-\delta_{j}\right)\right) \\
& \frac{\partial P_{i}}{\partial V_{i}}=2 V_{i} G_{i i}-\sum_{j \epsilon k(i)} V_{j}\left(G_{i j} \cos \left(\delta_{i}-\delta_{j}\right)+B_{i j} \sin \left(\delta_{i}-\delta_{j}\right)\right) \\
& \frac{\partial P_{i}}{\partial V_{j}}=V_{i}\left(G_{i j} \cos \left(\delta_{i}-\delta_{j}\right)+B_{i j} \sin \left(\delta_{i}-\delta_{j}\right)\right) \\
& \frac{\partial Q_{i}}{\partial \delta_{i}}=-V_{i} \sum_{j \epsilon k(i)} V_{j}\left(G_{i j} \cos \left(\delta_{i}-\delta_{j}\right)+B_{i j} \sin \left(\delta_{i}-\delta_{j}\right)\right) \\
& \frac{\partial Q_{i}}{\partial \delta_{j}}=V_{i} V_{j}\left(G_{i j} \cos \left(\delta_{i}-\delta_{j}\right)+B_{i j} \sin \left(\delta_{i}-\delta_{j}\right)\right) \\
& \frac{\partial Q_{i}}{\partial V_{i}}=-2 V_{i} B_{i i}-\sum_{j \in k(i)} V_{j}\left(G_{i j} \sin \left(\delta_{i}-\delta_{j}\right)-B_{i j} \cos \left(\delta_{i}-\delta_{j}\right)\right) \\
& \frac{\partial Q_{i}}{\partial V_{j}}=-V_{i}\left(G_{i j} \sin \left(\delta_{i}-\delta_{j}\right)-B_{i j} \cos \left(\delta_{i}-\delta_{j}\right)\right)
\end{aligned}
$$

For each iteration the following steps are performed.

1. Define

$$
\Delta y^{i}=\left[\begin{array}{c}
\Delta P_{j}^{i} \\
\Delta Q_{j}^{i}
\end{array}\right]=\left[b_{j}\right]-\left[f_{j}\left(x^{i}\right)\right]
$$


2. Calculate the Jacobian matrix.

3. Solve for

$$
\left[\begin{array}{cc}
\frac{\partial P^{i}}{\partial \delta^{i}} & \frac{\partial P^{i}}{\partial V^{i}} \\
\frac{\partial Q^{i}}{\partial \delta^{i}} & \frac{\partial Q^{i}}{\partial V^{i}}
\end{array}\right]\left[\begin{array}{c}
\Delta \delta_{j}^{i} \\
\Delta V_{j}^{i}
\end{array}\right]=\left[\begin{array}{c}
\Delta P_{j}^{i} \\
\Delta Q_{j}^{i}
\end{array}\right]
$$

4. Compute

$$
x^{i+1}=\left[\begin{array}{c}
\delta_{j}^{i+1} \\
V_{j}^{i+1}
\end{array}\right]=\left[\begin{array}{c}
\delta^{i} \\
V^{i}
\end{array}\right]+\left[\begin{array}{c}
\Delta \delta^{i} \\
\Delta V^{i}
\end{array}\right]
$$

Starting with $x^{0}$, the above steps are repeated until convergence is obtained or until the number of iterations exceeds a maximum. The convergence criterion is stated in terms of a threshold value. The real and reactive mismatch thresholds are specified as $\epsilon_{P}$ and $\epsilon_{Q}$. The power mismatch is the difference between specified and computed bus power injections or $\Delta y^{i}$. Convergence is assumed if the real elements of $\Delta y^{i}$ are all less than $\epsilon_{P}$, and the reactive elements of $\Delta y^{i}$ are all less than $\epsilon_{Q}$.

\subsubsection{Newton-Raphson Example}

Calculate $x^{i+1}$ for $i=0$ for the system in Figure 13, in Example 1, using the NewtonRaphson method. Start with $\delta_{2}^{0}=0, \delta_{3}^{0}=0$, and $V_{3}^{0}=1$ as initial values.

$$
\Delta y^{0}=\left[\begin{array}{c}
\Delta P_{j}^{0} \\
\Delta Q_{j}^{0}
\end{array}\right]=\left[\begin{array}{c}
2-(-.095) \\
-4-(.05) \\
-1.5-(-.55)
\end{array}\right]=\left[\begin{array}{c}
2.095 \\
-4.05 \\
-0.95
\end{array}\right]
$$

The Jacobian is calculated to be

$$
J=\left[\begin{array}{rrr}
18.05 & -8.55 & .95 \\
-8.55 & 15.55 & 1.55 \\
.95 & -1.45 & 14.45
\end{array}\right]
$$


Multiplying the first matrix and the inverse of the Jacobian gives

$$
\left[\begin{array}{l}
\Delta \delta_{2} \\
\Delta \delta_{3} \\
\Delta V_{3}
\end{array}\right]=\left[\begin{array}{rrr}
18.05 & -8.55 & .95 \\
-8.55 & 15.55 & 1.55 \\
.95 & -1.45 & 14.55
\end{array}\right]^{-1}\left[\begin{array}{r}
2.095 \\
-4.05 \\
-.95
\end{array}\right]
$$

The new values for $x^{i+1}$ are

$$
x^{1}=\left[\begin{array}{c}
\delta_{j}^{1} \\
V_{j}^{1}
\end{array}\right]=\left[\begin{array}{l}
0 \\
0 \\
1
\end{array}\right]+\left[\begin{array}{c}
.0024 \\
-.2500 \\
-.0910
\end{array}\right]=\left[\begin{array}{l}
.0024 \\
-.2500 \\
0.909
\end{array}\right]
$$

\subsubsection{Fast Decoupled Load Flow}

Fast power flow algorithms have been developed to give solutions in less time. These algorithms are based on a simplification of the Jacobian matrix. Attention is given to the exploitation of the loose physical interaction between real and reactive power flows in a power system, by mathematically decoupling $\frac{\partial P}{\partial \delta}$ and $\frac{\partial Q}{\partial V}$. The fast decoupled load flow method is suitable for routine accurate load flows and for outage-contingency evaluation studies performed on-line or off-line.

The fast decoupled load flow is a derivation from the Newton method [67]. It starts with a simplification of the Jacobian whereby the off-diagonal blocks $\frac{\partial P}{\partial V}$ and $\frac{\partial Q}{\partial \delta}$ are set to zero. The result is a block diagram of the Jacobian

$$
J=\left[\begin{array}{cc}
\frac{\partial P}{\partial \delta} & 0 \\
0 & \frac{\partial Q}{\partial V}
\end{array}\right]
$$

This reduces to two sets of decoupled equations:

$$
\begin{aligned}
& \frac{\partial P}{\partial \delta} \Delta \delta_{j}^{i}=\Delta P_{j}^{i} \\
& \frac{\partial Q}{\partial V} \Delta V_{j}^{i}=\Delta Q_{j}^{i}
\end{aligned}
$$

Further reduction in computer time is obtained by additional simplification of the Jacobian matrix. These simplifications come from the characteristics of a typical load 
flow problem. First, line and transformer resistances tend to be much smaller than the corresponding reactances, therefore $\left|G_{i j}\right| \ll\left|B_{i j}\right| i, j=1, \ldots, N$. Second, phase differences across a line or transformer $\left(\delta_{i}-\delta_{j}\right)$ are small because of thermal and stability limits. As a result $\cos \left(\delta_{i}-\delta_{j}\right) \approx 1.0$ and $\sin \left(\delta_{i}-\delta j\right) \approx 0$. From these facts the block diagonal terms are simplified as follows

$$
\begin{aligned}
& \frac{\partial P_{i}}{\partial \delta_{i}}=V_{i} \sum_{j \in k(i)}-B_{i j} \\
& \frac{\partial P_{i}}{\partial \delta_{j}}=V_{i} B_{i j} \\
& \frac{\partial Q_{i}}{\partial V_{i}}=-V_{i}\left(2 B_{S i i}+\sum_{j \in k(i)} B_{i j}\right) \\
& \frac{\partial Q_{i}}{\partial V_{j}}=V_{i} B_{i j}
\end{aligned}
$$

With the above modifications, $J_{1}$ and $J_{2}$ are constant matrices and do not have to be recalculated during successive iterations. The result is an iterative sequence of solutions of the form:

$$
\begin{gathered}
\delta_{j}^{k+1}=\delta_{j}^{k}+\left(B^{\prime}\right)^{-1} \frac{\Delta P_{j}^{k}}{V_{j}^{k}} \\
V_{j}^{k+1}=V_{j}^{k}+\left(B^{\prime \prime}\right)^{-1} \frac{\Delta Q_{j}^{k}}{V_{j}^{k}}
\end{gathered}
$$

The advantage of the fast decoupled load flow is that the Jacobian is replaced by two constant matrices $B^{\prime}$ and $B^{\prime \prime}$. These matrices are each half the size of the Jacobian matrix. Their inversion is performed only once, thus computational times and memory storage are reduced. Another advantage is that the region of convergence is larger for the decoupled load flow method than the Newton-Raphson method. The major disadvantage is that there is an increase in the number of iterations required for convergence over the Newton-Raphson method and that it is not so accurate. 


\subsubsection{Fast Decoupled Example}

Solve one iteration of the system in Figure 13, of example 1, using the fast decoupled load flow.

The decoupled matrices are:

$$
\begin{aligned}
B^{\prime} & =\left[\begin{array}{cc}
18.05 & -8.55 \\
-9 & 16
\end{array}\right] \\
B^{\prime \prime} & =\left[\begin{array}{l}
14
\end{array}\right] \\
\left(B^{\prime}\right)^{-1} & =\left[\begin{array}{ll}
.0755 & .0404 \\
.0425 & .0852
\end{array}\right] \\
\left(B^{\prime \prime}\right)^{-1} & =\left[\begin{array}{l}
.0714
\end{array}\right]
\end{aligned}
$$

The first iteration yields:

$$
\begin{aligned}
{\left[\begin{array}{l}
\delta_{2}^{1} \\
\delta_{3}^{1}
\end{array}\right] } & =\left[\begin{array}{l}
0 \\
0
\end{array}\right]+\left[\begin{array}{ll}
.0755 & .0404 \\
.0425 & .0852
\end{array}\right]\left[\begin{array}{c}
2.095 \\
-4.05
\end{array}\right] \\
& =\left[\begin{array}{l}
-.0052 \\
-.2561
\end{array}\right] \\
V_{3}^{1} & =1-.95 \div 14 \\
& =.9321
\end{aligned}
$$

\subsubsection{Comparison of methods}

The most commonly used method for solving the load flow is Newton-Raphson Iteration. It has the fastest convergence rate and the most accurate results. Its disadvantages are that a Jacobian has to be calculated at each iteration. The Gauss-Seidel takes the most iterations to converge but the calculations for each step are simpler. The Fast Decoupled algorithm is the fastest. For this reason it is used the most for contingency analysis. It has the disadvantage that the results are not so accurate. 


\begin{tabular}{|r|r|r|r|r|r|r|r|r|}
\hline \hline \multicolumn{2}{|c|}{ Newton-Raphson } & \multicolumn{3}{|c|}{ Gauss Seidel } & \multicolumn{3}{|c|}{ Fast Decoupled } \\
\hline$\delta_{2}$ & $\delta_{3}$ & $V_{3}$ & $\delta_{2}$ & $\delta_{3}$ & $V_{3}$ & $\delta_{2}$ & $\delta_{3}$ & $V_{3}$ \\
.0000 & .0000 & 1.0000 & .0000 & .0000 & 1.0000 & .0000 & .0000 & 1.0000 \\
.0024 & -.2500 & .9090 & -.8810 & -.2850 & .8960 & -.0052 & -.2561 & .9321 \\
-.0284 & -.3116 & .8500 & .1211 & -.2379 & .8450 & -.0154 & -.2862 & .8679 \\
-.0202 &.- .3131 & .8425 & -.2965 & -.3160 & .8630 & -.0180 & -.3034 & .8544 \\
-.0185 &.- .3123 & .8424 & .1805 & -.2760 & .8380 & -.0182 & -.3083 & .8470 \\
-.0185 & -.3123 & .8424 & -.0674 & -.3176 & .8519 & -.0184 & -.3107 & .8444 \\
\hline \hline
\end{tabular}

Table 4: Comparison of methods

Table 4 shows the results for the three methods used to solve the system of Figure 13.

\subsubsection{Control of Load Flow Variables}

In the load flow problem, automatic manipulation of some of the control variables is performed to insure convergence to a solution that satisfies the inequality constraints [17]. The inequality constraints are due to the physical properties of the power system elements. The generator operating characteristics require it to stay within certain limits. Constraining the real power generated is simple, since it is a known and fixed value. The reactive power generated is an unknown variable and must be kept within its minimum and maximum limits. At these buses containing generators the reactive power generation is computed after the initial load flow. If the generator's reactive power is outside its specified limits, then the bus is treated as a load bus with the reactive power set to its limit and the voltage set floating, requiring $V_{i}$ and $Q_{i}$ to exchange roles as knowns and unknowns. When $Q_{i}$ is within its limits then it is changed back to an unknown.

During the solution of the load flow problem, the transformer taps are adjusted 
to ensure voltage at a voltage controlled bus remains within limits. If the voltage is outside its limits, then the transformer tap settings nearest to the bus are increased or decreased until the voltage falls within the limits. The admittance values for the bus, $Y_{i j}$, are updated with the new tap position, and a new iteration of the solution is performed.

At the slack bus, if real power generation is outside its limits, the amount of power will be distributed among the remaining units and more load flow iterations are performed. If the reactive power generation violates its limit then either the slack bus can be changed to a different generator bus, or the voltage of the slack bus can be adjusted to a different constant (subject to its physical constraints), or reactive generation or load can be adjusted by switching of capacitor or inductor banks.

\subsection{Review of Previous Research}

A description of research done on the load flow problem and parallel processing techniques to solve it are given below. Most research done in this area has involved decomposing the network into sections and solving each network concurrently. The Electric Power Research Institute (EPRI) has sponsered projects mainly on the effect of array processors in the solution of the load flow. Other research projects are also given below. According to my study, an INMOS Transputer based load flow analysis, decomposed by each bus, has never been developed.

\subsubsection{Solutions by Decomposition}

Methods that reduce a large network of equations to many smaller decoupled subnetworks were studied at the University of Tokyo $[65,78]$. They propose a decomposed parallel processing technique to solve the nonlinear simultaneous equations of the load flow analysis.

In this method, a given system is decomposed into multiple subsystems by re- 
moving the interconnecting or tie lines. The voltage magnitudes and phase angles at the interconnecting nodes are fixed. The load flows in all subsystems are computed concurrently using local processors. The interconnecting lines are then re-connected. Variations in voltage magnitudes and phases that are produced are calculated by a coordinating processor using the interconnecting voltage sensitivities with respect to injection powers. The effect of the variations of interconnecting voltages on the voltage magnitudes and phases at non- interconnecting nodes in each subsystem is calculated using a local processor in each subsystem. This process is iterated until power and voltage constraints at all nodes are satisfied.

The first step in solving each subsystem is done using the Newton-Raphson algorithm in each processor in parallel. If the solution obtained does not satisfy the power equations for interconnecting nodes a correction equation is derived by calculating the Jacobian matrix also in parallel by the local processors. The matrix formed by the correction equations of each processor is called a mixed sensitivity matrix. The correction equations are used to reduce the power mismatch at interconnecting nodes as much as possible without increasing the power and voltage mismatches at noninterconnecting nodes of each subsystem. The correction equations are transmitted from the local processor to a coordinating processor. The coordinating processor then solves the interconnected system using the Newton-Raphson algorithm. After all values are modified each subsystem again solves individually with these new values. This process continues until a desired solution is obtained.

Calculations were performed using HITAC M-280 at the Computer Center of University of Tokyo. Conclusions obtained were that the proposed method and the Newton-Raphson method have the same convergence characteristics, the computing time is smaller than that of the Newton-Raphson method and is little effected by the number of subsystems.

Another decomposed load-flow algorithm can be found in [61]. This decomposed 
load flow is based on the fast decoupled load flow algorithm [67]. The solution is achieved by partitioning the system into a number of subsystems that are connected by tie lines. The $B^{\prime}$ and $B^{\prime \prime}$ matrices of the Fast Decoupled Algorithm are calculated for each area with reference to its own slack node with no consideration of the tie lines. This technique minimizes the amount of data needed to be transferred between the central program and the subsystem programs since there is no need for transmission of data related to buses connected to fictitious slack buses.

Data must be transfered at each iteration between the processors and the coordinating processor. The active and reactive power mismatches are transferred from each local proccessor to the coordinating processor. The new voltage magnitudes and phases are passed from the coordinating processor to each local processor. To minimize the data transfered it is shown that a fictious slack bus for each area should be created. This slack bus should be a bus that has the least ties with other ones of the fictitious slack buses of the subsystems.

Computation times, storage comparison and data transfer times are given in this article. The solution using this approach is obtained in half the time required by the regular decoupled load flow. Increasing the number of tie lines and areas increases the time required for the solution of the coordinating problem while reducing the time required in each subsystem.

In [60], another decomposed algorithm is given that uses the Newton-Raphson algorithm. It shows a way to reorganize the Jacobian matrix and then to decompose it into different sections. Results for each area are then calculated on separate processors, in parallel, using the Newton-Raphson algorithm.

Once each processor has done its calculations, then those columns of the Jacobian which are related to nodes connected to tielines are transmitted to the coordinating processor. The coordinating processor does its calculations and transmits the relevant subvectors to the appropriate area. This procedure continues until a solution is 
obtained.

Results show computation times between the decomposed simulator and the centralised simulator using a Perkin Elmer 3230 minicomputer. The size of each network and the amount of data needed to be transferred between the coordinating processor and the area simulators is also presented. It was found that the algorithm was well suited to parallel processing implementation using minicomputers interconnected by a fast communication medium or on a parallel microprocessor system.

In [7], a simulator is described that uses a decomposition technique to solve the load flow. This method exploits the physical configuration of the system yielding the natural uncoupling points. The technique is based on branch tearing, where a given network is decomposed at its loosely coupled branch points. Each cluster is solved on a different processor and the results combined by a central processor.

A two level architecture is given in this paper. A common bus is used to interconnect all processing nodes that form the first level. The second level is created by clustering the nodes into sets that can communicate on a local bus. Each processing node contains a processor, support hardware, local access memory and dual-port memory. The dual port memory is accessible to any processor via the common bus. The system built is based on the INTEL Multibus. An IBM-XT host microcomputer is the central processor used for coordination.

The decomposed load flow algorithms that have been studied are well suited for MIMD machines. Each processor does its calculations on the different subsystems in parallel. It has the advantage that each subsytem is small so that the processing time is less for each processor than for the system if solved as a whole. Another advantage is that for system expansion the processing time should not increase if additional local processors are added. It has the disadvantage that a coordinating processor is needed which adds additional time in message passing from the local processors to the coordinating processor and each is idle while the other performs 
its calculations. Also, a good method has to be used to sudivide the system into different sections. Therefore, the objectives for this type of algorithm would be to have low overall solution time, full utilization of the subsystem computers, and low interprocessor data transfer.

\subsubsection{Other Solutions}

In an actual power system, all of the generators and loads are running in parallel. Using this viewpoint, a prototype of a multiprocessor system is given in [71] to solve the load flow problem in parallel. The method uses the Gauss-Seidel iterative algorithm. In this method, each node can calculate its own equations at one time and parallel processing is used. No inverse matrix calculation is needed and it requires a small amount of data for the calculation.

The architecture proposed consists of several Local Processor Units (LPUs), a Main Processor Unit(MPU), a Control Bus and a Transfer Bus. Each LPU corresponds to each node or bus in the power system. A transfer bus corresponds to a node connection, and data will be transferred only between two nodes which are connected by the transfer bus. For large systems a common bus is used for data transfer. This increases the time for transferring data between nodes.

The results of comparing the multiprocessor system with a sequential machine (COSMO-700-II) is given in this paper. As the power system becomes larger, it is shown that the total time for the multiprocessor system becomes less than in the COSMO-700-II. Some of the problems found were that as the system became larger the number of LPUs needed increased, and that the Gauss iterative method requires many iterations to converge.

In [73], an algorithm is proposed to solve the load flow problem using elementalschemes. Elemental operations refer to operations performed on individual elements of a matrix. This exploits the parallelism existing when considering elemental operations 
and does not require natural clusters to exist. The algorithm is applied to the Fast Decoupled Load Flow and the results are provided in the paper.

The algorithm takes a given system and groups the factorization operations into blocks, where each block consists of the inversion of a diagonal element and the update operations involving this inverted element. Precedence rules are obtained from a simple directed path graph. Forward elimination and backward substitution that are part of the Fast Decoupled Load Flow algorithm are done similarly to the factorization.

The main problem with this kind of parallelism is the need to find efficient precedence rules among elemental computations that allow parallel execution without excessive overhead. This was shown in the results that are provided in this paper using two or three processors, efficiency was less for more processors because of increased overhead.

In [70], a method to develop asynchronous procedures is given. The algorithms used for the load flow solution used at the time of this article are synchronous, and cannot be broken into parallel processes for concurrent execution without inserting a number of synchronization points. The resulting delays and communication overheads grow with the number of processors. They develop concepts useful in thinking about asynchronous procedures, discuss a model after which they may be patterned, introduce some new convergence results and present some illustrations of how to construct asynchronous procedures. The Gauss-Seidel, modified for asynchronous processing, was run, and simulated results of the different states of the network given. The error shown is the average difference between the actual state of the network and the calculated state. As a processor is added, the error of the system decreases.

A general load flow algorithm of the Gauss iterative type (given in [81]) is well suited for parallel processing. A Jacobi or Gauss-Seidel type iteration is performed between a set of arbitrarily designated unique incoming branches at each bus and the 
other branches connected to the bus. This load flow algorithm directly uses complex voltage and power values, and is based on the electric power transmission system and the power transmission line rather than the network-analysis based ideas used in the conventional load flow algorithm.

\subsubsection{EPRI Research}

The Electrical Power Research Institute (EPRI) has sponsored projects to investigate new advanced computers and computational approaches, to demonstrate how they can be used to analyze power flows more quickly. In October 1980 in Dallas, utility engineers and computer staff, consultants, researchers, and EPRI staff gathered to exchange information on utility-related research and experiences with parallel processing [20]. Presentations in this conference introduced the concept of parallel processing and discussed its potential uses in power system planning and operations. Two utilities, Bonneville Power Administration and Northeast Utilities Service Company, gave their experience with array processors. The array processor worked up to ten times faster than the serial computers. However, they required assembly language programming to achieve such speed. The performance of a combination host computer and array processor approximately equaled that of serial computers, because of the time required for communication between the two machines.

A few projects sponsored by EPRI were spin offs from the above conference. In one of these projects, the objective was to develop a power flow computer program for use with an array processor computer [58]. Advantages and disadvantages of using array processors for solving on-line problems were to be evaluated.

A complete AC power flow code based on the fast decoupled algorithm was developed for the Floating Point Systems AP-120B array processor and its host computer. This program was able to solve a simple 118 bus power flow problem five times faster than a large computer alone could. The problem is that analyzing an isolated power 
flow is not a cost-effective task for an AP-120B. The data transfer time between host and array processor, together with the large input/output requirements, cause the array processor to be idle during most of the run. The array processor would be beneficial in the evaluation of the effect of many outages applied in sequence to a power system. In this problem, the array processor can solve a base case power flow plus the full $\mathrm{AC}$ power flow for a list of contingencies without returning control to or communicating with the host computer. It would be capable of evaluating at least 100 contingencies per minute for a 1000 bus power system.

In 1985 EPRI sponsored another research in the parallel solution of algebraic equations [53]. The approach taken was to use parallel processing to solve large sets of sparse simultaneous linear algebraic equations by breaking a single computation into a set of parallel computations that uses basic mathematical operations such as addition and multiplication. The researchers developed a new algorithm designed to subdivide matrices further than other methods. They used the program evaluation and review technique to determine the critical path for the required calculations, and developed a scheduling algorithm for a set of parallel processors that would minimize total computation time.

The algorithm developed uses an iterative approach to subdivide the matrix. The method repeatedly selects a set of partitions and schedules the use of the processors for each set until the results show no improvement over the previous iteration. The scheduling algorithm includes the computation time of the individual processors, and the communication time between processors. The major result from this project was that, when using the multiple factoring algorithm, the precedence requirements in the parallel solution are not the limiting factor. The algorithm overhead required by the extra control statements is responsible for most of the loss in efficiency. This is a function of how well the programs are written and the characteristics of the microprocessors used. 
Another project sponsored by EPRI investigated the use of an array processor for solving the power flow problem [19]. The objectives were to implement the Bonneville Power Administration power flow code on the Floating-Point Systems array processor, and to evaluate the ability of this processor to solve the power flow computations and power system network problems efficiently.

The Newton-Raphson solution algorithm portion of the power flow was redesigned. The solution code was run on a VAX 11/780 supermini computer, then on the VAX 11/780 using a model FPS-164 array processor, and again on the VAX 11/780 using a larger model FPS-264 array processor. The results obtained were that running the power flow program on the FPS-164 (including input/output) did not increase the run speed significantly. Using the faster model FPS-264 the total run, including input/output took one-fourth of the time of the original code.

A concurrent processing scheme based on commerically available microprocessors was the approach taken for power system analysis in [54]. Existing programs were run on a specially developed computer architecture. The objectives were to investigate the feasibility of using a concurrent processor architecture to solve the power system analysis problems, and to identify the most adequate computer architecture and estimate its cost effectiveness using the EPRI transmission system reliability methods program as a benchmark.

The project was implemented on a VAX 11/780 and on an IBM personal computer based on the Intel 8086 and 8087 microprocessors. Implementation and testing of a power system reliability program and its test cases demonstrated that a system of microcomputers has a cost effective advantage of a least five to one. Contingency analysis was suggested as a good application for this approach. 


\subsection{Load Flow Solution}

The parallel machine, PLF, described in the first sections will be used in solving of the load flow analysis problem. As previously stated, load flow is mathematically represented as a set of nonlinear sparse equations. The goal is to be able to solve these equations in a much faster time than done now. The next sections explain how the PLF is used to solve the load flow problem. The convergence of this system is also demonstrated.

\subsubsection{Parallel Machine Configuration}

For the particular problem being studied, the PLF system proceeds as follows. The Control Unit will have the information of the power system. It will treat each Processing Unit as being a bus in the system. This unit will set up the Switching Network according to the model of the power system being analyzed.

Each Processing Unit is numbered in ordered. The following bus information is given to each Processing Unit.

- type of bus-load, slack, generation, etc.

- bus number and name

- number of the buses connected to it-branches

- known values-voltages, loads, taps, etc.

- known values of buses connected to it-voltages,loads, taps, etc.

Each Processing Unit stores this information in its local memory. When the signal is sent, each Processing Unit starts to set up its communication lines. Each Processing Unit can simultaneously set up a sending line with another Processing Unit if they are connected. 
Once the circuit is set up and a node has all the information it needs, it can start its calculations. Depending on what type of bus it is represening, each Processing Unit will solve a different equation or set of equations. When a result is obtained, the Processing Unit sends the answer out through its sending circuits and reads incoming values from the receiving lines.

\subsubsection{Convergence of the System}

A general load flow algorithm of the Jacobi type is constructed in [82]. A region of convergence is defined for this algorithm using the concept of generalized block diagonal dominance for sets of quadratic algebraic equations. The PLF system uses the same type of algorithm and equations therefore will also converge.

The rate of convergence of this algorithm is shown in the plot of Figure 13. The number of iterations is plotted against the log 10 of the magnitude of the largest error at each iteration. 


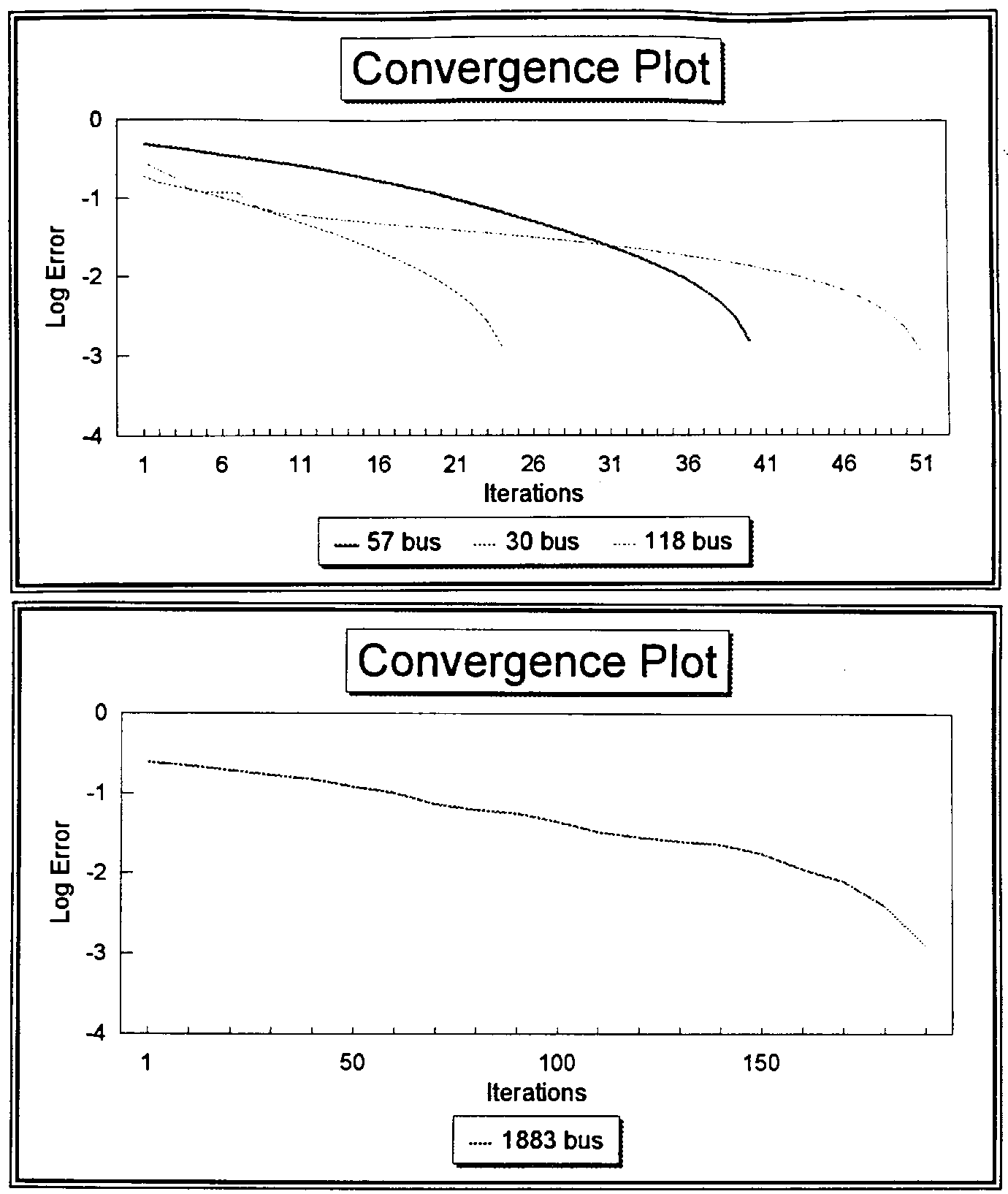

Figure 14: Rate of Convergence 


\section{LOADFLOW PERFORMANCE EVALUA- TION}

A software simulation of PLF was developed in order to demonstrate the feasibility of the system and evaluate the dynamic performance of the system. The simulation was written in FORTRAN since most power flow analysis at the time are written in FORTRAN. It is run on a Sun Computer. The simulation is capable of running a Newton Raphson power flow, Gauss-Seidel power flow, and the Parallel Load Flow (PLF) .

The test cases used were the following: 6-bus test case, 14-bus IEEE, 30-bus IEEE, 57-bus IEEE, 118-bus IEEE, and 1883-bus FPL test data [57]. The data were divided as given in the next section.

\subsection{Software Simulation}

This section explains the simulation for the analysis of the load flow. It shows how the data of a bus is represented at each Processing Unit. The information needed by each Processing Unit and the data that is shared is explained.

\subsubsection{Data Input}

Each bus has certain data that needs to be known to that Processing Unit only. This we will call the bus data record. It is the information that has to do with each bus independently of other buses. This data is input to each Processing Unit and has the following format:

- busno: the number given to each bus

- busname: the name given to each bus

- pload: load real power to be held constant and entered as MW 
- qload: load reactive power to be held constant and entered as MVAR

Each Processing Unit that is to represent a generator or plant bus must be specified in a generator data record. The generator data record has the following format:

- genbusno: the number of the bus were the generator is at

- pgen: generator real power output entered in MW

- qmax: maximum generator reactive power output entered in MVAR

- qmin: minimum generator reactive power output entered in MVAR

- vsched: regulated voltage set point entered in p.u.

For each line or branch to be represented in the load flow a line data record is needed. This data is later distributed to each Processing Unit that needs it. The line data record has the following format:

- frombus: branch "from bus" number

- tobus: branch "to bus" number

- $\mathrm{r}$ : branch resistance entered in p.u.

- $\mathrm{x}$ : branch reactance entered in p.u.

- bcap: total branch charging susceptance entered in p.u.

- linetype: the type of line either "line" if branch is a line or "tran" for a transformer branch. If it is a transformer then a transformer record follows immediately with the transformer tap information.

The transformer data record follows immediately after the line data record for the transformer branch. The format for the transformer branch data is the following: 
- tapbus: this is the tapped side of the transformer

- nontapbus: this is the nontap side of the transformer and the

- impedance side bus

- tapratio: transformer off-nominal turns ratio entered in p.u.

Each bus in the network that has a switch shunt admittance device must have a switched shunt data record. The format for the switched shunt data record is the following:

- capbusno: the bus number where the device is located

- cap: switch shunt admittance entered in MVAR.

\subsubsection{Data Types}

Once entered the data is distributed to each Processing Unit that needs it. The following information is needed at each Processing Unit:

- ties: the number of buses connected to this Processing Unit or the number of branches at the bus.

- connect: the bus numbers for each bus that has a line to it. This is a pointer table to the buses connected at that bus.

- busno: the number for the bus.

- busname: the name for the bus.

- psched: load real power for the bus.

- qsched: load reactive power for the bus.

- qqmax: if the bus is a generator this is the maximum reactive power. 
- qqmin: if the bus is a generator this is the minimum reactive power.

- volt: the complex voltage set to $1.0+\mathrm{j} 0.0$ if it is a non-generator bus or vsched if it is a generator bus.

- bustype: the type of bus either line, generator, transformer, or swing bus.

- vmag: real part of voltage or its magnitude.

- phase: imaginary part of volt or its phase.

- voltto: the voltage at the bus of the branch connected to that bus.

- tapinfo: the tap information.

- tranconnect: the buses that have transformers connected to that bus. A pointer to the transformers connected to that bus.

Each bus on the average has two to three branches connected to it. Therefore, for each Processing Unit, the length of a record will be one or two data items. There are three types of data. Constant bus information once entered is not changed. The bus data that are calculated change with each iteration. The bus data of connecting branches vary with each iteration.

\subsubsection{Data Calculated}

Once all the processors have the data they need, the equations for each bus need to be calculated. The calculations can be divided into two sets of equations.

The first step each process must take is to determine what bus type it is. For each bus type, different input data must be calculated. Depending on what components are connected to a bus the calculations will vary. The five basic components are considered in the following sections. 


\begin{tabular}{|c|c|c|}
\hline \hline Fixed Bus Data & Varying Bus Data & Varying Branch Data \\
\hline ties & volt & voltto \\
connect & phase & vmag \\
busno & vmag & phase \\
psched & & \\
qsched & & \\
qgen & & \\
\hline
\end{tabular}

Table 5: Data Type Per Bus

\subsubsection{Generators}

A specified amount of real power, $P_{G}$, is generated at a specified terminal voltage magnitude $V_{\text {sched }}$. The generator also has production or consumption of reactive, $Q_{G}$ depending on the excitation level (field currents). Limitations on these quantities are given:

$$
\begin{gathered}
q \min \leq q \leq q \max , \\
v \min \leq \text { volt } \leq \text { vmax. }
\end{gathered}
$$

The reactive limits are determined by excitation system limitations. These quantites are known to each bus. Terminal voltage limits are functions of service quality considerations and are usually a few percentage points off rated terminal voltage. These quantites are known at each processor.

\subsubsection{Transformers}

The impedance for a transformer branch has to be calculated from the data the processor has. Each bus has the tap ratio, if it is the tap side or non tap side, its 
resistance and its reactance. The following calculations are done per bus.

$$
\begin{array}{r}
Y_{i j}=t a p Y_{i j} \\
Y_{S i j}=-t a p(1-t a p) Y_{i j} \\
Y_{S j i}=(1-t) Y_{i j}
\end{array}
$$

where $i$ is the tap side bus and $j$ is the non tap side bus.

\subsubsection{Transmission Lines}

The impedance of a transmission line for each bus must also be calculated. Each processor knows its line series resistance and its reactance. The following calculations are done at each bus for all its transmission branches.

$$
Y_{i j}=\frac{1}{\frac{R_{i j}}{R_{i j}^{2}+X_{i j}^{2}}-j \frac{X_{i j}}{R_{i j}^{2}+X_{i j}^{2}}}
$$

\subsubsection{Load}

The load at each bus is a complex mix of small devices, appliances, lights, or larger components like furnaces, motors, and so on. A detailed knowledge of the load composition is not necessary. Real power consumption and its associated power factor is what is of interest at the bulk power level. This is the same as specifying real and reactive power consumption. Therefore, these two values are specified at each load bus.

\subsubsection{Shunt Capacitors and Inductors}

These devices are used for voltage control in the system. If real and reactive demand is high in a system, voltages will tend to decrease to unacceptable levels. Capacitors are switched on to increase reactive production and increase bus voltages. Under low real and reactive demand levels, line charging capacitance may be enough to increase 


\begin{tabular}{|r|r|r|r|r|}
\hline \hline buses & lines & generators & transformers & capacitance \\
6 & 11 & 3 & 0 & 0 \\
30 & 41 & 6 & 4 & 2 \\
57 & 80 & 7 & 17 & 3 \\
118 & 179 & 21 & 9 & 14 \\
1883 & 2004 & 222 & 149 & 59 \\
\hline \hline
\end{tabular}

Table 6: Test Case

voltages above its limits. Switching inductors on will create reactive load that will tend to reduce voltages to acceptable levels under valley load conditions.

\subsection{Results}

The Newton-Raphson load flow, Gauss-Seidel load flow and the PLF were run for the different scenarios. A six bus, 30 bus-IEEE, 57 bus-IEEE, 118 bus-IEEE and 1883 bus FPL systems were used for test cases. The data was broken down as shown in the Table 6:

Each test case was run to find the time of convergence, number of iterations, and the time to construct the $Y$ matrix. Table 7 shows the time for the convergence of the Newton Rahson and the PLF. The results show that an increase in the number of nodes of a system does not effect the solution time for the PLF system.

The times that are shown in the table are the times that it took each of the above algorithms to converge. The number shown is in seconds. It is the average or mean taken over running each case 25 times.

An advantage of the PLF architecture is that the $Y$ matrix does not have to be constructed. Each node figures its own impedance and does not need that value of the other nodes. This is a big savings in time when the number of buses increases. 


\begin{tabular}{||r||r|r||}
\hline \hline buses & $\mathrm{N}-\mathrm{R}$ & PLF \\
\hline \hline 6 & 1.07 & .020 \\
30 & 13.25 & .023 \\
57 & 31.29 & .027 \\
118 & 55.49 & .028 \\
1883 & 180.40 & .033 \\
\hline \hline
\end{tabular}

Table 7: Time for Convergence

Unit of time is in seconds.

\begin{tabular}{||r|r|r|r|r|r||}
\hline procedure & precision & case 1 & case 2 & case 3 & case 4 \\
\hline \hline Newton-Raphson & .001 & 3 & 3 & 4 & 3 \\
Newton-Raphson & .00001 & 6 & 6 & 7 & 6 \\
\hline PLF & .001 & 19 & 18 & 19 & 17 \\
PLF & .00001 & 25 & 27 & 31 & 28 \\
\hline \hline
\end{tabular}

Table 8: 30 Bus - Number of Iterations

For the 1883 FPL test case it takes .43 seconds to calculate the entire $Y$ matrix. For the PLF architecture it is the time for three multiplications and 3 additions.

For each case the data was changed to run different scenarios of the same number of buses. The number of iterations for each to converge were taken and the results are given below.

In conclusion, as the number of buses in the system increases, the time for convergence for the Newton-Raphson and for the Gauss-Seidel algorithm increases. The time for convergence for the PLF is maintained constant since the calculations are done in parallel. It does not matter how many buses are added as the calculation 


\begin{tabular}{||r|r|r|r|r|r||}
\hline \hline procedure & precision & case 1 & case 2 & case 3 & case 4 \\
\hline \hline Newton-Raphson & .001 & 3 & 4 & 4 & 3 \\
Newton-Raphson & .00001 & 8 & 8 & 9 & 8 \\
\hline PLF & .001 & 18 & 19 & 20 & 18 \\
PLF & .00001 & 47 & 47 & 53 & 46 \\
\hline
\end{tabular}

Table 9: 57 Bus - Number of Iterations

\begin{tabular}{||r|r|r|r|r|r||}
\hline \hline procedure & precision & case 1 & case 2 & case 3 & case 4 \\
\hline \hline Newton-Raphson & .001 & 4 & 5 & 5 & 5 \\
Newton-Raphson & .00001 & 10 & 10 & 11 & 11 \\
\hline PLF & .001 & 19 & 20 & 20 & 18 \\
PLF & .00001 & 45 & 49 & 50 & 51 \\
\hline \hline
\end{tabular}

Table 10: 118 Bus - Number of Iterations

\begin{tabular}{||r|r|r|r||}
\hline \hline procedure & precision & case 1 & case 2 \\
\hline \hline Newton-Raphson & .001 & 10 & 12 \\
Newton-Raphson & .00001 & 23 & 28 \\
\hline PLF & .001 & 78 & 87 \\
PLF & .00001 & 158 & 167 \\
\hline \hline
\end{tabular}

Table 11: FPL Test Case - Number of Iterations 
per node does not change. Each bus still has the same equation and the number of connections is still at an average of five. 


\section{CONCLUSION}

The PLF system is a new technique for solving a class of sparse non-linear equations. It consist of software and a parallel architecture used to solve this type of problem.

The software used to solve these equations is based on the Jacobi iterations. A successive overrelaxation method that uses an acceleration factor on the Jacobi iterations, reduce the number of iterations needed for convergence.

The architecture of PLF is a Multiple Instruction Multiple Data (MIMD) machine based on the INMOS Transputer and C004. It consist of Processing Units that perform the calculations. A Switching Networks that provides the communication of the data. The Control Unit sets up the network.

The Processsing Unit consist of a set of T800 Transputers. These Transputers have four input/output links. The architecture can be improved with new generation of Transputer that have more links.

The switching Network consist of a three stage Clos network made with C004s. With the new generation of $\mathrm{C} 004 \mathrm{~s}$ there can be an improvement. The network can also be simplfied by joining the Processing Units to each other. This depends on the specific problem being analyzed but it will decrease the communication time.

This architecture has been used to test for the solution of the load flow analysis. This network can be used to perform other power network analysis. Each bus has been represented by a Processing Unit. The switching network will represent the connectivity of the power network.

After testing a few networks, the proposed PLF system has proven to increase processing speed by a factor of 2000 as compared to present day techniques. Adding more nodes to this system does not effect the overall processing speed since each will perform in parallel. 


\subsection{Future Research}

There are other topics that are left for future research. Fault tolerance is an issue that has not been discussed here but is easily fitted into this architecture. Any node can be replicated by having more than one node solve for one equation.

Nonsparse systems of equations can also be run using this system. The Processing Unit would need to be modified to allow for more incoming data. The effects of this would have to be tested.

Other possibility of methods for solving the equations can also be developed. There might be other algorithms suited to run on this architecture.

This system can be incorporated into an open system environment. It can be used as a workstation and connected to a network of other workstations.

Larger systems can be developed for other applications. More nodes would have to be added. By adding more nodes the computation bandwidth and the communication bandwidth increase. The size of the problem is therefore not bounded by the computer architecture. 


\section{References}

[1] M. A. Ajiz and A. Jennings. A robust incomplete choleski-conjugate gradient algorithm. International Journal for Numerical Methods in Engineering, 20:949-966, 1984.

[2] Andres Antoniou. Digital Filters: Analysis and Design. MxGraw Hill, 1979.

[3] V. E. Benes. On rearrangeable three-stage connecting networks. The Bell System Technical Journal, pages 1481-1492, 1962.

[4] Dimitri P. Bertsekas and John N. Tsitsiklis. Parallel and Distributed Computation Numerical Methods. Prentice Hall, 1989.

[5] Cuy Blelloch. Applications and algorithms on the connection machine. Mit AI Laboratory, February 1987.

[6] Anjan Bose and Kevin A. Clements. Real-time modeling of power networks. Proceedings of the IEEE, 75(12):1607 to 1622, December 1987.

[7] G. E. Bridges, D. M. Burek, W. Pries, C. W. Lee, R.D.McLeod, A. M. Gole, and R. M. Mathur. A multiple processor based power system digital simulator. Fourth International Conference on AC DC Power Transmission, pages 477-482, September 1985.

[8] Richard L. Burden, J. Douglas Faires, and A. C. Reynolds. Numerical Analysis. Prindle, Weber and Schmidt, Boston, Massachusetts, 2 edition, 1981.

[9] C. Clos. A study of nonblocking switching networks. The Bell System Technical Journal, pages 406-424, May 1953.

[10] Thinking Machines Corporation. Introduction to data level parallelism. Technical Report TR86-14, Thinking Machines Corporation, April 1986.

[11] Thinking Machines Corporation. Connection machine model $\mathrm{cm}-2$ technical summary. Technical report, Thinking Machines Corporation, 245 First Street,Cambridge, Massachusetts 02142-1214, May 1989.

[12] M. Cosnard, M. H. Barton, and M. Vanneschi. Proceedings of the IFIPWG Conference of Parallel Processing. Elsevier Science Publishers B.V., 1988.

[13] A. Cosnuau and P. Leca. Scientific parallel computing with transputer networks. International Journal of High Speed Computing, 1:383-398, November 1989.

[14] M. L. Crow and M. lic. The parallel implementation of the waveform relaxation method for transient stability simulations. IEEE/PES Winter Meeeting, February 1990.

[15] M. L. Crow, M. lic, and J. White. Convergence properties of the waveform relaxation method as applied to electric power systems. IEEE Int'l Symposium on Circuits and Systems, May 1989.

[16] A.L. Davis and R.M. Keller. Data flow program graphs. IEEE Computer, pages 26-41, 1982.

[17] Atif S. Debs. Modern Power Systems Control and Operation. Kluwer Academic Publishers, 1988. 
[18] Angel L. DeCegama. Parallel Processing Architectures and VLSI Hardware. PrenticeHall Inc., 1989.

[19] B. Dembart, A. C. Mong, K. W. Neves, D. S. Dodson, and Y. M. Chan. Power flow calculations utilizing array processors. Technical Report EPRI EL-4642, Boeing Computer Services, 565 Andover Park West, Tukwila, Washington, June 1986.

[20] Electrical Power Research Institute, editor. Proceedings: Parallel Processing for Power System Planning and Operations, 3412 Hillview Avenue Palo Alto, California, October 1984. Electrical Power Research Institute, Electrical Power Research Institute. EL3775 .

[21] Olle I. Elgerd. Basic Electric Power Engineering. Addison-Wesley Publishing Company, 1977.

[22] M. K. Enns, W. F. Tinney, and F. L. Alvarado. Sparse matrix inverse factors. Summer Power Meeting, July 1988.

[23] Rolf-Dieter Fiebrich. Data parallel algorithms for engineering applications. E/G87-1, 1987.

[24] Carl-Erik Froberg. Numerical Mathematics, volume 1. The Benjamin/Cummings Publishing Co, Menlo Park, California, 2 edition, 1985.

[25] Carl Erik Froberg. Theory and Computer Applications. The Benjamin Cummings Publishing Co., Inc., 1985.

[26] J. Duncan Glover and Mulukutla Sarma. Power System Analysis and Design. PWS, 1987.

[27] Gene H. Golub and Charles F. Van Locia. Matrix Computation. John Hopkins University Press, 1985.

[28] A. Gomez and R. Betancourt. Implementation of the fast decoupled load flow on a vector computer. IEEE Power Engineering Proceedings, 1990.

[29] G. P. Granelli, M. Montagna, M. La Scala, and F. Torelli. Relaxation newton methods for transient stability analysis on a vector / parallel computer. IEEE Transactions on Power Systems, 9:637-643, May 1994.

[30] Charles A. Gross. Power Systems Analysis. Auburn University, 1973.

[31] Charles A. Gross. Power System Analysis. John Witey and Sons, 1979.

[32] H. H. Happ, C. Pottle, and K. A. Wirgam. Future computer technology for large power system simulation. Automatica, 15(6), November 1979.

[33] Oscar H. Tbarra and Myung Heekim. Fast parallel algorithms for solving triangular systems of linear equations on the hypercube. Journal of Parallel and Distributed Computing, 20:303-316, March 1994.

[34] M. Ilic-Spong and J. Zaborszky. A different approach to load flow. IEEE Transactions Power Apparatus and Systems, PAS-101, January 1982.

[35] Inmos. Transputer Architecture Reference Manual. INMOS Corporation, 1986.

[36] Inmos. The Transputer Family Product Information. Inmos, 1986. 
[37] Inmos. Transputer Reference Manual. Inmos, 1986.

[38] INMOS. IMS C00\& Programmable Link Switch, 1987.

[39] Inmos. Transputer Development System. Prentice Hall International Ltd, 66 Wood Lane End, Hemel Hempstead, Hertfordshire, 1988.

[40] S. Lennart Johnson. Data parallel programming and basic linear algebra subroutines. Mathematical Aspects of Scientific Software, March 1987.

[41] Q. Li, D. Barton, and N. Rishe. A linear equation solution algorithm on a coarsegrained distributed-memory parallel system. Proceedings of the Third Annual Parallel Symposium, pages 360-368, March 1989.

[42] Q. Li, W. B. Field, and D.Klein. Implementation of a transputer ring network and a deadlock prevention algorithm. The 3rd Us Occam User Group Meeting, September 1987.

[43] Q. Li, W. B. Field, and D. Klein. Channel design primitive in occam. The 3rd US Occam User Group Meeting, September 1987.

[44] Q. Li, W. B. Field, and D. Klein. A method for solving linear equations on a transputer system. The 3rd US Occam User Group Meeting, September 1987.

[45] Qiang Li. The architecture and the related control problems of a transputer based highly parallel database computer. Technical report, Florida International University, 1989.

[46] Qiang Li and Naphtali Rishe. A transputer t9000 family based architecture for parallel database machines. IPPS-93, pages 121-132, 1993.

[47] Maria Cereijo Martinez. A parallel technique for solving the power system load flow analysis. Interamerican Engineering Conference, 1990.

[48] Maria Cereijo Martinez. A parallel solution to the power system load flow analysis. Southcon92, 1992.

[49] Clare D. McGillem and George R. Cooper. Continuous and Discrete Signal and System Analysis. Holt, Rinehart and Winston, 1984.

[50] Robert A. Miller. Power System Operation. McGraw Hill Book Co., second edition, 1983.

[51] Jagdish J. Modi. Parallel Algorithms and Matrix Computation. Clarendon Press, 1988.

[52] O. A. Mohammed. Lecture notes on power flow. School of Engineering Florida International University, 1989.

[53] J. E. Van Ness. Multiple factoring in the parallel solution of algebraic equation. Technical Report EPRI EL-3893, Northwestern University, Evanston, Ilinois, March 1985.

[54] J. E. Van Ness and M. V. F. Pereira. Concurrent processors for computation of power system reliability. Technical Report EL-4598, Northwestern University, 3412 Hillview Ave Palo Alto, California, 1986.

[55] A. R. Newton and A. L. Sangiovanni-Vincentelli. Relaxation-based electrical simulation. IEEE Transactions on Computer Aided Design, CAD-3(4):308 to 331, October 1983. 
[56] J. M. Ortega and W. C. Rheinboldt. Iterative Solution of Nonlinear Equations in Several Variables. Academic Press, 1970.

[57] M. A. Pai. Computer Techniques in Power System Analysis. McGraw Hill Publishing Company, 1989.

[58] C. Pottle. Array and parallel processors in on-line computations. Technical Report EPRI EL-2363, Cornell University, Ithaca New York, April 1982.

[59] Elsevier Science Publisher, editor. Parallel Processing and Applications, L'Aquila Italy, September 1987. International Conference on Parallel Processing.

[60] M. Rafian and M. J. H. Sterling. Parallel processor algorithm for power system simulation. IEEE Proceddings, 135(4):285 to290, July 1988.

[61] M. Rafian, M. J. H. Sterling, and M. R. Irving. Decomposed load-flow algorithm suitable for parallel processor implementation. IEEE Proceedings, 132(6):281 to284, November 1985.

[62] Garry Rodrigue, editor. Parallel Processing for Scientific Computing, Los Angeles , California, December 1987. Siam.

[63] M. La Scala, G. Sblendorio, and R. Sbrizzai. Parallel-in-time implementation of transient stability simulations on a transputer network. IEEE Transactions on Power Systems, 9:1117-1125, May 1994.

[64] Fred C. Schweppe. Power system static-state estimation, part 1: Exact model. Transactions on Power Apparatus and Systems, January 1970.

[65] Y. Sekine and A. Yokoyama. A new load flow calculation suited for parallel processing. Proceedings of the 8th Power Systems Computational Conference, pages 529-533, 1984.

[66] Robert Stein and Jr. William Hunt. Electric Power System Components. Van Nostrand Reinbolh Co., 1979.

[67] B. Stott and O. Alsac. Fast decoupled load flow. IEEE Transactions on Power Apparatus and Systems, pages 859-869, May 1973.

[68] B. Stott, O. Alsac, and A. Monticelli. Security analysis and optimization. Proceedings of the IEEE, 12:1623-1644, 1987.

[69] Doron Tal, John Comfort, and Maria Martinez. The fast fourier transform as a test case for a systolic data flow machine. Conference on Parallel Processing, 1989.

[70] Sarosh N. Talukdar, Sam S. Pyo, and Theo C. Giras. Asynchronous procedures for parallel processing. IEEE Transactions on Power Apparatus and Systems, PAS102(11):3652-3659, November 1983.

[71] H. Taoka, S. Abe, and S. Takeda. Multiprocessor system for power system analysis. Real Time Programming, 1981.

[72] W. F. Tinney, V. Brandwajn, and S. M. Chan. Sparce vector methods. IEEE Transactions on Power Apparatus and Systems, PAS-104(2):295-301, February 1985.

[73] A. Torralba, A. Gomez, and L. G. Franquelo. Parallel solution of power analysis problems. E.T.S. Ing. Industriales Sevilla, Spain, 1988. 
[74] Lewis W. Tucker and George G. Robertson. Architecture and applications of the connection machine. IEEE Computer, page 26 to 38, August 1988.

[75] Daniel Tylavsky and Anjan Bose. Parallel processing in power systems computation. IEEE Transactions on Power Systems, 7(2):629-637, May 1992.

[76] R. S. Varga. Matrix Iterative Analysis. Prentice-Hall, 1962.

[77] Theodore Wildi. Electric Power Technology. John Wiley and Sons, 1981.

[78] A. Yokoyama and Y. Sekine. A new parallel processing method for load flow calculation using sensitivity matrix and its comparison with the diakoptics based method. Denki Gakkai Ronbunshi, 104-B(11):757-764, November 84.

[79] David Young and R. T. Gregory. A Survey of Numerical Mathematics. Addison-Wesley Publishing Company, 1973.

[80] J. Zaborszky, G. Huang, and K. W. Lu. A textured model for computationally efficient reactive power control and management. IEEE Transactions on Power Apparatus and Systems, PAS-104(7):1718 to 1727 , July 1985.

[81] J. Zaborszky, M. Llic-Spong, and K. W. Whang. Homogeneous, nonlinear, load-flow algorithm for HV-AC. Electrical Power and Energy Systems, 4(4):233 to 244, October 1982.

[82] J. Zaborszky, K. Prasad, K. Whang, M. Mic-Spong, et al. Towards a comprehensive analysis and operating practice of the large compound HV-AC-DC system. Technical report, US Department of Energy, February 1982.

[83] H. Zaininger, A. Wood, H. Clark, and J. Burns. Synthetic electric utility systems for evaluating advance technologies. Technical Report EPRI-EM-285, EPRI, Schenectady, N.Y., 1977. 


\section{A STOPPING ALGORITHM}

\section{ALGORITHM SYSTEM TERMINATION}

* Convergence is 1 and nonconvergence is 0

PAR $i=1$ to numnodes

$$
\operatorname{conv}(i)=0
$$

* Do until convergence of system

Do Until STOP

* Read in new values for children

For $j=1$ to numchild

Read child(j)

End For

* Perform calculations

If (converged) then $\operatorname{conv}(i)=1$

* Check if ther is node convergence

For $j=1$ to numchild

$$
\operatorname{conv}(i)=\operatorname{conv}(i) \cdot \operatorname{and} \cdot \operatorname{child}(j)
$$

End For

* Send message to parent

Send conv(i) to Parent Node

* Read Parent to see if it is a stop

Read Parent Node

End Do

End PAR 


\section{B SWITCH SETUP}

\section{ALGORITHM SWITCH SETUP}

tableof connections is null

connection is False

For $i=1, n$

While $j=1,8$ Or connection Do

If Connection $(i, j)$ in tableof connections

then connection

While $k=1,4$ Or not portavailable Do

If oport $t_{i, k}$ available

then portavailable

End While

If not portavailable

then make - port - available $\left(P U_{i}\right.$, output $)$.

While $k=1,4$ Or not portavailable Do

If iport $_{j, k}$ available

then portavailable

End While

If not portavailable

then make - port - available $\left(P U_{j}\right.$, input $)$.

Add To tableof connections connection oport $_{i, k}$ with iport $_{j, k}$

End While

End For 


\section{ALGORITHMS}

This section includes the parts of the algorithms used to test the PLF in Fortran.

Program Loadflow

MARIA CEREIJO MARTINEZ

A NEW PARALLEL TECHNIQUE FOR THE SOLUTION OF THE LOAD FLOW ANALYSIS

A PROGRAM WRITTEN FOR THE ABOVE DISSERTATION FOR THE REQUIREMENTS OF A DOCTOR OF PHILOSOPHY IN COMPUTER SCIENCE

FLORIDA INTERNATIONAL UNIVERSITY

MIAMI, FLORIDA

MARCH 1993

THIS PROGRAM CALCULATES THE POWER SYSTEM LOAD FLOW ANALYSIS. GAUSS-SEIDEL OR NEWTON-RAPHSON ALGORITHMS CAN BE RUN.

A SIMULATION OF A PARALLEL ALGORITHM, PLF, CAN ALSO RUN.

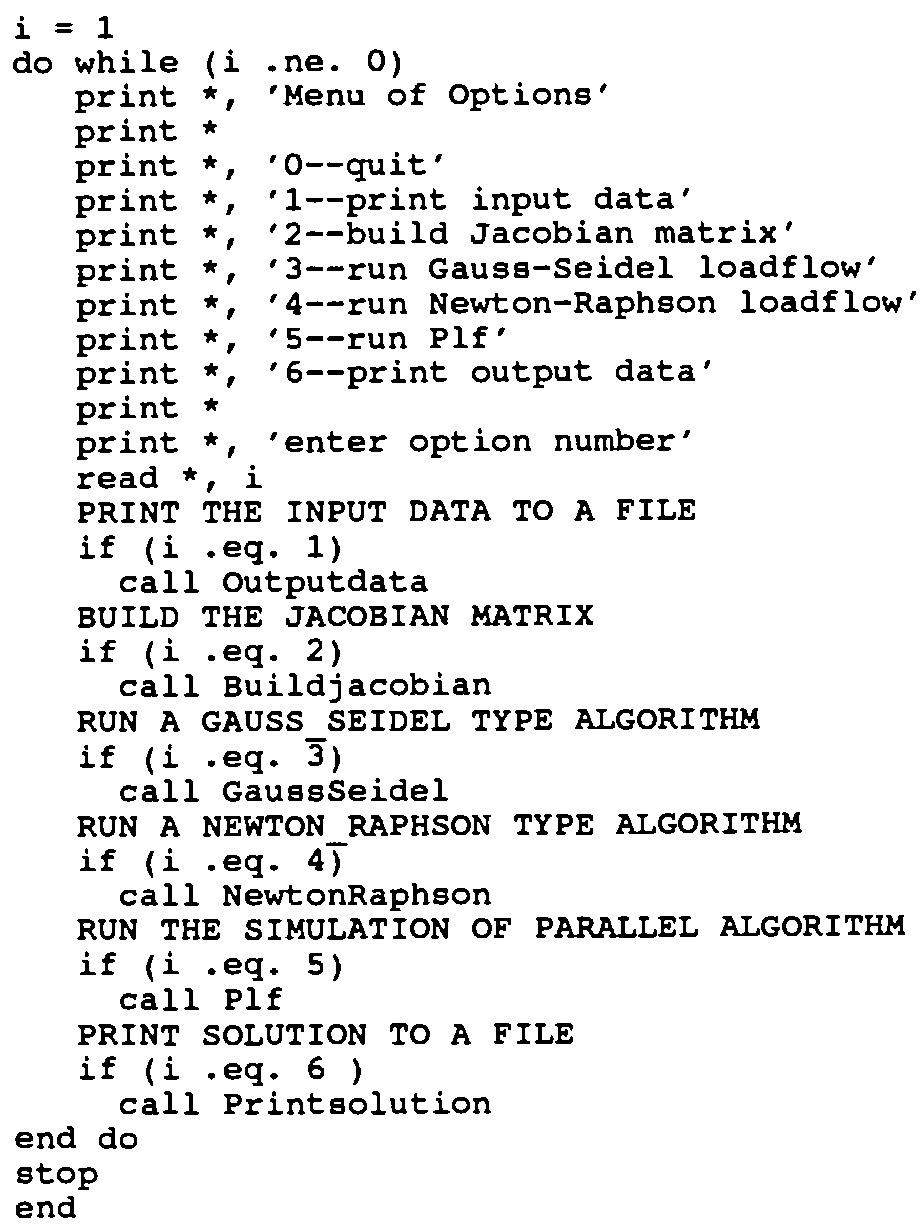




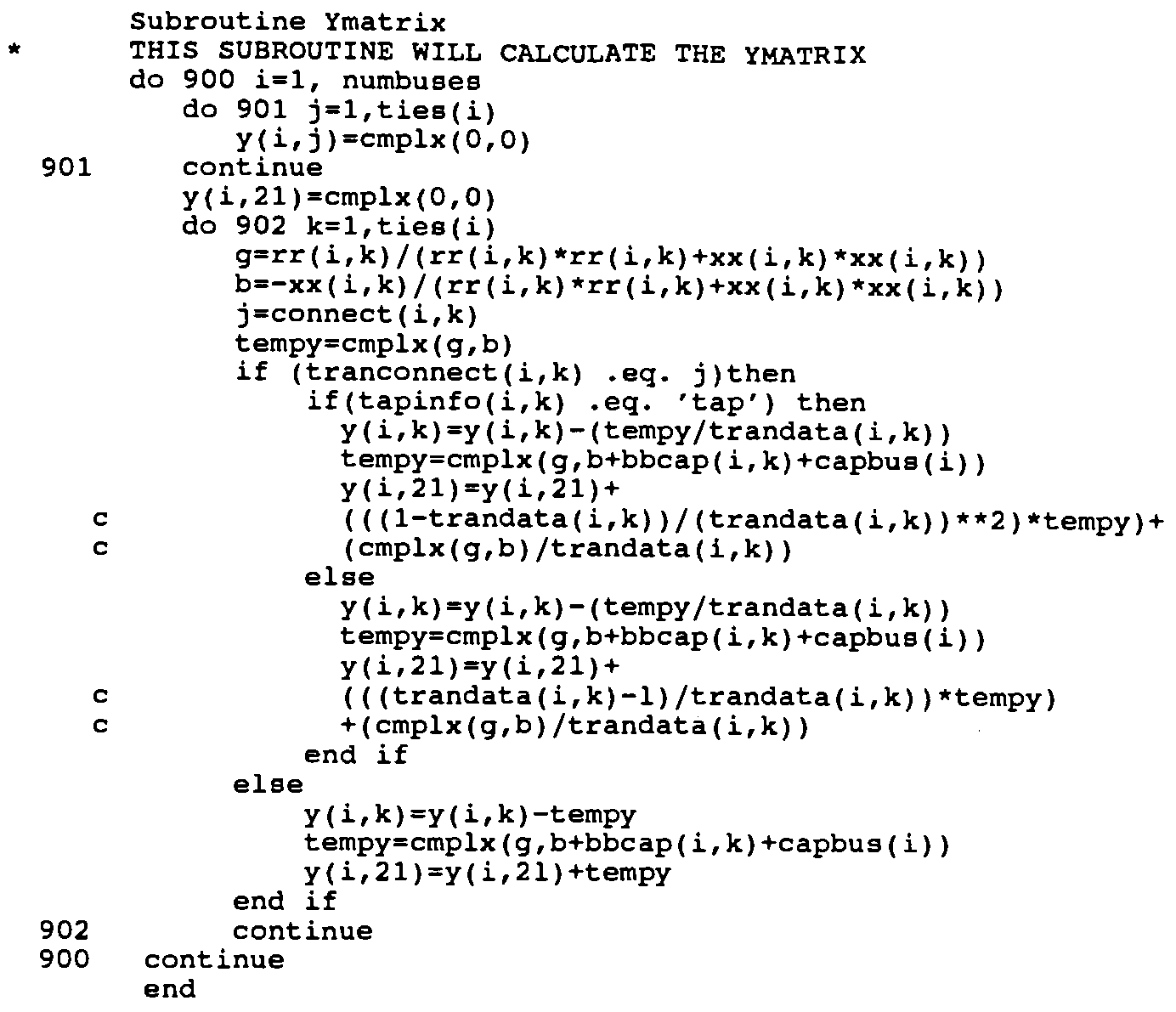




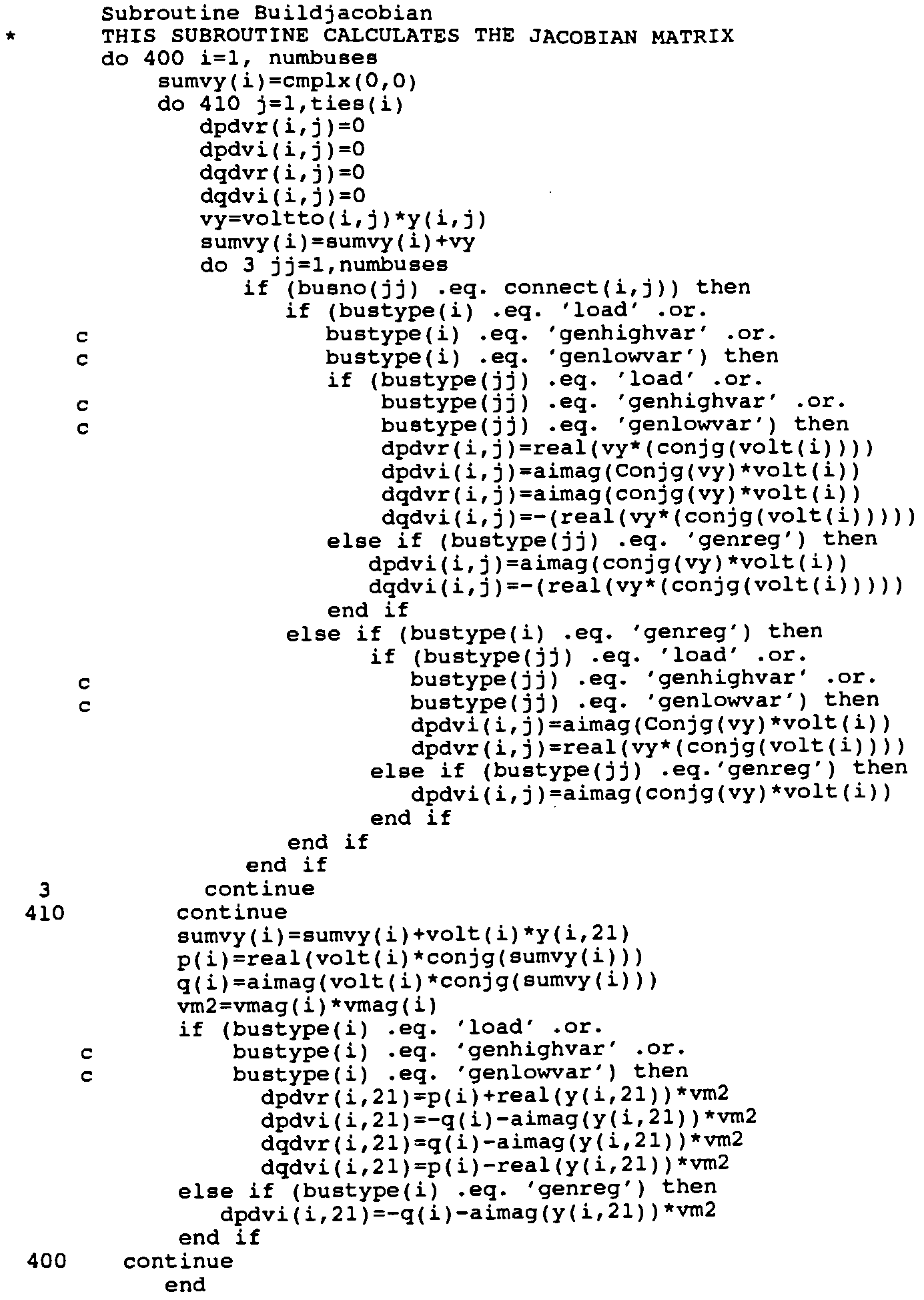


Subroutine NewtonRaphson

THIS SUBROUTINE IS A NEWTON-RAPHSON ALGORITHM. IT CALLS

JACOBIAN THEN DOES A GAUSSIAN ELIMINATION TO SOLVE

THE SYSTEM.

pmaxmismat $c h=0$

qmaxmismat $c h=0$

novarlimiting=' false'

iter $=0$

do while (iter .le. 75 .and. (pmaxmismatch .ge. 0001 .or.

c gmaxmismatch .ge. .0001 .or. novarlimiting .eq. 'false'))

iter $=$ iter +1

pmaxmismatch $=0$

qmaxmismatch $=0$

do $580 i=1$, numbuses

$\operatorname{delmag}(i)=0$

delphase $(i)=0$

$\operatorname{deltap}(i)=0$

$\operatorname{deltaq}(i)=0$

580

continue

call buildjacobian

do $500 \quad i=2$, numbuses

$\operatorname{deltap}(i)=$ ssched $(i)-p(i)$

if (abs(deltap(i)) .gt. pmaxmismatch) then

pmaxmismatch $=$ abs $(\operatorname{deltap}(i))$

maxpbus=busno (i)

end if

if (bustype(i) .eq. 'load' .or.

c bustype(i) .eq. 'genhighvar' .or.

c bustype(i) .eq. 'genlowvar') then

$\operatorname{deltaq}(i)=q \operatorname{seched}(i)-q(i)$

if (abs(deltaq(i)) .gt. qmaxmismatch) then

qmaxmismatch= abs(deltaq(i))

end if

500

if maxqbus=busno $(i)$

c

if (pmaxmismatch . It. .01 .and.

DO GAUSSIAN ELIMINATION

do $590 \quad i=1$, numbuses

if (bustype(i) .ne. 'swing') then

$j=21$

if (bustype(i) .eq. 'load' .or.

c bustype(i) .eq. 'genhighvar' .or.

c bustype(i) .eq. 'genlowvar') then

$\operatorname{detd}=\operatorname{dpdv} i(i, 21) * \operatorname{dqdvr}(i, 21)-\operatorname{dqdv} i(i, 21) * \operatorname{dpdvr}(i, 21)$

dll=dqdvr $(i, 21) /$ detd

dl2 $=-\operatorname{dpdvr}(i, 21) / \operatorname{detd}$

d2 $1=-\operatorname{dqdv} i(i, 21) / \operatorname{detd}$

$d 22=d p d v i(i, 21) / \operatorname{det} d$

else if (bustype(i) .eq. 'genreg') then

d1 $1=1 / \operatorname{dpdvi}(i, 21)$

d $12=0$

d $21=0$

d22 $=0$

end if

tdeltap $=$ d $11 * \operatorname{deltap}(i)+$ d12*deltaq $(i)$

tdeltaq $=d 21 * \operatorname{deltap}(i)+\mathrm{d} 22 * \operatorname{deltaq}(i)$

deltap $(i)=$ tdeltap

deltaq $(i)=t$ deltaq

do $588 k=1$, numbuses 
do $59 \mathrm{kk}=1$, ties (i)

if (busno(k) .eq. connect $(i, k k)$ ) then tdpdvi=d11*dpdvi $(i, k k)+d 12 * d q d v i(i, k k)$ tdpdvr $=d 11 * d p d v r(i, k k)+d 12 * \operatorname{dqdvr}(i, k k)$ tdqdvi $=d 21$ *dpdvi $(i, k k)+d 22 * d q d v i(i, k k)$ tdqdvr $=d 21 * d p d v r(i, k k)+d 22 * d q d v r(i, k k)$ dpdvi $(i, k k)=t d p d v i$ $\operatorname{dpdvr}(i, k k)=t \operatorname{dpdvr}$ dqdvi $(i, k k)=t d q d v i$ dqdvr $(i, k k)=t d q d v r$

end if continue do $58 j=1, t i e s(i)$

if (connect $(i, j)$. lt . busno(i)) then tapdvi=dpdvi $(i, j)$ tdpdvr=dpdvr $(i, j)$ tdqdvi=dqdvi $(i, j)$ tdqdvr=dqdvr $(i, j)$

do $60 j j=1$, numbuses if(busno(jj) .eq. connect(i,j)) then deltap $(i)=\operatorname{deltap}(i)-$ (tdpdvi*deltap (jj) +tdpdvr*deltaq (jj)) $\operatorname{deltaq}(i)=\operatorname{deltaq}(i)-$

end if (tdqdvi*deltap $(j j)+t d q d v r \star \operatorname{deltaq}(j j)$ )

continue do $589 k=j, t i e s(i)$

do $89 k \mathrm{k}=1$, numbuses if (busno(kk) .eq. j) then $\operatorname{dpdvi}(\mathbf{i}, k)=\operatorname{dpdvi}(\dot{i}, k)-$ (tdpdvi *dpdvi (kk, k) +tdpdvr*dqdvi (kk, k) ) $\operatorname{dpdvr}(i, k)=\operatorname{dpdvr}(i, k)-$ (tdpdvi*dpdvr $(\mathbf{k k}, \mathbf{k})+t \operatorname{dpdvr} \star \operatorname{dgdv} r(k \mathbf{k}, \mathbf{k})$ ) $\operatorname{dqdvi}(i, k)=\operatorname{dqdvi}(i, k)-$ (tdqdvi*dpdvi(kk,k)+tdqdvr*dqdvi(kk,k)) $\operatorname{dqdvr}(i, k)=\operatorname{dqdvr}(i, k)-$ end if (tdqdvi*dpdvr $(\mathbf{k k}, \mathbf{k})+t d q d v r \star d q d v r(k \mathbf{k}, k)$ )

continue continue end if continue end if continue DO BACK SUBSTITUTION

do $510 i=$ numbuses $, 1,-1$ delphase $(i)=\operatorname{deltap}(i)$

delmag $(i)=\operatorname{deltag}(i)$

if ( $i$. lt. numbuses) then do $520 j=i+1$, numbuses do $52 j j=1, t i e s(i)$ if (connect $(i, j j)$.eq. busno(j)) then delphase $(i)=$ delphase $(i)-$

c

c $(\operatorname{dpdvi}(i, j j) \star \operatorname{del} \operatorname{phase}(j)+\operatorname{dpdvr}(i, j j) * \operatorname{delmag}(j))$ delmag $(i)=\operatorname{delmag}(i)-$ (dqdvi $(i, j j)$ *delphase $(j)+\operatorname{dqdvr}(i, j j)$ adelmag $(j))$ end if continue continue end if 
do $530 i=1$, numbuses

phase $(i)=$ phase $(i)+$ delphase $(i)$

$\operatorname{vmag}(i)=\operatorname{vmag}(i) *(1+\operatorname{delmag}(i))$

$\operatorname{volt}(i)=\operatorname{cmplx}(\operatorname{vmag}(i) * \cos ($ phase $(i)), \operatorname{vmag}(i) \star \sin ($ phase $(i)))$

do $53 j=1$, numbuses

do 54 j $j=1$, ties $(j)$

if $($ connect $(j, j j)$.eq. busno(i)) then $\operatorname{voltto}(j, j j)=\operatorname{volt}(i)$

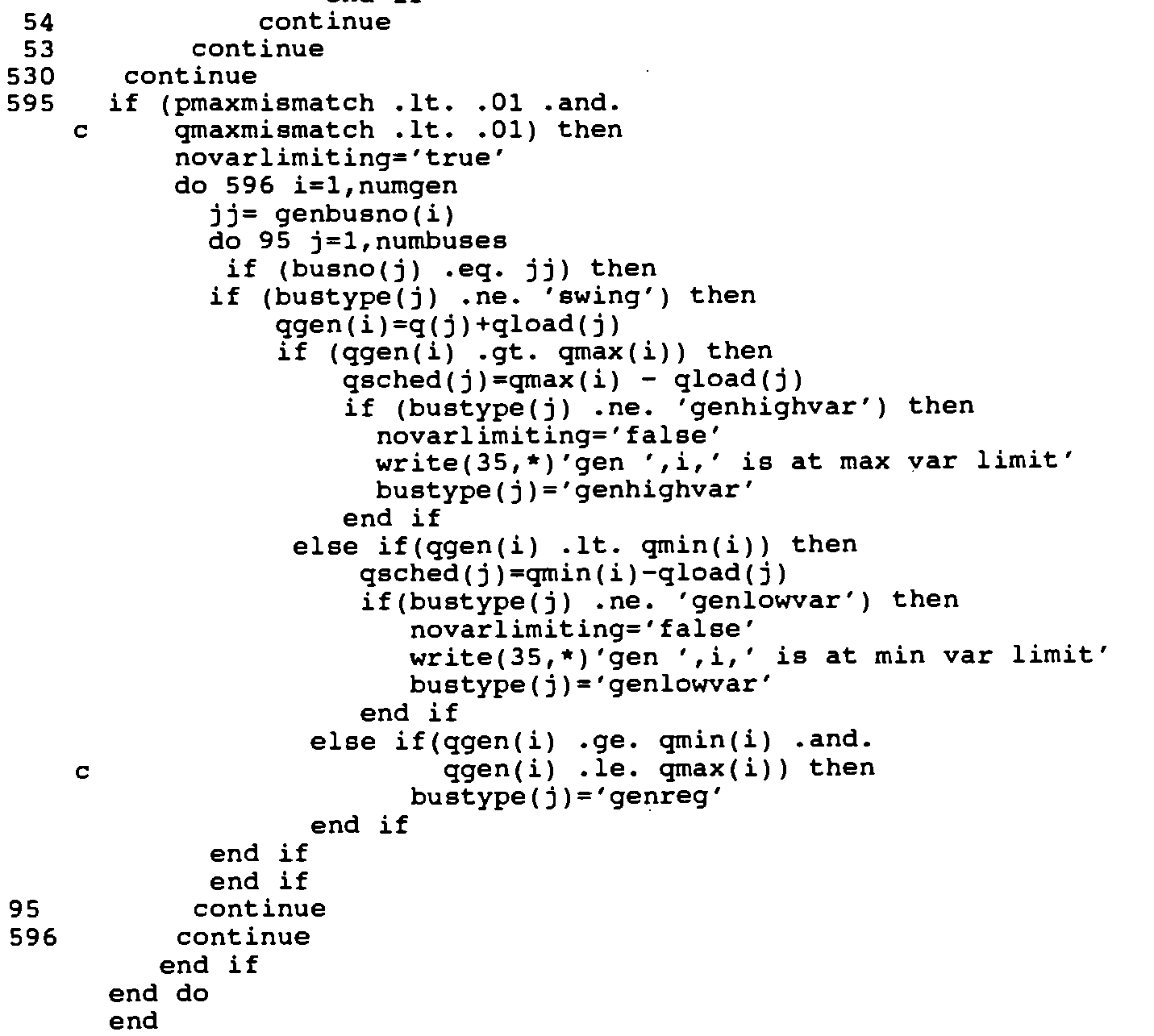


Subroutine plf

THIS SUBROUTINE WILL RUN THE GAUSS-SEIDEL ALGORITHM IN

PARALLEL USING THE PARALLEL DATA.

iter $=0$

pmaxmismatch $=0$

qmaxmismatch $=0$

novarlimiting =' false'

do while (iter .le. 150 . and. (pmaxmismatch .ge. .0001 or.

c qmaxmismatch .ge. .0001 .or. novarlimiting .eq. 'false'))

iter $=$ iter +1

pmaxmismatch $=0$

qmaxmismatch $=0$

perrorbus $=0$

gerrorbus $=0$

do $600 i=1$, numbuses

if (bustype(i) .ne. 'swing') then

oldv=volt $(i)$

pq=amplx (psched (i), qsched (i))

templ=conjg $(\mathrm{pq} / \mathrm{volt}(\mathrm{i}))$

soffdia $=\operatorname{cmplx}(0,0)$

ic $=99999$

do $610 j=1$, ties ( $i$ )

if (ic . ne. connect $(i, j))$ then

soffdia=soffdia+(voltto $(i, j) * y(i, j))$

$i c=$ connect $(i, j)$

610 continue

templ=templ-sof fdia

$e=t \operatorname{emp} 1 / y(i, 21)$

changevnew $=e-01 d v$

THIS IS AN ACCELERATOR FACTOR

$e=0 l d v+1.6 *$ changevnew

emag $=\operatorname{sqrt}(\operatorname{real}(e) * \operatorname{real}(e)+\operatorname{aimag}(e) * \operatorname{aimag}(e))$

ephase=atan (aimag (e)/real (e))

IF THE BUS IS A GENERATOR BUS THEN THE VOLTAGE MAGNITUDE

IS SET TO THE SCHEDULED MAGNITUDE AND A NEW REACTIVE

POWER IS CALCULATED

if (bustype(i) .eq. 'genreg') then

emag $=v m a g(i)$

e=cmplx (emag* cos (ephase), emag*sin (ephase) )

temp $1=y(i, 21) * e$

templ $1=$ templ+sof fdia

temp $1=$ conjg (templ)

temp $1=$ temp $1 * e$

end if

qsched $(i)=$ aimag (templ)

$\operatorname{volt}(i)=e$

$\operatorname{vmag}(i)=e m a g$

phase $(i)=e p h a s e$

end if

UPDATE THE VOLTTO MATRIX. THIS WOULD BE THE COMMUNICATION TO CONNECTING NODES.

do $660 \mathrm{k}=1$, ties (i)

do $663 \mathrm{kk}=1$, numbuses

if (busno(kk) .eq. k) then

do $666 \mathrm{kkk}=1$, ties(kk)

if (connect (kk,kkk) .eq. busno(i)) then

voltto $(k k, k k k)=\operatorname{volt}(i)$

666 end if continue

end if 


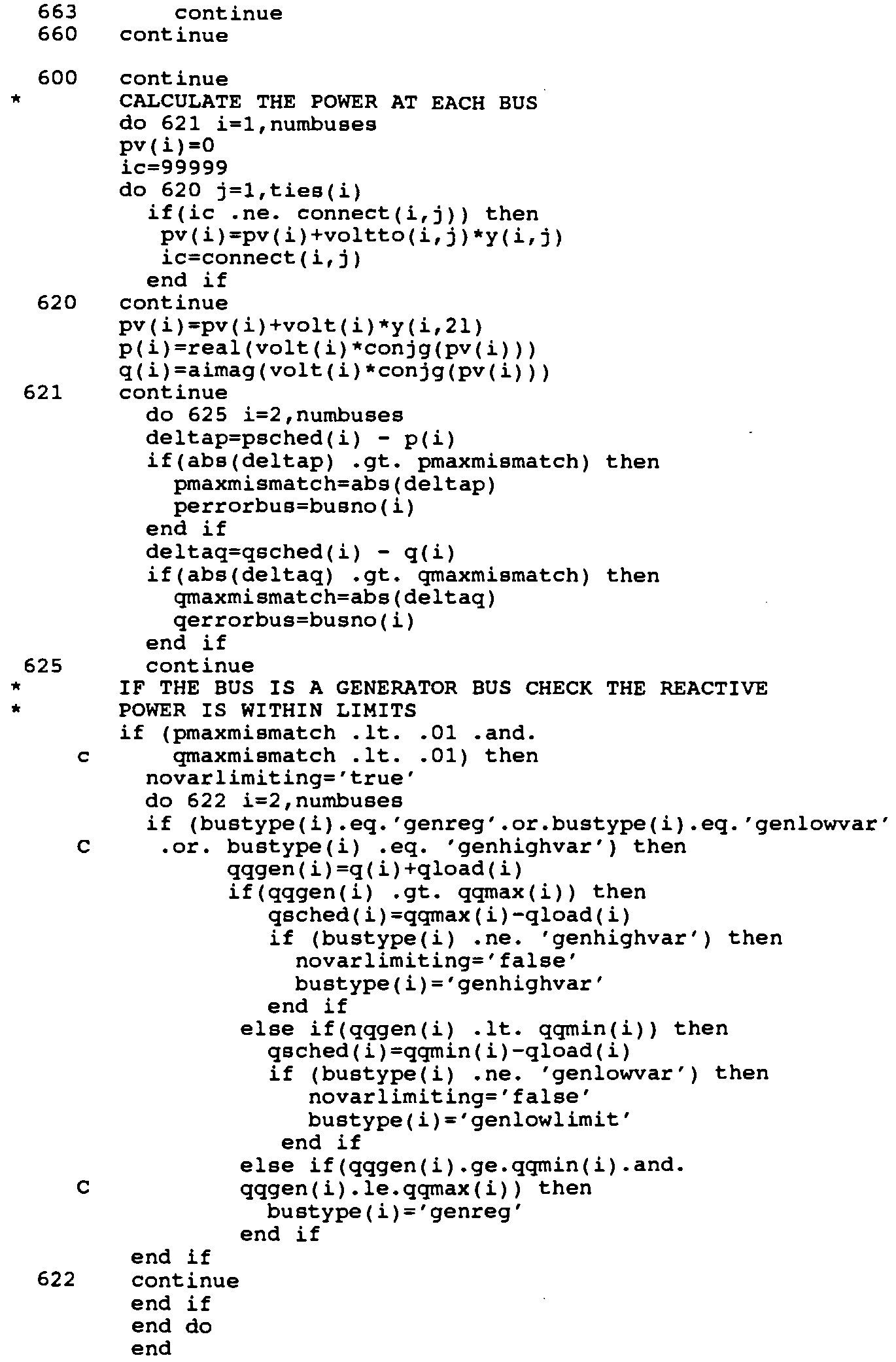


This section includes the Occam Programs used to simulate the PLF on the Transputers.

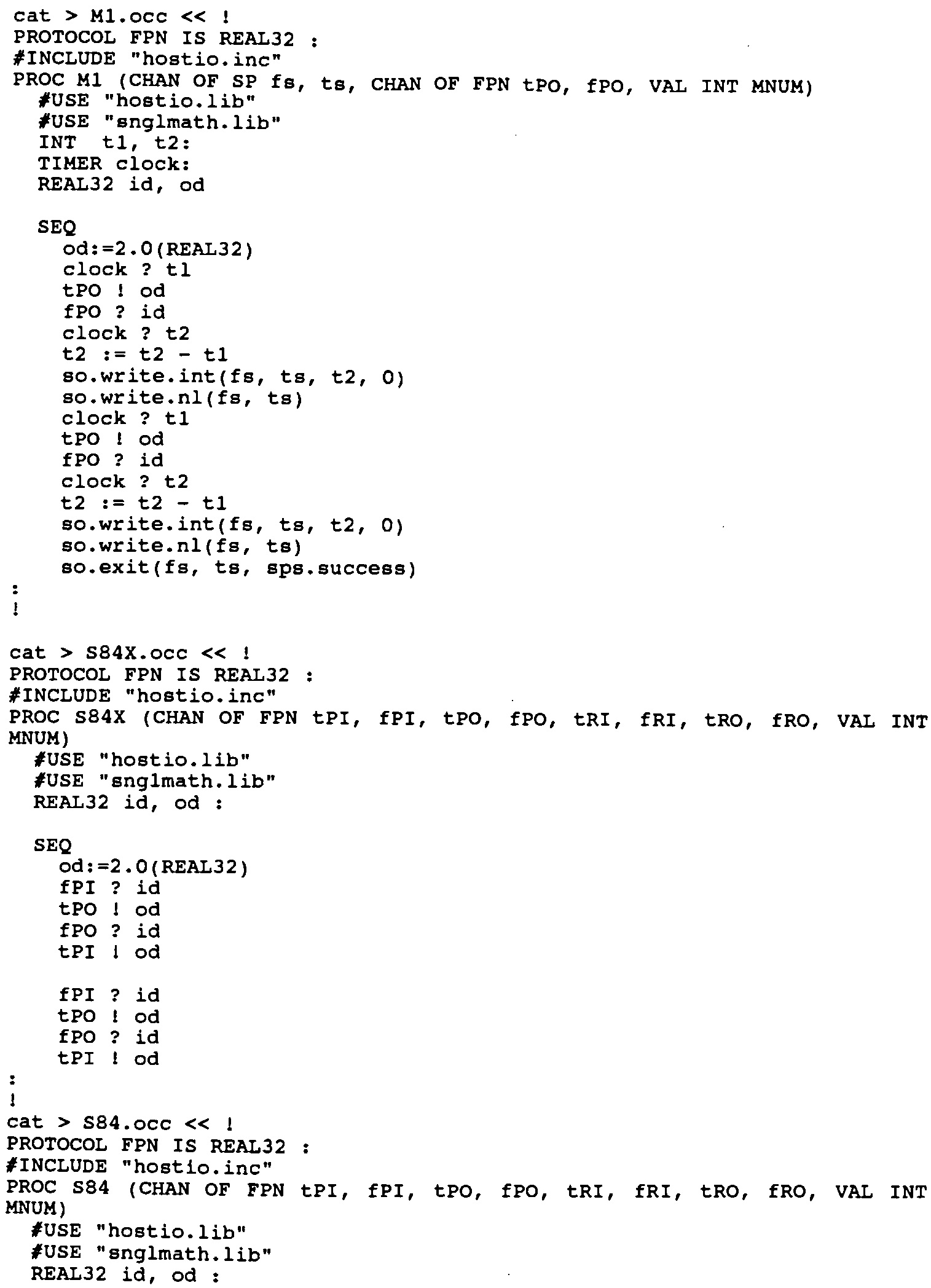




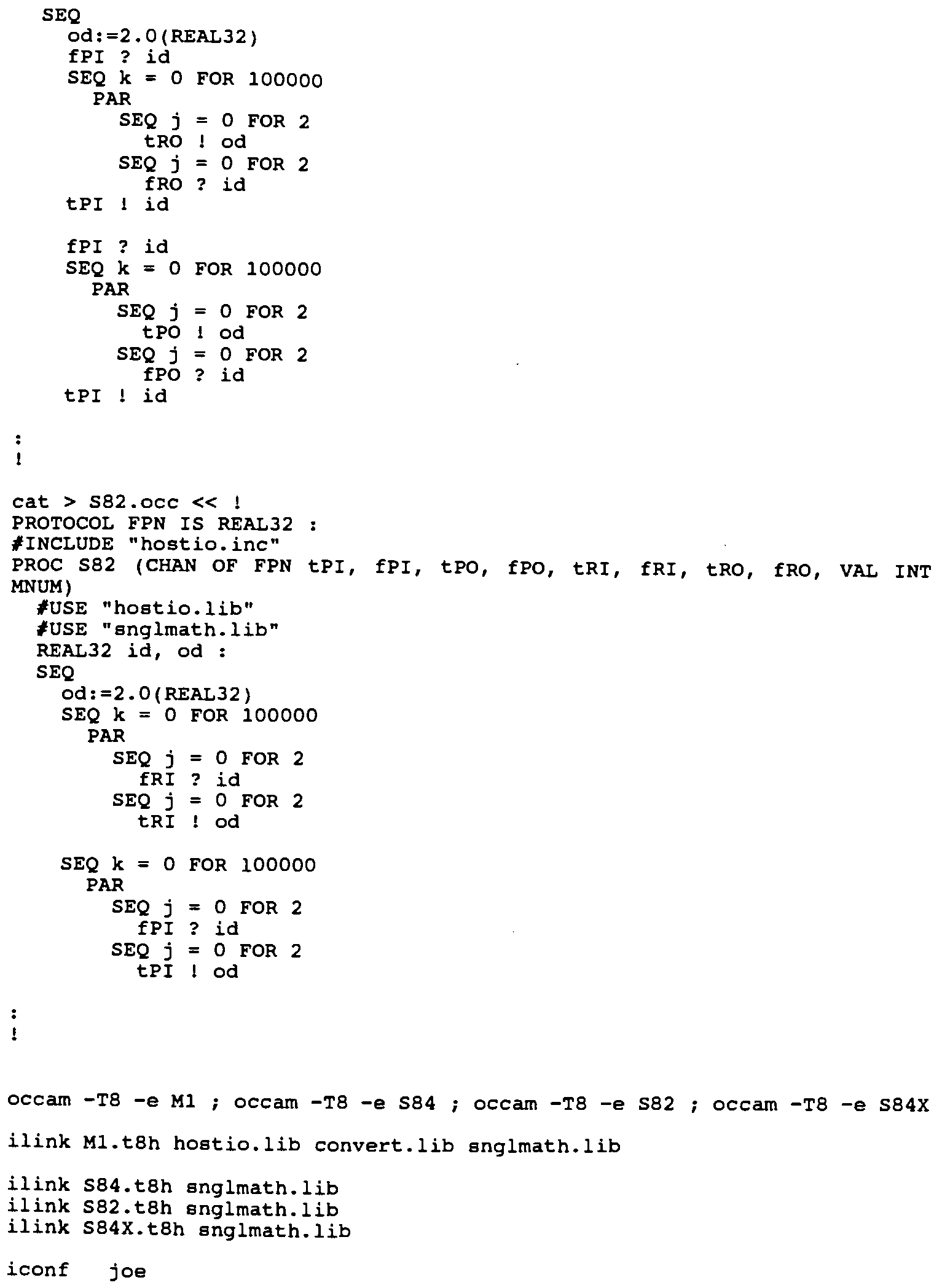




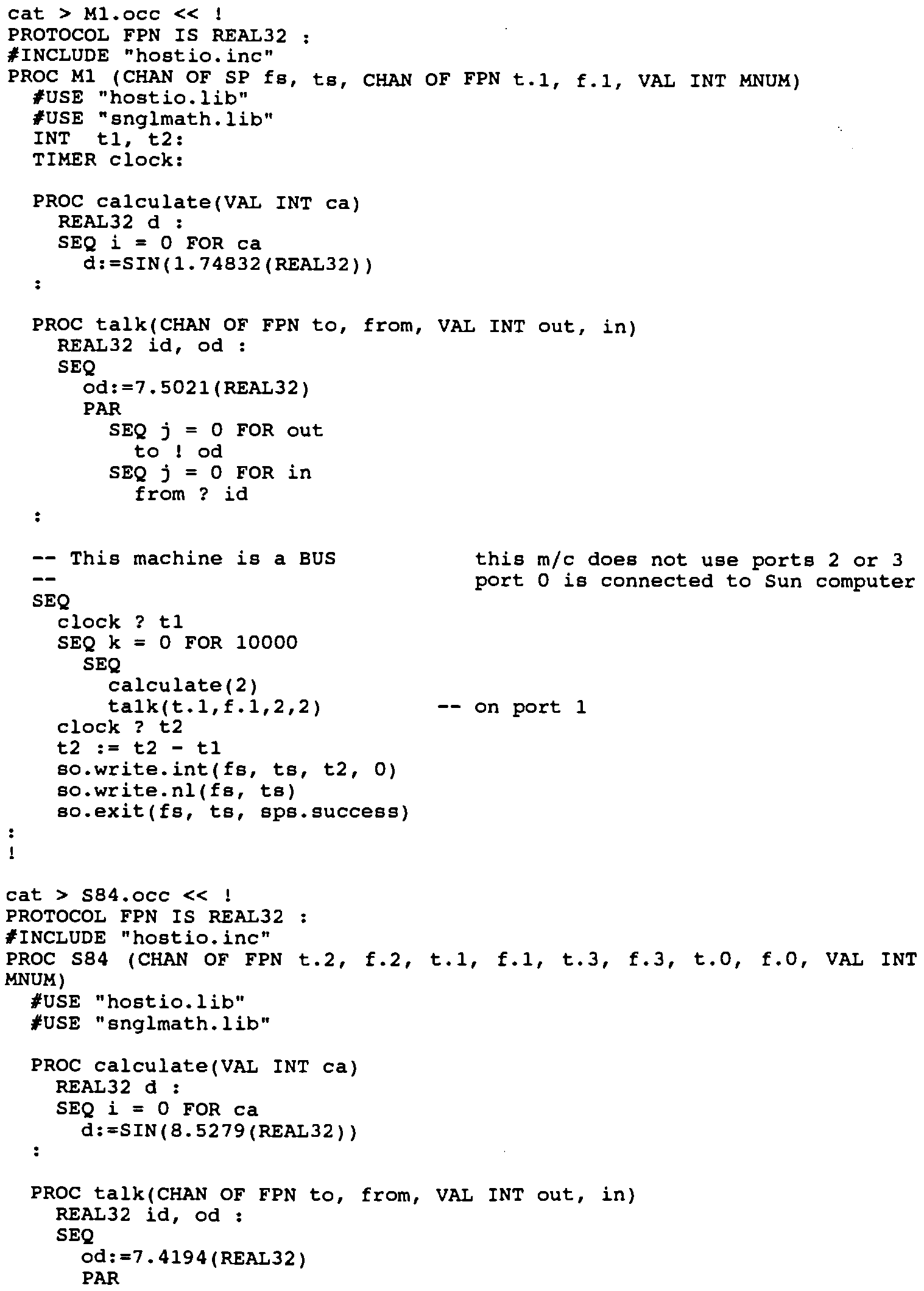

this $\mathrm{m} / \mathrm{c}$ does not use ports 2 or 3 port 0 is connected to sun computer 


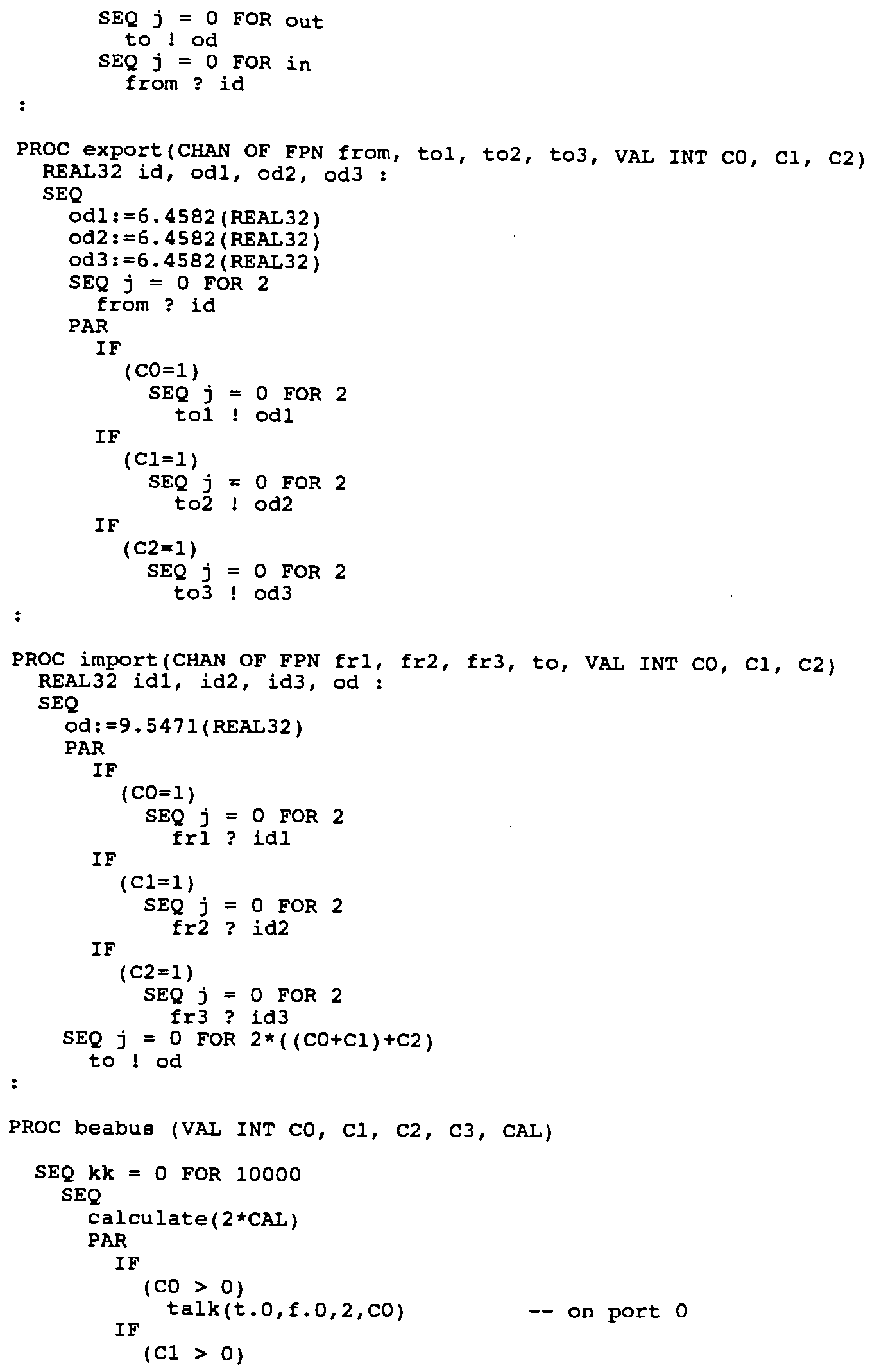




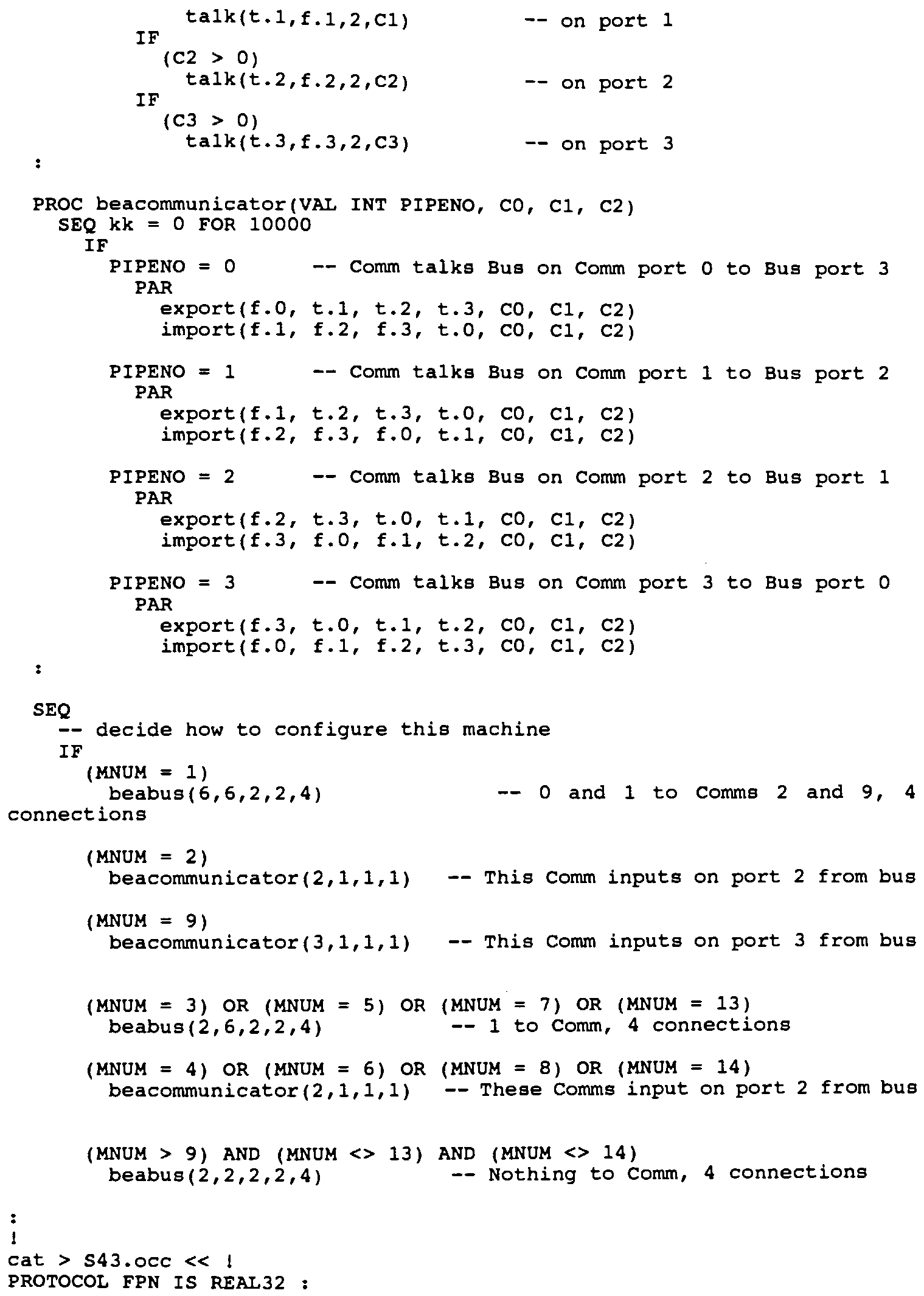




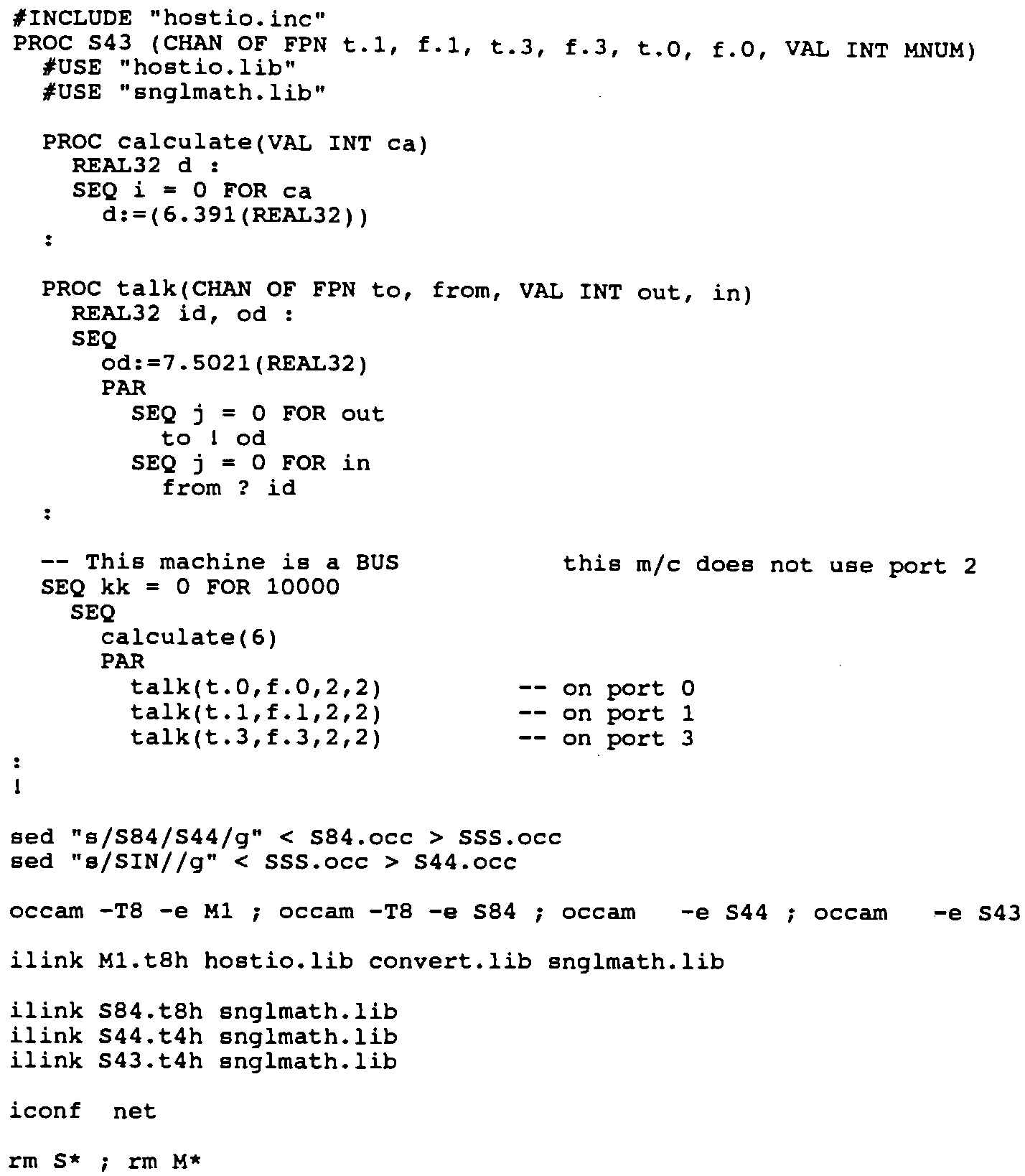




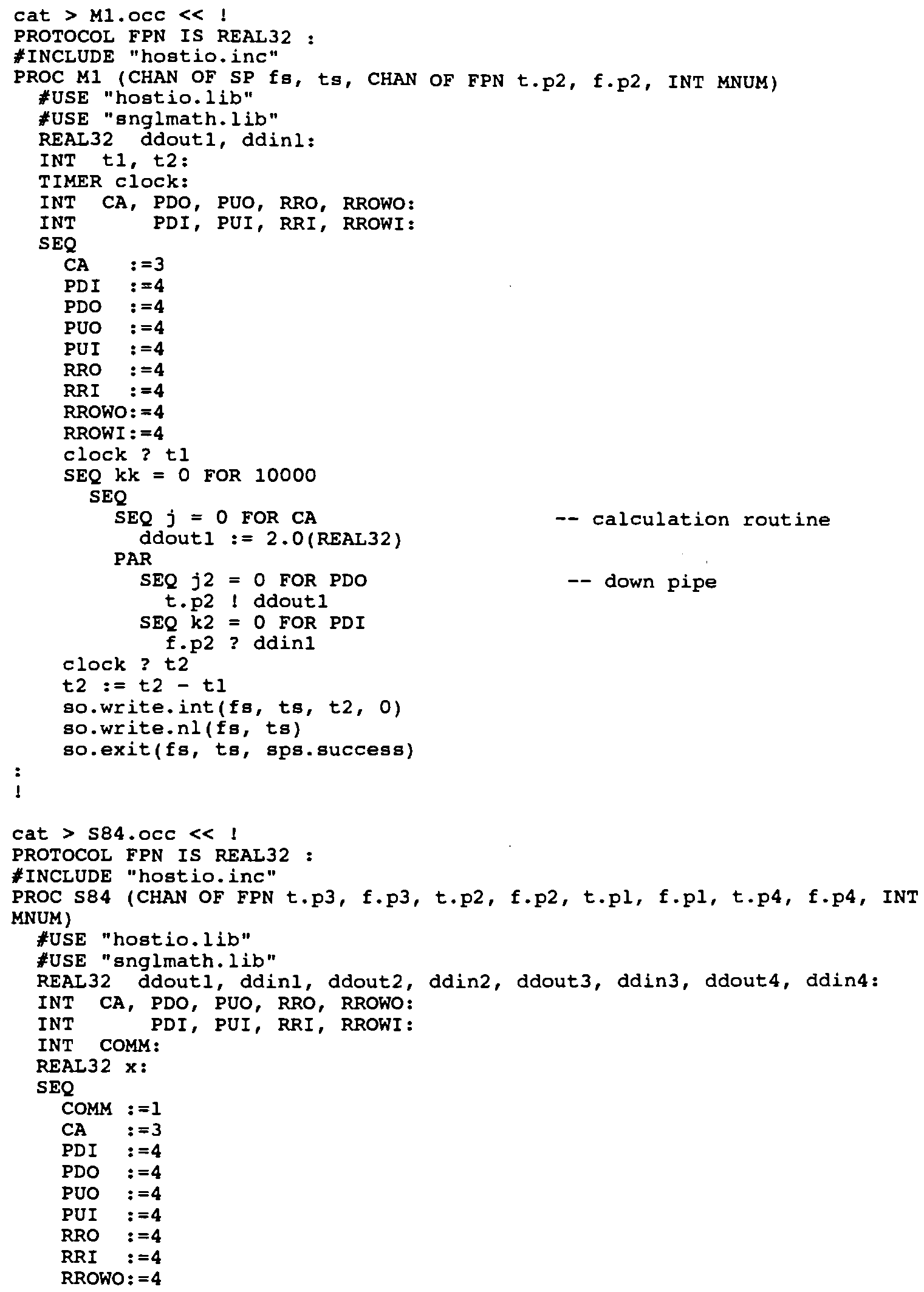




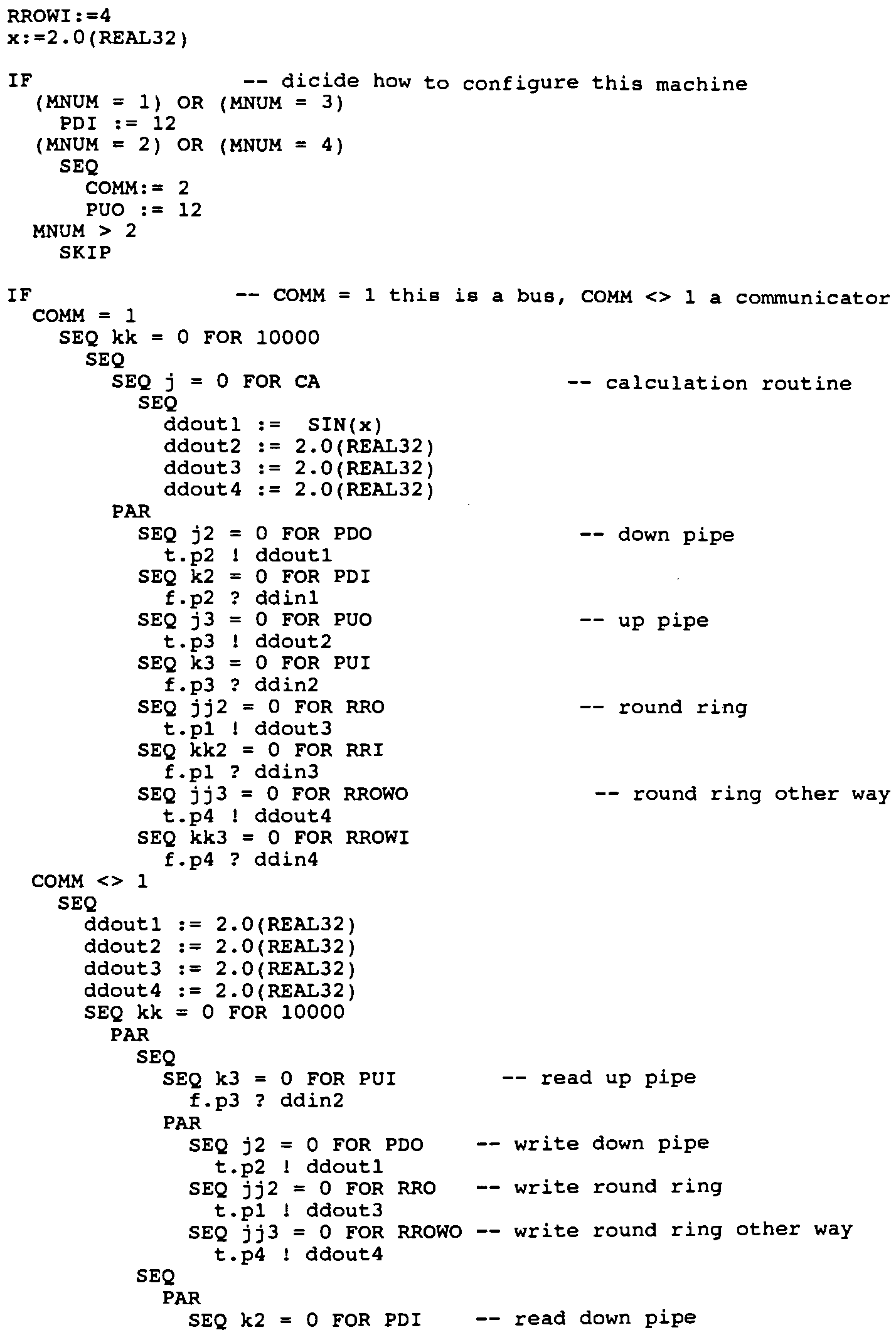




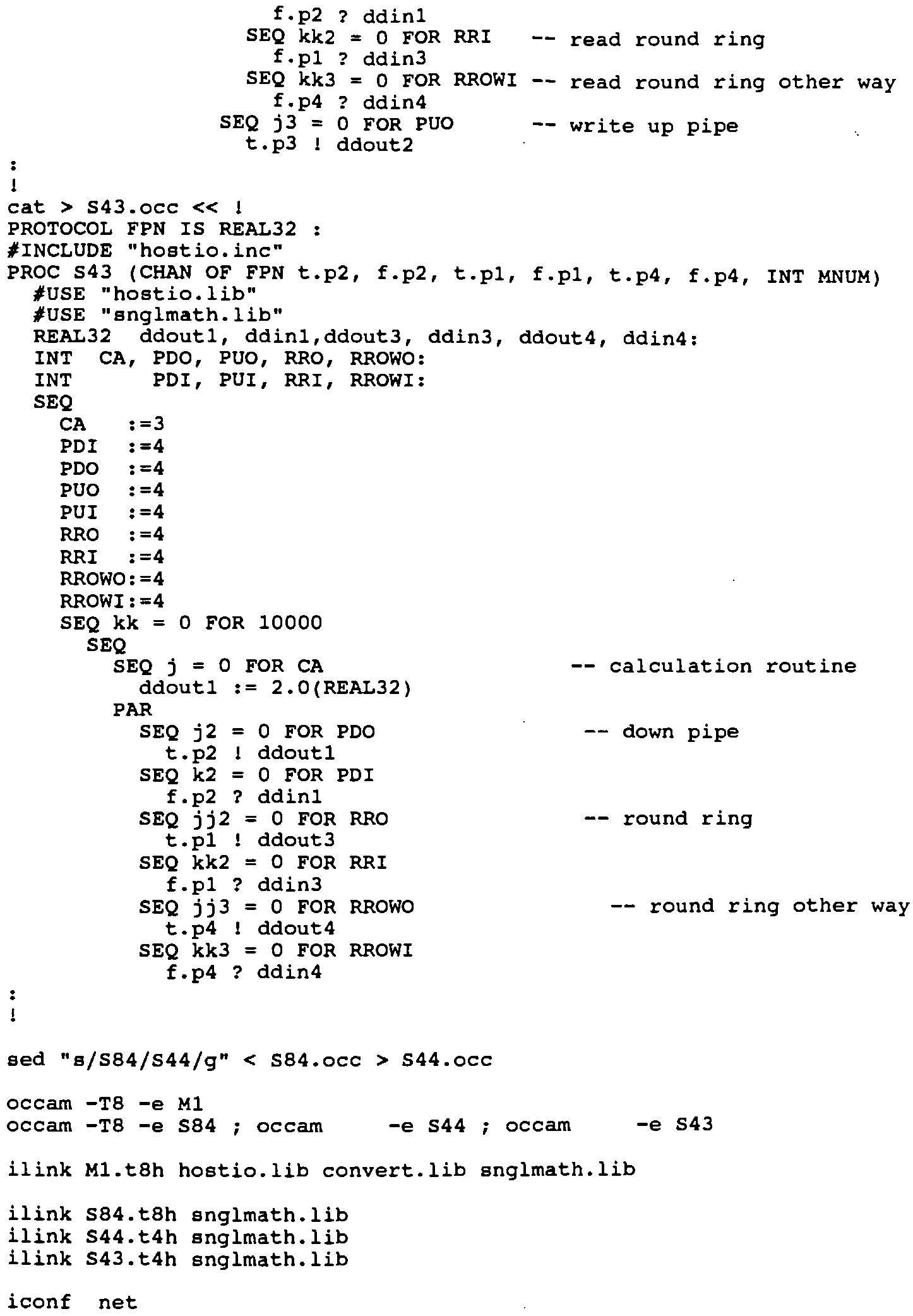




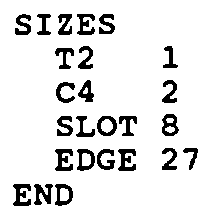

C4 0 , LINK 16 TO EDGE 0 C4 0 , LINK 17 TO EDGE 1

C4 0 , IINK 18 TO EDGE 2

C4 0 , LINK 19 TO EDGE 3

C4 0 , LINK 20 TO EDGE 4

C4 0 , LINK 21 TO EDGE 5

C4 0, LINK 22 TO EDGE 6

C4 0 , LINK 23 TO EDGE 7

C4 1, IINK 16 TO EDGE 8

C4 1, LINK 17 TO EDGE 9

C4 1 , LINK 18 TO EDGE 10

C4 1 , IINK 19 TO EDGE 11

C4 1 , LINK 20 TO EDGE 12

C4 1, LINK 21 TO EDGE 13

C4 1, LINK 22 TO EDGE 14 
C4 1, LINK 23 TO EDGE 15

C4 1, LINK 24 TO EDGE 16

C4 1, LINK 25 TO EDGE 17

C4 1, LINK 26 TO EDGE 18

C4 1, LINK 27 TO EDGE 19

C4 1, LINK 28 TO EDGE 20

C4 1, LINK 29 TO EDGE 21

C4 1, LINK 30 TO EDGE 22

C4 1, LINK 31 TO EDGE 23

C4 0 , LINK 0 TO EDGE 24

C4 0, LINK 31 TO EDGE 25

C4 1 , LINK 0 TO EDGE 26

END

DEF B012a

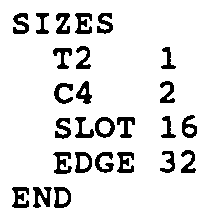




$$
\begin{aligned}
& \text { C4 1, LINK 5, I TO EDGE } 3,0 \\
& \text { C4 } 0 \text {, LINK 6,0 TO EDGE 4,I } \\
& \text { C4 1, LINK 6, I TO EDGE 4,0 } \\
& \text { C4 } 0 \text {, LINK 3,0 TO EDGE 5, I } \\
& \text { C4 1, LINK 3, I TO EDGE 5,0 } \\
& \text { C4 1, LINK 1,0 TO EDGE 6, I } \\
& \text { C4 } 0 \text {, LINK } 1, I \text { TO EDGE } 6,0 \\
& \text { C4 1, LINK 7,O TO EDGE 7,I } \\
& \text { C4 } 0 \text {, LINK 7, I TO EDGE 7,0 } \\
& \text { C4 1, LINK 29,0 TO EDGE 8, I } \\
& \text { C4 } 0 \text {, LINK } 29, \text { I TO EDGE } 8,0 \\
& \text { C4 1, LINK 30,0 TO EDGE 9,I } \\
& \text { C4 } 0 \text {, LINK } 30 \text {, I TO EDGE } 9,0 \\
& \text { C4 } 0, \text { LINK } 31,0 \text { TO EDGE 10, I } \\
& \text { C4 1, LINK 31, I TO EDGE 10,0 } \\
& \text { C4 } 0 \text {, LINK } 28,0 \text { TO EDGE } 11, \text { I } \\
& \text { C4 1, LINK } 28 \text {, I TO EDGE } 11,0 \\
& \text { C4 1, LINK 24, O TO EDGE 12, I } \\
& \text { C4 } 0 \text {, LINK 24, I TO EDGE } 12,0 \\
& \text { C4 } 0 \text {, LINK } 25,0 \text { TO EDGE } 13, \text { I } \\
& \text { C4 1, LINK 25, I TO EDGE } 13,0 \\
& \text { C4 } 0 \text {, LINK } 26,0 \text { TO EDGE } 14, I \\
& \text { C4 1, LINK 26, I TO EDGE } 14,0 \\
& \text { C4 1, LINK 27,0 TO EDGE 15, I } \\
& \text { C4 } 0 \text {, LINK 27, I TO EDGE 15, } 0 \\
& \text { C4 1, LINK } 17,0 \text { TO EDGE 16,I } \\
& \text { C4 } 0 \text {, LINK 17, I TO EDGE } 16,0 \\
& \text { C4 0, LINK 19,0 TO EDGE 17, I } \\
& \text { C4 1, LINK 19, I TO EDGE } 17,0 \\
& \text { C4 } 0 \text {, LINK } 22,0 \text { TO EDGE 18, I } \\
& \text { C4 1, LINK 22, I TO EDGE } 18,0 \\
& \text { C4 1, LINK } 23,0 \text { TO EDGE 19, I } \\
& \text { C4 0, LINK 23, I TO EDGE 19,0 } \\
& \text { C4 } 0 \text {, LINK } 16,0 \text { TO EDGE 20, I } \\
& \text { C4 } 1 \text {, LINK } 16 \text {, I TO EDGE } 20,0 \\
& \text { C4 } 0 \text {, LINK 18,0 TO EDGE 21, I } \\
& \text { C4 1, LINK 18, I TO EDGE } 21,0 \\
& \text { C4 1, LINK } 21,0 \text { TO EDGE } 22, \text { I } \\
& \text { C4 } 0 \text {, LINK } 21 \text {, I TO EDGE } 22,0 \\
& \text { C4 1, LINK 20,0 TO EDGE 23, I } \\
& \text { C4 } 0 \text {, LINK 20, I TO EDGE } 23,0
\end{aligned}
$$




$$
\begin{aligned}
& \text { C4 1, LINK 10,0 TO EDGE 24, I } \\
& \text { C4 } 0 \text {, LINK 10, I TO EDGE } 24,0 \\
& \text { C4 1, LINK } 13,0 \text { TO EDGE } 25 \text {, I } \\
& \text { C4 } 0 \text {, LINK } 13, \text { I TO EDGE } 25,0 \\
& \text { C4 1, LINK 14,0 TO EDGE 26, I } \\
& \text { C4 } 0 \text {, LINK 14, I TO EDGE 26,0 } \\
& \text { C4 1, LINK 11,0 TO EDGE } 27, \text { I } \\
& \text { C4 0, LINK 11, I TO EDGE } 27,0 \\
& \text { C4 } 0 \text {, LINK } 8,0 \text { TO EDGE 28, I } \\
& \text { C4 } 1 \text {, LINK } 8, \text { I TO EDGE } 28,0 \\
& \text { C4 0, LINK 9,0 TO EDGE 29, I } \\
& \text { C4 } 1 \text {, LINK 9, I TO EDGE } 29,0 \\
& \text { C4 } 0 \text {, LINK } 12,0 \text { TO EDGE } 30 \text {, I } \\
& \text { C4 } 1 \text {, LINK } 12, \text { I TO EDGE } 30,0 \\
& \text { C4 } 0 \text {, LINK } 15,0 \text { TO EDGE } 31, I \\
& \text { C4 } 1 \text {, LINK } 15, \text { I TO EDGE } 31,0
\end{aligned}
$$

END

DEF $\mathrm{BO} 12 \mathrm{~b}$

$$
\begin{array}{ll}
\text { SIZES } & \\
\text { T2 } & 1 \\
\text { C4 } & 2 \\
\text { SLOT } & 16 \\
\text { EDGE } & 32 \\
\text { END } &
\end{array}
$$

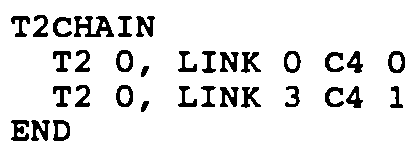

\section{HARDWIRE}

SLOT 0,LINK 2 TO SLOT 1,LINK 1 SLOT 1,LINK 2 TO SLOT 2,LINK 1 SLOT 2 ,LINK 2 TO SLOT 3,LINK 1 SLOT 3,LINK 2 TO SLOT 4,LINK 1 SLOT 4,LINK 2 TO SLOT 5,LINK 1 SLOT 5,LINK 2 TO SLOT 6,LINK 1 SLOT 6,LINK 2 TO SLOT 7,LINK 1 SLOT 7,LINK 2 TO SLOT 8,LINK 1 SLOT 8,LINK 2 TO SLOT 9,LINK 1 SLOT 9,LINK 2 TO SLOT 10,LINK 1 SLOT 10,LINK 2 TO SLOT 11,LINK 1 SLOT 11,IINK 2 TO SLOT 12,LINK 1 SLOT 12,LINK 2 TO SLOT 13,LINK 1 SLOT 13,IINK 2 TO SLOT 14,LINK 1 SLOT 14,LINK 2 TO SLOT 15,LINK 1

C4 0 , LINK 5, I TO SLOT 1 , LINK 0,0 C4 1 , IINK 5,O TO SLOT 1 , LINK $0, I$

C4 1, LINK 2,I TO SLOT 1, LINK 3,0 
C4 0, LINK 2,O TO SLOT 1 , LINK 3,I

C4 0, LINK 4,I TO SLOT 2, IINK 0,0

C4 1, LINK 4,0 TO SLOT 2, LINK 0, I

C4 1, IINK 0, I TO SLOT 2 , IINK 3,0 C4 0 , LINK 0,0 TO SLOT 2 , IINK 3,1

C4 0, LINK 19, I TO SLOT 3, LINK 0,0 C4 1, LINK 19,0 TO SLOT 3, LINK 0,I

C4 1, LINK 17, I TO SLOT 3 , LINK 3,0 C4 0 , LINK 17,0 TO SLOT 3 , LINK 3,I

C4 0 , IINK 18, I TO SLOT 4, LINK 0,0 C4 1 , LINK 18,0 TO SLOT 4 , IINK 0,1

C4 1, LINK 21, I TO SLOT 4, LINK 3,0 C4 0 , LINK 21,0 TO SLOT 4, IINK 3, I

C4 0 , IINK 3, I TO SLOT 5, LINK 0,0 C4 1 , LINK 3,0 TO SLOT 5, LINK 0, I

C4 1, LINK 1, I TO SLOT 5, LINK 3,0 C4 0 , IINK 1,0 TO SLOT 5, LINK 3,1

C4 0 , LINK 6,I TO SLOT 6, LINK 0,0 C4 1 , LINK 6,0 TO SLOT 6 , IINK 0,1

C4 1, LINK 7,I TO SLOT 6, LINK 3,0 C4 0 , LINK 7,0 TO SLOT 6, LINK 3, I

C4 0 , IINK 16, I TO SLOT 7 , LINK 0,0 C4 1 , LINK 16,0 TO SLOT 7 , LINK 0,1

C4 1, LINK 20, I TO SLOT 7, IINK 3,0 C4 0 , LINK 20,0 TO SLOT 7, LINK 3,I

C4 0 , LINK 9, I TO SLOT 8 , LINK 0,0 C4 1, LINK 9,0 TO SLOT 8, LINK 0,1

C4 1, LINK 14, I TO SLOT 8 , LINK 3,0 C4 0 , LINK 14,0 TO SLOT 8 , LINK 3, I

C4 0, LINK 28 , I TO SLOT 9, LINK 0,0 C4 1, LINK 28,0 TO SLOT 9, LINK 0,I

C4 1, LINK 29, I TO SLOT 9, IINK 3,0 C4 0, LINK 29,0 TO SLOT 9, LINK 3, I

C4 0 , LINK 31, I TO SLOT 10 , LINK 0,0 C4 1 , LINK 31,0 TO SLOT 10 , LINK 0, I

C4 1, LINK 30, I TO SLOT 10 , LINK 3,0 C4 0 , LINK 30,0 TO SLOT 10, LINK 3,I

C4 0 , LINK 15, I TO SLOT 11 , LINK 0,0 C4 1, IINK 15,0 TO SLOT 11 , LINK 0,1

C4 1, IINK 11, I TO SLOT 11 , LINK 3,0 C4 0, LINK 11,0 TO SLOT 11, LINK 3, I 
C4 0 , IINK 12 , I TO SLOT 12 , IINK 0,0 C4 1 , LINK 12,0 TO SLOT 12, LINK 0, I

C4 1, LINK 13, I TO SLOT 12 , IINK 3,0 C4 0 , IINK 13,0 TO SLOT 12, IINK 3,I

C4 0 , LINK 25, I TO SLOT 13, LINK 0,0 C4 1, LINK 25,0 TO SLOT 13, LINK 0,I

C4 1, LINK 27 , I TO SLOT 13 , LINK 3,0 C4 0 , LINK 27,0 TO SLOT 13, LINK 3, I

C4 0 , LINK 26, I TO SLOT 14 , IINK 0,0 C4 1, LINK 26,0 TO SLOT 14 , LINK 0, I

C4 1, LINK 24, I TO SLOT 14, IINK 3,0 C4 0 , IINK 24,0 TO SLOT 14, LINK 3,I

C4 0 , IINK 8, I TO SLOT 15 , IINK 0,0 C4 1 , IINK 8,0 TO SLOT 15 , IINK 0,1

C4 1, IINK 10, I TO SLOT 15, IINK 3,0 C4 0 , LINK 10,0 TO SLOT 15, IINK 3,I

C4 1, IINK 0,O TO EDGE $0, I$

C4 0 , IINK 0 , I TO EDGE 0,0

C4 1, IINK 2,O TO EDGE $1, I$

C4 0 , IINK 2, I TO EDGE 1,0

C4 0 , IINK 4,O TO EDGE 2,I

C4 1 , LINK 4, I TO EDGE 2,0

C4 0 , IINK 5,O TO EDGE $3, I$

C4 1, IINK 5, I TO EDGE 3,0

C4 0 , IINK 6,0 TO EDGE $4, I$

C4 1 , LINK 6, I TO EDGE 4,0

C4 0 , IINK 3,0 TO EDGE 5, I

C4 1 , IINK 3, I TO EDGE 5,0

C4 1 , IINK 1,0 TO EDGE $6, I$

C4 0 , LINK 1 , I TO EDGE 6,0

C4 1 , IINK 7,0 TO EDGE 7,I

C4 0 , LINK 7,I TO EDGE 7,0

C4 1, IINK 29,0 TO EDGE 8 , I

C4 0 , IINK 29, I TO EDGE 8,0

C4 1, LINK 30,0 TO EDGE 9,I

C4 0 , LINK 30, I TO EDGE 9,0

C4 0 , IINK 31,0 TO EDGE 10, I

C4 1, LINK 31, I TO EDGE 10,0

C4 0 , IINK 28,0 TO EDGE 11, I

C4 1 , IINK 28, I TO EDGE 11,0

C4 1, IINK 24,0 TO EDGE 12, I 
C4 0 , LINK 24, I TO EDGE 12,0

C4 0 , LINK 25, O TO EDGE 13, I

C4 1, LINK 25, I TO EDGE 13,0

C4 0 , LINK 26,0 TO EDGE 14, I C4 1, LINK 26, I TO EDGE 14,

C4 1, LINK 27,0 TO EDGE 15, I C4 0 , LINK $27, I$ TO EDGE 15,0

C4 1, LINK 17,0 TO EDGE 16 , I C4 0 , LINK 17, I TO EDGE 16 ,0

C4 0 , LINK 19,0 TO EDGE 17, I C4 1, LINK 19, I TO EDGE 17,0

C4 0 , LINK 22,0 TO EDGE 18, I C4 1 , LINK 22 , I TO EDGE 18,0

C4 1, LINK 23,0 TO EDGE 19, I C4 0 , LINK 23, I TO EDGE 19,0

C4 0 , LINK 16,0 TO EDGE 20,I C4 1, LINK 16, I TO EDGE 20,0

C4 0 , LINK 18,0 TO EDGE $21, I$ C4 1 , LINK 18, I TO EDGE 21,0

C4 1, LINK 21, O TO EDGE 22, I C4 0 , LINK 21 , I TO EDGE 22 ,0

C4 1, LINK 20,0 TO EDGE 23, I C4 0 , LINK 20,I TO EDGE 23,0

C4 1, LINK 10,0 TO EDGE 24, I C4 0 , LINK 10,I TO EDGE 24,0

C4 1, LINK 13,0 TO EDGE 25, I C4 0 , LINK 13, I TO EDGE 25,0

C4 1, LINK 14,0 TO EDGE 26, I C4 0 , IINK 14, I TO EDGE 26,0

C4 1, LINK 11,0 TO EDGE 27, I C4 0 , LINK 11, I TO EDGE 27,0

C4 0 , IINK 8,0 TO EDGE 28, I C4 1, LINK 8, I TO EDGE 28,0

C4 0, LINK 9,0 TO EDGE 29, I C4 1, LINK 9, I TO EDGE 29,0

C4 0 , IINK 12,0 TO EDGE 30, I C4 1, IINK 12, I TO EDGE 30,0

C4 0 , LINK 15,0 TO EDGE 31 , I C4 1 , LINK 15 , I TO EDGE 31,0

PIPE B014, B012a, B012a, B012b END 


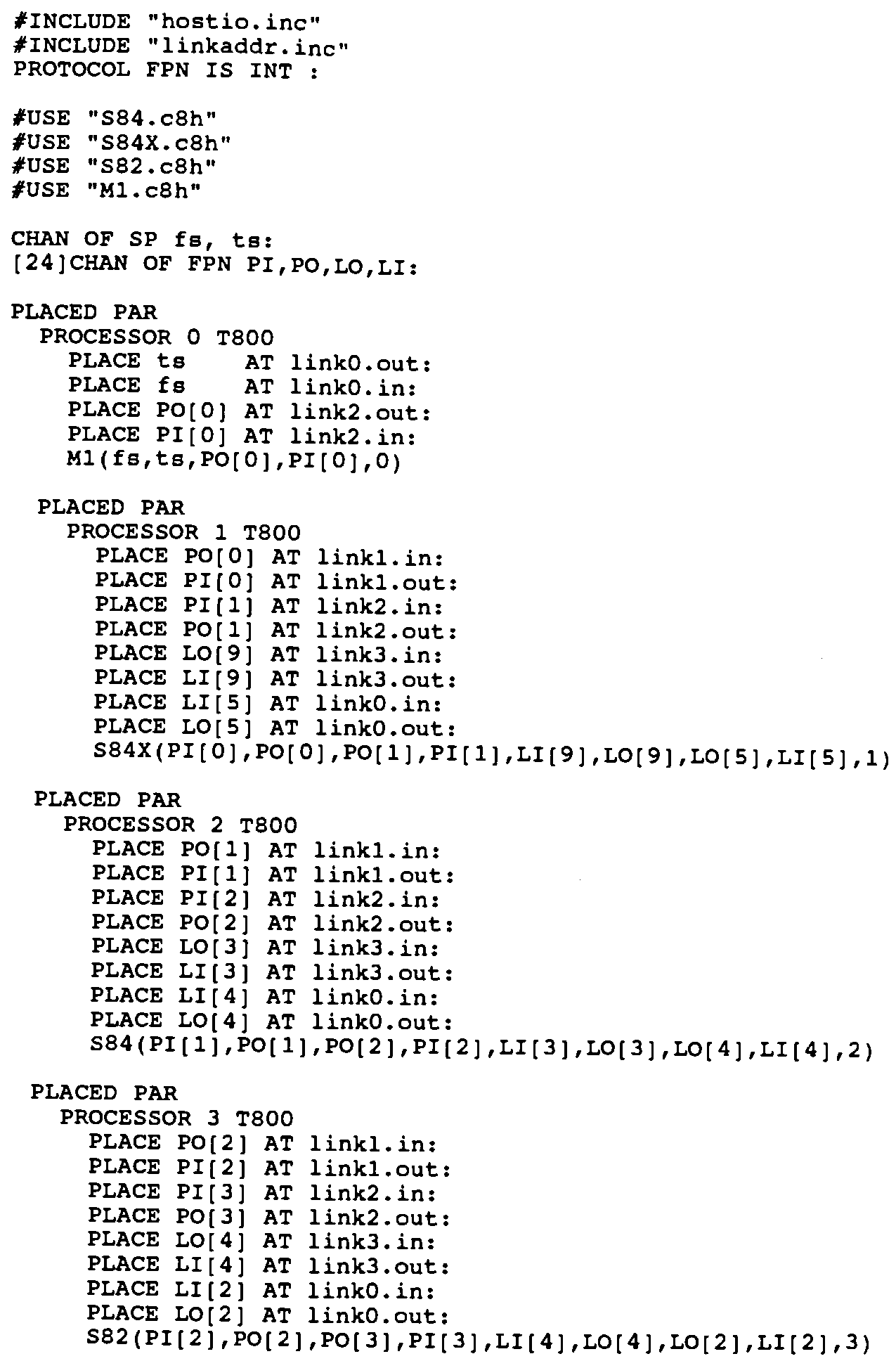


occam 2 product compiler (19th september 1988)

PROCESSOR 000 T8 1

SC 3112411240

CODE 280204528

$\begin{array}{lllll}\text { PROCESSOR } & 1 & 1 & \mathrm{~T} 8 & 1\end{array}$

SC $1 \quad \begin{array}{llll}48 & 48 & 0\end{array}$

CODE $36 \quad 28 \quad 68 \quad 0$

PROCESSOR 22 T8 1

SC $0 \quad 144 \quad 144 \quad 0$

CODE $36 \quad 64 \quad 1120$

$\begin{array}{lllll}\text { PROCESSOR } & 3 & 3 & \text { T8 } & 1\end{array}$

SC $2 \quad 128 \quad 128 \quad 0$

CODE $36 \quad 1001120$

LINK $02,1,1$

LINK 12,21

IINK 20,33

IINK 22,31 


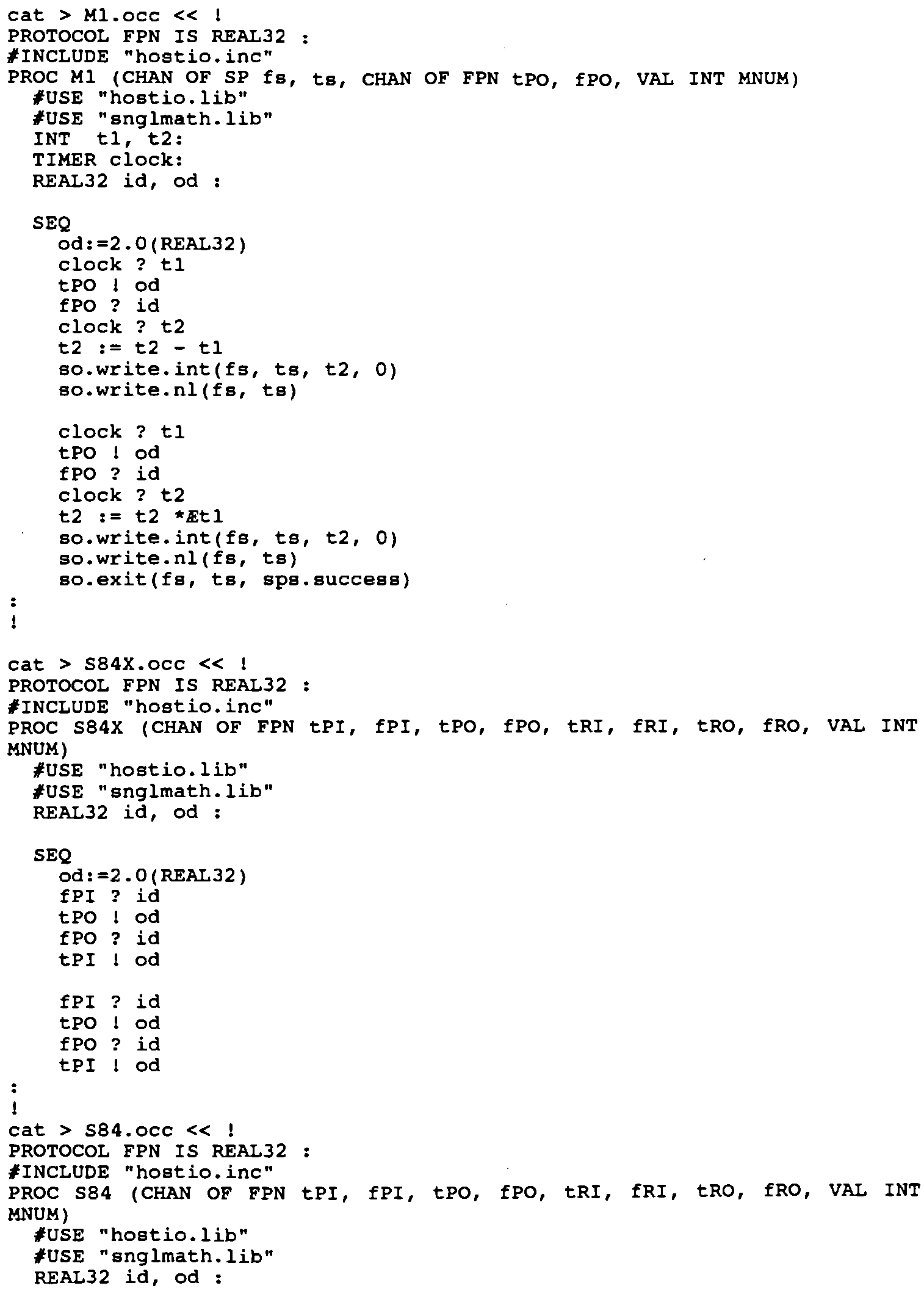




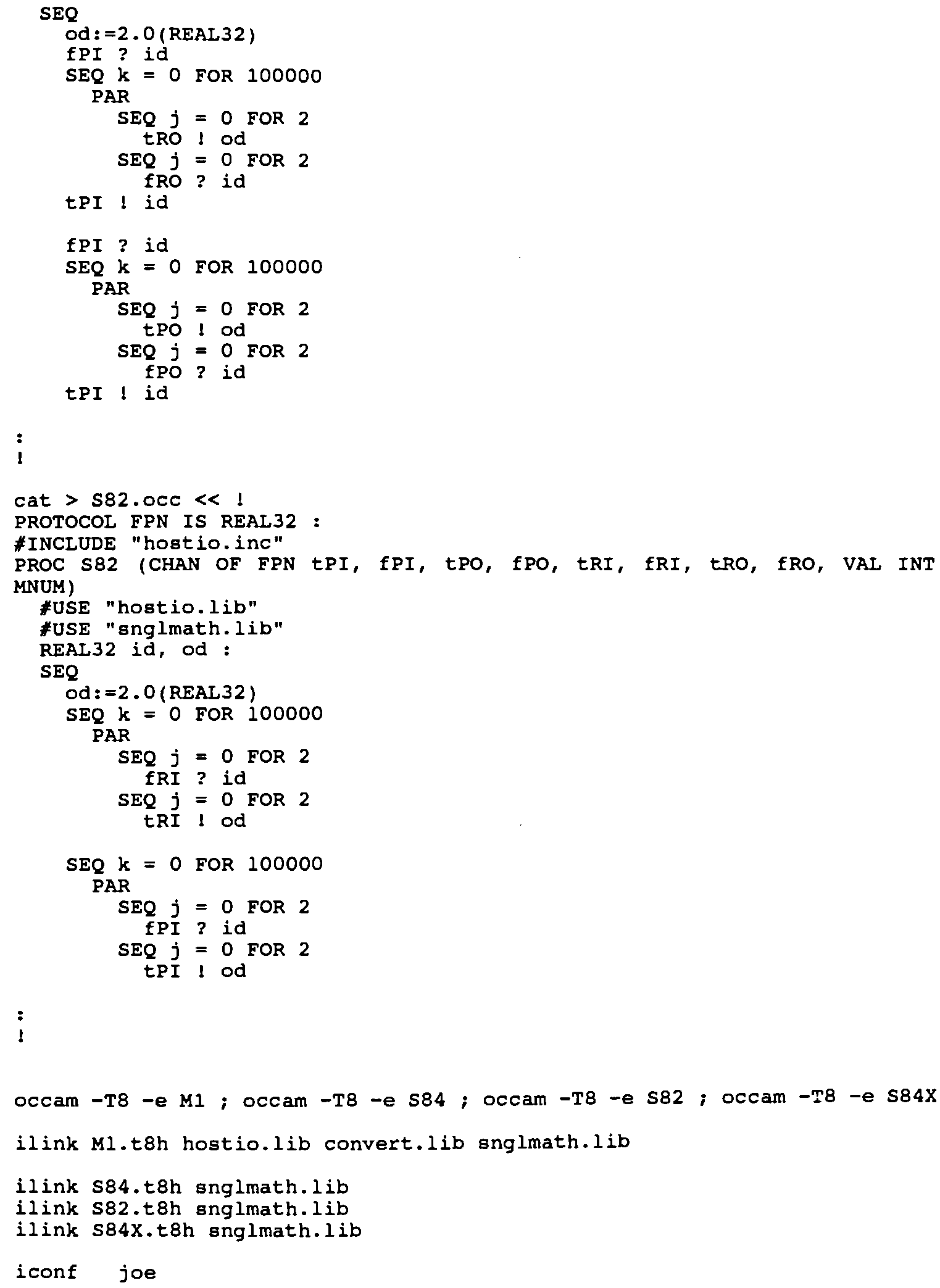




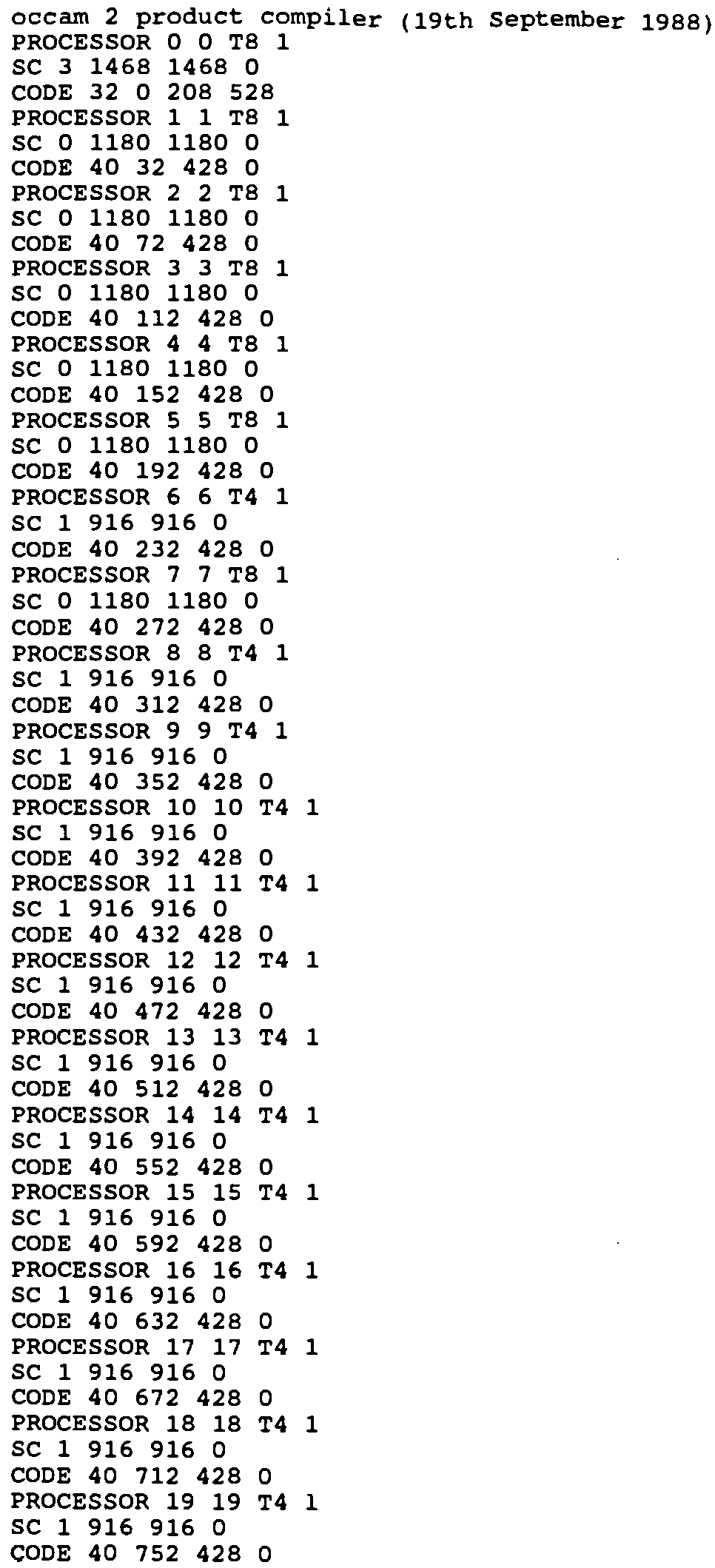


$\begin{array}{lllll}\text { PROCESSOR } 20 & 20 & \text { T4 } & 1\end{array}$

SC 19169160

CODE $40 \quad 792 \quad 428 \quad 0$

$\begin{array}{lllll}\text { PROCESSOR } & 21 & 21 & \text { T4 } & 1\end{array}$

SC 19169160

$\begin{array}{lllll}\text { CODE } & 40 & 832 & 428 & 0\end{array}$

$\begin{array}{lllll}\text { PROCESSOR } & 22 & 22 & \text { T4 } & 1\end{array}$

SC $2 \quad 176 \quad 176 \quad 0$

CODE $32 \quad 872 \quad 308 \quad 0$

LINK 02,11

LINK 10,53

LINK 12,21

LINK 13,90

LINK 20,43

LINK 22,31

LINK 23,30

LINK 32,41

LINK 33,40

LINK 42,51

LINK 50,93

LINK 52,61

LINK 60,83

LINK 62,7

LINK 63,70

LINK 72,81

LINK 73,80

LINK 82,91

LINK 92,101

LINK 100,113

LINK 102,111

LINK 103,220

LINK 110,123

LINK 112,121

LINK 120,133

LINK 122,131

LINK 130,143

LINK 132,141

LINK 140,153

LINK 142,151

LINK 150,163

LINK 152,161

LINK 160,173

LINK 162,171

LINK 170,183

IINK 172,181

LINK 180,193

LINK 182,191

LINK 190,203

LINK 192,201

LINK 200,213

LINK 202,211

IINK 210,223

LINK 212,221 


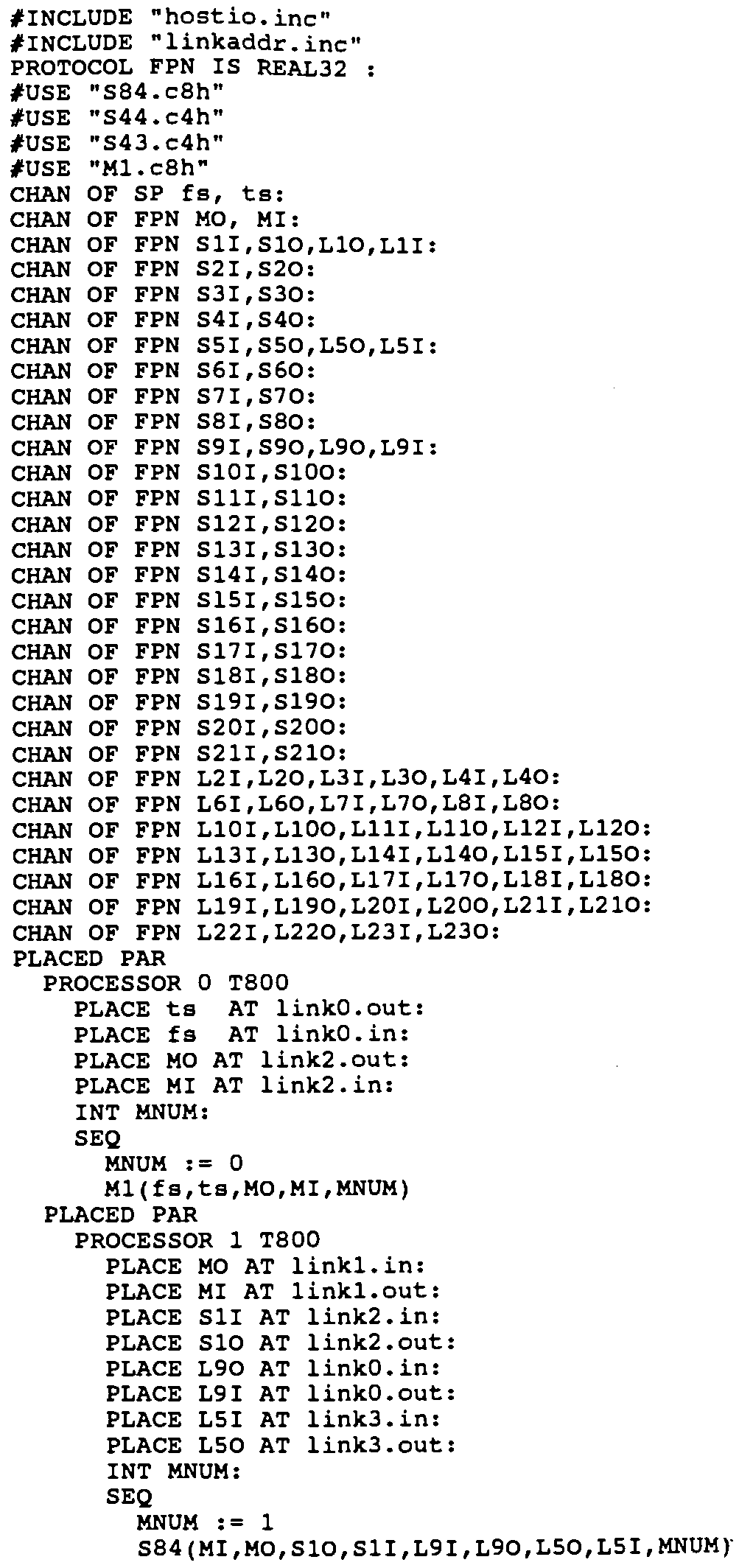




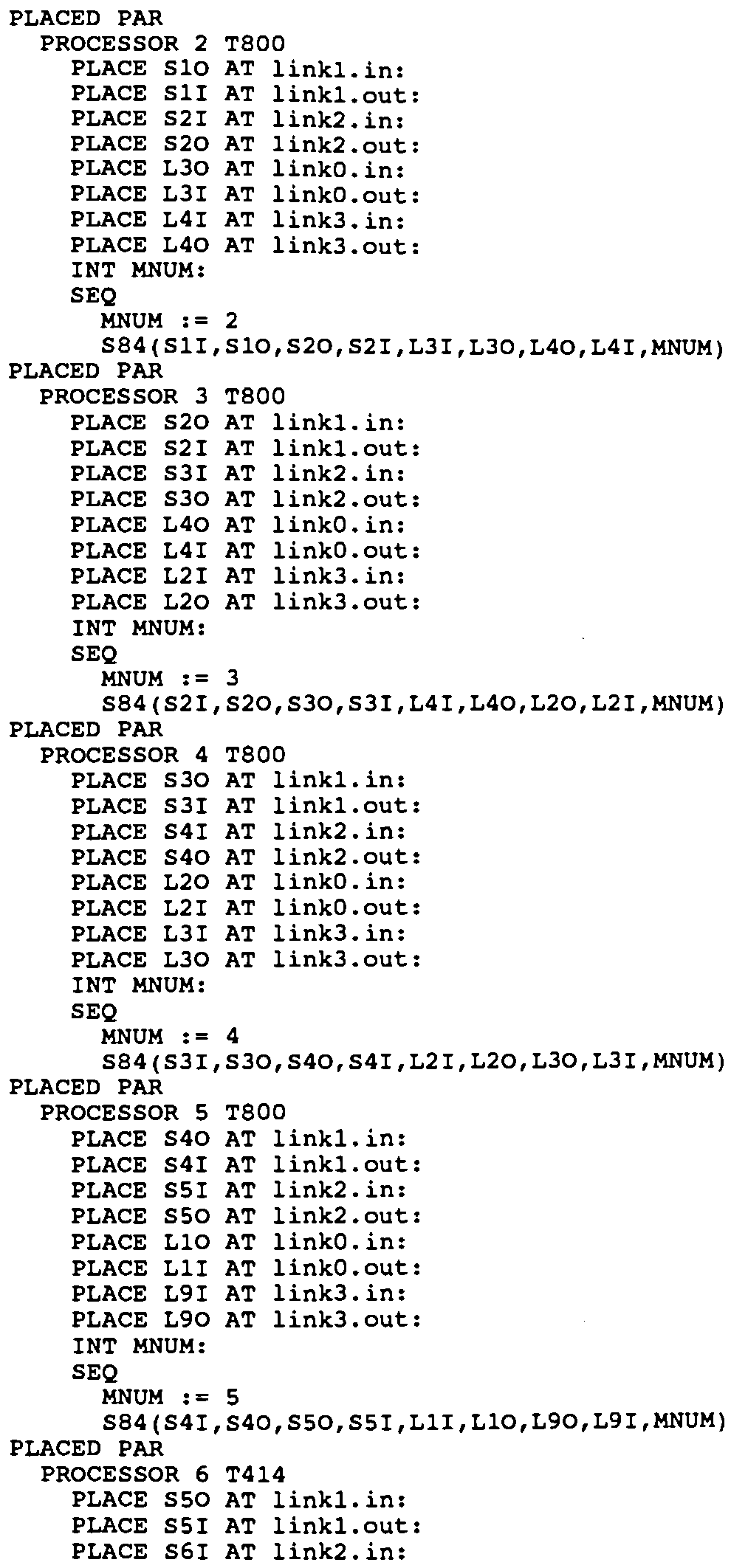




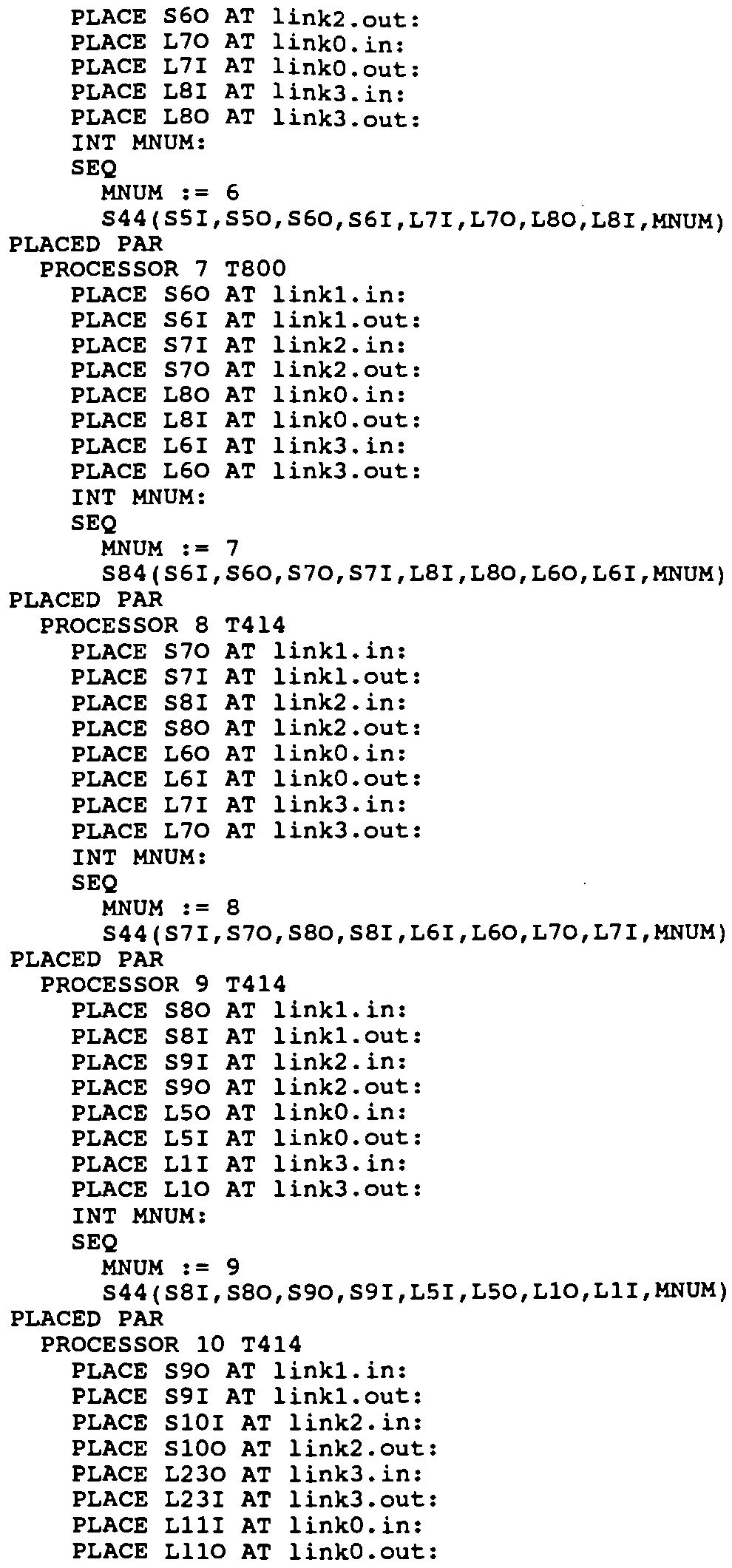




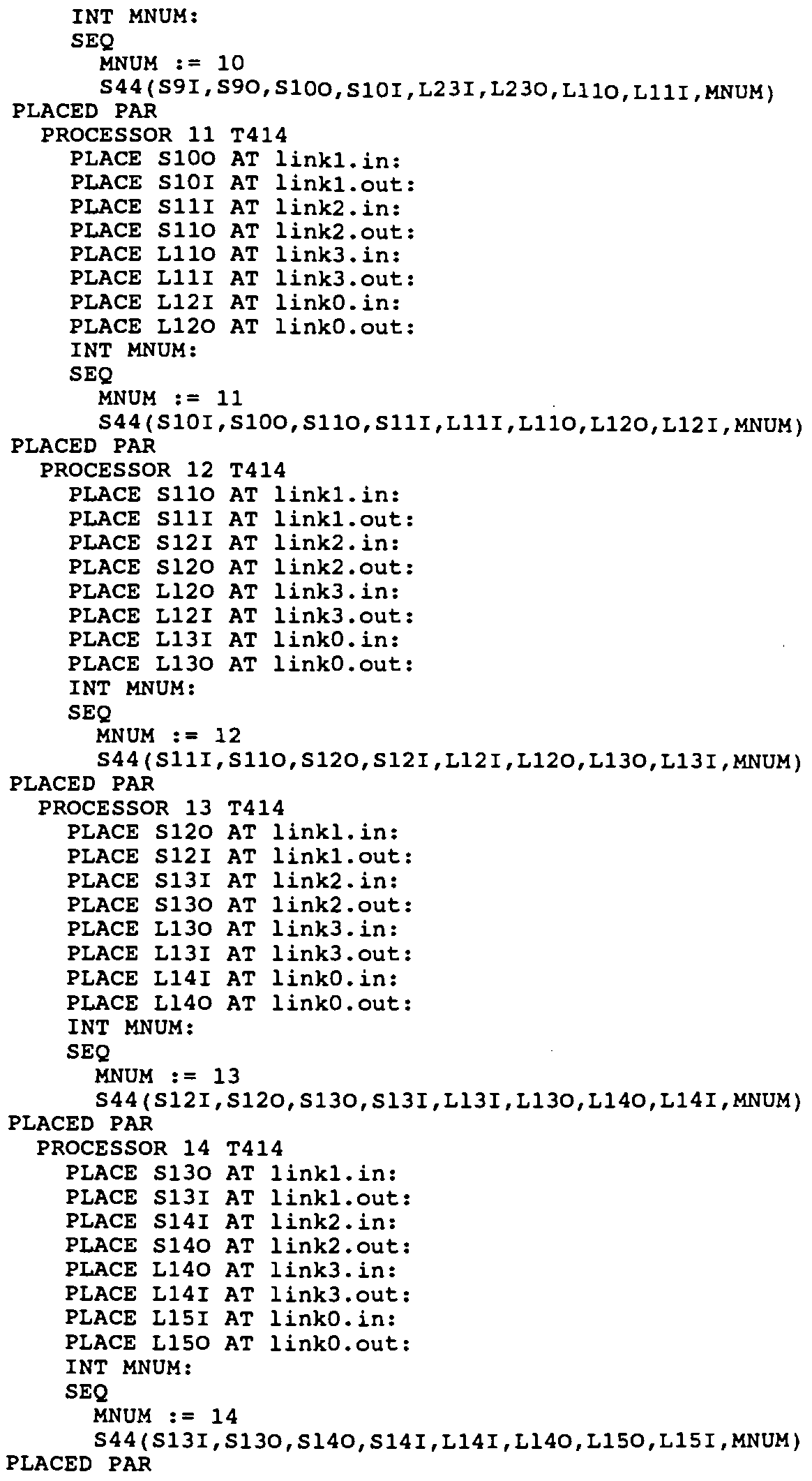




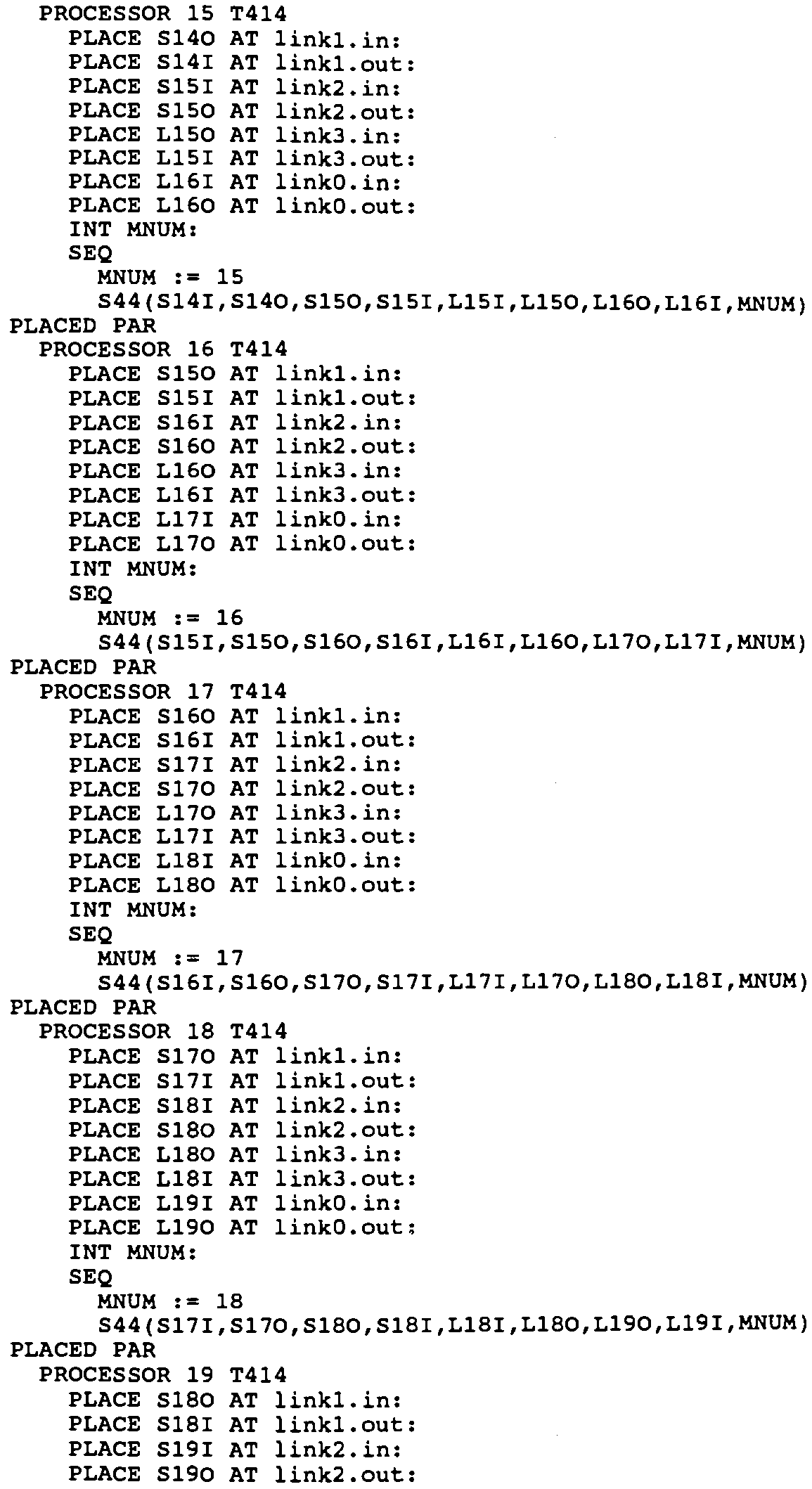







occam 2 product compiler (19th september 1988)

$\begin{array}{lllll}\text { PROCESSOR } & 0 & 0 & \text { T8 } & 1\end{array}$

SC 3180818080

CODE $28 \quad 0 \quad 204528$

PROCESSOR 111 T8 1 SC $0 \quad 13201320 \quad 0$

$\begin{array}{llllll}\text { CODE } & 48 & 28 & 428 & 20\end{array}$

PROCESSOR 22 T8 1

SC $0 \quad 1320 \quad 1320 \quad 0$

CODE $48 \quad 76 \quad 428 \quad 20$

$\begin{array}{lllll}\text { PROCESSOR } & 3 & \text { T8 } & 1\end{array}$

SC $0 \quad 13201320 \quad 0$

$\begin{array}{lllll}\text { CODE } & 48 & 124 & 428 & 20\end{array}$

PROCESSOR $4 \quad 4 \quad T 8 \quad 1$

SC $0 \quad 1320 \quad 1320 \quad 0$

CODE $48 \quad 172 \quad 428 \quad 20$

PROCESSOR 55 T8 1

SC $0 \quad 1320 \quad 1320 \quad 0$

CODE $48 \quad 220 \quad 428 \quad 20$

$\begin{array}{lllll}\text { PROCESSOR } & 6 & 6 & \text { T4 } & 1\end{array}$

SC $1716 \quad 716 \quad 0$

CODE $48 \quad 268 \quad 428 \quad 20$

$\begin{array}{lllll}\text { PROCESSOR } 7 & 7 & \text { T8 } & 1\end{array}$

SC $0 \quad 13201320 \quad 0$

CODE $48 \quad 316 \quad 428 \quad 20$

$\begin{array}{llllll}\text { PROCESSOR } & 8 & 8 & \text { T4 } & 1\end{array}$

SC 17167160

CODE $48 \quad 364 \quad 428 \quad 20$

PROCESSOR 999 T4 1

SC $1716716 \quad 0$

CODE $\begin{array}{llll}48 & 412 & 428 & 20\end{array}$

PROCESSOR $10 \quad 10$ T4 1

SC 17167160

CODE $48 \quad 460 \quad 428 \quad 20$

$\begin{array}{lllll}\text { PROCESSOR } & 11 & 11 & \text { T4 } & 1\end{array}$

SC 17167160

CODE $48 \quad 508 \quad 428 \quad 20$

$\begin{array}{lllll}\text { PROCESSOR } & 12 & 12 & \text { T4 } & 1\end{array}$

SC 17167160

CODE $48 \quad 556 \quad 428 \quad 20$

$\begin{array}{lllll}\text { PROCESSOR } & 13 & 13 & \text { T4 } & 1\end{array}$

SC 17167160

CODE $48 \quad 604 \quad 428 \quad 20$

$\begin{array}{llllll}\text { PROCESSOR } & 14 & 14 & \mathrm{~T} 4 & 1\end{array}$

SC 17167160

CODE $48 \quad 652 \quad 428 \quad 20$

PROCESSOR $15 \quad 15$ T4 1

SC 17167160

CODE $48 \quad 700 \quad 428 \quad 20$

PROCESSOR $16 \quad 16$ T4 1

SC 17167160

$\begin{array}{lllll}\text { CODE } & 48 & 748 & 428 & 20\end{array}$

$\begin{array}{llllll}\text { PROCESSOR } & 17 & 17 & \text { T4 } & 1\end{array}$

SC $1716716 \quad 0$

CODE $48 \quad 796 \quad 428 \quad 20$

$\begin{array}{lllll}\text { PROCESSOR } & 18 & 18 & \text { T4 } & 1\end{array}$

SC 17167160

CODE $48 \quad 844 \quad 428 \quad 20$

PROCESSOR $19 \quad 19$ T4 1

SC 17167160

CODE $48 \quad 892 \quad 428 \quad 20$ 
PROCESSOR $20 \quad 20$ T4 1

SC $1716716 \quad 0$

CODE $48 \quad 940 \quad 428 \quad 20$

$\begin{array}{lllll}\text { PROCESSOR } & 21 & 21 & \text { T4 } & 1\end{array}$

SC $1716 \quad 716 \quad 0$

CODE $\begin{array}{lllll}48 & 988 & 428 & 20\end{array}$

PROCESSOR $22 \quad 22$ T4 1

SC $2 \quad 172 \quad 1720$

CODE $32 \quad 1036 \quad 304 \quad 0$

IINK 02,11

IINK 10,53

IINK 12,21

LINK 13,90

IINK 20,43

IINK 22,31

IINK 23,30

LINK 32,41

IINK 33,40

IINK 42,51

LINK 50,93

LINK 52,61

IINK 60,83

LINK 62,7

LINK 63,70

LINK 72,81

LINK 73,80

LINK 82,91

IINK 92,101

IINK 100,113

IINK 102,111

LINK 103,220

IINK 110,123

LINK 11 2, 121

IINR 120,133

IINK 122,131

LINR 130,143

LINK 132,141

LINK 140,153

IINK 142,151

IINK 150,163

IINK 152,161

LINK 160,173

LINK 162,171

LINK 170,183

LINK 172,181

LINK 180,193

LINK 182,191

LINK 190,203

LINK 192,201

IINK 200,213

IINK 202,211

IINK 210,223

IINK 212,221 


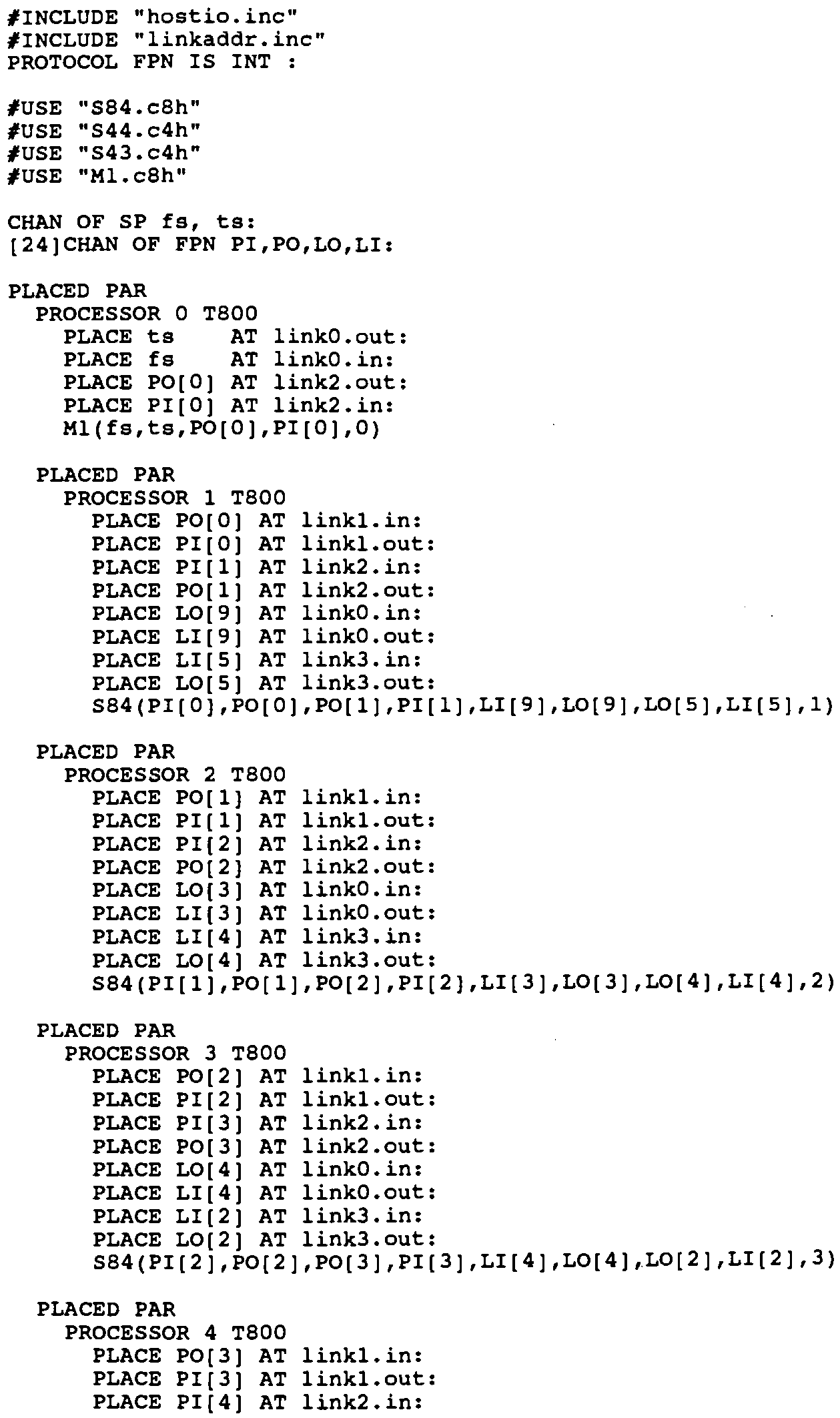


PLACE PO[4] AT link2.out:

PLACE LO[2] AT linko.in:

PLACE LI[2] AT link0.out:

PLACE LI[3] AT link3.in:

PLACE LO[3] AT link3.out:

S84 (PI [ 3], PO [3], PO [ 4], PI [ 4], LI [ 2], LO [2], LO [3], LI [ 3 ], 4)

\section{PLACED PAR}

PROCESSOR 5 T8O0

PLACE PO[4] AT linkl.in:

PLACE PI[4] AT link1.out:

PLACE PI[5] AT link2.in:

PLACE PO[5] AT link2.out:

PLACE LO[1] AT linko.in:

PLACE LI[1] AT link0.out:

PLACE LI[9] AT link3.in:

PLACE LO[9] AT link3.out:

S84 (PI [ 4], PO [4], PO [5], PI [5], LI [1], LO [1], LO [9], LI [9],5)

PLACED PAR

PROCESSOR 6 T414

PLACE PO[5] AT linkl.in:

PLACE PI [5] AT linkl.out:

PLACE PI [6] AT link2.in:

PLACE PO[6] AT link2.out:

PLACE LO[7] AT linko.in:

PLACE LI[7] AT link0.out:

PLACE LI [8] AT link3.in:

PLACE LO[8] AT link3.out:

S44 (PI [5], PO [5], PO [6], PI [6], LI [ 7], LO [ 7], LO [8], LI $[8], 6$ )

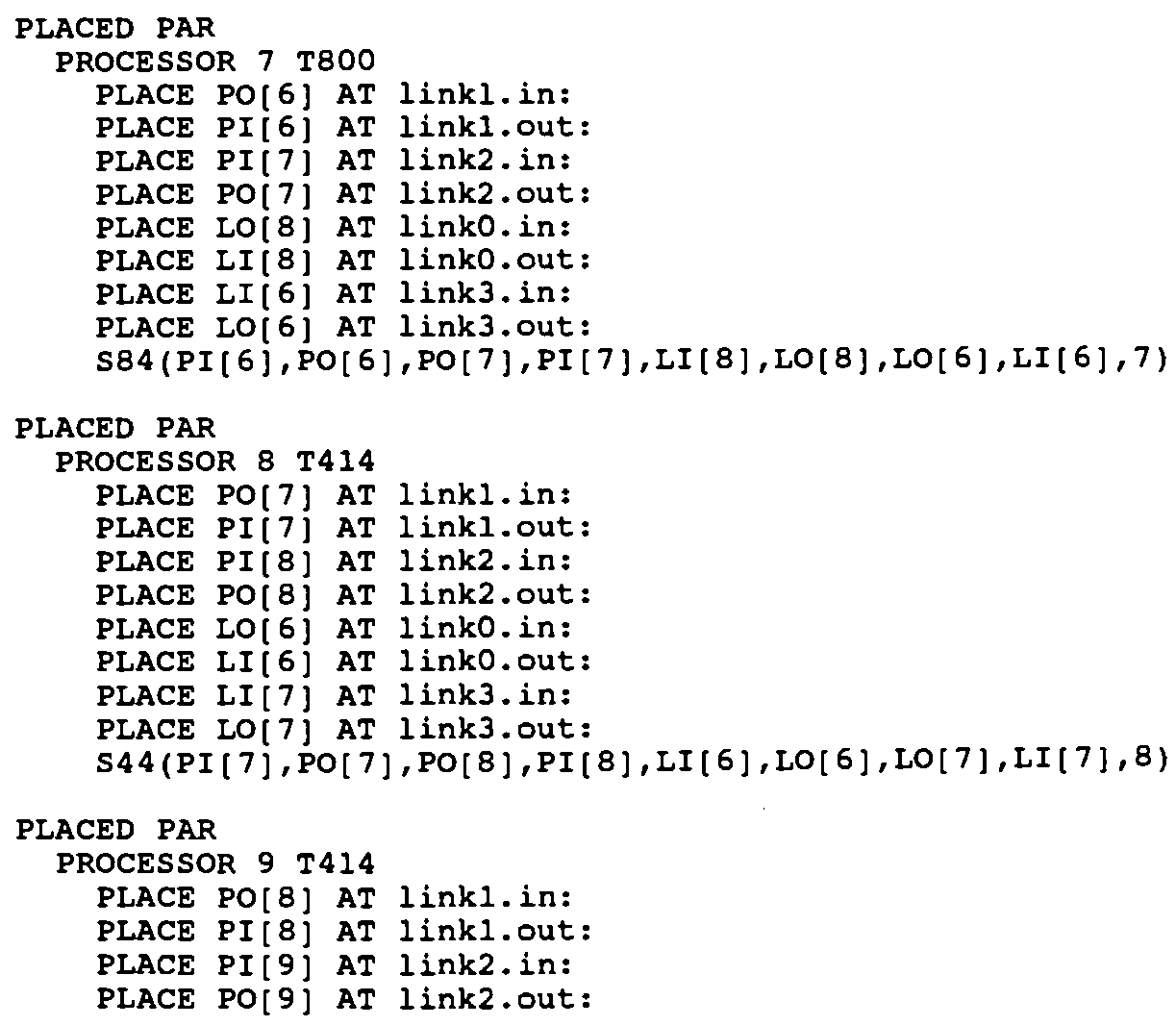


PLACE LO[5] AT linko.in:

PLACE LI[5] AT link0.out:

PLACE LI[1] AT link3.in:

PLACE LO[1] AT link3.out:

S44 (PI [8], PO [8], PO [9], PI [9], LI [5], LO [5], LO [1], LI [1],9)

\section{PLACED PAR}

PROCESSOR $10 \quad$ T414

PLACE PO[9] AT linkl.in:

PLACE PI[9] AT linkl.out:

PLACE PI[10] AT link2.in:

PLACE PO[10] AT link2.out:

PLACE LO[23] AT link3.in:

PLACE LI[23] AT link3.out:

PLACE LI[11] AT linko.in:

PLACE LO[11] AT link0.out:

S44 (PI [9], PO [9], PO[10], PI [10], LI [23], LO [23], LO[11], LI [11], 10)

PLACED PAR

PROCESSOR 11 T414

PLACE PO[10] AT linkl.in:

PLACE PI[10] AT link1.out:

PLACE PI [11] AT link2.in:

PLACE PO[11] AT link2.out:

PLACE LO[11] AT link3. in:

PLACE LI[11] AT link3.out:

PLACE LI[12] AT linko.in:

PLACE LO[12] AT link0.out:

S44 (PI [10], PO[10], PO[11], PI [11], LI [11], LO [11], LO [12], LI [12], 11)

\section{PLACED PAR}

PROCESSOR 12 T414

PLACE PO[11] AT linkl.in:

PLACE PI[11] AT link1.out:

PLACE PI[12] AT link2, in:

PLACE PO[12] AT link2.out:

PLACE LO[12] AT link3.in:

PLACE LI [12] AT link3.out:

PLACE LI[13] AT link0.in:

PLACE LO[13] AT link0.out:

S44 (PI [11], PO [11], PO [12] , PI [12], LI [12], LO [12], LO [13], LI [13], 12)

\section{PLACED PAR}

PROCESSOR 13 T414

PLACE PO[12] AT linkl.in:

PLACE PI[12] AT link1.out:

PLACE PI[13] AT link2.in:

PLACE PO [13] AT link2. out:

PLACE LO[13] AT link3. in:

PLACE LI[13] AT link3.out:

PLACE LI [14] AT link0. in:

PLACE LO[14] AT link0.out:

S44 ( PI [ 12], PO [12], PO [13], PI [ 13] , LI [ 13], LO [13], LO [14], LI [ 14], 13)

PLACED PAR

PROCESSOR $14 \quad$ T414

PLACE PO[13] AT linkl.in:

PLACE PI[13] AT link1.out:

PLACE PI[14] AT link2.in:

PLACE PO[14] AT link2.out:

PLACE LO[14] AT link3.in: 
PLACE LI[14] AT link3.out:

PLACE LI[15] AT linko.in:

PLACE LO[15] AT link0.out:

S44 ( PI [13], PO [13], PO [14], PI [ 14], LI [14], LO [14], LO [15], LI [15], 14)

PLACED PAR

PROCESSOR 15 T414

PLACE PO[14] AT linkl,in:

PLACE PI[14] AT link1. out:

PLACE PI[15] AT link2.in:

PLACE PO [15] AT link2. out:

PLACE LO[15] AT link3.in:

PLACE LI[15] AT link3.out:

PLACE LI[16] AT linko. in:

PLACE LO[16] AT linko.out:

S44 (PI [14], PO[14], PO[15], PI [15], LI [15], LO [15], LO [16], LI [16], 15)

PLACED PAR

PROCESSOR 16 T414

PLACE PO[15] AT linkl.in:

PLACE PI[15] AT linkl.out:

PLACE PI[16] AT link2.in:

PLACE PO[16] AT link2.out:

PLACE LO[16] AT link3.in:

PLACE LI[16] AT link3.out:

PLACE LI[17] AT linko.in:

PLACE LO[17] AT link0.out:

S44 (PI [15], PO [15], PO [16], PI [16], LI [16], LO [16], LO [17], LI [17], 16)

PLACED PAR

PROCESSOR $17 \quad$ T414

PLACE PO[16] AT linkl.in:

PLACE PI[16] AT link1.out:

PLACE PI[17] AT link2. in:

PLACE PO[17] AT link2, out:

PLACE LO[17] AT link3.in:

PLACE LI[17] AT link3.out:

PLACE LI[18] AT link0. in:

PLACE LO[18] AT link0.out:

S44 (PI [16], PO [16], PO [17], PI [17], LI [17], LO [17], LO [18], LI [18], 17)

PLACED PAR

PROCESSOR $18 \quad T 414$

PLACE PO[17] AT linkl.in:

PLACE PI[17] AT linkl.out:

PLACE PI[18] AT link2.in:

PLACE PO[18] AT link2.out:

PLACE LO[18] AT link3.in:

PLACE LI[18] AT link3.out:

PLACE LI[19] AT linko.in:

PLACE LO[19] AT link0.out:

S44(PI [17], PO [17], PO [18], PI [18], LI [18], LO [18], LO [19], LI [19], 18)

PLACED PAR

PROCESSOR 19 T414

PLACE PO[18] AT link1.in:

PLACE PI[18] AT linkl.out:

PLACE PI[19] AT link2. in:

PLACE PO[19] AT link2,out:

PLACE LO[19] AT link3. in:

PLACE LI[19] AT link3.out: 
PLACE LI[20] AT linko.in:

PLACE LO[20] AT link0.out:

S44 (PI [18], PO[ 18], PO [19], PI [19], LI [19], LO [19], LO[20], LI [20], 19)

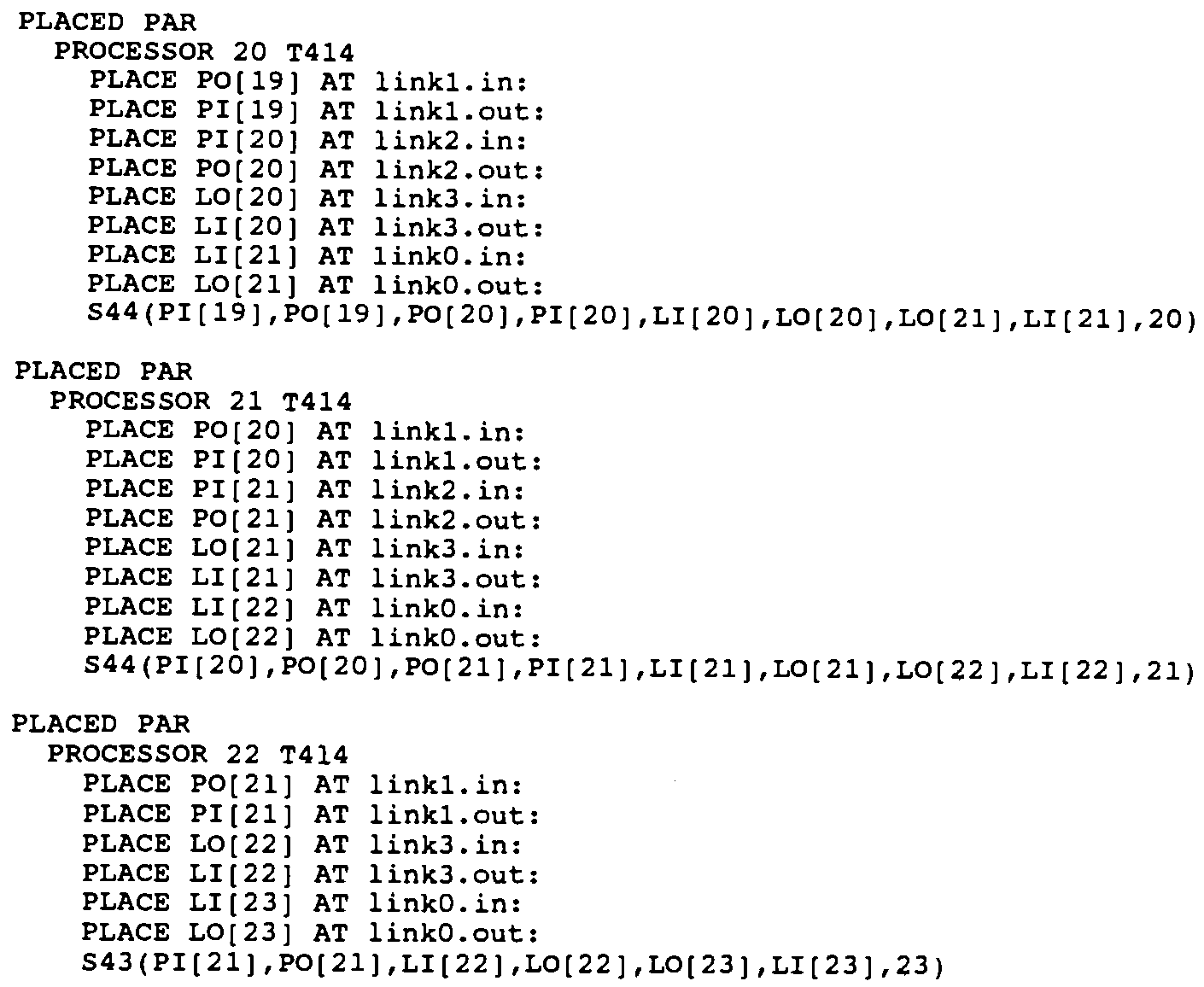




\section{OUTPUTS}

This section includes the results of the programs written to test the different systems.

Load Flow Solution

THIRTY BUS IEEE TEST SYSTEM

\begin{tabular}{|c|c|c|c|c|c|c|c|c|c|c|}
\hline $\begin{array}{l}\text { From } \\
\text { Bus }\end{array}$ & $\begin{array}{c}\text { n Volt } \\
\text { Mag }\end{array}$ & $\begin{array}{l}\text { Volt } \\
\text { Angle }\end{array}$ & $\begin{array}{l}\text { Mw } \\
\text { Load }\end{array}$ & $\begin{array}{l}\text { Mvar } \\
\text { Load }\end{array}$ & $\begin{array}{l}\text { Mw } \\
\text { Gen }\end{array}$ & $\begin{array}{l}\text { Mvar } \\
\text { Gen }\end{array}$ & $\begin{array}{l}\text { to } \\
\text { Bus }\end{array}$ & $\begin{array}{l}\text { Mw } \\
\text { Flow }\end{array}$ & $\begin{array}{l}\text { Mvar } \\
\text { Flow }\end{array}$ & $\begin{array}{l}\text { Mva } \\
\text { Flow }\end{array}$ \\
\hline 1 & 1.05 & 0.00 & 0.00 & 0.00 & 139.71 & -5.24 & & & & \\
\hline+ & & & & & & & 2 & 91.35 & -1.64 & 91.37 \\
\hline+ & & & & & & & 3 & 48.36 & -3.60 & 48.50 \\
\hline 2 & 1.03 & -2.76 & 21.70 & 12.70 & 57.56 & -6.20 & & & & \\
\hline+ & & & & & & & 1 & -89.90 & 0.26 & 89.90 \\
\hline+ & & & & & & & 4 & 29.17 & -9.03 & 30.53 \\
\hline+ & & & & & & & 5 & 58.04 & 1.67 & 58.07 \\
\hline+ & & & & & & & 6 & 38.55 & -11.81 & 40.32 \\
\hline 3 & 1.04 & -4.76 & 2.40 & 1.20 & & & & & & \\
\hline+ & & & & & & & 1 & -47.40 & 3.09 & 47.50 \\
\hline & & & & & & & 4 & 45.00 & -4.29 & 45.21 \\
\hline 4 & 1.03 & -5.70 & 7.60 & 1.60 & & & & & & \\
\hline+ & & & & & & & 2 & -28.69 & 6.57 & 29.43 \\
\hline+ & & & & & & & 3 & -44.75 & 4.12 & 44.94 \\
\hline+ & & & & & & & 6 & 42.09 & -13.13 & 44.09 \\
\hline+ & & & & & & & 12 & 23.75 & -31.66 & 39.58 \\
\hline 5 & 1.01 & -9.01 & 19.20 & 19.02 & 4.56 & 17.12 & & & & \\
\hline+ & & & & & & & 2 & -56.55 & 0.26 & 56.55 \\
\hline+ & & & & & & & 7 & -13.09 & -2.15 & 13.27 \\
\hline 6 & 1.03 & -6.72 & 0.00 & 0.00 & & & & & & \\
\hline+ & & & & & & & 2 & -37.69 & 10.43 & 39.10 \\
\hline+ & & & & & & & 4 & -41.87 & 12.93 & 43.82 \\
\hline+ & & & & & & & 7 & 36.33 & 10.51 & 37.82 \\
\hline+ & & & & & & & 8 & -1.46 & 20.39 & 20.44 \\
\hline+ & & & & & & & 9 & 19.0 & -44.68 & 48.56 \\
\hline+ & & & & & & & 10 & 14.4 & -25.34 & 29.15 \\
\hline+ & & & & & & & 8 & 11.27 & -2.30 & 11.50 \\
\hline 7 & 1.01 & -8.19 & 22.80 & 10.90 & & & & & & \\
\hline+ & & & & & & & 5 & 13.17 & 0.26 & 13.17 \\
\hline+ & & & & & & & 6 & -35.97 & -11.17 & 37.66 \\
\hline 8 & 1.02 & -6.55 & 30.03 & 10.89 & 35.00 & 1.75 & & & & \\
\hline+ & & & & & & & 6 & $1.51-$ & -21.17 & 21.22 \\
\hline+ & & & & & & & 28 & 3.0 & -7.09 & 7.90 \\
\hline 9 & 1.12 & -8.64 & 0.00 & 0.0 & & & & & & \\
\hline+ & & & & & & & 6 & -19.01 & 49.19 & 52.74 \\
\hline+ & & & & & & & 11 & -17.93 & 10.69 & 20.88 \\
\hline+ & & & & & & & 10 & 36.9 & -46.32 & 59.25 \\
\hline 10 & $1.17-$ & -10.42 & 5.80 & 2.00 & & & & & & \\
\hline+ & & & & & & & 6 & -14.41 & 29.65 & 32.96 \\
\hline+ & & & & & & & $9-$ & -36.94 & 49.40 & 61.68 \\
\hline+ & & & & & & & 20 & 10.83 & 13.16 & 17.04 \\
\hline
\end{tabular}




\begin{tabular}{|c|c|c|c|c|c|c|c|c|c|}
\hline+ & & & & & & 17 & 7.14 & 21.58 & 22.73 \\
\hline+ & & & & & & 21 & 18.30 & 13.52 & 22.75 \\
\hline+ & & & & & & 22 & 9.28 & 6.88 & 11.55 \\
\hline 11 & 1.10 & -6.91 & 0.00 & 0.00 & $\begin{array}{ll}17.93 & -9.97\end{array}$ & & & & \\
\hline+ & & & & & & 9 & 17.93 & -9.97 & 20.51 \\
\hline 12 & 1.11 & -8.55 & 11.20 & 7.50 & & & & & \\
\hline+ & & & & & & 4 & -23.75 & 35.17 & 42.44 \\
\hline+ & & & & & & 13 & -16.91 & 13.80 & 21.83 \\
\hline+ & & & & & & 1 & 6.90 & -1.17 & 7.0 \\
\hline+ & & & & & & 15 & 16.77 & -7.501 & 18.37 \\
\hline+ & & & & & & 16 & 5.79 & -12.90 & 14.21 \\
\hline 13 & 1.09 & -7.42 & 0.00 & 0.00 & $16.91-13.26$ & & & & \\
\hline+ & & & & & & 12 & 16.91 & -13.26 & 21.49 \\
\hline 14 & 1.10 & -9.45 & 6.20 & 1.60 & & & & & \\
\hline+ & & & & & & 12 & -6.85 & 1.27 & 6.97 \\
\hline+ & & & & & & 15 & 0.65 & -2.87 & 2.94 \\
\hline 15 & 1.10 & -9.81 & 8.20 & 2.50 & & & & & \\
\hline+ & & & & & & 12 & -16.59 & 7.86 & 18.36 \\
\hline+ & & & & & & 14 & -0.64 & 2.88 & 2.95 \\
\hline+ & & & & & & 18 & 4.43 & -7.39 & 8.62 \\
\hline+ & & & & & & 23 & 4.59 & -5.85 & 7.44 \\
\hline 16 & 1.12 & -9.65 & 3.50 & 1.80 & & & & & \\
\hline+ & & & & & & 12 & -5.63 & 13.31 & 14.45 \\
\hline+ & & & & & & 17 & 2.13 & -15.11 & 15.26 \\
\hline 17 & 1.15 & -10.38 & 9.00 & 5.80 & & & & & \\
\hline+ & & & & & & 16 & -1.98 & 15.46 & 15.59 \\
\hline+ & & & & & & 10 & -7.02 & -21.26 & 22.39 \\
\hline 18 & 1.12 & -10.63 & 3.20 & 0.90 & & & & & \\
\hline+ & & & & & & 15 & -4.37 & 7.52 & 8.70 \\
\hline+ & & & & & & 19 & 1.17 & -8.42 & 8.50 \\
\hline 19 & 1.12 & -10.94 & 9.50 & 3.40 & & & & & \\
\hline+ & & & & & & 18 & -1.13 & 8.50 & 8.57 \\
\hline+ & & & & & & 20 & -8.37 & -11.90 & 14.55 \\
\hline 20 & 1.13 & -10.87 & 2.20 & 0.70 & & & & & \\
\hline+ & & & & & & 19 & 8.43 & 12.01 & 14.67 \\
\hline+ & & & & & & 10 & -10.63 & -12.71 & 16.57 \\
\hline 21 & 1.15 & -10.81 & 17.50 & 11.20 & & & & & \\
\hline+ & & & & & & 10 & -18.17 & -13.24 & 22.48 \\
\hline+ & & & & & & 22 & 0.67 & 2.04 & 2.14 \\
\hline 22 & 1.15 & -10.80 & 0.00 & 0.00 & & & & & \\
\hline+ & & & & & & 10 & -9.21 & -6.73 & 11.41 \\
\hline+ & & & & & & 21 & -0.67 & -2.04 & 2.14 \\
\hline+ & & & & & & 24 & 9.88 & 8.77 & 13.21 \\
\hline 23 & 1.11 & -10.52 & 3.20 & 1.60 & & & & & \\
\hline+ & & & & & & 15 & -4.55 & 5.94 & 7.49 \\
\hline+ & & & & & & 24 & 1.35 & -7.54 & 7.66 \\
\hline 24 & 1.13 & -11.14 & 8.70 & 6.70 & & & & & \\
\hline+ & & & & & & 22 & -9.73 & -8.53 & 12.94 \\
\hline+ & & & & & & 23 & -1.29 & 7.67 & 7.78 \\
\hline+ & & & & & & 25 & 2.31 & 10.57 & 10.82 \\
\hline 25 & 1.09 & -10.57 & 0.00 & 0.00 & & & & & \\
\hline
\end{tabular}




\begin{tabular}{|c|c|c|c|c|c|c|c|c|}
\hline+ & & & & & 24 & -2.14 & -10.27 & 10.49 \\
\hline+ & & & & & 26 & 3.54 & 2.36 & 4.25 \\
\hline+ & & & & & 27 & -1.40 & 7.91 & 8.04 \\
\hline 26 & 1.08 & -10.93 & 3.50 & 2.30 & & & & \\
\hline+ & & & & & 25 & -3.50 & -2.30 & 4.19 \\
\hline 27 & 1.08 & -10.01 & 0.00 & 0.00 & & & & \\
\hline+ & & & & & 25 & 1.46 & -7.80 & 7.94 \\
\hline+ & & & & & 28 & -14.71 & 14.26 & 20.49 \\
\hline+ & & & & & 29 & 6.18 & 1.64 & 6.39 \\
\hline+ & & & & & 30 & 7.08 & 1.63 & 7.26 \\
\hline 28 & 1.03 & -7.10 & 0.00 & 0.00 & & & & \\
\hline+ & & & & & 27 & 14.71 & -12.88 & 19.55 \\
\hline+ & & & & & 8 & -3.46 & 2.73 & 4.41 \\
\hline+ & & & & & 6 & 11.25 & 0.99 & 11.29 \\
\hline 29 & 1.06 & -11.11 & 2.40 & 0.90 & & & & \\
\hline+ & & & & & 27 & -6.10 & -1.50 & 6.28 \\
\hline+ & & & & & 30 & 3.70 & 0.60 & 3.75 \\
\hline & 1.05 & -11.90 & 10.60 & 1.90 & & & & \\
\hline+ & & & & & 27 & -6.93 & -1.36 & 7.06 \\
\hline+ & & & & & 29 & -3.67 & -0.54 & 3.71 \\
\hline
\end{tabular}




\section{FIFTY SEVEN BUS IEEE TEST SYSTEM}

From Volt Volt Mw Mvar Mw Mvar to Mw Mva Mva Bus Mag Angle Load Load Gen Gen Bus Flow Flow Flow

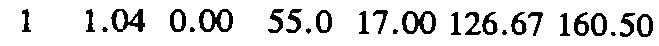

$$
\begin{aligned}
& + \\
& + \\
& + \\
& +
\end{aligned}
$$$$
+
$$

$\begin{array}{llll}2 & 1.85 & 26.96 & 27.02\end{array}$

$\begin{array}{llll}15 & 0.00 & -5.34 & 5.34\end{array}$

$\begin{array}{llll}16 & 0.00 & -2.95 & 2.95\end{array}$

$+$

$\begin{array}{llll}17 & 0.00 & -1.55 & 1.55\end{array}$

$\begin{array}{lllll}2 & 1.01 & 0.24 & 3.0088 .00 & 0.00-15.16\end{array}$

$$
\begin{array}{ccccc}
+ & 1 & -1.16-110.27 & 111.30 \\
+ & 3 & 0.00 & -.17 & 4.17
\end{array}
$$

\begin{tabular}{|c|c|c|c|c|c|c|c|}
\hline+ & & & & 3 & -1.97 & -4.91 & 5.30 \\
\hline+ & & & & 5 & 0.00 & -1.27 & 1.27 \\
\hline+ & & & & 6 & 0.00 & -1.71 & 1.71 \\
\hline+ & & & & 18 & 0.00 & 0.00 & 0.00 \\
\hline 5 & $0.98-0.31$ & 13.00 & 4.00 & & & & \\
\hline+ & & & & 4 & 0.00 & -1.25 & 1.25 \\
\hline+ & & & & 6 & 0.00 & -0.60 & 0.60 \\
\hline 6 & $0.99-1.33$ & 75.00 & 2.00 & & & & \\
\hline+ & & & & 4 & -23.16 & 10.67 & 25.50 \\
\hline+ & & & & 5 & 0.00 & -0.61 & 0.61 \\
\hline+ & & & & 7 & 0.00 & -1.36 & 1.36 \\
\hline+ & & & & 9 & 0.00 & -2.31 & 2.31 \\
\hline
\end{tabular}

$3 \quad 1.00-0.15 \quad 41.0021 .00$

$\begin{array}{rrrrr}+ & 2 & -27.14 & -31.18 & 41.34 \\ + & 4 & 0.00 & -1.89 & 1.89 \\ + & 15 & 0.00 & -2.70 & 2.70\end{array}$

\begin{tabular}{|c|c|c|c|c|}
\hline+ & 7 & 0.00 & -0.98 & 0.98 \\
\hline+ & 9 & 0.00 & -2.77 & 2.77 \\
\hline $1.00-1.20 \quad 121.00 \quad 26.00$ & & & & \\
\hline 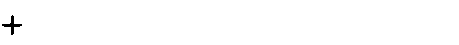 & 6 & 3.16 & 3.23 & 4.51 \\
\hline$t$ & 8 & 0.00 & -2.75 & 2.75 \\
\hline & 10 & 0.00 & -2.21 & 2.21 \\
\hline & 11 & 0.00 & -1.10 & 1.10 \\
\hline & 12 & 0.00 & -3.88 & 3.88 \\
\hline
\end{tabular}

$\begin{array}{lllll}4 & 0.99 & -0.25 & 0.00 & 0.00\end{array}$

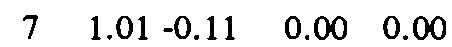

$\begin{array}{rrrrr}+ & 6 & 0.00 & -1.41 & 1.41 \\ + & 8 & 0.00 & -0.99 & 0.99 \\ + & 29 & 0.00 & 0.00 & 0.00\end{array}$

$\begin{array}{lllllll}8 & 1.00 & 5.08 & 150.00 & 22.00 & 450.00 & 29.56\end{array}$ 


$$
\begin{array}{rrrrr}
+ & 13 & 0.00 & -2.04 & 2.04 \\
+ & 55 & 0.00 & 0.00 & 0.00
\end{array}
$$

\begin{tabular}{|c|c|c|c|c|c|c|c|}
\hline+ & & & & 9 & 0.00 & -2.37 & 2.37 \\
\hline+ & & & & 12 & 0.00 & -1.77 & 1.77 \\
\hline+ & & & & 51 & 0.00 & 0.00 & 0.00 \\
\hline 11 & $1.00-0.18$ & 0.00 & 0.00 & & & & \\
\hline+ & & & & 9 & 0.00 & -1.10 & 1.10 \\
\hline+ & & & & 13 & 0.00 & -0.95 & 0.95 \\
\hline+ & & & & 41 & 0.00 & 0.00 & 0.00 \\
\hline+ & & & & 43 & 0.00 & 0.00 & 0.00 \\
\hline
\end{tabular}

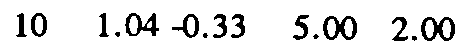

$12 \quad 1.01-1.11377 .0024 .00310 .00106 .94$

+
+
+
+
+

$\begin{array}{rrrr}9 & 0.00 & -3.98 & 3.98 \\ 10 & 0.00 & -1.69 & 1.69 \\ 13 & 0.00 & -3.11 & 3.11 \\ 16 & 0.00 & -1.11 & 1.11 \\ 17 & 0.00 & -2.45 & 2.45\end{array}$

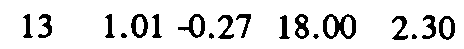

$+$

$+$

$+$

$+$

$+$

$+$

$\begin{array}{llll}14 & 1.03 & -0.45 & 10.50 \quad 5.30\end{array}$

$+$

$+$

$+$

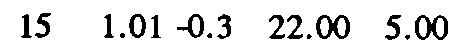

$+$

$+$

$+$

$+$

$+$

$\begin{array}{lllll}16 \quad 1.02 & -1.39 & 43.00 \quad 3.00\end{array}$

$+$

$+$

$\begin{array}{lllll}17 \quad 1.02 & -1.51 & 42.00 \quad 8.00\end{array}$

$+$

$+$

$\begin{array}{lllll}18 & 0.92 & -4.90 & 27.20 & 9.80\end{array}$

$\begin{array}{rrrr}9 & 0.00 & -2.07 & 2.07 \\ 11 & 0.00 & -0.96 & 0.96 \\ 12 & 0.00 & -3.08 & 3.08 \\ 14 & 0.00 & -0.56 & 0.56 \\ 15 & 0.00 & -1.17 & 1.17 \\ 49 & 0.00 & 0.00 & 0.00\end{array}$

$\begin{array}{rrrr}13 & 0.00 & -0.58 & 0.58 \\ 15 & 0.00 & -0.78 & 0.78 \\ 46 & 0.00 & 0.00 & 0.00\end{array}$

$\begin{array}{rccc}1 & -16.20 & -36.08 & 39.55 \\ 3 & 1.58 & 19.51 & 19.57 \\ 13 & 0.00 & -1.17 & 1.17 \\ 14 & 0.00 & -0.75 & 0.75 \\ 45 & 0.00 & 0.0 & 0.00\end{array}$

$\begin{array}{llll}1 & -14.24 & -10.96 & 17.96\end{array}$

$\begin{array}{llll}12 & 0.00 & -1.12 & 1.12\end{array}$

$\begin{array}{llll}1 & -28.30 & -12.45 & 30.91\end{array}$

$\begin{array}{lllll}12 & 0.00 & -2.48 & 2.48\end{array}$

$\begin{array}{llll}4 & 0.00 & 0.00 & 0.00\end{array}$ 
$\begin{array}{llll}19 & 0.00 & 0.00 & 0.00\end{array}$

$\begin{array}{lllll}19 & 0.99 & -0.45 & 3.30 & 0.60\end{array}$

$+$

$\begin{array}{llll}18 & 0.00 & 0.00 & 0.00\end{array}$

$\begin{array}{llll}20 & 0.00 & 0.00 & 0.00\end{array}$

$\begin{array}{lllll}20 & 1.01 & -0.67 & 2.30 & 1.00\end{array}$

$+$

$\begin{array}{llll}19 & 0.00 & 0.00 & 0.00\end{array}$

$\begin{array}{llll}21 & 2.45 & -6.05 & 6.53\end{array}$

$\begin{array}{lllll}21 & 0.99 & 0.17 & 0.00 & 0.00\end{array}$

$+$

$+$

$\begin{array}{llll}20 & 0.00 & 0.00 & 0.00\end{array}$

$\begin{array}{llll}22 & 0.00 & 0.00 & 0.00\end{array}$

$\begin{array}{lllll}22 & 1.00 & 0.00 & 0.00 & 0.00\end{array}$

+
+
+

$\begin{array}{llll}21 & -5.56 & -57.42 & 57.69\end{array}$

$\begin{array}{llll}23 & 0.00 & 0.00 & 0.00\end{array}$

$\begin{array}{llll}38 & 0.00 & 0.00 & 0.00\end{array}$

$\begin{array}{lllll}23 & 1.00 & -0.04 & 6.30 & 2.10\end{array}$

$+$

$\begin{array}{llll}22 & 0.00 & 0.00 & 0.00\end{array}$

$\begin{array}{llll}24 & 0.00 & -0.42 & 0.42\end{array}$

$\begin{array}{lllll}24 & 0.93 & 0.28 & 0.00 & 0.00\end{array}$

+
+
+

$\begin{array}{llll}23 & 0.00 & -0.37 & 0.37\end{array}$

$\begin{array}{llll}25 & 0.00 & 0.00 & 0.00\end{array}$

$\begin{array}{llll}26 & 0.00 & 0.00 & 0.00\end{array}$

$\begin{array}{lllll}25 & 0.86 & 3.34 & 6.30 & 3.20\end{array}$

$+$

$\begin{array}{llll}24 & 0.00 & 0.00 & 0.00\end{array}$

$\begin{array}{llll}30 & 0.00 & 0.00 & 0.00\end{array}$

$\begin{array}{lllll}26 & 1.04 & -0.17 & 0.00 & 0.00\end{array}$

$+$

$\begin{array}{llll}24 & 0.00 & 0.00 & 0.00\end{array}$

$\begin{array}{llll}27 & 0.00 & 0.00 & 0.00\end{array}$

$\begin{array}{lllll}27 & 1.00 & -0.36 & 9.30 & 0.50\end{array}$

$+$

$+$

$\begin{array}{llll}26 & 0.00 & 0.00 & 0.00\end{array}$

$\begin{array}{llll}28 & 0.00 & 0.00 & 0.00\end{array}$

$\begin{array}{lllll}28 & 1.00 & -0.06 & 4.60 & 2.30\end{array}$

$\begin{array}{lllll}+ & 27 & 0.00 & 0.00 & 0.00 \\ + & 29 & 0.00 & 0.00 & 0.00\end{array}$

$\begin{array}{lllll}29 & 0.98 & 0.04 & 17.00 & 2.60\end{array}$

$+$

$+$

$+$

$\begin{array}{llll}7 & 0.00 & 0.00 & 0.00\end{array}$

$\begin{array}{llll}28 & 0.00 & 0.00 & 0.00\end{array}$

$\begin{array}{llll}52 & 0.00 & 0.00 & 0.00\end{array}$

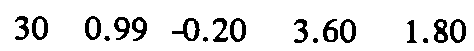

$+$

$25 \quad 0.00 \quad 0.00 \quad 0.00$ 
$\begin{array}{llll}31 & 0.00 & 0.00 & 0.00\end{array}$

$\begin{array}{lllll}31 & 0.98 & -0.68 & 5.80 & 2.90\end{array}$

$+$

$\begin{array}{llll}30 & 0.00 & 0.00 & 0.00\end{array}$

$\begin{array}{llll}32 & 0.00 & 0.00 & 0.00\end{array}$

$\begin{array}{lllll}32 & 1.00 & 0.04 & 1.60 & 0.80\end{array}$

+
+
+

$\begin{array}{llll}31 & 0.00 & 0.00 & 0.00\end{array}$

$\begin{array}{llll}33 & 0.00 & 0.00 & 0.00\end{array}$

$\begin{array}{llll}34 & 0.00 & 0.00 & 0.00\end{array}$

$\begin{array}{lllll}33 & 1.00 & -0.04 & 3.80 & 1.90\end{array}$

$+$

$\begin{array}{llll}32 & 0.00 & 0.00 & 0.00\end{array}$

$\begin{array}{lllll}34 & 1.00 & -0.07 & 0.00 & 0.00\end{array}$

$+$

$\begin{array}{llll}32 & 0.00 & 0.00 & 0.00\end{array}$

$\begin{array}{llll}35 & 0.00 & -0.16 & 0.16\end{array}$

$\begin{array}{lllll}35 & 1.00 & -0.07 & 6.00 & 3.00\end{array}$

$+$

$\begin{array}{llll}34 & 0.00 & -0.16 & 0.16\end{array}$

$\begin{array}{llll}36 & 0.00 & -0.08 & 0.08\end{array}$

$\begin{array}{lllll}36 & 1.00 & 0.00 & 0.00 & 0.00\end{array}$

$+$

$\begin{array}{llll}35 & 0.00 & -0.08 & 0.08\end{array}$

$\begin{array}{llll}37 & 0.00 & 0.00 & 0.00\end{array}$

$+$

$\begin{array}{llll}40 & 0.00 & 0.00 & 0.00\end{array}$

$\begin{array}{lllll}37 & 1.00 & 0.00 & 0.00 & 0.00\end{array}$

$+$

$+$

$\begin{array}{llll}36 & 0.00 & 0.00 & 0.00\end{array}$

$\begin{array}{llll}38 & 0.00 & -0.10 & 0.10\end{array}$

$\begin{array}{llll}39 & 0.00 & 0.00 & 0.00\end{array}$

$\begin{array}{lllll}38 & 1.00 & -0.08 & 14.00 & 7.00\end{array}$

+
+
+
+
+

$\begin{array}{rrrr}22 & 0.00 & 0.00 & 0.00 \\ 37 & 0.00 & -0.10 & 0.10 \\ 44 & 0.00 & -0.10 & 0.10 \\ 48 & 0.00 & 0.00 & 0.00 \\ 49 & 0.00 & -0.30 & 0.30\end{array}$

$\begin{array}{lllll}39 & 1.00 & -0.02 & 0.00 & 0.00\end{array}$
$+$
$+$
$\begin{array}{llll}37 & 0.00 & 0.00 & 0.00\end{array}$
$\begin{array}{llll}57 & 0.00 & 0.00 & 0.00\end{array}$
$\begin{array}{lllll}40 & 1.00 & -0.06 & 0.00 & 0.00\end{array}$
$+$
$\begin{array}{llll}36 & 0.00 & 0.00 & 0.00\end{array}$
$\begin{array}{llll}56 & 0.00 & -0.20 & 0.20\end{array}$
$\begin{array}{lllll}41 & 0.98 & -0.26 & 6.30 & 3.00\end{array}$
$\begin{array}{lllll}+ & 11 & 0.00 & 0.00 & 0.00 \\ + & 42 & 0.00 & 0.00 & 0.00 \\ + & 43 & 0.00 & 0.00 & 0.00\end{array}$ 


\begin{tabular}{|c|c|c|c|c|c|c|c|c|}
\hline+ & & & & & 56 & 0.00 & 0.00 & 0.00 \\
\hline 42 & 0.98 & -0.46 & 7.10 & 4.40 & & & & \\
\hline+ & & & & & 41 & 0.00 & 0.00 & 0.00 \\
\hline+ & & & & & 56 & 0.00 & 0.00 & 0.00 \\
\hline 43 & 0.97 & -0.13 & 2.00 & 1.00 & & & & \\
\hline+ & & & & & 11 & 0.0 & 0.00 & 0.00 \\
\hline+ & & & & & 41 & 0.00 & 0.00 & 0.00 \\
\hline 44 & 1.00 & -0.26 & 12.00 & 1.80 & & & & \\
\hline+ & & & & & 38 & 0.00 & -0.10 & 0.10 \\
\hline+ & & & & & 45 & 0.00 & -0.20 & 0.20 \\
\hline 45 & 0.97 & 0.30 & 0.00 & 0.00 & & & & \\
\hline+ & & & & & 15 & 0.00 & 0.00 & 0.00 \\
\hline+ & & & & & 44 & 0.00 & -0.19 & 0.19 \\
\hline 46 & 0.95 & 0.49 & 0.00 & 0.00 & & & & \\
\hline+ & & & & & 14 & 0.00 & 0.00 & 0.00 \\
\hline+ & & & & & 47 & 0.00 & -0.14 & 0.14 \\
\hline 47 & 0.99 & -0.23 & 29.70 & 11.60 & & & & \\
\hline+ & & & & & 46 & 0.00 & -0.16 & 0.16 \\
\hline+ & & & & & 48 & 0.00 & 0.00 & 0.00 \\
\hline 48 & 1.00 & 0.00 & 0.00 & 0.00 & & & & \\
\hline+ & & & & & 38 & 0.00 & 0.00 & 0.00 \\
\hline+ & & & & & 47 & 0.00 & 0.00 & 0.00 \\
\hline+ & & & & & 49 & 0.00 & -0.24 & 0.24 \\
\hline 49 & 0.97 & 0.36 & 18.00 & 8.50 & & & & \\
\hline+ & & & & & 13 & 0.00 & 0.00 & 0.00 \\
\hline+ & & & & & 38 & 0.00 & -0.28 & 0.28 \\
\hline+ & & & & & 48 & 0.00 & -0.22 & 0.22 \\
\hline+ & & & & & 50 & 0.00 & 0.00 & 0.00 \\
\hline 50 & 0.98 & -0.68 & 21.00 & 10.50 & & & & \\
\hline+ & & & & & 49 & 0.00 & 0.00 & 0.00 \\
\hline+ & & & & & 51 & 0.00 & 0.00 & 0.00 \\
\hline 51 & 0.94 & -0.20 & 18.00 & 5.30 & & & & \\
\hline+ & & & & & 10 & 0.00 & 0.00 & 0.00 \\
\hline+ & & & & & 50 & 0.00 & 0.00 & 0.00 \\
\hline 52 & 1.00 & -0.12 & 4.90 & 2.20 & & & & \\
\hline+ & & & & & 29 & 0.00 & 0.00 & 0.00 \\
\hline+ & & & & & 53 & 0.00 & 0.00 & 0.00 \\
\hline 53 & 0.98 & -0.49 & 20.00 & 10.00 & & & & \\
\hline+ & & & & & 52 & 0.00 & 0.00 & 0.00 \\
\hline
\end{tabular}


$\begin{array}{llll}54 & 0.00 & 0.00 & 0.00\end{array}$

$\begin{array}{lllll}54 & 0.99 & -0.20 & 4.10 & 1.40\end{array}$

$\begin{array}{lllll}+ & 53 & 0.00 & 0.00 & 0.00 \\ + & 55 & 0.00 & 0.00 & 0.00\end{array}$

$\begin{array}{lllll}55 & 0.94 & 0.31 & 6.80 & 3.40\end{array}$

$+$

$\begin{array}{rrrr}9 & 0.00 & 0.00 & 0.00 \\ 54 & 0.00 & 0.00 & 0.00\end{array}$

$\begin{array}{lllll}56 & 0.99 & -0.26 & 7.60 & 2.20\end{array}$

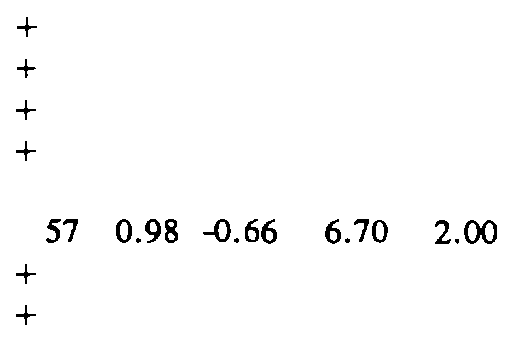

$\begin{array}{llll}40 & 0.00 & -0.20 & 0.20\end{array}$

$\begin{array}{llll}41 & 0.00 & 0.00 & 0.00\end{array}$

$\begin{array}{llll}42 & 0.00 & 0.00 & 0.00\end{array}$

$\begin{array}{llll}57 & 0.00 & 0.00 & 0.00\end{array}$

$\begin{array}{llll}39 & 0.00 & 0.00 & 0.00\end{array}$

$\begin{array}{llll}56 & 0.00 & 0.00 & 0.00\end{array}$ 
Load Flow Solution

118 BUS IEEE TEST SYSTEM

From Volt Volt Mw Mvar Mw Mvar to Mw Mvar Mva Bus Mag Angle Load Load Gen Gen Bus Flow Flow Flow $\begin{array}{llllll}1 & 0.95 & 0.00 & 51.00 & 27.00 & -12.543 .64\end{array}$

$+$

$\begin{array}{llll}2 & -17.78 & -11.21 & 21.03\end{array}$

$\begin{array}{lllll}3 & -45.76 & -12.11 & 47.34\end{array}$

$\begin{array}{lllll}2 & 0.97 & 0.91 & 20.00 & 9.00\end{array}$

$+$

$+$

$\begin{array}{llll}1 & 17.91 & 9.35 & 20.21\end{array}$

$\begin{array}{llll}12 & -37.91 & -18.35 & 42.12\end{array}$

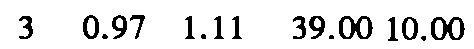

$\begin{array}{rrrrc}+ & 1 & 46.08 & 12.15 & 47.65 \\ + & 5 & -71.69 & -10.21 & 72.41 \\ + & 12 & -13.39 & -11.94 & 17.94\end{array}$

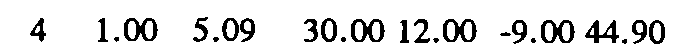

$\begin{array}{rrrrr}+ & 11 & 63.00 & 1.67 & 63.02\end{array}$

$\begin{array}{lllll}+ & 5 & -102.00 & 31.23 & 106.68\end{array}$

$\begin{array}{lllll}5 & 1.00 & 5.59 & 0.00 & 0.00\end{array}$

$\begin{array}{lrrrr}+ & 3 & 73.03 & 13.50 & 74.27 \\ + & 4 & 102.21 & -30.52 & 106.67 \\ + & 11 & 75.09 & -1.69 & 75.11 \\ + & 6 & 86.25 & 8.37 & 86.66 \\ + & 8 & -336.58 & -91.48 & 348.79\end{array}$

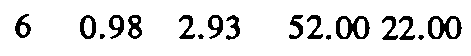

$\begin{array}{rrrrr}+ & 5 & -85.35 & -5.68 & 85.54 \\ + & 7 & 33.35 & -16.32 & 37.13\end{array}$

$\begin{array}{lllll}7 & 0.99 & 2.48 & 19.00 & 2.00\end{array}$

$+$

$\begin{array}{llll}6 & -33.29 & 16.10 & 36.97\end{array}$

$\begin{array}{lllll}+ & 12 & 14.29 & -18.10 & 23.06\end{array}$

$\begin{array}{lllllrll}8 & 1.03 & 10.56 & 28.00 & -158.20 & & & \\ + & & & & 30 & 76.20 & 49.66 & 90.96 \\ + & & & & 9 & -440.78 & -75.98 & 447.28 \\ + & & & & 5 & 336.58 & 123.67 & 358.58\end{array}$

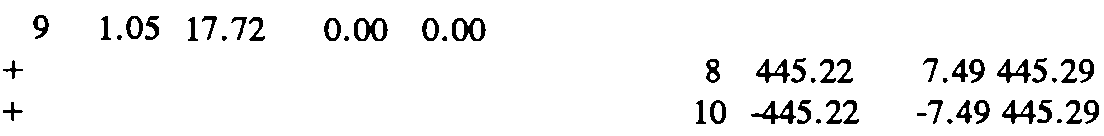

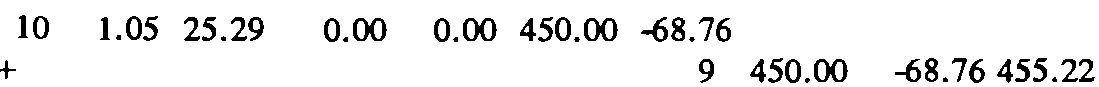

$\begin{array}{lllll}11 & 0.98 & 2.59 & 70.00 & 23.00\end{array}$ 


$\begin{array}{lrrrr}+ & 4 & -62.17 & -0.63 & 62.17 \\ + & 5 & -73.94 & 3.85 & 74.04 \\ + & 12 & 30.38 & -39.36 & 49.72 \\ + & 13 & 35.74 & 13.15 & 38.08\end{array}$

$\begin{array}{lllllll}12 & 0.99 & 2.10 & 47.00 & 10.00 & 102.10 & 109.79\end{array}$

+
+
+
+
+
+
+

$\begin{array}{llll}2 & 38.26 & 17.99 & 42.28\end{array}$

$\begin{array}{llll}3 & 13.54 & 8.53 & 16.00\end{array}$

$\begin{array}{llll}7 & -14.24 & 17.44 & 22.51\end{array}$

$\begin{array}{llll}11 & -30.23 & 39.37 & 49.64\end{array}$

$\begin{array}{llll}16 & 8.22 & 6.27 & 10.34\end{array}$

$\begin{array}{llll}117 & 20.15 & 5.20 & 20.81\end{array}$

$\begin{array}{llll}14 & 19.40 & 4.99 & 20.03\end{array}$

$\begin{array}{lllll}13 & 0.97 & 1.20 & 34.00 & 16.00\end{array}$

$\begin{array}{ccccl}+ & 11 & -35.40 & -13.82 & 38.00 \\ + & 15 & 1.40 & -2.18 & 2.59\end{array}$

$\begin{array}{lllll}14 & 0.98 & 1.37 & 14.00 & 1.00\end{array}$

$\begin{array}{lllll}+ & & & & \\ + & & & & \\ 15 & 0.96 & 1.03 & 90.00 & 30.00\end{array}$

$\begin{array}{llll}12 & -19.31 & -6.46 & 20.36\end{array}$

$\begin{array}{llll}15 & 5.31 & 5.46 & 7.61\end{array}$

$\begin{array}{llllll}+ & & & & \\ + & & & & \\ + & & & & \\ + & & & & \\ + & & & & \\ & & & & & \\ & 16 & 0.98 & 1.79 & 25.00 & 10.00\end{array}$

$+\quad \begin{array}{llll}12 & -8.19 & -8.25 & 11.63\end{array}$

$+\quad \begin{array}{lllll}17 & -16.81 & -1.75 & 16.90\end{array}$

$\begin{array}{lllll}17 & 0.9 & 3.59 & 11.00 & 3.00\end{array}$

$+$

$+$

$+$

$+$

$+$

$+$

$\begin{array}{llll}13 & -1.40 & -3.64 & 3.90\end{array}$

$\begin{array}{llll}14 & -5.25 & -10.02 & 11.32\end{array}$

$\begin{array}{lllll}17 & -104.97 & -26.79 & 108.33\end{array}$

$\begin{array}{lllll}19 & 12.47 & 13.06 & 18.05\end{array}$

$\begin{array}{llll}33 & 9.15 & -2.60 & 9.51\end{array}$

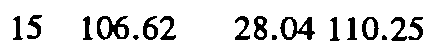

$\begin{array}{lllll}16 & 16.94 & -2.25 & 17.09\end{array}$

$\begin{array}{llll}113 & -3.30 & 7.41 & 8.11\end{array}$

$\begin{array}{lllll}18 & 81.05 & 39.50 & 90.16\end{array}$

$\begin{array}{lllll}31 & 14.59 & 7.92 & 16.61\end{array}$

$\begin{array}{llll}30 & -226.90 & 21.50 & 227.92\end{array}$

$\begin{array}{lllll}18 & 0.96 & 1.42 & 60.00 & 34.00\end{array}$

$+$

$\begin{array}{llll}17 & -80.02 & -36.50 & 87.95\end{array}$

$\begin{array}{llll}19 & 20.02 & 2.50 & 20.17\end{array}$

$\begin{array}{lllll}19 & 0.96 & 0.82 & 45.00 & 25.00\end{array}$
$+$
$+$
$\begin{array}{llll}15 & -12.42 & -13.83 & 18.59\end{array}$
$\begin{array}{llll}18 & -19.97 & -3.33 & 20.24\end{array}$
$+$
$\begin{array}{llll}20 & -10.69 & 2.45 & 10.96\end{array}$
$\begin{array}{llll}34 & -1.92 & -10.29 & 10.47\end{array}$ 


$$
\begin{aligned}
& \begin{array}{lllll}
20 & 0.95 & 1.67 & 18.00 & 3.00
\end{array} \\
& \begin{array}{llllll}
+ & 19 & 10.72 & -5.00 & 11.83
\end{array} \\
& \begin{array}{lllll}
21 & -28.72 & 2.00 & 28.79
\end{array} \\
& \begin{array}{lllll}
21 & 0.96 & 3.23 & 14.00 & 8.00
\end{array} \\
& \begin{array}{lllll}
+ & 20 & 28.89 & -3.19 & 29.07 \\
+ & 22 & -42.89 & -4.81 & 43.16
\end{array} \\
& \begin{array}{lllll}
22 & 0.97 & 5.75 & 10.00 & 5.00
\end{array} \\
& \begin{array}{lllll}
+ & 21 & 43.31 & 4.48 & 43.55
\end{array} \\
& \begin{array}{llll}
23 & -53.31 & -9.48 & 54.15
\end{array} \\
& \begin{array}{lllll}
23 & 1.01 & 10.57 & 7.00 & 3.00
\end{array} \\
& \begin{array}{ccccc}
+ & 22 & 54.37 & 10.42 & 55.35 \\
+ & 32 & 92.80 & 6.76 & 93.05 \\
+ & 24 & 10.31 & -2.50 & 10.61 \\
+ & 25 & -164.48 & -17.69 & 165.42
\end{array}
\end{aligned}
$$

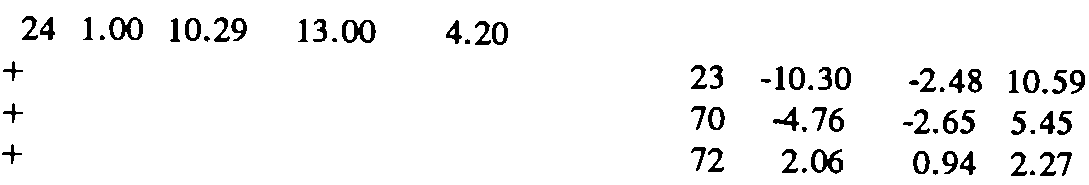

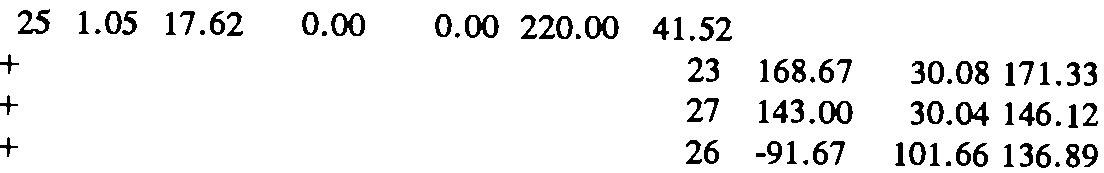

$$
\begin{aligned}
& \begin{array}{lllllll}
26 & 1.01 & 19.42 & 0.00 & 0.00 & 314.00 & 10.61
\end{array} \\
& \begin{array}{lllll}
+ & 25 & 91.67 & -95.43 & 132.33
\end{array} \\
& +\quad 30 \quad 222.33 \quad-11.02222 .60 \\
& \begin{array}{lllllll}
27 & 0.97 & 5.09 & 62.00 & 13.00 & -9.00 & -5.07
\end{array} \\
& + \\
& 25-136.64 \quad-15.46 \quad 137.52 \\
& \begin{array}{llll}
32 & 12.16 & -4.02 & 12.81
\end{array} \\
& \begin{array}{llll}
115 & 20.73 & 1.98 & 20.82
\end{array} \\
& \begin{array}{llll}
28 & 32.76 & -0.57 & 32.77
\end{array} \\
& \begin{array}{lllll}
28 & 0.96 & 3.37 & 17.00 & 7.00
\end{array} \\
& \begin{array}{rrrrr}
+ & 27 & -32.54 & -0.47 & 32.55 \\
+ & 29 & 15.54 & -6.53 & 16.86
\end{array} \\
& \begin{array}{cccccccccc}
29 & 0.96 & 2.38 & 24.00 & 4.00 & & & & \\
+ & & & & & & 28 & -15.47 & 4.61 & 16.14 \\
+ & & & & & & 31 & -8.53 & -8.61 & 12.12
\end{array} \\
& \begin{array}{lllll}
30 & 0.99 & 8.56 & 0.00 & 0.00
\end{array}
\end{aligned}
$$

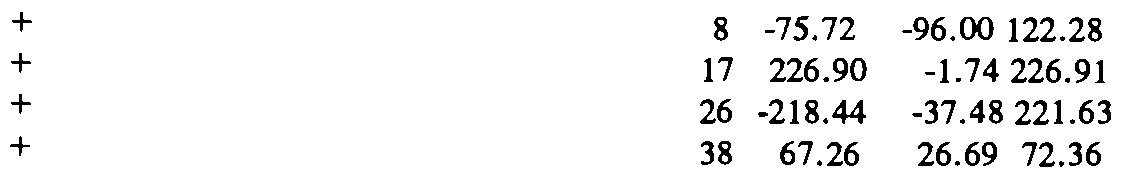


$\begin{array}{lllllll}31 & 0.97 & 2.50 & 43.00 & 27.00 & 7.00 & 31.54\end{array}$

$\begin{array}{ccccc}+ & 17 & -14.44 & -11.26 & 18.31 \\ + & 29 & 8.54 & 7.89 & 11.63 \\ + & 32 & -30.10 & 7.90 & 31.12\end{array}$

$\begin{array}{lllll}32 & 0.97 & 4.49 & 59.00 & 23.00\end{array}$

$\begin{array}{rrrrr}+ & 23 & -90.05 & -8.16 & 90.42 \\ + & 27 & -12.12 & 2.35 & 12.34 \\ + & 31 & 30.41 & -9.20 & 31.78 \\ + & 113 & 3.39 & -12.82 & 13.26 \\ + & 114 & 9.37 & 4.83 & 10.54\end{array}$

$\begin{array}{lllll}33 & 0.96 & 0.29 & 23.00 & 9.00\end{array}$

$+$

$+$

$\begin{array}{lllll}34 & 0.98 & 0.77 & 59.00 & 26.00\end{array}$

$+$

$+$

$+$

$+$

$\begin{array}{lllll}35 & 0.97 & 0.37 & 33.00 & 9.00\end{array}$

$+$

$+$

$\begin{array}{lllll}36 & 0.97 & 0.36 & 31.00 & 17.00\end{array}$

$+$

$+$

$\begin{array}{lllll}37 & 0.98 & 1.32 & 0.00 & 0.00\end{array}$

$$
+
$$$$
+
$$$$
+
$$

$+$

$+$

$+$

$\begin{array}{lllll}38 & 0.96 & 6.49 & 0.00 & 0.00\end{array}$

$+$

$+$

$+$

$\begin{array}{lllll}39 & 0.96 & -2.30 & 27.00 & 11.00\end{array}$

$+$

$+$

$\begin{array}{llllrrrrrr}40 & 0.97 & -3.48 & 20.00 & 23.00 & -46.00 & 44.43 & & & \\ + & & & & & & 37 & -42.69 & 10.83 & 44.05 \\ + & & & & & & 39 & -26.87 & 15.83 & 31.18\end{array}$

$$
\begin{array}{llll}
15 & -9.12 & -0.24 & 9.12
\end{array}
$$$$
\begin{array}{llll}
37 & -13.88 & -8.76 & 16.42
\end{array}
$$

$\begin{array}{cccl}19 & 1.97 & 4.55 & 4.96 \\ 36 & 30.58 & 16.55 & 34.78 \\ 37 & -92.54 & 15.64 & 93.86 \\ 43 & 0.99 & -9.35 & 9.40\end{array}$

$$
\begin{array}{llll}
36 & 0.53 & 0.02 & 0.53
\end{array}
$$$$
\begin{array}{llll}
37 & -33.53 & -9.02 & 34.72
\end{array}
$$

$\begin{array}{llll}34 & -30.47 & -16.74 & 34.77\end{array}$

$\begin{array}{llll}35 & -0.53 & -0.26 & 0.59\end{array}$

$\begin{array}{rrrl}33 & 13.99 & 5.70 & 15.11 \\ 34 & 92.78 & -15.70 & 94.10 \\ 35 & 33.67 & 8.40 & 34.70 \\ 38 & -239.47 & 68.22 & 249.00 \\ 39 & 55.08 & -4.80 & 55.29 \\ 40 & 43.95 & -11.27 & 45.37\end{array}$

$\begin{array}{llll}30 & -66.94 & -62.72 & 91.73\end{array}$

$\begin{array}{lllll}37 & 239.47 & -45.39 & 243.73\end{array}$

$\begin{array}{llll}65 & -172.53 & -63.88 & 183.98\end{array}$

$\begin{array}{llll}40 & 27.06 & -16.63 & 31.76\end{array}$ $\begin{array}{llll}37 & -54.06 & 5.63 & 54.35\end{array}$ 


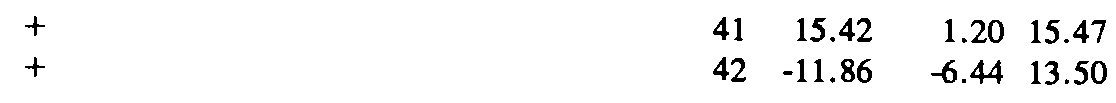

$\begin{array}{llllllllll}41 & 0.97 & -3.92 & 37.00 & 10.00 & & & & \\ + & & & & & 40 & -15.38 & -2.22 & 15.54 \\ + & & & & & & 42 & -21.62 & -7.78 & 22.97\end{array}$

$\begin{array}{lllllll}42 & 0.99 & -2.32 & 37.00 & 23.00 & -59.00 & 41.05\end{array}$

$\begin{array}{lllll}+ & 40 & 11.96 & 2.29 & 12.17 \\ + & 41 & 21.84 & 5.23 & 22.46\end{array}$

$\begin{array}{lllll}+ & 49 & -129.79 & 10.53 & 130.22\end{array}$

$\begin{array}{lllll}43 & 0.99 & 0.49 & 18.00 & 7.00\end{array}$

$\begin{array}{rrrrr}+ & 34 & -0.96 & 5.37 & 5.46\end{array}$

$\begin{array}{lllll}+ & 44 & -17.04 & -12.37 & 21.06\end{array}$

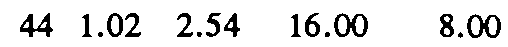

$\begin{array}{rlrrr}+ & 43 & 17.27 & 7.19 & 18.71\end{array}$

$\begin{array}{lllll}+ & 45 & -33.27 & 5.74 & 33.76\end{array}$

$\begin{array}{lllll}45 & 1.02 & 4.26 & 53.00 & 22.00\end{array}$

$\begin{array}{rrrrr}+ & 44 & 33.52 & -7.10 & 34.26 \\ + & 46 & -37.50 & -1.81 & 37.54\end{array}$

$\begin{array}{lllll}+ & 49 & -49.02 & 18.41 & 52.36\end{array}$

$\begin{array}{cccccccccc}46 & 1.04 & 6.9 & 9.00 & 8.30 & & & & \\ + & & & & 45 & 38.04 & 0.08 & 38.04 \\ + & & & & & 47 & -32.23 & 18.25 & 37.03 \\ + & & & & & 48 & -14.81 & 5.86 & 15.93\end{array}$

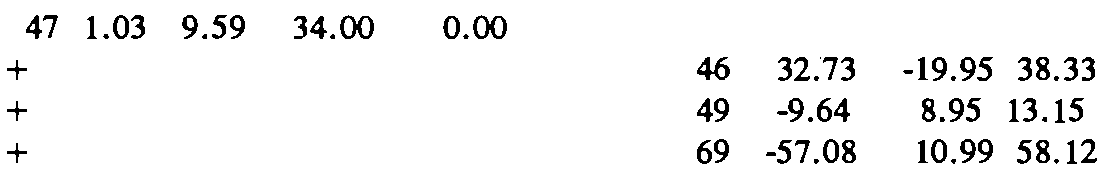

$\begin{array}{lllll}48 & 1.03 & 8.75 & 20.00 & 11.00\end{array}$

$\begin{array}{llllll}+ & 46 & 14.97 & -10.43 & 18.25\end{array}$

$\begin{array}{lllll}+ & 49 & -34.97 & 31.54 & 47.09\end{array}$

$\begin{array}{lllllll}49 & 1.02 & 10.02 & 87.00 & 30.00 & 204.00 & 37.56\end{array}$

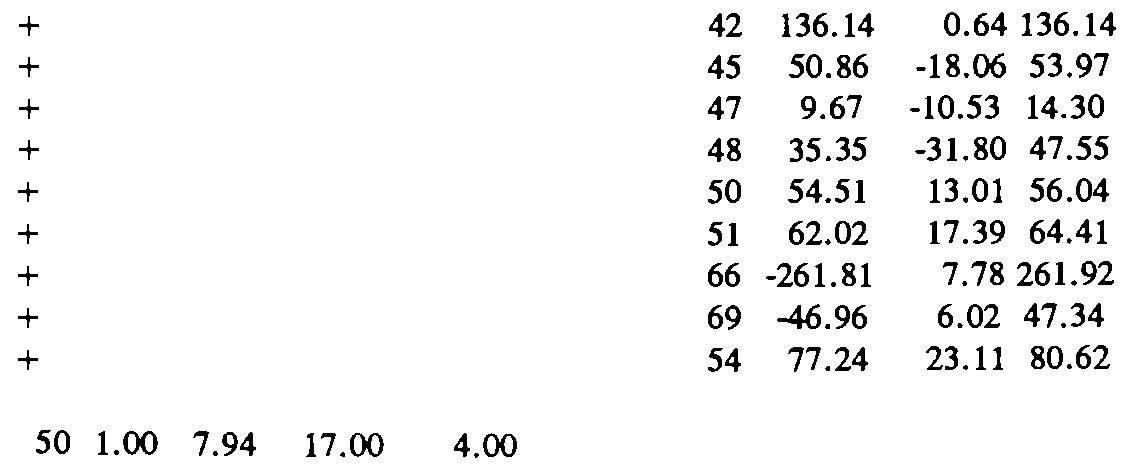


$\begin{array}{lllll}51 & 0.97 & 5.67 & 17.00 & 8.00\end{array}$

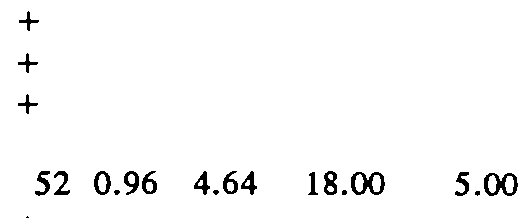

$+$

$+$

$\begin{array}{lllll}53 & 0.95 & 3.45 & 23.00 & 11.00\end{array}$

$+$

$+$

$\begin{array}{lllll}54 & 0.96 & 4.18 & 65.00 & 20.00\end{array}$

$+$

$+$

$+$

$+$

$+$

$\begin{array}{lllll}55 & 0.95 & 3.89 & 63.00 & 22.00\end{array}$

+
+
+

$\begin{array}{lllll}56 & 0.95 & 4.06 & 84.00 & 18.00\end{array}$

$+$

$+$

$+$

$+$

$+$

$\begin{array}{lllll}57 & 0.97 & 5.32 & 12.00 & 3.00\end{array}$

$+$

$+$

$\begin{array}{lllll}58 & 0.97 & 5.16 & 12.00 & 3.00\end{array}$

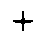

$+$

59

$+$

$+$

$+$

$+$

$+$

$+$ $\begin{array}{llll}49 & -60.07 & -15.31 & 61.99\end{array}$

$\begin{array}{llll}52 & 31.02 & 7.01 & 31.81\end{array}$

$\begin{array}{llll}58 & 12.04 & 0.30 & 12.04\end{array}$

$\begin{array}{llll}51 & -30.81 & -7.68 & 31.75\end{array}$

$\begin{array}{llll}53 & 12.81 & 2.68 & 13.08\end{array}$

$\begin{array}{llll}52 & -12.72 & -6.05 & 14.09\end{array}$

$\begin{array}{llll}54 & -10.28 & -4.95 & 11.41\end{array}$

$\begin{array}{llll}49 & -74.62 & -27.99 & 79.69\end{array}$

$\begin{array}{llll}53 & 10.31 & 2.30 & 10.56\end{array}$

$\begin{array}{llll}55 & 7.39 & 2.90 & 7.94\end{array}$

$\begin{array}{llll}56 & 22.43 & 9.70 & 24.44\end{array}$

$\begin{array}{lllll}59 & -30.52 & -6.91 & 31.29\end{array}$

$\begin{array}{llll}54 & -7.38 & -4.68 & 8.74\end{array}$

$\begin{array}{llll}56 & -20.91 & -9.21 & 22.84\end{array}$

$\begin{array}{llll}59 & -34.71 & -8.11 & 35.65\end{array}$

$\begin{array}{llll}54 & -22.41 & -10.30 & 24.66\end{array}$

$\begin{array}{llll}55 & 20.93 & 8.95 & 22.77\end{array}$

$\begin{array}{llll}57 & -23.79 & -8.52 & 25.27\end{array}$

$\begin{array}{llll}58 & 0.00 & -1.10 & 1.10\end{array}$

$\begin{array}{llll}59 & -58.73 & -7.03 & 59.15\end{array}$

$\begin{array}{llll}50 & -36.02 & -9.93 & 37.37\end{array}$

$\begin{array}{llll}56 & 24.02 & 6.93 & 25.00\end{array}$

$\begin{array}{cccc}51 & -12.00 & -1.87 & 12.15 \\ 56 & 0.00 & -1.13 & 1.13\end{array}$

61.03

$\begin{array}{llll}54 & 31.04 & 3.66 & 31.25\end{array}$

$\begin{array}{llll}55 & 35.36 & 5.75 & 35.82\end{array}$

$\begin{array}{llll}56 & 60.28 & 1.19 & 60.29\end{array}$

$\begin{array}{llll}60 & -44.25 & -0.66 & 44.25\end{array}$

$\begin{array}{llll}61 & -52.65 & 0.43 & 52.65\end{array}$

$\begin{array}{lllll}63 & -151.76 \quad 42.40 & 157.57\end{array}$ 


\begin{tabular}{|c|c|c|c|c|c|c|c|c|c|}
\hline 60 & 1.00 & 12.07 & 78.00 & 3.00 & & & & & \\
\hline+ & & & & & & 59 & 44.89 & -0.11 & 44.89 \\
\hline+ & & & & & & 61 & -112.76 & 2.56 & 112.79 \\
\hline+ & & & & & & 62 & -10.11 & -5.45 & 11.48 \\
\hline 61 & 1.00 & 12.94 & 0.00 & 0.00 & 160.00 & -9.78 & & & \\
\hline+ & & & & & & 59 & 53.59 & 0.03 & 53.59 \\
\hline+ & & & & & & 60 & 113.09 & -2.30 & 113.12 \\
\hline+ & & & & & & 62 & 25.37 & -9.35 & 27.04 \\
\hline+ & & & & & & 64 & -32.08 & 58.93 & 67.09 \\
\hline 62 & 1.00 & 12.36 & 77.00 & 14.00 & & & & & \\
\hline+ & & & & & & 60 & 10.12 & 4.06 & 10.91 \\
\hline+ & & & & & & 61 & -25.31 & 8.64 & 26.74 \\
\hline+ & & & & & & 66 & -37.35 & -14.77 & 40.17 \\
\hline+ & & & & & & 67 & -24.45 & -11.92 & 27.20 \\
\hline 63 & 0.97 & 11.68 & 0.00 & 0.00 & & & & & \\
\hline+ & & & & & & 59 & 151.76 & -32.92 & 155.29 \\
\hline+ & & & & & & 64 & -151.75 & -73.04 & 168.41 \\
\hline 64 & 0.99 & 13.43 & 0.00 & 0.00 & & & & & \\
\hline+ & & & & & & 61 & 32.08 & -57.74 & 66.06 \\
\hline+ & & & & & & 63 & 152.24 & 58.07 & 7162.94 \\
\hline+ & & & & & & 65 & -184.32 & -56.50 & 192.79 \\
\hline 65 & 1.00 & 16.59 & 0.00 & 0.00 & 391.00 & 132.12 & & & \\
\hline+ & & & & & & 38 & 175.48 & -4.47 & 175.54 \\
\hline+ & & & & & & 64 & 185.30 & 29.80 & 187.68 \\
\hline+ & & & & & & 68 & 24.18 & 34.55 & 42.17 \\
\hline+ & & & & & & 66 & 6.05 & -130.72 & 130.86 \\
\hline 66 & 1.05 & 16.48 & 39.00 & 18.00 & 392.00 & -7.11 & & & \\
\hline+ & & & & & & 49 & 267.69 & 16.86 & 268.22 \\
\hline+ & & & & & & 62 & 38.09 & 12.00 & 39.93 \\
\hline+ & & & & & & 65 & -6.05 & 136.59 & 136.72 \\
\hline+ & & & & & & 67 & 53.28 & 16.60 & 55.80 \\
\hline 67 & 1.02 & 13.81 & 28.00 & 7.00 & & & & & \\
\hline+ & & & & & & 62 & 24.63 & 9.56 & 26.42 \\
\hline+ & & & & & & 66 & -52.63 & -16.56 & 55.18 \\
\hline 68 & 0.99 & 16.42 & 0.00 & 0.00 & & & & & \\
\hline+ & & & & & & 65 & -24.11 & -97.49 & 100.43 \\
\hline+ & & & & & & 116 & 184.12 & 77.33 & 3199.70 \\
\hline+ & & & & & & 81 & -40.19 & -21.74 & 45.70 \\
\hline+ & & & & & & 69 & -119.73 & -156.65 & 5197.16 \\
\hline 69 & 1.05 & 18.70 & 0.00 & 0.00 & 514.30 & -9.22 & & & \\
\hline+ & & & & & & 47 & 59.85 & -9.54 & 60.60 \\
\hline+ & & & & & & 49 & 49.13 & -7.79 & 49.74 \\
\hline
\end{tabular}




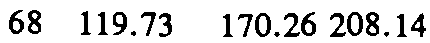

$\begin{array}{lllll}75 & 111.08 & 17.98 & 112.52\end{array}$

$\begin{array}{lllll}77 & 66.33 & 11.35 & 67.29\end{array}$

$\begin{array}{llll}70 & 107.99 & 15.43 & 109.09\end{array}$

$\begin{array}{lllll}70 & 1.00 & 11.55 & 66.00 & 20.00\end{array}$

+
+
+
+
+

$\begin{array}{llll}24 & 4.79 & -7.47 & 8.88\end{array}$

$\begin{array}{lllll}69 & -104.68 & -14.22 & 105.64\end{array}$

$\begin{array}{llll}71 & 16.04 & -13.78 & 21.14\end{array}$

$\begin{array}{lllll}74 & 16.98 & 7.24 & 18.46\end{array}$

$\begin{array}{llll}75 & 0.90 & 8.23 & 8.28\end{array}$

$\begin{array}{lllll}71 & 1.00 & 11.16 & 0.00 & 0.00\end{array}$

$+$

$+$

$+$

$\begin{array}{lllll}72 & 1.00 & 10.15 & 12.00 & 8.70\end{array}$

$+$

$+$

$\begin{array}{lllll}73 & 1.01 & 10.95 & 6.00 & -10.60\end{array}$

$+$

$\begin{array}{lllll}74 & 0.98 & 10.45 & 68.00 & 27.00\end{array}$

$+$

$+$

$\begin{array}{lllll}75 & 0.98 & 11.73 & 47.00 & 11.00\end{array}$

$+$

$+$

$+$

$+$

$+$

$\begin{array}{lllll}76 & 0.95 & 10.65 & 68.00 & 36.00\end{array}$

$+$

$+$

$\begin{array}{lllll}77 & 1.02 & 15.38 & 61.00 & 28.00\end{array}$

$+$

$+$

$+$

$+$

$+$

$+$

$\begin{array}{lllll}78 & 1.01 & 15.07 & 71.00 & 26.00\end{array}$

$+$

$+$ $\begin{array}{llll}70 & -16.00 & 13.07 & 20.66\end{array}$

$\begin{array}{llll}72 & 9.99 & -1.35 & 10.08\end{array}$

$\begin{array}{llll}73 & 6.01 & -11.72 & 13.17\end{array}$

$\begin{array}{llll}24 & -2.05 & -5.80 & 6.15\end{array}$

$\begin{array}{llll}71 & -9.94 & -2.90 & 10.36\end{array}$

$\begin{array}{llll}71 & -6.00 & 10.60 & 12.18\end{array}$

$\begin{array}{llll}70 & -16.84 & -10.05 & 19.61\end{array}$

$\begin{array}{llll}75 & -51.14 & 6.15 & 51.51\end{array}$

$\begin{array}{lllll}69 & -106.31 & -16.47 & 107.58\end{array}$

$\begin{array}{llll}70 & -0.86 & -11.63 & 11.66\end{array}$

$\begin{array}{llll}74 & 51.48 & -6.01 & 51.83\end{array}$

$\begin{array}{lllll}118 & 41.74 & 29.44 & 51.08\end{array}$

$\begin{array}{llll}77 & -33.03 & -6.34 & 33.63\end{array}$

$\begin{array}{llll}118 & -8.30 & -15.34 & 17.44\end{array}$

$\begin{array}{llll}77 & -59.69 & -20.66 & 63.16\end{array}$

$\begin{array}{cccc}69 & -65.01 & -18.11 & 67.49 \\ 75 & 33.71 & 3.64 & 33.91 \\ 76 & 61.60 & 23.46 & 65.92 \\ 78 & 45.56 & 1.03 & 45.57 \\ 80 & -137.88 & -32.31 & 141.61 \\ 82 & 1.05 & -5.72 & 5.81\end{array}$

$\begin{array}{llll}77 & -45.49 & -2.08 & 45.53\end{array}$

$\begin{array}{llll}79 & -25.51 & -23.92 & 34.97\end{array}$ 


\begin{tabular}{|c|c|c|c|c|c|c|c|c|}
\hline 79 & 1.02 & 15.34 & 39.00 & 32.00 & & & & \\
\hline+ & & & & & 78 & 25.57 & 23.55 & 34.76 \\
\hline+ & & & & & 80 & -64.57 & -13.91 & 66.05 \\
\hline 80 & 1.04 & 17.69 & 130.00 & $26.00 \quad 477.00$ & 62.71 & & & \\
\hline+ & & & & & 77 & 139.96 & 31.29 & 143.41 \\
\hline+ & & & & & 79 & 65.22 & 14.86 & 66.89 \\
\hline+ & & & & & 96 & 21.75 & 12.86 & 25.27 \\
\hline+ & & & & & 97 & 29.14 & 17.53 & 34.01 \\
\hline+ & & & & & 98 & 30.06 & 8.12 & 31.13 \\
\hline+ & & & & & 81 & 40.22 & 147.38 & 152.76 \\
\hline+ & & & & & 99 & 20.67 & 7.90 & 22.13 \\
\hline
\end{tabular}

$\begin{array}{lllll}81 & 0.99 & 16.91 & 0.00 & 0.00\end{array}$$$
+
$$$$
+
$$$$
\begin{array}{llll}
68 & 40.23 & -57.46 & 70.14
\end{array}
$$$$
\begin{array}{lllll}
80 & -40.22 & -139.91 & 145.58
\end{array}
$$

$\begin{array}{lllll}82 & 1.02 & 15.30 & 54.00 & 27.00\end{array}$

$$
+
$$$$
+
$$$$
\begin{array}{cccl}
77 & -1.05 & -2.72 & 2.92 \\
96 & -10.49 & 19.98 & 22.57 \\
83 & -42.46 & 17.68 & 45.99
\end{array}
$$

$\begin{array}{lllll}83 & 1.01 & 16.29 & 20.00 & 10.00\end{array}$

$\begin{array}{llll}82 & 42.69 & -20.82 & 47.50\end{array}$

$\begin{array}{llll}84 & -22.16 & 20.71 & 30.33\end{array}$

$\begin{array}{llll}85 & -40.53 & 20.95 & 45.62\end{array}$

$\begin{array}{lllll}84 & 1.00 & 18.72 & 11.00 & 7.00\end{array}$

$+$

$+$

$\begin{array}{llll}83 & 22.76 & -22.07 & 31.70\end{array}$

$\begin{array}{lllll}85 & -33.76 & 15.07 & 36.97\end{array}$

$\begin{array}{lllll}85 & 1.00 & 20.23 & 24.00 & 15.00\end{array}$

+
+
+
+
+

$\begin{array}{lllll}83 & 41.43 & -21.37 & 46.62\end{array}$

$\begin{array}{lllll}84 & 34.18 & -15.40 & 37.49\end{array}$

$\begin{array}{llll}86 & 21.16 & 3.52 & 21.46\end{array}$

$\begin{array}{llll}88 & -49.77 & 12.57 & 51.33\end{array}$

$\begin{array}{llll}89 & -70.99 & 5.68 & 71.22\end{array}$

\begin{tabular}{|c|c|c|c|c|c|c|c|c|}
\hline+ & & & & & 85 & -21.00 & -5.66 & 21.75 \\
\hline+ & & & & & 87 & 0.00 & -4.34 & 4.34 \\
\hline 87 & 0.99 & 18.79 & 0.00 & 0.00 & & & & \\
\hline+ & & & & & 86 & 0.00 & 0.00 & 0.00 \\
\hline 88 & 1.00 & 23.31 & 48.00 & 10.00 & & & & \\
\hline+ & & & & & 85 & 50.31 & -12.60 & 51.86 \\
\hline+ & & & & & 89 & -98.30 & 2.60 & 98.34 \\
\hline 89 & 1.01 & 27.32 & 0.00 & 0.00607 .00 & -9.90 & & & \\
\hline+ & & & & & 85 & 72.21 & -1.60 & 72.23 \\
\hline
\end{tabular}

$\begin{array}{lllll}86 & 0.99 & 18.82 & 21.00 & 10.00\end{array}$ 


$$
\begin{array}{ccccc}
+ & 88 & 99.65 & 2.39 & 99.68 \\
+ & 90 & 168.33 & 0.50168 .33 \\
+ & 92 & 266.75 & -11.18 & 266.99
\end{array}
$$

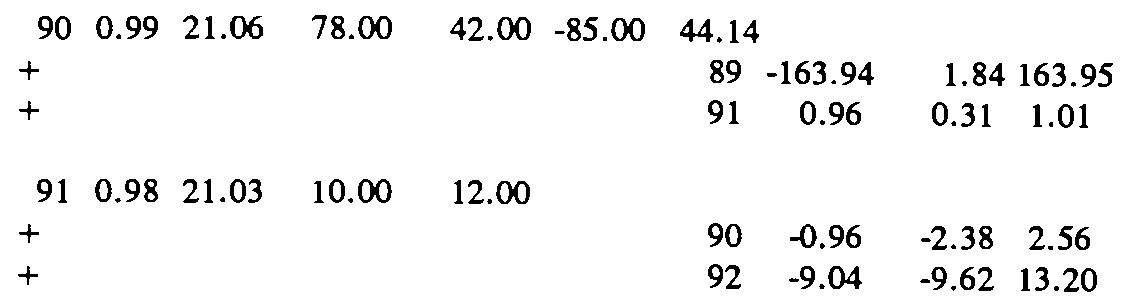

$\begin{array}{lllll}93 & 0.99 & 18.80 & 12.00 & 7.00\end{array}$

$\begin{array}{lllll}94 & 1.00 & 16.90 & 30.00 & 16.00\end{array}$

$\begin{array}{llll}92 & -45.93 & 14.11 & 48.04\end{array}$

$\begin{array}{llll}93 & -39.37 & 17.67 & 43.15\end{array}$

$\begin{array}{lllll}95 & 38.48 & 3.82 & 38.67\end{array}$

$\begin{array}{lllll}96 & 17.16 & -15.61 & 23.20\end{array}$

$\begin{array}{llll}100 & -0.34 & -35.98 & 35.98\end{array}$

$\begin{array}{lllll}95 & 0.99 & 15.96 & 42.00 & 31.00\end{array}$

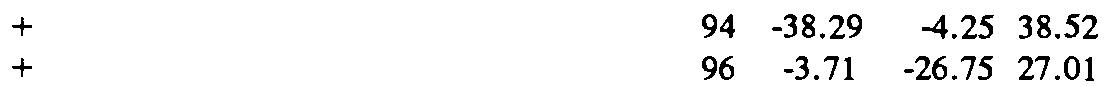

$\begin{array}{lllll}96 & 1.01 & 15.82 & 38.00 & 15.00\end{array}$

$+$

$+$

$+$

$+$

$+$

$\begin{array}{llll}80 & -21.51 & -16.83 & 27.31\end{array}$

$\begin{array}{llll}82 & 10.59 & -25.22 & 27.35\end{array}$

$\begin{array}{lllll}94 & -17.02 & 13.75 & 21.88\end{array}$

$\begin{array}{lllll}95 & 3.83 & 25.66 & 25.94\end{array}$

$\begin{array}{lllll}97 & -13.88 & -12.36 & 18.59\end{array}$

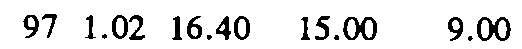

$+$

$+$

$\begin{array}{llll}80 & -28.94 & -19.18 & 34.72\end{array}$

$\begin{array}{llll}96 & 13.94 & 10.18 & 17.26\end{array}$

$\begin{array}{lllll}98 & 1.02 & 16.06 & 34.00 & 8.00\end{array}$

$\begin{array}{crrrl}+ & 80 & -29.84 & -10.17 & 31.52 \\ + & 100 & -4.16 & 2.17 & 4.69\end{array}$

$\begin{array}{lllll}99 & 1.01 & 15.62 & 42.00 \quad 15.60\end{array}$ 
$\begin{array}{rcccc}+ & 80 & -20.44 & -11.86 & 23.64 \\ + & 100 & -21.56 & -3.74 & 21.88\end{array}$

$\begin{array}{lllllll}100 & 1.02 & 16.57 & 27.00 & 18.00 & 252.00 & 7.6\end{array}$

$+$

$+$

$\begin{array}{llll}92 & -26.63 & 9.82 & 28.39\end{array}$

$\begin{array}{llll}94 & 0.54 & 30.48 & 30.49\end{array}$

$\begin{array}{llll}98 & 4.18 & -7.06 & 8.20\end{array}$

$\begin{array}{llll}99 & 21.64 & 1.89 & 21.72\end{array}$

$\begin{array}{llll}101 & -12.68 & 20.71 & 24.29\end{array}$

$\begin{array}{lllll}103 & 119.18 & -42.44 & 126.51\end{array}$

$\begin{array}{llll}104 & 57.79 & -10.70 & 58.77\end{array}$

$\begin{array}{llll}106 & 60.99 & -13.11 & 62.38\end{array}$

$\begin{array}{llll}101 & 0.99 & 17.84 & 22.00 \quad 18.00\end{array}$

$\begin{array}{rrrrr}+ & 100 & 12.86 & -23.22 & 26.54\end{array}$

$\begin{array}{lllll}+ & 102 & -34.86 & 5.22 & 35.25\end{array}$

$\begin{array}{lllll}102 & 0.99 & 20.20 & 5.00 & 3.00\end{array}$

$+$

$+$

$\begin{array}{llll}92 & -40.17 & 3.69 & 40.34\end{array}$

$\begin{array}{llll}101 & 35.17 & -6.69 & 35.80\end{array}$

$\begin{array}{lllll}103 & 1.02 & 12.77 & -17.00 & -24.00\end{array}$

$+$

$+$

$+$

$+$

$\begin{array}{llll}100 & -116.74 & 44.88 & 125.07\end{array}$

$\begin{array}{llll}110 & 58.71 & 2.30 & 58.75\end{array}$

$\begin{array}{llll}104 & 32.43 & -6.48 & 33.07\end{array}$

$\begin{array}{llll}105 & 42.61 & -16.70 & 45.76\end{array}$

$\begin{array}{lllll}104 & 1.01 & 9.81 & 38.00 & 25.00\end{array}$

$+$

$+$

$+$

$\begin{array}{llll}100 & -56.30 & 11.84 & 57.53\end{array}$

$\begin{array}{llll}103 & -31.95 & 3.97 & 32.19\end{array}$

$\begin{array}{llll}105 & 50.25 & -40.74 & 64.69\end{array}$

$\begin{array}{lllll}105 & 1.02 & 8.55 & 31.00 & 26.00\end{array}$

$+$

$+$

$+$

$+$

$+$

$\begin{array}{rrrr}103 & -41.57 & 15.59 & 44.39 \\ 104 & -49.86 & 41.24 & 64.70 \\ 106 & 8.20 & 18.59 & 20.32 \\ 107 & 26.80 & -5.78 & 27.42 \\ 108 & 25.42 & 9.34 & 27.08\end{array}$

$\begin{array}{lllll}106 & 1.01 & 8.45 & 43.00 & 16.00\end{array}$

$+$

$+$

$+$

$\begin{array}{llll}100 & -58.76 & 15.17 & 60.69\end{array}$

$\begin{array}{llll}105 & \mathbf{- 8 . 1 5} & -19.85 & 21.46\end{array}$

$\begin{array}{lllll}107 & 23.90 & -11.32 & 26.45\end{array}$

$\begin{array}{lllll}107 & 1.02 & 5.75 & 50.00 & 2.70\end{array}$

$+$

$+$

$\begin{array}{llll}105 & -26.43 & 2.12 & 26.52\end{array}$

$\begin{array}{llll}106 & -23.57 & 7.61 & 24.77\end{array}$

$\begin{array}{lllll}108 & 1.01 & 7.71 & 2.00 & 1.00\end{array}$

$+$

$+$

$\begin{array}{lllll}105 & -25.23 & -10.74 & 27.42\end{array}$

$\begin{array}{llll}109 & 23.23 & 9.74 & 25.19\end{array}$ 


$$
\begin{aligned}
& \begin{array}{lllll}
109 & 1.01 & 7.39 & 8.00 \quad 3.00
\end{array} \\
& \begin{array}{lllll}
+ & 108 & -23.16 & -10.33 & 25.36
\end{array} \\
& \begin{array}{lllll}
+ & 110 & 15.16 & 7.33 & 16.84
\end{array} \\
& \begin{array}{lllll}
110 & 1.00 & 6.86 & 39.00 & 30.00
\end{array} \\
& +\quad 103 \quad-57.41 \quad-0.9457 .41 \\
& +\quad \begin{array}{lllll}
+ & 109 & -15.08 & -9.13 & 17.63
\end{array} \\
& \begin{array}{lllll}
+ & 111 & -35.72 & 0.38 & 35.72
\end{array}
\end{aligned}
$$

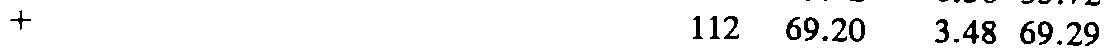

$$
\begin{aligned}
& \begin{array}{lllll}
111 & 1.00 & 8.43 & -36.00 & 1.40
\end{array} \\
& \begin{array}{lllll}
+ & 110 & 36.00 & -1.40 & 36.03
\end{array}
\end{aligned}
$$

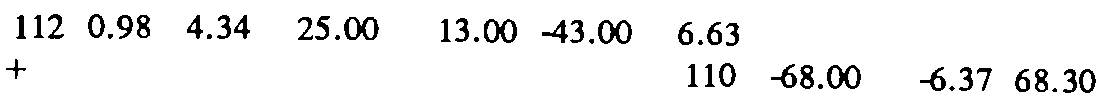

$$
\begin{aligned}
& \begin{array}{lllll}
113 & 0.99 & 3.69 & 0.00 & 0.00
\end{array} \\
& \begin{array}{lllll}
+ & 17 & 3.31 & -8.13 & 8.78
\end{array} \\
& \begin{array}{lllll}
+ & 32 & -3.31 & 8.13 & 8.78
\end{array}
\end{aligned}
$$

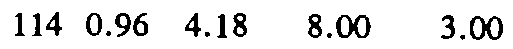

$$
\begin{aligned}
& \begin{array}{lllll}
+ & 32 & -9.35 & -6.26 & 11.25
\end{array} \\
& \begin{array}{lllll}
+ & 115 & 1.35 & 3.26 & 3.53
\end{array} \\
& \begin{array}{lllll}
115 & 0.96 & 4.18 & 22.00 & 7.00
\end{array} \\
& \begin{array}{lrrrr}
+ & 27 & -20.65 & -3.48 & 20.94 \\
+ & 114 & -1.35 & -3.52 & 3.77
\end{array} \\
& \begin{array}{rrrrr}
+ & 114 & -1.35 & -3.52 & 3.77
\end{array} \\
& \begin{array}{llll}
116 & 0.99 & 16.01 & 184.00 \quad 91.80
\end{array} \\
& \begin{array}{lllll}
+ & 68 & -183.99 & -91.80 & 205.62
\end{array} \\
& \begin{array}{rrrrrrrrr}
117 & 0.97 & 0.56 & 20.00 & 8.00 & & & & \\
+ & & & & & 12 & -20.00 & -8.00 & 21.54
\end{array}
\end{aligned}
$$

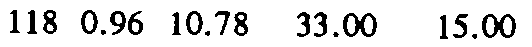

$$
\begin{aligned}
& + \\
& + \\
& \begin{array}{llll}
75 & -41.35 & -29.25 & 50.65
\end{array} \\
& \begin{array}{llll}
76 & 8.36 & 14.25 & 16.52
\end{array}
\end{aligned}
$$




\section{Load Flow Solution}

FPL data

Buses $=1883$ generators $=222$

From V V $\quad$ Mw Mvar Mw Mvar to Mw Mvar Mva

Bus Mag Angle Load Load Gen Gen Bus Flow Flow Flow

$\begin{array}{llllllll}1 & 1.03 & 0.00 & 0.00 & 0.00 & -97.02 & 1098.72\end{array}$

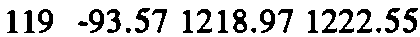

$\begin{array}{lllllll}2 & 1.03 & 13.58 & 0.00 & 0.00 & 367.00 & 1125.54\end{array}$

$119 \quad 380.32 \quad 1247.48 \quad 1304.17$

$\begin{array}{lllllll}3 & 1.01 & 12.27 & 0.00 & 0.00 & 666.00 & 1955.11\end{array}$

119700.982353 .192455 .38

$\begin{array}{lllllll}4 & 0.98 & 12.63 & 0.00 & 0.00 & 666.00 & 1745.15\end{array}$

$\begin{array}{lllll}5 & 0.59 & 0.13 & 0.00 & 0.00\end{array}$

119699.042121 .772233 .96

$\begin{array}{llll}40 & 255.75 & 190.37 & 318.82\end{array}$

$119-243.59-228.10 \quad 333.71$

$\begin{array}{lllll}6 & 0.19 & -60.76 & 33.04 & 9.64\end{array}$

$\begin{array}{llll}12 & 6.51 & -4.31 & 7.80\end{array}$

$\begin{array}{llll}20 & 30.80 & 18.81 & 36.09\end{array}$

$\begin{array}{llll}22 & -79.04 & -42.92 & 89.94\end{array}$

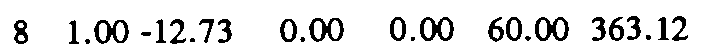

$\begin{array}{lllllll}35 & 62.80 & 404.32 & 409.17\end{array}$

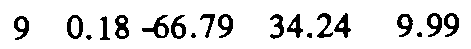

$\begin{array}{llll}12 & -43.34 & -18.89 & 47.27\end{array}$

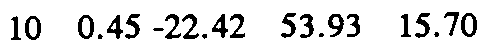

$\begin{array}{llll}40 & -56.89 & -23.10 & 61.40\end{array}$

$\begin{array}{llll}3303 & 57.52 & 10.37 & 58.45\end{array}$

$\begin{array}{lllllll}11 & 1.00 & -4.93 & 0.00 & 0.00 & 150.00 & 493.22\end{array}$

$12 \quad 0.19-62.15 \quad 0.00 \quad 0.00$

$\begin{array}{llll}35 & 153.58 & 547.92 & 569.04\end{array}$

$\begin{array}{llll}6 & -6.49 & 4.27 & 7.76\end{array}$

$\begin{array}{llll}9 & 44.57 & 23.75 & 50.50\end{array}$

$\begin{array}{llll}57 & -32.45 & -17.65 & 36.94\end{array}$

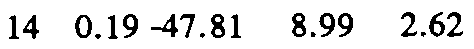

$\begin{array}{llll}61 & 46.68 & 20.57 & 51.01\end{array}$

$\begin{array}{llll}73 & -9.90 & -39.51 & 40.73\end{array}$

$\begin{array}{lllll}15 & 0.52 & -8.06 & 38.09 & 11.11\end{array}$

$110-122.01-117.89 \quad 169.66$

$\begin{array}{llll}118 & 100.89 & 97.00 & 139.95\end{array}$

$\begin{array}{lllll}16 & 0.17 & -65.97 & 37.58 & 10.96\end{array}$

$\begin{array}{llll}17 & -19.86 & -5.76 & 20.68\end{array}$

$\begin{array}{llll}21 & -4.14 & -10.28 & 11.09\end{array}$

$\begin{array}{llll}72 & -17.61 & -2.29 & 17.76\end{array}$

$\begin{array}{lllll}17 & 0.18 & -59.72 & 0.00 & 0.00\end{array}$

$\begin{array}{llll}16 & 20.10 & 7.78 & 21.55\end{array}$

$\begin{array}{llll}73 & -19.84 & -4.34 & 20.31\end{array}$

$\begin{array}{lllll}18 & 0.17 & -54.66 & 46.31 & 13.51\end{array}$

$\begin{array}{llll}33 & 106.63 & 35.69 & 112.44\end{array}$ 


$$
\begin{array}{rrrrrrrrrrr}
19 & 0.73 & -18.76 & 25.51 & 7.44 & 73 & -135.38 & -62.91 & 149.28 \\
20 & 0.18 & -63.21 & 9.07 & 2.65 & 76 & -25.39 & -3.50 & 25.63 \\
& & & & 6 & -30.24 & -16.85 & 34.61 \\
& & & & 21 & 24.76 & 7.77 & 25.95
\end{array}
$$

$\begin{array}{lllll}21 & 0.17 & -65.75 & 21.23 & 6.19\end{array}$

$\begin{array}{llll}16 & 4.21 & 10.51 & 11.33\end{array}$

$\begin{array}{llll}20 & -24.45 & -6.61 & 25.33\end{array}$

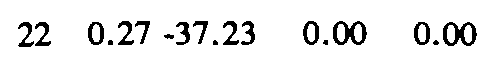

$\begin{array}{llll}6 & 80.87 & 103.63 & 131.45\end{array}$

$\begin{array}{lllll}65 & -83.84 & -113.69 & 141.26\end{array}$

$\begin{array}{lllll}23 & 0.18 & -51.08 & 42.37 & 12.36\end{array}$

$\begin{array}{llll}26 & 57.27 & 37.03 & 68.20\end{array}$

$\begin{array}{llll}41 & -91.63 & -54.56 & 106.64\end{array}$

$\begin{array}{lllll}24 & 0.14 & -52.86 & 35.10 & 10.24\end{array}$

$54-103.65-112.21 \quad 152.76$

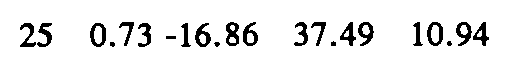

$\begin{array}{llll}611 & 62.76 & 90.54 & 110.16\end{array}$

$$
\begin{array}{rrrc}
28 & 12.02 & -13.29 & 17.92 \\
51 & -95.41 & -35.62 & 101.84 \\
76 & -14.35 & -21.90 & 26.19 \\
96 & 49.57 & 23.67 & 54.93
\end{array}
$$

$\begin{array}{lllll}26 & 0.18 & -54.64 & 30.82 & 8.99\end{array}$

$\begin{array}{llll}23 & -56.47 & -31.74 & 64.78\end{array}$

$\begin{array}{llll}85 & 26.79 & 22.85 & 35.21\end{array}$

$\begin{array}{lllll}27 & 0.39 & -27.63 & 37.15 & 10.84\end{array}$

$\begin{array}{llll}29 & -178.53 & -283.58 & 335.09\end{array}$

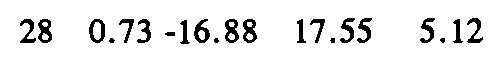

$\begin{array}{lllll}71 & 147.75 & 250.07 & 290.46\end{array}$

$\begin{array}{lllll}29 & 0.44 & -24.51 & 10.10 & 2.95\end{array}$

$\begin{array}{llll}25 & -11.38 & 11.00 & 15.83\end{array}$

$\begin{array}{lllll}27 & 183.65 & 330.31 & 377.93\end{array}$

$\begin{array}{llll}32 & -195.39 & -329.74 & 383.28\end{array}$

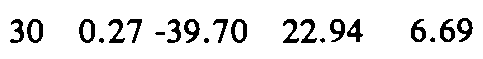

$\begin{array}{llll}65 & -96.26 & -114.29 & 149.43\end{array}$

$\begin{array}{llll}68 & 77.79 & 94.95 & 122.75\end{array}$

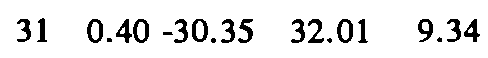

$\begin{array}{llll}50 & 124.70 & 167.95 & 209.18\end{array}$

$142-159.56-185.94 \quad 245.02$

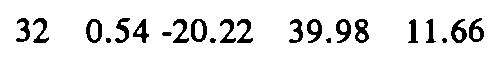

$\begin{array}{llll}29 & 208.93 & 421.18 & 470.15\end{array}$

$167-250.72-427.96 \quad 495.99$

$\begin{array}{lllll}33 & 0.15 & -61.00 & 42.12 & 12.28\end{array}$

$$
\begin{array}{cccc}
18 & -100.71 & -22.42 & 103.18 \\
39 & 58.78 & 24.57 & 63.71 \\
97 & -1.08 & -11.11 & 11.17
\end{array}
$$

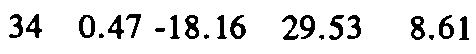

$35 \quad-3.96-107.13 \quad 107.21$ 
$\begin{array}{lllll}35 & 0.51 & -18.83 & 29.53 & 8.61\end{array}$

$\begin{array}{llll}38 & -19.76 & 90.70 & 92.83\end{array}$

$\begin{array}{lllll}36 & 0.30 & -34.68 & 32.87 & 9.59\end{array}$

$\begin{array}{cccc}8 & -53.74 & -201.53 & 208.58 \\ 11 & -143.12 & -252.30 & 290.07 \\ 34 & 5.72 & 116.21 & 116.35 \\ 38 & -5.00 & 117.19 & 117.30 \\ 75 & 5.23 & 115.11 & 115.23 \\ 90 & 83.22 & 28.41 & 87.94 \\ 93 & 82.95 & 27.77 & 87.47\end{array}$

$\begin{array}{llll}54 & 152.58 & 296.87 & 333.79\end{array}$

$\begin{array}{lllll}55 & 43.06 & 4.46 & 43.29\end{array}$

$\begin{array}{llll}58 & 16.28 & 1.22 & 16.32\end{array}$

$\begin{array}{llll}84 & 51.98 & -43.56 & 67.82\end{array}$

$\begin{array}{llll}88 & 57.33 & 2.01 & 57.37\end{array}$

$\begin{array}{lllll}100 & 40.12 & 63.67 & 75.26\end{array}$

$\begin{array}{llll}104 & -397.81 & -287.59 & 490.88\end{array}$

$\begin{array}{lllll}37 & 0.49 & -21.96 & 24.83 & 7.24\end{array}$

$\begin{array}{llll}90 & -60.21 & -33.35 & 68.82\end{array}$

$\begin{array}{llll}91 & 37.18 & 2.54 & 37.27\end{array}$

$\begin{array}{lllll}38 & 0.45 & -17.03 & 34.75 & 10.14\end{array}$

$\begin{array}{cccc}34 & 20.50 & -86.13 & 88.54 \\ 35 & 7.58 & -102.78 & 103.06 \\ 52 & -52.66 & -42.36 & 67.59 \\ 67 & 154.54 & 200.15 & 252.87 \\ 75 & 15.69 & -101.41 & 102.62 \\ 79 & 66.67 & 18.65 & 69.23 \\ 102 & -2.11 & -11.55 & 11.74 \\ 105 & -234.82 & 144.22 & 275.58\end{array}$

$\begin{array}{lllll}39 & 0.15 & -65.35 & 39.38 & 11.48\end{array}$

$\begin{array}{llll}33 & -57.74 & -19.13 & 60.82\end{array}$

$\begin{array}{llll}49 & 15.88 & 8.54 & 18.03\end{array}$

$\begin{array}{lllll}40 & 0.53 & -8.79 & 19.09 & 5.57\end{array}$

$\begin{array}{llll}5 & -252.88 & -132.96 & 285.70\end{array}$

$\begin{array}{llll}10 & 58.79 & 40.28 & 71.27\end{array}$

$\begin{array}{llll}56 & 80.72 & 44.56 & 92.21\end{array}$

$\begin{array}{llll}66 & 111.03 & 65.43 & 128.88\end{array}$

$\begin{array}{lllll}41 & 0.25 & -35.68 & 53.42 & 15.58\end{array}$

$\begin{array}{cccl}23 & 99.54 & 103.61 & 143.68 \\ 70 & 13.54 & -38.90 & 41.19 \\ 83 & 95.81 & 128.47 & 160.26 \\ 95 & -24.09 & -68.12 & 72.26 \\ 106 & -231.70 & -146.86 & 274.32\end{array}$

$\begin{array}{lllll}42 & 0.11 & -58.64 & 25.68 & 7.49\end{array}$

$611-101.59-234.65 \quad 255.70$

$\begin{array}{lllll}43 & 0.73 & -17.32 & 30.82 & 8.99\end{array}$

$\begin{array}{llll}96 & -9.62 & -18.57 & 20.91\end{array}$

$\begin{array}{llll}101 & -23.03 & 6.02 & 23.80\end{array}$

$\begin{array}{lllll}44 & 0.44 & -28.93 & 27.39 & 7.99\end{array}$ 
$46-113.41-163.59 \quad 199.05$

$\begin{array}{lllll}78 & 84.68 & 139.51 & 163.20\end{array}$

$\begin{array}{lllll}45 & 0.49 & -22.27 & 34.67 & 10.11\end{array}$

$\begin{array}{llll}60 & -37.53 & -15.94 & 40.78\end{array}$

$\begin{array}{lllll}46 & 0.49 & -26.99 & 28.93 & 8.44\end{array}$

$\begin{array}{llll}91 & 2.63 & -1.68 & 3.12\end{array}$

$\begin{array}{llll}44 & 118.48 & 183.76 & 218.64\end{array}$

$89-147.34-192.76 \quad 242.63$

$\begin{array}{lllll}47 & 0.30 & -37.27 & 31.16 & 9.09\end{array}$

$\begin{array}{llll}55 & -7.32 & -4.47 & 8.58\end{array}$

$\begin{array}{lllll}65 & -25.66 & -14.67 & 29.56\end{array}$

$\begin{array}{lllll}48 & 0.43 & -20.83 & 24.14 & 7.04\end{array}$

$\begin{array}{llll}79 & -20.14 & -11.57 & 23.22\end{array}$

$\begin{array}{lllll}49 & 0.14 & -67.93 & 42.37 & 12.36\end{array}$

$\begin{array}{llll}39 & -15.72 & -7.57 & 17.45\end{array}$

$\begin{array}{llll}94 & -28.34 & -5.12 & 28.80\end{array}$

$\begin{array}{lllll}50 & 0.32 & -37.80 & 49.82 & 14.53\end{array}$

$31-116.80-120.83 \quad 168.05$

$\begin{array}{llll}82 & 67.99 & 38.33 & 78.05\end{array}$

$\begin{array}{llll}84 & -10.73 & 44.68 & 45.95\end{array}$

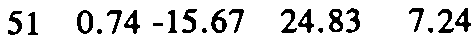

$\begin{array}{llll}25 & 95.82 & 37.69 & 102.97\end{array}$

$\begin{array}{llll}92 & 87.11 & 20.06 & 89.39\end{array}$

$\begin{array}{llll} & 107 & -338.20 & -148.13 \quad 369.22\end{array}$

$\begin{array}{llll}149 & 29.22 & 28.29 & 40.67\end{array}$

$\begin{array}{llll}480 & 98.62 & 29.19 & 102.85\end{array}$

$\begin{array}{lllll}52 & 0.48 & -13.90 & 14.38 & 4.19\end{array}$

$\begin{array}{llll}38 & 53.72 & 48.00 & 72.04\end{array}$

$\begin{array}{llll}66 & -67.28 & -52.13 & 85.11\end{array}$

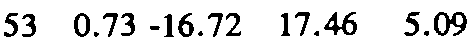

$\begin{array}{llll}76 & 36.64 & -4.31 & 36.89\end{array}$

$\begin{array}{llll}92 & -59.65 & -16.49 & 61.89\end{array}$

$\begin{array}{lllll}54 & 0.22 & -40.85 & 11.47 & 3.35\end{array}$

$\begin{array}{llll}24 & 125.30 & 210.86 & 245.28\end{array}$

$36-135.60-205.82 \quad 246.48$

$\begin{array}{lllll}55 & 0.30 & -36.65 & 35.53 & 10.36\end{array}$

$\begin{array}{llll}36 & -42.81 & -2.99 & 42.91\end{array}$

$\begin{array}{llll}47 & 7.36 & 4.53 & 8.64\end{array}$

$\begin{array}{lllll}56 & 0.48 & -13.62 & 29.79 & 8.69\end{array}$

$\begin{array}{llll}40 & -76.68 & -34.45 & 84.06\end{array}$

$\begin{array}{llll}80 & 48.20 & 26.78 & 55.14\end{array}$

$\begin{array}{lllll}57 & 0.28 & -38.93 & 17.89 & 5.22\end{array}$

$\begin{array}{llll}12 & 33.63 & 42.69 & 54.34\end{array}$

$\begin{array}{lllll}64 & -53.11 & -64.61 & 83.64\end{array}$

$\begin{array}{lllll}58 & 0.30 & -36.30 & 20.97 & 6.12\end{array}$

$\begin{array}{llll}36 & -16.24 & -0.89 & 16.26\end{array}$

$\begin{array}{lllll}65 & -5.72 & -4.78 & 7.45\end{array}$

$\begin{array}{lllll}59 & 0.73 & -16.06 & 32.70 & 9.54\end{array}$

$\begin{array}{llll}149 & -8.20 & -28.57 & 29.73\end{array}$ 


$$
\begin{array}{rrrrrrrrrr}
60 & 0.50 & -21.53 & 23.11 & 6.74 & 159 & -25.15 & 3.65 & 25.41 \\
& & & & 45 & 37.65 & 16.49 & 41.10 \\
61 & 0.18 & -51.73 & 22.85 & 6.67 & 93 & -57.99 & -27.62 & 64.23 \\
& & & & 14 & -45.14 & -17.49 & 48.41 \\
62 & 0.18 & -50.47 & 38.01 & 11.09 & 99 & 25.73 & 3.28 & 25.94 \\
& & & & 73 & -64.40 & -56.92 & 85.95 \\
63 & 0.12 & -59.97 & 26.54 & 7.74 & 83 & 33.24 & 41.13 & 52.88 \\
& & & & 83 & -24.39 & -46.17 & 52.22 \\
& & & & 611 & -3.15 & 30.48 & 30.64 \\
64 & 0.29 & -37.37 & 17.38 & 5.07 & & & & \\
& & & & 57 & 50.73 & 61.95 & 80.07 \\
& & & & 65 & -69.19 & -75.96 & 102.74
\end{array}
$$

$$
\begin{array}{lllll}
65 & 0.30 & -35.79 & 28.93 & 8.44
\end{array}
$$

$\begin{array}{llll}22 & 89.94 & 125.44 & 154.35\end{array}$

$\begin{array}{lllll}30 & 100.65 & 137.50 & 170.40\end{array}$

$\begin{array}{llll}47 & 25.84 & 15.64 & 30.21\end{array}$

$\begin{array}{lllll}58 & 5.70 & 4.75 & 7.42\end{array}$

$\begin{array}{lllll}64 & 69.79 & 80.99 & 106.91\end{array}$

$\begin{array}{llll}71 & -268.27 & -263.73 & 376.19\end{array}$

$\begin{array}{llll}78 & -52.95 & -91.06 & 105.34\end{array}$

$\begin{array}{lllll}66 & 0.52 & -9.70 & 29.77 & 8.68\end{array}$

$\begin{array}{llll}40 & -110.54 & -62.83 & 127.15\end{array}$

$\begin{array}{llll}52 & 69.27 & 61.99 & 92.96\end{array}$

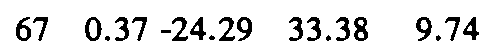

$38-146.19-146.71 \quad 207.11$

$\begin{array}{lllll}98 & 112.94 & 136.37 & 177.06\end{array}$

$\begin{array}{lllll}68 & 0.23 & -44.89 & 31.84 & 9.29\end{array}$

$\begin{array}{llll}30 & -74.19 & -75.47 & 105.84\end{array}$

$\begin{array}{llll}74 & 45.59 & 68.11 & 81.96\end{array}$

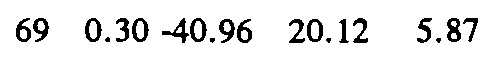

$\begin{array}{llll}82 & -23.98 & -35.10 & 42.51\end{array}$

$\begin{array}{llll}87 & -3.44 & 7.71 & 8.44\end{array}$

$\begin{array}{lllll}70 & 0.26 & -37.41 & 29.96 & 8.74\end{array}$

$\begin{array}{llll}41 & -13.12 & 41.57 & 43.59\end{array}$

$\begin{array}{llll}100 & -20.25 & -58.69 & 62.08\end{array}$

$\begin{array}{lllll}71 & 0.35 & -30.26 & 29.10 & 8.49\end{array}$

$27-141.70-216.62 \quad 258.85$

$\begin{array}{lllll}65 & 277.46 & 331.08 & 431.97\end{array}$

$114-167.53-104.22 \quad 197.31$

$\begin{array}{lllll}72 & 0.18 & -53.91 & 0.00 & 0.00\end{array}$

$$
\begin{array}{rrrr}
16 & 17.76 & 6.28 & 18.83 \\
99 & -11.04 & -11.11 & 15.66
\end{array}
$$

$\begin{array}{lllll}73 & 0.19 & -47.81 & 86.12 & 25.12\end{array}$

$$
\begin{array}{rrrr}
14 & 9.87 & 39.51 & 40.73 \\
17 & 19.96 & 8.99 & 21.90
\end{array}
$$


$\begin{array}{lllll}74 & 0.21 & -48.00 & 18.32 & 5.34\end{array}$

$\begin{array}{lllll}75 & 0.48 & -18.21 & 14.98 & 4.37\end{array}$

$\begin{array}{lllll}76 & 0.73 & -16.81 & 0.00 & 0.00\end{array}$

$\begin{array}{lllll}77 & 0.74 & -16.09 & 41.43 & 12.08\end{array}$

$\begin{array}{lllll}78 & 0.41 & -30.43 & 22.26 & 6.49\end{array}$

$\begin{array}{lllll}79 & 0.44 & -20.36 & 42.12 & 12.28\end{array}$

$\begin{array}{lllll}80 & 0.47 & -14.75 & 17.38 & 5.07\end{array}$

$\begin{array}{lllll}81 & 0.20 & -49.12 & 68.14 & 19.87\end{array}$

$\begin{array}{lllll}82 & 0.31 & -39.81 & 49.65 & 14.48\end{array}$

$\begin{array}{lllll}83 & 0.15 & -56.74 & 35.95 & 10.49\end{array}$

$\begin{array}{lllll}84 & 0.31 & -36.94 & 43.74 & 12.76\end{array}$

$\begin{array}{lllll}85 & 0.16 & -58.74 & 0.00 & 0.00\end{array}$

$\begin{array}{lllll}86 & 0.46 & -16.22 & 3.94 & 1.15\end{array}$

$\begin{array}{lllll}87 & 0.30 & -40.71 & 34.24 & 9.99\end{array}$ $\begin{array}{llll}18 & 147.72 & 90.50 & 173.24\end{array}$

$\begin{array}{lllll}62 & 65.13 & 62.36 & 90.17\end{array}$

$\begin{array}{lllll}81 & 54.63 & -35.37 & 65.08\end{array}$

$\begin{array}{llll}112 & -216.30 & -98.73 & 237.77\end{array}$

$\begin{array}{lllll}113 & -184.17 & -83.67 & 202.28\end{array}$

$\begin{array}{llll}68 & -44.09 & -58.73 & 73.44\end{array}$

$\begin{array}{llll}81 & 31.78 & 49.89 & 59.15\end{array}$

$\begin{array}{lllll}35 & -3.74 & -108.64 & 108.71\end{array}$

$\begin{array}{llll}38 & -14.65 & 109.12 & 110.10\end{array}$

$\begin{array}{llll}19 & 25.42 & 4.40 & 25.80\end{array}$

$\begin{array}{lllll}25 & 16.56 & 11.82 & 20.34\end{array}$

$\begin{array}{llll}53 & -35.20 & -0.50 & 35.21\end{array}$

$\begin{array}{llll}147 & 44.54 & 29.91 & 53.65\end{array}$

$\begin{array}{llll}480 & -89.05 & -56.55 & 105.48\end{array}$

$\begin{array}{llll}44 & -81.69 & -127.05 & 151.05\end{array}$

$\begin{array}{llll}65 & 59.34 & 128.15 & 141.22\end{array}$

$\begin{array}{llll}38 & -65.99 & -14.57 & 67.58\end{array}$

$\begin{array}{llll}48 & 20.25 & 11.79 & 23.44\end{array}$

$\begin{array}{llll}56 & -47.52 & -25.29 & 53.83\end{array}$

$\begin{array}{llll}86 & 30.33 & 20.76 & 36.75\end{array}$

$\begin{array}{llll}73 & -54.53 & 36.30 & 65.51\end{array}$

$\begin{array}{llll}74 & -31.54 & -47.59 & 57.10\end{array}$

$\begin{array}{llll}50 & -67.47 & -35.02 & 76.02\end{array}$

$\begin{array}{llll}69 & 24.30 & 37.08 & 44.33\end{array}$

$\begin{array}{llll}41 & -83.85 & -52.89 & 99.14\end{array}$

$\begin{array}{lllll}62 & -31.65 & -31.45 & 44.62\end{array}$

$\begin{array}{lllll}63 & 26.84 & 58.56 & 64.41\end{array}$

$\begin{array}{llll}94 & 50.48 & 18.30 & 53.69\end{array}$

$\begin{array}{llll}36 & -51.55 & 46.72 & 69.57\end{array}$

$\begin{array}{lllll}50 & 10.99 & -43.08 & 44.46\end{array}$

$\begin{array}{llll}26 & -26.26 & -19.35 & 32.61\end{array}$

$\begin{array}{llll}97 & 30.52 & 17.40 & 35.13\end{array}$

$\begin{array}{llll}80 & -30.05 & -19.69 & 35.92\end{array}$

$\begin{array}{llll}102 & 29.39 & 15.73 & 33.34\end{array}$

$\begin{array}{llll}69 & 3.43 & -7.72 & 8.44\end{array}$ 


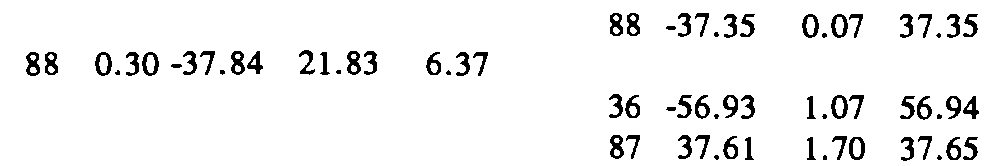

$\begin{array}{lllll}89 & 0.52 & -24.85 & 27.91 & 8.14\end{array}$

$\begin{array}{llll}46 & 150.58 & 212.86 & 260.74\end{array}$

$\begin{array}{llll}160 & -178.69-223.04 & 285.79\end{array}$

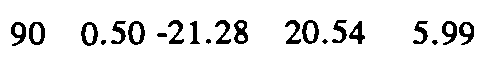

$\begin{array}{llll}35 & -82.61 & -25.54 & 86.47\end{array}$

$\begin{array}{llll}37 & 60.41 & 33.67 & 69.16\end{array}$

$\begin{array}{lllll}91 & 0.49 & -22.38 & 40.23 & 11.73\end{array}$

$\begin{array}{llll}37 & -37.13 & -2.32 & 37.20\end{array}$

$\begin{array}{llll}45 & -2.61 & 1.46 & 2.99\end{array}$

$\begin{array}{lllll}92 & 0.74 & -16.11 & 29.28 & 8.54\end{array}$

$\begin{array}{llll}51 & -86.91 & -19.41 & 89.05\end{array}$

$\begin{array}{llll}53 & 59.87 & 17.14 & 62.28\end{array}$

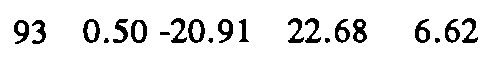

$\begin{array}{llll}35 & -82.37 & -24.44 & 85.92\end{array}$

$\begin{array}{llll}60 & 58.13 & 28.42 & 64.71\end{array}$

$\begin{array}{lllll}94 & 0.15 & -61.60 & 20.89 & 6.09\end{array}$

$\begin{array}{llll}49 & 28.93 & 8.58 & 30.18\end{array}$

$\begin{array}{llll}83 & -49.52 & -13.34 & 51.28\end{array}$

$\begin{array}{lllll}95 & 0.26 & -35.16 & 41.94 & 12.23\end{array}$

$\begin{array}{llll}41 & 24.76 & 72.03 & 76.17\end{array}$

$\begin{array}{llll}98 & -70.03 & -78.85 & 105.46\end{array}$

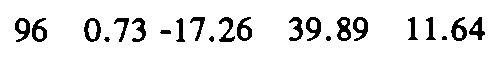

$\begin{array}{llll}25 & -49.47 & -23.35 & 54.71\end{array}$

$\begin{array}{llll}43 & 9.66 & 18.46 & 20.83\end{array}$

$\begin{array}{lllll}97 & 0.16 & -61.11 & 23.54 & 6.87\end{array}$

$\begin{array}{llll}33 & 1.13 & 11.47 & 11.52\end{array}$

$\begin{array}{llll}85 & -30.24 & -15.63 & 34.04\end{array}$

$\begin{array}{lllll}98 & 0.33 & -28.41 & 33.38 & 9.74\end{array}$

$67-109.41-114.36 \quad 158.26$

$\begin{array}{lllll}95 & 74.87 & 107.39 & 130.92\end{array}$

$\begin{array}{lllll}99 & 0.18 & -53.45 & 6.08 & 1.77\end{array}$

$\begin{array}{llll}61 & -26.61 & -4.37 & 26.97\end{array}$

$\begin{array}{llll}72 & 10.12 & 9.62 & 13.96\end{array}$

$\begin{array}{lllll}100 & 0.27 & -37.04 & 18.66 & 5.44\end{array}$

$\begin{array}{llll}36 & -38.73 & -56.23 & 68.28\end{array}$

$\begin{array}{lllll}70 & 20.59 & 60.79 & 64.18\end{array}$

$\begin{array}{lllll}101 & 0.73 & -17.22 & 27.99 & 8.16\end{array}$

$\begin{array}{llll}43 & 23.03 & -6.14 & 23.83\end{array}$

$\begin{array}{llll}136 & -49.60 & 0.77 & 49.60\end{array}$

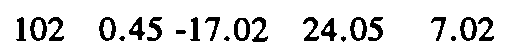

382688.08 ******* 2998.49

$\begin{array}{llll}86 & -29.29 & -15.29 & 33.04\end{array}$

$\begin{array}{lllll}103 & 0.78 & -9.98 & 6.51 & 1.90\end{array}$

$\begin{array}{llll}107 & 346.07 & 123.56 & 367.46\end{array}$

$182-338.05-143.70 \quad 367.32$ 
$\begin{array}{lllll}104 & 0.47 & -12.51 & 0.00 & 0.00\end{array}$

$\begin{array}{lllll}105 & 0.41 & -7.21 & 0.00 & 0.00\end{array}$

$$
\begin{array}{cccc}
36 & 405.30 & 649.41 & 765.50 \\
109 & -289.01 & -217.61 & 361.77 \\
111 & -63.92 & -340.22 & 346.17 \\
114 & 177.16 & 196.61 & 264.65 \\
121 & -71.10 & -85.33 & 111.07 \\
496 & -132.77 & -314.64 & 341.50
\end{array}
$$

$\begin{array}{llll}38 & 235.94 & -94.07 & 254.00\end{array}$

$\begin{array}{llll}109 & -67.25 & -282.75 & 290.64\end{array}$

$119-237.16-338.95 \quad 413.68$

$119-239.35-341.46416 .99$

$119-237.15-338.99 \quad 413.71$

$119-239.35-341.50 \quad 417.03$

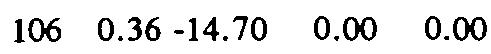

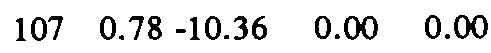

$\begin{array}{llll}41 & 236.49 & 317.78 & 396.12\end{array}$

$\begin{array}{llll}112 & 245.80 & 348.25 & 426.26\end{array}$

$115-173.16-319.04 \quad 363.01$

$\begin{array}{llll}117 & -125.22 & -179.65 & 218.98\end{array}$

$\begin{array}{llll}119-202.55 & -197.49 & 282.90\end{array}$

$\begin{array}{lllll}501 & -132.71 & -303.55 & 331.29\end{array}$

$\begin{array}{llll}610 & 207.70 & 311.95 & 374.77\end{array}$

$\begin{array}{llll}51 & 339.69 & 188.01 & 388.25\end{array}$ $103-347.36-148.98 \quad 377.96$

$\begin{array}{lllll}108 & 0.43 & -8.79 & 28.25 & 8.24\end{array}$

$\begin{array}{llll}116 & 217.90 & 253.02 & 333.92\end{array}$

$\begin{array}{lllll}109 & 0.54 & -5.45 & 0.00 & 0.00\end{array}$

$\begin{array}{llll}119 & -225.08 & -261.82 & 345.27\end{array}$

$\begin{array}{llll}104 & 296.73 & 285.44 & 411.74\end{array}$

$\begin{array}{lllll}105 & 76.86 & 369.61 & 377.52\end{array}$

$\begin{array}{llll}115 & 252.47 & 535.50 & 592.03\end{array}$

$119-139.59-107.36 \quad 176.10$

$120-478.38 * * * * * * * 1270.67$

$\begin{array}{lllll}110 & 0.54 & -6.34 & 88.17 & 25.72\end{array}$

$\begin{array}{llll}15 & 123.06 & 125.67 & 175.89\end{array}$

$119-215.13-147.07 \quad 260.60$

$\begin{array}{lllll}111 & 0.53 & -11.92 & 42.54 & 12.41\end{array}$

$\begin{array}{llll}104 & 68.26 & 384.16 & 390.18\end{array}$

$185-115.43-390.93 \quad 407.61$

$\begin{array}{lllll}112 & 0.31 & -18.11 & 0.00 & 0.00\end{array}$

$\begin{array}{llll}73 & 220.72 & 306.44 & 377.66\end{array}$

$106-231.29-317.97 \quad 393.19$

$\begin{array}{lllll}113 & 0.29 & -21.05 & 0.00 & 0.00\end{array}$

$\begin{array}{lllll}73 & 187.37 & 232.94 & 298.95\end{array}$

$\begin{array}{lll}610 & -178.16-246.51 \quad 304.15\end{array}$

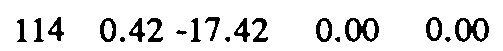

$\begin{array}{llll}71 & 169.77 & 168.12 & 238.93\end{array}$

$104-173.09-162.69 \quad 237.55$ 


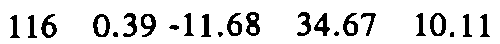

$\begin{array}{llll}106 & 178.22 & 369.49 & 410.23\end{array}$

$109-235.40-381.80 \quad 448.54$

$\begin{array}{lllll}117 & 0.38 & -13.39 & 32.87 & 9.59 \\ 118 & 0.49 & -10.78 & 19.77 & 5.77 \\ 119 & 0.64 & 3.72 & 0.00 & 0.00\end{array}$

$\begin{array}{llll}108 & -213.61 & -224.73 & 310.05\end{array}$

$\begin{array}{llll}117 & 190.08 & 215.20 & 287.13\end{array}$

$\begin{array}{llll}106 & 126.99 & 191.09 & 229.43\end{array}$

$116-188.03-200.76 \quad 275.06$

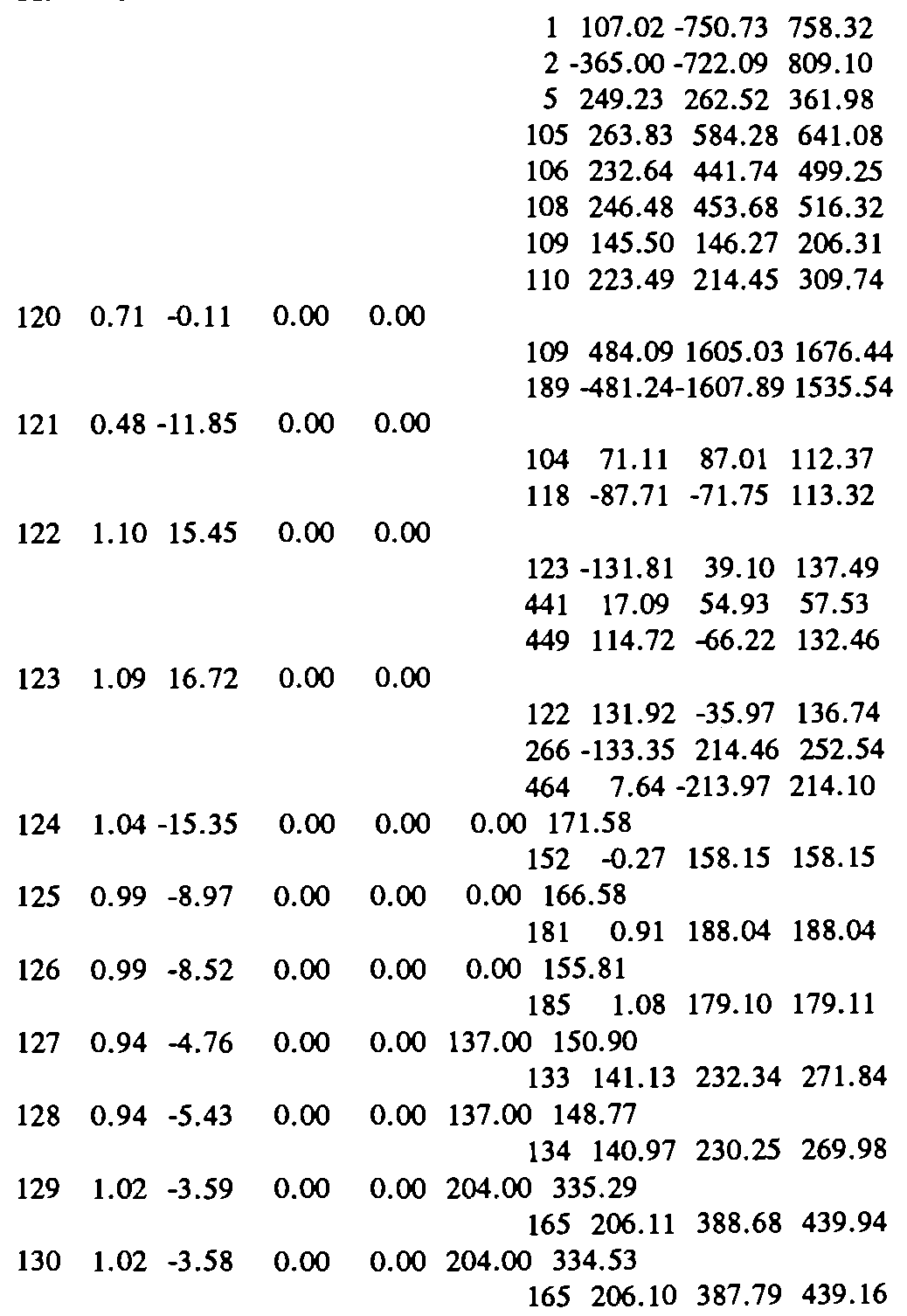

$\begin{array}{llll}15 & -99.84 & -88.48 & 133.40\end{array}$

$\begin{array}{lllll}121 & 87.67 & 73.84 & 114.62\end{array}$

$\begin{array}{llll}1 & 107.02 & -750.73 & 758.32\end{array}$

$\begin{array}{llll}2 & -365.00 & -722.09 & 809.10\end{array}$

$\begin{array}{lllll}5 & 249.23 & 262.52 & 361.98\end{array}$

$\begin{array}{lllll}105 & 263.83 & 584.28 & 641.08\end{array}$

$\begin{array}{llll}106 & 232.64 & 441.74 & 499.25\end{array}$

$\begin{array}{lllll}108 & 246.48 & 453.68 & 516.32\end{array}$

$\begin{array}{lllll}109 & 145.50 & 146.27 & 206.31\end{array}$

$109484.091605 .03 \quad 1676.44$

$189-481.24-1607.891535 .54$

$\begin{array}{llll}104 & 71.11 & 87.01 & 112.37\end{array}$

$\begin{array}{llll}123 & -131.81 & 39.10 & 137.49\end{array}$

$\begin{array}{llll}441 & 17.09 & 54.93 & 57.53\end{array}$

$\begin{array}{llll}449 & 114.72 & -66.22 & 132.46\end{array}$

$\begin{array}{llll}122 & 131.92 & -35.97 & 136.74\end{array}$

$\begin{array}{llll}266 & -133.35 & 214.46 & 252.54\end{array}$

.64 -213.97214 .10$

$\begin{array}{lll}387.79 & 439.16\end{array}$ 


\begin{tabular}{|c|c|c|c|c|c|c|c|c|}
\hline 131 & 0.97 & 0.63 & 0.00 & 0.00 & 367.003 & 326.95 & & \\
\hline & & & & & 185 & 5373.93 & 3502.25 & 626.16 \\
\hline 132 & 0.97 & 0.70 & 0.00 & 0.00 & 367.00 & 324.71 & & \\
\hline & & & & & 185 & 5373.83 & 498.56 & 623.14 \\
\hline 33 & 0.80 & -10.14 & 0.00 & 0.00 & & & & \\
\hline & & & & & 127 & $7-139.21$ & $1-187.04$ & 233.17 \\
\hline & & & & & 152 & 2138.47 & $7 \quad 169.24$ & 218.67 \\
\hline 134 & 0.80 & -10.81 & 27.71 & 8.08 & & & & \\
\hline & & & & & 128 & $8-139.15$ & $5-185.59$ & 231.97 \\
\hline & & & & & 152 & 2110.32 & 156.76 & 191.69 \\
\hline 35 & 1.48 & 23.37 & 13.41 & 3.91 & & & & \\
\hline & & & & & 472 & 2160.55 & -228.65 & 279.39 \\
\hline & & & & & 3014 & $14-175.96$ & $6 \quad 173.04$ & 246.79 \\
\hline 136 & 0.73 & -16.65 & 58.53 & 17.07 & & & & \\
\hline & & & & & 101 & 149.59 & -0.96 & 49.60 \\
\hline & & & & & 152 & $2-108.13$ & -10.26 & 108.62 \\
\hline 137 & 1.00 & -11.42 & 0.00 & 0.00 & & & & \\
\hline & & & & & 138 & $8 \quad 63.57$ & 16.35 & 65.63 \\
\hline & & & & & 143 & 3114.21 & 241.74 & 267.36 \\
\hline & & & & & 145 & $\begin{array}{ll}5 & 52.18\end{array}$ & 16.21 & 54.64 \\
\hline & & & & & 154 & 4234.41 & 354.46 & 424.96 \\
\hline & & & & & 163 & 3119.76 & 354.84 & 374.51 \\
\hline & & & & & 176 & $6-579.16$ & 615.08 & 844.83 \\
\hline & & & & & 497 & 7175.33 & 332.22 & 375.65 \\
\hline & & & & & 498 & $8-122.19$ & 50.33 & 132.15 \\
\hline & & & & & 533 & $3-64.02$ & -26.66 & 69.35 \\
\hline 138 & 1.00 & -11.44 & 25.55 & 7.45 & & & & \\
\hline & & & & & 137 & $7-63.56$ & -16.34 & 65.63 \\
\hline & & & & & 141 & 145.28 & 30.41 & 54.54 \\
\hline 139 & 0.93 & -12.03 & 45.83 & 13.37 & & & & \\
\hline & & & & & 157 & $7 \quad 47.38$ & 307.00 & 310.64 \\
\hline & & & & & 163 & $\begin{array}{ll}3 & -93.26\end{array}$ & -326.70 & 339.75 \\
\hline 140 & 0.83 & -10.42 & 23.10 & 6.74 & & & & \\
\hline & & & & & 150 & $\begin{array}{ll}0 & 169.07\end{array}$ & 166.16 & 237.06 \\
\hline & & & & & 165 & $5-190.63$ & $3-179.94$ & 262.14 \\
\hline 141 & 0.99 & -11.83 & 49.25 & 14.36 & & & & \\
\hline & & & & & 138 & $\begin{array}{ll}8 & -45.21\end{array}$ & -30.93 & 54.78 \\
\hline & & & & & 170 & $0 \quad 14.64$ & -31.57 & 34.80 \\
\hline & & & & & 230 & $\begin{array}{ll}0 & -20.30\end{array}$ & 55.20 & 58.81 \\
\hline 142 & 0.57 & -20.49 & 44.49 & 12.98 & & & & \\
\hline & & & & & 31 & 1179.01 & 300.07 & \\
\hline & & & & & 152 & $2-222.03$ & $3-312.49$ & 383.34 \\
\hline 143 & 0.95 & -12.21 & 35.58 & 10.38 & & & & \\
\hline & & & & & 137 & $7-11$ & & 256. \\
\hline & & & & & 153 & 376.89 & 223.83 & 236.67 \\
\hline 1 & 0.79 & -14.65 & 31.20 & 9.10 & & & & \\
\hline & & & & & 168 & $\begin{array}{ll}8 & -78.15\end{array}$ & & 254.2 \\
\hline & & & & & 169 & 947.66 & 230.51 & 235.38 \\
\hline & 0.99 & -11.82 & 4.75 & 1.39 & & & & \\
\hline
\end{tabular}




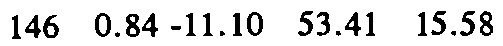

$\begin{array}{llll}137 & -52.06 & -16.88 & 54.73\end{array}$

$\begin{array}{llll}170 & 45.64 & 11.31 & 47.02\end{array}$

$\begin{array}{llll}157 & 48.72 & -254.30 & 258.93\end{array}$

$\begin{array}{lllll}161 & 230.91 & 329.48 & 402.34\end{array}$

$\begin{array}{llll}173 \quad 67.63 & -218.84 & 229.06\end{array}$

$\begin{array}{llll}179 & -211.20 & 201.43 & 291.86\end{array}$

$482-182.16-118.64 \quad 217.39$

$\begin{array}{lllll}147 & 0.73 & -16.51 & 61.35 & 17.90\end{array}$

$\begin{array}{llll}77 & -44.42 & -29.65 & 53.40\end{array}$

$\begin{array}{llll}156 & -16.52 & 8.01 & 18.36\end{array}$

$\begin{array}{lllll}148 & 0.87 & -14.54 & 44.79 & 13.06\end{array}$

$\begin{array}{llll}154 & -187.09 & -305.33 & 358.09\end{array}$

$\begin{array}{llll}155 & 141.15 & 354.90 & 381.94\end{array}$

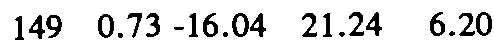

$\begin{array}{llll}51 & -29.16 & -28.46 & 40.75\end{array}$

$\begin{array}{llll}59 & 8.23 & 28.51 & 29.67\end{array}$

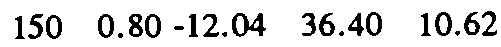

$140-167.07-155.64 \quad 228.34$

$\begin{array}{lllll}171 & 131.05 & 141.69 & 193.00\end{array}$

$\begin{array}{lllll}151 & 0.90 & -12.43 & 50.29 & 14.67\end{array}$

$\begin{array}{llll}153 & -19.87 & -213.20 & 214.12\end{array}$

$\begin{array}{lllll}157 & -30.65 & 189.77 & 192.23\end{array}$

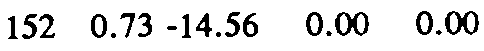

$124 \quad 1.72-111.64 \quad 111.65$

$133-137.61-143.97 \quad 199.16$

$134-109.76-136.12 \quad 174.86$

$\begin{array}{llll}136 & 108.64 & 13.06 & 109.43\end{array}$

$\begin{array}{llll}142 & 241.75 & 427.10 & 490.77\end{array}$

$\begin{array}{lllll}159 & 49.94 & -2.83 & 50.02\end{array}$

$\begin{array}{llll}160 & 224.27 & 349.03 & 414.87\end{array}$

$\begin{array}{llll}161 & -176.35 & -278.40 & 329.55\end{array}$

$\begin{array}{lllll}162 & -29.64 & -290.35 & 291.86\end{array}$

$\begin{array}{lllll}166 & 53.47 & -1.94 & 53.51\end{array}$

$\begin{array}{llll}167 & 306.96 & 605.78 \quad 679.12\end{array}$

$\begin{array}{llll}169 & -16.46 & -218.47 & 219.08\end{array}$

$\begin{array}{lllll}171 & -87.45 & -128.47 & 155.41\end{array}$

$\begin{array}{llll}181 & -424.82 & -185.21 & 463.44\end{array}$

$\begin{array}{lllll}153 & 0.92 & -12.51 & 55.26 & 16.12\end{array}$

$\begin{array}{llll}143 & -75.70 & -217.34 & 230.14\end{array}$

$\begin{array}{llll}151 & 20.67 & 217.76 & 218.73\end{array}$

$\begin{array}{lllll}154 & 0.92 & -13.62 & 36.32 & 10.59\end{array}$

$137-228.03-318.64 \quad 391.83$

$\begin{array}{llll}148 & 191.97 & 324.15 & 376.74\end{array}$

$\begin{array}{lllll}155 & 0.81 & -15.05 & 54.97 & 16.03\end{array}$

$148-134.85-331.11 \quad 357.51$

$\begin{array}{llll}162 & 79.80 & 318.16 & 328.02\end{array}$

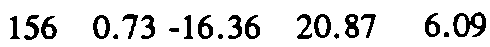

$\begin{array}{llll}147 & 16.52 & -8.22 & 18.46\end{array}$ 
$\begin{array}{lllll}157 & 0.88 & -12.05 & 69.90 & 20.39\end{array}$

$\begin{array}{lllll}158 & 0.90 & -13.33 & 34.17 & 9.97\end{array}$

$\begin{array}{lllll}159 & 0.73 & -15.88 & 24.74 & 7.21\end{array}$

$\begin{array}{lllll}160 & 0.56 & -22.50 & 24.07 & 7.02\end{array}$

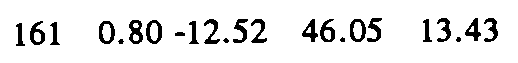

$\begin{array}{lllll}162 & 0.79 & -15.03 & 42.78 & 12.48\end{array}$

$\begin{array}{lllll}163 & 0.96 & -11.80 & 22.80 & 6.65\end{array}$

$\begin{array}{lllll}164 & 0.86 & -8.93 & 30.53 & 8.90\end{array}$

$\begin{array}{lllll}165 & 0.87 & -8.77 & 0.00 & 0.00\end{array}$

$\begin{array}{lllll}166 & 0.73 & -15.50 & 16.04 & 4.68\end{array}$

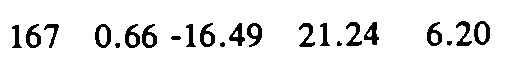

$\begin{array}{lllll}168 & 0.87 & -13.79 & 27.19 & 7.93\end{array}$

$\begin{array}{lllll}169 & 0.77 & -14.74 & 27.78 & 8.10\end{array}$

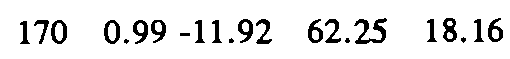

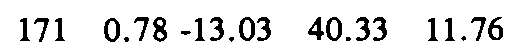

$\begin{array}{llll}166 & -37.35 & 2.87 & 37.46\end{array}$

$\begin{array}{lllll}139 & -45.23 & -293.32 & 296.79\end{array}$

$\begin{array}{lllll}146 & -46.96 & 268.44 & 272.52\end{array}$

$\begin{array}{lllll}151 & 31.30 & -186.26 & 188.87\end{array}$

$\begin{array}{lllll}173 & -8.38 & 230.70 & 230.86\end{array}$

$\begin{array}{llll}168 & 111.18 & 275.50 & 297.09\end{array}$ $\begin{array}{llll}497 & -145.96 & -292.52 & 326.91\end{array}$

$\begin{array}{llll}59 & 25.17 & -3.73 & 25.44\end{array}$

$\begin{array}{llll}152 & -49.80 & 2.93 & 49.89\end{array}$

$\begin{array}{llll}89 & 182.87 & 248.32 & 308.39\end{array}$

$152-207.96-242.94 \quad 319.79$

$\begin{array}{llll}146 & -228.60 & -310.12 & 385.27\end{array}$

$\begin{array}{llll}152 & 181.97 & 309.83 & 359.31\end{array}$

$\begin{array}{llll}152 & 34.35 & 310.82 & 312.72\end{array}$

$\begin{array}{llll}155 & -77.08 & -308.01 & 317.50\end{array}$

$137-117.66-341.76 \quad 361.44$

$\begin{array}{lllll}139 & 95.25 & 338.37 & 351.52\end{array}$

$\begin{array}{llll}165 & -226.58 & -181.89 & 290.55\end{array}$

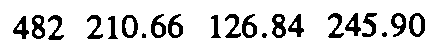

$\begin{array}{llll}129 & -203.92 & -312.61 & 373.24\end{array}$

$\begin{array}{llll}130 & -203.92 & -311.81 & 372.58\end{array}$

$\begin{array}{llll}140 & 193.08 & 192.53 & 272.67\end{array}$

$\begin{array}{lllll}164 & 226.70 & 182.98 & 291.33\end{array}$

$\begin{array}{llll}185 & -19.99 & 197.24 & 198.26\end{array}$

$\begin{array}{llll}152 & -53.34 & 2.10 & 53.38\end{array}$

$\begin{array}{llll}156 & 37.42 & -3.18 & 37.56\end{array}$

$\begin{array}{llll}32 & 271.59 & 540.88 & 605.24\end{array}$

$\begin{array}{llll}152 & -295.81 & -538.46 & 614.36\end{array}$

$\begin{array}{llll}144 & 82.23 & 267.02 & 279.39\end{array}$

$158-109.43-265.42 \quad 287.09$

$\begin{array}{lllll}144 & -46.61 & -223.94 & 228.73\end{array}$

$\begin{array}{llll}152 & 17.88 & 227.61 & 228.31\end{array}$

$\begin{array}{llll}141 & -14.64 & 31.22 & 34.48\end{array}$

$\begin{array}{llll}145 & -45.63 & -11.41 & 47.04\end{array}$ 


\begin{tabular}{|c|c|c|c|c|c|c|c|c|}
\hline & & & & & 150 & -130.00 & -136.16 & 188.25 \\
\hline & & & & & 152 & 89.26 & 137.89 & 164.26 \\
\hline 172 & 0.75 & -6.86 & 17.38 & 5.07 & & & & \\
\hline & & & & & 175 & -133.29 & 23.05 & 135.27 \\
\hline & & & & & 495 & 122.43 & -38.11 & 128.23 \\
\hline 173 & 0.86 & -11.69 & 56.45 & 16.47 & & & & \\
\hline & & & & & 146 & -66.97 & 224.33 & 234.12 \\
\hline & & & & & 157 & $9.53-$ & -224.13 & 224.33 \\
\hline 174 & 0.99 & -8.77 & 36.25 & 10.57 & & & & \\
\hline & & & & & 216 & -173.23 & 31.43 & 176.06 \\
\hline & & & & & 498 & 136.30 & -43.24 & 142.99 \\
\hline 175 & 0.75 & -5.47 & 0.00 & 0.00 & & & & \\
\hline & & & & & 172 & 133.85 & -23.41 & 135.88 \\
\hline & & & & & 180 & 11.58 & -297.66 & 297.88 \\
\hline & & & & & 181 & 128.61 & -98.34 & 161.90 \\
\hline & & & & & 187 & 158.08 & -94.47 & 184.16 \\
\hline & & & & & 189 & -923.57 & -293.71 & 969.15 \\
\hline & & & & & 206 & 139.39 & -100.65 & 171.93 \\
\hline & & & & & 500 & 12.82 & -294.60 & 294.88 \\
\hline & & & & & 501 & 175.59 & 654.45 & 677.60 \\
\hline & & & & & 502 & 191.44 & 526.34 & 560.07 \\
\hline 176 & 0.92 & -6.21 & 0.00 & 0.00 & & & & \\
\hline & & & & & 137 & 581.90 & -515.13 & 777.15 \\
\hline & & & & & 183 & 84.66 & 379.26 & 388.60 \\
\hline & & & & & 264 & 56.93 & -46.09 & 73.25 \\
\hline & & & & & 268 & -413.83 & -95.10 & 424.62 \\
\hline & & & & & 269 & -335.07 & -27.71 & 336.21 \\
\hline & & & & & 483 & 27.02 & 356.33 & 357.35 \\
\hline 177 & 0.76 & -8.81 & 34.09 & 9.94 & & & & \\
\hline & & & & & 181 & -52.58 & -160.81 & 169.19 \\
\hline & & & & & 271 & 30.47 & 122.52 & 126.25 \\
\hline 178 & 0.86 & -6.56 & 23.32 & 6.80 & & & & \\
\hline & & & & & 183 & $-5.74-$ & -350.15 & 350.20 \\
\hline & & & & & 500 & -4.88 & 326.13 & 326.16 \\
\hline 179 & 0.81 & -8.68 & 0.00 & 0.00 & & & & \\
\hline & & & & & 146 & 211.57 & -185.33 & 281.26 \\
\hline & & & & & 185 & -200.39 & 196.14 & 280.41 \\
\hline 180 & 0.78 & -5.87 & 27.71 & 8.08 & & & & \\
\hline & & & & & 175 & -9.85 & 309.83 & 309.99 \\
\hline & & & & & 483 & -19.22 & -317.24 & 317.82 \\
\hline 181 & 0.77 & -8.68 & 0.00 & 0.00 & & & & \\
\hline & & & & & 125 & 0.04 & -146.86 & 146.86 \\
\hline & & & & & 152 & 426.02 & 240.37 & 489.15 \\
\hline & & & & & 175 & -127.46 & 102.52 & 163.58 \\
\hline & & & & & 177 & 52.79 & 161.32 & 169.74 \\
\hline & & & & & 182 & 49.05 & -186.87 & 193.21 \\
\hline & & & & & 185 & -68.86 & -211.44 & 222.37 \\
\hline & & & & & 187 & -104.43 & 108.46 & 150.56 \\
\hline & & & & & 188 & -109.24 & -202.80 & 230.35 \\
\hline
\end{tabular}




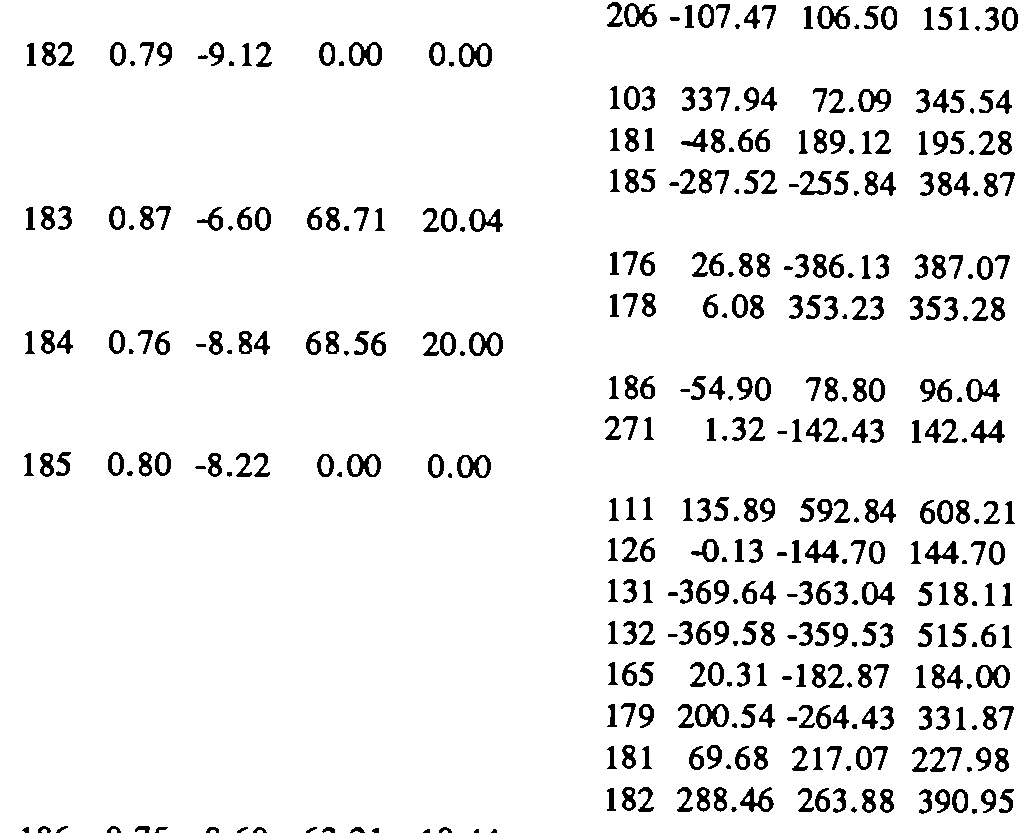

$\begin{array}{lllll}186 & 0.75 & -8.60 & 63.21 & 18.44\end{array}$

$\begin{array}{lllll}187 & 0.76 & -7.29 & 48.43 & 14.12\end{array}$

$\begin{array}{lllll}188 & 0.81 & -7.72 & 35.73 & 10.42\end{array}$

$\begin{array}{lllll}189 & 0.78 & 1.30 & 0.00 & 0.00\end{array}$

$\begin{array}{lllll}190 & 0.93 & -6.44 & 66.21 & 19.31\end{array}$

$\begin{array}{lllllll}191 & 1.01 & 2.46 & 0.00 & 0.00 & 0.00 & 12.91\end{array}$

$\begin{array}{lllllll}192 & 1.01 & 2.43 & 0.00 & 0.00 & 0.00 & 23.46\end{array}$

$\begin{array}{lllllll}193 & 1.00 & 10.79 & 0.00 & 0.00 & 272.00 & -69.17\end{array}$

$\begin{array}{lllllll}194 & 1.00 & 10.83 & 0.00 & 0.00 & 272.00 & -68.06\end{array}$

$\begin{array}{lllll}195 & 1.01 & 20.55 & 0.00 & 0.00\end{array}$

$\begin{array}{lllll}196 & 1.03 & 21.73 & 0.00 & 0.00\end{array}$ $\begin{array}{llll}212 & -1.04 & 3.10 & 3.27\end{array}$

$\begin{array}{llll}184 & 54.96 & -79.42 & 96.58\end{array}$

$\begin{array}{llll}495 & -93.12 & -13.13 & 94.04\end{array}$

$\begin{array}{llll}175 & -157.56 & 97.98 & 185.54\end{array}$

$\begin{array}{llll}181 & 104.60 & -107.39 & 149.91\end{array}$

$\begin{array}{llll}181 & 110.19 & 209.64 & 236.83\end{array}$

$258-147.63-219.20 \quad 264.28$

$\begin{array}{llll}120 & 483.65 & 1571.12 & 1643.88\end{array}$

$\begin{array}{lll}175 & 925.26 \quad 420.04 & 1016.14\end{array}$

275-1256.98 -876.09-670.98

$354-146.32-992.701003 .43$

$\begin{array}{llll}273 & -66.31 & -89.97 & 111.76\end{array}$

$\begin{array}{llll}531 & 1.56 & 56.83 & 56.86\end{array}$

$\begin{array}{llll}250 & 274.66 & 21.68 & 275.51\end{array}$

$\begin{array}{llll}250 & 274.61 & 22.01 & 275.49\end{array}$

$\begin{array}{llll}275 & 594.21 & -313.91 & 672.03\end{array}$

$275786.54-118.56 \quad 795.42$ 


\begin{tabular}{|c|c|c|c|c|c|c|c|c|}
\hline \multirow[t]{2}{*}{199} & 1.02 & 37.83 & 0.00 & 0.00 & \multirow{2}{*}{\multicolumn{2}{|c|}{272886.55}} & \multirow[b]{2}{*}{245.01} & \multirow[b]{2}{*}{919.78} \\
\hline & & & & & & & & \\
\hline 200 & 1.02 & 37.83 & 0.00 & 0.00 & $880.00-7$ & -71.92 & & \\
\hline \multirow{4}{*}{201} & & & & & 272 & 886.55 & 245.01 & 919.78 \\
\hline & 1.06 & 1.56 & 19.57 & 5.71 & & & & \\
\hline & & & & & 211 & -32.35 & 3.90 & 32.58 \\
\hline & 1.08 & 10.92 & & & 214 & 412.20 & 3.25 & 12.63 \\
\hline 202 & & & & & 208 & -2.17 & -0.58 & 2.24 \\
\hline \multirow[t]{4}{*}{203} & 0.99 & 0.78 & 36.82 & 10.74 & & & & \\
\hline & & & & & 204 & 25.26 & 7.55 & 26.36 \\
\hline & & & & & 215 & -27.93 & -12.18 & 30.47 \\
\hline & & & & & 253 & -36.52 & -32.11 & 48.62 \\
\hline \multirow[t]{2}{*}{204} & 0.99 & 0.58 & 25.59 & 7.47 & & & & \\
\hline & & & & & 203 & -25.21 & -7.49 & 26.30 \\
\hline \multirow[t]{3}{*}{205} & 1.09 & 10.70 & 0.00 & 0.00 & & & & \\
\hline & & & & & 209 & 35.40 & -3.27 & 35.55 \\
\hline & 077 & & & & 265 & -34.46 & 33.63 & 48.15 \\
\hline \multirow{2}{*}{206} & 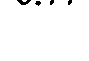 & -0.01 & 20.13 & 1.03 & 175 & & & \\
\hline & & & & & 181 & 107.41 & -106.26 & $\begin{array}{l}173.04 \\
151.09\end{array}$ \\
\hline \multirow[t]{3}{*}{207} & 1.00 & 1.43 & 33.02 & 9.63 & & & & \\
\hline & & & & & 212 & -90.03 & -30.36 & 95.01 \\
\hline & & & & & 215 & 55.49 & 20.67 & 59.22 \\
\hline \multirow[t]{3}{*}{208} & 1.09 & 11.22 & 23.12 & 6.74 & & & & \\
\hline & & & & & 202 & 2.37 & -0.24 & 2.38 \\
\hline & & & & & 213 & -25.45 & 0.06 & 25.45 \\
\hline \multirow[t]{3}{*}{209} & 1.08 & 9.16 & 9.91 & 2.89 & & & & \\
\hline & & & & & 205 & -35.26 & 3.79 & 35.46 \\
\hline & & & & & 277 & 25.25 & -6.54 & 26.08 \\
\hline \multirow[t]{3}{*}{210} & 1.07 & 5.25 & 10.81 & 3.16 & & & & \\
\hline & & & & & 211 & 33.39 & -2.82 & 33.51 \\
\hline & & & & & 277 & -44.62 & 7.09 & 45.18 \\
\hline \multirow[t]{3}{*}{211} & 1.06 & 2.27 & 0.99 & 0.29 & & & & \\
\hline & & & & & 201 & 32.39 & -3.70 & 32.61 \\
\hline & & & & & 210 & -33.08 & 3.82 & 33.30 \\
\hline \multirow[t]{5}{*}{212} & 1.01 & 2.51 & 23.20 & 6.77 & & & & \\
\hline & & & & & 191 & 0.37 & -1.68 & 1.72 \\
\hline & & & & & 192 & 1.04 & -3.08 & 3.26 \\
\hline & & & & & 207 & 90.49 & 32.28 & 96.08 \\
\hline & & & & & 250 & -111.48 & 50.37 & 122.33 \\
\hline \multirow[t]{3}{*}{213} & 1.09 & 11.97 & 0.00 & 0.00 & & & & \\
\hline & & & & & 208 & 25.59 & 0.04 & 25.59 \\
\hline & & & & & 270 & -24.66 & 19.75 & 31.59 \\
\hline \multirow[t]{3}{*}{214} & 1.06 & 0.87 & 11.89 & 3.47 & & & & \\
\hline & & & & & 201 & -12.24 & -3.56 & 12.74 \\
\hline & & & & & 251 & 0.09 & -3.81 & 3.81 \\
\hline \multirow[t]{2}{*}{215} & 0.99 & 1.13 & 28.24 & 8.23 & & & & \\
\hline & & & & & 203 & 27.99 & 12.22 & 30.54 \\
\hline
\end{tabular}




\begin{tabular}{|c|c|c|c|c|c|c|c|c|}
\hline 216 & 1.01 & 1.70 & 70.92 & 20.68 & 207 & -55.35 & -20.43 & 59.00 \\
\hline & & & & & 174 & 181.51 & -7.45 & 181.67 \\
\hline & & & & & 249 & -265.94 & $4-42.68$ & 269.34 \\
\hline \multirow[t]{3}{*}{217} & 0.98 & -10.20 & 32.44 & 9.46 & & & & \\
\hline & & & & & 220 & 97.93 & -46.59 & 108.45 \\
\hline & & & & & 233 & -131.87 & 745.65 & ; 139.55 \\
\hline \multirow[t]{3}{*}{218} & 0.99 & 4.96 & 25.43 & 7.42 & & & & \\
\hline & & & & & 242 & 11.01 & -18.00 & 21.10 \\
\hline & & & & & 250 & -37.63 & 10.31 & 39.02 \\
\hline \multirow[t]{3}{*}{219} & 1.01 & 1.89 & 31.62 & 9.22 & & & & \\
\hline & & & & & 231 & 63.87 & -14.51 & 65.50 \\
\hline & & & & & 253 & -100.36 & $5 \quad 2.61$ & 100.40 \\
\hline \multirow[t]{3}{*}{220} & 0.98 & -11.31 & 45.90 & 13.39 & & & & \\
\hline & & & & & 217 & -97.70 & 47.58 & 108.67 \\
\hline & & & & & 230 & 50.98 & -62.67 & 80.79 \\
\hline \multirow[t]{3}{*}{221} & 0.97 & -8.32 & 38.97 & 11.36 & & & & \\
\hline & & & & & 225 & -8.22 & 26.10 & 27.36 \\
\hline & & & & & 257 & -35.93 & -37.36 & 51.84 \\
\hline \multirow[t]{3}{*}{222} & 0.97 & -5.37 & 47.31 & 13.80 & & & & \\
\hline & & & & & 223 & 37.29 & 10.05 & 38.62 \\
\hline & & & & & 237 & -85.86 & -19.28 & 88.00 \\
\hline \multirow[t]{5}{*}{223} & 0.97 & -5.63 & 0.00 & 0.00 & & & & \\
\hline & & & & & 222 & -37.26 & -10.62 & 38.75 \\
\hline & & & & & 226 & 126.57 & -1.52 & 126.58 \\
\hline & & & & & 258 & -175.77 & 179.90 & 251.51 \\
\hline & & & & & 560 & 88.18 & 20.46 & 90.52 \\
\hline \multirow[t]{3}{*}{224} & 0.99 & -2.01 & 40.78 & 11.90 & $3.00 \quad 34$ & 4.33 & & \\
\hline & & & & & 227 & -897.89 & 496.21 & 2303.62 \\
\hline & & & & & 249 & -196.66 & -12.60 & 197.06 \\
\hline \multirow[t]{3}{*}{225} & 0.97 & -8.24 & 46.98 & 13.70 & & & & \\
\hline & & & & & 221 & 8.08 & -26.68 & 27.88 \\
\hline & & & & & 238 & -55.78 & 11.52 & 56.95 \\
\hline \multirow[t]{3}{*}{226} & 0.97 & -7.02 & 31.37 & 9.15 & & & & \\
\hline & & & & & 223 & -126.05 & 3.40 & 126.10 \\
\hline & & & & & 238 & 94.46 & -13.42 & 95.41 \\
\hline \multirow[t]{3}{*}{227} & 0.98 & -3.73 & 0.00 & 0.00 & & & & \\
\hline & & & & & 224 & -157.36 & -31.13 & 160.41 \\
\hline & & & & & 596 & 156.65 & 30.28 & 159.55 \\
\hline \multirow[t]{3}{*}{228} & 0.96 & -6.70 & 28.98 & 8.45 & & & & \\
\hline & & & & & 248 & 46.91 & 5.52 & 47.23 \\
\hline & & & & & 560 & -78.23 & -19.08 & 80.52 \\
\hline \multirow[t]{3}{*}{229} & 1.08 & 15.45 & 43.30 & 12.63 & & & & \\
\hline & & & & & 240 & -77.59 & 26.30 & 81.93 \\
\hline & & & & & 441 & 32.17 & -48.82 & 58.46 \\
\hline \multirow[t]{3}{*}{230} & $0.99-$ & -11.62 & 31.78 & 9.27 & & & & \\
\hline & & & & & 141 & 20.17 & -55.54 & 59.09 \\
\hline & & & & & 220 & -51.07 & 62.70 & 80.87 \\
\hline 231 & 1.01 & 1.73 & 24.02 & 7.01 & & & & \\
\hline
\end{tabular}




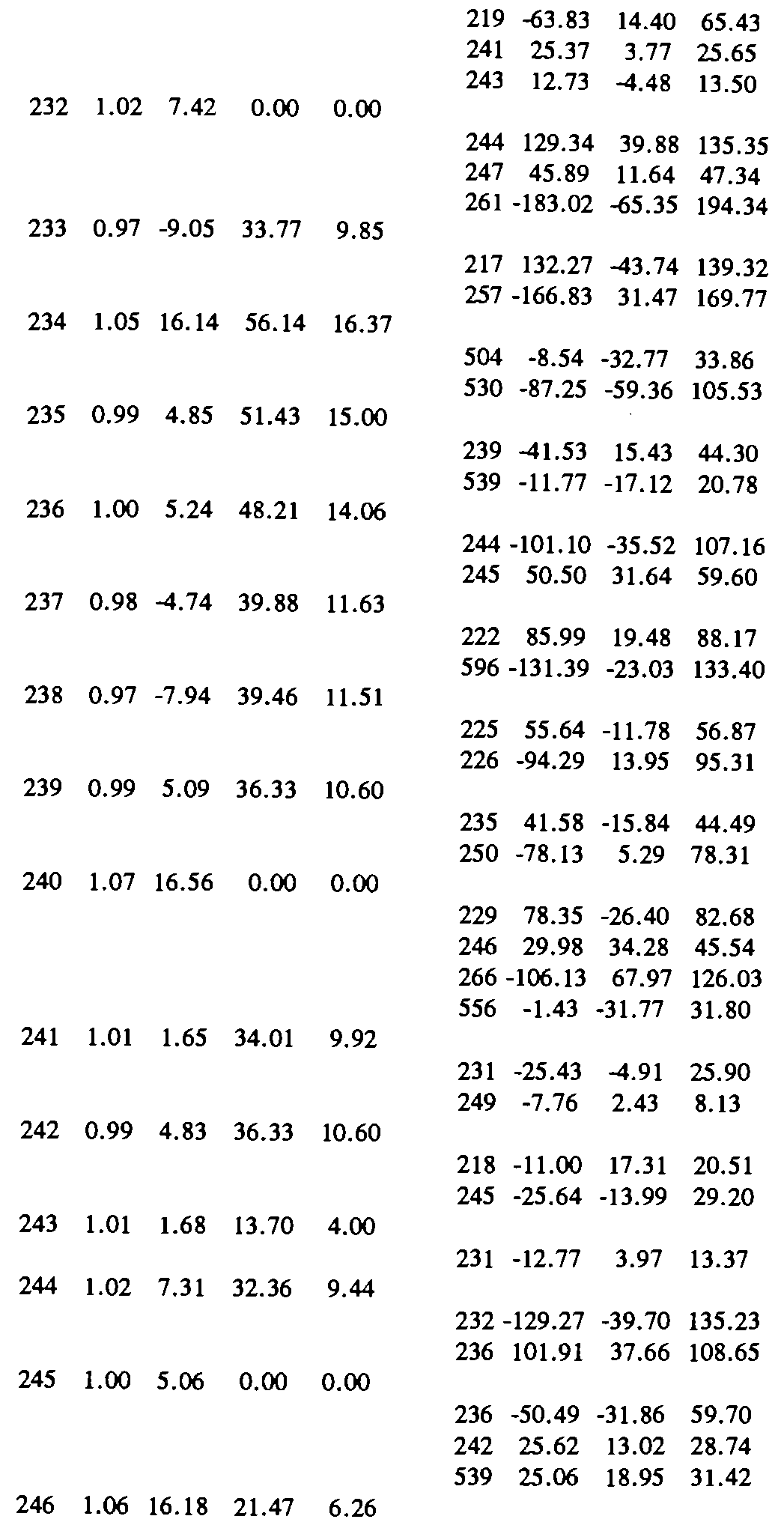




\begin{tabular}{|c|c|c|c|c|c|c|c|c|}
\hline & & & & & 240 & -29.54 & -35.68 & 46.32 \\
\hline & & & & & 504 & 7.28 & 27.43 & 28.38 \\
\hline \multirow[t]{2}{*}{247} & 1.01 & 6.70 & 45.98 & 13.41 & & & & \\
\hline & & & & & 232 & -45.62 & -13.06 & 47.45 \\
\hline \multirow[t]{2}{*}{248} & 0.96 & -6.90 & 47.64 & 13.89 & & & & \\
\hline & & & & & 228 & -47.03 & -5.80 & 47.39 \\
\hline \multirow[t]{7}{*}{249} & 1.01 & 1.77 & 0.00 & 0.00 & & & & \\
\hline & & & & & 216 & 266.01 & 43.01 & 269.47 \\
\hline & & & & & 224 & 198.73 & 23.93 & 200.17 \\
\hline & & & & & 241 & 7.71 & -4.05 & 8.71 \\
\hline & & & & & 250 & -114.94 & 53.53 & 126.80 \\
\hline & & & & & 251 & 4.17 & -43.06 & 43.26 \\
\hline & & & & & 268 & -344.84 & 118.01 & 364.47 \\
\hline \multirow[t]{9}{*}{250} & 0.99 & 5.57 & 0.00 & 0.00 & & & & \\
\hline & & & & & 193 & -273.84 & 3.35 & 273.86 \\
\hline & & & & & 194 & -273.79 & 3.24 & 273.81 \\
\hline & & & & & 212 & 111.76 & -43.47 & 119.92 \\
\hline & & & & & 218 & 37.93 & -11.27 & 39.57 \\
\hline & & & & & 239 & 78.29 & -5.32 & 78.47 \\
\hline & & & & & 249 & 115.91 & -48.10 & 125.50 \\
\hline & & & & & 580 & 205.84 & -95.11 & 226.75 \\
\hline & & & & & 600 & -10.11 & -40.86 & 42.09 \\
\hline \multirow[t]{4}{*}{251} & 1.06 & 0.86 & 0.00 & 0.00 & & & & \\
\hline & & & & & 214 & -0.09 & 3.82 & 3.82 \\
\hline & & & & & 249 & -4.60 & 37.84 & 38.12 \\
\hline & & & & & 479 & 3.46 & -38.00 & 38.15 \\
\hline \multirow[t]{2}{*}{252} & 1.05 & 15.68 & 47.88 & 13.97 & & & & \\
\hline & & & & & 530 & -48.88 & -9.42 & 49.78 \\
\hline \multirow[t]{4}{*}{253} & 1.01 & 2.20 & 0.00 & 0.00 & & & & \\
\hline & & & & & 203 & 36.59 & 33.82 & 49.82 \\
\hline & & & & & 219 & 100.51 & -2.43 & 100.54 \\
\hline & & & & & 254 & -135.89 & 125.56 & 185.02 \\
\hline \multirow[t]{3}{*}{254} & 1.00 & 2.95 & 55.40 & 16.16 & & & & \\
\hline & & & & & 253 & 136.43 & -123.38 & 183.95 \\
\hline & & & & & 538 & -192.89 & 107.00 & 220.58 \\
\hline \multirow[t]{3}{*}{255} & 1.11 & 13.78 & 39.58 & 11.54 & & & & \\
\hline & & & & & 449 & -85.20 & 71.25 & 111.07 \\
\hline & & & & & 457 & 43.37 & -88.01 & 98.12 \\
\hline \multirow[t]{2}{*}{256} & 1.07 & 15.01 & 29.72 & 8.67 & & & & \\
\hline & & & & & 441 & -30.35 & -10.90 & 32.25 \\
\hline \multirow[t]{4}{*}{257} & 0.97 & -8.28 & 0.00 & 0.00 & & & & \\
\hline & & & & & 221 & 35.92 & 37.29 & 51.78 \\
\hline & & & & & 233 & 167.10 & -29.68 & 169.72 \\
\hline & & & & & 273 & -192.02 & 275.78 & 336.04 \\
\hline \multirow[t]{5}{*}{258} & 0.94 & -3.36 & 0.00 & 0.00 & & & & \\
\hline & & & & & 188 & 152.28 & 250.35 & 293.02 \\
\hline & & & & & 223 & 176.23 & -166.65 & 242.55 \\
\hline & & & & & 262 & 292.18 & 17.74 & 292.72 \\
\hline & & & & & 268 & -622.16 & -161.83 & 642.87 \\
\hline
\end{tabular}




\begin{tabular}{|c|c|c|c|c|c|c|c|}
\hline \multirow[t]{3}{*}{259} & 1.04 & 17.30 & 0.00 & 0.00 & & & \\
\hline & & & & & $\begin{array}{lll}530 & 107.86\end{array}$ & $6-57.41$ & 122.19 \\
\hline & & & & & $532-112.58$ & $8-29.15$ & 116.29 \\
\hline \multirow[t]{3}{*}{260} & 1.05 & 12.24 & 0.08 & 0.02 & & & \\
\hline & & & & & $263 \quad 114.13$ & $3-23.68$ & 116.56 \\
\hline & & & & & $265-112.80$ & 16.03 & 113.93 \\
\hline \multirow[t]{3}{*}{261} & 1.03 & 9.38 & 0.00 & 0.00 & & & \\
\hline & & & & & $232 \quad 183.28$ & 72.61 & 197.13 \\
\hline & & & & & $263-183.23$ & $3-73.45$ & 197.40 \\
\hline \multirow[t]{3}{*}{262} & 0.93 & -4.65 & 24.52 & 7.15 & & & \\
\hline & & & & & $258-292.10$ & -14.48 & 292.46 \\
\hline & & & & & $273 \quad 265.99$ & 6.42 & 266.07 \\
\hline \multirow[t]{5}{*}{263} & 1.05 & 11.55 & 0.00 & 0.00 & & & \\
\hline & & & & & $260-113.16$ & 20.55 & 115.01 \\
\hline & & & & & $261 \quad 183.93$ & 71.36 & 197.29 \\
\hline & & & & & $266-495.04$ & 87.80 & 502.77 \\
\hline & & & & & $267 \quad 425.67$ & 165.47 & 456.70 \\
\hline \multirow[t]{3}{*}{264} & 0.92 & -6.31 & 27.78 & 8.10 & & & \\
\hline & & & & & $\begin{array}{ll}176 & -57.01\end{array}$ & 45.24 & 72.78 \\
\hline & & & & & $537 \quad 29.35$ & -56.01 & 63.24 \\
\hline \multirow[t]{4}{*}{265} & 1.05 & 12.63 & 0.00 & 0.00 & & & \\
\hline & & & & & $205 \quad 34.52$ & -31.49 & 46.73 \\
\hline & & & & & $260 \quad 113.64$ & -17.59 & 114.99 \\
\hline & & & & & $270-147.13$ & 22.40 & 148.82 \\
\hline \multirow[t]{10}{*}{266} & 1.05 & 18.59 & 0.00 & 0.00 & & & \\
\hline & & & & & $123 \quad 137.63$ & -211.14 & 252.04 \\
\hline & & & & & $240 \quad 106.25$ & -62.83 & 123.44 \\
\hline & & & & & 263502.08 & -47.58 & 504.33 \\
\hline & & & & & $268 \quad 435.40$ & 77.35 & 442.22 \\
\hline & & & & & $270 \quad 190.44$ & -31.39 & 193.01 \\
\hline & & & & & $272 \quad 234.85$ & 756.99 & 1881.71 \\
\hline & & & & & $276 \quad 167.82$ & -723.62 & 742.83 \\
\hline & & & & & $\begin{array}{lll}532 & 134.47\end{array}$ & 21.48 & 136.18 \\
\hline & & & & & $542 \quad 49.77$ & -223.29 & 228.77 \\
\hline \multirow[t]{3}{*}{267} & 1.04 & 9.53 & 29.23 & 8.52 & & & \\
\hline & & & & & $263-423.00$ & -154.05 & 450.17 \\
\hline & & & & & $268 \quad 396.99$ & 150.34 & 424.51 \\
\hline \multirow[t]{8}{*}{268} & 1.00 & 4.66 & 0.00 & 0.00 & & & \\
\hline & & & & & 176418.85 & 168.62 & 451.51 \\
\hline & & & & & $249 \quad 345.59$ & -99.13 & 359.52 \\
\hline & & & & & $258 \quad 630.49$ & 254.65 & 679.98 \\
\hline & & & & & $266-432.29$ & -11.69 & 432.45 \\
\hline & & & & & $267-394.99$ & -125.23 & 414.36 \\
\hline & & & & & $535-280.00$ & 85.14 & 292.65 \\
\hline & & & & & $599-315.15$ & 82.64 & 325.81 \\
\hline \multirow[t]{3}{*}{269} & 0.93 & -3.86 & 48.21 & 14.06 & & & \\
\hline & & & & & 176336.65 & 37.66 & 338.75 \\
\hline & & & & & $534-384.97$ & -50.81 & 388.30 \\
\hline
\end{tabular}




\begin{tabular}{|c|c|c|c|c|c|c|c|c|}
\hline & & & & & 213 & 24.74 & -17.84 & 30.50 \\
\hline & & & & & 265 & 150.04 & -29.19 & 152.85 \\
\hline & & & & & 266 & -188.16 & 25.82 & 189.93 \\
\hline \multirow[t]{3}{*}{271} & 0.76 & -8.87 & 22.95 & 6.69 & & & & \\
\hline & & & & & 177 & -30.70 & -122.87 & 126.64 \\
\hline & & & & & 184 & -1.45 & 142.42 & 142.43 \\
\hline \multirow[t]{4}{*}{272} & 0.99 & 31.65 & 8.67 & 2.53 & & & & \\
\hline & & & & & 199 & -884.98 & -144.39 & 896.68 \\
\hline & & & & & 200 & -884.98 & -144.39 & 896.68 \\
\hline & & & & & 266 & 1749.97 & -338.38 & 1782.38 \\
\hline \multirow[t]{4}{*}{273} & 0.93 & -6.29 & 0.00 & 0.00 & & & & \\
\hline & & & & & 190 & 66.05 & 88.88 & 110.74 \\
\hline & & & & & 257 & 192.71 & -257.23 & 321.41 \\
\hline & & & & & 262 & -266.64 & -3.63 & 266.67 \\
\hline \multirow[t]{3}{*}{274} & 1.05 & 12.11 & 0.00 & 0.00 & & & & \\
\hline & & & & & 275 & -1276.87 & 37.17 & 1099.75 \\
\hline & & & & & 535 & 1109.63 & -41.59 & 1110.41 \\
\hline \multirow[t]{7}{*}{275} & 1.05 & 16.36 & 0.00 & 0.00 & & & & \\
\hline & & & & & 189 & 1366.02 & 1445.87 & 1989.11 \\
\hline & & & & & 195 & -592.90 & 371.00 & 699.41 \\
\hline & & & & & 196 & -784.79 & 194.87 & 808.62 \\
\hline & & & & & 274 & 1124.20 & -92.86 & 1128.02 \\
\hline & & & & & 276 & -569.78 & -1320.53 & 1160.64 \\
\hline & & & & & 476 & -455.31 & -276.98 & 1440.85 \\
\hline \multirow[t]{4}{*}{276} & 1.10 & 17.92 & 0.00 & 0.00 & & & & \\
\hline & & & & & 266 & -167.31 & 762.11 & 780.26 \\
\hline & & & & & 275 & 593.82 & 963.32 & 1131.64 \\
\hline & & & & & 476 & $-379.02-$ & -1675.87 & 1366.87 \\
\hline \multirow[t]{3}{*}{277} & 1.07 & 6.21 & 0.00 & 0.00 & & & & \\
\hline & & & & & 209 & -24.65 & 6.86 & 25.59 \\
\hline & & & & & 210 & 44.79 & -6.55 & 45.26 \\
\hline \multirow[t]{2}{*}{278} & 1.00 & 5.31 & 0.00 & 0.00 & & & & \\
\hline & & & & & 344 & 20.92 & -74.82 & 77.69 \\
\hline \multirow[t]{2}{*}{279} & 1.15 & 6.43 & 0.00 & 0.00 & & & & \\
\hline & & & & & 309 & 138.89 & 32.61 & 142.66 \\
\hline \multirow[t]{2}{*}{280} & 1.02 & 7.43 & 0.00 & 0.00 & & & & \\
\hline & & & & & 309 & 359.57 & -463.17 & 586.36 \\
\hline \multirow[t]{2}{*}{281} & 1.38 & 20.39 & 0.00 & 0.00 & & & & \\
\hline & & & & & 349 & 522.87 & 651.52 & 835.39 \\
\hline \multirow[t]{2}{*}{282} & 1.38 & 21.36 & 0.00 & 0.00 & & & & \\
\hline & & & & & 349 & 802.65 & 651.44 & 1033.74 \\
\hline \multirow[t]{2}{*}{283} & 1.79 & 5.82 & 17.94 & 5.23 & & & & \\
\hline & & & & & 286 & -16.02 & 16.52 & 23.01 \\
\hline \multirow[t]{3}{*}{284} & 1.74 & 7.99 & 0.00 & 0.00 & & & & \\
\hline & & & & & 286 & 23.74 & -37.14 & 44.08 \\
\hline & & & & & 295 & -20.40 & 73.21 & 76.00 \\
\hline \multirow[t]{3}{*}{285} & 1.35 & 10.44 & 2.36 & 0.69 & & & & \\
\hline & & & & & 583 & -7.02 & 0.13 & 7.03 \\
\hline & & & & & 3231 & 5.54 & -0.08 & 5.54 \\
\hline
\end{tabular}




\begin{tabular}{|c|c|c|c|c|c|c|c|c|}
\hline 286 & 1.78 & 6.15 & 5.31 & 1.55 & & & & \\
\hline & & & & & 283 & 16.04 & -17.06 & 23.41 \\
\hline & & & & & 284 & -22.89 & 37.10 & 43.59 \\
\hline 287 & 1.36 & 10.81 & 0.00 & 0.00 & & & & \\
\hline & & & & & 353 & -4.63 & 40.30 & 40.56 \\
\hline & & & & & 583 & 9.26 & 1.24 & 9.34 \\
\hline 88 & 1.08 & -2.61 & 0.00 & 0.00 & & & & \\
\hline & & & & & 299 & 65.15 & 78.85 & 102.28 \\
\hline & & & & & 305 & 146.62 & 63.19 & 159.66 \\
\hline & & & & & 314 & -41.30 & -98.12 & 106.46 \\
\hline & & & & & 340 & -175.71 & -50.22 & 182.74 \\
\hline 89 & 0.99 & -5.92 & 44.13 & 12.87 & & & & \\
\hline & & & & & 299 & -49.27 & -12.27 & 50.77 \\
\hline 90 & 1.12 & 1.99 & 5.98 & 1.74 & & & & \\
\hline & & & & & 309 & -42.23 & -18.20 & 45.99 \\
\hline & & & & & 316 & 36.19 & 16.68 & 39.84 \\
\hline 91 & 1.83 & 10.41 & 20.97 & 6.12 & & & & \\
\hline & & & & & 313 & 2.35 & 28.95 & 29.05 \\
\hline 92 & 1.05 & -5.30 & 23.33 & 6.80 & & & & \\
\hline & & & & & 305 & -94.00 & -46.63 & 104.92 \\
\hline & & & & & 324 & 66.16 & 43.18 & 79.00 \\
\hline 93 & 1.82 & 10.63 & 47.67 & 13.90 & & & & \\
\hline & & & & & 307 & 5.37 & 9.83 & 11.20 \\
\hline & & & & & 594 & 170.62 & 420.48 & 453.77 \\
\hline 94 & 0.79 & -20.60 & 14.32 & 4.18 & & & & \\
\hline & & & & & 543 & 6.96 & -12.89 & 14.65 \\
\hline & & & & & 551 & -327.55 & -157.14 & 363.29 \\
\hline 95 & 1.68 & 8.62 & 0.00 & 0.00 & & & & \\
\hline & & & & & 284 & 20.51 & -70.60 & 73.52 \\
\hline & & & & & 297 & 108.13 & 114.91 & 157.78 \\
\hline & & & & & 329 & 114.33 & 161.73 & 198.06 \\
\hline & & & & & 342 & 83.95 & 880.10 & 884.09 \\
\hline 296 & 1.55 & 8.15 & 38.07 & 11.10 & & & & \\
\hline & & & & & 485 & 6.38 & 49.75 & 50.16 \\
\hline & & & & & 592 & 44.97 & 87.65 & 98.51 \\
\hline 297 & 1.68 & 8.61 & 6.99 & 2.04 & & & & \\
\hline & & & & & 295 & -108.12 & -114.91 & 157.78 \\
\hline & & & & & 330 & -87.53 & -125.61 & 153.10 \\
\hline 298 & 1.60 & 8.20 & 14.65 & 4.28 & & & & \\
\hline & & & & & 318 & -15.41 & -184.60 & 185.24 \\
\hline & & & & & 319 & 10.02 & 193.25 & 193.51 \\
\hline 299 & 1.00 & -5.58 & 0.00 & 0.00 & & & & \\
\hline & & & & & 288 & -64.74 & -76.93 & 100.55 \\
\hline & & & & & 289 & 49.02 & 11.48 & 50.35 \\
\hline & & & & & 320 & 37.56 & -110.23 & 116.45 \\
\hline & & & & & 343 & -202.97 & -30.20 & 205.21 \\
\hline & & & & & 551 & 359.52 & 295.21 & 465.19 \\
\hline & & & & & 559 & -184.28 & -32.54 & 187.13 \\
\hline
\end{tabular}




\begin{tabular}{|c|c|c|c|c|c|c|c|c|}
\hline & & & & & $\begin{array}{l}303 \\
309\end{array}$ & $\begin{array}{r}116.01 \\
-163.74\end{array}$ & $\begin{array}{r}-35.50 \\
27.14\end{array}$ & $\begin{array}{l}121.32 \\
165.97\end{array}$ \\
\hline \multirow[t]{4}{*}{301} & 1.78 & 10.67 & 59.13 & 17.25 & & & & \\
\hline & & & & & 315 & -38.04 & 625.23 & 626.39 \\
\hline & & & & & 321 & 28.95 & -202.15 & 204.21 \\
\hline & & & & & 325 & -11.75 & -265.97 & 266.23 \\
\hline \multirow[t]{3}{*}{303} & 1.13 & -1.84 & 46.66 & 13.61 & & & & \\
\hline & & & & & 300 & -115.91 & 35.79 & 121.32 \\
\hline & & & & & 314 & 68.37 & 88.46 & 111.80 \\
\hline \multirow[t]{3}{*}{304} & 1.55 & 8.35 & 37.90 & 11.06 & & & & \\
\hline & & & & & 319 & -39.29 & 14.69 & 41.95 \\
\hline & & & & & 331 & 23.70 & 19.11 & 30.44 \\
\hline \multirow[t]{3}{*}{305} & 1.07 & -3.88 & 54.16 & 15.80 & & & & \\
\hline & & & & & 288 & -146.15 & -60.60 & 158.21 \\
\hline & & & & & 292 & 93.87 & 46.87 & 104.93 \\
\hline \multirow[t]{2}{*}{306} & 1.13 & 2.17 & 33.44 & 9.75 & & & & \\
\hline & & & & & 322 & -34.88 & 4.18 & 35.13 \\
\hline \multirow[t]{3}{*}{307} & 1.82 & 10.63 & 25.27 & 7.37 & & & & \\
\hline & & & & & 293 & -5.35 & -10.91 & 12.15 \\
\hline & & & & & 330 & -2.16 & -5.00 & 5.45 \\
\hline \multirow[t]{3}{*}{308} & 1.83 & 10.46 & 32.17 & 9.38 & & & & \\
\hline & & & & & 330 & -53.78 & 82.68 & 98.64 \\
\hline & & & & & 554 & 53.01 & -23.97 & 58.18 \\
\hline \multirow[t]{9}{*}{309} & 1.13 & 2.78 & 0.00 & 0.00 & & & & \\
\hline & & & & & 279 & -138.60 & -23.33 & 140.56 \\
\hline & & & & & 280 & -356.93 & 546.12 & 652.42 \\
\hline & & & & & 290 & 42.47 & 15.82 & 45.32 \\
\hline & & & & & 300 & 165.49 & -20.80 & 166.79 \\
\hline & & & & & 335 & 55.31 & -16.14 & 57.62 \\
\hline & & & & & 344 & -58.91 & 143.41 & 155.04 \\
\hline & & & & & 595 & 195.17 & 69.63 & 207.22 \\
\hline & & & & & 3238 & 62.89 & -1.45 & 62.91 \\
\hline \multirow[t]{3}{*}{311} & 1.65 & 8.16 & 28.05 & 8.18 & & & & \\
\hline & & & & & 318 & 61.59 & 174.87 & 185.40 \\
\hline & & & & & 329 & -67.12 & -153.54 & 167.57 \\
\hline \multirow[t]{3}{*}{312} & 1.21 & 11.81 & 34.74 & 10.13 & & & & \\
\hline & & & & & 443 & 33.22 & -5.09 & 33.61 \\
\hline & & & & & 446 & -74.59 & -18.57 & 76.87 \\
\hline \multirow[t]{3}{*}{313} & 1.83 & 10.42 & 42.79 & 12.48 & & & & \\
\hline & & & & & 291 & -2.29 & -30.64 & 30.73 \\
\hline & & & & & 336 & -29.47 & 114.63 & 118.36 \\
\hline \multirow[t]{3}{*}{314} & 1.11 & -2.48 & 27.96 & 8.16 & & & & \\
\hline & & & & & 288 & 42.31 & 98.54 & 107.24 \\
\hline & & & & & 303 & -68.27 & -88.30 & 111.62 \\
\hline \multirow[t]{3}{*}{315} & 1.67 & 11.51 & 0.00 & 0.00 & & & & \\
\hline & & & & & 301 & 44.67 & -591.19 & 592.88 \\
\hline & & & & & 346 & -49.14 & 504.27 & 506.65 \\
\hline
\end{tabular}

$\begin{array}{lllll}316 & 1.11 & 1.41 & 14.07 & 4.10\end{array}$

$$
\begin{array}{llll}
290 & -35.73 & -18.37 & 40.17
\end{array}
$$




\begin{tabular}{|c|c|c|c|c|c|c|c|c|}
\hline & & & & & 479 & 21.70 & 21.74 & 30.72 \\
\hline \multirow[t]{4}{*}{317} & 1.53 & 8.59 & 0.00 & 0.00 & & & & \\
\hline & & & & & 334 & 36.46 & -53.55 & 64.79 \\
\hline & & & & & 337 & 51.09 & -58.11 & 77.38 \\
\hline & & & & & 348 & -73.82 & 132.64 & 151.80 \\
\hline \multirow[t]{3}{*}{318} & 1.63 & 8.08 & 34.87 & 10.17 & & & & \\
\hline & & & & & 298 & 16.82 & 185.17 & 185.93 \\
\hline & & & & & 311 & -61.12 & -175.00 & 185.37 \\
\hline \multirow[t]{5}{*}{319} & 1.55 & 8.43 & 0.00 & 0.00 & & & & \\
\hline & & & & & 298 & $-8.71-$ & -193.24 & 193.43 \\
\hline & & & & & 304 & 39.32 & -15.68 & 42.33 \\
\hline & & & & & 339 & 13.84 & 3.01 & 14.17 \\
\hline & & & & & 350 & -52.65 & 88.16 & 102.68 \\
\hline \multirow[t]{3}{*}{320} & 1.01 & -6.04 & 43.71 & 12.75 & & & & \\
\hline & & & & & 299 & -37.68 & 110.65 & 116.89 \\
\hline & & & & & 333 & -9.49 & -126.56 & 126.92 \\
\hline \multirow[t]{3}{*}{321} & 1.79 & 10.54 & 41.27 & 12.04 & & & & \\
\hline & & & & & 301 & -28.56 & 201.64 & 203.65 \\
\hline & & & & & 338 & -12.35 & -144.13 & 144.66 \\
\hline \multirow[t]{3}{*}{322} & 1.13 & 2.38 & 23.67 & 6.90 & & & & \\
\hline & & & & & 306 & 34.72 & -5.47 & 35.15 \\
\hline & & & & & 335 & -58.79 & -2.37 & 58.84 \\
\hline \multirow[t]{3}{*}{323} & 1.55 & 8.23 & 12.21 & 3.56 & & & & \\
\hline & & & & & 334 & -15.40 & 63.22 & 65.07 \\
\hline & & & & & 485 & 22.14 & -27.62 & 35.40 \\
\hline \multirow[t]{3}{*}{324} & 1.03 & -6.07 & 24.76 & 7.22 & & & & \\
\hline & & & & & 292 & -34.77 & -34.52 & 43.08 \\
\hline & & & & & 333 & 34.89 & 29.80 & 45.88 \\
\hline \multirow[t]{3}{*}{325} & 1.80 & 10.58 & 45.48 & 13.27 & & & & \\
\hline & & & & & 301 & 12.46 & 266.84 & 267.13 \\
\hline & & & & & 594 & -52.92 & -229.45 & 235.47 \\
\hline \multirow[t]{2}{*}{326} & 1.83 & 10.41 & 47.50 & 13.85 & & & & \\
\hline & & & & & 332 & 22.45 & 117.56 & 119.68 \\
\hline \multirow[t]{2}{*}{327} & 1.83 & 10.24 & 42.96 & 12.53 & & & & \\
\hline & & & & & 593 & -17.14 & 51.98 & 54.73 \\
\hline \multirow[t]{3}{*}{329} & 1.66 & 8.28 & 35.04 & 10.22 & & & & \\
\hline & & & & & 295 & -113.77 & -161.74 & 197.75 \\
\hline & & & & & 311 & 67.32 & 152.53 & 166.73 \\
\hline \multirow[t]{8}{*}{330} & 1.82 & 10.65 & 0.00 & 0.00 & & & & \\
\hline & & & & & 297 & 90.60 & 111.19 & 143.43 \\
\hline & & & & & 307 & 2.42 & -7.09 & 7.49 \\
\hline & & & & & 308 & 54.16 & -84.71 & 100.54 \\
\hline & & & & & 332 & 66.12 & -126.21 & 142.48 \\
\hline & & & & & 336 & 76.42 & -72.92 & 105.62 \\
\hline & & & & & 352 & -268.26 & 3742.94 & 3752.54 \\
\hline & & & & & 597 & 51.43 & 107.62 & 119.28 \\
\hline \multirow[t]{3}{*}{331} & 1.54 & 8.19 & 35.97 & 10.49 & & & & \\
\hline & & & & & 304 & -23.61 & -24.22 & 33.82 \\
\hline & & & & & 337 & -6.69 & 38.08 & 38.66 \\
\hline
\end{tabular}




\begin{tabular}{|c|c|c|c|c|c|c|c|c|}
\hline 332 & 1.83 & 10.41 & 38.66 & 11.28 & & & & \\
\hline & & & & & 326 & -22.37 & -117.86 & 119.96 \\
\hline & & & & & 330 & -65.83 & 124.23 & 140.59 \\
\hline 333 & 1.03 & -6.20 & 32.93 & 9.60 & & & & \\
\hline & & & & & 320 & 9.74 & 127.33 & 127.70 \\
\hline & & & & & 324 & -34.96 & -30.28 & 46.25 \\
\hline 334 & 1.54 & 8.36 & 13.31 & 3.88 & & & & \\
\hline & & & & & 317 & -36.49 & 51.27 & 62.93 \\
\hline & & & & & 323 & 15.29 & -66.10 & 67.85 \\
\hline 335 & 1.13 & 2.76 & 17.35 & 5.06 & & & & \\
\hline & & & & & 309 & -55.31 & 16.09 & 57.61 \\
\hline & & & & & 322 & 58.70 & 1.11 & 58.71 \\
\hline 336 & 1.83 & 10.48 & 20.80 & 6.07 & & & & \\
\hline & & & & & 313 & 29.66 & -115.51 & 119.25 \\
\hline & & & & & 330 & -76.17 & 71.61 & 104.54 \\
\hline 337 & 1.54 & 8.25 & 36.22 & 10.56 & & & & \\
\hline & & & & & 317 & -50.91 & 56.03 & 75.70 \\
\hline & & & & & 331 & 6.72 & -40.41 & 40.97 \\
\hline & & & & & 592 & 2.63 & -31.81 & 31.92 \\
\hline 338 & 1.80 & 10.50 & 38.91 & 11.35 & & & & \\
\hline & & & & & 321 & 12.45 & 141.94 & 142.49 \\
\hline & & & & & 597 & -49.18 & -109.89 & 120.39 \\
\hline 339 & 1.55 & 8.35 & 10.78 & 3.14 & & & & \\
\hline & & & & & 319 & -14.06 & -7.28 & 15.83 \\
\hline 340 & 1.11 & -0.08 & 16.84 & 4.91 & & & & \\
\hline & & & & & 288 & 176.70 & 56.88 & 185.63 \\
\hline & & & & & 595 & -195.99 & -64.45 & 206.31 \\
\hline
\end{tabular}

$\begin{array}{lllll}341 & 1.53 & 12.45 & 31.84 & 9.29\end{array}$

$\begin{array}{llll}346 & 211.81 & -852.18 & 878.11\end{array}$ $\begin{array}{llll}555 & -200.33 \quad 907.42 \quad 929.27\end{array}$

$\begin{array}{lllll}342 & 1.29 & 7.34 & 0.00 & 0.00\end{array}$

$$
\begin{array}{rrrr}
295 & -79.21 & -671.88 & 676.54 \\
344 & 352.55 & 725.71 & 806.81 \\
348 & -205.49 & -582.09 & 617.30 \\
352 & -300.79 & -513.17 & 594.83 \\
499 & -269.44 & -20.63 & 270.23 \\
3239 & 492.85 & 949.62 & 1069.90
\end{array}
$$

$\begin{array}{lllll}343 & 1.01 & -1.78 & 0.00 & 0.00\end{array}$

$\begin{array}{llll}299 & 203.43 & 44.25 & 208.19\end{array}$ $\begin{array}{llll}351 & -206.70 & -87.78 & 224.57\end{array}$

$\begin{array}{lllll}344 & 1.09 & 3.91 & 0.00 & 0.00\end{array}$

$\begin{array}{llll}278 & -20.73 & 81.81 & 84.39\end{array}$

$\begin{array}{llll}309 & 59.09 & -136.13 \quad 148.40\end{array}$

$342-336.41-610.60 \quad 697.14$

$\begin{array}{lllll}351 & 523.89 & 1115.37 & 1232.28\end{array}$ $3239-195.13-586.61 \quad 618.22$

$\begin{array}{lllll}345 & 1.38 & 15.14 & 12.21 & 3.56\end{array}$

$\begin{array}{llll}555 & 249.12 & -820.90 \quad 857.86\end{array}$

$\begin{array}{lllll}1556 & -255.13 & 826.14 & 864.63\end{array}$ 
$\begin{array}{lllll}346 & 1.55 & 12.13 & 0.00 & 0.00\end{array}$

$\begin{array}{lllll}315 & 50.55 & -466.94 & 469.66\end{array}$

$\begin{array}{llll}341 & -209.02 & 857.64 & 882.74\end{array}$

$\begin{array}{llll}553 & 171.88 & -304.13 & 349.34\end{array}$

$\begin{array}{lllll}347 & 1.33 & 16.02 & 12.63 & 3.68\end{array}$

$\begin{array}{llll}349 & -287.79 & 7.43 & 287.88\end{array}$

$\begin{array}{llll}353 & 281.89 & -0.20 & 281.89\end{array}$

$\begin{array}{lllll}348 & 1.50 & 9.37 & 0.00 & 0.00\end{array}$

$\begin{array}{lllll}317 & 73.91 & -128.66 & 148.38\end{array}$

$\begin{array}{lllll}342 & 205.57 & 648.14 & 679.96\end{array}$

$\begin{array}{llll}352 & -337.75 & -289.36 & 444.76\end{array}$

$\begin{array}{llll}552 & 71.58 & -186.32 \quad 199.60\end{array}$

$\begin{array}{lllll}349 & 1.33 & 18.65 & 0.00 & 0.00\end{array}$

$\begin{array}{llll}281 & -521.47 & -610.79 & 803.11\end{array}$

$282-800.50-589.06993 .88$

$\begin{array}{lllll}347 & 289.74 & -13.58 & 290.05\end{array}$

$\begin{array}{lllll}352 & 831.17 & 157.03 & 1393.89\end{array}$

$\begin{array}{lllll}1555 & 95.26 & 563.71 & 571.70\end{array}$

$\begin{array}{lllll}1725 & 58.56 & 519.82 & 523.11\end{array}$

$\begin{array}{lllll}350 & 1.53 & 8.97 & 0.00 & 0.00\end{array}$

$\begin{array}{llll}319 & 52.69 & -86.37 & 101.17\end{array}$

$\begin{array}{llll}552 & -48.93 & 197.57 & 203.54\end{array}$

$\begin{array}{lllll}351 & 1.06 & 3.34 & 0.00 & 0.00\end{array}$

$\begin{array}{llll}343 & 199.21 & 73.55 & 212.36\end{array}$

$344-521.92-675.801203 .95$

$\begin{array}{lllll}354 & 160.32 & 530.10 & 553.81\end{array}$

$\begin{array}{llll}535 & -64.64 & 19.93 & 67.64\end{array}$

$\begin{array}{llll}541 & 198.98 & 74.58 & 212.50\end{array}$

$\begin{array}{lllll}352 & 1.56 & 11.63 & 0.00 & 0.00\end{array}$

$330 \quad 284.70-1674.873217 .14$

$\begin{array}{llll}342 & 308.60 & 591.44 & 667.11\end{array}$

$\begin{array}{lllll}348 & 332.94 & 286.78 & 439.42\end{array}$

$\begin{array}{llllll}349 & -818.55 & 1374.78 & 1600.02\end{array}$

$\begin{array}{llll}553 & -129.66 & 341.85 & 365.61\end{array}$

$\begin{array}{lllll}353 & 1.31 & 11.10 & 0.00 & 0.00\end{array}$

$\begin{array}{llll}287 & 4.67 & -38.94 & 39.22\end{array}$

$\begin{array}{llll}347 & -279.35 & -12.68 & 279.63\end{array}$

$\begin{array}{llll}499 & 277.03 & 11.92 & 277.28\end{array}$

$\begin{array}{lllll}354 & 1.02 & 2.72 & 0.00 & 0.00\end{array}$

$\begin{array}{llll}189 & 52.15 & 768.86 & 770.63\end{array}$

$351-160.04-509.06 \quad 533.63$

$\begin{array}{lllll}356 & 1.47 & 28.35 & 0.00 & 0.00\end{array}$

$\begin{array}{llll}463 & 490.72 & -443.73 \quad 661.59\end{array}$

$\begin{array}{lllll}476 & 786.93 & -178.20 & 806.86\end{array}$

$\begin{array}{llll}477 & 224.47 & 93.77 & 243.27\end{array}$

$\begin{array}{llll}2498 & -436.82 & 199.04 & 480.03\end{array}$

$\begin{array}{llll}2499 & -799.24 & 141.29 & 811.63\end{array}$

$\begin{array}{lllll}357 & 1.60 & 23.90 & 0.00 & 0.00\end{array}$

$\begin{array}{llll}468 & 101.61 & 101.11 & 143.35\end{array}$ 


$\begin{array}{lllll}358 & 1.58 & 23.96 & 0.00 & 0.00 \\ 359 & 1.61 & 22.52 & 0.00 & 0.00 \\ 360 & 1.58 & 22.57 & 0.00 & 0.00 \\ 361 & 1.56 & 19.32 & 0.00 & 0.00 \\ 362 & 1.43 & 22.36 & 0.00 & 0.00 \\ 363 & 1.43 & 19.46 & 0.00 & 0.00 \\ 364 & 1.60 & 20.01 & 0.00 & 0.00 \\ 365 & 1.61 & 19.98 & 0.00 & 0.00 \\ 367 & 1.60 & 18.60 & 0.00 & 0.00\end{array}$

$\begin{array}{lllll}368 & 1.33 & 8.03 & 15.73 & 4.59\end{array}$

$\begin{array}{lllll}370 & 1.46 & 18.38 & 9.34 & 2.72\end{array}$

$\begin{array}{lllll}371 & 1.20 & 14.31 & 14.72 & 4.29\end{array}$

$\begin{array}{lllll}372 & 1.15 & 9.30 & 6.48 & 1.89\end{array}$

$\begin{array}{lllll}373 & 1.25 & 7.11 & 0.00 & 0.00\end{array}$

$\begin{array}{lllll}374 & 1.36 & 13.75 & 3.11 & 0.91\end{array}$

$\begin{array}{lllll}375 & 1.46 & 23.78 & 0.00 & 0.00\end{array}$

$\begin{array}{lllll}376 & 1.35 & 20.06 & 0.00 & 0.00\end{array}$

$\begin{array}{lllll}377 & 1.57 & 18.05 & 12.62 & 3.68\end{array}$ $\begin{array}{llll}468 & 101.57 & 62.85 & 119.44\end{array}$

$\begin{array}{llll}468 & 82.75 & 199.79 & 216.25\end{array}$

$\begin{array}{llll}468 & 82.66 & 114.31 & 141.07\end{array}$

$\begin{array}{llll}422 & 141.45 & 145.28 & 202.76\end{array}$

$\begin{array}{llll}461 & 366.68 & 216.13 \quad 425.63\end{array}$

$\begin{array}{llll}461 & 75.11 & 238.69 & 250.23\end{array}$

$469 \quad 311.97 \quad 577.71 \quad 656.56$

$469 \quad 320.65 \quad 613.60 \quad 692.33$

$\begin{array}{lllll}378 & 47.54 & 146.29 & 153.82\end{array}$

$\begin{array}{lllll}468 & -271.41 & 293.69 & 399.90\end{array}$

$\begin{array}{llll}474 & 223.40 & -353.56 & 418.23\end{array}$

$\begin{array}{llll}373 & 54.50 & 121.99 & 133.61\end{array}$

$\begin{array}{lllll}1069 & -61.02 & -91.38 & 109.88\end{array}$

$419-340.62-523.40 \quad 624.48$

$427-113.23-122.20 \quad 166.59$

$3301-133.48-180.36 \quad 224.38$

$409-111.43-115.50 \quad 160.49$

$3004-136.15-159.74209 .89$

$\begin{array}{lllll}368 & -53.82 & -118.27 & 129.93\end{array}$

$384-214.64-122.29247 .03$

$3148-391.58-353.61527 .61$

$\begin{array}{llll}379 & -30.67 & -10.88 & 32.55\end{array}$

$\begin{array}{llll}413 & 21.33 & -0.91 & 21.35\end{array}$

$\begin{array}{llll}380 & -155.01 & -119.81 & 195.92\end{array}$

$\begin{array}{llll}406 & 215.14 & 37.82 & 218.44\end{array}$

$\begin{array}{lllll}458 & -214.16 & 18.98 & 215.00\end{array}$

$\begin{array}{lllll}3007 & 147.29 & 66.50 & 161.61\end{array}$

$\begin{array}{llll}459 & -245.37 & -247.11 & 348.24\end{array}$

$\begin{array}{lllll}574 & 23.07 & -505.84 & 506.36\end{array}$

$\begin{array}{lllll}3002 & 132.87 & -44.78 & 140.21\end{array}$

$\begin{array}{llll}391 & -15.25 & -8.16 & 17.30\end{array}$ 
$\begin{array}{lllll}378 & 1.57 & 18.29 & 0.00 & 0.00\end{array}$

$\begin{array}{lllll}379 & 1.36 & 14.06 & 16.40 & 4.78\end{array}$

$\begin{array}{lllll}367 & -47.38 & -143.17 & 150.81\end{array}$

$\begin{array}{lllll}391 & 11.65 & -26.79 & 29.21\end{array}$

$\begin{array}{lllll}419 & 32.82 & 89.78 & 95.58\end{array}$

$\begin{array}{llll}426 & -26.21 & -66.57 & 71.55\end{array}$

$\begin{array}{cccccccccc}380 & 1.49 & 24.65 & 0.00 & 0.00 & & 374 & 30.87 & 7.57 & 31.79 \\ & & & & & 375 & -48.22 & -14.80 & 50.44 \\ & & & & & 381 & 24.60 & -15.15 & 28.89 \\ & & & & & 1900 & -183.13 & -115.22 & 216.36 \\ 381 & 1.50 & 23.80 & 24.40 & 7.11 & & & & \end{array}$

$\begin{array}{llll}380 & -23.56 & 9.09 & 25.25\end{array}$

$\begin{array}{lllll}382 & 1.38 & 15.50 & 0.00 & 0.00\end{array}$

$\begin{array}{llll}392 & -67.03 & -137.24 & 152.73\end{array}$

$\begin{array}{llll}395 & 64.14 & 15.28 & 65.94\end{array}$

$\begin{array}{llll}461 & -252.92 & 20.40 & 253.75\end{array}$

$\begin{array}{llll}572 & 23.18 & -101.94 & 104.54\end{array}$

$\begin{array}{llll}589 & 89.14 & 6.63 & 89.39\end{array}$

$\begin{array}{lllll}383 & 1.48 & 16.33 & 12.03 & 3.51\end{array}$

$\begin{array}{llll}394 & -47.81 & -52.60 & 71.08\end{array}$

$\begin{array}{llll}558 & 30.89 & 32.16 & 44.59\end{array}$

$\begin{array}{lllll}384 & 1.32 & 10.65 & 43.32 & 12.64\end{array}$

$\begin{array}{llll}373 & 217.86 & 139.71 & 258.81\end{array}$

$\begin{array}{llll}405 & -116.36 & 5.99 & 116.52\end{array}$

$\begin{array}{llll}570 & -151.36 & -12.34 & 151.87\end{array}$

$\begin{array}{lllll}385 & 1.36 & 14.05 & 24.14 & 7.04\end{array}$

$\begin{array}{llll}423 & 62.26 & 22.80 & 66.31\end{array}$

$\begin{array}{llll}589 & -92.72 & -14.27 & 93.81\end{array}$

$\begin{array}{lllll}386 & 1.43 & 17.53 & 4.88 & 1.42\end{array}$

$\begin{array}{llll}587 & -15.43 & -10.04 & 18.41\end{array}$

$\begin{array}{llll}3003 & -2.42 & -5.26 & 5.78\end{array}$

$\begin{array}{lllll}387 & 1.62 & 14.32 & 35.92 & 10.48\end{array}$

$\begin{array}{llll}393 & -44.40 & 8.87 & 45.28\end{array}$

$\begin{array}{llll}407 & -62.50 & 1.72 & 62.53\end{array}$

$\begin{array}{llll}420 & 45.39 & -11.59 & 46.84\end{array}$

$\begin{array}{lllll}388 & 1.48 & 16.52 & 1.51 & 0.44\end{array}$

$\begin{array}{llll}422 & -41.24 & -32.11 & 52.26\end{array}$

$\begin{array}{llll}1359 & 36.98 & 22.20 & 43.13\end{array}$

$\begin{array}{lllll}389 & 1.62 & 12.00 & 27.51 & 8.02\end{array}$

$\begin{array}{llll}613 & -31.41 & 42.84 & 53.12\end{array}$

$\begin{array}{lllll}390 & 1.62 & 14.28 & 30.62 & 8.93\end{array}$

$\begin{array}{llll}397 & -39.75 & -10.32 & 41.06\end{array}$

$\begin{array}{llll}415 & -22.04 & -39.41 & 45.15\end{array}$

$\begin{array}{lllll}391 & 1.57 & 18.23 & 10.01 & 2.92\end{array}$

$\begin{array}{llll}377 & 15.44 & 4.91 & 16.20\end{array}$

$\begin{array}{llll}378 & -11.62 & 26.40 & 28.84\end{array}$

$\begin{array}{lllll}392 & 1.38 & 15.51 & 14.22 & 4.15\end{array}$ 


\begin{tabular}{|c|c|c|c|c|c|c|c|c|}
\hline \multirow{3}{*}{393} & & & & & 382 & 67.05 & 137.28 & 152.78 \\
\hline & 1.62 & 14.66 & 26.84 & 7.83 & 387 & 44.36 & -10.95 & 45.69 \\
\hline & & & & & 430 & -88.22 & -38.64 & 96.31 \\
\hline \multirow[t]{3}{*}{394} & 1.49 & 16.50 & 28.94 & 8.44 & & & & \\
\hline & & & & & 383 & 47.92 & 51.77 & 70.54 \\
\hline & & & & & 401 & -79.17 & -42.82 & 90.01 \\
\hline \multirow[t]{3}{*}{395} & 1.37 & 14.85 & 2.61 & 0.76 & & & & \\
\hline & & & & & 382 & -63.97 & -15.96 & 65.93 \\
\hline & & & & & 416 & 57.86 & 8.87 & 58.54 \\
\hline \multirow[t]{3}{*}{396} & 1.51 & 18.97 & 5.97 & 1.74 & & & & \\
\hline & & & & & 419 & 83.64 & 79.10 & 115.12 \\
\hline & & & & & 425 & -95.21 & -87.63 & 129.40 \\
\hline \multirow[t]{3}{*}{397} & 1.62 & 14.35 & 35.50 & 10.35 & & & & \\
\hline & & & & & 390 & 39.77 & 9.69 & 40.93 \\
\hline & & & & & 430 & -71.82 & -10.17 & 72.53 \\
\hline \multirow[t]{3}{*}{398} & 1.52 & 22.03 & 10.10 & 2.94 & & & & \\
\hline & & & & & 468 & 139.03 & -236.39 & 274.24 \\
\hline & & & & & 3206 & -161.71 & 157.42 & 225.67 \\
\hline \multirow[t]{3}{*}{399} & 1.40 & 14.87 & 26.08 & 7.61 & & & & \\
\hline & & & & & 428 & -21.76 & -101.62 & 103.93 \\
\hline & & & & & 572 & -22.01 & 89.33 & 92.00 \\
\hline \multirow[t]{3}{*}{400} & 1.22 & 11.62 & 3.37 & 0.98 & & & & \\
\hline & & & & & 409 & 210.47 & 172.80 & 272.31 \\
\hline & & & & & 3006 & -224.37 & -187.00 & 292.08 \\
\hline \multirow[t]{3}{*}{401} & 1.49 & 16.73 & 6.73 & 1.96 & & & & \\
\hline & & & & & 394 & 79.24 & 42.46 & 89.90 \\
\hline & & & & & 421 & -93.70 & -61.87 & 112.29 \\
\hline \multirow[t]{2}{*}{402} & 1.38 & 20.73 & 2.86 & 0.83 & & & & \\
\hline & & & & & 411 & 133.10 & 67.68 & 149.32 \\
\hline \multirow[t]{2}{*}{403} & 1.59 & 19.90 & 21.87 & 6.38 & 3007 & -141.27 & -65.69 & 155.80 \\
\hline & & & & & 484 & -29.57 & -35.37 & 46.11 \\
\hline \multirow[t]{2}{*}{404} & 1.43 & 15.19 & 8.16 & 2.38 & & & & \\
\hline & & & & & 410 & -14.97 & -19.71 & 24.75 \\
\hline \multirow[t]{3}{*}{405} & 1.34 & 15.71 & 6.22 & 1.82 & & & & \\
\hline & & & & & 384 & 118.60 & -2.91 & 118.64 \\
\hline & & & & & 427 & -124.94 & 7.95 & 125.19 \\
\hline
\end{tabular}

$\begin{array}{lllll}406 & 1.42 & 21.09 & 19.52 & 5.69\end{array}$

$\begin{array}{lllll}407 & 1.62 & 14.45 & 29.86 & 8.71\end{array}$

$\begin{array}{lllll}408 & 1.62 & 19.04 & 17.67 & 5.15\end{array}$

$\begin{array}{lllll}409 & 1.17 & 9.71 & 6.90 & 2.01\end{array}$ $\begin{array}{llll}375 & -210.76 & -29.10 & 212.76\end{array}$

$\begin{array}{llll}3011 & 190.88 & 47.36 & 196.67\end{array}$

$$
\begin{array}{rrrr}
387 & 62.54 & -2.33 & 62.58 \\
430 & -90.46 & -3.20 & 90.52
\end{array}
$$

$\begin{array}{llll}426 & 44.31 & 58.20 & 73.15\end{array}$

$\begin{array}{llll}579 & -69.53 & 67.57 & 96.95\end{array}$

$\begin{array}{llll}372 & 112.49 & 117.70 & 162.81\end{array}$ 


\begin{tabular}{|c|c|c|c|c|c|c|c|c|}
\hline \multirow{4}{*}{410} & \multirow[b]{3}{*}{1.43} & \multirow[b]{3}{*}{15.23} & \multirow[b]{3}{*}{23.13} & \multirow[b]{3}{*}{6.75} & \multicolumn{3}{|c|}{$400-206.80-159.88$} & \multirow{2}{*}{$\begin{array}{l}261.39 \\
164.49\end{array}$} \\
\hline & & & & & 3001 & -133.65 & -95.89 & \\
\hline & & & & & & & & \\
\hline & & & & & 404 & 14.98 & 19.04 & 24.23 \\
\hline & & & & & 414 & -95.34 & -62.36 & 113.93 \\
\hline & & & & & 428 & 52.10 & 64.52 & 82.93 \\
\hline \multirow[t]{4}{*}{411} & 1.35 & 19.62 & 0.00 & 0.00 & & & & \\
\hline & & & & & 402 & -131.34 & -65.52 & 146.78 \\
\hline & & & & & 427 & 82.64 & 44.17 & 93.71 \\
\hline & & & & & 3009 & 41.64 & 7.53 & 42.31 \\
\hline \multirow[t]{3}{*}{412} & 1.61 & 12.33 & 34.58 & 10.08 & & & & \\
\hline & & & & & 584 & -44.26 & -8.79 & 45.12 \\
\hline & & & & & 613 & -228.15 & -133.21 & 264.19 \\
\hline \multirow[t]{3}{*}{413} & 1.35 & 13.57 & 10.52 & 3.07 & & & & \\
\hline & & & & & 374 & -21.28 & -0.11 & 21.28 \\
\hline & & & & & 423 & 10.00 & -4.24 & 10.86 \\
\hline \multirow[t]{4}{*}{414} & 1.44 & 15.51 & 0.00 & 0.00 & & & & \\
\hline & & & & & 410 & 95.46 & 62.25 & 113.97 \\
\hline & & & & & 465 & -64.75 & -34.09 & 73.18 \\
\hline & & & & & 558 & -28.83 & -43.42 & 52.12 \\
\hline \multirow[t]{3}{*}{415} & 1.62 & 14.30 & 50.30 & 14.67 & & & & \\
\hline & & & & & 390 & 22.03 & 38.87 & 44.68 \\
\hline & & & & & 430 & -65.86 & -5.32 & 66.08 \\
\hline \multirow[t]{3}{*}{416} & 1.37 & 14.54 & 14.05 & 4.10 & & & & \\
\hline & & & & & 379 & 48.30 & 13.57 & 50.17 \\
\hline & & & & & 395 & -57.82 & -9.37 & 58.58 \\
\hline \multirow[t]{3}{*}{417} & 1.61 & 12.80 & 58.46 & 17.05 & & & & \\
\hline & & & & & 478 & -122.82 & -20.45 & 124.51 \\
\hline & & & & & 584 & 38.64 & -17.63 & 42.47 \\
\hline \multirow[t]{3}{*}{418} & 1.39 & 16.63 & 26.67 & 7.78 & & & & \\
\hline & & & & & 419 & -280.39 & -251.88 & 376.92 \\
\hline & & & & & 3000 & 242.33 & 222.66 & 329.09 \\
\hline \multirow[t]{7}{*}{419} & 1.46 & 18.40 & 0.00 & 0.00 & & & & \\
\hline & & & & & 370 & 340.99 & 524.28 & 625.41 \\
\hline & & & & & 378 & -30.24 & -91.30 & 96.17 \\
\hline & & & & & 396 & -81.92 & -78.48 & 113.45 \\
\hline & & & & & 418 & 285.44 & 271.13 & 393.68 \\
\hline & & & & & 468 & -360.69 & -312.15 & 477.01 \\
\hline & & & & & 3012 & 46.55 & 20.82 & 50.99 \\
\hline \multirow[t]{2}{*}{420} & 1.62 & 14.07 & 48.54 & 14.16 & & & & \\
\hline & & & & & 387 & -45.37 & 9.68 & 46.39 \\
\hline \multirow[t]{3}{*}{421} & 1.50 & 16.87 & 16.49 & 4.81 & & & & \\
\hline & & & & & 401 & 93.79 & 61.26 & 112.02 \\
\hline & & & & & 422 & -106.12 & -47.22 & 116.15 \\
\hline \multirow[t]{5}{*}{422} & 1.50 & 17.40 & 0.00 & 0.00 & & & & \\
\hline & & & & & 361 & -141.10 & -135.51 & 195.63 \\
\hline & & & & & 388 & 41.50 & 27.22 & 49.63 \\
\hline & & & & & 421 & 106.40 & 47.16 & 116.38 \\
\hline & & & & & 469 & -46.13 & 4.97 & 46.40 \\
\hline
\end{tabular}




\begin{tabular}{|c|c|c|c|c|c|c|c|c|}
\hline & & & & & 1365 & 38.87 & 49.52 & 62.95 \\
\hline \multirow[t]{4}{*}{423} & 1.35 & 13.46 & 7.57 & 2.21 & & & & \\
\hline & & & & & 385 & -62.07 & -23.38 & 66.32 \\
\hline & & & & & 413 & -9.97 & 2.93 & 10.39 \\
\hline & & & & & 454 & 66.82 & 77.95 & 102.67 \\
\hline \multirow[t]{2}{*}{424} & 1.60 & 19.82 & 39.12 & 11.41 & & & & \\
\hline & & & & & 425 & -46.55 & 45.24 & 64.91 \\
\hline \multirow[t]{6}{*}{425} & 1.60 & 20.06 & 0.00 & 0.00 & & & & \\
\hline & & & & & 396 & 99.47 & 89.95 & 134.11 \\
\hline & & & & & 424 & 46.75 & -46.10 & 65.66 \\
\hline & & & & & 470 & -244.83 & 153.89 & 289.18 \\
\hline & & & & & 484 & 21.44 & 4.42 & 21.89 \\
\hline & & & & & 579 & 79.58 & -84.27 & 115.90 \\
\hline \multirow[t]{3}{*}{426} & 1.59 & 18.39 & 20.52 & 5.99 & & & & \\
\hline & & & & & 378 & 26.41 & 65.50 & 70.62 \\
\hline & & & & & 408 & -43.71 & -61.06 & 75.09 \\
\hline \multirow[t]{7}{*}{427} & 1.34 & 19.24 & 0.00 & 0.00 & & & & \\
\hline & & & & & 371 & 113.98 & 146.44 & 185.56 \\
\hline & & & & & 405 & 125.49 & -5.25 & 125.60 \\
\hline & & & & & 411 & -82.23 & -44.21 & 93.36 \\
\hline & & & & & 429 & 4.50 & -4.92 & 6.66 \\
\hline & & & & & 3002 & -172.39 & -113.42 & 206.35 \\
\hline & & & & & 3008 & 5.41 & -4.05 & 6.75 \\
\hline \multirow[t]{3}{*}{428} & 1.42 & 14.88 & 34.15 & 9.96 & & & & \\
\hline & & & & & 399 & 22.01 & 101.61 & 103.96 \\
\hline & & & & & 410 & -51.85 & -65.54 & 83.57 \\
\hline \multirow[t]{2}{*}{429} & 1.34 & 19.20 & 7.15 & 2.09 & & & & \\
\hline & & & & & 427 & -4.36 & 3.77 & 5.77 \\
\hline \multirow[t]{8}{*}{430} & 1.63 & 14.78 & 0.00 & 0.00 & & & & \\
\hline & & & & & 393 & 88.25 & 38.37 & 96.23 \\
\hline & & & & & 397 & 71.97 & 8.91 & 72.52 \\
\hline & & & & & 407 & 90.60 & 2.66 & 90.64 \\
\hline & & & & & 415 & 65.89 & 3.80 & 66.00 \\
\hline & & & & & 474 & -605.83 & -351.16 & 700.25 \\
\hline & & & & & 478 & 134.21 & 8.42 & 134.47 \\
\hline & & & & & 613 & 116.89 & 5.55 & 117.02 \\
\hline \multirow[t]{3}{*}{431} & 1.38 & 18.14 & 5.38 & 1.57 & & & & \\
\hline & & & & & 570 & 177.13 & 30.26 & 179.69 \\
\hline & & & & & 3011 & -191.87 & -55.14 & 199.63 \\
\hline \multirow[t]{3}{*}{432} & 1.24 & 11.54 & 35.92 & 10.48 & & & & \\
\hline & & & & & 440 & -28.85 & -140.94 & 143.87 \\
\hline & & & & & 442 & -20.79 & 117.37 & 119.20 \\
\hline \multirow[t]{3}{*}{433} & 1.29 & 11.55 & 8.33 & 2.43 & & & & \\
\hline & & & & & 451 & 42.01 & 83.98 & 93.90 \\
\hline & & & & & 453 & -62.32 & -83.28 & 104.02 \\
\hline \multirow[t]{4}{*}{434} & 1.31 & 13.88 & 0.00 & 0.00 & & & & \\
\hline & & & & & 438 & 111.61 & 44.04 & 119.98 \\
\hline & & & & & 439 & 152.85 & -9.36 & 153.13 \\
\hline & & & & & 460 & -275.47 & -24.57 & 276.57 \\
\hline
\end{tabular}




\begin{tabular}{|c|c|c|c|c|c|c|c|c|}
\hline \multirow[t]{2}{*}{435} & 1.31 & 13.27 & 17.83 & 5.20 & & & & \\
\hline & & & & & 439 & -27.99 & -13.51 & 31.08 \\
\hline \multirow[t]{3}{*}{436} & 1.31 & 13.16 & 14.72 & 4.29 & & & & \\
\hline & & & & & 439 & -147.23 & $3-20.10$ & 148.59 \\
\hline & & & & & 492 & 101.80 & -44.41 & 111.06 \\
\hline \multirow[t]{4}{*}{437} & 1.31 & 12.00 & 18.17 & 5.30 & & & & \\
\hline & & & & & 453 & 70.62 & 54.40 & 89.14 \\
\hline & & & & & 455 & -55.11 & 37.41 & 66.61 \\
\hline & & & & & 456 & -55.17 & -66.05 & 86.06 \\
\hline \multirow[t]{3}{*}{438} & 1.30 & 13.18 & 24.56 & 7.16 & & & & \\
\hline & & & & & 434 & -111.12 & $2-44.19$ & 119.58 \\
\hline & & & & & 452 & 83.18 & 50.09 & 97.10 \\
\hline \multirow[t]{4}{*}{439} & 1.31 & 13.34 & 0.00 & 0.00 & & & & \\
\hline & & & & & 434 & -152.50 & 9.96 & 152.82 \\
\hline & & & & & 435 & 28.00 & 12.77 & 30.77 \\
\hline & & & & & 436 & 147.30 & 20.20 & 148.68 \\
\hline \multirow[t]{5}{*}{440} & 1.26 & 11.55 & 19.85 & 5.79 & & & & \\
\hline & & & & & 432 & 29.12 & 141.30 & 144.27 \\
\hline & & & & & 444 & 11.09 & -134.80 & 135.26 \\
\hline & & & & & 447 & -0.16 & 77.73 & 77.73 \\
\hline & & & & & 536 & -68.84 & -68.89 & 97.39 \\
\hline \multirow[t]{4}{*}{441} & 1.08 & 15.33 & 19.98 & 5.83 & & & & \\
\hline & & & & & 122 & -16.20 & -56.59 & 58.87 \\
\hline & & & & & 229 & -32.03 & 48.51 & 58.13 \\
\hline & & & & & 256 & 30.50 & 9.22 & 31.86 \\
\hline \multirow[t]{3}{*}{442} & 1.23 & 11.71 & 55.01 & 16.05 & & & & \\
\hline & & & & & 432 & 20.96 & -117.48 & 119.33 \\
\hline & & & & & 446 & -75.03 & 102.66 & 127.16 \\
\hline \multirow[t]{3}{*}{443} & 1.21 & 11.62 & 13.96 & 4.07 & & & & \\
\hline & & & & & 312 & -33.16 & 3.81 & 33.38 \\
\hline & & & & & 445 & 17.16 & -10.76 & 20.26 \\
\hline \multirow[t]{3}{*}{444} & 1.27 & 11.45 & 24.31 & 7.09 & & & & \\
\hline & & & & & 440 & -10.96 & 134.90 & 135.34 \\
\hline & & & & & 451 & -13.62 & -123.16 & 123.91 \\
\hline \multirow[t]{2}{*}{445} & 1.21 & 11.51 & 19.85 & 5.79 & & & & \\
\hline & & & & & 443 & -17.17 & 9.24 & 19.50 \\
\hline \multirow[t]{6}{*}{446} & 1.22 & 12.43 & 0.00 & 0.00 & & & & \\
\hline & & & & & 312 & 74.84 & 16.74 & 76.69 \\
\hline & & & & & 442 & 75.42 & -102.68 & 127.40 \\
\hline & & & & & 450 & 58.06 & 79.15 & 98.16 \\
\hline & & & & & 464 & -247.09 & 64.97 & 255.49 \\
\hline & & & & & 488 & 36.36 & -90.52 & 97.55 \\
\hline \multirow[t]{3}{*}{447} & 1.24 & 11.66 & 37.69 & 10.99 & & & & \\
\hline & & & & & 440 & 0.27 & -79.16 & 79.16 \\
\hline & & & & & 488 & -40.55 & 81.44 & 90.97 \\
\hline \multirow[t]{3}{*}{448} & 1.16 & 12.35 & 11.19 & 3.26 & & & & \\
\hline & & & & & 481 & -21.12 & 73.36 & 76.34 \\
\hline & & & & & 573 & 5.94 & -83.76 & 83.97 \\
\hline 449 & 1.10 & 14.80 & 30.30 & 8.84 & & & & \\
\hline
\end{tabular}




\begin{tabular}{|c|c|c|c|c|c|c|c|c|}
\hline \multirow{3}{*}{450} & \multirow{3}{*}{1.20} & \multirow{3}{*}{11.97} & \multirow{3}{*}{45.26} & \multirow{3}{*}{13.20} & \multicolumn{2}{|c|}{$122-114.34$} & \multirow{2}{*}{$\begin{array}{r}66.85 \\
-71.02\end{array}$} & \multirow{2}{*}{$\begin{array}{l}132.45 \\
111.09\end{array}$} \\
\hline & & & & & 255 & 85.42 & & \\
\hline & & & & & & & & \\
\hline \multirow{4}{*}{451} & & & & & 446 & -57.88 & -79.77 & 98.56 \\
\hline & & & & & 573 & 9.95 & 80.50 & 81.11 \\
\hline & 1.28 & 11.44 & 34.32 & 10.01 & & & & \\
\hline & & & & & 433 & -42.65 & -95.22 & 104.34 \\
\hline \multirow{3}{*}{452} & & & & & 444 & 13.67 & 122.78 & 123.54 \\
\hline & 1.29 & 12.86 & 17.67 & 5.15 & & & & \\
\hline & & & & & 438 & -83.28 & -50.80 & 97.55 \\
\hline \multirow{3}{*}{453} & & & & & 536 & 66.07 & 60.17 & 89.37 \\
\hline & 1.31 & 11.18 & 10.32 & 4.76 & & & & \\
\hline & & & & & 433 & 62.37 & 82.72 & 103.59 \\
\hline \multirow{2}{*}{454} & 1.33 & 12.47 & 0.00 & 0.00 & 437 & -70.64 & -55.11 & 89.60 \\
\hline & & & & & 423 & -66.81 & -75.27 & 100.64 \\
\hline \multirow{3}{*}{455} & & & & & 456 & 62.08 & 44.54 & 76.41 \\
\hline & 1.31 & 12.42 & 37.85 & 11.04 & & & & \\
\hline & & & & & 437 & 55.21 & -38.54 & 67.33 \\
\hline \multirow{3}{*}{456} & & & & & 557 & -100.10 & 36.23 & 106.46 \\
\hline & 1.32 & 12.18 & 14.39 & 4.20 & & & & \\
\hline & & & & & 437 & 55.08 & 65.11 & 85.28 \\
\hline \multirow{4}{*}{457} & & & & & 454 & -62.29 & -45.94 & 77.40 \\
\hline & 1.13 & 13.01 & 12.11 & 3.53 & & & & \\
\hline & & & & & 255 & -43.26 & 87.61 & 97.71 \\
\hline & & & & & 481 & 29.65 & -77.06 & 82.57 \\
\hline \multirow[t]{2}{*}{458} & 1.46 & 26.21 & 0.00 & 0.00 & & & & \\
\hline & & & & & 375 & 214.66 & -9.88 & 214.89 \\
\hline \multirow{5}{*}{459} & & & & & 471 & -277.79 & -574.87 & 638.47 \\
\hline & 1.44 & 23.06 & 0.00 & 0.00 & & & & \\
\hline & & & & & 376 & 246.68 & 275.91 & 370.11 \\
\hline & & & & & 463 & -310.50 & -57.96 & 315.86 \\
\hline & & & & & 468 & $65.23-$ & -257.96 & 266.08 \\
\hline \multirow[t]{5}{*}{460} & 1.33 & 18.09 & 0.00 & 0.00 & & & & \\
\hline & & & & & 434 & 276.35 & 45.29 & 280.04 \\
\hline & & & & & 461 & -230.45 & -489.11 & 540.68 \\
\hline & & & & & 464 & 222.51 & 369.35 & 431.20 \\
\hline & & & & & 467 & -275.55 & 14.61 & 275.94 \\
\hline \multirow[t]{7}{*}{461} & 1.37 & 18.79 & 0.00 & 0.00 & & & & \\
\hline & & & & & 362 & $-365.82-$ & -186.01 & 410.39 \\
\hline & & & & & 363 & -74.81 & -228.45 & 240.38 \\
\hline & & & & & 382 & 253.26 & -5.84 & 253.33 \\
\hline & & & & & 460 & 231.35 & 498.94 & 549.97 \\
\hline & & & & & 465 & 75.06 & -484.70 & 490.48 \\
\hline & & & & & 2023 & -142.17 & -127.57 & 191.01 \\
\hline \multirow[t]{3}{*}{462} & 1.44 & 29.20 & 0.00 & 0.00 & & & & \\
\hline & & & & & 477 & $651.48-$ & -317.11 & 724.55 \\
\hline & 1.46 & 26.41 & 0.00 & & 3205 & -642.48 & 272.36 & 697.83 \\
\hline
\end{tabular}


$\begin{array}{lllll}464 & 1.20 & 15.79 & 0.00 & 0.00\end{array}$

$\begin{array}{lllll}465 & 1.47 & 17.65 & 0.00 & 0.00\end{array}$

$\begin{array}{lllll}466 & 1.58 & 25.23 & 7.57 & 2.21\end{array}$

$\begin{array}{lllll}467 & 1.33 & 19.59 & 0.00 & 0.00\end{array}$

$\begin{array}{lllll}468 & 1.54 & 21.50 & 0.00 & 0.00\end{array}$

$\begin{array}{lllll}469 & 1.50 & 18.10 & 0.00 & 0.00\end{array}$

$\begin{array}{lllll}470 & 1.56 & 22.66 & 0.00 & 0.00\end{array}$

$\begin{array}{lllll}471 & 1.46 & 26.23 & 0.00 & 0.00\end{array}$

$$
\begin{array}{cccc}
356 & -472.58 & 457.31 & 657.62 \\
459 & 315.46 & 42.65 & 318.33 \\
471 & 297.07 & 353.48 & 461.74 \\
475 & 64.34 & -216.84 & 226.18 \\
521 & 1.13 & 27.88 & 27.90 \\
1965 & -519.53 & -772.91 & 931.30 \\
3014 & 321.43 & -8.52 & 321.55
\end{array}
$$

$\begin{array}{llll}123 & 0.93 & 200.13 & 200.14\end{array}$ $\begin{array}{lllll}446 & 247.53 & -49.79 & 252.49\end{array}$

$\begin{array}{llll}460 & -216.87 & -355.33 & 416.28\end{array}$

$\begin{array}{llll}493 & -33.64 & 211.61 & 214.26\end{array}$

$\begin{array}{llll}414 & 89.76 & 345.88 & 509.45\end{array}$ $\begin{array}{lllll}461 & -73.44 & 495.99 & 501.40\end{array}$ $\begin{array}{llll}474 & 3.93 & -512.97 & 512.99\end{array}$

$\begin{array}{llll}473 & 586.15 & 75.29 & 590.96\end{array}$ $588-601.98-168.41625 .09$

$\begin{array}{llll}460 & 275.07 & -20.64 & 275.85\end{array}$ $\begin{array}{llll}476 & -532.22 & 209.83 & 572.09\end{array}$ $\begin{array}{llll}520 & -27.93 & 18.74 & 33.63\end{array}$

$\begin{array}{llll}576 & 20.02 & -369.72 & 370.27\end{array}$ $\begin{array}{llll}1233 & 259.08 & 150.90 & 299.82\end{array}$

$3577047.762980 .98 \quad 174.00$ $\begin{array}{lllll}358 & -101.45 & -56.90 & 116.32\end{array}$ $\begin{array}{lllll}359 & -82.53 & -189.61 & 206.79\end{array}$ $\begin{array}{lllll}360 & -82.56 & -109.82 & 137.39\end{array}$

$\begin{array}{lllll}367 & 278.03 & -305.38 & 412.99\end{array}$ $\begin{array}{llll}398 & -137.18 & 226.26 & 264.60\end{array}$ $\begin{array}{lllll}419 & 361.38 & 348.74 & 502.21\end{array}$ $\begin{array}{lllll}459 & -56.05 & 218.72 & 225.79\end{array}$ $473-329.84-116.59 \quad 349.84$ $\begin{array}{llll}474 & 260.09 & -333.58 & 423.00\end{array}$

$364-310.86-532.30 \quad 616.43$ $365-318.73-563.98 \quad 647.81$ $\begin{array}{llll}422 & 46.15 & -4.41 & 46.36\end{array}$

$\begin{array}{lllll}474 & 66.36 & -530.64 & 534.78\end{array}$ $\begin{array}{lllll}576 & -8.58 & 349.62 & 349.72\end{array}$ $\begin{array}{lllll}591 & 62.24 & -519.88 & 523.59\end{array}$ $\begin{array}{llll}1378 & 424.38 & 541.72 \quad 688.16\end{array}$ 4254654.874540 .884773 .90 $\begin{array}{llll}473 & -252.79 & -14.41 & 253.20\end{array}$

$\begin{array}{llll}458 & 278.06 & 575.33 \quad 639.00\end{array}$ 


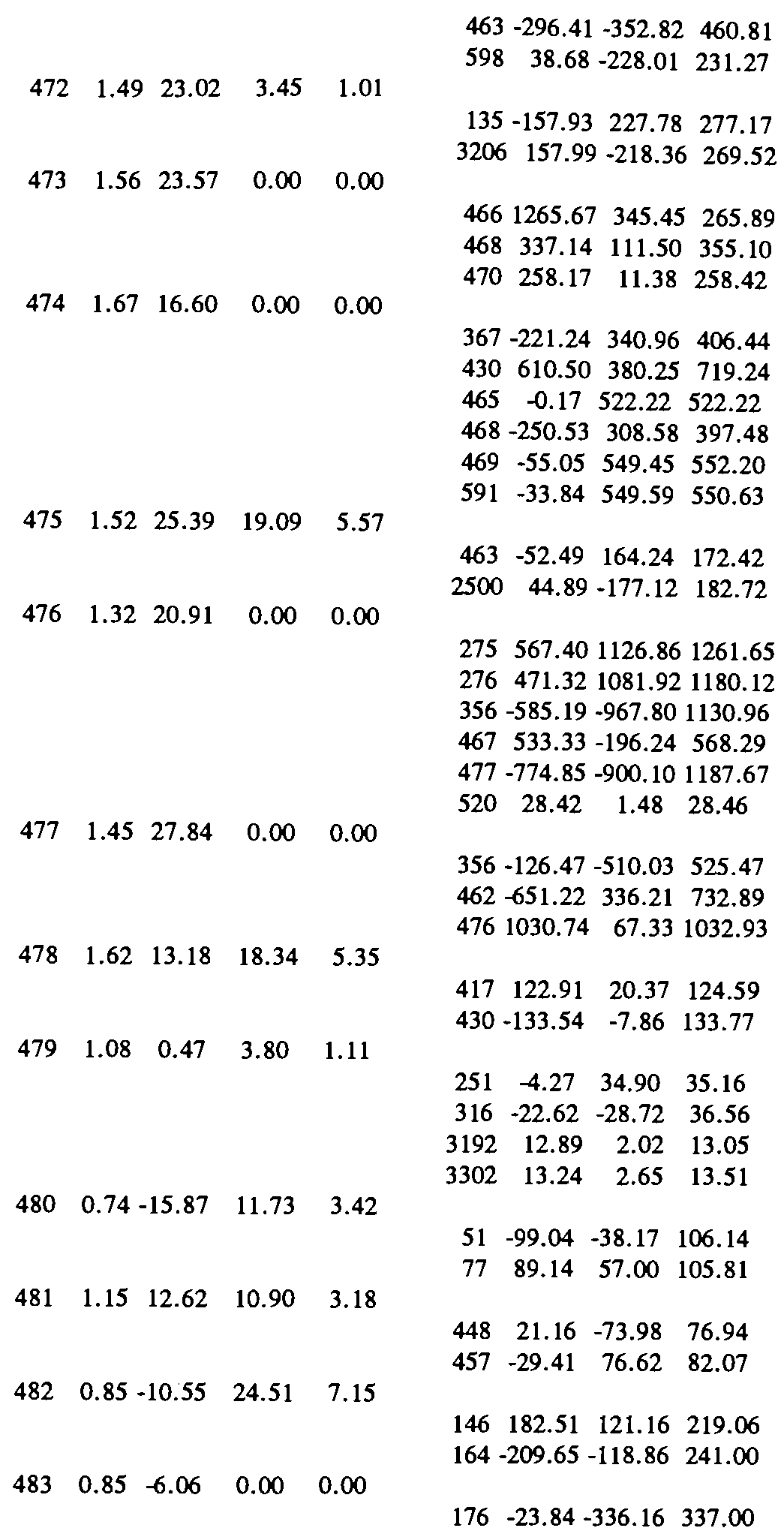




\begin{tabular}{|c|c|c|c|c|c|c|c|c|}
\hline \multirow[b]{2}{*}{484} & \multirow[b]{2}{*}{1.60} & \multirow[b]{2}{*}{19.97} & \multirow[b]{2}{*}{0.00} & \multirow[b]{2}{*}{0.00} & \multirow[t]{2}{*}{180} & 22.32 & \multirow[t]{2}{*}{337.88} & \multirow[t]{2}{*}{338.61} \\
\hline & & & & & & & & \\
\hline & & & & & 403 & 29.72 & 34.56 & 45.58 \\
\hline & & & & & 425 & -21.27 & -5.49 & 21.97 \\
\hline \multirow[t]{3}{*}{485} & 1.55 & 8.16 & 13.22 & 3.86 & & & & \\
\hline & & & & & 296 & -6.15 & -50.69 & 51.06 \\
\hline & & & & & 323 & -22.07 & 26.31 & 34.34 \\
\hline \multirow[t]{3}{*}{488} & 1.23 & 11.95 & 0.00 & 0.00 & & & & \\
\hline & & & & & 446 & -36.16 & 90.10 & 97.08 \\
\hline & & & & & 447 & 40.63 & -81.93 & 91.45 \\
\hline \multirow[t]{2}{*}{489} & 1.82 & 10.62 & 0.00 & 0.00 & & & & \\
\hline & & & & & 597 & -432.56 & 897.09 & 129.78 \\
\hline \multirow[t]{3}{*}{492} & 1.31 & 12.90 & 0.00 & 0.00 & & & & \\
\hline & & & & & 436 & -354.55 & 453.98 & 342.12 \\
\hline & & & & & 557 & 88.82 & -63.40 & 109.12 \\
\hline \multirow[t]{3}{*}{493} & 1.19 & 16.00 & 3.79 & 1.10 & & & & \\
\hline & & & & & $464^{\prime}$ & 7775.79 & 432.45 & 5298.07 \\
\hline & & & & & 542 & -32.35 & 222.39 & 224.73 \\
\hline \multirow[t]{3}{*}{495} & 0.75 & -8.53 & 12.63 & 3.68 & & & & \\
\hline & & & & & 172 & -121.78 & 36.47 & 127.12 \\
\hline & & & & & 186 & 93.14 & 12.97 & 94.03 \\
\hline \multirow[t]{3}{*}{496} & 0.61 & -8.57 & 36.32 & 10.59 & & & & \\
\hline & & & & & 104 & -786.08 & 567.87 & 167.98 \\
\hline & & & & & 502 & -169.05 & -434.13 & 465.88 \\
\hline \multirow[t]{3}{*}{497} & 0.95 & -12.43 & 24.07 & 7.02 & & & & \\
\hline & & & & & 137 & -172.36 & -313.64 & 357.88 \\
\hline & & & & & 158 & 148.61 & 309.47 & 343.30 \\
\hline \multirow[t]{3}{*}{498} & 0.99 & -9.82 & 13.74 & 4.01 & & & & \\
\hline & & & & & 137 & 122.46 & -47.93 & 131.51 \\
\hline & & & & & 174 & -136.21 & 45.08 & 143.47 \\
\hline \multirow[t]{3}{*}{499} & 1.30 & 10.46 & 0.00 & 0.00 & & & & \\
\hline & & & & & $342 ?$ & 3564.36 & $6-768.32$ & 127.43 \\
\hline & & & & & 353 & -273.81 & -13.36 & 274.14 \\
\hline \multirow[t]{3}{*}{500} & 0.80 & -6.03 & 0.00 & 0.00 & & & & \\
\hline & & & & & 175 & 15.65 & 843.98 & 187.23 \\
\hline & & & & & 178 & $6.83-$ & -308.39 & 308.46 \\
\hline 501 & 0.61 & -7.38 & 0.00 & 0.00 & & & & \\
\hline & & & & & 106 & 497.37 & 452.88 & 672.66 \\
\hline & & & & & 175 & -162.68 & -536.82 & 560.93 \\
\hline 502 & 0.64 & -7.84 & 0.00 & 0.00 & & & & \\
\hline & & & & & 175 & 1237.60 & 874.09 & 398.02 \\
\hline & & & & & 496 & 171.15 & 456.30 & 487.34 \\
\hline 504 & 1.06 & 16.16 & 0.00 & 0.00 & & & & \\
\hline & & & & & 234 & 8.95 & 32.21 & 33.43 \\
\hline & & & & & 246 & -6.94 & -28.10 & 28.95 \\
\hline 520 & 1.32 & 20.26 & 0.00 & 0.00 & & & & \\
\hline & & & & & 467 & -987.56 & -78.98 & 54.17 \\
\hline & & & & & 476 & -28.42 & -1.16 & 28.44 \\
\hline 521 & 1.46 & 26.40 & 0.00 & 0.00 & & & & \\
\hline
\end{tabular}




$$
\begin{array}{lllll}
522 & 0.98 & 7.74 & 0.00 & 0.00 \\
530 & 1.05 & 16.16 & 0.00 & 0.00
\end{array}
$$

$463-894.56 \quad 1876.987236 .14$

$535-312.482498 .782518 .24$

$\begin{array}{llll}234 & 87.27 & 59.38 & 105.56\end{array}$

$\begin{array}{llll}252 & 49.10 & 7.98 & 49.74\end{array}$

$\begin{array}{lllll}531 & 0.92 & -6.42 & 31.04 & 9.05\end{array}$

$\begin{array}{llll}259 & -107.72 & 60.12 & 123.36\end{array}$

$\begin{array}{rrrrrrrrrr}532 & 1.04 & 17.60 & 21.47 & 6.26 & & & & & \\ & & & & & 259 & 113.29 & 26.65 & 116.38 \\ & & & & & 266 & -132.79 & -26.74 & 135.46\end{array}$

$\begin{array}{lllllll}533 & 1.00 & -11.30 & 0.00 & 0.00 & 65.00 & 26.61\end{array}$

$\begin{array}{llll}190 & -2.35 & -59.89 & 59.93\end{array}$

$\begin{array}{lllll}534 & 0.96 & 4.02 & 0.00 & 0.00\end{array}$

$\begin{array}{llll}137 & 64.05 & 26.61 & 69.35\end{array}$

$\begin{array}{llll}269 & 389.66 & 87.29 & 399.32\end{array}$

$\begin{array}{llll}535 & -391.59 & -97.77 & 403.61\end{array}$

$\begin{array}{lllll}535 & 0.99 & 7.73 & 0.00 & 0.00\end{array}$

$\begin{array}{llll}268 & 278.75 & -77.72 & 289.39\end{array}$

$\begin{array}{lllll}274 & -954.87 & 119.31 & 1048.96\end{array}$

$\begin{array}{llll}351 & 38.60 & -91.46 & 99.28\end{array}$

$\begin{array}{llll}522 & -0.22 & 9.09 & 9.09\end{array}$

$\begin{array}{lllll}534 & 392.09 & 114.28 & 408.41\end{array}$

$\begin{array}{llll}599 & 316.60 & -77.34 & 325.91\end{array}$

$\begin{array}{lllll}536 & 1.28 & 12.21 & 0.00 & 0.00\end{array}$

$\begin{array}{llll}440 & 69.28 & 67.77 & 96.91\end{array}$

$\begin{array}{llll}452 & -66.25 & -61.99 & 90.72\end{array}$

$\begin{array}{lllll}537 & 0.92 & -6.38 & 0.00 & 0.00\end{array}$

$\begin{array}{llll}264 & -29.58 & 54.96 & 62.41\end{array}$

$\begin{array}{llll}531 & 24.91 & -38.39 & 45.77\end{array}$

$\begin{array}{lllll}538 & 1.00 & 3.66 & 12.05 & 3.52\end{array}$

$\begin{array}{llll}254 & 193.44 & -104.51 & 219.87\end{array}$

$\begin{array}{llll}580 & -206.67 & 100.99 & 230.03\end{array}$

$\begin{array}{lllll}539 & 0.99 & 4.95 & 14.45 & 4.21\end{array}$

$\begin{array}{llll}235 & 11.85 & 16.54 & 20.35\end{array}$

$\begin{array}{llll}245 & -25.00 & -19.18 & 31.51\end{array}$

$\begin{array}{lllll}541 & 1.05 & 2.28 & 11.03 & 3.22\end{array}$

$\begin{array}{llll}351 & -200.91 & -76.86 & 215.11\end{array}$

$\begin{array}{llll}559 & 182.72 & 72.75 & 196.68\end{array}$

$\begin{array}{lllll}542 & 1.11 & 17.33 & 9.08 & 2.65\end{array}$

$\begin{array}{llll}266 & -47.83 & 223.24 & 228.30\end{array}$

$\begin{array}{llll}493 & 37.36 & -224.48 & 227.56\end{array}$

$\begin{array}{lllll}543 & 0.79 & -20.61 & 0.00 & 0.00\end{array}$

$\begin{array}{llll}294 & -6.98 & 12.86 & 14.63\end{array}$

$3237-177.57 \quad-97.84 \quad 202.74$

$\begin{array}{lllll}551 & 0.92 & -9.64 & 14.65 & 4.28\end{array}$

$\begin{array}{llll}294 & 341.10 & 250.86 & 423.41\end{array}$

$299-352.99-251.36 \quad 433.34$ 


\begin{tabular}{|c|c|c|c|c|c|c|c|c|}
\hline \multirow[t]{3}{*}{552} & 1.50 & 9.27 & 7.58 & 2.21 & & & & \\
\hline & & & & & 348 & -72.64 & 183.44 & 197.30 \\
\hline & & & & & 350 & 44.62 & -211.28 & 215.94 \\
\hline \multirow[t]{3}{*}{553} & 1.55 & 11.92 & 10.02 & 2.92 & & & & \\
\hline & & & & & 346 & -172.07 & 301.59 & 347.23 \\
\hline & & & & & 352 & 128.46 & 5 -346.49 & 369.54 \\
\hline \multirow[t]{3}{*}{554} & 1.83 & 10.36 & 16.68 & 4.86 & & & & \\
\hline & & & & & 308 & -53.10 & 22.70 & 57.74 \\
\hline & & & & & 593 & 44.43 & -12.01 & 46.02 \\
\hline \multirow[t]{3}{*}{555} & 1.48 & 13.35 & 22.83 & 6.66 & & & & \\
\hline & & & & & 341 & 206.43 & -882.62 & 906.44 \\
\hline & & & & & 345 & -239.17 & 865.87 & 898.30 \\
\hline \multirow[t]{2}{*}{556} & 1.09 & 16.56 & 0.00 & 0.00 & & & & \\
\hline & & & & & 240 & 1.45 & 32.37 & 32.41 \\
\hline \multirow[t]{3}{*}{557} & 1.31 & 12.74 & 6.56 & 1.91 & & & & \\
\hline & & & & & 455 & 100.18 & -36.43 & 106.60 \\
\hline & & & & & 492 & -88.77 & 63.42 & 109.09 \\
\hline \multirow[t]{3}{*}{558} & 1.47 & 16.02 & 3.28 & 0.96 & & & & \\
\hline & & & & & 383 & -30.77 & -34.68 & 46.37 \\
\hline & & & & & 414 & 28.73 & 39.16 & 48.57 \\
\hline \multirow[t]{3}{*}{559} & 1.01 & -1.45 & 0.00 & 0.00 & & & & \\
\hline & & & & & 299 & 184.59 & 46.53 & 190.37 \\
\hline & & & & & 541 & -187.64 & -77.51 & 203.02 \\
\hline \multirow[t]{3}{*}{560} & 0.97 & -6.38 & 10.73 & 3.13 & & & & \\
\hline & & & & & 223 & -88.18 & -19.96 & 90.41 \\
\hline & & & & & 228 & 78.15 & 19.09 & 80.44 \\
\hline \multirow[t]{4}{*}{570} & 1.32 & 11.64 & 0.00 & 0.00 & & & & \\
\hline & & & & & 384 & 151.95 & 14.06 & 152.60 \\
\hline & & & & & 431 & -172.37 & -13.82 & 172.92 \\
\hline & & & & & 3013 & 20.34 & 1.66 & 20.41 \\
\hline \multirow[t]{2}{*}{571} & 1.56 & 17.43 & 14.64 & 4.27 & & & & \\
\hline & & & & & 591 & -32.01 & -75.33 & 81.85 \\
\hline \multirow[t]{3}{*}{572} & 1.40 & 15.01 & 10.60 & 3.09 & & & & \\
\hline & & & & & 382 & -22.72 & 101.52 & 104.03 \\
\hline & & & & & 399 & 22.16 & -89.76 & 92.45 \\
\hline \multirow[t]{3}{*}{573} & 1.19 & 11.98 & 19.09 & 5.57 & & & & \\
\hline & & & & & 448 & -6.36 & 81.68 & 81.93 \\
\hline & & & & & 450 & -9.98 & -81.15 & 81.76 \\
\hline \multirow[t]{3}{*}{574} & 1.39 & 19.96 & 0.00 & 0.00 & & & & \\
\hline & & & & & 376 & -22.66 & 518.11 & 518.61 \\
\hline & & & & & 2354 & $\begin{array}{r}43.97 \\
\end{array}$ & 46.13 & 63.73 \\
\hline \multirow[t]{3}{*}{576} & 1.48 & 18.24 & 9.42 & 2.75 & & & & \\
\hline & & & & & 467 & -17.54 & 371.48 & 371.89 \\
\hline & & & & & 469 & 10.04 & -349.95 & 350.09 \\
\hline \multirow[t]{3}{*}{579} & 1.61 & 19.35 & 15.14 & 4.42 & & & & \\
\hline & & & & & 408 & 69.65 & -67.81 & 97.21 \\
\hline & & & & & 425 & -79.19 & 84.40 & 115.74 \\
\hline \multirow[t]{2}{*}{580} & 1.00 & 4.26 & 0.00 & 0.00 & & & & \\
\hline & & & & & 250 & -205.13 & 99.52 & 227.99 \\
\hline
\end{tabular}


$\begin{array}{llll}538 & 207.01 & -98.83 & 229.39\end{array}$

$$
\begin{array}{rlllllllll}
583 & 1.36 & 10.76 & 0.00 & 0.00 & & & & \\
& & & & & 285 & 7.08 & -0.69 & 7.12 \\
& & & & & 287 & -9.26 & -1.35 & 9.36
\end{array}
$$

$\begin{array}{lllll}584 & 1.61 & 12.70 & 6.06 & 1.77\end{array}$

$\begin{array}{llll}412 & 44.06 & 6.62 & 44.55\end{array}$

$\begin{array}{llll}417 & -38.70 & 17.05 & 42.29\end{array}$

$\begin{array}{lllll}587 & 1.43 & 17.62 & 4.21 & 1.23\end{array}$

$\begin{array}{llll}386 & 15.41 & 8.74 & 17.72\end{array}$

$\begin{array}{llll}3010 & -44.90 & -38.77 & 59.32\end{array}$

$\begin{array}{lllll}588 & 1.59 & 26.17 & 78.47 & 22.89\end{array}$

$\begin{array}{llll}466 & 603.94 & 174.49 & 628.64\end{array}$ $\begin{array}{llll}1960 & -681.69 & -208.45 & 712.85\end{array}$

$\begin{array}{lllll}589 & 1.37 & 14.79 & 0.00 & 0.00\end{array}$

$\begin{array}{llll}382 & -88.91 & -6.64 & 89.16\end{array}$

$\begin{array}{llll}385 & 92.95 & 14.53 & 94.08\end{array}$

$\begin{array}{lllll}591 & 1.57 & 17.44 & 0.00 & 0.00\end{array}$

$\begin{array}{lllll}469 & -57.15 & 530.87 & 533.94\end{array}$

$\begin{array}{lllll}474 & 38.31 & -539.26 & 540.62\end{array}$

$\begin{array}{llll}571 & 31.97 & 73.16 & 79.84\end{array}$

$\begin{array}{lllll}592 & 1.55 & 8.14 & 0.00 & 0.00\end{array}$

$\begin{array}{llll}296 & -44.95 & -87.80 & 98.64\end{array}$

$\begin{array}{llll}337 & -2.99 & 26.24 & 26.41\end{array}$

$\begin{array}{lllll}593 & 1.83 & 10.28 & 0.00 & 0.00\end{array}$

$\begin{array}{llll}327 & 17.09 & -53.23 & 55.91\end{array}$

$\begin{array}{llll}554 & -44.77 & 10.79 & 46.05\end{array}$

$\begin{array}{lllll}594 & 1.82 & 10.61 & 0.00 & 0.00\end{array}$

$\begin{array}{llll}293 & -170.50 & -419.92 & 453.22\end{array}$

$\begin{array}{llll}325 & 53.27 & 229.47 & 235.57\end{array}$

$\begin{array}{lllll}595 & 1.11 & 0.72 & 0.00 & 0.00\end{array}$

$\begin{array}{llll}309 & -194.39 & -63.85 & 204.61\end{array}$

$\begin{array}{llll}340 & 196.24 & 66.92 & 207.34\end{array}$

$\begin{array}{lllll}596 & 0.98 & -4.63 & 29.01 & 8.46\end{array}$

$\begin{array}{llll}227 & -156.43 & -28.28 & 158.97\end{array}$

$\begin{array}{llll}237 & 131.40 & 23.22 & 133.44\end{array}$

$\begin{array}{lllll}597 & 1.82 & 10.60 & 0.00 & 0.00\end{array}$

$\begin{array}{llll}330 & -51.41 & -108.66 & 120.21\end{array}$

$\begin{array}{llll}338 & 49.01 & 107.83 & 118.45\end{array}$

$\begin{array}{llll}489 & -40.13 & -61.37 & 73.32\end{array}$

$\begin{array}{lllll}598 & 1.46 & 26.22 & 51.73 & 15.09\end{array}$

$\begin{array}{llll}471 & -38.57 & 227.71 & 230.95\end{array}$

$\begin{array}{lllll}599 & 0.99 & 5.99 & 0.00 & 0.00\end{array}$

$\begin{array}{llll}268 & 314.77 & -78.05 & 324.30\end{array}$

$\begin{array}{llll}535 & -317.11 & 82.45 & 327.65\end{array}$

$\begin{array}{lllllll}600 & 1.00 & 5.62 & 0.00 & 0.0 & 10.00 & 39.74\end{array}$

$\begin{array}{llll}250 & 10.00 & 39.72 & 40.96\end{array}$

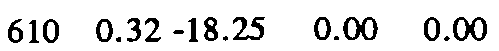

$106-202.55-266.91 \quad 335.06$

$\begin{array}{llll}113 & 169.26 & 256.25 & 307.10\end{array}$ 


\begin{tabular}{|c|c|c|c|c|c|c|c|c|}
\hline 611 & 0.11 & -58.17 & 0.00 & 0.00 & & & & \\
\hline & & & & & 24 & -57.14 & -68.01 & 88.82 \\
\hline & & & & & 42 & 101.59 & 240.04 & 260.65 \\
\hline & & & & & 63 & 3.66 & -27.30 & 27.54 \\
\hline 613 & 1.61 & 12.36 & 0.00 & 0.00 & & & & \\
\hline & & & & & 389 & 31.27 & -44.77 & 54.61 \\
\hline & & & & & 412 & 228.41 & 133.48 & 264.55 \\
\hline & & & & & 430 & -116.33 & -5.86 & 116.48 \\
\hline 701 & 1.16 & 22.53 & 0.00 & 0.00 & & & & \\
\hline & & & & & 801 & 1.37 & 25.10 & 25.14 \\
\hline 702 & 1.16 & 22.53 & 0.00 & 0.00 & & & & \\
\hline & & & & & 801 & 1.37 & 25.10 & 25.14 \\
\hline 703 & 1.19 & 26.07 & 0.00 & 0.00 & & & & \\
\hline & & & & & 758 & 93.37 & 89.38 & 129.25 \\
\hline 704 & 1.08 & 26.93 & 0.00 & 0.00 & & & & \\
\hline & & & & & 758 & 93.03 & 9.68 & 93.54 \\
\hline 705 & 1.24 & 23.74 & 0.00 & 0.00 & & & & \\
\hline & & & & & 754 & 42.26 & 137.50 & 143.85 \\
\hline 706 & 1.14 & 24.41 & 0.00 & 0.00 & & & & \\
\hline & & & & & 754 & 43.70 & 50.52 & 66.80 \\
\hline 707 & 1.24 & 22.14 & 0.00 & 0.00 & & & & \\
\hline & & & & & 768 & 29.19 & 140.59 & 143.59 \\
\hline 708 & 1.12 & 21.30 & 0.00 & 0.00 & & & & \\
\hline & & & & & 754 & 1.28 & 34.67 & 34.69 \\
\hline 709 & 1.11 & 20.62 & 0.00 & 0.00 & & & & \\
\hline & & & & & 768 & 1.27 & 34.50 & 34.53 \\
\hline 710 & 1.14 & 27.58 & 0.00 & 0.00 & & & & \\
\hline & & & & & 798 & 40.30 & 7.62 & 41.01 \\
\hline 712 & 1.22 & 23.50 & 0.00 & 0.00 & & & & \\
\hline & & & & & 755 & 113.17 & 219.86 & 247.27 \\
\hline 713 & 1.13 & 24.84 & 0.00 & 0.00 & & & & \\
\hline & & & & & 756 & 124.06 & 118.75 & 171.74 \\
\hline 716 & 1.11 & 31.95 & 0.00 & 0.00 & & & & \\
\hline & & & & & 800 & 511.34 & 82.56 & 517.96 \\
\hline 717 & 1.08 & 32.11 & 0.00 & 0.00 & & & & \\
\hline & & & & & 800 & 510.30 & -3.19 & 510.31 \\
\hline 718 & 1.18 & 25.47 & 0.00 & 0.00 & & & & \\
\hline & & & & & 801 & 215.99 & 170.10 & 274.92 \\
\hline 727 & 1.01 & 17.07 & 41.00 & 11.96 & & & & \\
\hline & & & & & 728 & -7.29 & -2.00 & 7.56 \\
\hline & & & & & 736 & -33.34 & -11.96 & 35.43 \\
\hline 728 & 1.01 & 17.23 & 61.36 & 17.90 & & & & \\
\hline & & & & & 727 & 7.28 & 1.85 & 7.52 \\
\hline & & & & & 733 & -68.66 & -19.68 & 71.43 \\
\hline 729 & 1.01 & 17.33 & 46.97 & 13.70 & & & & \\
\hline & & & & & 731 & -44.49 & -17.53 & 47.82 \\
\hline & & & & & 734 & -1.89 & 0.94 & 2.11 \\
\hline 730 & 1.05 & 14.78 & 14.85 & 4.33 & & & & \\
\hline & & & & & 908 & 4.90 & -10.80 & 11.86 \\
\hline
\end{tabular}




\begin{tabular}{|c|c|c|c|c|c|c|c|c|}
\hline & & & & & 1457 & 8.25 & -9.76 & 12.78 \\
\hline & & & & & 3044 & -28.51 & 14.67 & 32.06 \\
\hline \multirow[t]{4}{*}{731} & 1.02 & 18.38 & 0.00 & 0.00 & & & & \\
\hline & & & & & 729 & 44.68 & 18.40 & 48.32 \\
\hline & & & & & 742 & 11.56 & 8.89 & 14.58 \\
\hline & & & & & 764 & -57.10 & -51.03 & 76.58 \\
\hline \multirow[t]{3}{*}{732} & 1.03 & 18.99 & 36.70 & 10.70 & & & & \\
\hline & & & & & 735 & -2.92 & -0.30 & 2.93 \\
\hline & & & & & 739 & -33.38 & -11.95 & 35.45 \\
\hline \multirow[t]{5}{*}{733} & 1.03 & 19.90 & 53.04 & 15.47 & & & & \\
\hline & & & & & 728 & 69.41 & 23.15 & 73.17 \\
\hline & & & & & 735 & 41.66 & 10.75 & 43.02 \\
\hline & & & & & 765 & -58.56 & -49.62 & 76.75 \\
\hline & & & & & 803 & -111.63 & -154.73 & 190.80 \\
\hline \multirow[t]{3}{*}{734} & 1.01 & 17.35 & 7.94 & 2.32 & & & & \\
\hline & & & & & 729 & 1.88 & -1.02 & 2.14 \\
\hline & & & & & 736 & -9.95 & -0.76 & 9.98 \\
\hline \multirow[t]{3}{*}{735} & 1.03 & 19.05 & 38.29 & 11.17 & & & & \\
\hline & & & & & 732 & 2.93 & 0.15 & 2.93 \\
\hline & & & & & 733 & -41.50 & -10.24 & 42.75 \\
\hline \multirow[t]{6}{*}{736} & 1.01 & 17.61 & 39.32 & 11.47 & & & & \\
\hline & & & & & 727 & 33.41 & 12.21 & 35.57 \\
\hline & & & & & 734 & 9.95 & 0.62 & 9.97 \\
\hline & & & & & 744 & 43.89 & 14.40 & 46.20 \\
\hline & & & & & 746 & 40.50 & 13.19 & 42.59 \\
\hline & & & & & 805 & -174.02 & -179.98 & 250.36 \\
\hline \multirow[t]{3}{*}{737} & 1.00 & 17.09 & 44.83 & 13.07 & & & & \\
\hline & & & & & 741 & -69.91 & -18.87 & 72.41 \\
\hline & & & & & 745 & 25.47 & 3.71 & 25.74 \\
\hline \multirow[t]{3}{*}{738} & 1.04 & 16.83 & 0.00 & 0.00 & & & & \\
\hline & & & & & 740 & -31.45 & 13.24 & 34.12 \\
\hline & & & & & 3044 & 31.39 & -13.81 & 34.30 \\
\hline \multirow[t]{4}{*}{739} & 1.04 & 19.97 & 15.50 & 4.52 & & & & \\
\hline & & & & & 732 & 33.51 & 12.44 & 35.74 \\
\hline & & & & & 743 & -0.81 & 3.03 & 3.14 \\
\hline & & & & & 810 & -51.22 & -81.54 & 96.29 \\
\hline \multirow[t]{3}{*}{740} & 1.04 & 18.54 & 1.96 & 0.57 & & & & \\
\hline & & & & & 738 & 31.62 & -12.50 & 34.01 \\
\hline & & & & & 743 & -33.50 & 11.72 & 35.49 \\
\hline \multirow[t]{4}{*}{741} & 1.02 & 18.43 & 45.48 & 13.27 & & & & \\
\hline & & & & & 737 & 70.26 & 20.59 & 73.22 \\
\hline & & & & & 742 & 13.15 & -2.71 & 13.43 \\
\hline & & & & & 808 & -140.32 & -252.89 & 289.21 \\
\hline \multirow[t]{3}{*}{742} & 1.02 & 18.06 & 24.47 & 7.14 & & & & \\
\hline & & & & & 731 & -11.54 & -8.95 & 14.60 \\
\hline & & & & & 741 & -13.15 & 2.62 & 13.41 \\
\hline \multirow[t]{2}{*}{743} & 1.03 & 20.05 & 0.00 & 0.00 & & & & \\
\hline & & & & & $\begin{array}{l}739 \\
740\end{array}$ & $\begin{array}{r}0.84 \\
33.69\end{array}$ & $\begin{array}{l}-3.58 \\
-11.20\end{array}$ & $\begin{array}{l}3.68 \\
35.51\end{array}$ \\
\hline
\end{tabular}


$\begin{array}{lllll}744 & 1.00 & 16.85 & 34.74 & 10.13\end{array}$

$\begin{array}{llll}783 & -35.71 & -18.19 & 40.08\end{array}$

$\begin{array}{llll}736 & -43.76 & -13.87 & 45.91\end{array}$

$\begin{array}{llll}745 & 9.01 & 3.60 & 9.70\end{array}$

$\begin{array}{lllll}745 & 1.00 & 16.64 & 47.53 & 13.86\end{array}$

$\begin{array}{llll}737 & -25.45 & -3.64 & 25.71\end{array}$

$\begin{array}{llll}744 & -9.03 & -3.76 & 9.78\end{array}$

$\begin{array}{lllll}746 & 1.01 & 16.93 & 26.99 & 7.87\end{array}$

$\begin{array}{llll}746 & -13.17 & -6.17 & 14.55\end{array}$

$\begin{array}{lllll}754 & 1.06 & 21.29 & 0.00 & 0.00\end{array}$

$\begin{array}{llll}736 & -40.40 & -12.78 & 42.37\end{array}$

$\begin{array}{llll}745 & 13.17 & 6.03 & 14.49\end{array}$

$\begin{array}{lllll}705 & -41.25 & -116.27 & 123.37\end{array}$

$\begin{array}{llll}706 & -43.45 & -45.02 & 62.57\end{array}$

$\begin{array}{llll}708 & -1.22 & -33.01 & 33.04\end{array}$

$\begin{array}{lllll}765 & 46.61 & 36.41 & 59.14\end{array}$

$\begin{array}{llll}774 & 33.53 & 20.17 & 39.13\end{array}$

$\begin{array}{lllll}755 & 1.09 & 20.49 & 0.00 & 0.00\end{array}$

$712-111.63-191.44221 .61$

$\begin{array}{lllll}788 & 103.30 & 67.25 & 123.27\end{array}$

$\begin{array}{lllll}756 & 1.06 & 20.95 & 0.00 & 0.00\end{array}$

$713-123.20-102.86 \quad 160.50$

$\begin{array}{llll}792 & 117.50 & -18.52 & 118.95\end{array}$

$\begin{array}{lllll}757 & 1.07 & 19.89 & 28.20 & 8.23\end{array}$

$\begin{array}{llll}758 & -182.42 & -43.88 & 187.62\end{array}$

$\begin{array}{llll}761 & 68.16 & -0.90 & 68.17\end{array}$

$\begin{array}{lllll}785 & 58.32 & -8.95 & 59.00\end{array}$

$\begin{array}{llll}786 & 31.90 & 4.87 & 32.27\end{array}$

$\begin{array}{lllll}758 & 1.07 & 20.05 & 0.00 & 0.00\end{array}$

$\begin{array}{llll}703 & -92.44 & -71.51 & 116.87\end{array}$

$\begin{array}{llll}704 & -92.53 & 1.53 & 92.54\end{array}$

$\begin{array}{llll}757 & 182.72 & 44.43 & 188.04\end{array}$

$\begin{array}{lllll}759 & 1.05 & 20.47 & 53.33 & 15.55\end{array}$

$\begin{array}{llll}765 & -55.90 & 9.90 & 56.77\end{array}$

$\begin{array}{llll}779 & 3.73 & -33.28 & 33.49\end{array}$

$\begin{array}{lllll}760 & 1.06 & 19.05 & 25.59 & 7.46\end{array}$

$\begin{array}{llll}771 & -24.91 & -9.77 & 26.76\end{array}$

$\begin{array}{lllll}761 & 1.07 & 19.69 & 30.72 & 8.96\end{array}$

$\begin{array}{llll}757 & -68.91 & -18.15 & 71.26\end{array}$

$\begin{array}{llll}771 & 53.27 & 23.41 & 58.18\end{array}$

$\begin{array}{llll}788 & -12.82 & -25.92 & 28.91\end{array}$

$\begin{array}{lllll}762 & 1.06 & 19.57 & 37.64 & 10.98\end{array}$

$\begin{array}{llll}769 & -4.60 & 11.51 & 12.39\end{array}$

$\begin{array}{llll}775 & -29.43 & -51.36 & 59.20\end{array}$

$\begin{array}{lllll}763 & 1.04 & 20.61 & 27.36 & 7.98\end{array}$

$\begin{array}{llll}770 & 4.23 & 10.51 & 11.33\end{array}$

$\begin{array}{llll}774 & -30.86 & -21.67 & 37.71\end{array}$

$\begin{array}{lllll}764 & 1.05 & 20.04 & 50.15 & 14.63\end{array}$

$\begin{array}{llll}731 & 57.27 & 54.24 & 78.88\end{array}$ 


\begin{tabular}{|c|c|c|c|c|c|c|c|c|}
\hline & & & & & 769 & 45.53 & -23.73 & 51.34 \\
\hline & & & & & 787 & -32.86 & -8.07 & 33.84 \\
\hline & & & & & 792 & -116.29 & -27.83 & 119.58 \\
\hline \multirow[t]{6}{*}{765} & 1.05 & 20.79 & 0.00 & 0.00 & & & & \\
\hline & & & & & 733 & 58.67 & 51.28 & 77.92 \\
\hline & & & & & 754 & -46.32 & -36.26 & 58.82 \\
\hline & & & & & 759 & 55.94 & -10.62 & 56.94 \\
\hline & & & & & 778 & 16.04 & -38.16 & 41.40 \\
\hline & & & & & 803 & -92.27 & -146.31 & 172.97 \\
\hline \multirow[t]{2}{*}{766} & 1.00 & 21.55 & 35.11 & 10.24 & & & & \\
\hline & & & & & 780 & -35.00 & -12.16 & 37.06 \\
\hline \multirow[t]{3}{*}{767} & 1.00 & 21.19 & 32.59 & 9.51 & & & & \\
\hline & & & & & 772 & 41.18 & 29.12 & 50.44 \\
\hline & & & & & 780 & -72.16 & -42.00 & 83.49 \\
\hline \multirow[t]{5}{*}{768} & 1.06 & 20.61 & 0.00 & 0.00 & & & & \\
\hline & & & & & 707 & -28.07 & -119.11 & 122.37 \\
\hline & & & & & 709 & -1.21 & -32.86 & 32.88 \\
\hline & & & & & 778 & 22.86 & 46.24 & 51.58 \\
\hline & & & & & 783 & 2.94 & 19.98 & 20.20 \\
\hline \multirow[t]{3}{*}{769} & 1.06 & 19.58 & 39.41 & 11.49 & & & & \\
\hline & & & & & 762 & 3.61 & -26.18 & 26.43 \\
\hline & & & & & 764 & -45.51 & 23.58 & 51.26 \\
\hline \multirow[t]{3}{*}{770} & 1.04 & 20.58 & 28.11 & 8.20 & & & & \\
\hline & & & & & 763 & -4.12 & -11.11 & 11.85 \\
\hline & & & & & 783 & -23.53 & 1.99 & 23.62 \\
\hline \multirow[t]{4}{*}{771} & 1.06 & 19.26 & 45.01 & 13.13 & & & & \\
\hline & & & & & 760 & 25.23 & 9.01 & 26.79 \\
\hline & & & & & 761 & -52.93 & -23.64 & 57.97 \\
\hline & & & & & 789 & -16.80 & 2.01 & 16.92 \\
\hline \multirow[t]{5}{*}{772} & 0.99 & 20.66 & 22.79 & 6.65 & & & & \\
\hline & & & & & 767 & -40.88 & -29.42 & 50.37 \\
\hline & & & & & 777 & -2.99 & -16.42 & 16.69 \\
\hline & & & & & 3058 & 1.40 & 11.79 & 11.87 \\
\hline & & & & & 3059 & 26.91 & -16.88 & 31.77 \\
\hline \multirow[t]{4}{*}{773} & 1.07 & 19.97 & 47.35 & 13.81 & & & & \\
\hline & & & & & 776 & 20.90 & -3.85 & 21.26 \\
\hline & & & & & 788 & 11.29 & -4.74 & 12.24 \\
\hline & & & & & 806 & -81.32 & -88.91 & 120.49 \\
\hline \multirow[t]{3}{*}{774} & 1.05 & 20.86 & 2.15 & 0.63 & & & & \\
\hline & & & & & 754 & -33.10 & -20.72 & 39.05 \\
\hline & & & & & 763 & 31.14 & 21.38 & 37.77 \\
\hline \multirow[t]{5}{*}{775} & 1.06 & 19.59 & 41.00 & 11.96 & & & & \\
\hline & & & & & 762 & 30.71 & 35.16 & 46.68 \\
\hline & & & & & 788 & -17.04 & -56.92 & 59.42 \\
\hline & & & & & 789 & 8.18 & -44.49 & 45.23 \\
\hline & & & & & 807 & -68.49 & -191.23 & 203.12 \\
\hline \multirow[t]{3}{*}{776} & 1.07 & 19.72 & 50.71 & 14.79 & & & & \\
\hline & & & & & 773 & -20.62 & 2.98 & 20.84 \\
\hline & & & & & 785 & -26.15 & -27.37 & 37.86 \\
\hline
\end{tabular}


$\begin{array}{lllll}777 & 0.99 & 20.66 & 43.52 & 12.69\end{array}$

$\begin{array}{lllll}772 & 3.21 & 15.84 & 16.16\end{array}$

$\begin{array}{llll}3062 & -46.95 & -28.28 & 54.81\end{array}$

$\begin{array}{lllll}778 & 1.05 & 20.56 & 38.20 & 11.14\end{array}$

$\begin{array}{llll}765 & -15.97 & 37.97 & 41.19\end{array}$

$\begin{array}{llll}768 & -22.74 & -46.28 & 51.56\end{array}$

$\begin{array}{lllll}779 & 1.05 & 20.40 & 14.19 & 4.14\end{array}$

$\begin{array}{llll}759 & -3.64 & 32.29 & 32.50\end{array}$

$\begin{array}{llll}784 & -10.47 & -36.82 & 38.28\end{array}$

$\begin{array}{lllll}780 & 1.01 & 21.81 & 0.00 & 0.00\end{array}$

$\begin{array}{llll}766 & 35.30 & 11.92 & 37.26\end{array}$

$\begin{array}{llll}767 & 72.55 & 42.49 & 84.08\end{array}$

$\begin{array}{llll}809 & -163.91 & -252.35 & 300.91\end{array}$

$\begin{array}{llll}3062 & 54.49 & 24.65 & 59.81\end{array}$

$\begin{array}{lllll}781 & 1.04 & 22.63 & 0.00 & 0.00\end{array}$

$\begin{array}{llll}783 & 99.87 & -26.01 & 103.20\end{array}$

$\begin{array}{llll}809 & -103.88 & -147.89 & 180.73\end{array}$

$\begin{array}{lllll}782 & 1.04 & 20.82 & 0.47 & 0.14\end{array}$

$\begin{array}{llll}783 & -0.47 & -1.32 & 1.40\end{array}$

$\begin{array}{lllll}783 & 1.04 & 20.82 & 42.58 & 12.42\end{array}$

$\begin{array}{llll}743 & 35.74 & 18.82 & 40.39\end{array}$

$\begin{array}{llll}768 & -2.40 & -22.01 & 22.14\end{array}$

$\begin{array}{llll}770 & 23.76 & -2.51 & 23.90\end{array}$

$\begin{array}{llll}781 & -98.71 & 27.95 & 102.59\end{array}$

$\begin{array}{llll}782 & 0.71 & 0.50 & 0.87\end{array}$

$\begin{array}{lllll}784 & 1.06 & 20.39 & 54.26 & 15.83\end{array}$

$\begin{array}{llll}779 & 10.65 & 36.56 & 38.08\end{array}$

$\begin{array}{llll}787 & 34.18 & 0.87 & 34.19\end{array}$

$811-105.26-184.28 \quad 212.22$

$\begin{array}{lllll}785 & 1.07 & 19.80 & 28.02 & 8.17\end{array}$

$\begin{array}{llll}757 & -56.80 & -1.19 & 56.81\end{array}$

$\begin{array}{llll}776 & 32.37 & 0.97 & 32.39\end{array}$

$\begin{array}{lllll}786 & 1.07 & 19.70 & 32.87 & 9.59\end{array}$

$\begin{array}{llll}757 & -31.80 & -5.21 & 32.22\end{array}$

$\begin{array}{llll}788 & -0.76 & -4.70 & 4.76\end{array}$

$\begin{array}{lllll}787 & 1.05 & 20.14 & 0.00 & 0.00\end{array}$

$\begin{array}{llll}764 & 33.00 & 7.53 & 33.85\end{array}$

$\begin{array}{llll}784 & -33.40 & -2.39 & 33.49\end{array}$

$\begin{array}{lllll}788 & 1.07 & 19.70 & 34.93 & 10.19\end{array}$

$\begin{array}{llll}755 & -93.48 & -108.93 & 143.55\end{array}$

$\begin{array}{llll}761 & 18.41 & 1.28 & 18.45\end{array}$

$\begin{array}{lllll}773 & -10.49 & 2.85 & 10.87\end{array}$

$\begin{array}{llll}775 & 17.38 & 56.54 & 59.15\end{array}$

$\begin{array}{llll}786 & 1.07 & 3.97 & 4.11\end{array}$

$\begin{array}{llll}789 & 47.43 & 46.75 & 66.60\end{array}$

$\begin{array}{llll}790 & 4.38 & -20.36 & 20.82\end{array}$

$\begin{array}{lllll}789 & 1.06 & 19.49 & 36.61 & 10.68\end{array}$

$\begin{array}{llll}771 & 17.45 & -3.24 & 17.75\end{array}$

$\begin{array}{lllll}775 & -7.96 & 44.06 & 44.77\end{array}$ 


\begin{tabular}{|c|c|c|c|c|c|c|c|c|}
\hline \multirow{3}{*}{790} & & & & & 788 & -47.17 & -46.78 & 66.43 \\
\hline & 1.07 & 19.69 & 0.00 & 0.00 & & & & \\
\hline & & & & & 788 & 4.16 & 1.60 & 4.45 \\
\hline \multirow[t]{3}{*}{792} & 1.06 & 20.45 & 0.00 & 0.00 & & & & \\
\hline & & & & & 756 & -113.15 & 1.88 & 113.17 \\
\hline & & & & & 764 & 121.80 & 0.46 & 121.80 \\
\hline \multirow[t]{3}{*}{798} & 1.13 & 22.44 & 0.00 & 0.00 & & & & \\
\hline & & & & & 710 & -40.24 & -3.92 & 40.43 \\
\hline & & & & & 806 & 43.63 & -21.53 & 48.65 \\
\hline \multirow[t]{2}{*}{799} & 1.09 & 25.42 & 0.00 & 0.00 & & & & \\
\hline & & & & & 800 & 40.18 & -79.91 & 89.44 \\
\hline \multirow[t]{7}{*}{800} & 1.09 & 25.40 & 31.28 & 9.12 & & & & \\
\hline & & & & & 716 & -509.14 & -23.32 & 509.67 \\
\hline & & & & & 717 & -508.08 & 362.96 & 511.97 \\
\hline & & & & & 799 & -40.18 & 79.94 & 89.47 \\
\hline & & & & & 803 & 304.93 & -92.30 & 318.59 \\
\hline & & & & & 805 & 245.51 & -80.90 & 258.50 \\
\hline & & & & & 809 & 467.67 & 83.35 & 475.04 \\
\hline \multirow[t]{5}{*}{801} & 1.13 & 22.52 & 0.00 & 0.00 & & & & \\
\hline & & & & & 701 & -1.34 & -24.51 & 24.55 \\
\hline & & & & & 702 & -1.34 & -24.51 & 24.55 \\
\hline & & & & & 718 & -214.92 & -151.99 & 263.24 \\
\hline & & & & & 806 & 238.76 & 48.03 & 243.55 \\
\hline \multirow[t]{6}{*}{803} & 1.10 & 22.29 & 0.00 & 0.00 & & & & \\
\hline & & & & & 733 & 111.97 & 169.70 & 203.31 \\
\hline & & & & & 765 & 92.77 & 156.05 & 181.55 \\
\hline & & & & & 800 & -295.81 & 97.34 & 311.42 \\
\hline & & & & & 804 & 63.83 & 72.41 & 96.53 \\
\hline & & & & & 811 & 52.06 & -157.81 & 166.17 \\
\hline \multirow[t]{8}{*}{804} & 1.09 & 21.98 & 0.00 & 0.00 & & & & \\
\hline & & & & & 803 & -59.84 & -78.42 & 98.64 \\
\hline & & & & & 809 & -261.09 & 103.30 & 280.79 \\
\hline & & & & & 810 & 51.39 & -45.11 & 68.38 \\
\hline & & & & & 811 & $-2.58-$ & -121.00 & 121.03 \\
\hline & & & & & 818 & -407.02 & 45.59 & 409.57 \\
\hline & & & & & 963 & -86.20 & 129.44 & 155.52 \\
\hline & & & & & 1425 & 790.73 & -8.26 & 790.77 \\
\hline \multirow[t]{6}{*}{805} & 1.10 & 21.65 & 0.00 & 0.00 & & & & \\
\hline & & & & & 736 & 175.76 & 209.47 & 273.43 \\
\hline & & & & & 800 & -232.61 & 77.29 & 245.11 \\
\hline & & & & & 808 & 191.86 & -23.16 & 193.25 \\
\hline & & & & & 810 & 3.49 & 45.75 & 45.88 \\
\hline & & & & & 811 & -114.75 & -181.17 & 214.45 \\
\hline \multirow[t]{5}{*}{806} & 1.13 & 22.37 & 0.00 & 0.00 & & & & \\
\hline & & & & & 773 & 81.69 & 97.18 & 126.95 \\
\hline & & & & & 798 & -42.98 & 19.80 & 47.32 \\
\hline & & & & & 801 & -186.82 & -145.67 & 236.90 \\
\hline & & & & & 811 & 177.07 & 120.37 & 214.11 \\
\hline 807 & 1.11 & 20.42 & 0.00 & 0.00 & & & & \\
\hline
\end{tabular}




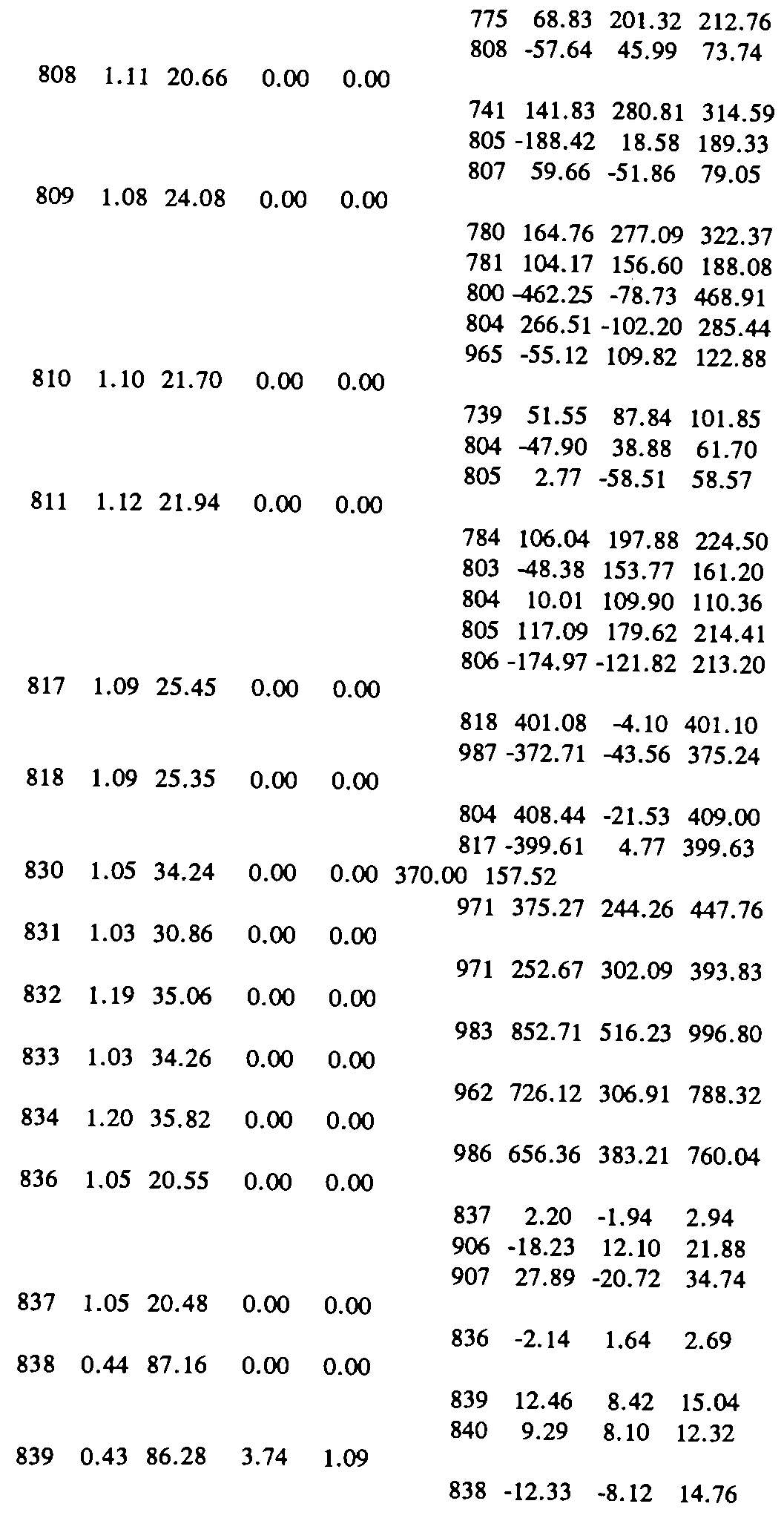




\begin{tabular}{|c|c|c|c|c|c|c|c|c|}
\hline \multirow[t]{3}{*}{840} & 0.42 & 86.02 & 0.00 & 0.00 & & & & \\
\hline & & & & & 838 & -9.02 & -7.67 & 11.84 \\
\hline & & & & & 841 & 379.89 & -115.52 & 397.06 \\
\hline \multirow{4}{*}{841} & & & & & 866 & 48.50 & 9.43 & 49.41 \\
\hline & 0.42 & 85.03 & 1.31 & 0.38 & & & & \\
\hline & & & & & 840 & -371.12 & 119.91 & 390.01 \\
\hline & & & & & 3083 & 123.11 & $1-45.28$ & $\begin{array}{ll}3131.17\end{array}$ \\
\hline \multirow[t]{4}{*}{842} & 1.31 & 14.29 & 0.00 & 0.00 & & & & \\
\hline & & & & & 875 & 0.51 & -16.63 & 16.64 \\
\hline & & & & & 1396 & -36.95 & 18.66 & 41.40 \\
\hline & & & & & 3026 & 36.52 & 20.03 & 41.65 \\
\hline \multirow[t]{4}{*}{843} & 1.31 & 16.55 & 3.64 & 1.06 & & & & \\
\hline & & & & & 844 & 20.05 & 10.73 & 22.74 \\
\hline & & & & & 914 & 16.01 & -25.40 & 30.03 \\
\hline & & & & & 967 & -41.03 & -71.99 & 82.86 \\
\hline \multirow[t]{4}{*}{844} & 1.30 & 16.36 & 0.00 & 0.00 & & & & \\
\hline & & & & & 843 & -19.94 & -10.84 & 22.70 \\
\hline & & & & & 3027 & 3.30 & -5.99 & 6.85 \\
\hline & & & & & 3028 & 17.09 & 5.29 & 17.88 \\
\hline \multirow[t]{4}{*}{845} & 1.15 & 14.26 & 0.00 & 0.00 & & & & \\
\hline & & & & & 846 & 9.33 & 3.25 & 9.88 \\
\hline & & & & & 890 & -38.13 & 41.02 & 56.00 \\
\hline & & & & & 1306 & 30.11 & -54.68 & 62.42 \\
\hline \multirow[t]{3}{*}{846} & 1.15 & 14.07 & 0.00 & 0.00 & & & & \\
\hline & & & & & 845 & -9.32 & -3.21 & 9.86 \\
\hline & & & & & 3328 & 15.50 & 60.39 & 62.35 \\
\hline \multirow[t]{4}{*}{847} & 1.18 & 14.78 & 0.00 & 0.00 & & & & \\
\hline & & & & & 899 & -14.08 & -38.84 & 41.31 \\
\hline & & & & & 909 & 12.68 & 36.21 & 38.37 \\
\hline & & & & & 3119 & 2.63 & 0.01 & 2.63 \\
\hline \multirow[t]{4}{*}{848} & 1.23 & 19.76 & 0.00 & 0.00 & & & & \\
\hline & & & & & 897 & $-7.09-$ & -111.31 & 111.53 \\
\hline & & & & & 918 & 10.45 & 100.24 & 100.79 \\
\hline & & & & & 3071 & 4.84 & 0.10 & 4.85 \\
\hline \multirow[t]{6}{*}{849} & 1.04 & 15.66 & 0.00 & 0.00 & & & & \\
\hline & & & & & 854 & 25.85 & -10.21 & 27.80 \\
\hline & & & & & 932 & -70.17 & 43.04 & 82.32 \\
\hline & & & & & 3041 & 4.68 & 32.89 & 33.22 \\
\hline & & & & & 3043 & 0.99 & -15.51 & 15.54 \\
\hline & & & & & 3047 & 40.53 & 8.27 & 41.36 \\
\hline \multirow[t]{4}{*}{850} & 1.02 & 11.98 & 0.00 & 0.00 & & & & \\
\hline & & & & & 851 & 2.71 & -1.55 & 3.12 \\
\hline & & & & & 854 & -17.72 & 12.36 & 21.61 \\
\hline & & & & & 878 & 14.61 & -17.76 & 23.00 \\
\hline \multirow[t]{2}{*}{851} & 1.02 & 11.96 & 2.80 & 0.82 & & & & \\
\hline & & & & & 850 & -2.71 & 1.53 & 3.11 \\
\hline \multirow{3}{*}{852} & 1.08 & 14.46 & 0.00 & 0.00 & & & & \\
\hline & & & & & 853 & -2.90 & 21.66 & 21.85 \\
\hline & & & & & 856 & 3.97 & -1.36 & 4.20 \\
\hline
\end{tabular}




\begin{tabular}{|c|c|c|c|c|c|c|c|c|}
\hline \multirow{3}{*}{853} & \multirow{3}{*}{1.08} & \multirow{3}{*}{14.62} & \multirow{4}{*}{0.00} & \multirow{3}{*}{0.00} & \multirow{2}{*}{$\begin{array}{l}1395 \\
3086\end{array}$} & 24.89 & 6.12 & \multirow{2}{*}{$\begin{array}{l}25.63 \\
24.60\end{array}$} \\
\hline & & & & & & -24.17 & 4.57 & \\
\hline & & & & & & & & \\
\hline \multirow{5}{*}{854} & & & & & 852 & 2.94 & -21.72 & 21.92 \\
\hline & & & & & 3043 & 0.54 & 14.38 & 14.39 \\
\hline & & & & & 3072 & -3.24 & 5.72 & 6.57 \\
\hline & 1.03 & 13.57 & 7.00 & 2.04 & & & & \\
\hline & & & & & 849 & -25.20 & 10.54 & 27.31 \\
\hline & & & & & 850 & 18.26 & -12.30 & 22.01 \\
\hline \multirow[t]{2}{*}{855} & 1.08 & 14.40 & 0.93 & 0.27 & & & & \\
\hline & & & & & 856 & -0.98 & -0.86 & 1.30 \\
\hline \multirow[t]{4}{*}{856} & 1.08 & 14.41 & 0.00 & 0.00 & & & & \\
\hline & & & & & 852 & -3.98 & 1.27 & 4.17 \\
\hline & & & & & 855 & 0.98 & 0.41 & 1.06 \\
\hline & & & & & 3089 & 2.72 & -0.01 & 2.72 \\
\hline \multirow[t]{6}{*}{857} & 1.11 & 17.85 & 0.00 & 0.00 & & & & \\
\hline & & & & & 860 & 34.83 & -33.31 & 48.19 \\
\hline & & & & & 889 & 50.16 & -23.24 & 55.28 \\
\hline & & & & & 891 & 46.47 & -16.39 & 49.28 \\
\hline & & & & & 924 & -4.97 & 53.06 & 53.30 \\
\hline & & & & & 969 & -132.63 & -139.20 & 192.27 \\
\hline \multirow[t]{4}{*}{858} & 1.31 & 16.25 & 0.00 & 0.00 & & & & \\
\hline & & & & & 901 & -6.60 & 36.94 & 37.52 \\
\hline & & & & & 914 & 4.41 & -22.13 & 22.57 \\
\hline & & & & & 3025 & 3.78 & 25.15 & 25.44 \\
\hline \multirow[t]{4}{*}{859} & 0.92 & 15.84 & 0.00 & 0.00 & & & & \\
\hline & & & & & 874 & -26.29 & -67.82 & 72.74 \\
\hline & & & & & 917 & 27.51 & 60.15 & 66.14 \\
\hline & & & & & 3120 & 9.23 & -4.52 & 10.28 \\
\hline \multirow[t]{4}{*}{860} & 1.11 & 17.82 & 0.00 & 0.00 & & & & \\
\hline & & & & & 857 & -34.81 & 33.33 & 48.19 \\
\hline & & & & & 861 & 36.52 & 5.79 & 36.98 \\
\hline & & & & & 3091 & 3.20 & -1.00 & 3.35 \\
\hline \multirow[t]{3}{*}{861} & 1.10 & 16.50 & 9.81 & 2.86 & & & & \\
\hline & & & & & 860 & -36.04 & -5.34 & 36.44 \\
\hline & & & & & 865 & 26.60 & 1.80 & 26.66 \\
\hline \multirow[t]{3}{*}{862} & 1.06 & 15.57 & 5.32 & 1.55 & & & & \\
\hline & & & & & 863 & -3.39 & -10.17 & 10.72 \\
\hline & & & & & 905 & 5.84 & -4.77 & 7.54 \\
\hline \multirow[t]{3}{*}{863} & 1.06 & 15.51 & 2.71 & 0.79 & & & & \\
\hline & & & & & 862 & 3.46 & 10.03 & 10.61 \\
\hline & & & & & 900 & -6.61 & -10.56 & 12.45 \\
\hline \multirow[t]{4}{*}{864} & 1.08 & 18.55 & 0.00 & 0.00 & & & & \\
\hline & & & & & 887 & -6.34 & -12.78 & 14.27 \\
\hline & & & & & 3073 & 18.96 & -4.58 & 19.50 \\
\hline & & & & & 3090 & -3.28 & 9.20 & 9.77 \\
\hline 865 & 1.09 & 15.79 & 0.00 & 0.00 & & & & \\
\hline & & & & & 861 & -26.39 & -1.74 & 26.45 \\
\hline & & & & & 3074 & 0.78 & -4.78 & 4.85 \\
\hline
\end{tabular}




\begin{tabular}{|c|c|c|c|c|c|c|c|c|}
\hline \multirow[b]{2}{*}{866} & \multirow[b]{2}{*}{0.37} & \multirow[b]{2}{*}{60.89} & \multirow[b]{2}{*}{9.53} & \multirow[b]{2}{*}{2.78} & \multirow[t]{2}{*}{3086} & \multirow[t]{2}{*}{27.42} & \multirow[t]{2}{*}{-10.14} & \multirow[t]{2}{*}{29.24} \\
\hline & & & & & & & & \\
\hline \multirow{5}{*}{867} & & & & & 840 & -41.39 & 10.33 & 42.66 \\
\hline & & & & & 3042 & 31.55 & -8.88 & 32.78 \\
\hline & & & & & 3084 & 4.59 & 0.87 & 4.67 \\
\hline & 1.02 & 11.72 & 3.45 & 1.01 & & & & \\
\hline & & & & & 878 & -3.48 & -1.45 & 3.77 \\
\hline \multirow[t]{3}{*}{868} & 0.96 & 18.86 & 2.71 & 0.79 & & & & \\
\hline & & & & & 869 & 9.38 & -30.03 & 31.46 \\
\hline & & & & & 3080 & -11.10 & 25.65 & 27.95 \\
\hline \multirow[t]{4}{*}{869} & 0.96 & 18.60 & 0.00 & 0.00 & & & & \\
\hline & & & & & 868 & -9.28 & 30.15 & 31.55 \\
\hline & & & & & 2996 & 6.54 & -30.00 & 30.71 \\
\hline & & & & & 3075 & 2.66 & -0.40 & 2.69 \\
\hline \multirow[t]{5}{*}{870} & 1.33 & 11.92 & 2.24 & 0.65 & & & & \\
\hline & & & & & 871 & -11.35 & 41.77 & 43.29 \\
\hline & & & & & 939 & -57.40 & -45.70 & 73.37 \\
\hline & & & & & 1031 & 13.25 & 0.03 & 13.25 \\
\hline & & & & & 3138 & 53.22 & 11.01 & 54.35 \\
\hline \multirow[t]{4}{*}{871} & 1.30 & 12.50 & 0.00 & 0.00 & & & & \\
\hline & & & & & 870 & 11.61 & -41.38 & 42.97 \\
\hline & & & & & 879 & -14.34 & 40.23 & 42.71 \\
\hline & & & & & 3031 & 2.61 & -0.21 & 2.61 \\
\hline \multirow[t]{4}{*}{872} & 1.30 & 15.85 & 0.00 & 0.00 & & & & \\
\hline & & & & & 873 & 13.36 & -15.43 & 20.41 \\
\hline & & & & & 881 & -34.93 & 4.20 & 35.18 \\
\hline & & & & & 912 & 20.00 & 4.19 & 20.44 \\
\hline \multirow[t]{4}{*}{873} & 1.30 & 15.10 & 0.00 & 0.00 & & & & \\
\hline & & & & & 872 & -13.16 & 15.28 & 20.17 \\
\hline & & & & & 875 & 8.78 & -16.50 & 18.69 \\
\hline & & & & & 3032 & 4.16 & 0.71 & 4.22 \\
\hline \multirow[t]{3}{*}{874} & 0.99 & 15.53 & 3.45 & 1.01 & & & & \\
\hline & & & & & 859 & 28.58 & 72.13 & 77.59 \\
\hline & & & & & 909 & -32.39 & -58.96 & 67.27 \\
\hline \multirow[t]{3}{*}{875} & 1.32 & 14.04 & 8.97 & 2.62 & & & & \\
\hline & & & & & 842 & -0.36 & 16.40 & 16.40 \\
\hline & & & & & 873 & -8.47 & 16.11 & 18.20 \\
\hline \multirow[t]{4}{*}{876} & 0.93 & 21.60 & 0.00 & 0.00 & & & & \\
\hline & & & & & 880 & -22.44 & -4.64 & 22.92 \\
\hline & & & & & 884 & 17.34 & 0.47 & 17.35 \\
\hline & & & & & 3076 & 6.79 & 1.44 & 6.94 \\
\hline \multirow[t]{2}{*}{877} & 1.02 & 11.57 & 11.02 & 3.21 & & & & \\
\hline & & & & & 878 & -11.22 & 15.93 & 19.49 \\
\hline 878 & 1.02 & 11.78 & 0.00 & 0.00 & & & & \\
\hline & & & & & 850 & -14.55 & 17.79 & 22.98 \\
\hline & & & & & 867 & 3.51 & 1.29 & 3.73 \\
\hline & & & & & 877 & 11.28 & -15.93 & 19.52 \\
\hline 879 & 1.27 & 13.38 & 6.07 & 1.77 & & & & \\
\hline & & & & & 871 & 14.68 & -39.76 & 42.38 \\
\hline
\end{tabular}




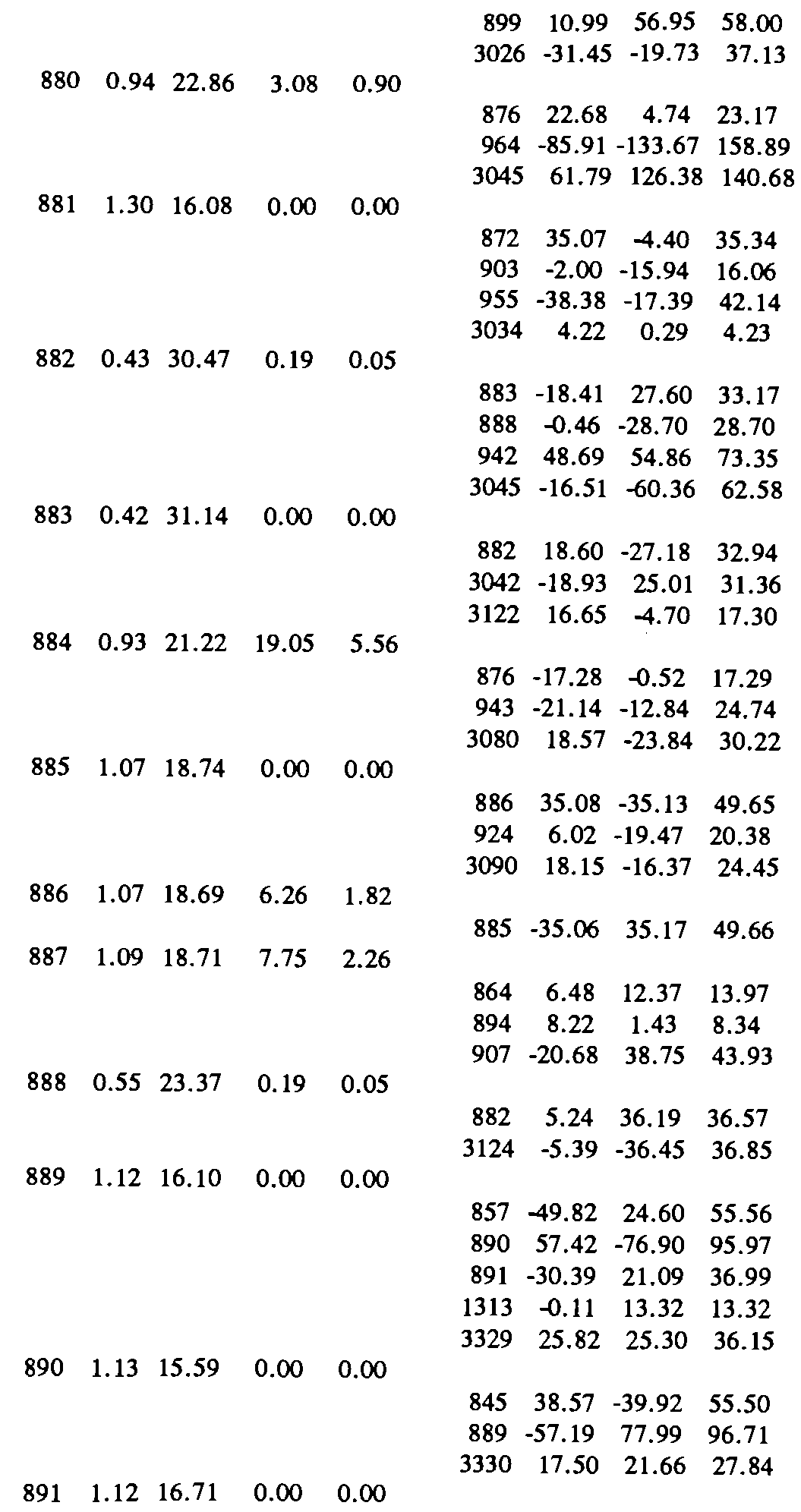

$\begin{array}{llll}857 & -46.28 & 17.05 & 49.33\end{array}$ 


\begin{tabular}{|c|c|c|c|c|c|c|c|c|}
\hline & & & & & $\begin{array}{r}889 \\
3331\end{array}$ & $\begin{array}{r}30.50 \\
14.83\end{array}$ & $\begin{array}{r}-20.87 \\
-1359\end{array}$ & $\begin{array}{r}36.96 \\
20.12\end{array}$ \\
\hline \multirow{5}{*}{893} & 1.20 & 4.79 & 168 & 0.49 & 3331 & 14.83 & $\begin{array}{ll}3 & 13.59\end{array}$ & \\
\hline & & & 1.00 & & 895 & -18.27 & -42.69 & 46.43 \\
\hline & & & & & 922 & -18.50 & -15.99 & 24.45 \\
\hline & & & & & 3138 & -43.92 & $\begin{array}{ll}2 & -5.15\end{array}$ & 44.22 \\
\hline & & & & & 3140 & -39.04 & $\begin{array}{ll}4 & -44.57\end{array}$ & 59.25 \\
\hline \multirow[t]{4}{*}{894} & 1.08 & 18.26 & 0.00 & 0.00 & & & & \\
\hline & & & & & 887 & -8.06 & -1.88 & 8.28 \\
\hline & & & & & 3030 & 2.39 & -0.74 & 2.50 \\
\hline & & & & & 3033 & 6.32 & 0.57 & 6.35 \\
\hline \multirow[t]{4}{*}{895} & 1.24 & 4.53 & 0.00 & 0.00 & & & & \\
\hline & & & & & 893 & 19.08 & 34.65 & 47.64 \\
\hline & & & & & 1036 & -22.02 & $\begin{array}{ll}2 & -44.14\end{array}$ & 49.33 \\
\hline & & & & & 3137 & 1.64 & 0.28 & 1.66 \\
\hline \multirow[t]{2}{*}{896} & 1.24 & 19.62 & 5.60 & 1.63 & & & & \\
\hline & & & & & 897 & -5.74 & -3.68 & 6.81 \\
\hline \multirow[t]{5}{*}{897} & 1.24 & 19.64 & 0.00 & 0.00 & & & & \\
\hline & & & & & 848 & 7.45 & 112.75 & 113.00 \\
\hline & & & & & 896 & 5.80 & 3.51 & 6.78 \\
\hline & & & & & 902 & 20.64 & -65.65 & 68.81 \\
\hline & & & & & 975 & -38.14 & -108.73 & 115.23 \\
\hline \multirow[t]{3}{*}{898} & 1.19 & 17.12 & 2.43 & 0.71 & & & & \\
\hline & & & & & 909 & 35.84 & 56.60 & 66.99 \\
\hline & & & & & 974 & -37.79 & -67.66 & 77.50 \\
\hline \multirow[t]{4}{*}{899} & 1.19 & 14.65 & 0.00 & 0.00 & & & & \\
\hline & & & & & 847 & 14.36 & 39.14 & 41.69 \\
\hline & & & & & 879 & -9.03 & -54.35 & 55.10 \\
\hline & & & & & 3118 & 12.85 & $\begin{array}{ll}5 & -23.85\end{array}$ & 27.09 \\
\hline \multirow[t]{4}{*}{900} & 1.08 & 15.54 & 0.00 & 0.00 & & & & \\
\hline & & & & & 863 & 6.79 & 10.20 & 12.25 \\
\hline & & & & & 909 & -8.38 & -13.99 & 16.31 \\
\hline & & & & & 3123 & 6.12 & -3.01 & 6.82 \\
\hline \multirow[t]{4}{*}{901} & 1.29 & 17.10 & 0.00 & 0.00 & & & & \\
\hline & & & & & 858 & 7.04 & -36.62 & 37.29 \\
\hline & & & & & 902 & -9.26 & 34.17 & 35.41 \\
\hline & & & & & 3035 & 3.21 & 0.07 & 3.21 \\
\hline \multirow[t]{3}{*}{902} & 1.28 & 17.73 & 8.97 & 2.62 & & & & \\
\hline & & & & & 897 & -18.88 & 67.79 & 70.37 \\
\hline & & & & & 901 & 9.59 & -33.95 & 35.27 \\
\hline \multirow[t]{4}{*}{903} & 1.34 & 15.40 & 0.00 & 0.00 & & & & \\
\hline & & & & & 881 & 2.53 & 14.86 & 15.07 \\
\hline & & & & & 914 & -29.42 & 147.36 & 150.27 \\
\hline & & & & & 3128 & 2.56 & 0.44 & 2.60 \\
\hline \multirow[t]{4}{*}{904} & 1.04 & 13.12 & 0.00 & 0.00 & & & & \\
\hline & & & & & 916 & -78.80 & -53.53 & 95.27 \\
\hline & & & & & 1565 & 73.69 & 51.82 & 90.09 \\
\hline & & & & & 3046 & 3.46 & -0.16 & 3.46 \\
\hline & 1.06 & 15.53 & 0.00 & 0.00 & & & & \\
\hline
\end{tabular}




\begin{tabular}{|c|c|c|c|c|c|c|c|c|}
\hline & & & & & 862 & -5.83 & 4.74 & 7.52 \\
\hline & & & & & 3121 & 3.27 & -3.21 & 4.58 \\
\hline & & & & & 3157 & 2.25 & -0.95 & 2.44 \\
\hline \multirow[t]{6}{*}{906} & 1.05 & 21.24 & 7.00 & 2.04 & & & & \\
\hline & & & & & 836 & 18.39 & -12.22 & 22.08 \\
\hline & & & & & 907 & 21.65 & -15.42 & 26.59 \\
\hline & & & & & 918 & -0.02 & -83.19 & 83.19 \\
\hline & & & & & 920 & -31.19 & 385.76 & 387.01 \\
\hline & & & & & 972 & -10.00 & -341.43 & 341.58 \\
\hline \multirow[t]{4}{*}{907} & 1.06 & 19.96 & 22.69 & 6.62 & & & & \\
\hline & & & & & 836 & -27.77 & 20.87 & 34.74 \\
\hline & & & & & 887 & 21.04 & -37.84 & 43.30 \\
\hline & & & & & 906 & -21.40 & 15.43 & 26.39 \\
\hline \multirow[t]{4}{*}{908} & 1.06 & 14.55 & 0.00 & 0.00 & & & & \\
\hline & & & & & 730 & -4.87 & 10.63 & 11.70 \\
\hline & & & & & 3048 & 7.37 & -3.48 & 8.15 \\
\hline & & & & & 3050 & -5.48 & -17.36 & 18.20 \\
\hline \multirow[t]{6}{*}{909} & 1.11 & 15.55 & 2.71 & 0.79 & & & & \\
\hline & & & & & 847 & -11.31 & -34.97 & 36.75 \\
\hline & & & & & 874 & 36.47 & 65.64 & 75.09 \\
\hline & & & & & 898 & -34.79 & -52.75 & 63.18 \\
\hline & & & & & 900 & 8.69 & 13.69 & 16.22 \\
\hline & & & & & 3126 & 8.21 & -11.70 & 14.30 \\
\hline \multirow[t]{3}{*}{910} & 1.02 & 12.49 & 2.43 & 0.71 & & & & \\
\hline & & & & & 911 & 26.20 & -1.41 & 26.24 \\
\hline & & & & & 3047 & -32.19 & -5.47 & 32.65 \\
\hline \multirow[t]{4}{*}{911} & 1.02 & 12.43 & 0.00 & 0.00 & & & & \\
\hline & & & & & 910 & -26.19 & 1.41 & 26.23 \\
\hline & & & & & 1565 & 20.57 & -0.98 & 20.59 \\
\hline & & & & & 3049 & 5.60 & 0.62 & 5.63 \\
\hline \multirow[t]{3}{*}{912} & 1.29 & 15.76 & 30.35 & 8.85 & & & & \\
\hline & & & & & 872 & -19.98 & -4.23 & 20.42 \\
\hline & & & & & 913 & -10.55 & -7.14 & 12.74 \\
\hline \multirow[t]{3}{*}{913} & 1.30 & 15.79 & 0.00 & 0.00 & & & & \\
\hline & & & & & 912 & 10.56 & 7.10 & 12.73 \\
\hline & & & & & 3028 & -10.28 & -4.83 & 11.36 \\
\hline \multirow[t]{4}{*}{914} & 1.34 & 15.41 & 5.23 & 1.52 & & & & \\
\hline & & & & & 843 & -15.47 & 25.00 & 29.40 \\
\hline & & & & & 858 & -4.14 & 21.86 & 22.25 \\
\hline & & & & & 903 & 29.44 & -147.30 & 150.21 \\
\hline \multirow[t]{3}{*}{915} & 1.10 & 15.12 & 14.57 & 4.25 & & & & \\
\hline & & & & & 916 & -34.28 & -0.56 & 34.28 \\
\hline & & & & & 3050 & 19.33 & 20.57 & 28.23 \\
\hline \multirow[t]{4}{*}{916} & 1.10 & 16.05 & 10.37 & 3.02 & & & & \\
\hline & & & & & 904 & 80.25 & 60.18 & 100.31 \\
\hline & & & & & 915 & 34.44 & 0.81 & 34.45 \\
\hline & & & & & 976 & -128.11 & -128.64 & 181.55 \\
\hline \multirow[t]{2}{*}{917} & 0.83 & 16.54 & 0.00 & 0.00 & & & & \\
\hline & & & & & 859 & -24.18 & -55.09 & 60.16 \\
\hline
\end{tabular}




\begin{tabular}{|c|c|c|c|c|c|c|c|c|}
\hline & & & & & 3124 & 23.89 & 54.03 & 59.07 \\
\hline & & & & & 3127 & 1.91 & -0.64 & 2.01 \\
\hline \multirow[t]{4}{*}{918} & 1.20 & 19.87 & 0.00 & 0.00 & & & & \\
\hline & & & & & 848 & -9.99 & -98.39 & 98.90 \\
\hline & & & & & 906 & 2.57 & 94.22 & 94.25 \\
\hline & & & & & 919 & 11.46 & -5.46 & 12.70 \\
\hline \multirow[t]{2}{*}{919} & 1.20 & 19.81 & 7.56 & 2.21 & & & & \\
\hline & & & & & 918 & -11.45 & 5.42 & 12.67 \\
\hline \multirow[t]{4}{*}{920} & 0.87 & 26.37 & 0.00 & 0.00 & & & & \\
\hline & & & & & 906 & 54.41 & -316.46 & 321.11 \\
\hline & & & & & 921 & 46.64 & -70.32 & 84.38 \\
\hline & & & & & 3083 & -36.49 & 264.14 & 266.65 \\
\hline \multirow[t]{2}{*}{921} & 0.87 & 26.20 & 9.34 & 2.72 & & & & \\
\hline & & & & & 920 & -46.45 & 70.51 & 84.44 \\
\hline \multirow[t]{3}{*}{922} & 1.20 & 4.79 & 0.00 & 0.00 & & & & \\
\hline & & & & & 893 & 18.50 & 16.00 & 24.46 \\
\hline & & & & & 923 & 4.16 & 0.80 & 4.24 \\
\hline \multirow[t]{2}{*}{923} & 1.20 & 4.73 & 5.32 & 1.55 & & & & \\
\hline & & & & & 922 & -4.16 & -0.98 & 4.27 \\
\hline \multirow[t]{4}{*}{924} & 1.09 & 18.15 & 0.00 & 0.00 & & & & \\
\hline & & & & & 857 & 5.17 & -52.32 & 52.58 \\
\hline & & & & & 885 & -5.81 & 19.20 & 20.06 \\
\hline & & & & & 925 & 10.11 & -49.59 & 50.61 \\
\hline \multirow[t]{2}{*}{925} & 1.09 & 18.12 & 3.45 & 1.01 & & & & \\
\hline & & & & & 924 & -10.09 & 49.61 & 50.63 \\
\hline \multirow[t]{5}{*}{926} & 0.93 & 22.89 & 0.00 & 0.00 & & & & \\
\hline & & & & & 927 & 10.92 & 1.29 & 11.00 \\
\hline & & & & & 946 & 125.53 & 149.49 & 195.21 \\
\hline & & & & & 2999 & 4.25 & -0.64 & 4.29 \\
\hline & & & & & 3056 & -135.12 & $2-159.88$ & 209.33 \\
\hline \multirow[t]{2}{*}{927} & 0.93 & 22.77 & 10.09 & 2.94 & & & & \\
\hline & & & & & 926 & -10.75 & -1.75 & 10.89 \\
\hline \multirow[t]{4}{*}{928} & 0.84 & 22.22 & 0.00 & 0.00 & & & & \\
\hline & & & & & 929 & 10.99 & 1.47 & 11.09 \\
\hline & & & & & 931 & -48.08 & -173.99 & 180.51 \\
\hline & & & & & 938 & 42.57 & 166.86 & 172.20 \\
\hline \multirow[t]{2}{*}{929} & 0.84 & 22.05 & 10.18 & 2.97 & & & & \\
\hline & & & & & 928 & -10.83 & -1.96 & 11.01 \\
\hline \multirow[t]{4}{*}{931} & 0.93 & 22.71 & 10.46 & 3.05 & & & & \\
\hline & & & & & 928 & 51.63 & 190.72 & 197.58 \\
\hline & & & & & 943 & 20.74 & -19.55 & 28.50 \\
\hline & & & & & 950 & -79.86 & -177.50 & 194.64 \\
\hline \multirow[t]{3}{*}{932} & 1.00 & 20.43 & 20.64 & 6.02 & & & & \\
\hline & & & & & 849 & 70.55 & -35.57 & 79.01 \\
\hline & & & & & 933 & -90.79 & 28.90 & 95.28 \\
\hline \multirow[t]{4}{*}{933} & 1.00 & 23.72 & 0.00 & 0.00 & & & & \\
\hline & & & & & 932 & 93.12 & -25.15 & 96.45 \\
\hline & & & & & 947 & -4.14 & 24.75 & 25.09 \\
\hline & & & & & 960 & -19.81 & 2.36 & 19.95 \\
\hline
\end{tabular}




\begin{tabular}{|c|c|c|c|c|c|c|c|c|}
\hline & & & & & 968 & -119.59 & -123.91 & 172.21 \\
\hline & & & & & 3063 & 51.68 & -0.22 & 51.68 \\
\hline \multirow[t]{4}{*}{934} & 0.77 & 20.88 & 0.00 & 0.00 & & & & \\
\hline & & & & & 937 & 132.93 & 258.34 & 290.53 \\
\hline & & & & & 938 & -134.46 & -271.23 & 302.73 \\
\hline & & & & & 3057 & 14.93 & -8.19 & 17.03 \\
\hline \multirow[t]{7}{*}{935} & 0.61 & 16.16 & 0.00 & 0.00 & & & & \\
\hline & & & & & 937 & -112.85 & -194.79 & 225.12 \\
\hline & & & & & 942 & 138.24 & 243.77 & 280.23 \\
\hline & & & & & 942 & 138.24 & 243.77 & 280.23 \\
\hline & & & & & 942 & 138.23 & 243.77 & 280.24 \\
\hline & & & & & 942 & 138.23 & 243.77 & 280.24 \\
\hline & & & & & 970 & -383.41 & -591.52 & 704.91 \\
\hline \multirow[t]{2}{*}{936} & 0.67 & 18.29 & 8.97 & 2.62 & & & & \\
\hline & & & & & 937 & -7.61 & -5.23 & 9.23 \\
\hline \multirow[t]{4}{*}{937} & 0.67 & 18.34 & 0.00 & 0.00 & & & & \\
\hline & & & & & 934 & -126.98 & -222.90 & 256.53 \\
\hline & & & & & 935 & 117.08 & 220.31 & 249.49 \\
\hline & & & & & 936 & 7.66 & 5.08 & 9.19 \\
\hline \multirow[t]{4}{*}{938} & 0.82 & 22.10 & 2.15 & 0.63 & & & & \\
\hline & & & & & 928 & -41.62 & -162.57 & 167.82 \\
\hline & & & & & 934 & 137.73 & 292.66 & 323.45 \\
\hline & & & & & 3060 & -100.31 & $1-130.33$ & 164.46 \\
\hline \multirow[t]{6}{*}{939} & 1.37 & 13.80 & 0.00 & 0.00 & & & & \\
\hline & & & & & 870 & 57.55 & 48.98 & 75.57 \\
\hline & & & & & 948 & 39.39 & 11.95 & 41.16 \\
\hline & & & & & 973 & -189.36 & -43.55 & 194.30 \\
\hline & & & & & 1056 & 28.07 & -23.55 & 36.64 \\
\hline & & & & & 3147 & 61.69 & -49.09 & 78.84 \\
\hline \multirow[t]{4}{*}{940} & 1.00 & 22.24 & 0.00 & 0.00 & & & & \\
\hline & & & & & 941 & 110.31 & 27.90 & 113.78 \\
\hline & & & & & 945 & -19.17 & 14.57 & 24.08 \\
\hline & & & & & 963 & -93.42 & -204.54 & 224.87 \\
\hline \multirow[t]{4}{*}{941} & 0.99 & 21.07 & 0.00 & 0.00 & & & & \\
\hline & & & & & 940 & -109.73 & -26.16 & 112.80 \\
\hline & & & & & 3058 & 28.40 & -6.81 & 29.21 \\
\hline & & & & & 3061 & 84.37 & 13.39 & 85.43 \\
\hline \multirow[t]{6}{*}{942} & 0.35 & 19.07 & 0.00 & 0.00 & & & & \\
\hline & & & & & 882 & -47.80 & -36.03 & 59.86 \\
\hline & & & & & 935 & -72.05 & -144.27 & 161.26 \\
\hline & & & & & 935 & -72.05 & -144.27 & 161.26 \\
\hline & & & & & 935 & -72.06 & -144.27 & 161.26 \\
\hline & & & & & 935 & -72.06 & -144.27 & 161.26 \\
\hline \multirow[t]{3}{*}{943} & 0.93 & 21.94 & 0.00 & 0.00 & & & & \\
\hline & & & & & 884 & 21.16 & 13.22 & 24.95 \\
\hline & & & & & 931 & -20.21 & 18.50 & 27.40 \\
\hline \multirow[t]{3}{*}{945} & 0.99 & 22.85 & 0.00 & 0.00 & & & & \\
\hline & & & & & 940 & 20.24 & -17.47 & 26.74 \\
\hline & & & & & 3063 & -35.57 & -0.02 & 35.57 \\
\hline
\end{tabular}




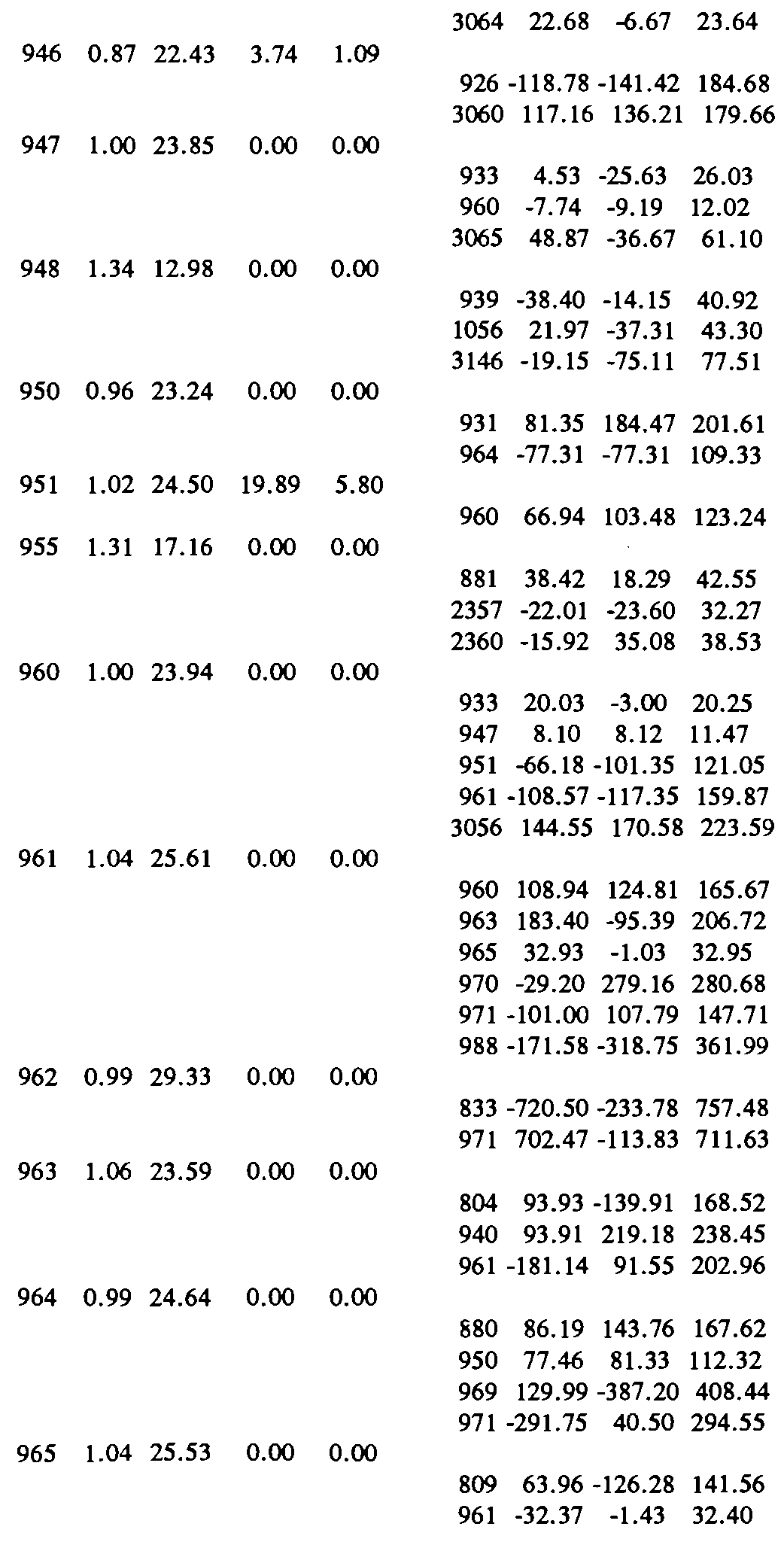




\begin{tabular}{|c|c|c|c|c|c|c|c|c|}
\hline \multirow{3}{*}{966} & \multirow{3}{*}{1.25} & \multirow{3}{*}{21.10} & \multirow{3}{*}{90.21} & \multirow[b]{2}{*}{26.31} & 968 & \multicolumn{2}{|c|}{$341.16-166.82$} & \multirow[t]{2}{*}{379.76} \\
\hline & & & & & & & & \\
\hline & & & & & 972 & 30.64 & -299.03 & 300.60 \\
\hline \multirow[t]{5}{*}{967} & 1.36 & 17.64 & 0.00 & 0.00 & & & & \\
\hline & & & & & 843 & 41.11 & 75.42 & 85.90 \\
\hline & & & & & 973 & 133.89 & -102.06 & 168.35 \\
\hline & & & & & 975 & -176.46 & 205.25 & 270.68 \\
\hline & & & & & 2361 & 4.39 & -125.11 & 125.19 \\
\hline \multirow[t]{3}{*}{968} & 1.04 & 25.51 & 0.00 & 0.00 & & & & \\
\hline & & & & & 933 & 119.88 & 132.23 & 178.48 \\
\hline & & & & & 965 & -341.15 & 166.88 & 379.78 \\
\hline \multirow[t]{9}{*}{969} & 1.18 & 20.54 & 0.00 & 0.00 & & & & \\
\hline & & & & & 857 & 133.20 & 153.65 & 203.35 \\
\hline & & & & & 964 & -115.88 & 438.25 & 453.31 \\
\hline & & & & & 972 & -59.48 & -244.04 & 251.19 \\
\hline & & & & & 984 & -468.49 & 191.93 & 506.28 \\
\hline & & & & & 1372 & 256.51 & $1-206.49$ & 329.30 \\
\hline & & 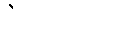 & & & 1374 & 286.31 & $1-233.49$ & 369.45 \\
\hline & & & & & 1388 & 183.98 & $8-309.87$ & 360.37 \\
\hline & & & & & 3100 & -195.01 & 1320.01 & 374.75 \\
\hline \multirow[t]{4}{*}{970} & 0.91 & 27.14 & 0.00 & 0.00 & & & & \\
\hline & & & & & 935 & 396.94 & 984.01 & 1061.05 \\
\hline & & & & & 961 & 36.31 & -269.49 & 271.92 \\
\hline & & & & & 971 & -427.98 & -741.88 & 856.48 \\
\hline \multirow[t]{9}{*}{971} & 0.98 & 28.85 & 0.00 & 0.00 & & & & \\
\hline & & & & & 830 & -373.47 & -195.78 & 421.68 \\
\hline & & & & & 831 & -251.28 & -279.80 & 376.07 \\
\hline & & & & & 961 & 107.47 & -126.91 & 166.30 \\
\hline & & & & & 962 & -697.13 & 118.17 & 707.07 \\
\hline & & & & & 964 & 296.81 & -32.53 & 298.59 \\
\hline & & & & & 970 & 437.50 & 806.95 & 917.91 \\
\hline & & & & & 974 & 250.33 & -374.31 & 450.30 \\
\hline & & & & & 3100 & 208.56 & $6-285.15$ & 353.28 \\
\hline \multirow[t]{6}{*}{972} & 1.25 & 21.09 & 0.00 & 0.00 & & & & \\
\hline & & & & & 906 & 13.08 & 408.23 & 408.44 \\
\hline & & & & & 966 & -30.57 & 299.16 & 300.72 \\
\hline & & & & & 969 & 63.43 & 231.34 & 239.88 \\
\hline & & & & & 1373 & 170.55 & $5-298.55$ & 343.83 \\
\hline & & & & & 3204 & -280.25 & $5-419.30$ & 504.33 \\
\hline \multirow[t]{5}{*}{973} & 1.38 & 15.44 & 0.00 & 0.00 & & & & \\
\hline & & & & & 939 & 189.56 & 49.35 & 195.88 \\
\hline & & & & & 967 & -134.98 & 74.42 & 154.14 \\
\hline & & & & & 974 & -178.06 & 575.27 & 602.19 \\
\hline & & & & & 1085 & 121.61 & $1-236.73$ & 266.14 \\
\hline \multirow[t]{4}{*}{974} & 1.24 & 18.44 & 0.00 & 0.00 & & & & \\
\hline & & & & & 898 & 38.00 & 71.87 & 81.30 \\
\hline & & & & & 971 & -225.53 & 470.21 & 521.50 \\
\hline & & & & & 973 & 187.23 & -533.27 & 565.18 \\
\hline 975 & 1.30 & 20.41 & 0.00 & 0.00 & & & & \\
\hline
\end{tabular}




\begin{tabular}{|c|c|c|c|c|c|c|c|c|}
\hline & & & & & 897 & 38.39 & 114.33 & 120.60 \\
\hline & & & & & 967 & $178.96-$ & -216.83 & 281.15 \\
\hline & & & & & 3204 & -211.43 & 158.31 & 264.13 \\
\hline \multirow[t]{3}{*}{976} & 1.15 & 18.04 & 0.00 & 0.00 & & & & \\
\hline & & & & & 916 & 128.64 & 138.50 & 189.02 \\
\hline & & & & & 1229 & -124.10 & -75.24 & 145.12 \\
\hline \multirow[t]{4}{*}{982} & 1.16 & 24.12 & 0.00 & 0.00 & & & & \\
\hline & & & & & $983-$ & -878.50 & 181.73 & 897.10 \\
\hline & & & & & 984 & 501.79 & -204.90 & 542.01 \\
\hline & & & & & 1247 & 484.07 & -125.94 & 500.19 \\
\hline \multirow[t]{5}{*}{983} & 1.13 & 29.73 & 0.00 & 0.00 & & & & \\
\hline & & & & & 832 & -846.07 & -410.04 & 940.20 \\
\hline & & & & & 982 & 943.55 & -298.50 & 989.64 \\
\hline & & & & & $986-$ & -596.36 & -81.37 & 601.88 \\
\hline & & & & & 987 & 606.59 & 258.30 & 659.29 \\
\hline \multirow[t]{3}{*}{984} & 1.16 & 24.00 & 0.00 & 0.00 & & & & \\
\hline & & & & & 969 & 470.35 & -160.31 & 496.92 \\
\hline & & & & & 982 & -501.57 & 206.00 & 542.23 \\
\hline \multirow[t]{3}{*}{986} & 1.13 & 29.86 & 0.00 & 0.00 & & & & \\
\hline & & & & & 834 & -652.52 & -294.82 & 716.03 \\
\hline & & & & & 983 & 597.71 & 79.72 & 603.00 \\
\hline \multirow[t]{4}{*}{987} & 1.10 & 27.05 & 0.00 & 0.00 & & & & \\
\hline & & & & & 817 & 432.82 & -78.64 & 439.91 \\
\hline & & & & & 983. & -562.79 & -378.10 & 678.01 \\
\hline & & & & & 988 & 213.62 & 358.93 & 417.69 \\
\hline \multirow[t]{3}{*}{988} & 1.10 & 27.02 & 0.00 & 0.00 & & & & \\
\hline & & & & & 961 & 172.81 & 340.93 & 382.23 \\
\hline & & & & & 987 & -213.48 & -358.20 & 416.99 \\
\hline \multirow[t]{2}{*}{996} & 1.19 & 4.54 & 0.00 & 0.00 & & & & \\
\hline & & & & & 1080 & 8.89 & -5.79 & 10.61 \\
\hline \multirow[t]{2}{*}{997} & 2.01 & -10.03 & 0.00 & 0.00 & & & & \\
\hline & & & & & 1049 & 14.22 & 93.97 & 95.04 \\
\hline \multirow[t]{2}{*}{998} & 1.50 & -6.63 & 0.00 & 0.00 & & & & \\
\hline & & & & & 1051 & 6.14 & 21.07 & 21.95 \\
\hline \multirow[t]{2}{*}{999} & 1.55 & -1.77 & 0.00 & 0.00 & & & & \\
\hline & & & & & 1052 & 6.47 & 97.57 & 97.79 \\
\hline \multirow[t]{2}{*}{1000} & 1.42 & 1.95 & 0.00 & 0.00 & & & & \\
\hline & & & & & 1037 & -0.38 & -8.95 & 8.96 \\
\hline \multirow[t]{2}{*}{1001} & 1.64 & 11.07 & 0.00 & 0.00 & & & & \\
\hline & & & & & 1070 & 33.47 & 145.31 & 149.12 \\
\hline \multirow[t]{2}{*}{1002} & 1.49 & 11.23 & 0.00 & 0.00 & & & & \\
\hline & & & & & 1070 & 32.27 & 49.42 & 59.02 \\
\hline 1003 & 1.54 & 10.76 & 0.00 & 0.00 & 1070 & 74.26 & 200.00 & 213.34 \\
\hline \multirow[t]{2}{*}{1004} & 1.61 & 15.19 & 0.00 & 0.00 & & & & \\
\hline & & & & & 1086 & 119.48 & 268.17 & 293.58 \\
\hline \multirow[t]{2}{*}{1005} & 1.68 & 14.92 & 0.00 & 0.00 & & & & \\
\hline & & & & & 1086 & 57.95 & 204.91 & 212.94 \\
\hline 1006 & 1.67 & -8.89 & 0.00 & 0.00 & & & & \\
\hline
\end{tabular}




\begin{tabular}{|c|c|c|c|c|c|c|c|c|}
\hline \multirow{3}{*}{1007} & & & & & 1026 & 7.48 & 11.70 & 13.89 \\
\hline & 1.93 & -9.17 & 0.00 & 0.00 & & & & \\
\hline & & & & & 1026 & 7.31 & 96.83 & 97.10 \\
\hline \multirow[t]{4}{*}{1011} & 1.31 & 9.67 & 0.00 & 0.00 & & & & \\
\hline & & & & & 1027 & -6.10 & -1.87 & 6.38 \\
\hline & & & & & 1028 & 0.65 & -0.62 & 0.90 \\
\hline & & & & & 3135 & 2.20 & -0.14 & 2.20 \\
\hline \multirow[t]{3}{*}{1012} & 1.43 & -1.08 & 5.14 & 1.50 & & & & \\
\hline & & & & & 1020 & 15.64 & -7.11 & 17.18 \\
\hline & & & & & 1037 & -22.73 & 5.53 & 23.39 \\
\hline \multirow[t]{3}{*}{1013} & 1.49 & -3.23 & 2.80 & 0.82 & & & & \\
\hline & & & & & 1018 & -39.11 & 64.31 & 75.27 \\
\hline & & & & & 1026 & 29.53 & -66.40 & 72.67 \\
\hline \multirow[t]{2}{*}{1014} & 1.44 & 1.70 & 5.42 & 1.58 & & & & \\
\hline & & & & & 1037 & -6.19 & -1.37 & 6.34 \\
\hline \multirow[t]{2}{*}{1015} & 1.43 & -3.12 & 4.84 & 1.25 & & & & \\
\hline & & & & & 1016 & -13.05 & -0.40 & 13.06 \\
\hline \multirow[t]{4}{*}{1016} & 1.43 & -3.05 & 0.00 & 0.00 & & & & \\
\hline & & & & & 1015 & 13.07 & 0.42 & 13.08 \\
\hline & & & & & 1017 & -4.14 & -8.67 & 9.61 \\
\hline & & & & & 1020 & -11.81 & 6.61 & 13.53 \\
\hline \multirow[t]{3}{*}{1017} & 1.43 & -3.05 & 3.27 & 0.95 & & & & \\
\hline & & & & & 1016 & 4.11 & 8.54 & 9.48 \\
\hline & & & & & 1035 & -4.44 & -8.84 & 9.89 \\
\hline \multirow[t]{5}{*}{1018} & 1.48 & -2.58 & 0.00 & 0.00 & & & & \\
\hline & & & & & 1013 & 39.43 & -63.53 & 74.77 \\
\hline & & & & & 1024 & 131.35 & 37.34 & 136.56 \\
\hline & & & & & 1042 & 11.54 & 8.20 & 14.16 \\
\hline & & & & & 1082 & -184.28 & 63.65 & 194.97 \\
\hline \multirow[t]{4}{*}{1019} & 1.40 & -6.46 & 0.00 & 0.00 & & & & \\
\hline & & & & & 1021 & -29.92 & 39.99 & 49.95 \\
\hline & & & & & 1032 & 23.81 & 8.75 & 25.37 \\
\hline & & & & & 1054 & 0.15 & -71.83 & 71.83 \\
\hline \multirow[t]{3}{*}{1020} & 1.43 & -1.98 & 5.04 & 1.47 & & & & \\
\hline & & & & & 1012 & -15.68 & 6.62 & 17.02 \\
\hline & & & & & 1016 & 11.61 & -7.69 & 13.93 \\
\hline \multirow[t]{4}{*}{1021} & 1.37 & -2.82 & 0.00 & 0.00 & & & & \\
\hline & & & & & 1019 & 31.27 & -38.19 & 49.36 \\
\hline & & & & & 3154 & -25.14 & -12.14 & 27.92 \\
\hline & & & & & 3160 & -50.71 & 29.56 & 58.69 \\
\hline \multirow[t]{3}{*}{1022} & 1.37 & 3.60 & 17.00 & 4.96 & & & & \\
\hline & & & & & 1036 & -25.88 & -8.88 & 27.36 \\
\hline & & & & & 3156 & -2.84 & -2.36 & 3.69 \\
\hline \multirow[t]{3}{*}{1023} & 1.35 & -5.96 & 0.00 & 0.00 & & & & \\
\hline & & & & & 1025 & -48.91 & 28.84 & 56.78 \\
\hline & & & & & 1030 & 40.66 & -32.05 & 51.77 \\
\hline \multirow[t]{3}{*}{1024} & 1.45 & -4.08 & 0.00 & 0.00 & & & & \\
\hline & & & & & 1018 & -129.57 & -33.51 & 133.84 \\
\hline & & & & & 3173 & 6.84 & 1.77 & 7.07 \\
\hline
\end{tabular}




\begin{tabular}{|c|c|c|c|c|c|c|c|c|}
\hline & & & & & 3180 & 117.42 & 31.68 & 121.62 \\
\hline \multirow[t]{4}{*}{1025} & 1.35 & -5.23 & 0.00 & 0.00 & & & & \\
\hline & & & & & 1023 & 49.11 & -28.35 & 56.71 \\
\hline & & & & & 1045 & -95.53 & 11.94 & 96.28 \\
\hline & & & & & 3174 & 4.78 & 1.70 & 5.08 \\
\hline \multirow[t]{6}{*}{1026} & 1.63 & -9.79 & 0.00 & 0.00 & & & & \\
\hline & & & & & 1006 & -7.47 & -11.29 & 13.54 \\
\hline & & & & & 1007 & -7.08 & -81.95 & 82.26 \\
\hline & & & & & 1013 & -24.75 & 73.47 & 77.53 \\
\hline & & & & & 1044 & -0.60 & .78 .45 & 78.45 \\
\hline & & & & & 3176 & 31.74 & 99.59 & 104.53 \\
\hline \multirow[t]{4}{*}{1027} & 1.32 & 9.84 & 4.76 & 1.39 & & & & \\
\hline & & & & & 1011 & 6.09 & 1.36 & 6.24 \\
\hline & & & & & 1056 & -16.36 & -54.07 & 56.49 \\
\hline & & & & & 3139 & 2.68 & 6.88 & 7.38 \\
\hline \multirow[t]{2}{*}{1028} & 1.31 & 9.64 & 1.21 & 0.35 & & & & \\
\hline & & & & & 1011 & -0.68 & 0.09 & 0.68 \\
\hline \multirow[t]{4}{*}{1029} & 1.41 & -7.74 & 0.00 & 0.00 & & & & \\
\hline & & & & & 1033 & 27.95 & 51.92 & 58.97 \\
\hline & & & & & 1076 & -112.67 & -4.52 & 112.76 \\
\hline & & & & & 3176 & -152.49 & -181.52 & 237.07 \\
\hline \multirow[t]{4}{*}{1030} & 1.36 & -7.38 & 0.00 & 0.00 & & & & \\
\hline & & & & & 1023 & -40.31 & 32.89 & 52.03 \\
\hline & & & & & 1033 & -3.27 & -46.31 & 46.42 \\
\hline & & & & & 3177 & -21.12 & -8.23 & 22.66 \\
\hline \multirow[t]{4}{*}{1031} & 1.30 & 10.14 & 0.00 & 0.00 & & & & \\
\hline & & & & & 870 & -12.90 & -1.53 & 12.99 \\
\hline & & & & & 3136 & -5.79 & -10.07 & 11.62 \\
\hline & & & & & 3139 & -0.30 & -8.03 & 8.04 \\
\hline \multirow[t]{3}{*}{1032} & 1.39 & -6.85 & 8.59 & 2.51 & & & & \\
\hline & & & & & 1019 & -23.80 & -8.88 & 25.40 \\
\hline & & & & & 3155 & -21.58 & -9.74 & 23.67 \\
\hline \multirow[t]{4}{*}{1033} & 1.39 & -7.65 & 0.00 & 0.00 & & & & \\
\hline & & & & & 1029 & -27.42 & -51.17 & 58.05 \\
\hline & & & & & 1030 & 3.45 & 46.74 & 46.87 \\
\hline & & & & & 3178 & -27.30 & -4.07 & 27.60 \\
\hline \multirow[t]{2}{*}{1034} & 1.44 & -3.29 & 3.92 & 1.14 & & & & \\
\hline & & & & & 1035 & -5.61 & -1.72 & 5.87 \\
\hline \multirow[t]{4}{*}{1035} & 1.45 & -3.10 & 0.00 & 0.00 & & & & \\
\hline & & & & & 1017 & 4.19 & 7.82 & 8.87 \\
\hline & & & & & 1034 & 5.40 & 1.06 & 5.50 \\
\hline & & & & & 1042 & -9.87 & -8.36 & 12.94 \\
\hline \multirow[t]{5}{*}{1036} & 1.38 & 4.10 & 18.86 & 5.50 & & & & \\
\hline & & & & & 895 & 24.73 & 47.34 & 53.41 \\
\hline & & & & & 1022 & 26.00 & 8.77 & 27.44 \\
\hline & & & & & 1083 & -129.08 & -54.66 & 140.17 \\
\hline & & & & & 3153 & 57.41 & -25.98 & 63.01 \\
\hline \multirow[t]{2}{*}{1037} & 1.45 & 1.99 & 7.56 & 2.21 & & & & \\
\hline & & & & & 1000 & 0.38 & 9.19 & 9.20 \\
\hline
\end{tabular}




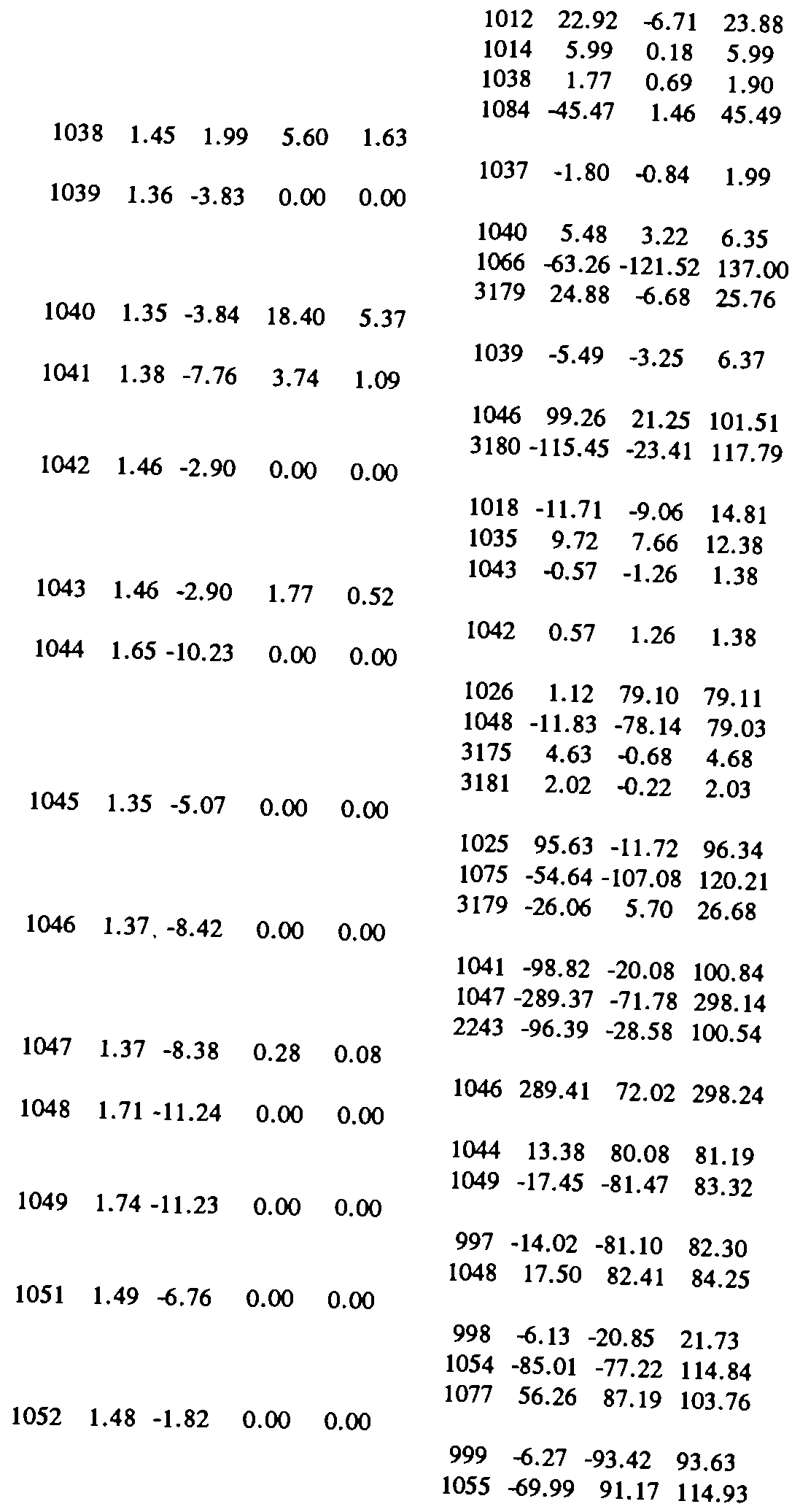




\begin{tabular}{|c|c|c|c|c|c|c|c|c|}
\hline 1053 & 1.41 & -4.46 & 0.00 & 0.00 & 3165 & 43.92 & -13.71 & 46.01 \\
\hline \multirow{8}{*}{1054} & \multirow{7}{*}{1.50} & \multirow{8}{*}{-6.64} & \multirow{7}{*}{0.00} & \multirow{7}{*}{0.00} & 1057 & 101.03 & 9.22 & 101.45 \\
\hline & & & & & 1075 & -27.68 & -2.30 & 27.78 \\
\hline & & & & & 2257 & -101.50 & -16.43 & 3102.82 \\
\hline & & & & & & & & \\
\hline & & & & & 1019 & 0.08 & 76.65 & 76.65 \\
\hline & & & & & 1051 & 85.38 & 77.57 & 115.36 \\
\hline & & & & & 3158 & -42.29 & 35.13 & 54.97 \\
\hline & & & & & 3163 & -42.38 & 35.56 & 55.32 \\
\hline \multirow{4}{*}{1055} & 1.48 & -1.43 & 0.00 & 0.00 & & & & \\
\hline & & & & & 1052 & 70.31 & -90.87 & 114.89 \\
\hline & & & & & 3162 & -2.59 & -2.45 & 3.56 \\
\hline & & & & & 3163 & 87.47 & -74.83 & 115.11 \\
\hline \multirow[t]{8}{*}{1056} & 1.37 & 10.39 & 0.00 & 0.00 & 3166 & -159.70 & 167.77 & 7231.62 \\
\hline & & & & & 939 & -26.61 & 15.90 & 31.00 \\
\hline & & & & & 948 & -20.37 & 31.82 & 37.78 \\
\hline & & & & & 1027 & 16.48 & 56.45 & 58.81 \\
\hline & & & & & 1070 & 9.05 & -37.32 & 38.40 \\
\hline & & & & & 1071 & 177.41 & 90.82 & 199.31 \\
\hline & & & & & 2503 & -81.46 & -80.02 & 114.18 \\
\hline & & & & & 2505 & -76.44 & -35.23 & 84.17 \\
\hline \multirow[t]{4}{*}{1057} & 1.40 & -4.79 & 0.00 & 0.00 & & & & \\
\hline & & & & & 1053 & -100.75 & -8.93 & 101.15 \\
\hline & & & & & 3187 & -94.41 & -111.25 & 145.91 \\
\hline & & & & & 3188 & 17.97 & -69.01 & 71.31 \\
\hline \multirow[t]{4}{*}{1058} & 1.46 & -6.83 & 0.00 & 0.00 & & & & \\
\hline & & & & & 1077 & -71.67 & -94.84 & 118.87 \\
\hline & & & & & 3164 & -79.20 & -26.20 & 83.42 \\
\hline & & & & & 3188 & -1.01 & 71.42 & 71.42 \\
\hline 1059 & 1.40 & $-2.0 Z$ & 12.00 & 3.31 & & & & \\
\hline \multirow[t]{2}{*}{1060} & 1.21 & 4.46 & 33.90 & 9.89 & 3165 & -109.18 & -22.05 & 111.39 \\
\hline & & & & & 1061 & 132.59 & 195.39 & 236.13 \\
\hline \multirow[t]{3}{*}{1061} & 1.20 & 4.46 & 25.12 & 7.33 & $1063-$ & -185.58 & -161.39 & 245.94 \\
\hline & & & & & $1060-$ & -131.09 & -193.32 & 233.58 \\
\hline & & & & & 1079 & 84.12 & 170.31 & 189.95 \\
\hline \multirow[t]{2}{*}{1062} & 1.19 & 4.47 & 5.42 & 1.58 & & & & \\
\hline & 1.26 & & & & $1079-$ & $-148.82-$ & -231.48 & 275.19 \\
\hline \multirow{3}{*}{1063} & & 8.81 & .00 & 0.60 & 1060 & 189.08 & 171.75 & 255.44 \\
\hline & & & & & $1071-$ & $-192.93-$ & -175.70 & 260.94 \\
\hline & & & & & 1072 & -2.11 & -5.64 & 6.02 \\
\hline \multirow[t]{4}{*}{1066} & 1.43 & -2.44 & 0.00 & 0.00 & & & & \\
\hline & & & & & 1039 & 63.64 & 129.87 & 144.63 \\
\hline & & & & & 1075 & 72.05 & -14.69 & 73.53 \\
\hline & & & & & $3189-$ & -135.92 & 8.49 & 136.18 \\
\hline
\end{tabular}




\begin{tabular}{|c|c|c|c|c|c|c|c|c|}
\hline \multirow[t]{3}{*}{1067} & \multirow[t]{3}{*}{1.44} & \multirow[t]{3}{*}{1.59} & \multirow[t]{2}{*}{7.19} & \multirow[t]{2}{*}{2.10} & \multirow{2}{*}{\multicolumn{2}{|c|}{$1073-177.98$}} & \multirow[b]{2}{*}{-14.31} & \multirow[b]{2}{*}{178.55} \\
\hline & & & & & & & & \\
\hline & & & & & 3189 & 148.41 & -3.10 & 148.44 \\
\hline 1068 & 1.34 & 8.25 & 0.19 & 0.05 & & & & \\
\hline \multirow{7}{*}{1069} & & & & & 1071 & -9.28 & -14.83 & 17.50 \\
\hline & 1.40 & 9.10 & 0.00 & 0.00 & & & & \\
\hline & & & & & 368 & 61.63 & 91.71 & 110.50 \\
\hline & & & & & 1070 & -20.90 & -257.77 & 258.62 \\
\hline & & & & & 1071 & 32.00 & 55.95 & 64.45 \\
\hline & & & & & 1085 & -131.46 & -74.29 & 151.00 \\
\hline & & & & & 3149 & 31.81 & 53.51 & 62.25 \\
\hline \multirow[t]{8}{*}{1070} & 1.41 & 9.06 & 3.64 & 1.06 & & & & \\
\hline & & & & & 1001 & -33.15 & -123.90 & 128.26 \\
\hline & & & & & 1002 & -32.22 & -45.49 & 55.75 \\
\hline & & & & & 1003 & -73.34 & -180.89 & 195.19 \\
\hline & & & & & 1056 & -9.09 & 32.00 & 33.26 \\
\hline & & & & & 1069 & 21.21 & 259.23 & 260.10 \\
\hline & & & & & 3147 & -53.15 & 47.22 & 71.10 \\
\hline & & & & & 3166 & 178.64 & -139.50 & 226.65 \\
\hline \multirow[t]{6}{*}{1071} & 1.34 & 8.27 & 41.74 & 12.18 & & & & \\
\hline & & & & & 1056 & -176.12 & -83.88 & 195.08 \\
\hline & & & & & 1063 & 195.93 & 192.15 & 274.42 \\
\hline & & & & & 1068 & 9.21 & 14.31 & 17.02 \\
\hline & & & & & 1069 & -32.48 & -60.59 & 68.75 \\
\hline & & & & & 3149 & -32.53 & -61.39 & 69.47 \\
\hline \multirow[t]{2}{*}{1072} & 1.26 & 5.87 & 1.31 & 0.38 & & & & \\
\hline & & & & & 1063 & 1.96 & 4.90 & 5.27 \\
\hline \multirow{2}{*}{1073} & 1.45 & 2.07 & 0.00 & 0.00 & & & & \\
\hline & & & & & 1067 & 178.15 & 15.32 & 178.81 \\
\hline \multirow{4}{*}{1075} & 1.42 & -3.89 & & & 2506 & -148.68 & 59.54 & 160.16 \\
\hline & & 500 & 0.00 & 0.00 & 1045 & 54.94 & 113.39 & 126.00 \\
\hline & & & & & 1053 & 27.13 & 0.21 & 27.13 \\
\hline & & & & & 1066 & -71.76 & 14.08 & 73.12 \\
\hline \multirow[t]{3}{*}{1076} & 1.42 & -4.25 & 0.00 & 0.00 & & & & \\
\hline & & & & & 1029 & 113.04 & 11.46 & 113.62 \\
\hline & & & & & 2253 & -123.14 & 19.54 & 124.68 \\
\hline \multirow{4}{*}{1077} & 1.48 & -6.76 & 0.00 & 0.00 & & & & \\
\hline & & & & & 1051 & -56.01 & -87.01 & 103.48 \\
\hline & & & & & 1058 & 72.28 & 95.54 & 119.80 \\
\hline & & & & & 1078 & -3.18 & -5.60 & 6.44 \\
\hline \multirow[t]{2}{*}{1078} & 1.48 & -6.73 & 0.00 & 0.00 & & & & \\
\hline & & & & & 1077 & 1.88 & 2.44 & 3.09 \\
\hline \multirow{3}{*}{1079} & 1.19 & 4.49 & 0.00 & 0.00 & & & & \\
\hline & & & & & 1061 & $-83.63-$ & -169.67 & 189.16 \\
\hline & & & & & 1062 & 148.87 & 231.74 & 275.44 \\
\hline \multirow{2}{*}{1080} & & & & & $1080-$ & -104.02 & -140.70 & 174.98 \\
\hline & & & & & 996 & -8.89 & 5.79 & 10.61 \\
\hline
\end{tabular}




\begin{tabular}{|c|c|c|c|c|c|c|c|c|}
\hline \multirow[b]{2}{*}{1082} & \multirow[b]{2}{*}{1.44} & \multirow[b]{2}{*}{4.59} & \multirow[b]{2}{*}{0.00} & \multirow[b]{2}{*}{0.00} & 1079 & \multirow[t]{2}{*}{104.04} & \multirow[t]{2}{*}{140.81} & \multirow[t]{2}{*}{175.08} \\
\hline & & & & & & & & \\
\hline & & & & & $\begin{array}{l}1018 \\
1083\end{array}$ & $\begin{array}{r}185.18 \\
-206.03\end{array}$ & $\begin{array}{c}-38.96 \\
-9.09\end{array}$ & $\begin{array}{r}189.23 \\
206\end{array}$ \\
\hline & & & & & & & -9.09 & 206.23 \\
\hline & & & & & 1084 & 24.79 & -66.99 & 71.43 \\
\hline & & & & & 2240 & -26.54 & 68.30 & 73.27 \\
\hline \multirow[t]{4}{*}{1083} & 1.45 & 8.97 & 0.00 & 0.00 & & & & \\
\hline & & & & & 1036 & 129.79 & 68.50 & 146.76 \\
\hline & & & & & 1082 & 198.59 & -33.80 & 201.45 \\
\hline & & & & & 1085 & -334.30 & -14.35 & 334.61 \\
\hline \multirow[t]{4}{*}{1084} & 1.45 & 3.70 & 0.00 & 0.00 & & & & \\
\hline & & & & & 1037 & 45.51 & -0.11 & 45.51 \\
\hline & & & & & 1082 & -39.48 & -1.53 & 39.51 \\
\hline & & & & & 2507 & -20.41 & 5.26 & 21.08 \\
\hline \multirow[t]{6}{*}{1085} & 1.46 & 12.93 & 0.00 & 0.00 & & & & \\
\hline & & & & & $973-$ & 121.76 & 212.03 & 244.50 \\
\hline & & & & & 1069 & 132.12 & 86.79 & 158.08 \\
\hline & & & & & 1083 & 334.56 & 6.27 & 334.62 \\
\hline & & & & & 1086 & -186.78 & -182.98 & 261.47 \\
\hline & & & & & 2502 & -204.37 & -175.47 & 269.37 \\
\hline \multirow[t]{4}{*}{1086} & 1.47 & 12.98 & 0.00 & 0.00 & & & & \\
\hline & & & & & 1004 & -117.97 & -239.48 & 3266.96 \\
\hline & & & & & 1005 & -56.60 & -177.02 & 185.85 \\
\hline & & & & & 1085 & 186.72 & 182.50 & 261.10 \\
\hline \multirow[t]{2}{*}{1087} & 1.28 & 21.74 & 60.14 & 17.54 & & & & \\
\hline & & & & & 3204 & -37.95 & -67.74 & 77.64 \\
\hline \multirow[t]{2}{*}{1097} & 1.34 & 6.11 & 0.00 & 0.00 & & & & \\
\hline & & & & & 1213 & 2.12 & 45.97 & 46.02 \\
\hline 1098 & 1.29 & 3.84 & 0.00 & 0.00 & & & & \\
\hline \multirow[t]{2}{*}{1099} & 1.33 & 22.52 & 0.00 & 0.00 & 1129 & 0.31 & 21.73 & 21.74 \\
\hline & & & & & 1155 & 92.19 & 58.79 & 109.34 \\
\hline \multirow[t]{2}{*}{1100} & 1.44 & 21.94 & 0.00 & 0.00 & & & & \\
\hline & & & & & 1155 & 92.20 & 124.89 & 155.23 \\
\hline \multirow[t]{2}{*}{1101} & 1.27 & 22.83 & 0.00 & 0.00 & & & & \\
\hline & & & & & 1155 & 92.19 & 25.97 & 95.78 \\
\hline \multirow[t]{4}{*}{1107} & 1.29 & 4.97 & 0.00 & 0.00 & & & & \\
\hline & & & & & 1108 & -7.60 & -0.01 & 7.60 \\
\hline & & & & & 1154 & 14.00 & -16.73 & 21.82 \\
\hline & & & & & 1157 & -27.84 & 13.52 & 30.95 \\
\hline \multirow[t]{2}{*}{1108} & 1.29 & 4.98 & 4.39 & 1.28 & & & & \\
\hline & & & & & 1107 & 7.60 & 0.01 & 7.60 \\
\hline 1109 & 1.11 & 6.11 & 0.00 & 0.00 & & & & \\
\hline & & & & & 1110 & -2.58 & -2.98 & 3.94 \\
\hline & & & & & 1161 & 1.16 & -2.25 & 2.53 \\
\hline & & & & & 1192 & -8.26 & -5.49 & 9.92 \\
\hline 1110 & 1.11 & 6.12 & 0.00 & 0.00 & & & & \\
\hline & & & & & 1109 & 2.57 & 2.93 & 3.90 \\
\hline
\end{tabular}




\begin{tabular}{|c|c|c|c|c|c|c|c|c|}
\hline 1112 & 1.39 & 3.69 & 0.00 & 0.00 & 1162 & -8.97 & -0.67 & 8.99 \\
\hline & & & & & 1144 & -23.80 & 16.59 & 29.01 \\
\hline & & & & & 1156 & 11.08 & -19.31 & 22.26 \\
\hline & & & & & 1162 & 8.17 & 0.88 & 8.22 \\
\hline 1114 & 1.09 & 4.66 & 5.14 & 1.50 & & & & \\
\hline 1115 & 1.06 & 6.00 & 0.00 & 0.00 & 1117 & -43.08 & -53.91 & 69.01 \\
\hline & & & & & 1116 & 3.01 & 1.53 & 3.37 \\
\hline & & & & & 1170 & -98.75 & -47.27 & 109.48 \\
\hline 1116 & 1.06 & & & & 1181 & 86.98 & 42.97 & 97.02 \\
\hline 1117 & 1.09 & 4.68 & 0.00 & 0.00 & 1115 & -3.01 & -1.55 & 3.38 \\
\hline & & & & & 1114 & 43.08 & 53.95 & 69.04 \\
\hline 1118 & 1.07 & 4.70 & 0.00 & 0.00 & 1177 & -21.41 & -23.55 & 31.83 \\
\hline & & & & & 1119 & 0.78 & -1.76 & 1.92 \\
\hline & & & & & 1147 & -15.04 & -38.99 & 41.79 \\
\hline 1119 & 1.07 & 4.70 & 3.45 & 1.01 & 1153 & -27.06 & -19.48 & 33.34 \\
\hline 1120 & 1.28 & 6.00 & 5.60 & 1.63 & 1118 & -0.78 & 1.71 & 1.88 \\
\hline & & & & & 1131 & -1.56 & -0.88 & 1.79 \\
\hline 1121 & 1.09 & 6.63 & 0.00 & 0.00 & 1157 & -4.94 & -1.10 & 5.06 \\
\hline & & & & & 1122 & -51.58 & -57.91 & 77.55 \\
\hline & & & & & 1142 & -26.92 & -69.03 & 74.09 \\
\hline 1122 & 1.09 & 6.64 & & & 1204 & -21.00 & 22.83 & 31.02 \\
\hline 1123 & 1.08 & 6.10 & 1.68 & 0.49 & 1121 & 51.59 & 57.93 & 77.57 \\
\hline 1125 & 1.08 & 6.18 & 0.37 & 0.11 & 1127 & -12.52 & -6.58 & 14.14 \\
\hline 1126 & 1.07 & 6.05 & 0.00 & 0.00 & 1128 & -9.00 & -4.17 & 9.91 \\
\hline 1127 & 1.08 & 6.12 & 0.00 & 0.00 & 1195 & -77.91 & -76.32 & 109.06 \\
\hline & & & & & 1123 & 12.52 & 6.56 & 14.13 \\
\hline & & & & & 1128 & -46.36 & -38.60 & 60.32 \\
\hline & & & & & 1195 & 28.32 & 29.18 & 40.66 \\
\hline 1128 & 1.08 & 6.20 & 0.00 & 0.00 & & & & \\
\hline & & & & & 1125 & 9.00 & 4.15 & 9.91 \\
\hline & & & & & 1127 & 46.45 & 38.76 & 60.50 \\
\hline 1129 & 1.26 & 5.85 & 1083 & & 1173 & -59.77 & -48.79 & 77.15 \\
\hline & & 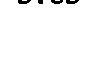 & 10.00 & 3.10 & 1098 & -0.55 & -21.22 & 21.23 \\
\hline & & & & & 1130 & 20.14 & -21.52 & 29.48 \\
\hline & & & & & 1144 & 13.64 & -83.48 & 84.59 \\
\hline
\end{tabular}




\begin{tabular}{|c|c|c|c|c|c|c|c|c|}
\hline \multirow{2}{*}{1130} & & & & 327 & 1236 & -111.43 & 155.55 & 191.34 \\
\hline & 1.27 & 5.25 & 11.21 & 3.27 & 1129 & -20.02 & 21.59 & 29.44 \\
\hline & & & & & 1150 & 8.95 & -24.67 & 26.24 \\
\hline \multirow[t]{4}{*}{1131} & 1.28 & 6.07 & 0.00 & 0.00 & & & & \\
\hline & & & & & 1120 & 1.50 & 0.14 & 1.51 \\
\hline & & & & & 1132 & -6.14 & -0.82 & 6.20 \\
\hline & & & & & 1157 & -11.55 & -2.88 & 11.91 \\
\hline \multirow[t]{2}{*}{1132} & 1.28 & 6.08 & 3.27 & 0.95 & & & & \\
\hline & & & & & 1131 & 6.14 & 0.79 & 6.19 \\
\hline \multirow[t]{3}{*}{1133} & 1.08 & 6.83 & 0.00 & 0.00 & & & & \\
\hline & & & & & $\begin{array}{l}1170 \\
1173\end{array}$ & 102.51 & $\begin{array}{r}47.81 \\
-19.30\end{array}$ & $\begin{array}{l}113.11 \\
31.49\end{array}$ \\
\hline & & & & & 1230 & -137.65 & -65.32 & 152.36 \\
\hline 1134 & 1.13 & 12.22 & 5.60 & 1.63 & & & & \\
\hline \multirow[t]{3}{*}{1135} & 1.13 & 12.24 & 0.00 & 0.00 & 1135 & -113.37 & -170.20 & 204.50 \\
\hline & & & & & 1134 & 113.40 & 170.36 & 204.65 \\
\hline & & & & & 1137 & 20.04 & 10.69 & 22.71 \\
\hline \multirow{2}{*}{1136} & 1.08 & 722 & 1588 & & 1155 & -69.44 & -81.54 & 107.10 \\
\hline & 1.00 & & 15.00 & 4.03 & 1204 & -20.68 & -9.45 & 22.74 \\
\hline \multirow[t]{4}{*}{1137} & 1.12 & 11.52 & 6.44 & 1.88 & & & & \\
\hline & & & & & 1135 & -19.93 & -10.81 & 22.67 \\
\hline & & & & & 1344 & 41.00 & 25.20 & 48.12 \\
\hline & 124 & & & & 1350 & -30.45 & -20.86 & 36.91 \\
\hline \multirow{2}{*}{1138} & 1.24 & 12.08 & 11.02 & 3.21 & 1158 & -37.23 & 16.07 & 40.55 \\
\hline & & & & & 1302 & 23.87 & -24.56 & 34.25 \\
\hline \multirow[t]{3}{*}{1139} & 1.11 & 5.06 & 0.00 & 0.00 & & & & \\
\hline & & & & & 1175 & 39.70 & -13.87 & 42.06 \\
\hline & & & & & 1192 & -41.12 & 12.34 & 42.93 \\
\hline 1140 & 1.28 & 0.30 & 0.00 & 0.00 & 1157 & -12.28 & -6.90 & 14.09 \\
\hline \multirow[t]{4}{*}{1141} & 1.13 & 6.60 & 0.00 & 0.00 & & & & \\
\hline & & & & & 1149 & -66.76 & -68.17 & 95.42 \\
\hline & & & & & 1168 & 55.11 & 63.69 & 84.23 \\
\hline & & & & & 1397 & 2.63 & 1.30 & 2.94 \\
\hline \multirow[t]{3}{*}{1142} & 1.10 & 6.73 & 0.00 & 0.00 & & & & \\
\hline & & & & & 1121 & 27.01 & 69.56 & 74.62 \\
\hline & & & & & 1182 & -41.88 & -72.97 & 84.13 \\
\hline \multirow{3}{*}{1143} & 127 & 1126 & 10.27 & 300 & 1197 & 7.68 & 1.35 & 7.79 \\
\hline & & & & & 1151 & 65.16 & -61.94 & 89.90 \\
\hline & & & & & 1155 & -76.41 & 57.15 & 95.42 \\
\hline
\end{tabular}




\begin{tabular}{|c|c|c|c|c|c|c|c|c|}
\hline & & & & & 1112 & 23.83 & -16.57 & 29.02 \\
\hline & & & & & 1129 & -11.97 & 90.88 & 91.66 \\
\hline & & & & & 1190 & 5.40 & -11.48 & 12.68 \\
\hline & & & & & 3332 & -26.12 & -65.11 & 70.15 \\
\hline \multirow[t]{4}{*}{1145} & 1.29 & 7.13 & 3.08 & 0.90 & & & & \\
\hline & & & & & 1157 & 18.20 & 4.04 & 18.64 \\
\hline & & & & & 1187 & -27.30 & -8.81 & 28.69 \\
\hline & & & & & 1200 & 2.91 & -0.24 & 2.92 \\
\hline \multirow[t]{2}{*}{1147} & 1.07 & 4.71 & 6.26 & 1.82 & & & & \\
\hline & & & & & 1118 & 15.04 & 39.00 & 41.80 \\
\hline \multirow[t]{3}{*}{1148} & 1.12 & 6.64 & 0.00 & 0.00 & & & & \\
\hline & & & & & 1149 & -27.94 & -64.97 & 70.72 \\
\hline & & & & & 1173 & 26.06 & 62.97 & 68.15 \\
\hline \multirow[t]{7}{*}{1149} & 1.14 & 6.89 & 1.12 & 0.33 & & & & \\
\hline & & & & & 1141 & 67.33 & 69.40 & 96.69 \\
\hline & & & & & 1148 & 28.17 & 66.16 & 71.90 \\
\hline & & & & & 1171 & 5.38 & 1.17 & 5.50 \\
\hline & & & & & 1177 & 33.80 & 28.04 & 43.92 \\
\hline & & & & & 1218 & -30.42 & -43.08 & 52.74 \\
\hline & & & & & 1232 & -106.11 & -26.27 & 109.32 \\
\hline \multirow[t]{3}{*}{1150} & 1.30 & 4.27 & 13.91 & 4.06 & & & & \\
\hline & & & & & 1130 & -8.75 & 24.69 & 26.19 \\
\hline & & & & & 1154 & -8.65 & 15.12 & 17.42 \\
\hline \multirow[t]{4}{*}{1151} & 1.31 & 8.64 & 25.22 & 7.35 & & & & \\
\hline & & & & & 1143 & -64.20 & 66.19 & 92.21 \\
\hline & & & & & 1187 & 33.50 & 8.89 & 34.66 \\
\hline & & & & & 1196 & 4.74 & 0.06 & 4.75 \\
\hline \multirow[t]{3}{*}{1152} & 1.05 & 5.67 & 22.88 & 6.67 & & & & \\
\hline & & & & & 1184 & 32.22 & 12.30 & 34.49 \\
\hline & & & & & 1204 & -72.51 & -30.31 & 78.59 \\
\hline \multirow[t]{4}{*}{1153} & 1.08 & 5.07 & 0.00 & 0.00 & & & & \\
\hline & & & & & 1118 & 27.10 & 19.61 & 33.45 \\
\hline & & & & & 1176 & -32.39 & -22.88 & 39.66 \\
\hline & & & & & 3103 & 1.88 & -0.28 & 1.90 \\
\hline \multirow[t]{4}{*}{1154} & 1.29 & 4.37 & 0.00 & 0.00 & & & & \\
\hline & & & & & 1107 & -13.94 & 16.61 & 21.69 \\
\hline & & & & & 1150 & 8.66 & -15.16 & 17.46 \\
\hline & & & & & 3104 & 7.57 & 0.60 & 7.59 \\
\hline \multirow[t]{8}{*}{1155} & 1.24 & 14.83 & 4.30 & 1.25 & & & & \\
\hline & & & & & 1099 & -92.06 & -42.59 & 101.44 \\
\hline & & & & & 1100 & -91.97 & -96.74 & 133.48 \\
\hline & & & & & 1101 & -92.08 & -12.50 & 92.92 \\
\hline & & & & & 1135 & 71.62 & 91.43 & 116.14 \\
\hline & & & & & 1143 & 77.66 & -51.51 & 93.19 \\
\hline & & & & & 1158 & 39.77 & -16.43 & 43.03 \\
\hline & & & & & 1234 & 72.93 & -88.58 & 114.74 \\
\hline \multirow[t]{3}{*}{1156} & 1.41 & 2.44 & 14.57 & 4.25 & & & & \\
\hline & & & & & 1112 & -11.02 & 18.86 & 21.84 \\
\hline & & & & & 1205 & -4.01 & 32.24 & 32.49 \\
\hline
\end{tabular}




\begin{tabular}{|c|c|c|c|c|c|c|c|c|}
\hline \multirow[t]{7}{*}{1157} & 1.28 & 6.38 & 28.67 & 8.36 & & & & \\
\hline & & & & & 1107 & 28.08 & -13.33 & 31.09 \\
\hline & & & & & 1120 & 4.87 & -0.10 & 4.87 \\
\hline & & & & & 1131 & 11.52 & 2.45 & 11.78 \\
\hline & & & & & 1140 & 12.29 & 6.88 & 14.08 \\
\hline & & & & & 1145 & -18.21 & -4.46 & 18.75 \\
\hline & & & & & 1182 & -59.49 & 171.26 & 181.30 \\
\hline \multirow[t]{3}{*}{1158} & 1.24 & 13.46 & 2.99 & 0.87 & & & & \\
\hline & & & & & 1138 & 37.36 & -15.81 & 40.57 \\
\hline & & & & & 1155 & -39.53 & 16.97 & 43.02 \\
\hline \multirow[t]{2}{*}{1159} & 1.08 & 5.21 & 0.00 & 0.00 & & & & \\
\hline & & & & & 1176 & -7.00 & -2.87 & 7.56 \\
\hline \multirow[t]{4}{*}{1160} & 1.22 & 5.04 & 0.00 & 0.00 & & & & \\
\hline & & & & & 1129 & -39.89 & -62.20 & 73.89 \\
\hline & & & & & 1193 & 33.50 & 60.16 & 68.86 \\
\hline & & & & & 3105 & 4.54 & 0.32 & 4.55 \\
\hline \multirow[t]{3}{*}{1161} & 1.11 & 6.10 & 0.00 & 0.00 & & & & \\
\hline & & & & & 1109 & -1.16 & 2.21 & 2.49 \\
\hline & & & & & 3106 & 3.05 & 0.82 & 3.16 \\
\hline \multirow[t]{4}{*}{1162} & 1.38 & 3.46 & 0.00 & 0.00 & & & & \\
\hline & & & & & 1111 & 8.96 & 0.64 & 8.99 \\
\hline & & & & & 1112 & -8.21 & -1.33 & 8.32 \\
\hline & & & & & 3195 & 1.61 & 0.06 & 1.61 \\
\hline \multirow[t]{3}{*}{1163} & 1.07 & 5.67 & 0.00 & 0.00 & & & & \\
\hline & & & & & 1168 & -52.39 & -60.12 & 79.74 \\
\hline & & & & & 1172 & 49.04 & 58.71 & 76.50 \\
\hline \multirow[t]{2}{*}{1164} & 1.04 & 4.99 & 18.30 & 5.34 & & & & \\
\hline & & & & & 1179 & -53.37 & -9.41 & 54.19 \\
\hline \multirow[t]{2}{*}{1165} & 1.04 & 5.16 & 5.42 & 1.58 & & & & \\
\hline & & & & & 1181 & -18.61 & -6.01 & 19.56 \\
\hline \multirow[t]{2}{*}{1166} & 1.04 & 4.99 & 3.27 & 0.95 & & & & \\
\hline & & & & & 1183 & -77.97 & -64.14 & 100.97 \\
\hline \multirow[t]{2}{*}{1167} & 1.10 & 6.13 & 0.19 & 0.05 & & & & \\
\hline & & & & & 1168 & -6.20 & -2.00 & 6.51 \\
\hline \multirow[t]{4}{*}{1168} & 1.10 & 6.15 & 0.00 & 0.00 & & & & \\
\hline & & & & & 1141 & -54.24 & -61.84 & 82.25 \\
\hline & & & & & 1163 & 53.27 & 62.02 & 81.75 \\
\hline & & & & & 1167 & 6.20 & 1.98 & 6.50 \\
\hline 1169 & 1.07 & 6.54 & 8.31 & 2.42 & & & & \\
\hline \multirow{4}{*}{1170} & & & & & 1170 & -10.20 & -3.14 & 10.67 \\
\hline & 1.08 & 0.62 & 0.00 & 0.00 & & & & \\
\hline & & & & & 1113 & $\begin{array}{r}99.49 \\
10270\end{array}$ & 48.90 & 110.85 \\
\hline & & & & & 1130 & -102.29 & -47.28 & 112.69 \\
\hline \multirow[t]{2}{*}{1171} & 1.14 & & & & 1169 & 10.21 & 3.07 & 10.66 \\
\hline & & & & & 1149 & -5.38 & -1.31 & 5.54 \\
\hline \multirow[t]{3}{*}{1172} & 1.05 & 5.56 & 1.40 & 0.41 & & & & \\
\hline & & & & & 1163 & -48.20 & -57.77 & 75.23 \\
\hline & & & & & 1209 & 8.69 & 9.09 & 12.58 \\
\hline
\end{tabular}




\begin{tabular}{|c|c|c|c|c|c|c|c|c|}
\hline \multirow[t]{4}{*}{1173} & 1.08 & 6.26 & 0.00 & 0.00 & & & & \\
\hline & & & & & 1128 & 59.82 & 48.92 & 77.27 \\
\hline & & & & & 1133 & -24.76 & 19.45 & 31.49 \\
\hline & & & & & 1148 & -25.64 & -61.03 & 66.20 \\
\hline \multirow[t]{2}{*}{1174} & 1.05 & 5.51 & 4.02 & 1.17 & & & & \\
\hline & & & & & 1188 & -8.93 & -2.58 & 9.30 \\
\hline \multirow[t]{4}{*}{1175} & 1.12 & 3.75 & 4.86 & 1.42 & & & & \\
\hline & & & & & 1139 & -39.55 & 14.43 & 42.10 \\
\hline & & & & & 1193 & 19.93 & -20.79 & 28.80 \\
\hline & & & & & 3107 & 1.80 & 1.45 & 2.31 \\
\hline \multirow[t]{4}{*}{1176} & 1.08 & 5.23 & 0.00 & 0.00 & & & & \\
\hline & & & & & 1153 & 32.41 & 22.96 & 39.72 \\
\hline & & & & & 1159 & 6.99 & 2.85 & 7.55 \\
\hline & & & & & 1201 & -67.43 & -76.61 & 102.06 \\
\hline \multirow[t]{3}{*}{1177} & 1.10 & 5.01 & 12.79 & 3.73 & & & & \\
\hline & & & & & 1117 & 21.45 & 23.69 & 31.96 \\
\hline & & & & & 1149 & -33.45 & -26.60 & 42.74 \\
\hline \multirow[t]{4}{*}{1179} & 1.04 & 5.01 & 0.00 & 0.00 & & & & \\
\hline & & & & & 1164 & 53.40 & 9.43 & 54.22 \\
\hline & & & & & 1181 & -80.17 & -39.26 & 89.27 \\
\hline & & & & & 1183 & 21.01 & 20.64 & 29.45 \\
\hline \multirow[t]{4}{*}{1181} & 1.05 & 5.18 & 0.00 & 0.00 & & & & \\
\hline & & & & & 1115 & -86.16 & -41.13 & 95.47 \\
\hline & & & & & 1165 & 18.62 & 6.02 & 19.57 \\
\hline & & & & & 1179 & 80.47 & 39.69 & 89.72 \\
\hline \multirow[t]{4}{*}{1182} & 1.21 & 8.50 & 3.83 & 1.12 & & & & \\
\hline & & & & & 1142 & 43.43 & 80.68 & 91.63 \\
\hline & & & & & 1157 & 61.87 & -159.24 & 170.84 \\
\hline & & & & & 1235 & -110.17 & 49.12 & 120.62 \\
\hline \multirow[t]{3}{*}{1183} & 1.04 & 5.00 & 0.00 & 0.00 & & & & \\
\hline & & & & & 1166 & 77.98 & 64.19 & 101.00 \\
\hline & & & & & 1179 & -21.00 & -20.62 & 29.44 \\
\hline \multirow[t]{3}{*}{1184} & 1.05 & 5.62 & 0.00 & 0.00 & & & & \\
\hline & & & & & 1152 & -32.21 & -12.27 & 34.47 \\
\hline & & & & & 1188 & 33.82 & 16.16 & 37.48 \\
\hline \multirow[t]{3}{*}{1187} & 1.30 & 7.42 & 7.47 & 2.18 & & & & \\
\hline & & & & & 1145 & 27.32 & 8.76 & 28.69 \\
\hline & & & & & 1151 & -33.39 & -8.76 & 34.52 \\
\hline \multirow[t]{4}{*}{1188} & 1.05 & 5.55 & 0.00 & 0.00 & & & & \\
\hline & & & & & 1174 & 8.94 & 2.57 & 9.30 \\
\hline & & & & & 1184 & -33.78 & -16.17 & 37.45 \\
\hline & & & & & 1209 & -6.51 & -32.61 & 33.26 \\
\hline \multirow[t]{4}{*}{1189} & 1.32 & 4.70 & 10.27 & 3.00 & & & & \\
\hline & & & & & 1129 & -26.21 & 120.35 & 123.17 \\
\hline & & & & & 1190 & -2.78 & -89.05 & 89.09 \\
\hline & & & & & 1203 & 17.91 & -34.17 & 38.57 \\
\hline \multirow[t]{3}{*}{1190} & 1.39 & 3.66 & 31.75 & 9.26 & & & & \\
\hline & & & & & 1144 & -5.47 & 11.02 & 12.30 \\
\hline & & & & & 1189 & 4.47 & 93.04 & 93.15 \\
\hline
\end{tabular}




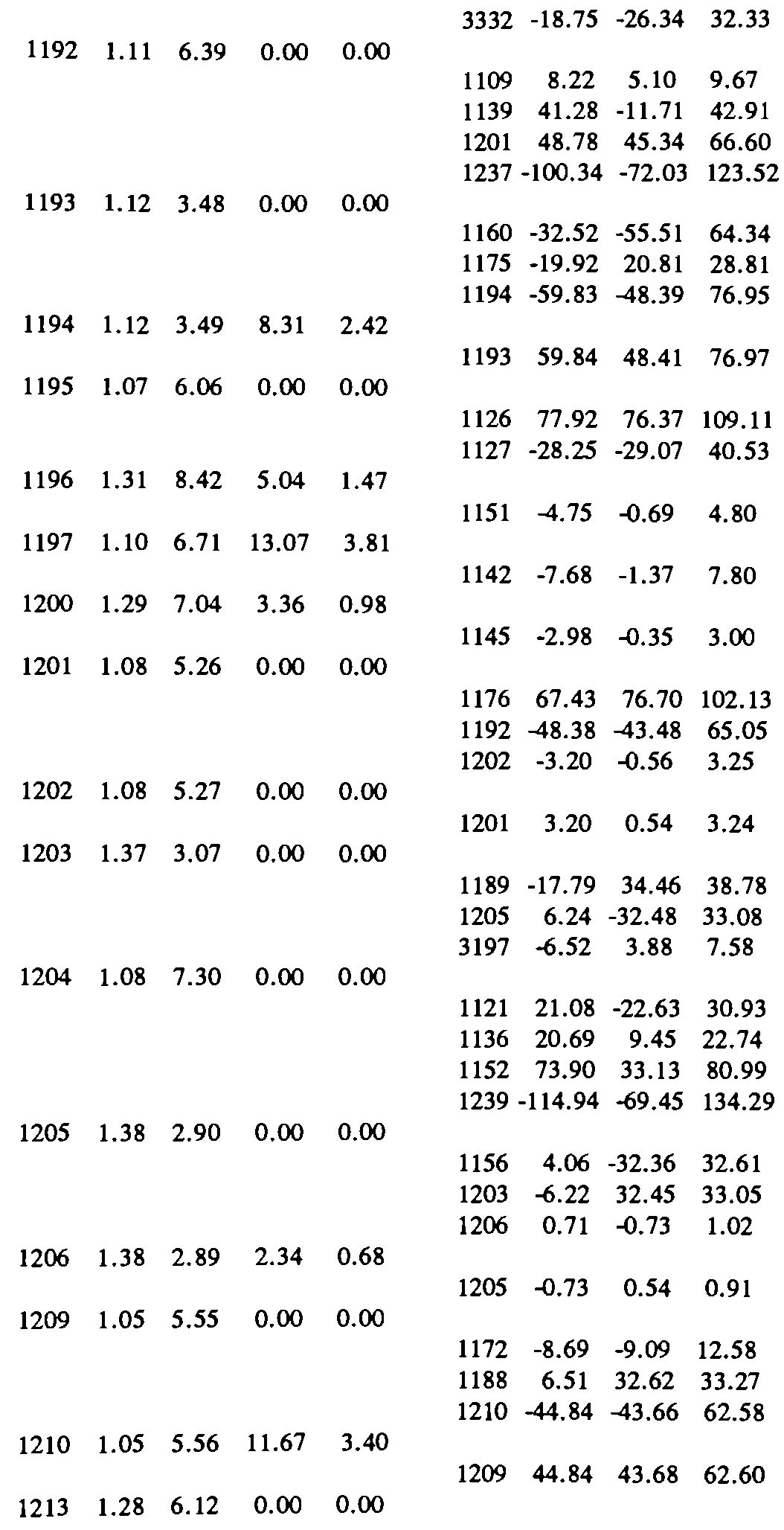




\begin{tabular}{|c|c|c|c|c|c|c|c|c|}
\hline & & & & & 1097 & -2.02 & -44.01 & 44.06 \\
\hline & & & & & 1129 & 10.63 & 27.63 & 29.60 \\
\hline & & & & & 1217 & -6.51 & 72.93 & 73.22 \\
\hline \multirow[t]{3}{*}{1217} & 1.20 & 7.77 & 5.04 & 1.47 & & & & \\
\hline & & & & & 1213 & 7.92 & -70.66 & 71.11 \\
\hline & & & & & 1218 & $3-14.04$ & 69.81 & 71.21 \\
\hline \multirow[t]{6}{*}{1218} & 1.19 & 8.26 & 0.00 & 0.00 & & & & \\
\hline & & & & & 1149 & 30.55 & 45.54 & 54.84 \\
\hline & & & & & 1217 & 14.50 & -68.94 & 70.45 \\
\hline & & & & & 1219 & 22.67 & 5.87 & 23.42 \\
\hline & & & & & 1221 & 4.96 & 1.00 & 5.06 \\
\hline & & & & & 1232 & -82.43 & 70.07 & 108.19 \\
\hline \multirow[t]{2}{*}{1219} & 1.18 & 8.18 & 25.31 & 7.38 & & & & \\
\hline & & & & & 1218 & -22.65 & -6.06 & 23.45 \\
\hline \multirow[t]{2}{*}{1220} & 1.19 & 8.22 & 3.36 & 0.98 & & & & \\
\hline & & & & & 1221 & -12.99 & -9.20 & 15.92 \\
\hline \multirow[t]{3}{*}{1221} & 1.19 & 8.24 & 0.00 & 0.00 & & & & \\
\hline & & & & & 1218 & -4.97 & -1.25 & 5.13 \\
\hline & & & & & 1220 & 12.97 & 9.07 & 15.83 \\
\hline \multirow[t]{4}{*}{1229} & 1.16 & 18.66 & 0.00 & 0.00 & & & & \\
\hline & & & & & 976 & 125.03 & 70.50 & 143.53 \\
\hline & & & & & 1248 & -460.26 & 36.27 & 461.69 \\
\hline & & & & & 2201 & 339.18 & -66.28 & 345.59 \\
\hline \multirow[t]{5}{*}{1230} & 1.13 & 11.58 & 0.00 & 0.00 & & & & \\
\hline & & & & & 1133 & 138.04 & 80.15 & 159.63 \\
\hline & & & & & 1232 & 264.83 & -315.19 & 411.68 \\
\hline & & & & & 1609 & -78.57 & 363.24 & 371.64 \\
\hline & & & & & 2201 & -331.23 & -109.69 & 348.92 \\
\hline \multirow[t]{3}{*}{1231} & 1.25 & 15.41 & 0.00 & 0.00 & & & & \\
\hline & & & & & 1233 & -253.84 & -163.52 & 301.95 \\
\hline & & & & & 1235 & 250.87 & 155.82 & 295.32 \\
\hline \multirow[t]{7}{*}{1232} & 1.16 & 9.92 & 0.00 & 0.00 & & & & \\
\hline & & & & & 1149 & 106.38 & 32.40 & 111.21 \\
\hline & & & & & 1218 & 82.59 & -66.19 & 105.84 \\
\hline & & & & & 1230 & -262.89 & 323.83 & 417.11 \\
\hline & & & & & 1235 & -136.84 & -86.91 & 162.11 \\
\hline & & & & & 1236 & 110.44 & -178.27 & 209.70 \\
\hline & & & & & 1237 & 97.30 & 25.87 & 100.68 \\
\hline \multirow[t]{4}{*}{1233} & 1.28 & 16.83 & 3.92 & 1.14 & & & & \\
\hline & & & & & 467 & -258.22 & -154.62 & 300.98 \\
\hline & & & & & 1231 & 254.78 & 163.40 & 302.68 \\
\hline & & & & & 2900 & -1.03 & -12.20 & 12.24 \\
\hline \multirow[t]{4}{*}{1234} & 1.26 & 13.81 & 0.00 & 0.00 & & & & \\
\hline & & & & & 1155 & -72.76 & 91.65 & 117.02 \\
\hline & & & & & 1235 & 165.98 & 180.65 & 245.33 \\
\hline & & & & & 1381 & -87.14 & -108.79 & 139.39 \\
\hline \multirow[t]{3}{*}{1235} & 1.19 & 11.43 & 0.00 & 0.00 & & & & \\
\hline & & & & & 1182 & 110.46 & -42.62 & 118.40 \\
\hline & & & & & 1231 & -248.75 & -153.29 & 292.19 \\
\hline
\end{tabular}




\begin{tabular}{|c|c|c|c|c|c|c|c|c|}
\hline & & & & & $\begin{array}{l}1232 \\
1234\end{array}$ & $\begin{array}{r}135.97 \\
-165.27\end{array}$ & $\begin{array}{r}75.66 \\
-187.71\end{array}$ & $\begin{array}{l}155.61 \\
250.09 \\
271\end{array}$ \\
\hline \multirow[t]{3}{*}{1236} & 1.21 & 7.82 & 0.00 & 0.00 & 1044 & 165.07 & 332.81 & 371.50 \\
\hline & & & & & 1129 & 111.66 & -145.07 & 183.06 \\
\hline & & & & & 1232 & -111.54 & 167.76 & 201.45 \\
\hline \multirow[t]{3}{*}{1237} & 1.15 & 8.51 & 0.00 & 0.00 & & & & \\
\hline & & & & & 1192 & 100.59 & 78.00 & 127.28 \\
\hline & & & & & 1232 & -99.36 & -42.99 & 108.26 \\
\hline \multirow[t]{4}{*}{1239} & 1.14 & 11.02 & 0.00 & 0.00 & & & & \\
\hline & & & & & 1204 & 115.53 & 80.48 & 140.80 \\
\hline & & & & & 1609 & -96.83 & 156.54 & 184.06 \\
\hline & & & & & 1644 & -19.73 & -187.73 & 188.76 \\
\hline \multirow[t]{3}{*}{1247} & 1.16 & 22.36 & 0.00 & 0.00 & & & & \\
\hline & & & & & 982 & -437.07 & -83.41 & 444.96 \\
\hline & & & & & 1248 & 482.14 & -49.81 & 484.71 \\
\hline \multirow{2}{*}{1248} & & 22.20 & 0.00 & 0.00 & 1229 & 461.87 & -7.37 & 461.93 \\
\hline & & & & & 1247 & -481.97 & 50.69 & 484.63 \\
\hline \multirow[t]{2}{*}{1261} & 1.32 & 13.41 & 0.00 & 0.00 & & & & \\
\hline & & & & & 1318 & -0.04 & -0.27 & 0.28 \\
\hline \multirow[t]{2}{*}{1262} & 1.45 & 18.56 & 0.00 & 0.00 & & & & \\
\hline & & & & & 1385 & 51.51 & 85.36 & 99.70 \\
\hline 1263 & 1.50 & 18.44 & 0.00 & 0.00 & & & & \\
\hline \multirow[t]{2}{*}{1264} & 1.45 & 18.57 & 0.00 & 0.00 & 1000 & 31.11 & $152 . / 5$ & 101.29 \\
\hline & & & & & 1386 & 51.51 & 82.45 & 97.22 \\
\hline \multirow[t]{2}{*}{1265} & 1.53 & 18.36 & 0.00 & 0.00 & & & & \\
\hline & & & & & 1386 & 51.96 & 198.42 & 205.11 \\
\hline 1266 & 1.39 & 18.67 & 0.00 & 0.00 & 1207 & & & \\
\hline \multirow[t]{2}{*}{1267} & 1.52 & 18.37 & 0.00 & 0.00 & 1001 & 41.15 & 3.02 & 47.23 \\
\hline & & & & & 1387 & 51.93 & 197.26 & 203.98 \\
\hline \multirow[t]{2}{*}{1268} & 1.12 & 9.76 & 5.42 & 1.58 & & & & \\
\hline & & & & & 1323 & -6.09 & -2.65 & 6.64 \\
\hline \multirow[t]{2}{*}{1269} & 1.75 & 17.09 & 0.00 & 0.00 & & & & \\
\hline & & & & & 1327 & 23.89 & 212.37 & 213.71 \\
\hline \multirow[t]{2}{*}{1270} & 1.60 & 18.80 & 0.00 & 0.00 & & & & \\
\hline & 1.66 & 18.73 & 0.00 & 0.00 & 1365 & 78.50 & 181.95 & 198.16 \\
\hline 1271 & 2.00 & ני. & . & & 1365 & 79.60 & 275.00 & 286.29 \\
\hline \multirow[t]{2}{*}{1272} & 1.50 & 15.96 & 0.00 & 0.00 & & & & \\
\hline & & & & & 1327 & 0.17 & 7.78 & 7.79 \\
\hline \multirow[t]{2}{*}{1273} & 1.56 & 17.18 & 0.00 & 0.00 & & & & \\
\hline & & & & & 1365 & 5.46 & 121.10 & 121.22 \\
\hline \multirow[t]{4}{*}{1274} & 1.13 & 9.97 & 0.00 & 0.00 & & & & \\
\hline & & & & & 1275 & -65.84 & -116.88 & 134.15 \\
\hline & & & & & 1323 & -141.35 & -215.72 & 257.91 \\
\hline & & & & & 1390 & -31.39 & -64.99 & 72.17 \\
\hline
\end{tabular}


$\begin{array}{lllll}1275 & 1.13 & 9.98 & 5.51 & 1.61\end{array}$

$\begin{array}{lllll}1276 & 1.10 & 10.13 & 0.00 & 0.00\end{array}$

$\begin{array}{lllll}1277 & 1.23 & 11.61 & 0.00 & 0.00\end{array}$

$\begin{array}{lllll}1278 & 1.23 & 11.47 & 10.74 & 3.13\end{array}$

$\begin{array}{lllll}1279 & 1.15 & 15.51 & 0.00 & 0.00\end{array}$

$\begin{array}{lllll}1280 & 1.18 & 11.13 & 1.77 & 0.52\end{array}$

$\begin{array}{lllll}1281 & 1.37 & 13.47 & 53.33 & 15.55\end{array}$

$\begin{array}{lllll}1282 & 1.27 & 11.41 & 26.05 & 7.60\end{array}$

$\begin{array}{lllll}1283 & 1.34 & 15.30 & 10.93 & 3.19\end{array}$

$\begin{array}{lllll}1284 & 1.21 & 11.16 & 47.81 & 13.95\end{array}$

$\begin{array}{lllll}1285 & 1.33 & 12.82 & 8.03 & 2.34\end{array}$

$\begin{array}{lllll}1286 & 1.20 & 11.37 & 10.18 & 2.97\end{array}$

$\begin{array}{lllll}1287 & 1.37 & 13.17 & 51.18 & 14.93\end{array}$

$\begin{array}{lllll}1288 & 1.17 & 10.71 & 34.37 & 10.02\end{array}$

$\begin{array}{lllll}1289 & 1.16 & 14.25 & 18.12 & 5.28\end{array}$ $\begin{array}{llll}280 & 69.16 & 95.35 & 117.79\end{array}$

$\begin{array}{llll}1302 & -78.64 & -98.33 & 125.91\end{array}$

$\begin{array}{llll}1281 & -18.06 & 8.40 & 19.92\end{array}$

$\begin{array}{lllll}1303 & -4.20 & 20.56 & 20.98\end{array}$

$\begin{array}{llll}1336 & -29.88 & 30.60 & 42.77\end{array}$

$\begin{array}{llll}1274 & 65.86 & 116.95 & 134.22\end{array}$

$1346-127.78-157.20 \quad 202.58$

$\begin{array}{llll}1278 & 10.06 & 1.06 & 10.12\end{array}$

$\begin{array}{llll}1340 & -21.66 & -120.56 & 122.49\end{array}$

$\begin{array}{lllll}1341 & -8.88 & -14.03 & 16.61\end{array}$

$\begin{array}{llll}1277 & -10.04 & -1.20 & 10.11\end{array}$

$\begin{array}{lllll}1289 & 31.52 & -19.07 & 36.84\end{array}$

$\begin{array}{llll}1299 & 26.15 & 30.56 & 40.22\end{array}$

$\begin{array}{llll}1372 & -67.81 & -130.51 & 147.07\end{array}$

$\begin{array}{llll}3079 & 5.03 & 17.34 & 18.06\end{array}$

$\begin{array}{llll}1286 & -68.00 & -94.31 & 116.27\end{array}$

$\begin{array}{llll}1324 & -2.45 & -2.62 & 3.59\end{array}$

$\begin{array}{llll}1345 & 63.19 & 89.66 & 109.69\end{array}$

$\begin{array}{llll}1287 & 18.11 & -8.62 & 20.05\end{array}$

$\begin{array}{lllll}1307 & -40.20 & 35.78 & 53.82\end{array}$

$\begin{array}{llll}1308 & 71.61 & 11.63 & 72.55\end{array}$

$\begin{array}{llll}1311 & -45.18 & 35.67 & 57.57\end{array}$

$\begin{array}{lllll}1321 & 49.63 & 53.20 & 72.75\end{array}$

$\begin{array}{llll}1384 & -112.25 & 39.62 & 119.03\end{array}$

$\begin{array}{llll}1317 & -19.92 & 12.16 & 23.33\end{array}$

$\begin{array}{lllll}1338 & -23.57 & 22.51 & 32.59\end{array}$

$\begin{array}{llll}1355 & 15.57 & 19.93 & 25.29\end{array}$

$\begin{array}{llll}3029 & -18.62 & -16.08 & 24.60\end{array}$

$\begin{array}{llll}1324 & 6.81 & 34.33 & 35.00\end{array}$

$\begin{array}{llll}1331 & -56.09 & 1.49 & 56.11\end{array}$

$\begin{array}{llll}1298 & -5.90 & -25.92 & 26.58\end{array}$

$\begin{array}{llll}1347 & -4.33 & 17.55 & 18.08\end{array}$

$1331-104.70-102.13 \quad 146.26$

$\begin{array}{llll}1351 & 65.20 & 82.14 & 104.87\end{array}$ 


\begin{tabular}{|c|c|c|c|c|c|c|c|c|}
\hline & & & & & 1279 & -31.31 & 19.46 & 36.86 \\
\hline & & & & & 1290 & 12.51 & -27.15 & 29.89 \\
\hline \multirow[t]{6}{*}{1290} & 1.17 & 14.01 & 0.00 & 0.00 & & & & \\
\hline & & & & & 1289 & -12.45 & 27.19 & 29.91 \\
\hline & & & & & 1326 & 44.22 & -8.28 & 44.99 \\
\hline & & & & & 1332 & 38.06 & -51.93 & 64.39 \\
\hline & & & & & 1374 & -109.78 & -137.25 & 175.75 \\
\hline & & & & & 3081 & 33.69 & 20.21 & 39.29 \\
\hline \multirow[t]{3}{*}{1291} & 1.23 & 11.57 & 23.25 & 6.78 & & & & \\
\hline & & & & & 1310 & -48.08 & -83.18 & 96.07 \\
\hline & & & & & 1316 & 22.99 & 72.13 & 75.71 \\
\hline \multirow[t]{3}{*}{1292} & 1.40 & 15.48 & 37.73 & 11.00 & & & & \\
\hline & & & & & 1293 & -28.67 & 95.57 & 99.77 \\
\hline & & & & & 1294 & -9.80 & -50.76 & 51.70 \\
\hline \multirow[t]{6}{*}{1293} & 1.37 & 16.21 & 0.00 & 0.00 & & & & \\
\hline & & & & & 1292 & 29.24 & -93.31 & 97.79 \\
\hline & & & & & 1322 & 41.28 & 17.37 & 44.78 \\
\hline & & & & & 1360 & -21.66 & -195.28 & 196.48 \\
\hline & & & & & 1373 & -81.40 & -4.59 & 81.53 \\
\hline & & & & & 3029 & 17.71 & 7.92 & 19.40 \\
\hline \multirow[t]{3}{*}{1294} & 1.45 & 15.47 & 44.64 & 13.02 & & & & \\
\hline & & & & & 1292 & 10.33 & 51.64 & 52.66 \\
\hline & & & & & 1327 & -55.77 & -67.79 & 87.78 \\
\hline \multirow[t]{4}{*}{1295} & 1.32 & 11.77 & 44.27 & 12.91 & & & & \\
\hline & & & & & 1321 & -46.38 & -15.29 & 48.83 \\
\hline & & & & & 1335 & 9.43 & -50.15 & 51.03 \\
\hline & & & & & 1338 & -9.79 & 117.55 & 117.96 \\
\hline \multirow[t]{4}{*}{1296} & 1.22 & 12.81 & 18.49 & 5.39 & & & & \\
\hline & & & & & 1297 & -2.86 & -40.51 & 40.61 \\
\hline & & & & & 1305 & -4.14 & 67.11 & 67.24 \\
\hline & & & & & 1328 & -13.11 & -38.93 & 41.07 \\
\hline \multirow[t]{4}{*}{1297} & 1.23 & 12.75 & 21.11 & 6.16 & & & & \\
\hline & & & & & 1296 & 2.94 & 40.64 & 40.75 \\
\hline & & & & & 1309 & 34.54 & -35.54 & 49.56 \\
\hline & & & & & 1343 & -58.88 & -11.77 & 60.04 \\
\hline \multirow[t]{4}{*}{1298} & 1.34 & 12.83 & 23.72 & 6.92 & & & & \\
\hline & & & & & 1285 & 5.96 & 25.83 & 26.50 \\
\hline & & & & & 1314 & 4.68 & -23.25 & 23.72 \\
\hline & & & & & 1336 & -34.59 & -10.12 & 36.04 \\
\hline \multirow[t]{3}{*}{1299} & 1.08 & 14.95 & 5.14 & 1.50 & & & & \\
\hline & & & & & 1279 & -24.70 & -29.01 & 38.10 \\
\hline & & & & & 3077 & -21.36 & -38.91 & 44.39 \\
\hline \multirow[t]{4}{*}{1300} & 1.15 & 13.94 & 0.00 & 0.00 & & & & \\
\hline & & & & & 1306 & -11.30 & -10.86 & 15.67 \\
\hline & & & & & 3078 & 0.33 & -8.94 & 8.95 \\
\hline & & & & & 3087 & 4.86 & 0.57 & 4.90 \\
\hline \multirow[t]{3}{*}{1301} & 1.13 & 15.94 & 2.89 & 0.84 & & & & \\
\hline & & & & & 1356 & -2.38 & 14.73 & 14.92 \\
\hline & & & & & 3079 & -0.62 & -17.24 & 17.25 \\
\hline
\end{tabular}


$\begin{array}{lllll}1302 & 1.25 & 12.33 & 18.21 & 5.31\end{array}$

$\begin{array}{crcl}1138 & -23.80 & 24.60 & 34.23 \\ 1286 & 79.98 & 103.20 & 130.56 \\ 1304 & 24.17 & 37.70 & 44.79 \\ 1342 & -100.73 & -75.37 & 125.81\end{array}$

$\begin{array}{lllll}1303 & 1.36 & 13.35 & 27.92 & 8.14\end{array}$

$\begin{array}{llll}1287 & 4.27 & -20.80 & 21.23\end{array}$

$\begin{array}{llll}1336 & -32.86 & 10.82 & 34.59\end{array}$

$\begin{array}{lllll}1304 & 1.23 & 12.00 & 27.36 & 7.98\end{array}$

$\begin{array}{lllll}1302 & -24.01 & -37.34 & 44.39\end{array}$

$\begin{array}{llll}1331 & -3.43 & 28.99 & 29.20\end{array}$

$\begin{array}{lllll}1305 & 1.18 & 13.27 & 13.91 & 4.06\end{array}$

$\begin{array}{llll}1296 & 4.55 & -65.52 & 65.67\end{array}$

$\begin{array}{lllll}1306 & -18.17 & 60.83 & 63.48\end{array}$

$\begin{array}{lllll}1306 & 1.16 & 14.02 & 0.00 & 0.00\end{array}$

$\begin{array}{llll}845 & -30.00 & 54.97 & 62.63\end{array}$

$\begin{array}{llll}1300 & 11.38 & 10.77 & 15.67\end{array}$

$\begin{array}{llll}1305 & 18.53 & -59.53 & 62.35\end{array}$

$\begin{array}{lllll}1307 & 1.36 & 13.94 & 6.07 & 1.77\end{array}$

$\begin{array}{llll}1281 & 40.32 & -35.46 & 53.69\end{array}$

$\begin{array}{llll}1311 & -47.57 & 29.34 & 55.89\end{array}$

$\begin{array}{lllll}1308 & 1.35 & 11.79 & 53.51 & 15.61\end{array}$

$\begin{array}{llll}1281 & -71.18 & -9.80 & 71.85\end{array}$

$\begin{array}{llll}1321 & -20.14 & 54.29 & 57.90\end{array}$

$\begin{array}{llll}1335 & 36.14 & 11.16 & 37.82\end{array}$

$\begin{array}{lllll}1309 & 1.24 & 11.84 & 12.98 & 3.79\end{array}$

$\begin{array}{llll}1297 & -34.32 & 36.11 & 49.82\end{array}$

$\begin{array}{llll}1341 & 18.79 & 19.37 & 26.99\end{array}$

$\begin{array}{lllll}1310 & 1.27 & 12.24 & 23.72 & 6.92\end{array}$

$\begin{array}{llll}1291 & 48.71 & 86.24 & 99.05\end{array}$

$\begin{array}{lllll}1318 & -72.68 & -93.96 & 118.79\end{array}$

$\begin{array}{lllll}1311 & 1.36 & 14.31 & 39.32 & 11.47\end{array}$

$\begin{array}{llll}1281 & 45.39 & -35.07 & 57.36\end{array}$

$\begin{array}{llll}1307 & 47.65 & -29.09 & 55.83\end{array}$

$\begin{array}{llll}1337 & 35.24 & -29.52 & 45.97\end{array}$

$1378-183.52-227.05 \quad 291.95$

$\begin{array}{lllll}1312 & 1.25 & 11.51 & 27.83 & 8.12\end{array}$

$\begin{array}{llll}1334 & 4.19 & 73.56 & 73.68\end{array}$

$\begin{array}{llll}1338 & -42.82 & -7.65 & 43.49\end{array}$

$\begin{array}{llll}1355 & 8.63 & -15.13 & 17.42\end{array}$

$\begin{array}{lllll}1313 & 1.11 & 16.20 & 0.00 & 0.00\end{array}$

$\begin{array}{llll}889 & 0.19 & -13.57 & 13.58\end{array}$

$\begin{array}{llll}1356 & 34.86 & -51.45 & 62.15\end{array}$

$\begin{array}{lllll}1314 & 1.37 & 12.44 & 14.29 & 4.17\end{array}$

$\begin{array}{llll}1298 & -4.59 & 22.82 & 23.27\end{array}$

$\begin{array}{llll}1337 & -10.40 & -28.86 & 30.67\end{array}$

$\begin{array}{lllll}1315 & 1.30 & 13.52 & 52.67 & 15.36\end{array}$

$\begin{array}{llll}1317 & 48.76 & 29.15 & 56.81\end{array}$

$\begin{array}{lllll}1321 & 54.96 & -81.83 & 98.57\end{array}$ 


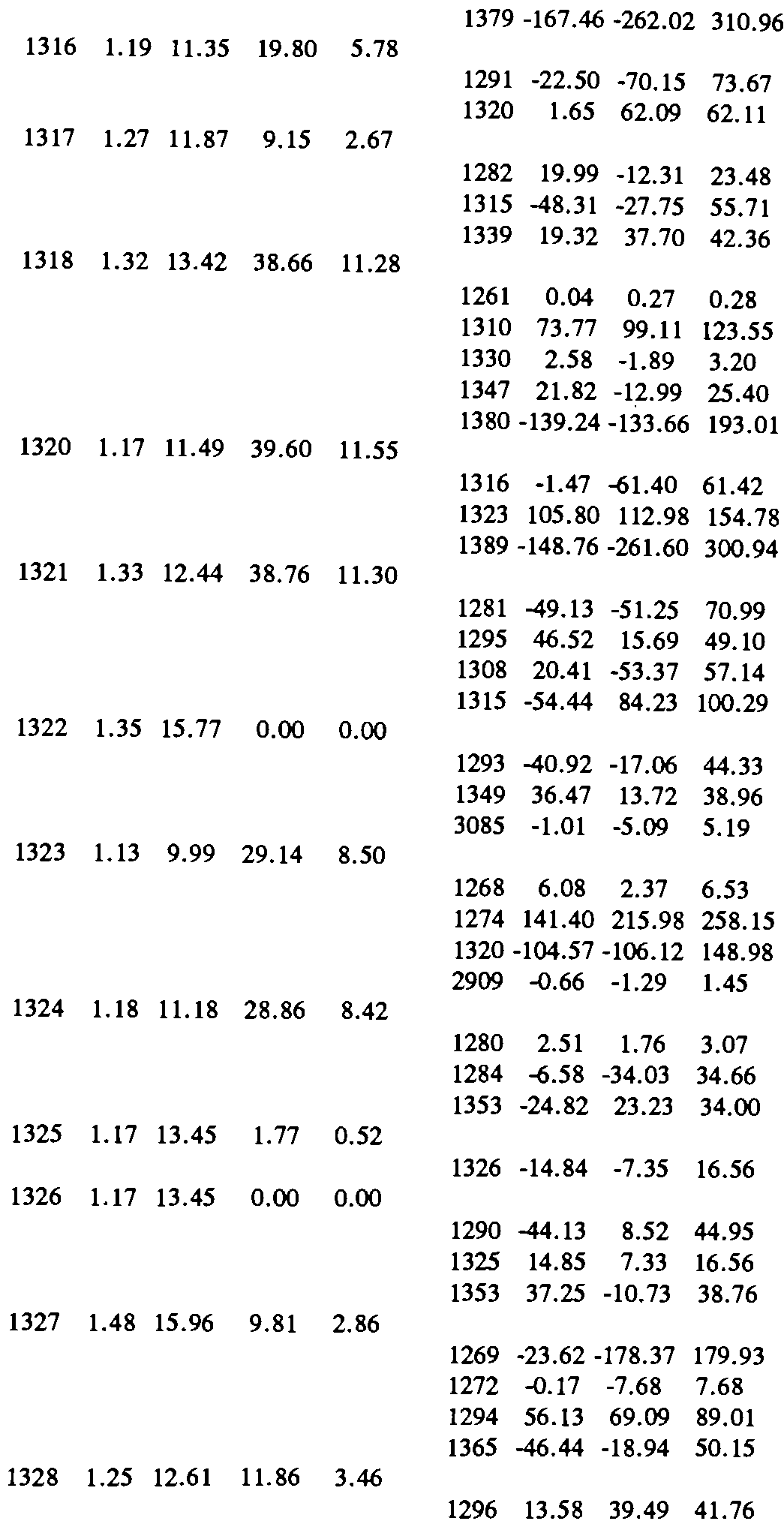




\begin{tabular}{|c|c|c|c|c|c|c|c|c|}
\hline \multirow{4}{*}{1329} & 126 & 1283 & 000 & 00 & 1329 & -27.22 & -8.94 & 28.65 \\
\hline & & & & & 1328 & 27.31 & 8.98 & 28.75 \\
\hline & & & & & 1349 & -34.66 & -14.98 & 37.76 \\
\hline & & & & & 3088 & 0.62 & -3.48 & 3.53 \\
\hline \multirow[t]{2}{*}{1330} & 1.32 & 12.95 & 2.52 & 0.74 & & & & \\
\hline & & & & & 1318 & -2.38 & -0.45 & 2.42 \\
\hline \multirow[t]{6}{*}{1331} & 1.22 & 12.24 & 6.44 & 1.88 & & & & \\
\hline & & & & & 1284 & 56.31 & -0.77 & 56.32 \\
\hline & & & & & 1288 & 105.95 & 108.73 & 151.81 \\
\hline & & & & & 1304 & 3.53 & -29.07 & 29.28 \\
\hline & & & & & 1338 & -35.54 & -98.96 & 105.14 \\
\hline & & & & & 1381 & -140.24 & -176.87 & 225.72 \\
\hline \multirow[t]{3}{*}{1332} & 1.21 & 11.77 & 33.43 & 9.75 & & & & \\
\hline & & & & & 1290 & -37.31 & 54.80 & 66.30 \\
\hline & & & & & 1334 & -0.26 & -75.44 & 75.44 \\
\hline \multirow[t]{2}{*}{1333} & 1.22 & 11.69 & 3.64 & 1.06 & & & & \\
\hline & & & & & 1334 & -11.24 & -10.72 & 15.53 \\
\hline \multirow[t]{4}{*}{1334} & 1.22 & 11.71 & 0.00 & 0.00 & & & & \\
\hline & & & & & 1312 & -3.84 & -72.22 & 72.32 \\
\hline & & & & & 1332 & 0.34 & 75.79 & 75.79 \\
\hline & & & & & 1333 & 11.25 & 10.71 & 15.53 \\
\hline \multirow[t]{3}{*}{1335} & 1.35 & 11.31 & 46.41 & 13.54 & & & & \\
\hline & & & & & 1295 & -9.21 & 50.88 & 51.71 \\
\hline & & & & & 1308 & -36.07 & -11.02 & 37.71 \\
\hline \multirow[t]{5}{*}{1336} & 1.36 & 13.86 & 0.00 & 0.00 & & & & \\
\hline & & & & & 1287 & 30.02 & -30.37 & 42.70 \\
\hline & & & & & 1298 & 34.76 & 10.17 & 36.22 \\
\hline & & & & & 1303 & 32.95 & -10.82 & 34.68 \\
\hline & & & & & 1382 & -101.86 & -90.60 & 136.33 \\
\hline \multirow[t]{3}{*}{1337} & 1.38 & 12.53 & 25.22 & 7.35 & & & & \\
\hline & & & & & 1311 & -34.89 & 30.34 & 46.24 \\
\hline & & & & & 1314 & 10.46 & 28.74 & 30.59 \\
\hline \multirow[t]{6}{*}{1338} & 1.26 & 12.53 & 19.89 & 5.80 & & & & \\
\hline & & & & & 1282 & 23.74 & -22.42 & 32.65 \\
\hline & & & & & 1295 & 10.86 & -112.33 & 112.85 \\
\hline & & & & & 1312 & 42.98 & 8.09 & 43.73 \\
\hline & & & & & 1331 & 36.20 & 101.22 & 107.50 \\
\hline & & & & & 1383 & -141.93 & -195.36 & 241.47 \\
\hline \multirow[t]{3}{*}{1339} & 1.25 & 11.58 & 14.19 & 4.14 & & & & \\
\hline & & & & & 1317 & -19.12 & -37.35 & 41.96 \\
\hline & & & & & 1340 & 4.57 & 32.24 & 32.56 \\
\hline \multirow[t]{4}{*}{1340} & 1.23 & 11.62 & 2.15 & 0.63 & & & & \\
\hline & & & & & 1277 & 21.66 & 120.66 & 122.59 \\
\hline & & & & & 1339 & -4.48 & -32.15 & 32.46 \\
\hline & & & & & 1341 & -8.08 & -12.28 & 14.70 \\
\hline \multirow[t]{3}{*}{1341} & 1.24 & 11.76 & 0.00 & 0.00 & & & & \\
\hline & & & & & 1277 & 8.94 & 13.78 & 16.43 \\
\hline & & & & & 1309 & -18.77 & -19.39 & 26.99 \\
\hline
\end{tabular}




\begin{tabular}{|c|c|c|c|c|c|c|c|c|}
\hline & & & & & $\begin{array}{l}1340 \\
3082\end{array}$ & $\begin{array}{l}8.12 \\
3.32\end{array}$ & $\begin{array}{c}11.96 \\
0.48\end{array}$ & $\begin{array}{l}14.46 \\
3.35\end{array}$ \\
\hline \multirow[t]{3}{*}{1342} & 1.26 & 12.83 & 0.00 & 0.00 & & & & \\
\hline & & & & & 1302 & 101.05 & 76.91 & 126.99 \\
\hline & & & & & 3353 & -99.97 & -74.54 & 124.69 \\
\hline \multirow[t]{3}{*}{1343} & 1.25 & 15.35 & 0.00 & 0.00 & & & & \\
\hline & & & & & 1297 & 59.50 & 14.15 & 61.15 \\
\hline & & & & & 1388 & -70.37 & -219.46 & 230.47 \\
\hline \multirow[t]{3}{*}{1344} & 1.10 & 10.15 & 8.12 & 2.37 & & & & \\
\hline & & & & & 1137 & -40.70 & -24.10 & 47.30 \\
\hline & & & & & 1346 & -15.19 & -69.12 & 70.77 \\
\hline \multirow[t]{3}{*}{1345} & 1.15 & 10.54 & 24.56 & 7.16 & & & & \\
\hline & & & & & 1280 & -62.33 & -86.74 & 106.81 \\
\hline & & & & & 1346 & 37.52 & 79.75 & 88.14 \\
\hline \multirow[t]{4}{*}{1346} & 1.10 & 10.15 & 0.00 & 0.00 & & & & \\
\hline & & & & & 1276 & 127.81 & 157.37 & 202.73 \\
\hline & & & & & 1344 & 15.19 & 69.17 & 70.81 \\
\hline & & & & & 1345 & -36.56 & -76.63 & 84.91 \\
\hline \multirow[t]{4}{*}{1347} & 1.33 & 12.95 & 0.00 & 0.00 & & & & \\
\hline & & & & & 1285 & 4.38 & -17.78 & 18.31 \\
\hline & & & & & 1318 & -21.74 & 12.81 & 25.23 \\
\hline & & & & & 1348 & 15.06 & -0.35 & 15.06 \\
\hline \multirow[t]{2}{*}{1348} & 1.33 & 12.86 & 17.18 & 5.01 & & & & \\
\hline & & & & & 1347 & -15.05 & 0.26 & 15.05 \\
\hline \multirow[t]{4}{*}{1349} & 1.26 & 13.02 & 0.00 & 0.00 & & & & \\
\hline & & & & & 1322 & -34.66 & -12.53 & 36.86 \\
\hline & & & & & 1329 & 34.80 & 15.10 & 37.94 \\
\hline & & & & & 3093 & 1.16 & -0.82 & 1.42 \\
\hline \multirow[t]{4}{*}{1350} & 1.13 & 11.76 & 0.00 & 0.00 & & & & \\
\hline & & & & & 1137 & 30.50 & 20.96 & 37.01 \\
\hline & & & & & 3081 & -31.87 & -20.18 & 37.72 \\
\hline & & & & & 3092 & 1.56 & -0.56 & 1.66 \\
\hline \multirow[t]{3}{*}{1351} & 1.16 & 10.43 & 15.04 & 4.39 & & & & \\
\hline & & & & & 1288 & -64.96 & -81.09 & 103.90 \\
\hline & & & & & 1390 & 44.69 & 68.65 & 81.91 \\
\hline \multirow[t]{4}{*}{1353} & 1.17 & 11.79 & 0.00 & 0.00 & & & & \\
\hline & & & & & 1324 & 24.92 & -23.12 & 33.99 \\
\hline & & & & & 1326 & -37.06 & 11.18 & 38.71 \\
\hline & & & & & 1354 & -5.19 & -16.52 & 17.32 \\
\hline \multirow[t]{2}{*}{1354} & 1.17 & 11.79 & 5.70 & 1.66 & & & & \\
\hline & & & & & 1353 & 5.19 & 16.50 & 17.30 \\
\hline \multirow[t]{3}{*}{1355} & 1.26 & 11.09 & 24.75 & 7.22 & & & & \\
\hline & & & & & 1282 & -15.50 & -20.09 & 25.37 \\
\hline & & & & & 1312 & -8.61 & 14.73 & 17.06 \\
\hline \multirow[t]{3}{*}{1356} & 1.11 & 16.18 & 11.11 & 3.24 & & & & \\
\hline & & & & & 1301 & 2.45 & -15.04 & 15.24 \\
\hline & & & & & 1313 & -34.86 & 51.48 & 62.17 \\
\hline \multirow[t]{2}{*}{1357} & 1.49 & 17.11 & 17.56 & 5.12 & & & & \\
\hline & & & & & 1365 & -19.84 & -16.75 & 25.96 \\
\hline
\end{tabular}




\begin{tabular}{|c|c|c|c|c|c|c|c|c|}
\hline \multirow[t]{3}{*}{1359} & 1.47 & 16.28 & 43.89 & 12.80 & & & & \\
\hline & & & & & 388 & -36.93 & -23.57 & 43.81 \\
\hline & & & & & 1364 & -6.86 & 10.24 & 12.32 \\
\hline \multirow[t]{3}{*}{1360} & 1.46 & 16.43 & 0.00 & 0.00 & & & & \\
\hline & & & & & 1293 & 22.25 & 207.97 & 209.16 \\
\hline & & & & & 1363 & -18.48 & $3-41.96$ & 45.85 \\
\hline \multirow[t]{3}{*}{1361} & 1.41 & 16.68 & 15.13 & 4.41 & & & & \\
\hline & & & & & 1362 & 45.48 & 3144.59 & 151.57 \\
\hline & & & & & 1365 & -63.47 & -159.52 & 171.68 \\
\hline \multirow[t]{3}{*}{1362} & 1.39 & 16.62 & 0.00 & 0.00 & & & & \\
\hline & & & & & 1361 & -45.00 & -143.21 & 150.11 \\
\hline & & & & & 1378 & 35.97 & -97.80 & 104.20 \\
\hline \multirow[t]{4}{*}{1363} & 1.47 & 16.45 & 20.26 & 5.91 & & & & \\
\hline & & & & & 1360 & 18.40 & 40.92 & 44.86 \\
\hline & & & & & 1364 & 13.19 & -19.98 & 23.94 \\
\hline & & & & & 1365 & -54.27 & -36.38 & 65.33 \\
\hline \multirow[t]{3}{*}{1364} & 1.47 & 16.37 & 8.12 & 2.37 & & & & \\
\hline & & & & & 1359 & 6.68 & -11.91 & 13.66 \\
\hline & & & & & 1363 & -13.52 & 18.79 & 23.15 \\
\hline \multirow[t]{9}{*}{1365} & 1.49 & 17.18 & 0.00 & 0.00 & & & & \\
\hline & & & & & 422 & -38.93 & -50.64 & 63.88 \\
\hline & & & & & 1270 & -77.79 & -167.14 & 184.36 \\
\hline & & & & & 1271 & -78.21 & -245.29 & 257.45 \\
\hline & & & & & 1273 & $-5.23-$ & -116.00 & 116.12 \\
\hline & & & & & 1327 & 46.49 & 20.13 & 50.66 \\
\hline & & & & & 1357 & 19.70 & 15.70 & 25.19 \\
\hline & & & & & 1361 & 65.44 & 166.43 & 178.83 \\
\hline & & & & & 1363 & 53.74 & 34.03 & 63.61 \\
\hline \multirow[t]{4}{*}{1372} & 1.23 & 17.07 & 0.00 & 0.00 & & & & \\
\hline & & & & & 969 & -256.57 & 205.45 & 328.69 \\
\hline & & & & & 1279 & 68.28 & 140.61 & 156.31 \\
\hline & & & & & 1381 & 184.93 & -247.29 & 308.79 \\
\hline \multirow[t]{4}{*}{1373} & 1.37 & 17.47 & 0.00 & 0.00 & & & & \\
\hline & & & & & 972 & -175.57 & 282.03 & 332.21 \\
\hline & & & & & 1293 & 81.50 & 6.40 & 81.75 \\
\hline & & & & & 1375 & 89.55 & -163.46 & 186.38 \\
\hline \multirow[t]{4}{*}{1374} & 1.24 & 16.36 & 0.00 & 0.00 & & & & \\
\hline & & & & & 969 & -287.28 & 238.43 & 373.34 \\
\hline & & & & & 1290 & 110.20 & 150.03 & 186.15 \\
\hline & & & & & 1381 & 172.00 & -244.24 & 298.72 \\
\hline \multirow[t]{7}{*}{1375} & 1.39 & 17.01 & 0.00 & 0.00 & & & & \\
\hline & & & & & 1373 & -93.57 & 152.19 & 178.66 \\
\hline & & & & & 1378 & 118.02 & -188.46 & 222.36 \\
\hline & & & & & 1384 & 246.27 & 142.70 & 284.62 \\
\hline & & & & & 1385 & -93.07 & -61.39 & 111.49 \\
\hline & & & & & 1386 & -92.93 & -97.21 & 134.48 \\
\hline & & & & & 1387 & -90.86 & -21.42 & 93.35 \\
\hline
\end{tabular}

$\begin{array}{lllll}1376 & 1.38 & 15.37 & 55.19 & 16.10\end{array}$

$1378-334.64-439.83 \quad 552.66$ 


\begin{tabular}{|c|c|c|c|c|c|c|c|c|}
\hline \multirow[t]{7}{*}{1378} & 1.42 & 16.09 & 0.00 & 0.00 & 1379 & 265.63 & 374.57 & 459.19 \\
\hline & & & & & 469 & -417.98 & -511.90 & 660.87 \\
\hline & & & & & 1311 & 184.40 & 243.27 & 305.26 \\
\hline & & & & & 1362 & -35.86 & 100.37 & 106.58 \\
\hline & & & & & 1375 & -123.21 & 171.93 & 211.52 \\
\hline & & & & & 1376 & 335.40 & 446.70 & 558.61 \\
\hline & & & & & 1382 & 79.32 & 118.90 & 142.92 \\
\hline \multirow[t]{5}{*}{1379} & 1.36 & 15.08 & 0.00 & 0.00 & & & & \\
\hline & & & & & 1315 & 168.00 & 279.37 & 326.00 \\
\hline & & & & & 1376 & -265.59 & -372.87 & 457.79 \\
\hline & & & & & 1383 & 224.21 & 483.84 & 533.27 \\
\hline & & & & & 1388 & -118.91 & 322.32 & 343.55 \\
\hline \multirow[t]{4}{*}{1380} & 1.39 & 16.08 & 0.00 & 0.00 & & & & \\
\hline & & & & & 1318 & 139.87 & 147.33 & 203.15 \\
\hline & & & & & 1382 & 64.17 & -167.82 & 179.67 \\
\hline & & & & & 2026 & -209.37 & 317.29 & 380.15 \\
\hline \multirow[t]{7}{*}{1381} & 1.29 & 14.55 & 0.00 & 0.00 & & & & \\
\hline & & & & & 1234 & 84.10 & 92.53 & 125.04 \\
\hline & & & & & 1331 & 140.56 & 192.78 & 238.59 \\
\hline & & & & & 1372 & -190.15 & 240.33 & 306.46 \\
\hline & & & & & 1374 & -177.67 & 235.94 & 295.36 \\
\hline & & & & & 1383 & -56.65 & -593.31 & 596.01 \\
\hline & & & & & 2021 & 174.30 & -50.18 & 181.38 \\
\hline \multirow[t]{4}{*}{1382} & 1.40 & 15.84 & 51.74 & 15.09 & & & & \\
\hline & & & & & 1336 & 102.15 & 97.37 & 141.13 \\
\hline & & & & & 1378 & -81.63 & -128.37 & 152.12 \\
\hline & & & & & 1380 & -65.80 & 161.71 & 174.58 \\
\hline \multirow[t]{6}{*}{1383} & 1.33 & 14.52 & 0.00 & 0.00 & & & & \\
\hline & & & & & 1338 & 142.63 & 211.38 & 255.00 \\
\hline & & & & & 1379 & -224.67 & -476.34 & 526.67 \\
\hline & & & & & 1381 & 56.78 & 604.60 & 607.26 \\
\hline & & & & & 1384 & -138.95 & -210.25 & 252.01 \\
\hline & & & & & 2020 & 160.70 & 60.59 & 171.74 \\
\hline \multirow[t]{4}{*}{1384} & 1.35 & 15.09 & 0.00 & 0.00 & & & & \\
\hline & & & & & 1281 & 112.40 & -36.12 & 118.06 \\
\hline & & & & & 1375 & -251.65 & -155.97 & 296.07 \\
\hline & & & & & 1383 & 136.07 & 201.39 & 243.05 \\
\hline \multirow[t]{4}{*}{1385} & 1.39 & 17.23 & 0.00 & 0.00 & & & & \\
\hline & & & & & 1262 & -51.28 & -80.67 & 95.59 \\
\hline & & & & & 1263 & -51.22 & -141.18 & 150.18 \\
\hline & & & & & 1375 & 93.07 & 61.90 & 111.78 \\
\hline \multirow[t]{4}{*}{1386} & 1.39 & 17.23 & 0.00 & 0.00 & & & & \\
\hline & & & & & 1264 & -51.29 & -77.99 & 93.35 \\
\hline & & & & & 1265 & -51.10 & -180.43 & 187.52 \\
\hline & & & & & 1375 & 92.93 & 97.96 & 135.03 \\
\hline \multirow[t]{3}{*}{1387} & 1.39 & 17.22 & 0.00 & 0.00 & & & & \\
\hline & & & & & 1266 & -49.19 & -1.91 & 49.22 \\
\hline & & & & & 1267 & -51.07 & -179.35 & 186.48 \\
\hline
\end{tabular}




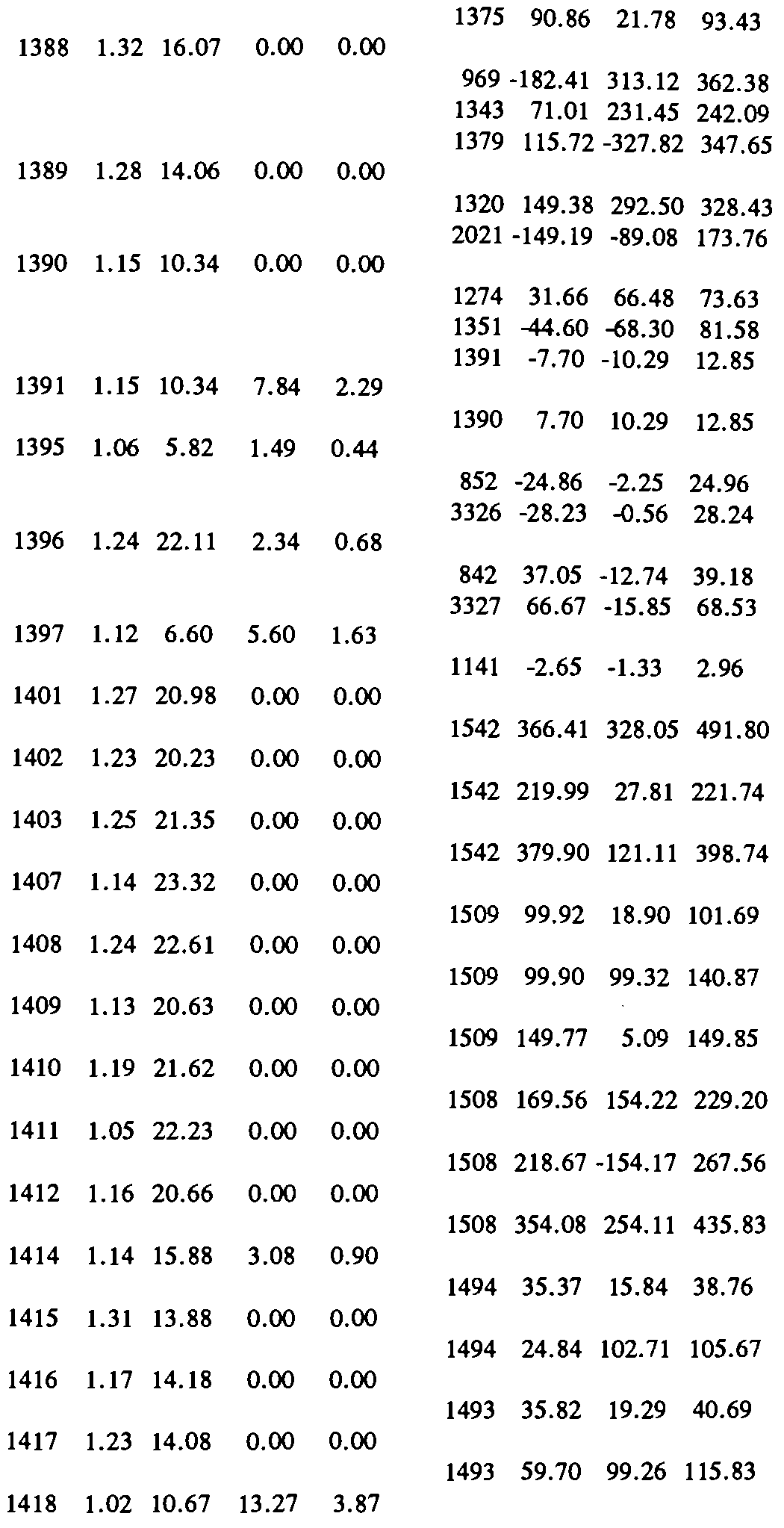




\begin{tabular}{|c|c|c|c|c|c|c|c|c|}
\hline & & & & & 1435 & -53.53 & -49.61 & 72.99 \\
\hline & & & & & 1674 & -9.43 & -10.87 & 14.39 \\
\hline \multirow[t]{3}{*}{1421} & 1.02 & 9.90 & 23.75 & 6.93 & & & & \\
\hline & & & & & 1430 & 7.53 & -5.55 & 9.35 \\
\hline & & & & & 1435 & -32.83 & -3.14 & 32.98 \\
\hline \multirow[t]{3}{*}{1422} & 1.06 & 13.65 & 12.25 & 3.57 & & & & \\
\hline & & & & & 1426 & -5.14 & -3.03 & 5.97 \\
\hline & & & & & 1429 & -7.66 & -1.63 & 7.83 \\
\hline \multirow[t]{3}{*}{1423} & 1.05 & 12.11 & 12.92 & 3.77 & & & & \\
\hline & & & & & 1427 & -81.10 & -23.60 & 84.46 \\
\hline & & & & & 1428 & 67.02 & 18.05 & 69.41 \\
\hline \multirow[t]{3}{*}{1424} & 1.06 & 13.23 & 25.36 & 7.40 & & & & \\
\hline & & & & & 1427 & -93.49 & -30.03 & 98.19 \\
\hline & & & & & 1436 & 66.78 & 20.20 & 69.77 \\
\hline \multirow[t]{6}{*}{1425} & 1.09 & 19.00 & 0.00 & 0.00 & & & & \\
\hline & & & & & 804 & -786.13 & 44.15 & 787.37 \\
\hline & & & & & 1427 & 174.47 & 39.73 & 178.94 \\
\hline & & & & & 1483 & 221.84 & -250.11 & 334.31 \\
\hline & & & & & 1664 & 176.41 & 17.97 & 177.32 \\
\hline & & & & & 1744 & 208.39 & 70.34 & 219.95 \\
\hline \multirow[t]{4}{*}{1426} & 1.06 & 13.84 & 0.00 & 0.00 & & & & \\
\hline & & & & & 1422 & 5.00 & 2.49 & 5.59 \\
\hline & & & & & 1675 & 81.72 & 31.11 & 87.44 \\
\hline & & & & & 1744 & -115.17 & -91.94 & 147.37 \\
\hline \multirow[t]{4}{*}{1427} & 1.07 & 14.22 & 0.00 & 0.00 & & & & \\
\hline & & & & & 1423 & 81.56 & 26.68 & 85.81 \\
\hline & & & & & 1424 & 93.76 & 31.76 & 98.99 \\
\hline & & & & & 1425 & -174.15 & -24.63 & 175.88 \\
\hline \multirow[t]{3}{*}{1428} & 1.04 & 11.31 & 53.68 & 15.66 & & & & \\
\hline & & & & & 1423 & -66.90 & -17.17 & 69.07 \\
\hline & & & & & 1467 & 12.89 & 1.41 & 12.96 \\
\hline \multirow{2}{*}{1429} & 1.06 & 13.83 & 0.18 & 1.80 & & & & \\
\hline & & & & & 1422 & 7.59 & 1.34 & 7.71 \\
\hline \multirow{3}{*}{1430} & & & & & 1654 & -13.88 & -3.30 & 14.27 \\
\hline & 1.02 & 9.10 & 17.43 & 3.09 & & 750 & & \\
\hline & & & & & $\begin{array}{l}1421 \\
1520\end{array}$ & $\begin{array}{r}-1.59 \\
-10.05\end{array}$ & $\begin{array}{c}5.38 \\
-10.64\end{array}$ & $\begin{array}{l}9.30 \\
14.64\end{array}$ \\
\hline \multirow[t]{6}{*}{1431} & 1.05 & 9.81 & 0.00 & 0.00 & & & & \\
\hline & & & & & 1449 & 116.53 & 49.69 & 126.69 \\
\hline & & & & & 1453 & -16.69 & -72.81 & 74.70 \\
\hline & & & & & 1476 & -108.74 & -15.97 & 109.91 \\
\hline & & & & & 1477 & -93.38 & -54.81 & 108.28 \\
\hline & & & & & 1478 & 29.83 & 49.63 & 57.91 \\
\hline \multirow[t]{3}{*}{1432} & 1.04 & 9.80 & 29.74 & 8.68 & & & & \\
\hline & & & & & 1434 & 20.17 & -22.63 & 30.32 \\
\hline & & & & & 1633 & -52.80 & 11.63 & 54.06 \\
\hline \multirow[t]{3}{*}{1433} & 1.04 & 11.30 & 14.60 & 4.26 & & & & \\
\hline & & & & & 1436 & -67.07 & -19.08 & 69.73 \\
\hline & & & & & 1438 & 50.28 & 12.23 & 51.75 \\
\hline
\end{tabular}




\begin{tabular}{|c|c|c|c|c|c|c|c|c|}
\hline 1434 & 1.04 & 9.59 & 10.68 & 3.12 & & & & \\
\hline & & & & & 1432 & -20.17 & 22.66 & 30.34 \\
\hline & & & & & 1478 & -54.13 & -75.86 & 93.19 \\
\hline 1435 & 1.02 & 10.68 & 11.66 & 3.40 & & & & \\
\hline & & & & & 1418 & 53.53 & 49.64 & 73.01 \\
\hline & & & & & 1421 & 32.80 & 3.28 & 32.97 \\
\hline & & & & & 1675 & -69.32 & -23.83 & 73.30 \\
\hline 1436 & 1.05 & 12.40 & 0.00 & 0.00 & & & & \\
\hline & & & & & 1424 & -66.56 & -19.24 & 69.28 \\
\hline & & & & & 1433 & 67.29 & 20.32 & 70.29 \\
\hline 1437 & 1.04 & 11.76 & 25.50 & 7.44 & & & & \\
\hline & & & & & 1633 & 44.44 & 5.13 & 44.74 \\
\hline & & & & & 1676 & -71.68 & -15.08 & 73.25 \\
\hline 1438 & 1.04 & 10.90 & 11.31 & 3.30 & & & & \\
\hline & & & & & 1433 & -50.22 & -11.94 & 51.62 \\
\hline & & & & & 1633 & -9.10 & -46.37 & 47.26 \\
\hline 1439 & 1.06 & 12.61 & 14.22 & 4.15 & & & & \\
\hline & & & & & 1440 & -29.83 & -10.92 & 31.76 \\
\hline & 1.06 & 12.64 & 0.00 & 0.00 & & & & \\
\hline & & & & & 1439 & 29.41 & 9.54 & 30.91 \\
\hline & & & & & 1476 & 105.67 & 24.94 & 108.58 \\
\hline & & & & & 1484 & -156.14 & -53.24 & 164.97 \\
\hline
\end{tabular}

$\begin{array}{lllll}1441 & 1.03 & 8.17 & 12.36 & 3.60\end{array}$

$\begin{array}{llll}1562 & 36.40 & 17.56 & 40.41\end{array}$

$\begin{array}{llll}1678 & -52.46 & -23.11 & 57.32\end{array}$

$\begin{array}{lllll}1442 & 1.02 & 6.95 & 16.96 & 4.95\end{array}$

$\begin{array}{llll}1443 & -8.84 & -3.39 & 9.47\end{array}$

$\begin{array}{llll}1444 & -46.66 & -5.70 & 47.01\end{array}$

$\begin{array}{lllll}1443 & 1.02 & 6.96 & 0.00 & 0.00\end{array}$

$\begin{array}{llll}1442 & 8.84 & 3.38 & 9.46\end{array}$

$\begin{array}{llll}1444 & -55.51 & -27.28 & 61.85\end{array}$

$\begin{array}{llll}1452 & 6.48 & 6.17 & 8.95\end{array}$

$\begin{array}{lllll}1444 & 1.02 & 6.99 & 0.00 & 0.00\end{array}$

$\begin{array}{llll}1442 & 46.43 & 5.16 & 46.72\end{array}$

$\begin{array}{lllll}1443 & 55.52 & 27.30 & 61.87\end{array}$

$\begin{array}{llll}1549 & -61.52 & -21.83 & 65.28\end{array}$

$\begin{array}{lllll}1445 & 1.05 & 11.31 & 0.00 & 0.00\end{array}$

$\begin{array}{llll}1446 & 99.75 & 34.35 & 105.50\end{array}$

$\begin{array}{llll}1745 & -101.83 & -39.75 & 109.31\end{array}$

$\begin{array}{lllll}1446 & 1.02 & 7.36 & 23.98 & 6.99\end{array}$

$\begin{array}{llll}1445 & -99.22 & -26.68 & 102.74\end{array}$

$\begin{array}{llll}1451 & 22.37 & 4.36 & 22.79\end{array}$

$\begin{array}{llll}1567 & 13.41 & 2.61 & 13.66\end{array}$

$\begin{array}{llllllllll}1447 & 1.08 & 9.89 & 12.15 & 3.54 & & & & & \\ & & & & & 1453 & 25.69 & 75.90 & 80.13\end{array}$

$1500-114.75-132.19 \quad 175.05$

$\begin{array}{lllll}1448 & 1.04 & 8.95 & 0.00 & 0.00\end{array}$

$1454 \quad-66.09-30.42 \quad 72.76$

$\begin{array}{llll}1678 & 56.71 & 24.34 & 61.71\end{array}$ 


\begin{tabular}{|c|c|c|c|c|c|c|c|c|}
\hline \multirow[t]{2}{*}{1449} & 1.02 & 7.51 & 14.38 & 4.20 & 1431 & -115.23 & $3-43.98$ & $8 \quad 123.34$ \\
\hline & & & & & 1549 & 23.26 & 1.64 & 23.32 \\
\hline \multirow[t]{3}{*}{1450} & 1.02 & 7.18 & 7.14 & 2.08 & & & & \\
\hline & & & & & 1562 & -37.97 & -17.84 & 41.95 \\
\hline & & & & & 1567 & -48.35 & -20.78 & 52.63 \\
\hline \multirow[t]{3}{*}{1451} & 1.02 & 7.35 & 0.00 & 0.00 & & & & \\
\hline & & & & & 1446 & -22.37 & -4.36 & 22.79 \\
\hline & & & & & 1456 & 40.75 & 12.00 & 42.48 \\
\hline \multirow[t]{3}{*}{1452} & 1.02 & 6.90 & 10.48 & 3.06 & & & & \\
\hline & & & & & 1443 & -6.51 & -6.29 & 9.05 \\
\hline & & & & & 1568 & -82.21 & -30.51 & 87.69 \\
\hline \multirow[t]{3}{*}{1453} & 1.07 & 9.80 & 11.24 & 3.28 & & & & \\
\hline & & & & & 1431 & 16.91 & 73.70 & 75.62 \\
\hline & & & & & 1447 & -25.58 & -75.22 & 79.45 \\
\hline \multirow[t]{3}{*}{1454} & 1.04 & 9.11 & 12.01 & 3.50 & & & & \\
\hline & & & & & 1448 & 66.13 & 30.64 & 72.89 \\
\hline & & & & & 1477 & -75.71 & -32.60 & 82.43 \\
\hline \multirow[t]{3}{*}{1455} & 1.08 & 17.74 & 0.00 & 0.00 & & & & \\
\hline & & & & & 1508 & 17.44 & -99.75 & 101.26 \\
\hline & & & & & 1664 & -23.66 & 49.40 & 54.77 \\
\hline \multirow[t]{3}{*}{1456} & 1.02 & 7.17 & 0.00 & 0.00 & & & & \\
\hline & & & & & 1451 & -40.72 & -11.90 & 42.42 \\
\hline & & & & & 1568 & 40.06 & 11.51 & 41.69 \\
\hline \multirow[t]{3}{*}{1457} & 1.06 & 14.42 & 0.00 & 0.00 & & & & \\
\hline & & & & & 730 & -8.26 & 9.49 & 12.59 \\
\hline & & & & & 1654 & 7.88 & -10.31 & 12.97 \\
\hline \multirow[t]{3}{*}{1458} & 1.03 & 12.09 & 17.75 & 5.18 & & & & \\
\hline & & & & & 1470 & 62.63 & 33.74 & 71.14 \\
\hline & & & & & 1654 & -81.31 & -40.39 & 90.79 \\
\hline \multirow[t]{3}{*}{1459} & 1.05 & 13.70 & 2.01 & 0.58 & & & & \\
\hline & & & & & 1654 & -59.77 & -29.59 & 66.69 \\
\hline & & & & & 1660 & 56.82 & 27.01 & 62.91 \\
\hline \multirow[t]{3}{*}{1461} & 1.02 & 10.90 & 27.82 & 8.11 & & & & \\
\hline & & & & & 1472 & 46.55 & 46.69 & 65.93 \\
\hline & & & & & 1596 & -76.41 & -57.00 & 95.33 \\
\hline \multirow[t]{3}{*}{1462} & 0.99 & 10.37 & 0.00 & 0.00 & & & & \\
\hline & & & & & 1463 & 7.82 & 40.40 & 41.15 \\
\hline & & & & & 1469 & -37.64 & -72.92 & 82.06 \\
\hline \multirow[t]{3}{*}{1463} & 0.98 & 10.35 & 20.04 & 5.85 & & & & \\
\hline & & & & & 1462 & -7.78 & -40.18 & 40.92 \\
\hline & & & & & 1590 & -12.02 & 34.74 & 36.76 \\
\hline \multirow{3}{*}{1464} & 1.09 & 11.18 & 13.94 & 4.07 & & & & \\
\hline & & & & & 1494 & -124.36 & -77.31 & 146.43 \\
\hline & & & & & 1655 & 30.00 & 14.35 & 33.25 \\
\hline \multirow{2}{*}{1465} & 1.01 & 10.22 & נפ.9 & 4.00 & 1473 & -12.87 & -33.30 & 3571 \\
\hline & & & & & 1630 & -5.36 & 24.21 & 24.79 \\
\hline 400 & 1.07 & 13.24 & 0.00 & 0.00 & & & & \\
\hline
\end{tabular}




\begin{tabular}{|c|c|c|c|c|c|c|c|c|}
\hline \multirow[t]{5}{*}{1467} & 1.04 & 11.09 & 17.64 & 5.14 & & & & \\
\hline & & & & & 1428 & -12.92 & -1.57 & 13.01 \\
\hline & & & & & 1466 & -98.24 & -70.32 & 120.82 \\
\hline & & & & & 1481 & 51.70 & 46.33 & 69.42 \\
\hline & & & & & 1520 & 41.23 & 19.84 & 45.75 \\
\hline \multirow[t]{4}{*}{1468} & 1.04 & 11.24 & 15.09 & 4.40 & & & & \\
\hline & & & & & 1501 & -95.59 & -89.06 & 130.65 \\
\hline & & & & & 1674 & 20.04 & 13.01 & 23.89 \\
\hline & & & & & 1747 & 56.39 & -9.99 & 57.27 \\
\hline \multirow[t]{3}{*}{1469} & 0.99 & 10.38 & 0.00 & 0.00 & & & & \\
\hline & & & & & 1462 & 37.65 & 72.97 & 82.11 \\
\hline & & & & & 1663 & -21.19 & -55.08 & 59.02 \\
\hline \multirow[t]{3}{*}{1470} & 1.02 & 11.09 & 14.17 & 4.13 & & & & \\
\hline & & & & & 1458 & -62.38 & -32.42 & 70.31 \\
\hline & & & & & 1471 & 47.36 & 27.50 & 54.76 \\
\hline \multirow[t]{3}{*}{1471} & 1.01 & 10.60 & 13.83 & 4.03 & & & & \\
\hline & & & & & 1470 & -47.22 & -26.99 & 54.39 \\
\hline & & & & & 1663 & -15.48 & -31.75 & 35.32 \\
\hline \multirow[t]{3}{*}{1472} & 1.01 & 10.58 & 13.06 & 3.81 & & & & \\
\hline & & & & & 1461 & -46.45 & -46.15 & 65.48 \\
\hline & & & & & 1746 & -16.80 & -11.72 & 20.49 \\
\hline \multirow[t]{3}{*}{1473} & 1.02 & 10.30 & 23.97 & 6.99 & & & & \\
\hline & & & & & 1465 & 12.91 & 33.45 & 35.86 \\
\hline & & & & & 1481 & -40.28 & -43.80 & 59.51 \\
\hline \multirow[t]{3}{*}{1475} & 1.06 & 12.34 & 0.00 & 0.00 & & & & \\
\hline & & & & & 1477 & 107.07 & 21.36 & 109.18 \\
\hline & & & & & 1484 & -111.31 & -31.94 & 115.80 \\
\hline \multirow[t]{3}{*}{1476} & 1.06 & 12.39 & 0.00 & 0.00 & & & & \\
\hline & & & & & 1431 & 108.90 & 21.04 & 110.91 \\
\hline & & & & & 1440 & -105.64 & -24.94 & 108.54 \\
\hline \multirow[t]{7}{*}{1477} & 1.05 & 9.83 & 0.00 & 0.00 & & & & \\
\hline & & & & & 1431 & 93.39 & 54.86 & 108.32 \\
\hline & & & & & 1454 & 75.92 & 33.69 & 83.06 \\
\hline & & & & & 1475 & -106.94 & -16.51 & 108.21 \\
\hline & & & & & 1482 & 3.15 & 40.63 & 40.75 \\
\hline & & & & & 1485 & 1.74 & -13.79 & 13.90 \\
\hline & & & & & 1486 & -28.60 & -65.48 & 71.45 \\
\hline \multirow[t]{3}{*}{1478} & 1.04 & 9.60 & 15.41 & 4.49 & & & & \\
\hline & & & & & 1431 & -29.70 & -49.09 & 57.38 \\
\hline & & & & & 1434 & 54.14 & 75.90 & 93.23 \\
\hline \multirow[t]{3}{*}{1479} & 1.07 & 10.27 & 14.03 & 4.09 & & & & \\
\hline & & & & & 1655 & -62.97 & -43.85 & 76.74 \\
\hline & & & & & 1726 & 46.47 & 37.94 & 59.99 \\
\hline \multirow{2}{*}{1481} & 1.03 & 10.40 & 13.98 & 4.08 & 1467 & -51.37 & -45.10 & 68.36 \\
\hline & & & & & 1473 & 40.33 & 43.98 & 59.67 \\
\hline
\end{tabular}

$\begin{array}{llll}1467 & 98.27 & 76.06 & 124.27\end{array}$

$\begin{array}{lllll}1501 & -27.02 & -58.16 & 64.14\end{array}$

$\begin{array}{llll}1509 & -118.77 & -64.25 & 135.03\end{array}$

$\begin{array}{llll}1428 & -12.92 & -1.57 & 13.01\end{array}$

$\begin{array}{llll}1466 & -98.24 & -70.32 & 120.82\end{array}$

$\begin{array}{lllll}1481 & 51.70 & 46.33 & 69.42\end{array}$

45.75

$\begin{array}{lllll}1501 & -95.59 & -89.06 & 130.65\end{array}$

$\begin{array}{llll}1674 & 20.04 & 13.01 & 23.89\end{array}$

$\begin{array}{llll}1462 & 37.65 & 72.97 & 82.11\end{array}$

59.02

$\begin{array}{llll}1458 & -62.38 & -32.42 & 70.31\end{array}$

$\begin{array}{llll}1471 & 47.36 & 27.50 & 54.76\end{array}$

$\begin{array}{llll}1470 & -47.22 & -26.99 & 54.39\end{array}$

$\begin{array}{lllll}1461 & -46.45 & -46.15 & 65.48\end{array}$

$\begin{array}{llll}1746 & -16.80 & -11.72 & 20.49\end{array}$

$\begin{array}{llll}1465 & 12.91 & 33.45 & 35.86\end{array}$

$\begin{array}{llll}1477 & 107.07 & 21.36 & 109.18\end{array}$

$1484-111.31 \quad-31.94 \quad 115.80$

$\begin{array}{llll}1431 & 108.90 & 21.04 & 110.91\end{array}$

$\begin{array}{llll}1431 & 93.39 & 54.86 & 108.32\end{array}$

$\begin{array}{llll}1454 & 75.92 & 33.69 & 83.06\end{array}$

$\begin{array}{llll}1475 & -106.94 & -16.51 & 108.21\end{array}$

$\begin{array}{llll}1482 & 3.15 & 40.63 & 40.75\end{array}$

$\begin{array}{lllll}1485 & 1.74 & -13.79 & 13.90\end{array}$

$\begin{array}{llll}1431 & -29.70 & -49.09 & 57.38\end{array}$ 


\begin{tabular}{|c|c|c|c|c|c|c|c|c|}
\hline \multirow[t]{3}{*}{1482} & 1.04 & 9.91 & 9.29 & 2.71 & & & & \\
\hline & & & & & 1477 & -3.07 & -40.46 & 40.57 \\
\hline & & & & & 1496 & -6.25 & 37.59 & 38.11 \\
\hline \multirow[t]{4}{*}{1483} & 1.12 & 17.54 & 0.00 & 0.00 & & & & \\
\hline & & & & & 1425 & -221.28 & 252.40 & 335.66 \\
\hline & & & & & 1484 & 314.71 & 167.87 & 356.68 \\
\hline & & & & & 1558 & -91.00 & -386.81 & 397.37 \\
\hline \multirow[t]{6}{*}{1484} & 1.07 & 12.71 & 0.00 & 0.00 & & & & \\
\hline & & & & & 1440 & 156.20 & 53.39 & 165.07 \\
\hline & & & & & 1475 & 111.45 & 32.18 & 116.00 \\
\hline & & & & & 1483 & -312.86 & -134.39 & 340.51 \\
\hline & & & & & 1501 & -72.01 & -29.66 & 77.88 \\
\hline & & & & & 1745 & 83.67 & 16.73 & 85.33 \\
\hline \multirow[t]{3}{*}{1485} & 1.06 & 9.71 & 14.60 & 4.26 & & & & \\
\hline & & & & & 1477 & -1.73 & 13.56 & 13.67 \\
\hline & & & & & 1726 & -80.33 & -62.67 & 101.89 \\
\hline \multirow[t]{3}{*}{1486} & 1.07 & 10.03 & 12.02 & 3.50 & & & & \\
\hline & & & & & 1477 & 28.86 & 66.55 & 72.54 \\
\hline & & & & & 1489 & -41.36 & -70.42 & 81.67 \\
\hline \multirow[t]{3}{*}{1488} & 1.09 & 10.07 & 24.83 & 7.24 & & & & \\
\hline & & & & & 1491 & -119.57 & -115.64 & 166.34 \\
\hline & & & & & 1500 & 78.97 & 98.92 & 126.58 \\
\hline \multirow[t]{3}{*}{1489} & 1.09 & 10.42 & 23.86 & 6.96 & & & & \\
\hline & & & & & 1486 & 41.62 & 71.80 & 82.99 \\
\hline & & & & & 1497 & -65.79 & -78.93 & 102.76 \\
\hline \multirow[t]{3}{*}{1490} & 1.10 & 10.69 & 9.02 & 2.63 & & & & \\
\hline & & & & & 1494 & -65.85 & -20.25 & 68.89 \\
\hline & & & & & 1666 & 52.64 & 14.50 & 54.61 \\
\hline \multirow[t]{4}{*}{1491} & 1.09 & 10.24 & 25.14 & 7.33 & & & & \\
\hline & & & & & 1488 & 119.80 & 113.17 & 164.80 \\
\hline & & & & & 1493 & -94.52 & -106.03 & 142.05 \\
\hline & & & & & 1666 & -51.67 & -16.29 & 54.18 \\
\hline \multirow[t]{3}{*}{1492} & 1.12 & 15.81 & 0.00 & 0.00 & & & & \\
\hline & & & & & 1494 & 109.24 & 26.04 & 112.30 \\
\hline & & & & & 1509 & -107.68 & 41.15 & 115.27 \\
\hline \multirow[t]{7}{*}{1493} & 1.14 & 11.68 & 0.00 & 0.00 & & & & \\
\hline & & & & & 1416 & -35.71 & -17.27 & 39.67 \\
\hline & & & & & 1417 & -59.16 & -89.62 & 107.39 \\
\hline & & & & & 1491 & 95.72 & 112.68 & 147.85 \\
\hline & & & & & 1497 & 65.70 & 82.62 & 105.56 \\
\hline & & & & & 1537 & -52.56 & -9.51 & 53.42 \\
\hline & & & & & 1667 & -16.81 & -80.26 & 82.00 \\
\hline \multirow[t]{7}{*}{1494} & 1.10 & 11.47 & 0.00 & 0.00 & & & & \\
\hline & & & & & 1414 & -35.31 & -12.65 & 37.51 \\
\hline & & & & & 1415 & -24.55 & -85.54 & 88.99 \\
\hline & & & & & 1464 & 125.35 & 67.57 & 142.40 \\
\hline & & & & & 1490 & 66.03 & 21.13 & 69.33 \\
\hline & & & & & 1492 & -108.82 & -17.37 & 110.20 \\
\hline & & & & & 1495 & -23.62 & -22.74 & 32.79 \\
\hline
\end{tabular}




\begin{tabular}{|c|c|c|c|c|c|c|c|c|}
\hline 1495 & 1.11 & 11.62 & 22.35 & 6.52 & & & & \\
\hline & & & & & 1494 & 23.65 & 22.79 & 32.84 \\
\hline & & & & & 1510 & -45.15 & -28.67 & 53.48 \\
\hline \multirow[t]{3}{*}{1496} & 1.03 & 10.15 & 14.73 & 4.30 & & & & \\
\hline & & & & & 1482 & 6.34 & -37.34 & 37.87 \\
\hline & & & & & 1747 & -82.42 & -15.75 & 83.92 \\
\hline \multirow[t]{3}{*}{1497} & 1.12 & 11.15 & 0.42 & 0.12 & & & & \\
\hline & & & & & 1489 & 66.29 & 81.46 & 105.03 \\
\hline & & & & & 1493 & -65.23 & -80.62 & 103.70 \\
\hline \multirow[t]{4}{*}{1498} & 0.29 & -6.80 & 24.79 & 7.23 & & & & \\
\hline & & & & & 1513 & -134.28 & -217.60 & 255.70 \\
\hline & & & & & 1526 & 117.77 & 264.94 & 289.94 \\
\hline & & & & & 1539 & -8.53 & -54.74 & 55.39 \\
\hline \multirow[t]{3}{*}{1499} & 0.68 & 6.36 & 0.00 & 0.00 & & & & \\
\hline & & & & & 1513 & 18.30 & 55.67 & 58.60 \\
\hline & & & & & 1669 & -18.92 & -56.00 & 59.11 \\
\hline \multirow[t]{3}{*}{1500} & 1.08 & 9.92 & 12.44 & 3.63 & & & & \\
\hline & & & & & 1447 & 114.77 & 132.33 & 175.17 \\
\hline & & & & & 1488 & -78.34 & -104.00 & 130.20 \\
\hline \multirow[t]{5}{*}{1501} & 1.07 & 13.24 & 0.00 & 0.00 & & & & \\
\hline & & & & & 1466 & 27.03 & 58.18 & 64.15 \\
\hline & & & & & 1468 & 95.77 & 95.67 & 135.37 \\
\hline & & & & & 1484 & 72.54 & 28.95 & 78.10 \\
\hline & & & & & 1509 & -162.99 & -73.10 & 178.63 \\
\hline \multirow[t]{3}{*}{1502} & 1.15 & 14.18 & 15.39 & 4.49 & & & & \\
\hline & & & & & 1518 & -13.48 & -20.82 & 24.81 \\
\hline & & & & & 1730 & -2.32 & 15.89 & 16.06 \\
\hline \multirow[t]{3}{*}{1503} & 0.99 & 10.75 & 0.00 & 0.00 & & & & \\
\hline & & & & & 1569 & -3.23 & -6.56 & 7.31 \\
\hline & & & & & 1597 & 3.24 & 6.07 & 6.88 \\
\hline \multirow[t]{3}{*}{1504} & 0.78 & 7.66 & 16.30 & 4.75 & & & & \\
\hline & & & & & 1515 & 83.66 & 204.98 & 221.39 \\
\hline & & & & & 1590 & -100.25 & -210.13 & 232.82 \\
\hline \multirow[t]{3}{*}{1505} & 1.08 & 10.17 & 14.85 & 4.33 & & & & \\
\hline & & & & & 1525 & 12.39 & 3.53 & 12.88 \\
\hline & & & & & 1741 & -36.84 & -17.07 & 40.60 \\
\hline \multirow[t]{4}{*}{1507} & 1.08 & 10.07 & 0.00 & 0.00 & & & & \\
\hline & & & & & 1516 & -8.64 & -3.44 & 9.30 \\
\hline & & & & & 1517 & 3.43 & 4.89 & 5.97 \\
\hline & & & & & 1522 & 3.44 & 6.19 & 7.08 \\
\hline \multirow[t]{9}{*}{1508} & 1.12 & 17.27 & 0.00 & 0.00 & & & & \\
\hline & & & & & 1410 & -168.89 & -131.67 & 214.15 \\
\hline & & & & & 1411 & -217.76 & 183.63 & 284.85 \\
\hline & & & & & 1412 & -353.16 & -223.09 & 417.73 \\
\hline & & & & & 1455 & -11.34 & 78.28 & 79.10 \\
\hline & & & & & 1509 & 43.79 & -14.49 & 46.12 \\
\hline & & & & & 1512 & 334.72 & 361.57 & 492.72 \\
\hline & & & & & 1534 & -103.86 & -497.43 & 508.16 \\
\hline & & & & & 1649 & 205.77 & -32.76 & 208.36 \\
\hline
\end{tabular}




\begin{tabular}{|c|c|c|c|c|c|c|c|c|}
\hline \multirow{8}{*}{1509} & 1.12 & 1649 & 000 & 0.00 & 1731 & 267.86 & -34.57 & 270.08 \\
\hline & & 10.43 & & 0.00 & 1407 & -99.40 & -6.74 & 99.63 \\
\hline & & & & & 1408 & -99.25 & -79.55 & 127.20 \\
\hline & & & & & 1409 & -149.17 & 5.70 & 149.28 \\
\hline & & & & & 1466 & 121.31 & 68.52 & 139.33 \\
\hline & & & & & 1492 & 108.17 & -40.88 & 115.64 \\
\hline & & & & & 1501 & 167.57 & 81.01 & 186.12 \\
\hline & & & & & 1508 & -43.76 & 15.15 & 46.31 \\
\hline \multirow[t]{3}{*}{1510} & 1.13 & 12.93 & 1.98 & 0.58 & & & & \\
\hline & & & & & 1495 & 45.50 & 29.87 & 54.43 \\
\hline & & & & & 1521 & -49.04 & -31.81 & 58.46 \\
\hline \multirow[t]{3}{*}{1511} & 0.95 & 13.44 & 0.00 & 0.00 & & & & \\
\hline & & & & & 1512 & -174.88 & -581.49 & 607.22 \\
\hline & & & & & 1513 & 174.48 & 549.59 & 576.62 \\
\hline \multirow[t]{4}{*}{1512} & 1.04 & 14.47 & 0.00 & 0.00 & & & & \\
\hline & & & & & 1508 & -324.10 & -333.59 & 465.11 \\
\hline & & & & & 1511 & 183.18 & 632.11 & 658.11 \\
\hline & & & & & 1609 & 147.08 & -302.95 & 336.76 \\
\hline \multirow[t]{6}{*}{1513} & 0.66 & 6.12 & 11.60 & 3.38 & & & & \\
\hline & & & & & 1498 & 189.46 & 557.57 & 588.87 \\
\hline & & & & & 1499 & -18.03 & -54.20 & 57.12 \\
\hline & & & & & 1511 & -170.13 & -366.02 & 403.63 \\
\hline & & & & & 1514 & -13.14 & -69.64 & 70.87 \\
\hline & & & & & 1581 & 0.69 & -57.87 & 57.88 \\
\hline \multirow[t]{4}{*}{1514} & 0.70 & 6.19 & 0.00 & 0.00 & & & & \\
\hline & & & & & 1513 & 13.72 & 72.96 & 74.24 \\
\hline & & & & & 1608 & -6.55 & -41.08 & 41.60 \\
\hline & & & & & 1707 & -7.39 & -32.25 & 33.09 \\
\hline \multirow[t]{3}{*}{1515} & 0.50 & -0.10 & 12.51 & 3.65 & & & & \\
\hline & & & & & 1504 & -69.93 & -121.66 & 140.32 \\
\hline & & & & & 1540 & 57.41 & 117.77 & 131.02 \\
\hline \multirow[t]{3}{*}{1516} & 1.08 & 10.12 & 0.00 & 0.00 & & & & \\
\hline & & & & & 1507 & 8.66 & 3.38 & 9.30 \\
\hline & & & & & 1525 & -12.19 & -7.07 & 14.09 \\
\hline \multirow[t]{2}{*}{1517} & 1.04 & 8.49 & 3.40 & 0.99 & & & & \\
\hline & & & & & 1507 & -3.43 & -4.61 & 5.74 \\
\hline \multirow[t]{3}{*}{1518} & 1.16 & 14.35 & 5.27 & 1.54 & & & & \\
\hline & & & & & 1502 & 13.70 & 20.79 & 24.90 \\
\hline & & & & & 1648 & -20.30 & -23.37 & 30.96 \\
\hline \multirow[t]{3}{*}{1519} & 1.13 & 13.27 & 0.00 & 0.00 & & & & \\
\hline & & & & & 1521 & -0.28 & -16.48 & 16.48 \\
\hline & & & & & 1589 & -2.82 & 20.35 & 20.54 \\
\hline \multirow[t]{3}{*}{1520} & 1.03 & 9.95 & 31.61 & 9.22 & & & & \\
\hline & & & & & 1430 & 10.04 & 10.46 & 14.50 \\
\hline & & & & & 1467 & -40.94 & -19.06 & 45.16 \\
\hline \multirow[t]{3}{*}{1521} & 1.14 & 13.26 & 0.00 & 0.00 & & & & \\
\hline & & & & & 1510 & 49.15 & 32.15 & 58.73 \\
\hline & & & & & 1519 & 0.31 & 16.43 & 16.44 \\
\hline
\end{tabular}




\begin{tabular}{|c|c|c|c|c|c|c|c|c|}
\hline \multirow{3}{*}{1522} & & & & & 1587 & -47.80 & $0-46.82$ & 66.91 \\
\hline & 1.03 & 8.48 & 3.40 & 0.99 & & & & \\
\hline & & & & & 1507 & -3.43 & -5.80 & 6.74 \\
\hline \multirow[t]{2}{*}{1523} & 1.03 & 7.27 & 6.13 & 1.79 & & & & \\
\hline & & & & & 1525 & -6.17 & -5.42 & 8.21 \\
\hline \multirow[t]{3}{*}{1524} & 0.88 & 8.37 & 14.71 & 4.29 & & & & \\
\hline & & & & & 1531 & -102.27 & $7-145.43$ & 3177.79 \\
\hline & 1.08 & 10.15 & $0 \mathrm{M}$ & 00 & 1656 & 86.82 & 2140.41 & 165.09 \\
\hline \multirow{3}{*}{1525} & & 10.10 & & 0.00 & 1505 & -12.38 & -3.55 & 12.88 \\
\hline & & & & & 1516 & 12.19 & 7.05 & 14.08 \\
\hline & & & & & 1523 & 6.17 & 6.00 & 8.61 \\
\hline \multirow[t]{3}{*}{1528} & 1.15 & 13.31 & 6.44 & 1.88 & & & & \\
\hline & & & & & 1536 & -72.33 & $3 \quad-16.74$ & 74.24 \\
\hline & & & & & 1537 & 64.22 & 13.91 & 65.71 \\
\hline 1529 & 1.05 & 8.86 & 2.91 & 0.85 & & & & \\
\hline \multirow{2}{*}{1530} & 1.04 & 8.89 & 2.91 & 0.85 & 1741 & -2.94 & -3.84 & 4.84 \\
\hline & & & & & 1741 & -2.94 & -4.41 & 5.30 \\
\hline \multirow[t]{3}{*}{1531} & 0.95 & 10.04 & 0.00 & 0.00 & & & & \\
\hline & & & & & 1524 & 105.51 & $1 \quad 159.56$ & 191.29 \\
\hline & 1.12 & 16.88 & 0.00 & & 1535 & -105.58 & $8-159.60$ & 191.36 \\
\hline \multirow{2}{*}{1532} & & & & & 1534 & -185.49 & -237.44 & 301.30 \\
\hline & & & & & 1535 & 183.52 & 200.91 & 272.11 \\
\hline \multirow[t]{3}{*}{1533} & 1.12 & 16.33 & 0.00 & 0.00 & & & & \\
\hline & & & & & 1536 & 101.15 & -92.25 & 136.90 \\
\hline & & & & & 1649 & -122.99 & 41.38 & 129.76 \\
\hline \multirow[t]{4}{*}{1534} & 1.13 & 17.37 & 0.00 & 0.00 & & & & \\
\hline & & & & & 1508 & 104.90 & 502.62 & 513.44 \\
\hline & & & & & 1532 & 185.87 & 238.28 & 302.20 \\
\hline & & & & & 1542 & -288.54 & -738.63 & 792.98 \\
\hline \multirow[t]{3}{*}{1535} & 1.02 & 11.76 & 75.51 & 22.02 & & & & \\
\hline & & & & & 1531 & 108.16 & 174.49 & 205.29 \\
\hline & & & & & 1532 & -182.80 & -167.34 & 247.83 \\
\hline \multirow[t]{4}{*}{1536} & 1.16 & 14.04 & 57.23 & 16.69 & & & & \\
\hline & & & & & 1528 & 72.47 & 17.61 & 74.58 \\
\hline & & & & & 1533 & -100.97 & 99.74 & 141.93 \\
\hline & & & & & 1708 & -29.17 & -92.19 & 96.69 \\
\hline \multirow[t]{3}{*}{1537} & 1.14 & 12.39 & 12.31 & 3.59 & & & & \\
\hline & & & & & 1493 & 52.65 & 10.00 & 53.59 \\
\hline & & & & & 1528 & -64.01 & -12.99 & 65.31 \\
\hline \multirow[t]{3}{*}{1539} & 0.33 & -7.23 & 10.62 & 3.10 & & & & \\
\hline & & & & & 1498 & 10.26 & 62.80 & 63.63 \\
\hline & & & & & 1540 & -21.06 & -66.06 & 69.33 \\
\hline \multirow[t]{3}{*}{1540} & 0.36 & -6.52 & 29.44 & 8.59 & & & & \\
\hline & & & & & 1515 & -51.46 & -81.09 & 96.04 \\
\hline & & & & & 1539 & 22.15 & 72.62 & 75.92 \\
\hline 1541 & 1.20 & 15.27 & 0.06 & 0.02 & & & & \\
\hline
\end{tabular}




\begin{tabular}{|c|c|c|c|c|c|c|c|c|}
\hline & & & & & 1554 & 18.02 & 30.83 & 35.71 \\
\hline & & & & & 1563 & -21.89 & -32.15 & 38.89 \\
\hline \multirow[t]{12}{*}{1542} & 1.23 & 18.67 & 0.00 & 0.00 & & & & \\
\hline & & & & & 1401 & -365.65 & -301.71 & 474.05 \\
\hline & & & & & 1402 & -219.81 & -21.74 & 220.89 \\
\hline & & & & & 1403 & -379.33 & -101.63 & 392.70 \\
\hline & & & & & 1534 & 295.00 & 798.02 & 850.80 \\
\hline & & & & & 1555 & 13.39 & -480.97 & 481.16 \\
\hline & & & & & 1556 & 273.55 & -733.75 & 783.08 \\
\hline & & & & & 1558 & 236.74 & 489.84 & 544.04 \\
\hline & & & & & 1591 & 251.66 & 414.90 & 485.26 \\
\hline & & & & & 1601 & 334.67 & 213.19 & 396.80 \\
\hline & & & & & 1662 & -391.10 & -166.63 & 425.12 \\
\hline & & & & & 1725 & -53.76 & -506.60 & 509.45 \\
\hline \multirow[t]{3}{*}{1544} & 1.22 & 15.47 & 16.24 & 4.74 & & & & \\
\hline & & & & & 1561 & 28.19 & 27.76 & 39.57 \\
\hline & & & & & 1605 & -45.16 & -31.92 & 55.30 \\
\hline \multirow[t]{4}{*}{1545} & 0.77 & 6.23 & 0.00 & 0.00 & & & & \\
\hline & & & & & 1548 & 18.77 & 38.39 & 42.73 \\
\hline & & & & & 1640 & -46.41 & -104.01 & 113.89 \\
\hline & & & & & 1669 & 26.32 & 64.29 & 69.46 \\
\hline \multirow[t]{3}{*}{1546} & 1.19 & 15.04 & 0.00 & 0.00 & & & & \\
\hline & & & & & 1637 & 8.14 & 21.92 & 23.39 \\
\hline & & & & & 1657 & -8.93 & -22.16 & 23.89 \\
\hline \multirow[t]{3}{*}{1547} & 1.20 & 15.39 & 5.83 & 1.70 & & & & \\
\hline & & & & & 1557 & -26.30 & -33.36 & 42.48 \\
\hline & & & & & 1563 & 19.16 & 31.36 & 36.75 \\
\hline \multirow[t]{3}{*}{1548} & 0.75 & 5.74 & 0.00 & 0.00 & & & & \\
\hline & & & & & 1545 & -18.56 & -37.38 & 41.73 \\
\hline & & & & & 1707 & 17.90 & 37.08 & 41.17 \\
\hline \multirow[t]{3}{*}{1549} & 1.02 & 7.51 & 9.92 & 2.89 & & & & \\
\hline & & & & & 1444 & 61.63 & 22.43 & 65.58 \\
\hline & & & & & 1449 & -23.26 & -1.64 & 23.32 \\
\hline \multirow[t]{3}{*}{1550} & 1.17 & 14.66 & 0.00 & 0.00 & & & & \\
\hline & & & & & 1552 & 102.45 & 78.64 & 129.15 \\
\hline & & & & & 1559 & -105.91 & -80.96 & 133.31 \\
\hline \multirow[t]{3}{*}{1551} & 1.18 & 14.93 & 7.64 & 2.23 & & & & \\
\hline & & & & & 1559 & -29.67 & -26.45 & 39.74 \\
\hline & & & & & 1648 & 19.22 & 22.59 & 29.66 \\
\hline \multirow[t]{3}{*}{1552} & 1.15 & 13.56 & 17.04 & 4.97 & & & & \\
\hline & & & & & 1550 & -101.92 & -75.32 & 126.73 \\
\hline & & & & & 1587 & 19.23 & 19.76 & 27.57 \\
\hline \multirow[t]{3}{*}{1554} & 1.18 & 15.02 & 14.00 & 4.08 & & & & \\
\hline & & & & & 1541 & -18.01 & -30.77 & 35.65 \\
\hline & & & & & 1559 & -3.03 & 22.57 & 22.77 \\
\hline \multirow[t]{4}{*}{1555} & 1.25 & 18.48 & 0.00 & 0.00 & & & & \\
\hline & & & & & 349 & $-92.37-$ & -555.17 & 562.80 \\
\hline & & & & & 1542 & -11.35 & 482.23 & 482.37 \\
\hline & & & & & 1557 & 101.96 & 30.78 & 106.51 \\
\hline
\end{tabular}




\begin{tabular}{|c|c|c|c|c|c|c|c|c|}
\hline \multirow{2}{*}{1556} & 1.29 & 17.21 & 0.00 & 0.00 & & & & \\
\hline & & & & & 345 & 265.97 & $7-773.39$ & 817.85 \\
\hline & & & & & 1542 & -267.35 & 5769.15 & 5814.29 \\
\hline \multirow[t]{4}{*}{1557} & 1.24 & - 15.99 & 21.09 & 6.15 & & & & \\
\hline & & & & & 1547 & 26.76 & 33.92 & 43.21 \\
\hline & & & & & 1555 & -101.81 & $1-25.97$ & $7 \quad 105.07$ \\
\hline & & & & & 1605 & 29.91 & 48.68 & 57.13 \\
\hline \multirow[t]{4}{*}{1558} & 1.20 & 18.18 & 0.00 & 0.00 & & & & \\
\hline & & & & & 1483 & 94.71 & 400.21 & 1411.26 \\
\hline & & & & & 1542 & -235.20 & $0-481.18$ & 8535.58 \\
\hline & & & & & 1637 & 139.78 & $8 \quad 36.80$ & 144.55 \\
\hline \multirow[t]{5}{*}{1559} & 1.18 & 15.03 & 0.00 & 0.00 & & & & \\
\hline & & & & & 1550 & 106.11 & 82.14 & $4 \quad 134.18$ \\
\hline & & & & & 1551 & 29.67 & 26.48 & 39.77 \\
\hline & & & & & 1554 & 3.03 & -22.58 & 22.78 \\
\hline & & & & & 1637 & -175.82 & $2-105.07$ & 7204.83 \\
\hline \multirow[t]{4}{*}{1560} & 1.11 & 15.87 & 0.00 & 0.00 & & & & \\
\hline & & & & & 1580 & 124.92 & 2113.72 & 2168.93 \\
\hline & & & & & 1591 & -49.99 & -74.98 & 90.11 \\
\hline & & & & & 1649 & -94.37 & -23.20 & 97.18 \\
\hline \multirow[t]{3}{*}{1561} & 1.21 & 15.30 & 16.50 & 4.81 & & & & \\
\hline & & & & & 1544 & -28.04 & -27.66 & 39.39 \\
\hline & & & & & 1657 & 11.38 & 22.63 & 25.33 \\
\hline \multirow[t]{3}{*}{1562} & 1.02 & 7.65 & 0.00 & 0.00 & & & & \\
\hline & & & & & 1441 & -36.33 & -17.25 & 40.22 \\
\hline & & & & & 1450 & 38.01 & 18.11 & 42.11 \\
\hline \multirow[t]{3}{*}{1563} & 1.20 & 15.31 & 0.00 & 0.00 & & & & \\
\hline & & & & & 1541 & 21.89 & 32.18 & 38.92 \\
\hline & & & & & 1547 & -19.12 & -31.32 & 36.69 \\
\hline \multirow[t]{4}{*}{1565} & 1.01 & 11.45 & 0.00 & 0.00 & & & & \\
\hline & & & & & 904 & -72.98 & -48.43 & 87.58 \\
\hline & & & & & 911 & -20.49 & 0.86 & 20.51 \\
\hline & & & & & 1737 & 39.33 & 12.87 & 41.38 \\
\hline \multirow[t]{3}{*}{1566} & 1.00 & 10.94 & 16.31 & 4.76 & & & & \\
\hline & & & & & 1569 & 7.66 & 6.93 & 10.33 \\
\hline & & & & & 1736 & -25.27 & -12.22 & 28.07 \\
\hline \multirow[t]{3}{*}{1567} & 1.02 & 7.19 & 12.70 & 3.70 & & & & \\
\hline & & & & & 1446 & -13.44 & -2.71 & 13.71 \\
\hline & & & & & 1450 & 48.35 & 20.80 & 52.64 \\
\hline \multirow[t]{3}{*}{1568} & 1.02 & 6.92 & 7.41 & 2.16 & & & & \\
\hline & & & & & 1452 & 82.22 & 30.55 & 87.71 \\
\hline & & & & & 1456 & -40.04 & -11.38 & 41.63 \\
\hline \multirow[t]{3}{*}{1569} & 1.00 & 10.82 & 4.72 & 1.38 & & & & \\
\hline & & & & & 1503 & 3.34 & 5.73 & 6.64 \\
\hline & & & & & 1566 & -7.67 & -7.10 & 10.45 \\
\hline \multirow[t]{3}{*}{1571} & 0.97 & 6.92 & 0.87 & 0.25 & & & & \\
\hline & & & & & 1617 & 10.23 & 33.88 & 35.39 \\
\hline & & & & & 1743 & -12.72 & -35.80 & 37.99 \\
\hline 1572 & 1.00 & 7.10 & 10.08 & 2.94 & & & & \\
\hline
\end{tabular}




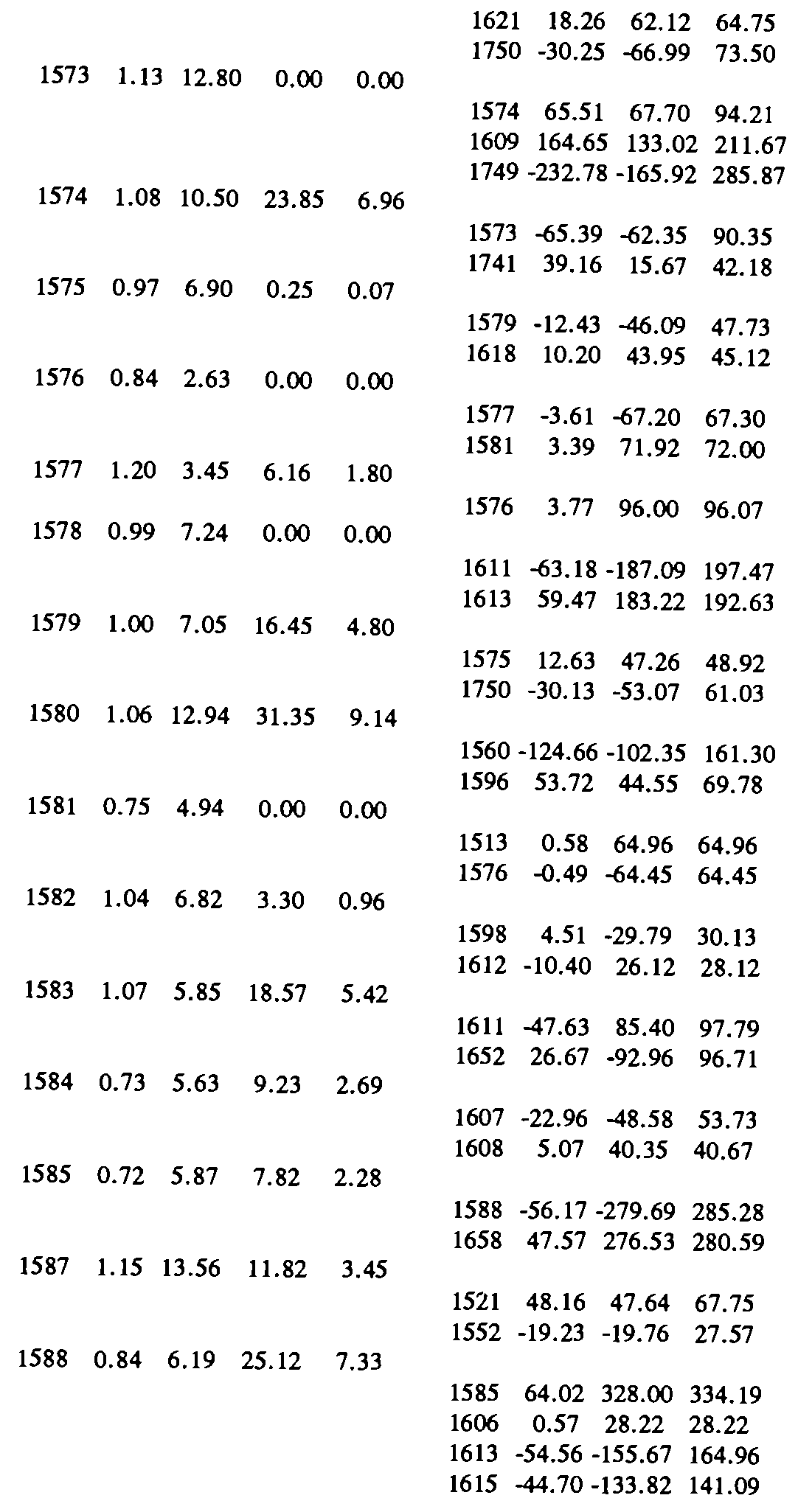




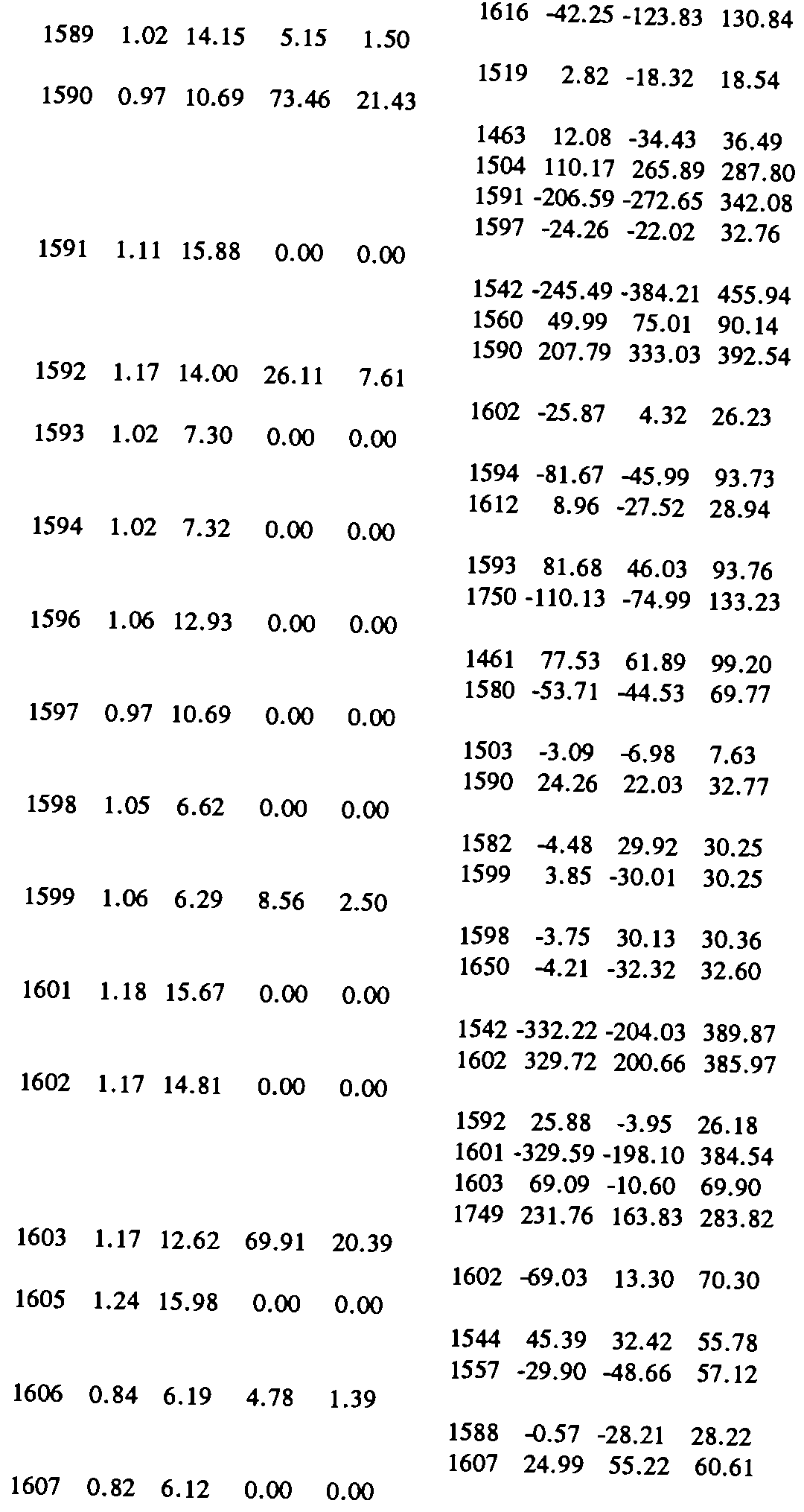




\begin{tabular}{|c|c|c|c|c|c|c|c|c|}
\hline & & & & & 1584 & 25.36 & 54.63 & 60.23 \\
\hline & & & & & 1606 & -24.56 & -54.11 & 59.42 \\
\hline \multirow{2}{*}{1608} & 0.73 & 5.65 & 3.04 & 0.89 & 1514 & & & \\
\hline & & & & & $\begin{array}{l}1514 \\
1584\end{array}$ & $\begin{array}{r}7.25 \\
-5.05\end{array}$ & 42.74 & 43.35 \\
\hline \multirow[t]{9}{*}{1609} & 1.11 & 11.92 & 0.00 & 0.00 & & -5.05 & -40.29 & 40.61 \\
\hline & & & & & 1230 & 79.18 & -359.26 & 367.88 \\
\hline & & & & & 1239 & 96.87 & -156.64 & 184.18 \\
\hline & & & & & 1512 & -141.29 & 318.50 & 348.43 \\
\hline & & & & & 1573 & -165.12 & -134.33 & 212.85 \\
\hline & & & & & 1611 & 123.85 & 134.27 & 182.67 \\
\hline & & & & & 1619 & 101.67 & 7.22 & 101.93 \\
\hline & & & & & 1731 & -223.58 & 29.87 & 225.56 \\
\hline & & & & & 1750 & 125.83 & 140.16 & 188.36 \\
\hline \multirow[t]{4}{*}{1611} & 1.03 & 7.57 & 12.16 & 3.55 & & & & \\
\hline & & & & & 1578 & 64.31 & 194.13 & 204.50 \\
\hline & & & & & 1583 & 48.37 & -81.25 & 94.56 \\
\hline & & & & & 1609 & -123.42 & -114.95 & 168.66 \\
\hline \multirow[t]{3}{*}{1612} & 1.03 & 6.99 & 0.00 & 0.00 & & & & \\
\hline & & & & & 1582 & 10.42 & -26.07 & 28.07 \\
\hline & & & & & 1593 & -8.94 & 27.64 & 29.05 \\
\hline \multirow[t]{3}{*}{1613} & 0.97 & 7.09 & 0.00 & 0.00 & & & & \\
\hline & & & & & 1578 & -58.37 & -178.29 & 187.61 \\
\hline & & & & & 1588 & 59.92 & 179.81 & 189.53 \\
\hline \multirow[t]{3}{*}{1615} & 0.93 & 6.53 & 12.90 & 3.76 & & & & \\
\hline & & & & & 1588 & 48.76 & 148.82 & 156.60 \\
\hline & & & & & 1621 & -63.64 & -154.87 & 167.43 \\
\hline \multirow[t]{4}{*}{1616} & 0.91 & 6.66 & 0.00 & 0.00 & & & & \\
\hline & & & & & 1588 & 44.51 & 134.00 & 141.20 \\
\hline & & & & & 1621 & -27.50 & -103.00 & 106.61 \\
\hline & & & & & 1696 & -19.41 & -31.67 & 37.14 \\
\hline \multirow[t]{5}{*}{1617} & 0.96 & 6.88 & 0.00 & 0.00 & & & & \\
\hline & & & & & 1571 & -10.17 & -33.66 & 35.16 \\
\hline & & & & & 1618 & -25.46 & -59.05 & 64.31 \\
\hline & & & & & 1621 & 22.42 & 128.65 & 130.59 \\
\hline & & & & & 2160 & -77.85 & -130.15 & 151.66 \\
\hline \multirow[t]{3}{*}{1618} & 0.96 & 6.89 & 0.31 & 0.09 & & & & \\
\hline & & & & & 1575 & -10.11 & -43.55 & 44.71 \\
\hline & & & & & 1617 & 25.47 & 59.11 & 64.36 \\
\hline \multirow[t]{3}{*}{1619} & 1.11 & 10.35 & 0.00 & 0.00 & & & & \\
\hline & & & & & $1609-$ & -105.13 & -20.24 & 107.06 \\
\hline & & & & & 1620 & 103.49 & 65.10 & 122.26 \\
\hline \multirow[t]{6}{*}{1620} & 1.07 & 7.59 & 21.12 & 6.16 & & & & \\
\hline & & & & & 1619 & -103.33 & -58.26 & 118.62 \\
\hline & & & & & 1622 & 26.78 & 5.47 & 27.34 \\
\hline & & & & & 1629 & 25.78 & 17.37 & 31.08 \\
\hline & & & & & 1641 & -33.63 & -61.00 & 69.66 \\
\hline & & & & & 1734 & 37.15 & 17.12 & 40.91 \\
\hline 1621 & 0.96 & 6.88 & 0.00 & 0.00 & & & & \\
\hline
\end{tabular}




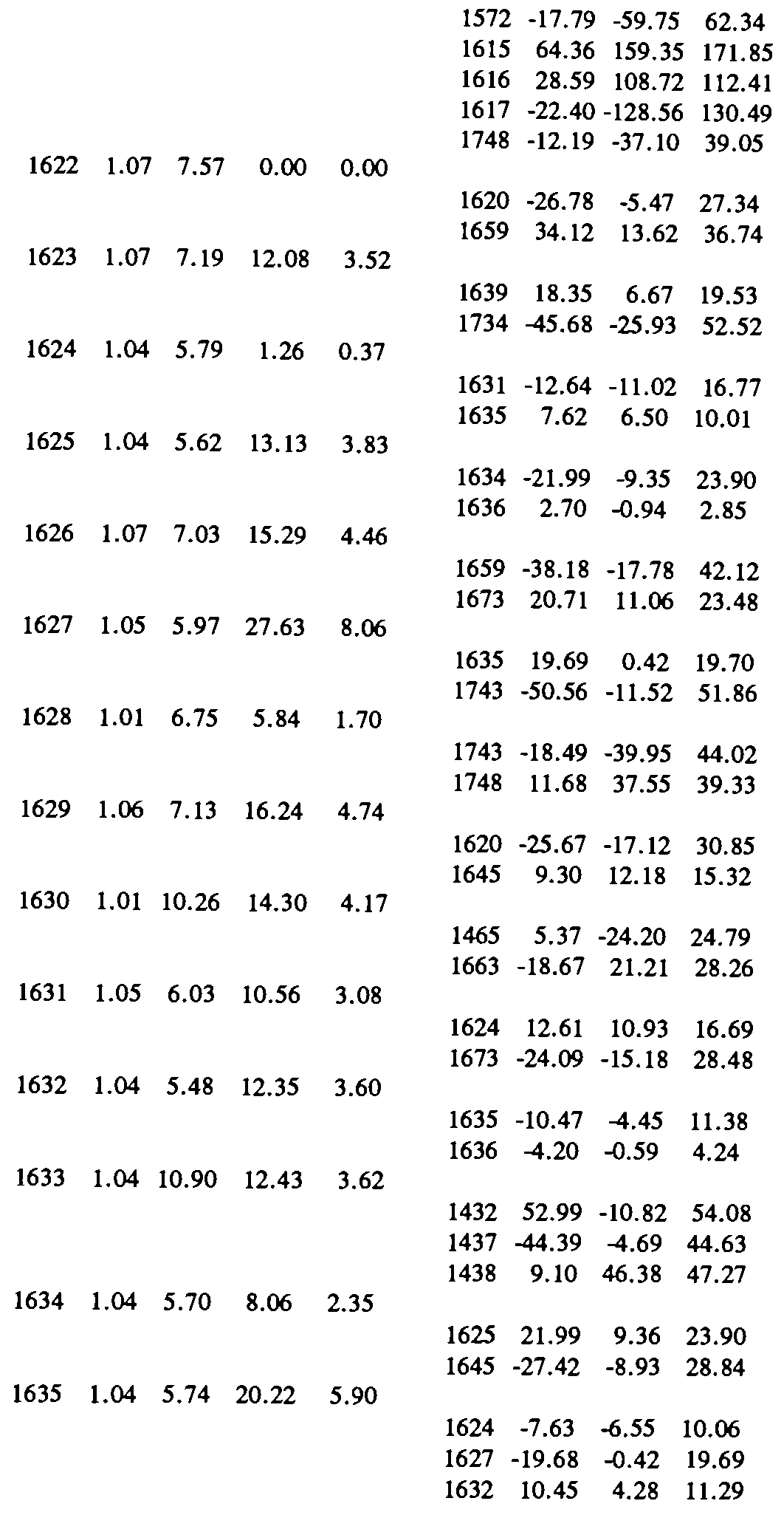




\begin{tabular}{|c|c|c|c|c|c|c|c|c|}
\hline \multirow[t]{2}{*}{1636} & 1.04 & 5.55 & 0.00 & 0.00 & & & & \\
\hline & & & & & 1625 & -2.74 & 0.72 & 2.84 \\
\hline & & & & & 1632 & 4.16 & 0.46 & 4.19 \\
\hline \multirow[t]{4}{*}{1637} & 1.18 & . 15.06 & 0.00 & 0.00 & & & & \\
\hline & & & & & 1546 & -8.07 & -22.02 & 23.45 \\
\hline & & & & & 1558 & -139.61 & $1-28.74$ & 142.54 \\
\hline & & & & & 1559 & 175.85 & 5105.22 & 204.93 \\
\hline \multirow[t]{3}{*}{1638} & 1.18 & 9.36 & 6.95 & 2.03 & & & & \\
\hline & & & & & 1642 & 40.03 & 69.37 & 80.09 \\
\hline & & & & & 2121 & -48.04 & -72.70 & 87.14 \\
\hline \multirow[t]{3}{*}{1639} & 1.07 & 7.07 & 0.93 & 0.27 & & & & \\
\hline & & & & & 1623 & -18.35 & -6.69 & 19.54 \\
\hline & & & & & 1699 & 17.98 & 7.35 & 19.42 \\
\hline \multirow[t]{3}{*}{1640} & 0.81 & 6.44 & 13.06 & 3.81 & & & & \\
\hline & & & & & 1545 & 48.45 & 109.61 & 119.84 \\
\hline & & & & & 1656 & -89.13 & -139.93 & 165.90 \\
\hline \multirow[t]{3}{*}{1641} & 1.09 & 7.87 & 0.00 & 0.00 & & & & \\
\hline & & & & & 1620 & 33.87 & 62.06 & 70.70 \\
\hline & & & & & 1642 & -36.16 & -64.76 & 74.17 \\
\hline \multirow[t]{3}{*}{1642} & 1.10 & 8.00 & 4.64 & 1.35 & & & & \\
\hline & & & & & 1638 & -38.91 & -64.15 & 75.03 \\
\hline & & & & & 1641 & 36.27 & 65.28 & 74.68 \\
\hline \multirow[t]{3}{*}{1643} & 1.15 & 10.69 & 0.00 & 0.00 & & & & \\
\hline & & & & & 1644 & -150.98 & -158.65 & 219.01 \\
\hline & & & & & 1645 & 145.19 & 196.38 & 244.22 \\
\hline \multirow[t]{4}{*}{1644} & 1.16 & 10.97 & 0.00 & 0.00 & & & & \\
\hline & & & & & 1235 & -163.90 & -328.64 & 367.24 \\
\hline & & & & & 1239 & 19.93 & 184.11 & 185.19 \\
\hline & & & & & 1643 & 151.17 & 157.73 & 218.48 \\
\hline \multirow[t]{5}{*}{1645} & 1.05 & 6.90 & 0.00 & 0.00 & & & & \\
\hline & & & & & 1629 & -9.34 & -12.21 & 15.37 \\
\hline & & & & & 1634 & 27.49 & 9.33 & 29.03 \\
\hline & & & & & 1643 & -144.62 & -170.74 & 223.76 \\
\hline & & & & & 1743 & 46.31 & 36.68 & 59.08 \\
\hline \multirow[t]{3}{*}{1648} & 1.16 & 14.44 & 0.00 & 0.00 & & & & \\
\hline & & & & & 1518 & 20.33 & 23.38 & 30.98 \\
\hline & & & & & 1551 & -19.22 & -22.57 & 29.65 \\
\hline \multirow[t]{4}{*}{1649} & 1.12 & 16.36 & 0.00 & 0.00 & & & & \\
\hline & & & & & 1508 & -204.00 & 30.50 & 206.26 \\
\hline & & & & & 1533 & 123.00 & -41.38 & 129.78 \\
\hline & & & & & 1560 & 94.69 & 17.48 & 96.29 \\
\hline \multirow[t]{2}{*}{1650} & 1.21 & 7.14 & 4.27 & 1.25 & & & & \\
\hline & & & & & 1599 & 4.26 & 36.96 & 37.21 \\
\hline \multirow[t]{2}{*}{1651} & 1.16 & 4.97 & 14.92 & 4.35 & & & & \\
\hline & & & & & 1652 & -8.03 & 134.62 & 134.86 \\
\hline \multirow{2}{*}{1652} & 1.08 & 3.40 & 18.00 & 5.25 & 1583 & -26.34 & 94.45 & 98.05 \\
\hline & & & & & 1651 & 8.44 & -125.67 & 125.95 \\
\hline
\end{tabular}

$\begin{array}{lllll}1654 & 1.06 & 14.29 & 10.12 & 2.95\end{array}$ 


\begin{tabular}{|c|c|c|c|c|c|c|c|c|}
\hline & & & & & 1429 & 13.85 & 2.98 & 14.17 \\
\hline & & & & & 1457 & -7.87 & 10.17 & 12.86 \\
\hline & & & & & 1458 & 82.07 & 44.41 & 93.32 \\
\hline & & & & & 1459 & 59.92 & 30.30 & 67.14 \\
\hline & & & & & 1664 & -156.48 & -49.45 & 164.10 \\
\hline \multirow[t]{3}{*}{1655} & 1.09 & 11.18 & 12.82 & 3.74 & & & & \\
\hline & & & & & 1464 & -30.00 & -14.34 & 33.25 \\
\hline & & & & & 1479 & 63.32 & 45.37 & 77.89 \\
\hline \multirow[t]{3}{*}{1656} & 0.81 & 6.46 & 12.58 & 3.67 & & & & \\
\hline & & & & & 1524 & -84.72 & -127.45 & 153.04 \\
\hline & & & & & 1640 & 89.18 & 140.13 & 166.10 \\
\hline \multirow[t]{3}{*}{1657} & 1.20 & 15.17 & 2.77 & 0.81 & & & & \\
\hline & & & & & 1546 & 8.98 & 21.95 & 23.72 \\
\hline & & & & & 1561 & -11.34 & -22.71 & 25.38 \\
\hline \multirow[t]{3}{*}{1658} & 0.19 & 4.40 & 2.83 & 0.82 & & & & \\
\hline & & & & & 1526 & 11.89 & 73.25 & 74.21 \\
\hline & & & & & 1585 & -14.72 & -74.11 & 75.56 \\
\hline \multirow[t]{3}{*}{1659} & 1.07 & 7.46 & 0.00 & 0.00 & & & & \\
\hline & & & & & 1622 & -34.11 & -13.57 & 36.71 \\
\hline & & & & & 1626 & 38.25 & 18.06 & 42.30 \\
\hline \multirow[t]{3}{*}{1660} & 1.04 & 12.38 & 16.97 & 4.95 & & & & \\
\hline & & & & & 1459 & -56.45 & -25.60 & 61.98 \\
\hline & & & & & 1679 & 39.36 & 20.35 & 44.31 \\
\hline \multirow[t]{2}{*}{1662} & 1.26 & 21.47 & 0.00 & 0.00 & & & & \\
\hline & & & & & 1542 & 391.79 & 190.04 & 435.45 \\
\hline \multirow[t]{6}{*}{1663} & 1.01 & 10.60 & 9.64 & 2.81 & & & & \\
\hline & & & & & 1469 & 21.37 & 56.04 & 59.98 \\
\hline & & & & & 1471 & 15.48 & 31.76 & 35.33 \\
\hline & & & & & 1630 & 18.69 & -21.16 & 28.23 \\
\hline & & & & & 1679 & -33.87 & -18.21 & 38.45 \\
\hline & & & & & 1746 & -2.55 & -18.91 & 19.08 \\
\hline \multirow[t]{4}{*}{1664} & 1.08 & 17.75 & 0.00 & 0.00 & & & & \\
\hline & & & & & 1425 & -175.90 & -23.23 & 177.42 \\
\hline & & & & & 1455 & 23.66 & -49.39 & 54.76 \\
\hline & & & & & 1654 & 156.65 & 60.14 & 167.80 \\
\hline \multirow[t]{3}{*}{1666} & 1.09 & 10.38 & 6.30 & 1.84 & & & & \\
\hline & & & & & 1490 & -52.59 & -14.25 & 54.48 \\
\hline & & & & & 1491 & 51.70 & 16.40 & 54.24 \\
\hline \multirow[t]{3}{*}{1667} & 1.16 & 11.49 & 0.00 & 0.00 & & & & \\
\hline & & & & & 1493 & 17.35 & 81.47 & 83.30 \\
\hline & & & & & 1668 & -16.39 & -70.90 & 72.77 \\
\hline \multirow[t]{2}{*}{1668} & 1.36 & 13.23 & 3.00 & 0.87 & & & & \\
\hline & & & & & 1667 & 16.70 & 83.75 & 85.40 \\
\hline \multirow[t]{3}{*}{1669} & 0.74 & 6.15 & 4.92 & 1.43 & & & & \\
\hline & & & & & 1499 & 20.79 & 60.63 & 64.10 \\
\hline & & & & & 1545 & -25.38 & -61.81 & 66.81 \\
\hline \multirow[t]{3}{*}{1673} & 1.06 & 6.33 & 5.76 & 1.68 & & & & \\
\hline & & & & & 1626 & -20.71 & -11.06 & 23.47 \\
\hline & & & & & 1631 & 24.09 & 15.24 & 28.51 \\
\hline
\end{tabular}




\begin{tabular}{|c|c|c|c|c|c|c|c|c|}
\hline \multirow[t]{3}{*}{1674} & 1.03 & 10.75 & 11.60 & 3.38 & 1700 & -8.57 & -5.15 & 10.00 \\
\hline & & & & & 1418 & 9.40 & 10.79 & 14.31 \\
\hline & & & & & 1468 & -20.01 & -13.02 & 23.87 \\
\hline \multirow[t]{3}{*}{1675} & 1.03 & 11.66 & 12.22 & 3.56 & & & & \\
\hline & & & & & 1426 & -81.09 & -27.70 & 85.69 \\
\hline & & & & & 1435 & 69.52 & 25.07 & 73.90 \\
\hline \multirow[t]{3}{*}{1676} & 1.05 & 12.39 & 12.15 & 3.55 & & & & \\
\hline & & & & & 1437 & 71.80 & 15.85 & 73.53 \\
\hline & & & & & 1744 & -83.69 & -19.12 & 85.85 \\
\hline \multirow[t]{3}{*}{1678} & 1.04 & 8.56 & 6.99 & 2.04 & & & & \\
\hline & & & & & 1441 & 52.55 & 23.51 & 57.57 \\
\hline & & & & & 1448 & -56.60 & -23.91 & 61.44 \\
\hline \multirow[t]{3}{*}{1679} & 1.02 & 11.44 & 5.54 & 1.62 & & & & \\
\hline & & & & & 1660 & -39.08 & -19.70 & 43.76 \\
\hline & & & & & 1663 & 34.05 & 18.70 & 38.84 \\
\hline \multirow[t]{3}{*}{1696} & 0.92 & 6.52 & 0.00 & 0.00 & & & & \\
\hline & & & & & 1616 & 19.66 & 31.85 & 37.43 \\
\hline & & & & & 1697 & -18.40 & -22.96 & 29.43 \\
\hline \multirow[t]{2}{*}{1697} & 1.03 & 11.40 & 8.75 & 2.55 & & & & \\
\hline & & & & & 1696 & 18.46 & 27.55 & 33.16 \\
\hline \multirow[t]{3}{*}{1699} & 1.06 & 6.77 & 4.66 & 1.36 & & & & \\
\hline & & & & & 1639 & -17.91 & -7.43 & 19.39 \\
\hline & & & & & 1700 & 12.51 & 4.77 & 13.39 \\
\hline \multirow[t]{3}{*}{1700} & 1.06 & 6.61 & 5.13 & 1.50 & & & & \\
\hline & & & & & 1673 & 8.61 & 4.76 & 9.84 \\
\hline & & & & & 1699 & -12.47 & -4.91 & 13.40 \\
\hline \multirow[t]{3}{*}{1707} & 0.74 & 5.69 & 10.12 & 2.95 & & & & \\
\hline & & & & & 1514 & 8.20 & 33.93 & 34.91 \\
\hline & & & & & 1548 & -17.71 & -36.64 & 40.70 \\
\hline \multirow[t]{3}{*}{1708} & 1.17 & 14.10 & 0.00 & 0.00 & & & & \\
\hline & & & & & 1536 & 29.29 & 92.78 & 97.30 \\
\hline & & & & & 1709 & -29.74 & -123.82 & 127.35 \\
\hline \multirow[t]{2}{*}{1709} & 1.24 & 14.73 & 6.68 & 1.95 & & & & \\
\hline & & & & & 1708 & 30.11 & 131.71 & 135.11 \\
\hline \multirow[t]{3}{*}{1725} & 1.26 & 18.64 & 0.00 & 0.00 & & & & \\
\hline & & & & & 349 & $-53.72-$ & -511.18 & 513.99 \\
\hline & & & & & 1542 & 57.08 & 510.10 & 513.28 \\
\hline \multirow[t]{3}{*}{1726} & 1.06 & 9.73 & 7.55 & 2.20 & & & & \\
\hline & & & & & 1479 & -46.24 & -37.22 & 59.36 \\
\hline & & & & & 1485 & 80.35 & 62.72 & 101.93 \\
\hline \multirow[t]{3}{*}{1730} & 1.14 & 14.40 & 38.65 & 11.27 & & & & \\
\hline & & & & & 1502 & 2.51 & -16.22 & 16.41 \\
\hline & & & & & 1731 & -40.36 & 41.41 & 57.83 \\
\hline \multirow[t]{4}{*}{1731} & 1.12 & 15.43 & 0.00 & 0.00 & & & & \\
\hline & & & & & 1508 & -263.73 & 34.91 & 266.03 \\
\hline & & & & & 1609 & 226.78 & -28.72 & 228.59 \\
\hline & & & & & 1730 & 40.39 & -39.97 & 56.83 \\
\hline 1734 & 1.07 & 7.25 & 0.00 & 0.00 & & & & \\
\hline
\end{tabular}




\begin{tabular}{|c|c|c|c|c|c|c|c|c|}
\hline \multirow[b]{2}{*}{1736} & \multirow[b]{2}{*}{1.01} & \multirow[b]{2}{*}{11.41} & \multirow[b]{2}{*}{22.11} & \multirow[b]{2}{*}{6.45} & \multirow{2}{*}{$\begin{array}{l}1620 \\
1623\end{array}$} & \multirow{2}{*}{$\begin{array}{r}-37.12 \\
45.70\end{array}$} & \multirow{2}{*}{$\begin{array}{r}-16.94 \\
25.99\end{array}$} & \multirow{2}{*}{$\begin{array}{l}40.80 \\
52.57\end{array}$} \\
\hline & & & & & & & & \\
\hline & & & & & 1566 & 25.42 & 12.32 & 28.25 \\
\hline & & & & & 1737 & -101.58 & $\begin{array}{ll}-53.84 \\
\end{array}$ & 114.97 \\
\hline 1737 & 1.01 & 11.44 & 3.32 & 0.97 & & & & \\
\hline & & & & & 1565 & -39.33 & -12.86 & 41.37 \\
\hline 1741 & 1.08 & 10.26 & 0.00 & 0.00 & 1736 & 101.60 & 53.90 & 115.01 \\
\hline & & & & & 1505 & 36.85 & 17.11 & 40.63 \\
\hline & & & & & 1529 & 2.94 & 4.04 & 5.00 \\
\hline & & & & & 1530 & 2.94 & 4.65 & 5.50 \\
\hline 1743 & 1.05 & 6.89 & 12.01 & 3.50 & 1574 & -39.12 & -15.54 & 42.09 \\
\hline & & & & & 1571 & 13.61 & 37.78 & 40.16 \\
\hline & & & & & 1627 & 50.65 & 12.20 & 52.10 \\
\hline & & & & & 1628 & 19.00 & 41.17 & 45.34 \\
\hline 1744 & 1.06 & 13.86 & 26.63 & 7.77 & 1645 & -46.31 & -36.66 & 59.06 \\
\hline & & & & & 1425 & -207.95 & -49.99 & 213.87 \\
\hline & & & & & 1426 & 115.19 & 92.04 & 147.44 \\
\hline & & & & & 1676 & 84.11 & 21.40 & 86.79 \\
\hline 1745 & 1.07 & 12.69 & 10.60 & 3.09 & & & & \\
\hline & & & & & 1445 & 102.59 & 40.61 & 110.33 \\
\hline 1746 & 1.01 & 10.59 & 12.41 & 3.62 & 1484 & -83.66 & -16.72 & 85.31 \\
\hline & & & & & 1472 & 16.81 & 11.72 & 20.49 \\
\hline 1747 & 1.03 & 10.17 & 11.52 & 3.36 & 1663 & 2.59 & 18.90 & 19.07 \\
\hline & & & & & 1468 & -56.12 & 10.90 & 57.17 \\
\hline 1748 & 0.97 & 6.94 & 0.00 & 0.00 & 1496 & 82.43 & 15.78 & 83.93 \\
\hline & & & & & 1621 & 12.27 & 37.40 & 39.36 \\
\hline 1749 & 1.15 & 14.20 & 0.00 & 0.00 & 1628 & -11.03 & -36.19 & 37.84 \\
\hline & & & & & 1573 & 234.56 & 167.85 & 288.43 \\
\hline 1750 & 1.02 & 7.34 & 0.00 & 0.00 & 1602 & -231.02 & -163.27 & 282.89 \\
\hline & & & & & 1572 & 30.56 & 68.35 & 74.88 \\
\hline & & & & & 1579 & 30.33 & 53.97 & 61.91 \\
\hline & & & & & 1594 & 110.14 & 75.07 & 133.29 \\
\hline & & & & & 1609 & -125.21 & -118.85 & 172.64 \\
\hline & 1.01 & 32.14 & 0.00 & 0.60 & 1846 & 43.47 & 65.34 & 78.48 \\
\hline 1771 & 1.75 & 31.22 & 0.00 & 0.00 & & & & \\
\hline 1772 & 1.62 & 31.78 & 0.00 & 0.00 & 1847 & 41.45 & 127.24 & 133.82 \\
\hline 1773 & 1.70 & 33.16 & 0.00 & 0.00 & 1846 & 134.70 & 96.11 & 165.47 \\
\hline
\end{tabular}




\begin{tabular}{|c|c|c|c|c|c|c|c|c|}
\hline \multirow{2}{*}{$\begin{array}{l}1774 \\
1775\end{array}$} & 1.67 & 32.60 & 0.00 & 0.00 & 1935 & 394.77 & 440.70 & 591.66 \\
\hline & 1.67 & 29.65 & 0.00 & 0.00 & 1935 & 264.75 & 283.35 & 387.79 \\
\hline 1776 & 1.62 & 30.52 & 0.00 & 0.00 & 1967 & 152.68 & 675.85 & 692.88 \\
\hline 1777 & 1.76 & 30.51 & 0.00 & 0.00 & 1888 & 26.27 & 25.98 & 36.95 \\
\hline 1778 & 1.66 & 31.25 & 0.00 & 0.00 & 1888 & 28.31 & 118.06 & 121.41 \\
\hline 1779 & 1.69 & 29.65 & 0.00 & 0.00 & 1888 & 46.39 & 62.58 & 77.90 \\
\hline 1780 & 1.62 & 32.00 & 0.00 & 0.00 & 1888 & 32.14 & 144.27 & 147.80 \\
\hline 1781 & 1.70 & 35.15 & 0.00 & 0.00 & 1888 & 160.23 & 80.14 & 179.15 \\
\hline 1782 & 1.69 & 35.06 & 0.00 & 0.00 & 1970 & 709.38 & 774.47 & 1050.25 \\
\hline 1783 & 1.64 & 32.93 & 0.00 & 0.00 & 1970 & 679.79 & 677.38 & 959.67 \\
\hline 1784 & 1.62 & 32.49 & 0.00 & 0.00 & 1846 & 110.57 & 75.45 & 133.86 \\
\hline 1785 & 1.62 & 31.32 & 0.00 & 0.00 & 1847 & 110.45 & 46.60 & 119.88 \\
\hline \multirow[t]{2}{*}{1800} & 1.60 & 29.01 & 2.52 & 1.07 & 1935 & 63.22 & 46.05 & 78.22 \\
\hline & 1.61 & 29.05 & 12.42 & 5.29 & 1879 & -4.42 & -4.39 & 6.23 \\
\hline \multirow{2}{*}{1803} & & 29.00 & 12.42 & & 1840 & -24.90 & -94.55 & 97.78 \\
\hline & & & & & 1879 & 10.94 & 56.30 & 57.35 \\
\hline \multirow[t]{2}{*}{1806} & 1.58 & 29.10 & 30.75 & 13.10 & & & & \\
\hline & & & & & $\begin{array}{l}1858 \\
1870\end{array}$ & $\begin{array}{r}-64.72 \\
32.73\end{array}$ & $\begin{array}{r}8.25 \\
-42.99\end{array}$ & $\begin{array}{r}65.24 \\
54.03\end{array}$ \\
\hline \multirow[t]{3}{*}{1809} & 1.56 & 29.02 & 21.87 & 9.32 & & & & \\
\hline & & & & & $\begin{array}{l}1813 \\
1846\end{array}$ & $\begin{array}{r}33.45 \\
-164.54\end{array}$ & $\begin{array}{l}-10.65 \\
-37.23\end{array}$ & $\begin{array}{c}35.11 \\
168.69\end{array}$ \\
\hline & & & & & 1876 & 92.35 & -19.75 & 94.44 \\
\hline \multirow[t]{3}{*}{1813} & 1.56 & 28.86 & 15.60 & 6.64 & & & & \\
\hline & & & & & $\begin{array}{l}1809 \\
1853\end{array}$ & $\begin{array}{c}-33.46 \\
-7.95\end{array}$ & $\begin{array}{r}10.58 \\
-75.81\end{array}$ & $\begin{array}{c}35.09 \\
76.23\end{array}$ \\
\hline & & & & & 1894 & -13.46 & -77.49 & 78.65 \\
\hline \multirow[t]{3}{*}{1816} & 1.56 & 28.76 & 12.56 & 5.35 & & & & \\
\hline & & & & & 1876 & 44.06 & 22.30 & 49.38 \\
\hline & & & & & 1888 & -78.87 & -121.01 & 144.45 \\
\hline \multirow[t]{2}{*}{1817} & 1.56 & 28.76 & 12.56 & 5.35 & & & & \\
\hline & & & & & $\begin{array}{l}1876 \\
1888\end{array}$ & $\begin{array}{r}44.49 \\
-78.14\end{array}$ & $\begin{array}{c}22.01 \\
-121.50\end{array}$ & $\begin{array}{c}49.64 \\
144.46\end{array}$ \\
\hline \multirow[t]{2}{*}{1823} & 1.51 & 27.42 & 40.63 & 17.31 & & & & \\
\hline & & & & & 1832 & -44.15 & -43.76 & 62.16 \\
\hline
\end{tabular}




\begin{tabular}{|c|c|c|c|c|c|c|c|c|}
\hline & & & & & 1835 & -15.49 & -38.54 & 41.54 \\
\hline & & & & & 1861 & 47.91 & 16.37 & 50.63 \\
\hline & & & & & 1873 & 26.32 & 7.90 & 27.48 \\
\hline & & & & & 1911 & -8.83 & 2.10 & 9.08 \\
\hline & & & & & 1954 & -49.50 & -135.88 & 144.61 \\
\hline \multirow[t]{5}{*}{1826} & 1.57 & 28.86 & 17.84 & 7.60 & & & & \\
\hline & & & & & 1847 & -63.82 & -37.53 & 74.04 \\
\hline & & & & & 1853 & 21.62 & 3.28 & 21.87 \\
\hline & & & & & 1888 & -60.94 & -153.74 & 165.38 \\
\hline & & & & & 1894 & 17.17 & 3.35 & 17.49 \\
\hline \multirow[t]{3}{*}{1829} & 1.55 & 28.57 & 55.93 & 23.83 & & & & \\
\hline & & & & & 1846 & -70.18 & -24.77 & 74.43 \\
\hline & & & & & 1897 & 11.93 & -16.61 & 20.45 \\
\hline \multirow[t]{3}{*}{1832} & 1.54 & 28.14 & 37.95 & 16.17 & & & & \\
\hline & & & & & 1823 & 44.57 & 44.42 & 62.92 \\
\hline & & & & & 1876 & -83.25 & -71.39 & 109.67 \\
\hline \multirow[t]{3}{*}{1835} & 1.52 & 27.47 & 46.10 & 19.64 & & & & \\
\hline & & & & & 1823 & 15.68 & 37.51 & 40.65 \\
\hline & & & & & 1850 & -60.96 & -62.98 & 87.65 \\
\hline \multirow[t]{3}{*}{1838} & 1.57 & 28.78 & 32.49 & 13.84 & & & & \\
\hline & & & & & 1882 & -14.48 & -47.19 & 49.36 \\
\hline & & & & & 1888 & -25.95 & -0.02 & 25.95 \\
\hline \multirow[t]{3}{*}{1840} & 1.64 & 29.14 & 16.95 & 7.22 & & & & \\
\hline & & & & & 1803 & 25.18 & 95.89 & 99.14 \\
\hline & & & & & 1918 & -40.53 & 117.58 & 124.37 \\
\hline \multirow[t]{3}{*}{1843} & 1.56 & 28.61 & 0.93 & 0.40 & & & & \\
\hline & & & & & 1867 & -33.06 & -38.22 & 50.54 \\
\hline & & & & & 1897 & 29.77 & 2.91 & 29.91 \\
\hline \multirow[t]{7}{*}{1846} & 1.58 & 29.94 & 24.77 & 10.55 & & & & \\
\hline & & & & & 1770 & -43.47 & -60.20 & 74.25 \\
\hline & & & & & 1772 & -134.48 & -89.60 & 161.60 \\
\hline & & & & & 1783 & -110.29 & -67.11 & 129.10 \\
\hline & & & & & 1809 & 169.78 & 13.17 & 170.29 \\
\hline & & & & & 1829 & 70.83 & 26.19 & 75.51 \\
\hline & & & & & 1891 & 20.91 & -14.12 & 25.23 \\
\hline \multirow[t]{6}{*}{1847} & 1.58 & 29.45 & 0.00 & 0.00 & & & & \\
\hline & & & & & 1771 & -41.00 & -113.75 & 120.91 \\
\hline & & & & & 1784 & -110.22 & -39.74 & 117.17 \\
\hline & & & & & 1826 & 64.03 & 38.15 & 74.53 \\
\hline & & & & & 1864 & 89.06 & 44.45 & 99.53 \\
\hline & & & & & 1905 & -6.34 & -99.75 & 99.95 \\
\hline \multirow[t]{5}{*}{1850} & 1.53 & 27.92 & 42.03 & 17.91 & & & & \\
\hline & & & & & 1835 & 61.15 & 63.67 & 88.28 \\
\hline & & & & & 1876 & -60.20 & -53.26 & 80.38 \\
\hline & & & & & 1897 & -57.49 & -58.57 & 82.07 \\
\hline & & & & & 1921 & 14.18 & 37.56 & 40.15 \\
\hline \multirow[t]{3}{*}{1853} & 1.57 & 28.83 & 41.74 & 17.78 & & & & \\
\hline & & & & & 1813 & 8.39 & 69.95 & 70.45 \\
\hline & & & & & 1826 & -21.26 & -9.67 & 23.36 \\
\hline
\end{tabular}


$\begin{array}{lllll}1856 & 1.58 & 29.90 & 33.22 & 14.15\end{array}$

$\begin{array}{lllll}1858 & 1.59 & 30.11 & 0.00 & 0.00\end{array}$

$$
\begin{array}{cccc}
1858 & -40.26 & -40.07 & 56.80 \\
1891 & 4.49 & -12.00 & 12.81
\end{array}
$$

$\begin{array}{llll}1806 & 64.93 & -7.62 & 65.38\end{array}$

$\begin{array}{lllll}1856 & 40.33 & 40.21 & 56.95\end{array}$

$\begin{array}{lllll}1882 & 117.21 & -129.97 & 175.01\end{array}$

$1963-219.29-159.73 \quad 271.29$

$\begin{array}{lllll}1861 & 1.50 & 26.78 & 48.17 & 20.52\end{array}$

$\begin{array}{llll}1823 & -47.71 & -16.14 & 50.37\end{array}$

$\begin{array}{lllll}1864 & 1.57 & 29.13 & 23.50 & 10.01\end{array}$

$\begin{array}{llll}1847 & -88.66 & -44.31 & 99.12\end{array}$

$\begin{array}{lllll}1867 & 1.56 & 28.78 & 33.53 & 14.28\end{array}$

$\begin{array}{llll}1867 & 64.75 & 33.37 & 72.85\end{array}$

$\begin{array}{llll}1843 & 33.20 & 38.29 & 50.68\end{array}$

$\begin{array}{lllll}1870 & 1.59 & 28.81 & 45.85 & 19.53\end{array}$

$\begin{array}{llll}1864 & -64.48 & -32.97 & 72.42\end{array}$

$\begin{array}{llll}1806 & -32.61 & 43.16 & 54.09\end{array}$

$\begin{array}{llll}1882 & -13.18 & -63.52 & 64.87\end{array}$

$\begin{array}{lllll}1873 & 1.50 & 26.88 & 26.53 & 11.30\end{array}$

$\begin{array}{llll}1823 & -26.18 & -8.39 & 27.49\end{array}$

$\begin{array}{lllll}1876 & 1.56 & 28.70 & 43.93 & 18.71\end{array}$

$\begin{array}{llll}1809 & -93.79 & 8.80 & 94.20\end{array}$

$\begin{array}{llll}1816 & -44.80 & -27.92 & 52.79\end{array}$

$\begin{array}{llll}1817 & -44.80 & -27.92 & 52.79\end{array}$

$\begin{array}{lllll}1832 & 83.51 & 72.80 & 110.79\end{array}$

$\begin{array}{llll}1850 & 60.51 & 54.44 & 81.39\end{array}$

$\begin{array}{llll}1897 & 15.72 & 6.89 & 17.16\end{array}$

$\begin{array}{lllll}1879 & 1.61 & 29.04 & 25.49 & 10.86\end{array}$

$\begin{array}{llll}1800 & 4.60 & 3.97 & 6.08\end{array}$

$\begin{array}{lllll}1803 & -10.85 & -56.16 & 57.20\end{array}$

$\begin{array}{llll}1945 & -15.92 & 75.11 & 76.77\end{array}$

$\begin{array}{lllll}1882 & 1.61 & 28.84 & 43.67 & 18.60\end{array}$

$\begin{array}{llll}1838 & 14.97 & 47.42 & 49.72\end{array}$

$\begin{array}{llll}1858 & -116.06 & 133.84 & 177.15\end{array}$

$\begin{array}{llll}1870 & 13.45 & 64.02 & 65.41\end{array}$

$\begin{array}{lllll}1885 & 34.39 & 50.19 & 60.84\end{array}$

$\begin{array}{llll}1947 & 11.25 & 87.65 & 88.37\end{array}$

$\begin{array}{lllll}1885 & 1.59 & 28.51 & 48.69 & 20.74\end{array}$

$\begin{array}{llll}1882 & -34.18 & -49.70 & 60.32\end{array}$

$\begin{array}{llll}1888 & -17.80 & 27.56 & 32.81\end{array}$

$\begin{array}{lllll}1888 & 1.57 & 28.88 & 52.04 & 22.17\end{array}$

$\begin{array}{llll}1776 & -26.27 & -24.53 & 35.94\end{array}$

$\begin{array}{lllll}1777 & -28.31 & -104.83 & 108.59\end{array}$

$\begin{array}{lllll}1778 & -46.39 & -57.46 & 73.85\end{array}$

$\begin{array}{lllll}1779 & -31.81 & -134.28 & 138.00\end{array}$

$\begin{array}{llll}1780 & -159.86 & -69.34 & 174.25\end{array}$

$\begin{array}{lllll}1816 & 78.79 & 116.55 & 140.68\end{array}$

$\begin{array}{llll}1817 & 78.79 & 116.55 & 140.68\end{array}$ 


\begin{tabular}{|c|c|c|c|c|c|c|c|c|}
\hline & & & & & 1826 & 60.53 & 151.07 & 162.74 \\
\hline & & & & & 1838 & 28.15 & -8.50 & 29.40 \\
\hline & & & & & 1885 & 15.69 & -35.37 & 38.69 \\
\hline \multirow[t]{3}{*}{1891} & 1.58 & 29.86 & 28.66 & 12.21 & & & & \\
\hline & & & & & 1846 & -19.79 & 10.63 & 22.47 \\
\hline & & & & & 1856 & -4.48 & 11.75 & 12.57 \\
\hline \multirow[t]{3}{*}{1894} & 1.57 & 28.84 & 30.86 & 13.14 & & & & \\
\hline & & & & & 1813 & 13.09 & 74.34 & 75.49 \\
\hline & & & & & 1826 & -17.60 & -5.73 & 18.51 \\
\hline \multirow[t]{6}{*}{1897} & 1.56 & 28.45 & 0.00 & 0.00 & & & & \\
\hline & & & & & 1829 & -11.96 & 16.41 & 20.30 \\
\hline & & & & & 1843 & -29.75 & -2.96 & 29.90 \\
\hline & & & & & 1850 & 57.81 & 59.46 & 82.93 \\
\hline & & & & & 1876 & -15.77 & -7.15 & 17.32 \\
\hline & & & & & 1973 & 3.37 & -20.84 & 21.11 \\
\hline \multirow[t]{3}{*}{1900} & 1.54 & 25.91 & 0.00 & 0.00 & & & & \\
\hline & & & & & 380 & 186.34 & 121.72 & 222.57 \\
\hline & & & & & 1933 & -183.98 & 69.14 & 196.54 \\
\hline \multirow[t]{3}{*}{1901} & 1.59 & 27.79 & 30.21 & 12.87 & & & & \\
\hline & & & & & 1937 & 109.79 & -105.62 & 152.35 \\
\hline & & & & & 1947 & -148.90 & 31.74 & 152.24 \\
\hline \multirow[t]{3}{*}{1902} & 1.59 & 30.00 & 0.28 & 0.12 & & & & \\
\hline & & & & & 1931 & 49.58 & 24.44 & 55.28 \\
\hline & & & & & 1935 & -48.74 & -92.37 & 104.45 \\
\hline \multirow[t]{3}{*}{1903} & 1.51 & 27.59 & 34.67 & 14.77 & & & & \\
\hline & & & & & 1911 & 10.05 & 26.08 & 27.95 \\
\hline & & & & & 1933 & -46.77 & -78.69 & 91.54 \\
\hline \multirow[t]{3}{*}{1905} & 1.61 & 29.52 & 0.00 & 0.00 & & & & \\
\hline & & & & & 1847 & 6.34 & 101.78 & 101.98 \\
\hline & & & & & 1918 & -9.97 & 126.47 & 126.86 \\
\hline \multirow[t]{3}{*}{1907} & 1.60 & 29.71 & 12.42 & 5.29 & & & & \\
\hline & & & & & 1918 & 49.15 & -129.69 & 138.69 \\
\hline & & & & & 1935 & -62.47 & 53.48 & 82.24 \\
\hline \multirow[t]{2}{*}{1909} & 1.58 & 28.90 & 35.49 & 15.12 & & & & \\
\hline & & & & & 1945 & -35.77 & -22.48 & 42.25 \\
\hline \multirow[t]{3}{*}{1911} & 1.51 & 27.53 & 0.00 & 0.00 & & & & \\
\hline & & & & & 1823 & 8.83 & -2.08 & 9.07 \\
\hline & & & & & 1903 & -10.05 & -26.01 & 27.88 \\
\hline \multirow[t]{3}{*}{1913} & 1.56 & 30.05 & 29.55 & 12.59 & & & & \\
\hline & & & & & 1925 & 86.44 & -64.55 & 107.88 \\
\hline & & & & & 1957 & -114.48 & -132.31 & 174.96 \\
\hline \multirow[t]{4}{*}{1915} & 1.56 & 28.91 & 22.32 & 9.51 & & & & \\
\hline & & & & & 1927 & 100.21 & 80.96 & 128.83 \\
\hline & & & & & 1935 & -143.77 & -124.08 & 3 189.91 \\
\hline & & & & & 1943 & 19.84 & -7.45 & 21.19 \\
\hline \multirow[t]{3}{*}{1916} & 1.57 & 27.30 & 0.00 & 0.00 & & & & \\
\hline & & & & & 1923 & 20.18 & -58.08 & 61.49 \\
\hline & & & & & 1960 & -20.61 & -151.10 & 152.50 \\
\hline 1918 & 1.61 & 29.57 & 0.00 & 0.00 & & & & \\
\hline
\end{tabular}




\begin{tabular}{|c|c|c|c|c|c|c|c|c|}
\hline & & & & & 1840 & $40.53-$ & -114.77 & 121.72 \\
\hline & & & & & 1905 & 10.05 & -126.04 & 126.44 \\
\hline & & & & & 1907 & -49.05 & 130.36 & 139.28 \\
\hline \multirow[t]{3}{*}{1921} & 1.52 & 27.76 & 0.00 & 0.00 & & & & \\
\hline & & & & & 1850 & -14.18 & -37.23 & 39.84 \\
\hline & & & & & 1933 & 12.21 & -5.90 & 13.56 \\
\hline \multirow[t]{3}{*}{1923} & 1.57 & 27.15 & 70.46 & 30.02 & & & & \\
\hline & & & & & 1916 & -20.12 & 58.43 & 61.80 \\
\hline & & & & & 1942 & -53.84 & -129.73 & 140.46 \\
\hline \multirow[t]{3}{*}{1925} & 1.57 & 29.65 & 29.33 & 12.50 & & & & \\
\hline & & & & & 1913 & -86.30 & 65.40 & 108.28 \\
\hline & & & & & 1931 & 56.68 & -85.69 & 102.74 \\
\hline \multirow[t]{4}{*}{1927} & 1.54 & 28.17 & 0.00 & 0.00 & & & & \\
\hline & & & & & 1915 & -99.86 & -78.56 & 127.06 \\
\hline & & & & & 1933 & 65.74 & 50.20 & 82.71 \\
\hline & & & & & 1939 & 31.82 & -9.77 & 33.29 \\
\hline \multirow[t]{4}{*}{1931} & 1.58 & 29.36 & 33.40 & 14.23 & & & & \\
\hline & & & & & 1902 & -49.46 & -23.69 & 54.84 \\
\hline & & & & & 1925 & -56.56 & 86.44 & 103.30 \\
\hline & & & & & 1947 & 71.51 & -62.92 & 95.26 \\
\hline \multirow[t]{7}{*}{1933} & 1.52 & 27.70 & 0.00 & 0.00 & & & & \\
\hline & & & & & 1900 & 183.98 & -62.63 & 194.35 \\
\hline & & & & & 1903 & 46.93 & 79.35 & 92.19 \\
\hline & & & & & 1921 & -12.21 & 5.91 & 13.57 \\
\hline & & & & & 1927 & -65.60 & -49.26 & 82.04 \\
\hline & & & & & 1945 & -92.94 & -98.57 & 135.48 \\
\hline & & & & & 1965 & -56.12 & -125.30 & 137.30 \\
\hline \multirow[t]{10}{*}{1935} & 1.59 & 30.06 & 5.99 & 2.55 & & & & \\
\hline & & & & & 1773 & -393.24 & -393.89 & 556.59 \\
\hline & & & & & 1774 & -264.75 & -259.31 & 370.59 \\
\hline & & & & & 1785 & -63.15 & -43.91 & 76.92 \\
\hline & & & & & 1902 & 48.79 & 92.68 & 104.73 \\
\hline & & & & & 1907 & 62.56 & -52.91 & 81.94 \\
\hline & & & & & 1915 & 144.63 & 129.98 & 194.46 \\
\hline & & & & & 1945 & 146.54 & 45.93 & 153.57 \\
\hline & & & & & 1947 & 112.02 & 7.45 & 112.27 \\
\hline & & & & & 1967 & 197.16 & 89.19 & 216.40 \\
\hline \multirow[t]{3}{*}{1937} & 1.59 & 27.48 & 47.44 & 20.21 & & & & \\
\hline & & & & & 1901 & -109.58 & 106.53 & 152.83 \\
\hline & & & & & 1941 & 63.35 & 45.90 & 78.23 \\
\hline \multirow[t]{2}{*}{1939} & 1.54 & 28.08 & 32.99 & 14.05 & & & & \\
\hline & & & & & 1927 & -31.82 & 9.82 & 33.30 \\
\hline \multirow[t]{3}{*}{1941} & 1.59 & 27.33 & 35.85 & 15.27 & & & & \\
\hline & & & & & 1937 & -63.31 & -45.61 & 78.03 \\
\hline & & & & & 1942 & 27.80 & 39.11 & 47.98 \\
\hline \multirow[t]{4}{*}{1942} & 1.58 & 27.21 & 0.00 & 0.00 & & & & \\
\hline & & & & & 1923 & 53.94 & 130.38 & 141.10 \\
\hline & & & & & 1941 & -27.77 & -38.88 & 47.77 \\
\hline & & & & & 1944 & -22.01 & -54.12 & 58.43 \\
\hline
\end{tabular}




\begin{tabular}{|c|c|c|c|c|c|c|c|c|}
\hline 1943 & 1.56 & 28.82 & 20.39 & 8.69 & & & & \\
\hline \multirow{3}{*}{1944} & 1.60 & 27.43 & 0.00 & 0.00 & 1915 & -19.83 & 7.48 & 21.20 \\
\hline & & & & & 1942 & 22.03 & 54.76 & 59.03 \\
\hline & & & & & 1960 & -22.30 & -70.93 & 74.35 \\
\hline \multirow[t]{5}{*}{1945} & 1.58 & 29.21 & 0.00 & 0.00 & & & & \\
\hline & & & & & 1879 & 15.92 & -74.03 & 75.72 \\
\hline & & & & & 1909 & 35.82 & 22.78 & 42.45 \\
\hline & & & & & 1933 & 93.88 & 104.96 & 140.82 \\
\hline & & & & & 1935 & -146.17 & -43.45 & 152.49 \\
\hline \multirow[t]{6}{*}{1947} & 1.59 & 28.73 & 0.00 & 0.00 & & & & \\
\hline & & & & & 1882 & -11.25 & -86.30 & 87.03 \\
\hline & & & & & 1901 & 149.48 & -29.30 & 152.32 \\
\hline & & & & & 1931 & -71.24 & 64.09 & 95.83 \\
\hline & & & & & 1935 & -111.63 & -4.82 & 111.74 \\
\hline & & & & & 1969 & 44.97 & -48.16 & 65.89 \\
\hline \multirow[t]{3}{*}{1954} & 1.56 & 28.09 & 0.00 & 0.00 & & & & \\
\hline & & & & & 1823 & 49.50 & 140.97 & 149.41 \\
\hline & & & & & 1965 & -48.79 & 25.47 & 55.04 \\
\hline \multirow[t]{4}{*}{1957} & 1.61 & 31.41 & 0.00 & 0.00 & & & & \\
\hline & & & & & 1913 & 114.48 & 138.83 & 179.95 \\
\hline & & & & & 1963 & 220.06 & -291.90 & 365.56 \\
\hline & & & & & 1970 & -331.32 & 63.75 & 337.39 \\
\hline \multirow[t]{6}{*}{1960} & 1.61 & 27.50 & 0.00 & 0.00 & & & & \\
\hline & & & & & 588 & 684.32 & 223.79 & 719.99 \\
\hline & & & & & 1916 & 20.61 & 155.08 & 156.44 \\
\hline & & & & & 1944 & 22.35 & 71.36 & 74.78 \\
\hline & & & & & 1969 & -203.48 & 78.13 & 217.96 \\
\hline & & & & & 1970 & -522.89 & 59.85 & 526.30 \\
\hline \multirow[t]{3}{*}{1963} & 1.61 & 31.29 & 0.00 & 0.00 & & & & \\
\hline & & & & & 1858 & 219.29 & 166.78 & 275.50 \\
\hline & & & & & 1957 & -219.86 & 292.84 & 366.19 \\
\hline \multirow[t]{7}{*}{1965} & 1.56 & 28.27 & 0.00 & 0.00 & & & & \\
\hline & & & & & 463 & 526.35 & 830.05 & 982.87 \\
\hline & & & & & 1933 & 56.12 & 128.69 & 140.39 \\
\hline & & & & & 1954 & 48.80 & -25.29 & 54.97 \\
\hline & & & & & 1967 & -88.92 & -81.33 & 120.50 \\
\hline & & & & & 1970 & -468.13 & -140.23 & 488.68 \\
\hline & & & & & 1973 & -72.21 & -83.12 & 110.10 \\
\hline \multirow[t]{6}{*}{1967} & 1.58 & 29.01 & 0.00 & 0.00 & & & & \\
\hline & & & & & 1775 & -151.42 & -636.77 & 654.53 \\
\hline & & & & & 1935 & -196.97 & -84.79 & 214.45 \\
\hline & & & & & 19005 & 89.13 & 83.66 & 122.25 \\
\hline & & & & & 1969 & 157.87 & -182.33 & 241.18 \\
\hline & & & & & 1973 & 71.09 & 62.97 & 94.97 \\
\hline \multirow[t]{4}{*}{1969} & 1.60 & 28.25 & 0.00 & 0.00 & & & & \\
\hline & & & & & 1947 & -44.95 & 48.96 & 66.46 \\
\hline & & & & & 1960 & 203.89 & -75.23 & 217.32 \\
\hline & & & & & 1967 & -157.47 & 186.80 & 244.31 \\
\hline
\end{tabular}




\begin{tabular}{|c|c|c|c|c|c|c|c|c|}
\hline 1970 & 1.61 & 32.19 & 0.00 & 0.00 & & & & \\
\hline & & & & & 1781 & -706.85 & -695.83 & 991.87 \\
\hline & & & & & 1782 & -677.64 & -610.85 & 912.32 \\
\hline & & & & & 1957 & 331.73 & -59.14 & 336.96 \\
\hline & & & & & 1960 & 526.11 & -16.87 & 526.38 \\
\hline & & & & & 1965 & 471.52 & 177.21 & 503.72 \\
\hline 1973 & 1.56 & 28.40 & 0.00 & 0.00 & & & & \\
\hline & & & & & 1897 & -3.37 & 20.94 & 21.21 \\
\hline & & & & & 1965 & 72.25 & 83.56 & 110.46 \\
\hline & & & & & 1967 & -70.96 & -61.53 & 93.92 \\
\hline 1997 & 1.53 & 19.67 & 0.00 & 0.00 & & & & \\
\hline & & & & & 2006 & 62.37 & 283.67 & 290.45 \\
\hline 1998 & 1.48 & 21.62 & 0.00 & 0.00 & & & & \\
\hline & & & & & 2023 & 217.78 & 302.90 & 373.06 \\
\hline 1999 & 1.46 & 20.12 & 0.00 & 0.00 & & & & \\
\hline & & & & & 2023 & 179.77 & 401.58 & 439.98 \\
\hline 2000 & 1.30 & 12.24 & 49.50 & 14.44 & & & & \\
\hline & & & & & 2009 & -13.70 & -24.63 & 28.19 \\
\hline & & & & & 2010 & -69.89 & -34.31 & 77.86 \\
\hline & & & & & 2014 & -23.29 & -54.21 & 59.00 \\
\hline 2001 & 1.31 & 12.92 & 52.30 & 15.25 & & & & \\
\hline & & & & & 2010 & 107.18 & 10.17 & 107.66 \\
\hline & & & & & 2012 & 2.10 & 42.24 & 42.29 \\
\hline & & & & & 2020 & -167.87 & -88.04 & 189.55 \\
\hline 2002 & 1.31 & 12.58 & 24.28 & 7.08 & & & & \\
\hline & & & & & 2005 & -95.50 & -50.95 & 108.25 \\
\hline & & & & & 2009 & 64.35 & 31.71 & 71.74 \\
\hline 2003 & 1.30 & 12.82 & 42.03 & 12.26 & & & & \\
\hline & & & & & 2004 & -54.52 & 29.72 & 62.09 \\
\hline & & & & & 2007 & 8.23 & -52.73 & 53.36 \\
\hline 2004 & 1.29 & 13.21 & 54.17 & 15.80 & & & & \\
\hline & & & & & 2003 & 54.59 & -29.29 & 61.95 \\
\hline & & & & & 2015 & 25.85 & -38.10 & 46.04 \\
\hline & & & & & 2021 & -136.54 & 43.22 & 143.22 \\
\hline 2005 & 1.32 & 13.30 & 34.55 & 10.08 & & & & \\
\hline & & & & & 2002 & 95.85 & 52.68 & 109.37 \\
\hline & & & & & 2006 & -86.89 & -8.75 & 87.33 \\
\hline & & & & & 2007 & 76.09 & 61.86 & 98.06 \\
\hline & & & & & 2013 & 92.15 & 59.02 & 109.43 \\
\hline & & & & & 2022 & -196.25 & 30.39 & 198.59 \\
\hline 2006 & 1.37 & 18.25 & 0.00 & 0.00 & & & & \\
\hline & & & & & 1997 & -62.08 & -252.39 & 259.91 \\
\hline & & & & & 2005 & 88.58 & 16.75 & 90.15 \\
\hline & & & & & 2023 & -32.33 & -16.07 & 36.10 \\
\hline 2007 & 1.31 & 12.63 & 42.03 & 12.26 & & & & \\
\hline & & & & & 2003 & -8.12 & 53.23 & 53.85 \\
\hline & & & & & 2005 & -75.82 & -60.19 & 96.80 \\
\hline & & & & & 2011 & 106.06 & 33.62 & 111.26 \\
\hline & & & & & 2013 & -83.88 & -66.33 & 106.94 \\
\hline
\end{tabular}




\begin{tabular}{|c|c|c|c|c|c|c|c|c|}
\hline \multirow[t]{2}{*}{2008} & 1.30 & 12.24 & 79.38 & 23.15 & \multirow{2}{*}{\multicolumn{2}{|c|}{$2011-120.51$}} & \multirow[b]{2}{*}{-82.68} & \multirow[b]{2}{*}{146.14} \\
\hline & & & & & & & & \\
\hline \multirow{3}{*}{2009} & & & & & 2014 & & -60.34 & 66.08 \\
\hline & 1.30 & 12.29 & 55.10 & 16.07 & & & & \\
\hline & & & & & 2000 & 13.72 & 24.72 & 28.27 \\
\hline \multirow{3}{*}{2010} & & & & & 2002 & -64.16 & -31.21 & 71.35 \\
\hline & 1.30 & 12.31 & 50.43 & 14.71 & & & & \\
\hline & & & & & 2000 & 69.93 & 34.43 & 77.94 \\
\hline \multirow{3}{*}{2011} & & & & & 2001 & -106.99 & -9.00 & 107.37 \\
\hline & 1.01 & 12.01 & 0.00 & 0.00 & & & & \\
\hline & & & & & 2007 & -106.01 & -33.37 & 111.13 \\
\hline \multirow[t]{3}{*}{2012} & 1.30 & 12.94 & 23.35 & 6.81 & 2008 & 120.00 & 83.64 & 146.81 \\
\hline & & & & & 2001 & -2.07 & -42.04 & 42.09 \\
\hline & & & & & 2015 & -24.62 & 25.33 & 35.32 \\
\hline \multirow[t]{3}{*}{2013} & 1.32 & 12.94 & 13.07 & 3.81 & & & & \\
\hline & & & & & 2005 & -91.93 & -58.09 & 108.75 \\
\hline & & & & & 2007 & 84.08 & 67.23 & 107.66 \\
\hline \multirow[t]{3}{*}{2014} & 1.30 & 12.24 & 7.47 & 2.18 & & & & \\
\hline & & & & & 2000 & 23.30 & 54.25 & 59.04 \\
\hline & & & & & 2008 & 26.96 & 60.37 & 66.12 \\
\hline \multirow[t]{3}{*}{2015} & 1.30 & 13.03 & 5.60 & 1.63 & & & & \\
\hline & & & & & 2004 & -25.82 & 38.30 & 46.19 \\
\hline & & & & & 2012 & 24.63 & -25.26 & 35.28 \\
\hline \multirow[t]{3}{*}{2020} & 1.33 & 14.37 & 0.00 & 0.00 & & & & \\
\hline & & & & & 1383 & -160.94 & -61.34 & 172.23 \\
\hline & & & & & 2001 & 168.16 & 93.71 & 192.51 \\
\hline \multirow[t]{5}{*}{2021} & 1.29 & 14.44 & 0.00 & 0.00 & & & & \\
\hline & & & & & 1381 & -174.45 & 49.74 & 181.40 \\
\hline & & & & & 1389 & 148.66 & 87.43 & 172.46 \\
\hline & & & & & 2004 & 136.70 & -40.05 & 142.45 \\
\hline & & & & & 2024 & -99.49 & -38.13 & 106.54 \\
\hline \multirow[t]{4}{*}{2022} & 1.32 & 15.79 & 0.00 & 0.00 & & & & \\
\hline & & & & & 2005 & 197.10 & -21.78 & 198.30 \\
\hline & & & & & 2024 & 130.02 & 163.44 & 208.85 \\
\hline & & & & & 2026 & -352.70 & -371.08 & 511.95 \\
\hline \multirow[t]{6}{*}{2023} & 1.38 & 18.94 & 0.00 & 0.00 & & & & \\
\hline & & & & & 461 & 142.05 & 126.70 & 190.35 \\
\hline & & & & & 1998 & -215.26 & -271.67 & 346.61 \\
\hline & & & & & 1999 & -177.70 & -375.91 & 415.80 \\
\hline & & & & & 2006 & 32.39 & 16.59 & 36.39 \\
\hline & & & & & 2026 & 182.03 & 25.71 & 183.84 \\
\hline \multirow[t]{5}{*}{2024} & 1.29 & 15.03 & 0.00 & 0.00 & & & & \\
\hline & & & & & 2021 & 99.67 & 39.40 & 107.18 \\
\hline & & & & & 2022 & -129.25 & -158.13 & 204.24 \\
\hline & & & & & 2200 & 6.06 & 181.31 & 181.41 \\
\hline & & & & & 3351 & 17.74 & -47.01 & 50.25 \\
\hline \multirow[t]{2}{*}{2025} & 1.44 & 19.04 & 0.00 & 0.00 & & & & \\
\hline & & & & & 2026 & 399.01 & 601.51 & 721.82 \\
\hline
\end{tabular}


$\begin{array}{lllll}2026 & 1.37 & 17.05 & 0.00 & 0.00\end{array}$

\begin{tabular}{|c|c|c|c|c|c|c|c|c|}
\hline 2101 & 1.26 & 11.58 & 0.00 & 0.00 & 2025 & -397.58 & -556.27 & 683.75 \\
\hline 2102 & 1.29 & 14.15 & 0.00 & 0.00 & 2120 & 1.12 & 81.93 & 81.94 \\
\hline 2103 & 1.28 & 18.12 & 0.00 & 0.00 & 2120 & 99.85 & 144.52 & 175.66 \\
\hline 2104 & 1.52 & 9.90 & 0.00 & 0.00 & 2200 & 402.67 & 411.45 & 575.71 \\
\hline 2105 & 1.37 & 9.91 & 0.00 & 0.00 & 2121 & 0.13 & 105.28 & 105.28 \\
\hline 2106 & 1.53 & 9.91 & 0.00 & 0.00 & 2121 & 0.39 & 61.76 & 61.77 \\
\hline 2107 & 1.30 & 9.92 & 0.00 & 0.00 & 2121 & 0.31 & 114.24 & 114.24 \\
\hline 2120 & 1.21 & 11.62 & 0.00 & 0.00 & 2121 & 0.04 & 53.50 & 53.50 \\
\hline & & & & & 2101 & -1.01 & -78.33 & 78.34 \\
\hline & & & & & 2102 & -99.47 & -131.20 & 164.64 \\
\hline & & & & & 2121 & 115.50 & -64.40 & 132.23 \\
\hline & & & & & 2150 & 9.17 & 1.95 & 9.37 \\
\hline & & & & & 2151 & 51.34 & 42.36 & 66.55 \\
\hline & & & & & 2153 & 30.93 & 15.49 & 34.60 \\
\hline & & & & & 2200 & -113.62 & 21.50 & 115.64 \\
\hline
\end{tabular}

$\begin{array}{llll}1380 & 209.57 & -312.93 & 376.62\end{array}$

$\begin{array}{llll}2022 & 355.66 & 391.00 \quad 528.56\end{array}$

$\begin{array}{lllll}2023 & -181.11 & -19.51 & 182.16\end{array}$

$2025-397.58-556.27 \quad 683.75$

$\begin{array}{lllll}2121 & 1.22 & 10.07 & 30.63 & 8.93\end{array}$

$\begin{array}{rrrl}1638 & 48.65 & 75.55 & 89.86 \\ 2104 & 0.14 & -84.70 & 84.70 \\ 2105 & -0.20 & -54.95 & 54.95 \\ 2106 & 0.00 & -91.10 & 91.10 \\ 2107 & 0.08 & -50.14 & 50.14 \\ 2120 & -115.04 & 68.29 & 133.78 \\ 2122 & 35.21 & 80.29 & 87.68\end{array}$

$\begin{array}{lllll}2122 & 1.17 & 10.24 & 0.00 & 0.00\end{array}$

$$
\begin{array}{rrrr}
2121 & -33.46 & -76.94 & 83.90 \\
2151 & -17.45 & -22.84 & 28.75 \\
2155 & 29.28 & 19.83 & 35.36 \\
2157 & 23.02 & 16.73 & 28.46 \\
2161 & -3.76 & 59.32 & 59.44
\end{array}
$$

$\begin{array}{lllll}2150 & 1.20 & 11.27 & 9.34 & 2.72\end{array}$

$\begin{array}{llll}2120 & -9.16 & -1.89 & 9.35\end{array}$

$\begin{array}{lllll}2151 & 1.18 & 10.60 & 36.42 & 10.62\end{array}$

$\begin{array}{lllll}2152 & 1.18 & 10.29 & 21.67 & 6.32\end{array}$

$\begin{array}{rrrc}2120 & -51.03 & -40.59 & 65.20 \\ 2122 & 17.52 & 23.23 & 29.09 \\ 2152 & 12.45 & -3.40 & 12.91 \\ 2154 & -15.84 & 10.20 & 18.84\end{array}$




\begin{tabular}{|c|c|c|c|c|c|c|c|c|}
\hline & & & & & 2151 & -12.44 & 3.47 & 12.91 \\
\hline \multirow[t]{2}{*}{2153} & 1.19 & 10.50 & 21.85 & 6.37 & 2153 & -9.38 & -9.59 & 13.42 \\
\hline & & & & & 2120 & -30.78 & -14.67 & 34.10 \\
\hline & & & & & 2152 & 9.40 & 9.68 & 13.49 \\
\hline \multirow[t]{4}{*}{2154} & 1.18 & 11.03 & 25.40 & 7.41 & & & & \\
\hline & & & & & 2151 & 15.86 & -10.05 & 18.78 \\
\hline & & & & & 2156 & 63.18 & 44.96 & 77.54 \\
\hline & & & & & 2201 & -103.11 & 23.90 & 105.84 \\
\hline \multirow[t]{3}{*}{2155} & 1.16 & 9.87 & 24.56 & 7.16 & & & & \\
\hline & & & & & 2122 & -29.23 & -19.51 & 35.14 \\
\hline & & & & & 2156 & 4.68 & 12.74 & 13.57 \\
\hline \multirow[t]{5}{*}{2156} & 1.15 & 9.81 & 27.46 & 8.01 & & & & \\
\hline & & & & & 2154 & -62.78 & -42.70 & 75.93 \\
\hline & & & & & 2155 & -4.67 & -12.66 & 13.49 \\
\hline & & & & & 2157 & -4.03 & -12.49 & 13.12 \\
\hline & & & & & 2158 & 43.17 & 59.23 & 73.29 \\
\hline \multirow[t]{3}{*}{2157} & 1.16 & 9.85 & 19.70 & 5.75 & & & & \\
\hline & & & & & 2122 & -22.97 & -16.46 & 28.26 \\
\hline & & & & & 2156 & 4.04 & 12.56 & 13.19 \\
\hline \multirow[t]{3}{*}{2158} & 1.13 & 9.30 & 24.28 & 7.08 & & & & \\
\hline & & & & & 2156 & -42.90 & -57.76 & 71.94 \\
\hline & & & & & 2159 & 18.52 & 50.98 & 54.24 \\
\hline \multirow[t]{3}{*}{2159} & 1.11 & 9.07 & 8.50 & 2.48 & & & & \\
\hline & & & & & 2158 & -18.29 & -49.72 & 52.98 \\
\hline & & & & & 2160 & 9.44 & 47.15 & 48.09 \\
\hline \multirow[t]{4}{*}{2160} & 1.08 & 9.06 & 11.86 & 3.46 & & & & \\
\hline & & & & & 1617 & 82.23 & 150.02 & 171.08 \\
\hline & & & & & 2159 & -9.27 & -46.26 & 47.18 \\
\hline & & & & & 2161 & -84.75 & -106.82 & 136.36 \\
\hline \multirow[t]{4}{*}{2161} & 1.14 & 10.56 & 28.76 & 8.39 & & & & \\
\hline & & & & & 2122 & 3.99 & -57.99 & 58.13 \\
\hline & & & & & 2160 & 86.23 & 114.72 & 143.52 \\
\hline & & & & & 2202 & -118.02 & -49.44 & 127.96 \\
\hline \multirow[t]{6}{*}{2200} & 1.20 & 15.55 & 0.00 & 0.00 & & & & \\
\hline & & & & & 2024 & $-4.10-$ & -167.59 & 167.64 \\
\hline & & & & & 2103 & -394.35 & -368.29 & 539.58 \\
\hline & & & & & 2120 & 113.83 & -13.55 & 114.63 \\
\hline & & & & & 2201 & 101.23 & 241.92 & 262.25 \\
\hline & & & & & 2202 & 117.83 & 70.84 & 137.48 \\
\hline \multirow[t]{5}{*}{2201} & 1.16 & 15.14 & 0.00 & 0.00 & & & & \\
\hline & & & & & 1229 & -336.63 & 80.76 & 346.18 \\
\hline & & & & & 1230 & 332.98 & 126.27 & $7 \quad 356.12$ \\
\hline & & & & & 2154 & 103.31 & -16.25 & 104.58 \\
\hline & & & & & 2200 & -100.22 & -234.80 & 255.29 \\
\hline \multirow[t]{3}{*}{2202} & 1.19 & 15.09 & 0.00 & 0.00 & & & & \\
\hline & & & & & 2161 & 118.32 & 60.96 & 133.11 \\
\hline & & & & & 2200 & -117.63 & -69.44 & 136.60 \\
\hline 2231 & 1.51 & 1.91 & 0.00 & 0.00 & & & & \\
\hline
\end{tabular}




\begin{tabular}{|c|c|c|c|c|c|c|c|c|}
\hline \multirow[t]{2}{*}{2232} & 1.47 & 2.18 & 0.00 & 0.00 & 2241 & 49.31 & 122.13 & 131.71 \\
\hline & & & & & 2241 & 203.34 & 164.62 & 261.62 \\
\hline \multirow[t]{2}{*}{2235} & 1.70 & -5.30 & 0.00 & 0.00 & & & & \\
\hline & & & & & 2242 & -0.52 & 123.27 & 123.27 \\
\hline 2236 & 1.53 & -5.25 & 0.00 & 0.00 & & & & \\
\hline \multirow{2}{*}{2237} & 1.61 & -3.84 & 0.00 & 0.00 & 2242 & 0.67 & 372.55 & 372.55 \\
\hline & & & & & 2242 & 40.85 & 160.23 & 165.36 \\
\hline \multirow[t]{4}{*}{2240} & 1.42 & 4.86 & 0.00 & 0.00 & & & & \\
\hline & & & & & 1082 & 24.34 & -79.64 & 83.28 \\
\hline & & & & & 2241 & 452.38 & $3-70.34$ & 457.82 \\
\hline & & & & & 2501 & -481.19 & 177.34 & 512.83 \\
\hline \multirow[t]{6}{*}{2241} & 1.44 & 0.79 & 0.00 & 0.00 & & & & \\
\hline & & & & & 2231 & -49.10 & -115.05 & 125.08 \\
\hline & & & & & 2232 & -202.96 & -156.30 & 256.17 \\
\hline & & & & & 2240 & -451.55 & 103.50 & 463.26 \\
\hline & & & & & 2253 & 430.11 & l -19.53 & 430.56 \\
\hline & & & & & 2260 & 261.71 & 22.81 & 262.70 \\
\hline \multirow[t]{8}{*}{2242} & 1.46 & -5.18 & 0.00 & 0.00 & & & & \\
\hline & & & & & 2235 & 0.68 & -106.03 & 106.03 \\
\hline & & & & & 2236 & -0.22 & -354.53 & 354.53 \\
\hline & & & & & 2237 & -40.30 & -143.97 & 149.50 \\
\hline & & & & & 2243 & 95.22 & 107.30 & 143.46 \\
\hline & & & & & 2244 & -16.83 & 50.63 & 53.35 \\
\hline & & & & & 2255 & -18.33 & 56.82 & 59.70 \\
\hline & & & & & 2261 & -30.29 & 54.67 & 62.50 \\
\hline \multirow[t]{3}{*}{2243} & 1.38 & -7.68 & 0.00 & 0.00 & & & & \\
\hline & & & & & 1046 & 96.99 & 30.06 & 101.54 \\
\hline & & & & & 2242 & -94.67 & -97.73 & 136.07 \\
\hline \multirow[t]{4}{*}{2244} & 1.43 & -4.22 & 0.00 & 0.00 & & & & \\
\hline & & & & & 2242 & 17.31 & -49.29 & 52.24 \\
\hline & & & & & 2255 & -7.34 & 15.61 & 17.25 \\
\hline & & & & & 2256 & -40.14 & 23.41 & 46.47 \\
\hline \multirow[t]{5}{*}{2251} & 1.42 & -3.92 & 21.48 & 6.26 & & & & \\
\hline & & & & & 2253 & -80.00 & 5.81 & 80.21 \\
\hline & & & & & 2255 & 23.44 & -20.36 & 31.05 \\
\hline & & & & & 2256 & -81.15 & -35.63 & 88.63 \\
\hline & & & & & 2269 & -12.09 & -11.58 & 16.74 \\
\hline \multirow[t]{2}{*}{2252} & 1.42 & -3.96 & 46.69 & 13.62 & & & & \\
\hline & & & & & 2253 & -55.89 & -16.45 & 58.26 \\
\hline \multirow[t]{7}{*}{2253} & 1.43 & -3.61 & 24.28 & 7.08 & & & & \\
\hline & & & & & 1076 & 123.50 & -18.58 & 124.89 \\
\hline & & & & & 2241 & -424.45 & 552.13 & 427.64 \\
\hline & & & & & 2251 & 80.16 & -5.38 & 80.34 \\
\hline & & & & & 2252 & 56.05 & 16.87 & 58.53 \\
\hline & & & & & 2256 & 93.03 & -4.16 & 93.12 \\
\hline & & & & & 2261 & 37.77 & -53.83 & 65.76 \\
\hline
\end{tabular}

$\begin{array}{lllll}2254 & 1.42 & -4.03 & 28.95 & 8.44\end{array}$ 


\begin{tabular}{|c|c|c|c|c|c|c|c|c|}
\hline \multirow{5}{*}{2255} & \multirow{5}{*}{1.43} & \multirow{5}{*}{-4.18} & \multirow{5}{*}{0.00} & \multirow{5}{*}{0.00} & \multirow{2}{*}{\multicolumn{2}{|c|}{$\begin{array}{ll}2255 & -0.15 \\
2257 & -80.98\end{array}$}} & \multirow{2}{*}{$\begin{array}{r}-26.20 \\
30.63\end{array}$} & 26.20 \\
\hline & & & & & & & & 86.58 \\
\hline & & & & & 2259 & 9.39 & -30.72 & 32.12 \\
\hline & & & & & 2262 & -8.95 & -8.42 & 12.29 \\
\hline & & & & & & & & \\
\hline & & & & & 2242 & 18.90 & -55.28 & 58.42 \\
\hline & & & & & 2244 & 7.35 & -15.59 & 17.24 \\
\hline & & & & & 2251 & -23.39 & 20.50 & 31.10 \\
\hline & & & & & 2254 & 0.22 & 26.39 & 26.39 \\
\hline & & & & & 2259 & 9.15 & 33.93 & 35.14 \\
\hline 2256 & 1.43 & -3.85 & 28.95 & 8.44 & & & & \\
\hline & & & & & 2244 & 40.25 & -23.12 & 46.42 \\
\hline & & & & & 2251 & 81.20 & 35.77 & 88.73 \\
\hline & & & & & 2253 & -92.97 & 4.55 & 93.08 \\
\hline 2257 & 1.41 & -3.50 & 42.03 & 12.26 & & & & \\
\hline & & & & & 1053 & 101.63 & 17.63 & 103.15 \\
\hline & & & & & 2254 & 81.11 & -29.83 & 86.43 \\
\hline & & & & & 2258 & -227.89 & -0.53 & 227.89 \\
\hline 2258 & 1.42 & -2.46 & 22.41 & 6.54 & & & & \\
\hline & & & & & 2257 & 228.51 & 4.65 & 228.56 \\
\hline & & & & & 2260 & -254.46 & -12.54 & 254.76 \\
\hline 2259 & 1.42 & -4.16 & 30.82 & 8.99 & & & & \\
\hline & & & & & 2254 & -9.35 & 30.83 & 32.22 \\
\hline & & & & & 2255 & -9.10 & -33.79 & 35.00 \\
\hline 2260 & 1.43 & -1.21 & 15.88 & 4.63 & & & & \\
\hline & & & & & 2241 & -260.28 & -13.55 & 260.63 \\
\hline & & & & & 2258 & 255.33 & 18.18 & 255.97 \\
\hline 2261 & 1.43 & -3.91 & 15.88 & 4.63 & & & & \\
\hline & & & & & 2242 & 30.93 & -53.01 & 61.37 \\
\hline & & & & & 2253 & -37.63 & 54.21 & 65.99 \\
\hline 2262 & 1.42 & -4.01 & 7.47 & 2.18 & & & & \\
\hline & & & & & 2254 & 8.95 & 8.43 & 12.30 \\
\hline 2269 & 1.43 & -3.91 & 12.14 & 3.54 & & & & \\
\hline & & & & & 2251 & 12.09 & 11.59 & 16.75 \\
\hline 2351 & 1.38 & 21.74 & 0.00 & 0.00 & & & & \\
\hline & & & & & 2354 & 80.01 & 38.81 & 88.93 \\
\hline 2352 & 1.37 & 20.60 & 0.00 & 0.00 & & & & \\
\hline & & & & & 2354 & 152.12 & 81.63 & 172.64 \\
\hline 2353 & 1.34 & 18.85 & 0.00 & 0.00 & & & & \\
\hline & & & & & 2354 & -0.02 & -0.43 & 0.43 \\
\hline 2354 & 1.34 & 18.85 & 0.00 & 0.00 & & & & \\
\hline & & & & & 574 & -43.24 & -48.48 & 64.96 \\
\hline & & & & & 2351 & -79.70 & -33.81 & 86.58 \\
\hline & & & & & 2352 & -151.75 & -75.57 & 169.53 \\
\hline & & & & & 2353 & 0.02 & 0.43 & 0.43 \\
\hline & & & & & 2355 & 108.95 & 45.98 & 118.25 \\
\hline & & & & & 2356 & 90.34 & 44.33 & 100.63 \\
\hline & & & & & 2363 & 73.41 & 68.85 & 100.65 \\
\hline 2355 & 1.34 & 18.38 & 38.66 & 11.28 & & & & \\
\hline
\end{tabular}




\begin{tabular}{|c|c|c|c|c|c|c|c|c|}
\hline \multirow[b]{3}{*}{2356} & \multirow[b]{3}{*}{1.33} & \multirow[b]{3}{*}{17.94} & \multirow[b]{3}{*}{22.60} & \multirow[b]{3}{*}{6.59} & \multicolumn{2}{|c|}{$2354-108.76$} & \multirow{3}{*}{$\begin{array}{r}-44.85 \\
40.09\end{array}$} & \multirow{2}{*}{$\begin{array}{l}117.65 \\
81.35\end{array}$} \\
\hline & & & & & 2357 & 70.79 & & \\
\hline & & & & & & & & \\
\hline & & & & & 2354 & -90.01 & -42.40 & 99.50 \\
\hline \multirow{7}{*}{2357} & & & & & 2357 & 67.24 & 37.74 & 77.10 \\
\hline & 1.32 & 17.28 & 0.00 & 0.00 & & & & \\
\hline & & & & & 955 & 22.17 & 22.85 & 31.84 \\
\hline & & & & & 2355 & -70.46 & -38.13 & 80.11 \\
\hline & & & & & 2356 & -67.05 & -36.62 & 76.40 \\
\hline & & & & & 2358 & 80.05 & 80.74 & 113.70 \\
\hline & & & & & 2359 & 37.30 & 31.42 & 48.77 \\
\hline \multirow[t]{2}{*}{2358} & 1.22 & 12.82 & 78.45 & 22.88 & & & & \\
\hline & & & & & 2357 & -79.72 & -68.77 & 105.29 \\
\hline \multirow[t]{3}{*}{2359} & 1.31 & 16.95 & 34.93 & 10.19 & & & & \\
\hline & & & & & 2357 & -37.23 & -31.00 & 48.45 \\
\hline & & & & & 2364 & 1.32 & 17.42 & 17.47 \\
\hline \multirow[t]{6}{*}{2360} & 1.30 & 17.46 & 0.00 & 0.00 & & & & \\
\hline & & & & & 955 & 16.26 & -36.49 & 39.95 \\
\hline & & & & & 2361 & -11.31 & -114.80 & 115.35 \\
\hline & & & & & 2362 & -22.26 & -28.42 & 36.10 \\
\hline & & & & & 2363 & -24.47 & -32.74 & 40.87 \\
\hline & & & & & 2364 & 31.63 & -15.88 & 35.39 \\
\hline \multirow[t]{3}{*}{2361} & 1.36 & 17.61 & 0.00 & 0.00 & & & & \\
\hline & & & & & 967 & -4.43 & 124.27 & 124.35 \\
\hline & & & & & 2360 & 11.52 & 120.01 & 120.56 \\
\hline \multirow[t]{4}{*}{2362} & 1.31 & 17.57 & 0.00 & 0.00 & & & & \\
\hline & & & & & 2360 & 22.29 & 28.57 & 36.23 \\
\hline & & & & & 2363 & -30.20 & -43.56 & 53.00 \\
\hline & & & & & 3275 & 4.15 & -6.06 & 7.35 \\
\hline \multirow[t]{4}{*}{2363} & 1.31 & 17.63 & 20.17 & 5.88 & & & & \\
\hline & & & & & 2354 & -72.98 & -65.58 & 98.12 \\
\hline & & & & & 2360 & 24.52 & 33.01 & 41.12 \\
\hline & & & & & 2362 & 30.22 & 43.69 & 53.13 \\
\hline \multirow[t]{3}{*}{2364} & 1.30 & 16.96 & 33.99 & 9.91 & & & & \\
\hline & & & & & 2359 & -1.32 & -17.40 & 17.45 \\
\hline & & & & & 2360 & -31.58 & 16.20 & 35.49 \\
\hline \multirow[t]{6}{*}{2476} & 1.04 & 38.87 & 90.00 & 0.00 & & & & \\
\hline & & & & & 3402 & 276.98 & -139.96 & 310.33 \\
\hline & & & & & 3409 & 935.29 & 309.08 & 985.04 \\
\hline & & & & & 3411 & 308.36 & -424.01 & 524.28 \\
\hline & & & & & 3436 & 204.40 & 198.64 & 285.02 \\
\hline & & & & & 3489 & 1346.24 & 4118.80 & 01351.47 \\
\hline \multirow[t]{2}{*}{2491} & 1.44 & 35.28 & 0.00 & 0.00 & & & & \\
\hline & & & & & 2582 & 779.02 & 961.24 & 1237.28 \\
\hline \multirow[t]{2}{*}{2492} & 1.41 & 35.39 & 0.00 & 0.00 & & & & \\
\hline & & & & & 2498 & 791.89 & 882.27 & 1185.53 \\
\hline \multirow[t]{2}{*}{2498} & 1.34 & 32.79 & 0.00 & 0.00 & & & & \\
\hline & & & & & $\begin{array}{r}356 \\
2492\end{array}$ & $\begin{array}{r}531.05 \\
-789.88\end{array}$ & $\begin{array}{l}-684.67 \\
-803.47\end{array}$ & $\begin{array}{c}866.48 \\
1126.71\end{array}$ \\
\hline
\end{tabular}




\begin{tabular}{|c|c|c|c|c|c|c|c|c|}
\hline & & & & & 2499 & 55.46 & -845.15 & 846.97 \\
\hline & & & & & 2520 & -514.95 & 5806.19 & 956.62 \\
\hline & & & & & 2526 & 713.48 & 8140.57 & 727.19 \\
\hline & & & & & 2582 & -16.37 & -321.42 & 321.84 \\
\hline 2499 & 1.43 & 32.36 & 0.00 & 0.00 & & & & \\
\hline & & & & & 356 & 858.14 & -436.44 & 962.75 \\
\hline & & & & & 2498 & -52.26 & 899.96 & 901.47 \\
\hline & & & & & 3501 & -805.12 & 2447.28 & 921.02 \\
\hline 2500 & 1.55 & 25.09 & 0.00 & 0.00 & & & & \\
\hline & & & & & 475 & -41.60 & 170.08 & 175.09 \\
\hline & & & & & 3429 & -60.32 & -118.83 & 133.27 \\
\hline & & & & & 3450 & 89.36 & -202.75 & 221.56 \\
\hline 2501 & 1.39 & 11.95 & 0.00 & 0.00 & & & & \\
\hline & & & & & 2240 & 489.54 & $4-114.33$ & 502.72 \\
\hline & & & & & 2871 & -525.72 & 2268.73 & 590.42 \\
\hline & & & & & 3438 & -209.96 & $\begin{array}{ll}6 & -47.82\end{array}$ & 215.34 \\
\hline & & & & & 3456 & 112.14 & $4-0.30$ & 112.14 \\
\hline & & & & & 3480 & 130.68 & 0.34 & 130.68 \\
\hline 2502 & 1.53 & 15.47 & 0.00 & 0.00 & & & & \\
\hline & & & & & 1085 & 205.05 & 5171.23 & 267.14 \\
\hline & & & & & 2503 & 167.23 & $3-56.73$ & 176.59 \\
\hline & & & & & 3438 & 78.63 & 233.25 & 246.15 \\
\hline & & & & & 3440 & -455.90 & 0 505.93 & 681.03 \\
\hline 2503 & 1.56 & 12.49 & 219.00 & 46.40 & & & & \\
\hline & & & & & 1056 & 89.09 & 90.23 & 126.80 \\
\hline & & & & & 2502 & -166.81 & 66.46 & 179.56 \\
\hline & & & & & 2504 & 22.78 & 5.75 & 23.49 \\
\hline & & & & & 3458 & -101.11 & 1155.28 & 185.30 \\
\hline & & & & & 3462 & -62.09 & -4.99 & 62.29 \\
\hline 2504 & 1.55 & 12.18 & 23.90 & 6.90 & & & & \\
\hline & & & & & 2503 & -22.72 & -5.60 & 23.40 \\
\hline 2505 & 1.43 & 11.90 & 10.90 & 1.80 & & & & \\
\hline & & & & & 1056 & 78.58 & 37.12 & 86.91 \\
\hline & & & & & 3462 & -89.17 & -36.76 & 96.45 \\
\hline 2506 & 1.45 & 3.47 & 44.10 & 13.00 & & & & \\
\hline & & & & & 1073 & 150.01 & -56.57 & 160.32 \\
\hline & & & & & 3456 & -95.55 & 94.69 & 134.52 \\
\hline & & & & & 3472 & -21.27 & 52.15 & 56.32 \\
\hline & & & & & 3483 & -9.77 & 11.84 & 15.35 \\
\hline 2507 & 1.45 & 4.00 & 0.00 & 0.00 & & & & \\
\hline & & & & & 1084 & 15.52 & -25.52 & 29.87 \\
\hline & & & & & 3480 & -15.44 & 21.08 & 26.13 \\
\hline & & & & & 3483 & -2.30 & 23.78 & 23.89 \\
\hline 2520 & 1.23 & 36.54 & 0.00 & 0.00 & & & & \\
\hline & & & & & 2498 & 521.07 & $7-708.72$ & 879.66 \\
\hline & & & & & 3408 & 578.34 & 4395.82 & 1130.81 \\
\hline & & & $\therefore$ & & 3425 & 537.18 & 709.69 & 890.07 \\
\hline 2526 & 1.32 & 28.80 & 0.00 & 0.00 & & & & \\
\hline & & & & & 2498 & -710.23 & $\begin{array}{ll}3 & -89.26\end{array}$ & 715.82 \\
\hline
\end{tabular}




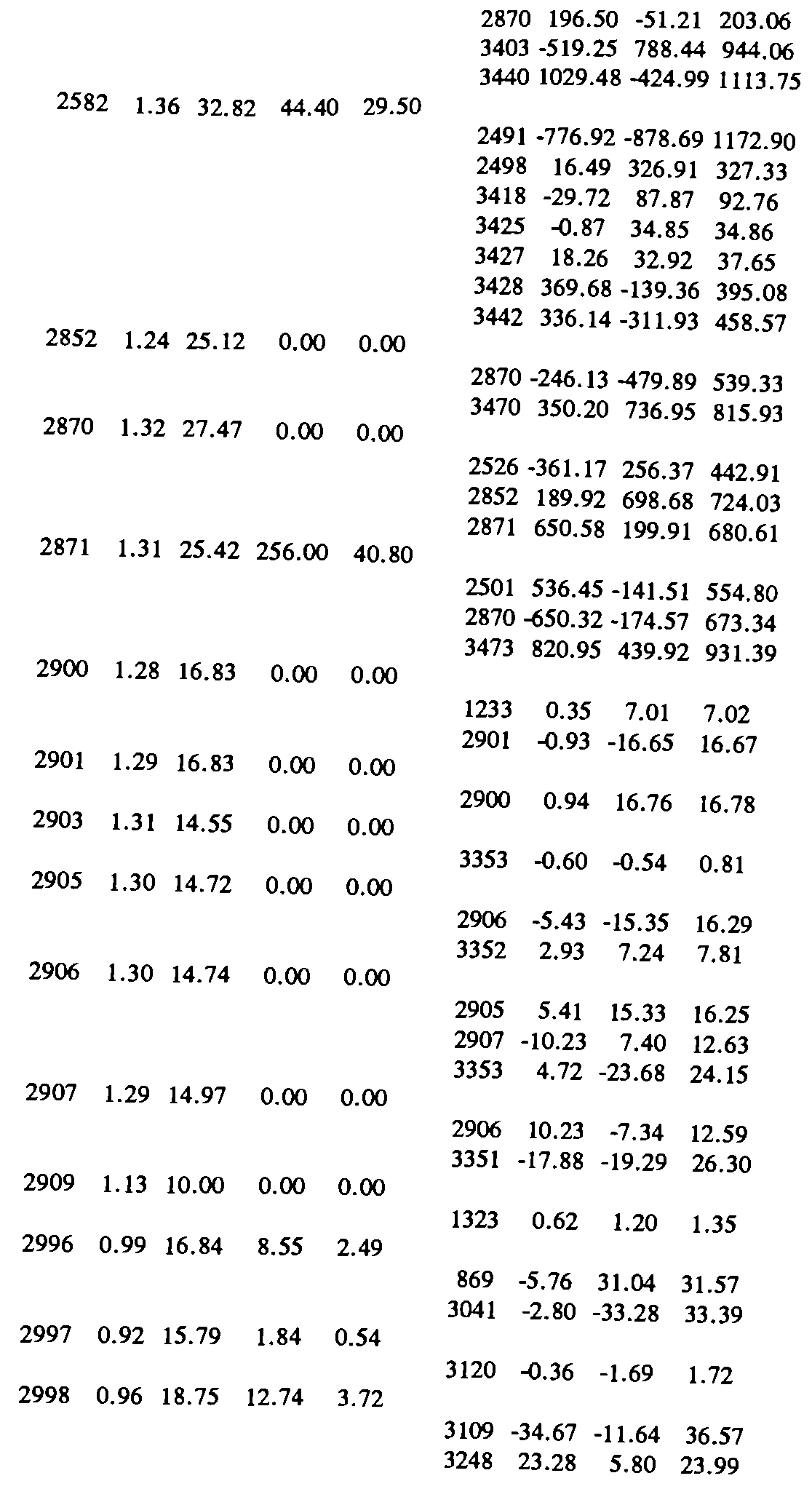




\begin{tabular}{|c|c|c|c|c|c|c|c|c|}
\hline 2999 & 0.93 & 22.83 & 3.30 & 0.96 & & & & \\
\hline & & & & & 926 & -4.20 & 0.30 & 4.21 \\
\hline 3000 & 1.35 & 15.48 & 8.89 & 2.59 & & & & \\
\hline & & & & & 418 & -239.22 & -211.82 & 319.52 \\
\hline & & & & & 3006 & 231.47 & 213.11 & 314.63 \\
\hline 3001 & 1.17 & 9.73 & 0.00 & 0.00 & & & & \\
\hline & & & & & 409 & 133.71 & 96.01 & 164.61 \\
\hline & & & & & 3186 & 2.96 & 0.44 & 2.99 \\
\hline 3002 & 1.35 & 20.02 & 3.37 & 0.98 & & & & \\
\hline & & & & & 376 & -132.86 & 44.86 & 140.22 \\
\hline & & & & & 427 & 173.03 & 116.88 & 208.81 \\
\hline 3003 & 1.43 & 17.53 & 1.72 & 0.50 & & & & \\
\hline & & & & & 386 & 2.42 & 4.58 & 5.18 \\
\hline 3004 & 1.15 & 9.32 & 0.00 & 0.00 & & & & \\
\hline & & & & & 372 & 136.18 & 159.91 & 210.04 \\
\hline & & & & & 3266 & 0.70 & 0.30 & 0.76 \\
\hline 3006 & 1.27 & 13.01 & 3.37 & 0.98 & & & & \\
\hline & & & & & 400 & 227.56 & 198.69 & 302.09 \\
\hline & & & & & 3000 & -225.22 & -190.30 & 294.85 \\
\hline 3007 & 1.42 & 22.55 & 4.23 & 1.23 & & & & \\
\hline & & & & & 375 & -145.22 & -62.84 & 158.24 \\
\hline & & & & & 402 & 144.14 & 70.93 & 160.65 \\
\hline 3008 & 1.34 & 19.06 & 5.88 & 1.71 & & & & \\
\hline & & & & & 427 & -5.07 & 2.14 & 5.50 \\
\hline 3009 & 1.34 & 19.21 & 0.00 & 0.00 & & & & \\
\hline & & & & & 411 & -41.44 & -8.08 & 42.22 \\
\hline & & & & & 3193 & 42.92 & 11.31 & 44.39 \\
\hline 3010 & 1.43 & 17.66 & 0.00 & 0.00 & & & & \\
\hline & & & & & 587 & 44.97 & 38.79 & 59.39 \\
\hline & & & & & 3012 & -49.22 & -28.99 & 57.12 \\
\hline & & & & & 3264 & 12.12 & -1.98 & 12.29 \\
\hline 3011 & 1.39 & 18.75 & 2.65 & 0.77 & & & & \\
\hline & & & & & 406 & -188.68 & -39.74 & 192.82 \\
\hline & & & & & 431 & 192.44 & 57.34 & 200.80 \\
\hline 3012 & 1.45 & 17.97 & 2.58 & 0.75 & & & & \\
\hline & & & & & 419 & -46.24 & -21.27 & 50.90 \\
\hline & & & & & 3010 & 49.66 & 29.74 & 57.89 \\
\hline 3013 & 1.32 & 11.43 & 15.20 & 4.43 & & & & \\
\hline & & & & & 570 & -20.49 & -2.16 & 20.61 \\
\hline & & & & & 3194 & 6.93 & 33.81 & 34.51 \\
\hline 3014 & 1.46 & 24.47 & 0.00 & 0.00 & & & & \\
\hline & & & & & 135 & 180.65 & -171.35 & 248.99 \\
\hline & & & & & 463 & -319.36 & 9.65 & 319.50 \\
\hline & & & & & 3054 & 29.27 & 9.87 & 30.89 \\
\hline & & & & & 3133 & 110.29 & 163.17 & 196.95 \\
\hline 3015 & 1.47 & 30.75 & 0.00 & 0.00 & & & & \\
\hline & & & & & 3205 & 2.60 & 88.20 & 88.24 \\
\hline 3016 & 1.28 & 10.55 & 5.30 & 1.55 & & & & \\
\hline & & & & & 3017 & -0.14 & -1.55 & 1.56 \\
\hline
\end{tabular}




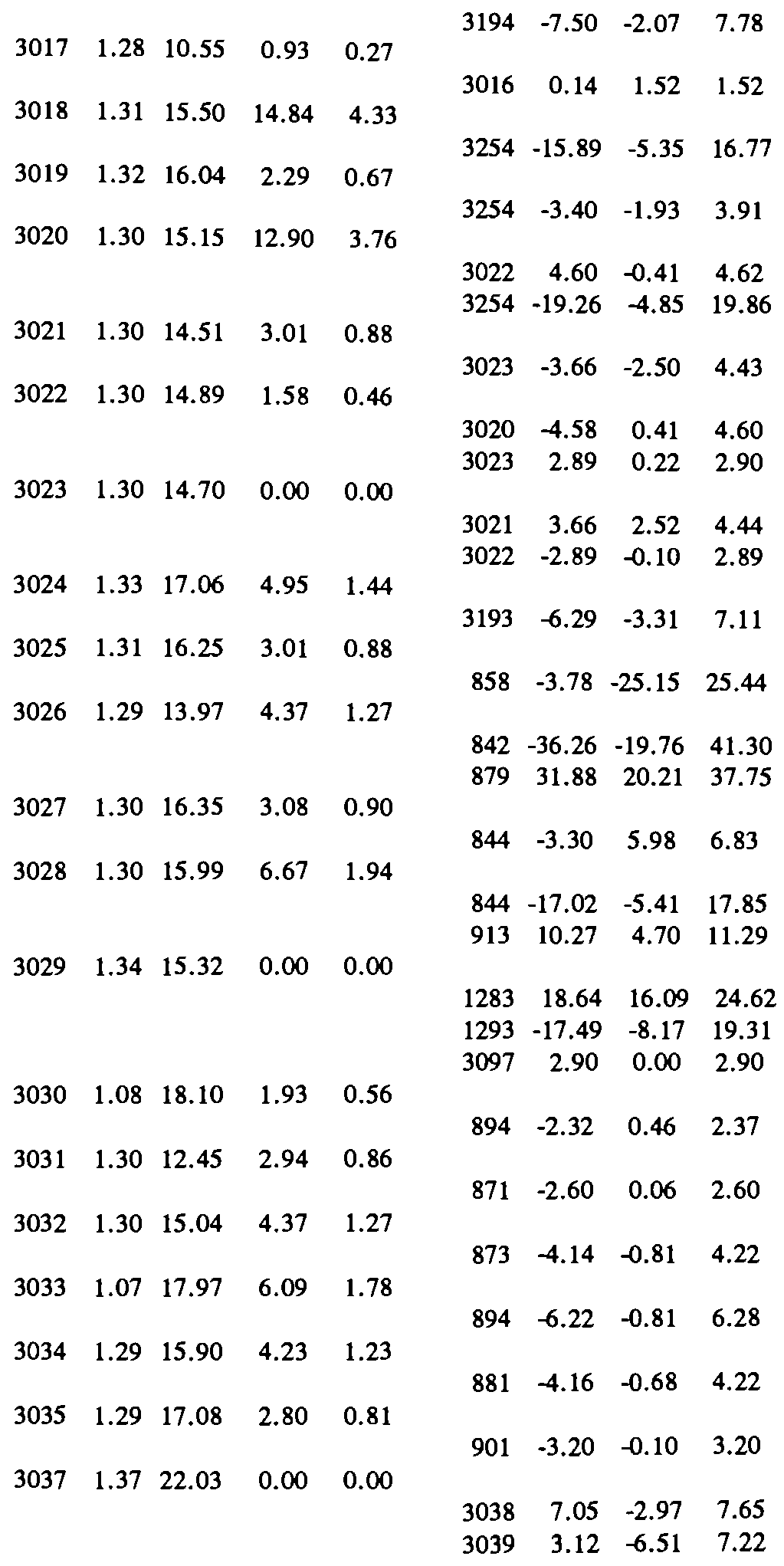




\begin{tabular}{|c|c|c|c|c|c|c|c|c|}
\hline \multirow{2}{*}{3038} & \multirow{2}{*}{1.37} & 21.95 & 9.39 & 2.74 & 3133 & \multicolumn{2}{|c|}{$-44.89-130.98$} & 138.46 \\
\hline & & & & & 3037 & -7.11 & 2.80 & 7.65 \\
\hline \multirow[t]{3}{*}{3039} & 1.38 & 21.87 & 0.00 & 0.00 & & & & \\
\hline & & & & & 3037 & -3.11 & 6.55 & 7.25 \\
\hline & & & & & 3040 & 3.04 & -0.25 & 3.05 \\
\hline \multirow[t]{2}{*}{3040} & 1.37 & 21.58 & 3.44 & 1.00 & & & & \\
\hline & 1.03 & 15.68 & 0.28 & 0.08 & 3039 & -3.10 & 0.10 & 3.10 \\
\hline \multirow{2}{*}{3041} & & & & & 849 & -4.64 & -32.78 & 33.10 \\
\hline & & & & & 2996 & 3.62 & 34.60 & 34.79 \\
\hline \multirow[t]{3}{*}{3042} & 0.37 & 52.57 & 3.07 & 0.90 & & & & \\
\hline & & & & & 866 & -29.90 & 13.32 & 32.73 \\
\hline & & & & & 883 & 23.28 & -14.31 & 27.32 \\
\hline \multirow[t]{3}{*}{3043} & 1.06 & 15.07 & 1.12 & 0.33 & & & & \\
\hline & & & & & 849 & -0.82 & 15.28 & 15.30 \\
\hline & & & & & 853 & -0.49 & -14.60 & 14.61 \\
\hline \multirow[t]{3}{*}{3044} & 1.05 & 15.55 & 2.79 & 0.81 & & & & \\
\hline & & & & & 730 & 28.57 & -14.35 & 31.97 \\
\hline & & & & & 738 & -31.27 & 14.47 & 34.46 \\
\hline \multirow[t]{3}{*}{3045} & 0.62 & 25.44 & 4.97 & 1.45 & & & & \\
\hline & & & & & 880 & -37.27 & -86.22 & 93.93 \\
\hline & & & & & 882 & 31.76 & 85.44 & 91.15 \\
\hline \multirow[t]{2}{*}{3046} & 1.04 & 13.07 & 4.02 & 1.17 & & & & \\
\hline & & & & & 904 & -3.50 & -0.35 & 3.52 \\
\hline \multirow[t]{3}{*}{3047} & 1.03 & 14.54 & 7.71 & 2.25 & & & & \\
\hline & & & & & 849 & -40.34 & -7.63 & 41.05 \\
\hline & & & & & 910 & 32.47 & 6.33 & 33.09 \\
\hline \multirow[t]{2}{*}{3048} & 1.06 & 14.54 & 9.22 & 2.69 & & & & \\
\hline & & & & & 908 & -7.37 & 3.47 & 8.14 \\
\hline \multirow[t]{2}{*}{3049} & 1.02 & 12.40 & 6.71 & 1.96 & & & & \\
\hline & & & & & 911 & -5.60 & -0.64 & 5.63 \\
\hline \multirow[t]{3}{*}{3050} & 1.09 & 14.77 & 13.80 & 4.03 & & & & \\
\hline & & & & & 908 & 5.57 & 17.48 & 18.35 \\
\hline & & & & & 915 & -19.26 & -20.38 & 28.04 \\
\hline \multirow[t]{3}{*}{3051} & 1.37 & 22.01 & 18.35 & 5.35 & & & & \\
\hline & & & & & 3052 & 40.33 & 26.85 & 48.45 \\
\hline & & & & & 3133 & -114.28 & $8-173.91$ & 208.10 \\
\hline 3052 & 1.36 & 21.75 & 0.00 & 0.00 & & & & \\
\hline & & & & & 3051 & -40.42 & -26.72 & 48.45 \\
\hline & & & & & 3053 & 34.65 & ; 13.99 & 37.37 \\
\hline 053 & 1.36 & 21.62 & 13.69 & 3.99 & & & & \\
\hline & & & & & 3052 & -34.73 & $3-13.97$ & 37.43 \\
\hline & & & & & 3068 & 13.60 & -0.80 & 13.63 \\
\hline 3054 & 1.46 & 23.86 & 0.00 & 0.00 & & & & \\
\hline & & & & & 3014 & -29.26 & -9.52 & 30.77 \\
\hline & & & & & 3055 & 27.09 & 21.88 & 34.82 \\
\hline 3055 & 1.45 & 23.65 & 0.00 & 0.00 & & & & \\
\hline & & & & & 3054 & -27.07 & $7-21.94$ & 34.84 \\
\hline
\end{tabular}




\begin{tabular}{|c|c|c|c|c|c|c|c|c|}
\hline & & & & & 3067 & 12.10 & -6.30 & 13.64 \\
\hline \multirow[t]{3}{*}{3056} & 0.95 & 23.04 & 0.00 & 0.00 & 3141 & -1.99 & -6.13 & 0.47 \\
\hline & & & & & 926 & 137.90 & 163.91 & 214.20 \\
\hline & & & & & 960 & -139.54 & -159.89 & 212.22 \\
\hline \multirow[t]{2}{*}{3057} & 0.77 & 20.84 & 9.11 & 2.66 & & & & \\
\hline & & & & & 934 & -14.92 & 8.17 & 17.01 \\
\hline \multirow[t]{3}{*}{3058} & 0.99 & 20.66 & 28.22 & 8.23 & & & & \\
\hline & & & & & 772 & -1.27 & -12.07 & 12.14 \\
\hline & & & & & 941 & -28.30 & 6.59 & 29.06 \\
\hline 3059 & 0.99 & 20.64 & 24.25 & 7.07 & & & & \\
\hline \multirow{3}{*}{3060} & 0.85 & 22.28 & 8.61 & 2.51 & $1 / 2$ & -26.90 & 16.87 & 31.76 \\
\hline & & & & & 938 & 104.48 & 135.76 & 171.31 \\
\hline & & & & & 946 & -115.06 & -133.47 & 176.22 \\
\hline \multirow[t]{4}{*}{3061} & 0.99 & 20.99 & 24.53 & 7.16 & & & & \\
\hline & & & & & 941 & -84.28 & -13.29 & 85.33 \\
\hline & & & & & 3109 & 49.13 & 15.98 & 51.67 \\
\hline & & & & & 3111 & 9.31 & 1.82 & 9.49 \\
\hline \multirow[t]{3}{*}{3062} & 1.01 & 21.67 & 4.64 & 1.35 & & & & \\
\hline & & & & & 777 & 47.30 & 28.49 & 55.21 \\
\hline & & & & & 780 & -54.40 & -24.59 & 59.70 \\
\hline \multirow[t]{3}{*}{3063} & 0.99 & 23.04 & 14.31 & 4.17 & & & & \\
\hline & & & & & 933 & -51.23 & 0.24 & 51.23 \\
\hline & & & & & 945 & 35.81 & -0.40 & 35.82 \\
\hline \multirow[t]{2}{*}{3064} & 0.99 & 22.82 & 19.22 & 5.61 & & & & \\
\hline & & & & & 945 & -22.67 & 6.66 & 23.62 \\
\hline \multirow[t]{2}{*}{3065} & 1.00 & 23.83 & 21.96 & 6.41 & & & & \\
\hline & & & & & 947 & -48.86 & 36.68 & 61.10 \\
\hline \multirow[t]{3}{*}{3067} & 1.45 & 23.61 & 4.73 & 1.38 & & & & \\
\hline & & & & & 3055 & -12.12 & 6.26 & 13.64 \\
\hline & & & & & 3070 & 8.60 & -8.83 & 12.32 \\
\hline \multirow[t]{3}{*}{3068} & 1.36 & 21.57 & 0.00 & 0.00 & & & & \\
\hline & & & & & 3053 & -13.64 & 0.77 & 13.66 \\
\hline & & & & & 3271 & 8.68 & -10.23 & 13.42 \\
\hline \multirow[t]{2}{*}{3070} & 1.45 & 23.57 & 11.18 & 3.26 & & & & \\
\hline & & & & & 3067 & -8.64 & 8.73 & 12.28 \\
\hline \multirow[t]{2}{*}{3071} & 1.22 & 19.46 & 4.59 & 1.34 & & & & \\
\hline & & & & & 848 & -4.73 & -0.58 & 4.76 \\
\hline \multirow[t]{3}{*}{3072} & 1.08 & 14.71 & 3.47 & 1.01 & & & & \\
\hline & & & & & 853 & 3.23 & -5.77 & 6.61 \\
\hline & & & & & 3267 & -6.59 & 5.89 & 8.84 \\
\hline \multirow[t]{4}{*}{3073} & 1.07 & 17.22 & 0.78 & 0.23 & & & & \\
\hline & & & & & 864 & -18.63 & 4.69 & 19.21 \\
\hline & & & & & 3098 & 6.64 & 0.33 & 6.64 \\
\hline & & & & & 3152 & 12.53 & -6.44 & 14.09 \\
\hline \multirow[t]{2}{*}{3074} & 1.09 & 15.77 & 1.34 & 0.39 & & & & \\
\hline & & & & & 865 & -0.78 & 4.77 & 4.84 \\
\hline 3075 & 0.96 & 18.56 & 2.46 & 0.72 & & & & \\
\hline
\end{tabular}




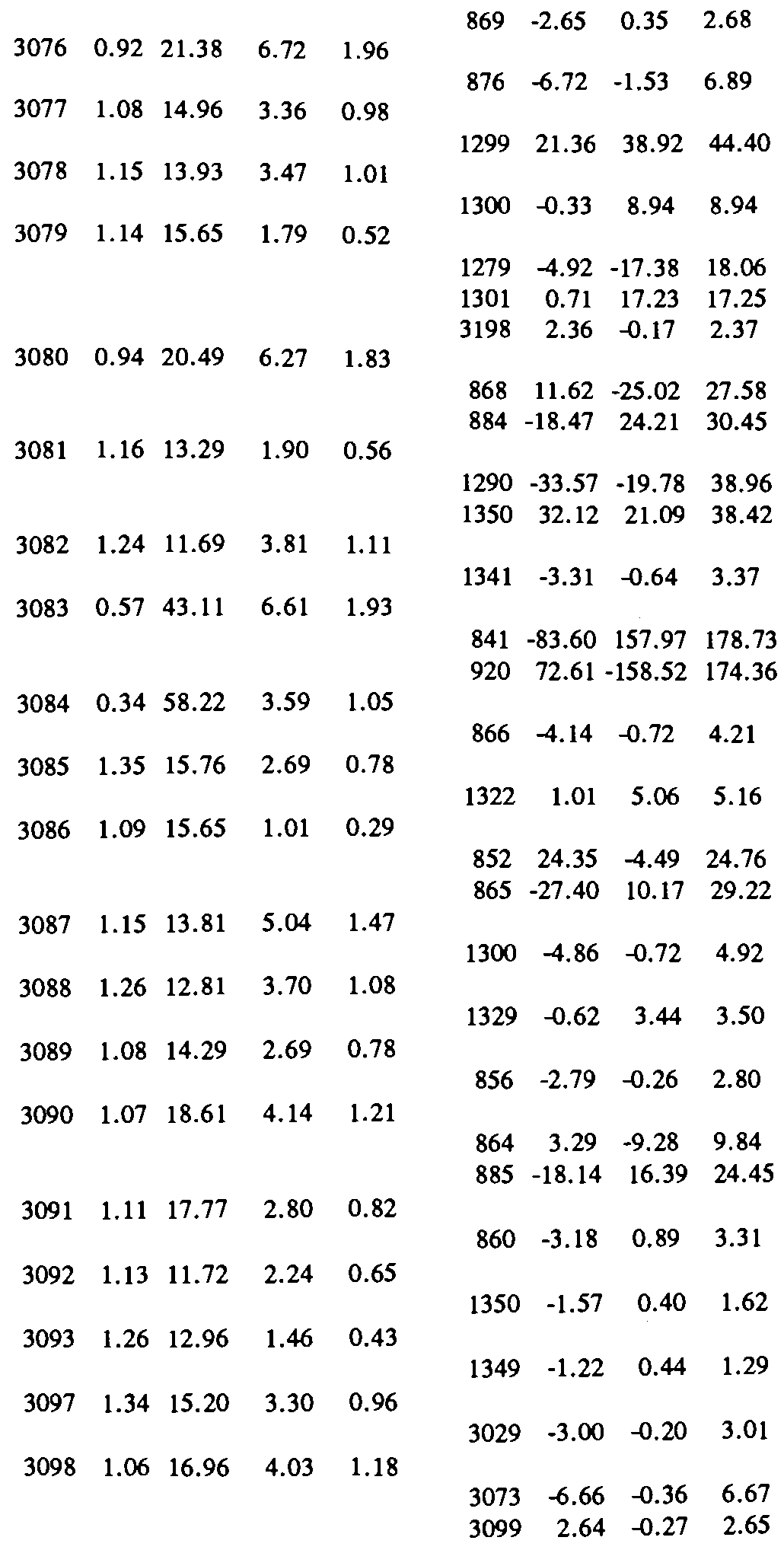




\begin{tabular}{|c|c|c|c|c|c|c|c|c|}
\hline 3099 & 1.06 & 16.75 & 2.24 & 0.65 & & & & \\
\hline \multirow[t]{3}{*}{3100} & 1.13 & 22.30 & 0.00 & 0.00 & 3098 & -2.73 & 0.07 & 2.73 \\
\hline & & & & & 969 & 196.78 & -308.74 & 366.12 \\
\hline & & & & & 971 & -201.61 & 327.80 & 384.84 \\
\hline 3101 & 1.64 & -10.56 & 3.94 & 1.15 & & & & \\
\hline 3102 & 1.36 & -7.48 & 5.53 & 1.61 & 3175 & -5.87 & -0.53 & 5.89 \\
\hline 3103 & 1.08 & 4.90 & 2.07 & 0.60 & 3177 & -10.63 & -2.67 & 10.96 \\
\hline \multirow[t]{2}{*}{3104} & 1.28 & 3.91 & 0.00 & 0.00 & 1153 & -1.99 & -0.19 & 2.00 \\
\hline & & & & & 1154 & -7.64 & -1.00 & 7.71 \\
\hline 3105 & 1.22 & 4.99 & 5.65 & 1.65 & 3272 & 5.50 & 0.26 & 5.51 \\
\hline 3106 & 1.10 & 6.07 & 3.66 & 1.07 & 1160 & -4.57 & -0.43 & 4.59 \\
\hline 3107 & 1.12 & 3.75 & 8.60 & 2.51 & 1161 & -3.06 & -0.91 & 3.20 \\
\hline \multirow[t]{4}{*}{3109} & 0.97 & 19.11 & 0.00 & 0.00 & 1175 & -1.80 & -1.46 & 2.32 \\
\hline & & & & & 2998 & 34.72 & 11.88 & 36.69 \\
\hline & & & & & 3061 & -48.80 & -14.28 & 50.85 \\
\hline & & & & & 3110 & 14.20 & 2.54 & 14.42 \\
\hline 3110 & 0.97 & 18.96 & 13.69 & 3.99 & & & & \\
\hline 3111 & 0.98 & 20.48 & 9.22 & 2.69 & 3109 & -14.26 & -2.67 & 14.51 \\
\hline \multirow[t]{2}{*}{3113} & 0.73 & 17.14 & 1.84 & 0.54 & 3061 & -9.40 & -2.05 & 9.62 \\
\hline & & & & & 3124 & -1.63 & -0.62 & 1.74 \\
\hline 3114 & 0.73 & 16.97 & 3.75 & 1.09 & 3124 & -3.40 & -1.34 & 3.65 \\
\hline \multirow[t]{2}{*}{3118} & 1.19 & 14.63 & 1.51 & 0.44 & & & & \\
\hline & & & & & 899 & -12.85 & 23.85 & 27.09 \\
\hline \multirow[t]{2}{*}{3119} & 1.16 & 14.65 & 2.56 & 0.75 & & & & \\
\hline & & & & & 847 & -2.58 & -0.41 & 2.61 \\
\hline \multirow[t]{3}{*}{3120} & 0.92 & 15.77 & 2.70 & 0.79 & & & & \\
\hline & & & & & 859 & -9.23 & 4.52 & 10.27 \\
\hline & & & & & 2997 & 0.36 & 1.66 & 1.70 \\
\hline 3121 & 1.06 & 15.49 & 1.51 & 0.44 & & & & \\
\hline \multirow[t]{2}{*}{3122} & 0.42 & 30.91 & 3.68 & 1.07 & 905 & -3.26 & 3.18 & 4.55 \\
\hline & & & & & 883 & -16.62 & 4.76 & 17.29 \\
\hline \multirow[t]{2}{*}{3123} & 1.08 & 15.49 & 3.35 & 0.98 & & & & \\
\hline & & & & & 900 & -6.12 & 2.98 & 6.81 \\
\hline \multirow[t]{4}{*}{3124} & 0.73 & 17.53 & 2.17 & 0.63 & & & & \\
\hline & & & & & 888 & 12.11 & 47.28 & 48.80 \\
\hline & & & & & 917 & -20.21 & -48.12 & 52.20 \\
\hline & & & & & 3113 & 1.52 & 0.43 & 1.58 \\
\hline
\end{tabular}




\begin{tabular}{|c|c|c|c|c|c|c|c|c|}
\hline 3126 & 1.11 & 15.52 & 1.84 & 0.54 & 3114 & 3.34 & 1.24 & 3.56 \\
\hline 3127 & 0.83 & 16.44 & 1.12 & 0.33 & 909 & -8.21 & 11.70 & 14.29 \\
\hline 128 & 1.33 & 15.38 & 2.37 & 0.69 & 917 & -1.90 & 0.53 & 1.97 \\
\hline 3130 & 1.39 & -0.70 & 3.18 & 0.93 & 903 & -2.54 & -0.66 & 2.62 \\
\hline 3132 & 1.38 & -7.58 & 3.94 & 1.15 & 3185 & -7.19 & -1.61 & 7.37 \\
\hline 3133 & 1.37 & 22.06 & 0.00 & 0.00 & 3134 & -5.55 & -1.31 & 5.70 \\
\hline 3134 & 1.38 & -7.37 & 11.06 & 3.22 & $\begin{array}{l}3014 \\
3037 \\
3051\end{array}$ & $\begin{array}{r}-110.00 \\
44.89 \\
114.36\end{array}$ & $\begin{array}{c}-148.86 \\
131.20 \\
174.39\end{array}$ & $\begin{array}{c}185.09 \\
138.67 \\
208.54\end{array}$ \\
\hline & & & & & $\begin{array}{l}3132 \\
3244\end{array}$ & $\begin{array}{r}5.34 \\
-17.17\end{array}$ & $\begin{array}{r}1.16 \\
-3.77\end{array}$ & $\begin{array}{l}5.46 \\
17.58\end{array}$ \\
\hline 3135 & 1.31 & 9.62 & 3.04 & 0.89 & 1011 & -2.24 & -0.08 & 2.25 \\
\hline 3137 & 1.24 & 4.50 & 2.47 & 0.72 & 1031 & 5.79 & 10.06 & 11.61 \\
\hline 3138 & 1.28 & 9.26 & 5.61 & 1.64 & 895 & -1.67 & -0.46 & 1.73 \\
\hline & & & & & $\begin{array}{l}870 \\
893\end{array}$ & $\begin{array}{r}-51.54 \\
46.07\end{array}$ & $\begin{array}{r}-8.63 \\
8.24\end{array}$ & $\begin{array}{l}52.26 \\
46.80\end{array}$ \\
\hline 3139 & 1.31 & 9.90 & 3.04 & 0.89 & $\begin{array}{l}1027 \\
1031\end{array}$ & $\begin{array}{r}-2.72 \\
0.25\end{array}$ & $\begin{array}{r}-7.20 \\
7.60\end{array}$ & $\begin{array}{l}7.70 \\
7.61\end{array}$ \\
\hline 140 & 1.20 & 4.80 & 6.37 & 1.86 & 893 & 39.04 & 44.59 & 59.27 \\
\hline 141 & 1.45 & 23.64 & 3.58 & 1.04 & & & & \\
\hline 146 & 1.35 & 12.98 & 2.76 & 0.81 & 3055 & 1.98 & 6.11 & 6.43 \\
\hline 3147 & 1.40 & 10.42 & 7.23 & 2.11 & 948 & 19.16 & 75.14 & 77.54 \\
\hline & & & & & $\begin{array}{c}939 \\
1070\end{array}$ & $\begin{array}{r}-60.29 \\
53.28\end{array}$ & $\begin{array}{l}50.23 \\
-47.52\end{array}$ & $\begin{array}{c}78.47 \\
71.40\end{array}$ \\
\hline 3148 & 1.25 & 7.12 & 3.04 & 0.89 & 373 & 391.76 & 353.97 & 527.99 \\
\hline 3149 & 1.39 & 8.92 & 1.43 & 0.42 & & & & \\
\hline & & & & & 1069 & -32.04 & -54.28 & 63.03 \\
\hline 3152 & 1.07 & 16.38 & 1.68 & 0.49 & 1071 & 31.96 & 60.31 & 68.26 \\
\hline & & & & & 3073 & -12.52 & 6.54 & 14.12 \\
\hline 153 & 1.38 & 1.91 & 1.12 & 0.33 & 3267 & 10.55 & -6.22 & 12.25 \\
\hline & & & & & 1036 & -56.24 & 27.74 & 62.71 \\
\hline
\end{tabular}




\begin{tabular}{|c|c|c|c|c|c|c|c|c|}
\hline 3154 & 1.37 & -2.79 & 0.90 & 0.26 & 3160 & 54.42 & -27.99 & 61.20 \\
\hline 3155 & 1.39 & -6.82 & 1.50 & 0.44 & 1021 & 25.15 & 12.15 & 27.93 \\
\hline 3156 & 1.37 & 3.60 & 3.15 & 0.92 & 1032 & 21.58 & 9.74 & 23.68 \\
\hline 3157 & 1.06 & 15.24 & 1.87 & 0.55 & 1022 & 2.83 & 2.32 & 3.65 \\
\hline 3158 & 1.50 & -6.14 & 0.90 & 0.26 & 905 & -2.13 & 0.04 & 2.13 \\
\hline 3159 & 1.37 & -0.69 & 2.77 & 0.81 & $\begin{array}{l}1054 \\
3163\end{array}$ & $\begin{array}{r}42.27 \\
-42.09\end{array}$ & $\begin{array}{r}-35.36 \\
37.40\end{array}$ & $\begin{array}{l}55.11 \\
56.30\end{array}$ \\
\hline 3160 & 1.37 & -0.45 & 0.00 & 0.00 & 3160 & -3.58 & -0.43 & 3.60 \\
\hline & & & & & $\begin{array}{l}1021 \\
3153 \\
3159\end{array}$ & $\begin{array}{r}51.87 \\
-53.24 \\
3.36\end{array}$ & $\begin{array}{c}-27.79 \\
30.07 \\
0.20\end{array}$ & $\begin{array}{l}58.84 \\
61.14 \\
3.36\end{array}$ \\
\hline 3161 & 1.46 & 1.81 & 1.50 & 0.44 & 3166 & -4.14 & $-1,33$ & \\
\hline 3162 & 1.48 & -1.43 & 2.02 & 0.59 & 1055 & 2.36 & 1.81 & 2.98 \\
\hline 3163 & 1.49 & -5.02 & 3.52 & 1.03 & $\begin{array}{l}1054 \\
1055 \\
3158\end{array}$ & $\begin{array}{r}42.30 \\
-84.39 \\
42.27\end{array}$ & $\begin{array}{r}-36.22 \\
78.80 \\
-36.86\end{array}$ & $\begin{array}{c}55.68 \\
115.46 \\
56.09\end{array}$ \\
\hline 3164 & 1.46 & -6.80 & 2.10 & 0.61 & 1058 & 79.22 & 26.22 & 83.44 \\
\hline 3165 & 1.48 & -1.97 & 3.30 & 0.96 & $\begin{array}{l}1052 \\
1059\end{array}$ & $\begin{array}{l}-43.92 \\
109.24\end{array}$ & $\begin{array}{l}13.62 \\
22.13\end{array}$ & $\begin{array}{l}45.98 \\
111.45\end{array}$ \\
\hline 3166 & 1.46 & 1.87 & 0.00 & 0.00 & $\begin{array}{l}1055 \\
1070-\end{array}$ & $\begin{array}{r}166.72 \\
-166.37\end{array}$ & $\begin{array}{r}-157.26 \\
164.04\end{array}$ & $\begin{array}{l}229.19 \\
233.64\end{array}$ \\
\hline 3171 & 1.38 & -0.81 & 6.28 & 1.83 & 3161 & 3.73 & 0.84 & 3.82 \\
\hline 3172 & 1.34 & -5.62 & 8.71 & 2.54 & 3185 & -8.23 & -2.00 & 8.47 \\
\hline 3173 & 1.43 & -4.11 & 7.12 & 2.08 & 3246 & -9.67 & -2.12 & 9.90 \\
\hline 3174 & 1.35 & -5.28 & 6.28 & 1.83 & 1024 & -6.87 & -2.05 & 7.17 \\
\hline 3175 & 1.65 & -10.36 & 1.59 & 0.46 & 1025 & -4.85 & -1.89 & 5.21 \\
\hline & & & & & 1044 & -4.84 & 0.27 & 4.85 \\
\hline 176 & 1.41 & -7.70 & 10.22 & 2.98 & 3101 & 5.62 & 0.36 & 5.63 \\
\hline & & & & & $\begin{array}{l}1026 \\
1029\end{array}$ & $\begin{array}{c}-24.81 \\
152.52\end{array}$ & $\begin{array}{c}-88.22 \\
181.80\end{array}$ & $\begin{array}{l}91.64 \\
237.31\end{array}$ \\
\hline
\end{tabular}




\begin{tabular}{|c|c|c|c|c|c|c|c|c|}
\hline \multirow[t]{3}{*}{3177} & 1.36 & -7.36 & 8.71 & 2.54 & & & & \\
\hline & & & & & 1030 & 21.11 & 8.23 & 22.66 \\
\hline & & & & & 3102 & 10.60 & 2.66 & 10.93 \\
\hline \multirow[t]{2}{*}{3178} & 1.39 & -7.62 & 6.28 & 1.83 & & & & \\
\hline & & & & & 1033 & 27.31 & 4.07 & 27.61 \\
\hline \multirow[t]{3}{*}{3179} & 1.35 & -4.33 & 2.35 & 0.68 & & & & \\
\hline & & & & & 1039 & -24.86 & 6.73 & 25.75 \\
\hline & & & & & 1045 & 26.09 & -5.60 & 26.69 \\
\hline \multirow{3}{*}{3180} & 1.43 & -4.97 & 3.94 & 1.15 & & & & \\
\hline & & & & & 1024 & -116.48 & -29.61 & 120.18 \\
\hline & & & & & 1041 & 118.28 & 29.69 & 121.95 \\
\hline 3181 & 1.65 & -10.28 & 3.94 & 1.15 & & & & \\
\hline \multirow[t]{4}{*}{3182} & 1.07 & -0.71 & 0.00 & 0.00 & 1044 & -2.30 & -0.31 & 2.32 \\
\hline & & & & & 3184 & 3.49 & 0.61 & 3.55 \\
\hline & & & & & 3191 & 6.77 & 1.87 & 7.02 \\
\hline & & & & & 3192 & -10.89 & -1.99 & 11.07 \\
\hline \multirow[t]{2}{*}{3183} & 1.08 & -0.16 & 2.08 & 0.61 & & & & \\
\hline & & & & & 3192 & -2.33 & -0.55 & 2.40 \\
\hline \multirow[t]{2}{*}{3184} & 1.06 & -1.01 & 3.61 & 1.05 & & & & \\
\hline & & & & & 3182 & -3.61 & -0.73 & 3.68 \\
\hline \multirow[t]{4}{*}{3185} & 1.39 & -0.61 & 0.00 & 0.00 & & & & \\
\hline & & & & & 3130 & 7.16 & 1.59 & 7.33 \\
\hline & & & & & 3171 & 8.19 & 1.94 & 8.42 \\
\hline & & & & & 3245 & -14.59 & -3.35 & 14.97 \\
\hline \multirow[t]{2}{*}{3186} & 1.17 & 9.50 & 3.37 & 0.98 & & & & \\
\hline & & & & & 3001 & -3.00 & -0.44 & 3.04 \\
\hline \multirow[t]{3}{*}{3187} & 1.40 & -4.76 & 0.00 & 0.00 & & & & \\
\hline & & & & & 1057 & 94.41 & 111.36 & 145.99 \\
\hline & & & & & 3246 & 18.01 & 72.12 & 74.34 \\
\hline \multirow[t]{4}{*}{3188} & 1.43 & -6.26 & 0.00 & 0.00 & & & & \\
\hline & & & & & 1057 & -17.24 & 69.69 & 71.80 \\
\hline & & & & & 1058 & 1.35 & -71.13 & 71.14 \\
\hline & & & & & 3244 & 20.98 & 63.84 & 67.20 \\
\hline \multirow[t]{4}{*}{3189} & 1.44 & -0.05 & 0.00 & 0.00 & & & & \\
\hline & & & & & 1066 & 136.14 & -4.62 & 136.21 \\
\hline & & & & & 1067 & -148.09 & 6.19 & 148.21 \\
\hline & & & & & 3245 & 17.16 & 85.94 & 87.64 \\
\hline \multirow[t]{2}{*}{3191} & 1.06 & -0.91 & 6.91 & 2.02 & & & & \\
\hline & & & & & 3182 & -6.79 & -1.87 & 7.04 \\
\hline \multirow[t]{4}{*}{3192} & 1.08 & -0.07 & 0.00 & 0.00 & & & & \\
\hline & & & & & 479 & -12.89 & -1.89 & 13.03 \\
\hline & & & & & 3182 & 10.84 & 2.02 & 11.03 \\
\hline & & & & & 3183 & 2.23 & 0.48 & 2.29 \\
\hline \multirow[t]{4}{*}{3193} & 1.33 & 17.23 & 0.00 & 0.00 & & & & \\
\hline & & & & & 3009 & -42.92 & -9.74 & 44.01 \\
\hline & & & & & 3024 & 6.26 & 3.25 & 7.05 \\
\hline & & & & & 3254 & 36.27 & 7.20 & 36.98 \\
\hline 3194 & 1.29 & 11.17 & 0.00 & 0.00 & & & & \\
\hline
\end{tabular}




\begin{tabular}{|c|c|c|c|c|c|c|c|c|}
\hline & & & & & $\begin{array}{l}3013 \\
3016\end{array}$ & $\begin{array}{r}-6.93 \\
7.46\end{array}$ & $\begin{array}{c}-33.05 \\
2.01\end{array}$ & $\begin{array}{l}33.77 \\
7.73\end{array}$ \\
\hline 3195 & 1.38 & 3.44 & 2.63 & 0.77 & & & & \\
\hline 3197 & 1.37 & 3.08 & 3.43 & 1.00 & 1162 & -1.65 & -0.21 & 1.67 \\
\hline 3198 & 1.14 & 15.58 & 2.58 & 0.75 & 1203 & 6.43 & -4.44 & 7.82 \\
\hline 3201 & 1.53 & 32.72 & 0.00 & 0.00 & 3079 & -2.37 & 0.10 & 2.38 \\
\hline 3202 & 1.54 & 32.58 & 0.00 & 0.00 & 3205 & 880.55 & 1115.46 & 1421.13 \\
\hline 3203 & 0.77 & -21.58 & 17.92 & 5.23 & 3205 & 869.12 & 1238.26 & 1512.83 \\
\hline & & & & & 3237 & -46.78 & -12.55 & 48.43 \\
\hline 3204 & 1.29 & 21.76 & 0.00 & 0.00 & 3256 & 11.57 & 2.81 & 11.91 \\
\hline & & & & & $\begin{array}{c}972 \\
975 \\
1087\end{array}$ & $\begin{array}{r}282.36 \\
212.53 \\
37.97\end{array}$ & $\begin{array}{r}429.64 \\
-159.28 \\
67.73\end{array}$ & $\begin{array}{c}514.12 \\
265.59 \\
77.65\end{array}$ \\
\hline 3205 & 1.42 & 30.73 & 0.00 & 0.00 & 3205 & -554.66 & -263.18 & 613.92 \\
\hline & & & & & $\begin{array}{c}462 \\
3015\end{array}$ & $\begin{array}{c}644.10 \\
-2.54\end{array}$ & $\begin{array}{l}-260.64 \\
-85.45\end{array}$ & $\begin{array}{l}694.84 \\
85.49\end{array}$ \\
\hline & & & & & 3201 & -856.14 & 354.12 & 1324.44 \\
\hline & & & & & 3202 & -841.79 & 2987.23 & 1401.66 \\
\hline 3206 & 1.51 & 22.33 & 0.00 & 0.00 & 3204 & 557.95 & 368.23 & 668.51 \\
\hline & & & & & 398 & 161.72 & -161.71 & 228.70 \\
\hline 3211 & 0.77 & -18.00 & 25.32 & 7.39 & 472 & -155.24 & 215.65 & 265.72 \\
\hline & & & & & 3253 & -55.02 & -18.19 & 57.94 \\
\hline & & & & & 3262 & 24.52 & 5.32 & 25.09 \\
\hline 3214 & 0.75 & -19.77 & 0.00 & 0.00 & & & & \\
\hline & & & & & 3215 & 45.73 & 15.70 & 48.35 \\
\hline 3215 & 0.74 & -22.89 & 14.68 & 4.28 & 3220 & -47.22 & -17.59 & 50.39 \\
\hline & & & & & 3214 & -45.55 & -12.93 & 47.35 \\
\hline & & & & & 3216 & 30.20 & 9.84 & 31.77 \\
\hline 3216 & $0.71-$ & -27.12 & 30.47 & 8.89 & & & & \\
\hline 3220 & 0.77 & -17.82 & 7.39 & 2.16 & 3215 & -31.02 & -7.73 & 31.97 \\
\hline & & & & & 3214 & 47.57 & 19.58 & 51.45 \\
\hline & & & & & 3253 & -56.48 & -23.18 & 61.05 \\
\hline 3221 & 0.78 & -16.12 & 26.33 & 7.68 & & & & \\
\hline 3222 & 0.79 & -14.97 & 17.36 & 5.07 & 3225 & -30.20 & -12.49 & 32.68 \\
\hline & & & & & 3223 & -95.51 & -47.73 & 106.77 \\
\hline 3223 & 0.79 & -14.77 & 0.00 & 0.00 & $32 \angle 4$ & 61.88 & 19.55 & 64.90 \\
\hline
\end{tabular}




\begin{tabular}{|c|c|c|c|c|c|c|c|c|}
\hline 3224 & 0.79 & -15.52 & 11.65 & 3.40 & 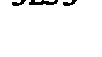 & 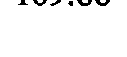 & 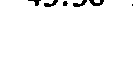 & 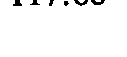 \\
\hline & & & & & 3222 & -61.85 & -18.91 & 64.68 \\
\hline & & & & & 3225 & 47.90 & 12.81 & 49.58 \\
\hline 3225 & 0.79 & -15.88 & 22.52 & 6.57 & & & & \\
\hline & & & & & 3221 & 30.18 & 12.60 & 32.71 \\
\hline & & & & & 3224 & -47.88 & -12.52 & 49.49 \\
\hline 3228 & 0.79 & -15.07 & 20.39 & 5.95 & & & & \\
\hline & & & & & 3223 & -18.95 & -4.27 & 19.42 \\
\hline & & & & & 3236 & 5.68 & 0.20 & 5.68 \\
\hline & & & & & 3252 & -8.76 & -2.14 & 9.02 \\
\hline 3230 & 1.06 & 2.16 & 0.00 & 0.00 & & & & \\
\hline & & & & & 3223 & 264.10 & 313.61 & 410.00 \\
\hline & & & & & 3239 & -257.70 & -206.50 & 330.23 \\
\hline 3231 & 1.34 & 9.89 & 5.20 & 1.52 & & & & \\
\hline & & & & & 285 & -5.46 & -0.69 & 5.50 \\
\hline 3232 & 1.13 & 1.76 & 0.00 & 0.00 & & & & \\
\hline & & & & & 3233 & 30.06 & -4.77 & 30.44 \\
\hline & & & & & 3235 & 30.23 & 7.93 & 31.25 \\
\hline & & & & & 3238 & -64.22 & -1.25 & 64.23 \\
\hline 3233 & 1.13 & 0.18 & 26.33 & 7.68 & & & & \\
\hline & & & & & 3232 & -31.33 & 4.06 & 31.60 \\
\hline & & & & & 3234 & 3.96 & -4.74 & 6.18 \\
\hline 3234 & 1.13 & -0.18 & 4.59 & 1.34 & & & & \\
\hline & & & & & 3233 & -5.50 & 3.15 & 6.34 \\
\hline 3235 & 1.13 & 1.60 & 31.26 & 9.12 & & & & \\
\hline & & & & & 3232 & -30.36 & -7.99 & 31.39 \\
\hline 3236 & 0.79 & -15.23 & 6.50 & 1.89 & & & & \\
\hline & & & & & 3228 & -6.09 & -0.27 & 6.09 \\
\hline 3237 & 0.79 & -20.54 & 0.00 & 0.00 & & & & \\
\hline & & & & & 543 & 177.62 & 98.17 & 202.94 \\
\hline & & & & & 3203 & 47.07 & 13.51 & 48.97 \\
\hline 3238 & 1.13 & 2.28 & 0.00 & 0.00 & & & & \\
\hline & & & & & 309 & -63.02 & 0.85 & 63.02 \\
\hline & & & & & 3232 & 64.06 & 1.50 & 64.08 \\
\hline 3239 & 1.10 & 3.99 & 0.00 & 0.00 & & & & \\
\hline & & & & & 342 & -470.62 & -795.91 & 924.64 \\
\hline & & & & & 344 & 196.05 & 591.69 & 623.32 \\
\hline & & & & & 3230 & 258.32 & 221.05 & 339.99 \\
\hline 3244 & 1.40 & -6.69 & 2.95 & 0.86 & & & & \\
\hline & & & & & 3134 & 17.14 & 3.86 & 17.57 \\
\hline & & & & & 3188 & -20.89 & -62.00 & 65.42 \\
\hline 3245 & 1.39 & -0.34 & 2.35 & 0.68 & & & & \\
\hline & & & & & 3185 & 14.56 & 3.38 & 14.95 \\
\hline & & & & & 3189 & -17.05 & -83.20 & 84.93 \\
\hline
\end{tabular}


$\begin{array}{lllll}3246 & 1.36 & -5.12 & 0.00 & 0.00\end{array}$

$$
\begin{array}{lllll}
3248 & 0.96 & 18.32 & 22.91 & 6.68 \\
3249 & 1.36 & -5.17 & 5.53 & 1.61 \\
3251 & 0.79 & -15.05 & 22.29 & 6.50 \\
3252 & 0.79 & -14.87 & 0.00 & 0.00
\end{array}
$$$$
\begin{array}{llll}
3172 & 9.64 & 2.09 & 9.86
\end{array}
$$$$
\begin{array}{llll}
3187 & -17.89 & -69.78 & 72.03
\end{array}
$$$$
\begin{array}{llll}
3249 & 3.80 & 1.42 & 4.06
\end{array}
$$$$
\begin{array}{llll}
2998 & -23.25 & -5.62 & 23.92
\end{array}
$$$$
\begin{array}{llll}
3246 & -3.83 & -1.44 & 4.09
\end{array}
$$$$
\begin{array}{llll}
3252 & -26.50 & -12.16 & 29.16
\end{array}
$$$$
\begin{array}{llll}
3223 & -27.00 & -2.03 & 27.08
\end{array}
$$$$
\begin{array}{llll}
3228 & 8.63 & 2.10 & 8.88
\end{array}
$$$$
\begin{array}{lllll}
3253 & 0.78 & -16.85 & 0.00 & 0.00
\end{array}
$$$$
\begin{array}{llll}
3251 & 26.47 & 12.23 & 29.16
\end{array}
$$$$
\begin{array}{llll}
3211 & 55.06 & 19.27 & \mathbf{5 8 . 3 3}
\end{array}
$$$$
\begin{array}{llll}
3220 & 56.51 & 24.25 & 61.50
\end{array}
$$

$\begin{array}{lllll}3254 & 1.32 & 16.16 & 0.00 & 0.00\end{array}$

$\begin{array}{llll}3223 & -108.79 & -38.60 & 115.44\end{array}$

$\begin{array}{llll}3018 & 15.87 & 5.49 & 16.80\end{array}$

$\begin{array}{llll}3019 & 3.34 & 1.82 & 3.80\end{array}$

$\begin{array}{llll}3020 & 19.31 & 5.08 & 19.97\end{array}$

$\begin{array}{llll}3193 & -36.09 & -6.56 & 36.68\end{array}$

$\begin{array}{lllll}3256 & 0.77 & -21.76 & 17.92 & 5.23\end{array}$

$\begin{array}{llll}3203 & -11.70 & -2.82 & 12.04\end{array}$

$\begin{array}{lllll}3262 & 0.77 & -18.28 & 26.55 & 7.74\end{array}$

$\begin{array}{llll}3211 & -24.58 & -5.26 & 25.13\end{array}$

$\begin{array}{lllll}3264 & 1.43 & 17.54 & 3.51 & 1.02\end{array}$

$\begin{array}{lllll}3265 & 1.43 & 17.43 & 9.75 & 2.84\end{array}$

$\begin{array}{llll}3010 & -12.20 & 1.99 & 12.36\end{array}$

$\begin{array}{llll}3265 & 7.66 & -2.35 & 8.01\end{array}$

$\begin{array}{lllll}3266 & 1.15 & 9.33 & 2.08 & 0.61\end{array}$

$\begin{array}{llll}3264 & -8.01 & 1.90 & 8.23\end{array}$

$\begin{array}{llll}3004 & -0.73 & -0.44 & 0.86\end{array}$

$\begin{array}{lllll}3267 & 1.07 & 15.17 & 3.47 & 1.01\end{array}$

$\begin{array}{llll}3072 & 6.55 & -5.88 & 8.80\end{array}$

$\begin{array}{llll}3152 & -10.64 & 6.26 & 12.35\end{array}$

$\begin{array}{lllll}3271 & 1.36 & 21.53 & 16.13 & 4.70\end{array}$

$\begin{array}{llll}3068 & -8.71 & 10.19 & 13.40\end{array}$

$\begin{array}{lllll}3272 & 1.28 & 3.84 & 3.34 & 0.98\end{array}$

$\begin{array}{llll}3104 & -5.53 & -0.29 & 5.54\end{array}$

$\begin{array}{llll}3273 & 2.99 & 0.33 & 3.01\end{array}$

$\begin{array}{lllll}3273 & 1.28 & 3.68 & 3.34 & 0.98\end{array}$

$\begin{array}{llll}3272 & -3.18 & -0.46 & 3.22\end{array}$

$\begin{array}{lllll}3275 & 1.31 & 17.56 & 5.30 & 1.55\end{array}$

$\begin{array}{llll}2362 & -4.16 & 5.97 & 7.28\end{array}$

$\begin{array}{lllll}3301 & 1.20 & 14.33 & 5.69 & 1.66\end{array}$

$\begin{array}{llll}371 & 133.51 & 180.51 & 224.51\end{array}$

$\begin{array}{lllll}3302 & 1.08 & 0.39 & 14.11 & 4.11\end{array}$ 


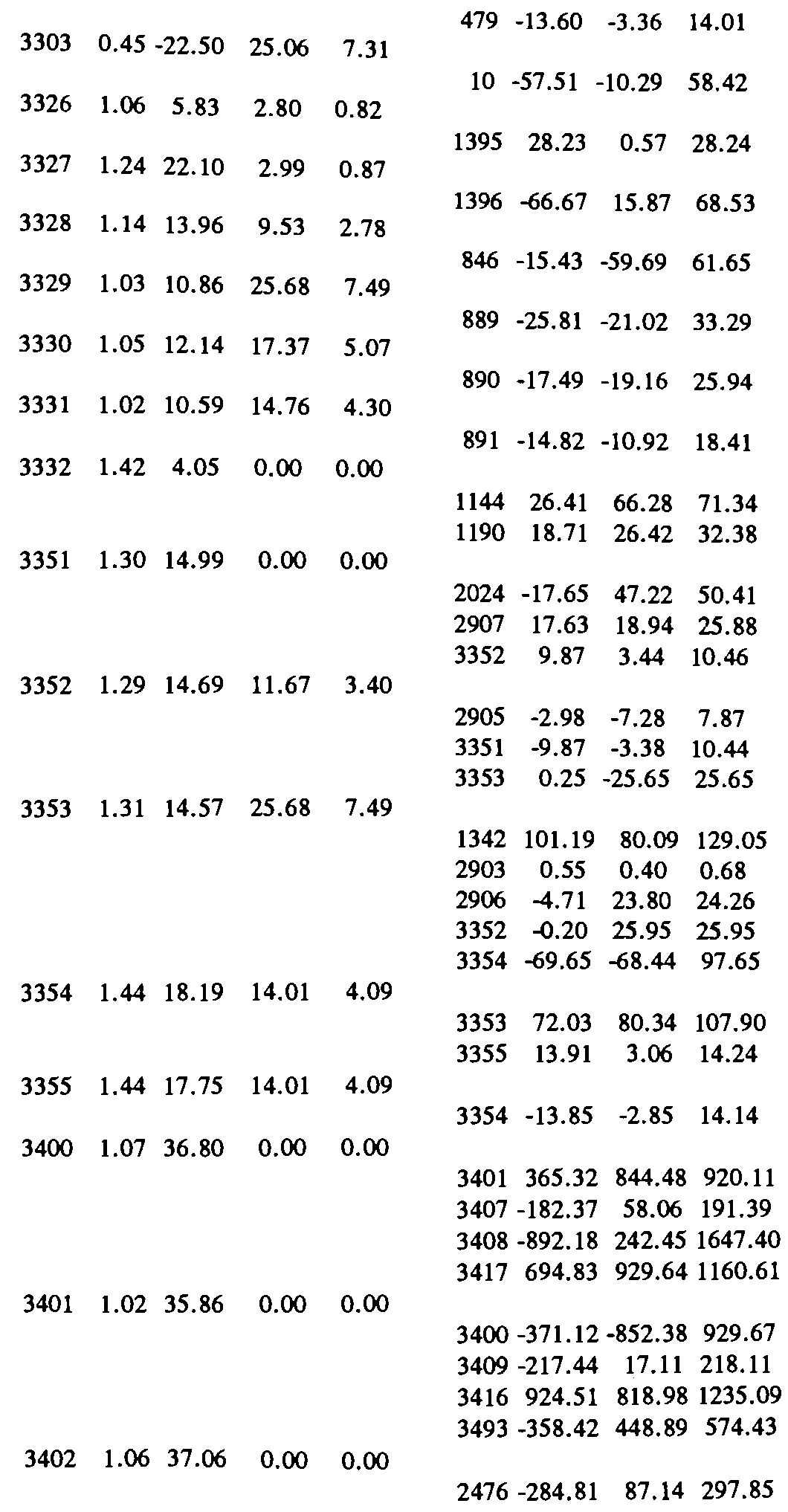




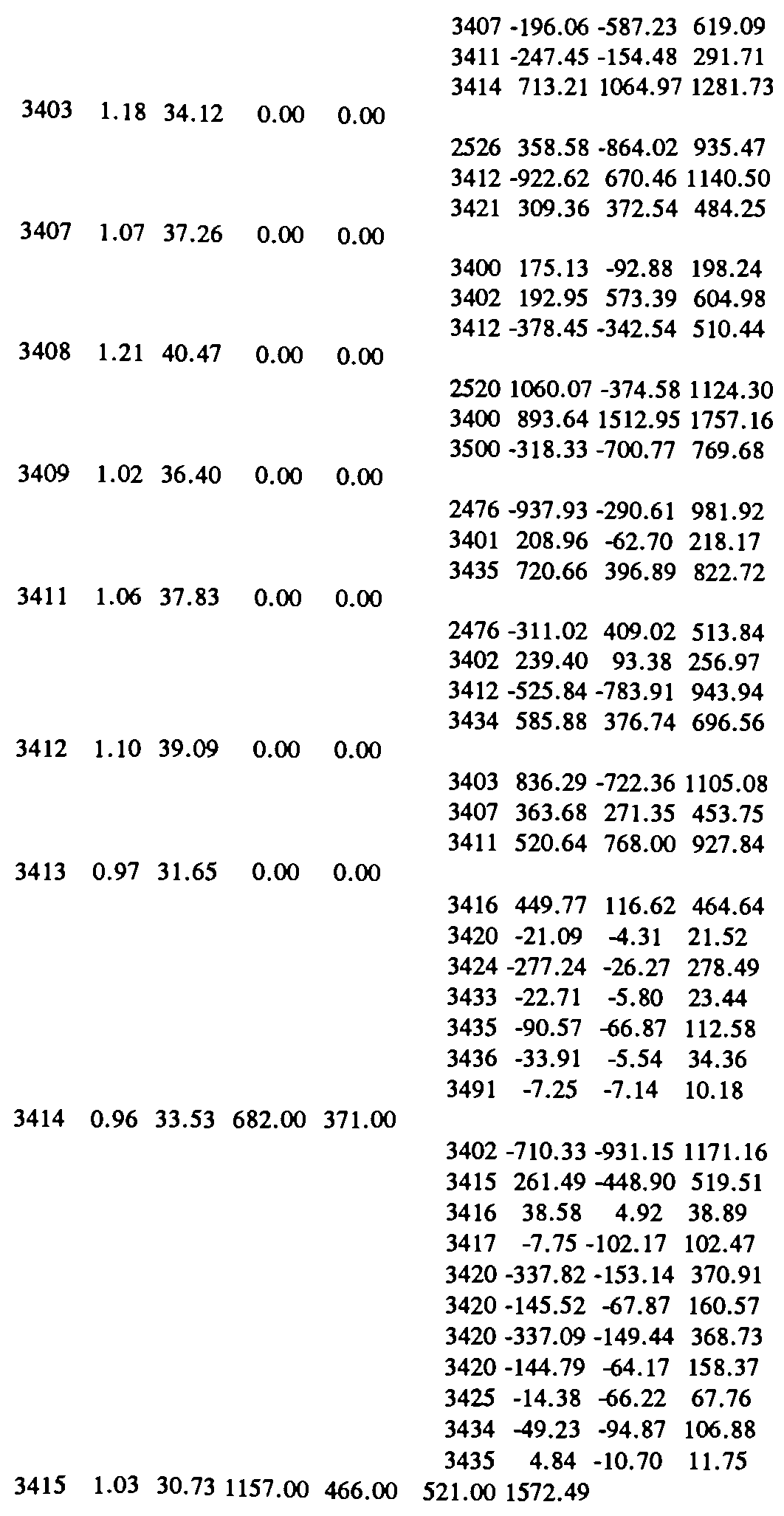




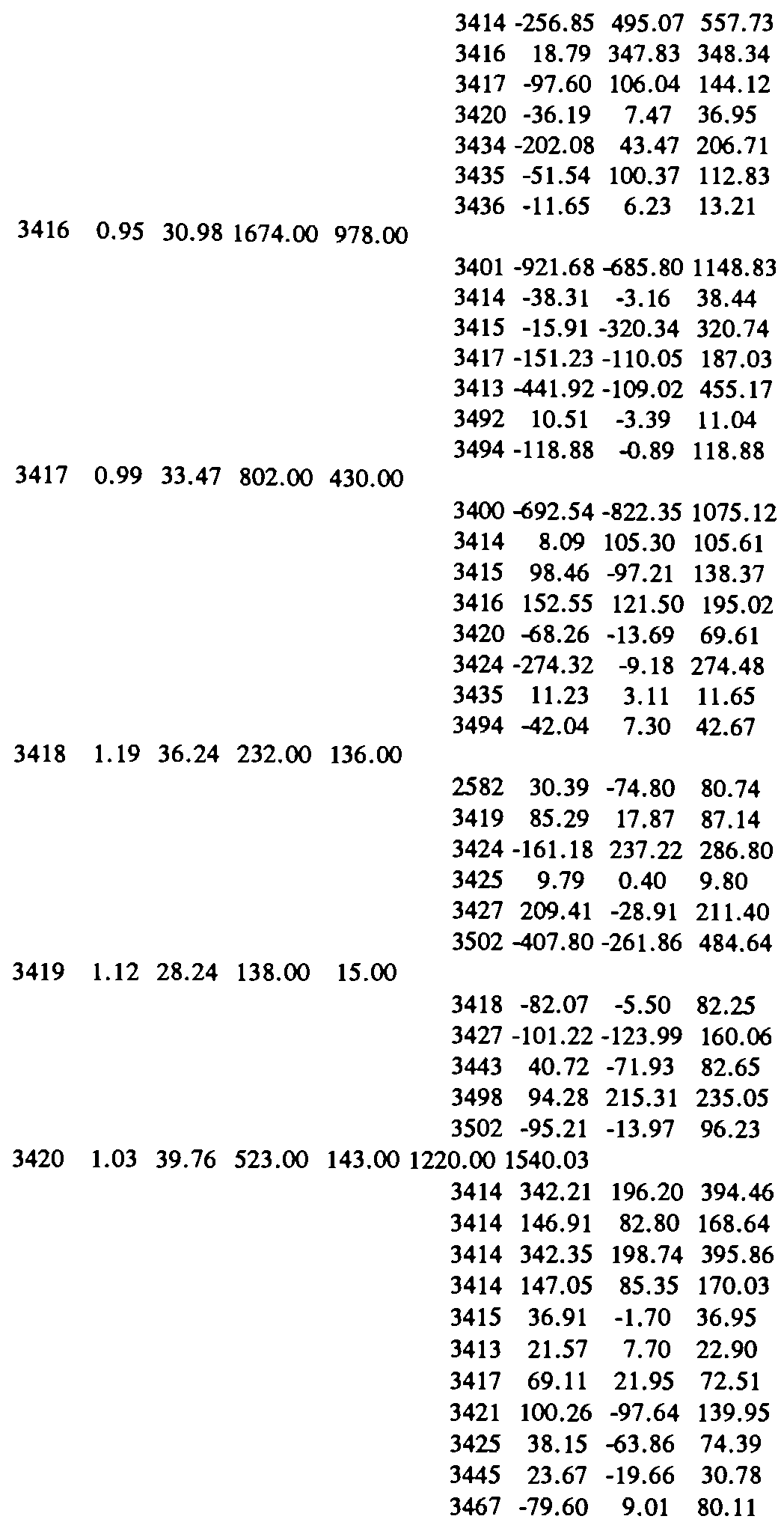


$\begin{array}{lllll}3421 & 1.15 & 32.92 & 414.00 & 198.00\end{array}$

$\begin{array}{lllll}3422 & 1.25 & 25.53 & 37.30 & 20.10\end{array}$

$$
\begin{array}{cccc}
3403 & -309.03 & -356.64 & 471.90 \\
3420 & -97.44 & 120.69 & 155.12 \\
3422 & 187.59 & -160.07 & 246.61 \\
3425 & -23.25 & -13.87 & 27.08 \\
3445 & 81.81 & -10.43 & 82.48 \\
3467 & -281.78 & 203.54 & 347.61 \\
3468 & 25.03 & 32.84 & 41.29 \\
& & & \\
3421 & -185.83 & 184.82 & 262.09 \\
3425 & -56.31 & 60.50 & 82.66 \\
3440 & -38.61 & -135.53 & 140.92 \\
3448 & 238.69 & -94.10 & 256.57 \\
& & & \\
3438 & 252.07 & -86.09 & 266.37 \\
3440 & -380.24 & 181.10 & 421.16 \\
3446 & 101.00 & -107.76 & 147.69 \\
4 & & & \\
3413 & 283.08 & 87.48 & 296.29 \\
3417 & 279.79 & 58.76 & 285.89 \\
3418 & 165.45 & -201.81 & 260.96 \\
3425 & 343.15 & -295.99 & 453.17 \\
3494 & 17.98 & 21.94 & 28.37 \\
3502 & -22.73 & -265.43 & 266.40
\end{array}
$$

$\begin{array}{lllll}3423 & 1.39 & 19.47 & 14.50 & 11.70\end{array}$

$\begin{array}{lllll}3424 & 1.03 & 43.41 & 1000.00 \quad 613.00\end{array}$

$\begin{array}{lllll}3425 & 1.18 & 34.60 & 645.00 & 349.00\end{array}$

$2520-536.14-660.58 \quad 850.77$

$\begin{array}{llll}2582 & 1.69 & -30.08 & 30.13\end{array}$

$\begin{array}{lllll}3414 & 16.04 & 81.16 & 82.73\end{array}$

$\begin{array}{llll}3418 & -9.73 & -0.12 & 9.73\end{array}$

$\begin{array}{llll}3420 & -36.69 & 76.23 & 84.60\end{array}$

$\begin{array}{lllll}3421 & 23.43 & 14.92 & 27.78\end{array}$

$\begin{array}{llll}3422 & 61.45 & -47.98 & 77.96\end{array}$

$\begin{array}{lllll}3424 & -334.07 & 392.40 & 515.34\end{array}$

$\begin{array}{llll}3427 & 10.75 & -6.82 & 12.73\end{array}$

$\begin{array}{lllll}3440 & 158.31 & -164.92 & 228.61\end{array}$

$\begin{array}{lllll}3427 & 1.19 & 30.35 & 0.00 & 0.00\end{array}$

$\begin{array}{llll}2582 & -17.16 & -28.02 & 32.86\end{array}$

$\begin{array}{lllll}3418 & -207.57 & 38.19 & 211.05\end{array}$

$\begin{array}{llll}3419 & 100.16 & 124.06 & 159.45\end{array}$

$\begin{array}{lllll}3425 & -10.31 & 7.67 & 12.85\end{array}$

$3449 \quad 134.41-117.94 \quad 178.82$

$\begin{array}{lllll}3428 & 1.38 & 30.10 & 17.30 & 14.90\end{array}$

$\begin{array}{lllll}2582 & -368.42 & 156.72 & 400.37\end{array}$

$\begin{array}{llll}3441 & 239.92 & -129.16 & 272.48\end{array}$

$\begin{array}{lllll}3451 & 108.76 & -24.39 & 111.46\end{array}$

$\begin{array}{lllll}3429 & 1.55 & 25.12 & 5.40 & -1.30\end{array}$

$\begin{array}{llll}2500 & 60.22 & 118.70 & 133.11\end{array}$

$\begin{array}{llll}3430 & -62.57 & -39.37 & 73.93\end{array}$

$\begin{array}{lllll}3430 & 1.56 & 25.89 & 0.00 & 0.00\end{array}$ 


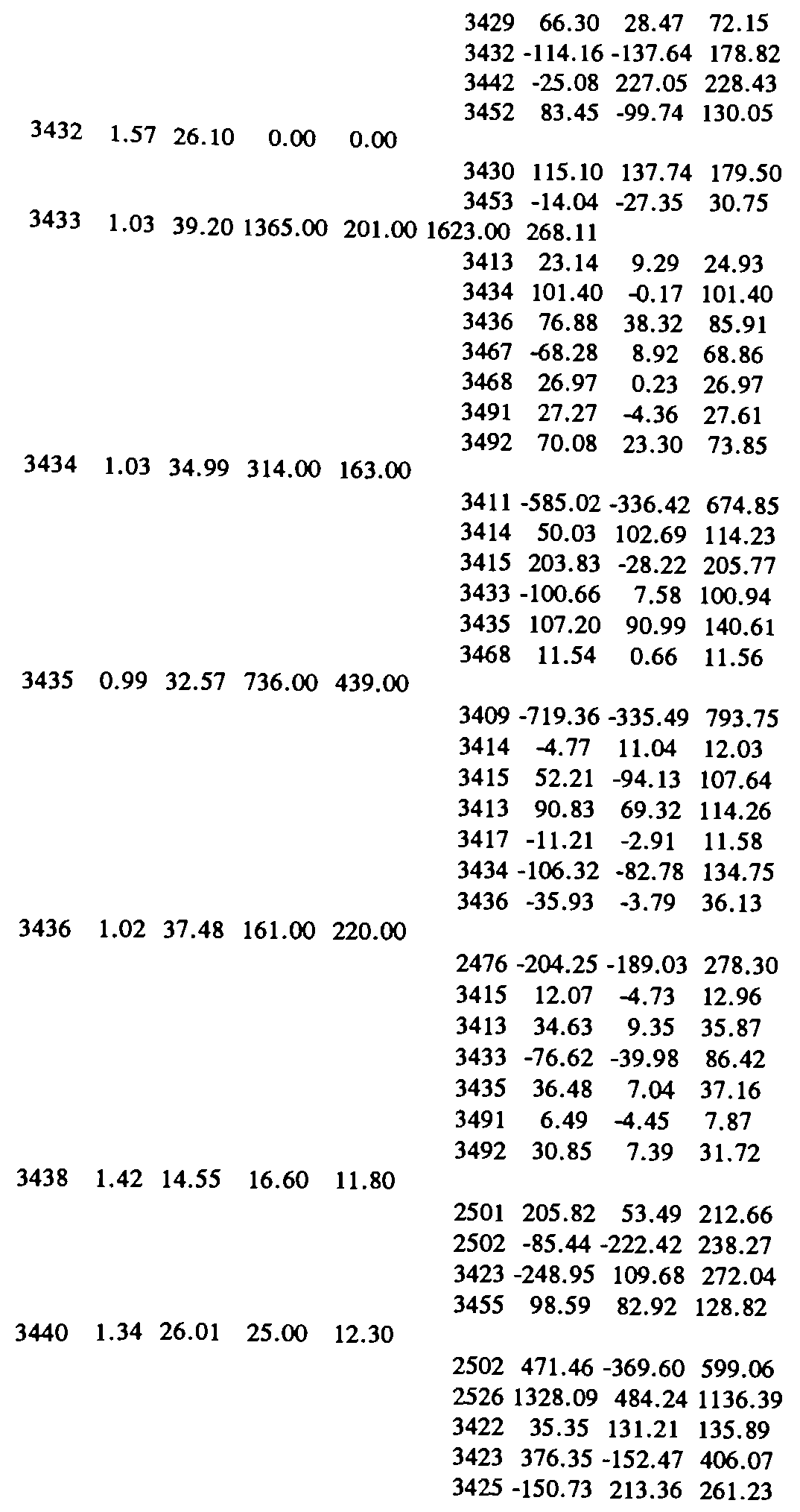




\begin{tabular}{|c|c|c|c|c|c|c|c|c|}
\hline & & & & & 3441 & -70.34 & -144.75 & 160.94 \\
\hline \multirow{6}{*}{3441} & & & & & 3458 & 332.49 & -100.71 & 347.40 \\
\hline & 1.41 & 26.86 & 0.00 & 0.00 & & & & \\
\hline & & & & & 3428 & -237.90 & 138.99 & 275.53 \\
\hline & & & & & 3440 & 71.68 & 143.24 & 160.18 \\
\hline & & & & & 3442 & -13.53 & -136.20 & 136.87 \\
\hline & & & & & 3457 & 183.53 & -150.22 & 237.16 \\
\hline \multirow[t]{5}{*}{3442} & 1.48 & 26.76 & 1.90 & 1.30 & & & & \\
\hline & & & & & 2582 & -327.65 & 375.82 & 498.59 \\
\hline & & & & & 3430 & 25.97 & -219.94 & 221.46 \\
\hline & & & & & 3441 & 9.09 & 136.90 & 137.20 \\
\hline & & & & & 3463 & 274.53 & -300.00 & 406.65 \\
\hline \multirow[t]{4}{*}{3443} & 1.16 & 26.92 & 24.00 & 52.80 & & & & \\
\hline & & & & & 3419 & -40.58 & 75.69 & 85.88 \\
\hline & & & & & 3444 & 19.97 & -106.14 & 108.01 \\
\hline & & & & & 3449 & -3.37 & -22.45 & 22.70 \\
\hline \multirow[t]{6}{*}{3444} & 1.63 & 18.95 & 118.00 & 45.90 & & & & \\
\hline & & & & & 3443 & -7.10 & 151.33 & 151.50 \\
\hline & & & & & 3449 & -25.36 & 78.61 & 82.60 \\
\hline & & & & & 3452 & -97.15 & 77.53 & 124.29 \\
\hline & & & & & 3464 & -25.73 & -122.28 & 124.96 \\
\hline & & & & & 3499 & 35.77 & 184.83 & 188.26 \\
\hline \multirow[t]{5}{*}{3445} & 1.15 & 31.39 & 410.00 & 73.20 & & & & \\
\hline & & & & & 3420 & -22.75 & 25.34 & 34.05 \\
\hline & & & & & 3421 & -81.36 & 12.58 & 82.33 \\
\hline & & & & & 3467 & -56.36 & 41.22 & 69.83 \\
\hline & & & & & 3468 & 26.63 & 43.76 & 51.22 \\
\hline \multirow[t]{6}{*}{3446} & 1.44 & 17.34 & 181.00 & 112.00 & & & & \\
\hline & & & & & 3423 & -100.74 & 115.80 & 153.48 \\
\hline & & & & & 3447 & 138.96 & - $\quad-7.59$ & 139.16 \\
\hline & & & & & 3448 & -25.75 & 56.05 & 61.68 \\
\hline & & & & & 3455 & 36.47 & 4.64 & 36.76 \\
\hline & & & & & 3458 & -25.57 & 32.90 & 41.67 \\
\hline \multirow[t]{3}{*}{3447} & 1.44 & 14.50 & 179.00 & 80.30 & & & & \\
\hline & & & & & 3446 & -138.83 & 13.84 & 139.52 \\
\hline & & & & & 3448 & -40.03 & 94.63 & 102.75 \\
\hline \multirow[t]{5}{*}{3448} & 1.27 & 22.48 & 175.00 & 53.30 & & & & \\
\hline & & & & & 3422 & -237.93 & 3108.77 & 261.61 \\
\hline & & & & & 3446 & 27.05 & -47.21 & 54.41 \\
\hline & & & & & 3447 & 46.64 & -77.92 & 90.81 \\
\hline & & & & & 3458 & -10.29 & -35.64 & 37.09 \\
\hline \multirow[t]{6}{*}{3449} & 1.26 & 26.54 & 148.00 & 39.70 & & & & \\
\hline & & & & & 3427 & -133.84 & 4134.22 & 189.55 \\
\hline & & & & & 3443 & 3.82 & 24.31 & 24.60 \\
\hline & & & & & 3444 & 27.44 & -57.62 & 63.82 \\
\hline & & & & & 3451 & -29.22 & -39.56 & 49.19 \\
\hline & & & & & 3464 & -16.37 & -100.94 & 102.26 \\
\hline \multirow[t]{2}{*}{3450} & 1.64 & 23.53 & 60.60 & 19.40 & & & & \\
\hline & & & & & 2500 & -88.71 & 217.18 & 234.60 \\
\hline
\end{tabular}




\begin{tabular}{|c|c|c|c|c|c|c|c|c|}
\hline \multirow[t]{4}{*}{3451} & 1.39 & 27.74 & 46.00 & 19.50 & . & 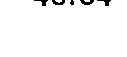 & 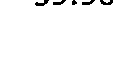 & 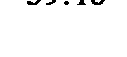 \\
\hline & & & & & 3428 & -108.57 & 29.08 & 112.40 \\
\hline & & & & & 3449 & 31.37 & 44.39 & 54.35 \\
\hline & & & & & 3464 & 31.25 & -92.07 & 97.23 \\
\hline \multirow[t]{7}{*}{3452} & 1.59 & 25.14 & 32.80 & 10.70 & & & & \\
\hline & & & & & 3430 & -83.31 & 102.23 & 131.88 \\
\hline & & & & & 3444 & 102.04 & -64.76 & 120.86 \\
\hline & & & & & 3450 & 18.30 & -38.11 & 42.27 \\
\hline & & & & & 3453 & -140.04 & 62.30 & 153.27 \\
\hline & & & & & 3454 & 3.62 & 49.12 & 49.25 \\
\hline & & & & & 3464 & 64.62 & -123.14 & 139.06 \\
\hline \multirow[t]{4}{*}{3453} & 1.58 & 26.20 & 20.20 & 9.80 & & & & \\
\hline & & & & & 3432 & 14.05 & 27.49 & 30.87 \\
\hline & & & & & 3452 & 140.48 & -59.37 & 152.51 \\
\hline & & & & & 3454 & 164.79 & 3.95 & 164.84 \\
\hline \multirow[t]{3}{*}{3454} & 1.57 & 25.18 & 169.00 & 59.10 & & & & \\
\hline & & & & & 3452 & -3.56 & -48.71 & 48.84 \\
\hline & & & & & 3453 & -164.16 & -0.98 & 164.16 \\
\hline \multirow[t]{5}{*}{3455} & 1.38 & 12.53 & 149.00 & 71.30 & & & & \\
\hline & & & & & 3438 & -98.42 & -77.03 & 124.98 \\
\hline & & & & & 3446 & -35.02 & -1.49 & 35.05 \\
\hline & & & & & 3456 & 43.20 & -26.68 & 50.78 \\
\hline & & & & & 3458 & -58.99 & 34.31 & 68.24 \\
\hline \multirow[t]{4}{*}{3456} & 1.39 & 9.54 & 55.00 & 48.20 & & & & \\
\hline & & & & & 2501 & -111.94 & 5.01 & 112.05 \\
\hline & & & & & 2506 & 100.88 & -80.77 & 129.23 \\
\hline & & & & & 3455 & -42.23 & 29.24 & 51.36 \\
\hline \multirow[t]{4}{*}{3457} & 1.49 & 23.36 & 87.30 & 49.40 & & & & \\
\hline & & & & & 3441 & -182.72 & 169.24 & 249.06 \\
\hline & & & & & 3458 & 59.87 & 82.72 & 102.11 \\
\hline & & & & & 3462 & 37.68 & -106.47 & 112.94 \\
\hline \multirow[t]{7}{*}{3458} & 1.37 & 22.13 & 177.00 & 87.00 & & & & \\
\hline & & & & & 2503 & 110.41 & -119.60 & 162.77 \\
\hline & & & & & 3440 & -331.08 & 125.33 & 354.01 \\
\hline & & & & & 3446 & 26.82 & -29.13 & 39.59 \\
\hline & & & & & 3448 & 11.32 & 38.32 & 39.95 \\
\hline & & & & & 3455 & 63.67 & -23.91 & 68.01 \\
\hline & & & & & 3457 & -56.87 & -75.14 & 94.24 \\
\hline \multirow[t]{6}{*}{3462} & 1.68 & 17.77 & 110.00 & 30.10 & & & & \\
\hline & & & & & 2503 & 66.24 & 11.54 & 67.24 \\
\hline & & & & & 2505 & 99.99 & 53.80 & 113.55 \\
\hline & & & & & 3450 & -46.52 & 39.18 & 60.82 \\
\hline & & & & & 3457 & -30.79 & 124.33 & 128.09 \\
\hline & & & & & 3463 & -199.52 & 186.20 & 272.91 \\
\hline \multirow[t]{2}{*}{3463} & 1.63 & 21.79 & 28.40 & 11.60 & & & & \\
\hline & & & & & $\begin{array}{l}3442 \\
3462\end{array}$ & $\begin{array}{r}-272.53 \\
205.31\end{array}$ & $\begin{array}{r}355.31 \\
-166.32\end{array}$ & $\begin{array}{l}447.79 \\
264.22\end{array}$ \\
\hline
\end{tabular}


$\begin{array}{lllll}3464 & 1.71 & 18.52 & 42.20 & 12.10\end{array}$

$3464 \quad 41.35-127.61 \quad 134.14$

$\begin{array}{llll}3444 & 27.96 & 128.11 & 131.12\end{array}$

$\begin{array}{lllll}3449 & 41.13 & 132.65 & 138.88\end{array}$

$\begin{array}{lllll}3451 & -19.78 & 117.85 & 119.49\end{array}$

$\begin{array}{lllll}3452 & -53.57 & 139.94 & 149.85\end{array}$

$\begin{array}{llll}3463 & -35.70 & 136.16 & 140.76\end{array}$

$\begin{array}{lllllll}3465 & 1.04 & 12.36 & 1706.00 & 530.00 & 2273.00 & 802.00\end{array}$

$\begin{array}{llll}3466 & 740.09 & -33.21 & 740.84\end{array}$

$\begin{array}{llll}3467 & -318.37 & 112.24 & 337.57\end{array}$

$\begin{array}{llll}3468 & -29.33 & 18.98 & 34.93\end{array}$

$\begin{array}{llll}3469 & 79.97 & -4.55 & 80.10\end{array}$

$\begin{array}{llll}3488 & 115.79 & 56.90 & 129.02\end{array}$

$\begin{array}{llll}3492 & -24.92 & 17.56 & 30.49\end{array}$

$\begin{array}{lllllll}3466 & 1.05 & 7.43 & 119.00 & 87.90 & 666.00 & 367.79\end{array}$

$\begin{array}{llll}3465 & -739.39 & 97.24 & 745.76\end{array}$

$\begin{array}{lllll}3487 & 1767.73 & 370.65 & 1806.17\end{array}$

$\begin{array}{lllll}3490 & -481.22 & 44.51 & 483.28\end{array}$

$\begin{array}{lllllll}3467 & 1.04 & 45.68 & 1324.00 & 613.00 & 2851.00 & 468.40\end{array}$

$\begin{array}{llll}3420 & 80.43 & -0.75 & 80.43\end{array}$

$\begin{array}{llll}3421 & 289.67 & -123.41 & 314.86\end{array}$

$\begin{array}{llll}3433 & 69.12 & -1.16 & 69.13\end{array}$

$\begin{array}{llll}3445 & 58.79 & -23.62 & 63.36\end{array}$

$\begin{array}{llll}3465 & 327.68 & 81.14 & 337.57\end{array}$

$\begin{array}{lllll}3468 & 104.41 & 1.77 & 104.43\end{array}$

$\begin{array}{llll}3469 & 142.22 & 25.13 & 144.42\end{array}$

$\begin{array}{llll}3470 & 404.69 & -110.81 & 419.59\end{array}$

$\begin{array}{llll}3492 & 49.83 & 7.11 & 50.34\end{array}$

$\begin{array}{lllll}3468 & 1.00 & 28.99 & 1251.00 & 125.00\end{array}$

$\begin{array}{llll}3421 & -23.73 & -27.07 & 36.00\end{array}$

$\begin{array}{llll}3433 & -25.68 & 4.39 & 26.06\end{array}$

$\begin{array}{llll}3434 & -11.21 & 0.53 & 11.22\end{array}$

$\begin{array}{llll}3445 & -24.83 & -37.20 & 44.73\end{array}$

$\begin{array}{llll}3465 & 32.27 & -9.42 & 33.62\end{array}$

$\begin{array}{llll}3467 & -96.74 & 27.23 & 100.50\end{array}$

$\begin{array}{llll}3470 & 37.96 & -133.57 & 138.86\end{array}$

$\begin{array}{llll}3473 & 23.69 & -24.68 & 34.21\end{array}$

$\begin{array}{lllllll}3469 & 1.04 & 6.70 & 569.00 & 397.00 & 511.00 & 526.53\end{array}$

$\begin{array}{llll}3465 & -79.14 & 12.40 & 80.10\end{array}$

$\begin{array}{llll}3467 & -126.36 & 69.92 & 144.42\end{array}$

$\begin{array}{llll}3470 & -241.06 & -36.11 & 243.75\end{array}$

$\begin{array}{llll}3476 & 27.58 & -4.47 & 27.94\end{array}$

$\begin{array}{lllll}3484 & 186.88 & 22.05 & 188.17\end{array}$

$\begin{array}{llll}3496 & 153.09 & 67.44 & 167.28\end{array}$

$\begin{array}{llll}3497 & 20.42 & -1.70 & 20.49\end{array}$

$\begin{array}{lllll}3470 & 1.19 & 23.94 & 332.00 & 165.00\end{array}$

$\begin{array}{llll}2852 & -349.34 & -697.79 & 780.35\end{array}$

$\begin{array}{lllll}3467 & -383.31 & 289.42 & 480.30\end{array}$

$\begin{array}{lllll}3468 & -31.00 & 162.23 & 165.17\end{array}$ 


\begin{tabular}{|c|c|c|c|c|c|c|c|c|}
\hline & & & & & 3469 & 251.30 & 121.25 & 279.02 \\
\hline & & & & & 3473 & 198.61 & -12.80 & 199.02 \\
\hline \multirow[t]{3}{*}{3472} & 1.21 & 13.15 & 301.00 & 57.40 & & & & \\
\hline & & & & & 2506 & 24.85 & -39.98 & 47.07 \\
\hline & & & & & 3473 & -322.89 & 342.23 & 470.51 \\
\hline \multirow[t]{8}{*}{3473} & 1.18 & 14.43 & 203.00 & 107.00 & & & & \\
\hline & & & & & 2871 & $-803.09-$ & -249.36 & 840.92 \\
\hline & & & & & 3468 & -19.80 & 35.31 & 40.48 \\
\hline & & & & & 3470 & -169.46 & 44.54 & 175.21 \\
\hline & & & & & 3472 & 323.21 & -327.50 & 460.14 \\
\hline & & & & & 3476 & 132.87 & 67.32 & 148.95 \\
\hline & & & & & 3478 & 205.98 & 21.92 & 207.14 \\
\hline & & & & & 3497 & 135.56 & 17.97 & 136.75 \\
\hline \multirow[t]{7}{*}{3476} & 1.04 & -4.54 & 1219.00 & 511.00 & 1627.00 & 612.39 & & \\
\hline & & & & & 3469 & -26.29 & 9.81 & 28.06 \\
\hline & & & & & 3473 & -130.12 & -18.03 & 131.36 \\
\hline & & & & & 3477 & -179.10 & 38.06 & 183.10 \\
\hline & & & & & 3484 & 209.37 & 3.99 & 209.41 \\
\hline & & & & & 3486 & 695.55 & 47.26 & 697.16 \\
\hline & & & & & 3497 & -158.59 & 44.42 & 164.69 \\
\hline \multirow[t]{3}{*}{3477} & 1.04 & 4.63 & 854.00 & 345.00 & 998.00 & 132.64 & & \\
\hline & & & & & 3476 & 192.00 & 1.86 & 192.01 \\
\hline & & & & & 3478 & -40.93 & -170.86 & 175.69 \\
\hline \multirow[t]{4}{*}{3478} & 1.16 & 5.46 & 223.00 & 64.80 & & & & \\
\hline & & & & & 3473 & -202.28 & 10.26 & 202.54 \\
\hline & & & & & 3477 & 43.68 & 193.86 & 198.72 \\
\hline & & & & & 3480 & -70.94 & -226.78 & 237.62 \\
\hline \multirow[t]{5}{*}{3480} & 1.38 & 6.99 & 75.10 & 11.60 & & & & \\
\hline & & & & & 2501 & -130.18 & 10.87 & 130.64 \\
\hline & & & & & 2507 & 15.79 & -19.36 & 24.98 \\
\hline & & & & & 3478 & 71.93 & 271.33 & 280.70 \\
\hline & & & & & 3481 & 112.62 & -320.58 & $8 \quad 339.79$ \\
\hline \multirow[t]{3}{*}{3481} & 1.45 & 5.92 & 144.00 & 21.20 & & & & \\
\hline & & & & & 3480 & -111.61 & 337.64 & 4355.61 \\
\hline & & & & & 3483 & 142.56 & -8.18 & 142.79 \\
\hline \multirow[t]{4}{*}{3483} & 1.44 & 4.03 & 134.00 & 26.10 & & & & \\
\hline & & & & & 2506 & 9.83 & -11.67 & 15.26 \\
\hline & & & & & 2507 & 2.30 & -23.68 & 23.79 \\
\hline & & & & & 3481 & -141.55 & 12.81 & 142.12 \\
\hline \multirow[t]{7}{*}{3484} & 1.04 & -21.95 & 1153.00 & 1008.00 & 758.00 & 1396.79 & & \\
\hline & & & & & 3469 & -174.55 & 70.26 & 188.16 \\
\hline & & & & & 3476 & -200.11 & 58.56 & 208.51 \\
\hline & & & & & 3486 & -0.25 & 0.03 & 0.26 \\
\hline & & & & & 3488 & -19.36 & 47.85 & 51.62 \\
\hline & & & & & 3496 & 58.03 & 191.50 & 200.10 \\
\hline & & & & & 3497 & -60.89 & 20.59 & 64.27 \\
\hline \multirow[t]{3}{*}{3485} & 1.02 & -29.45 & 0.00 & 0.00 & & & & \\
\hline & & & & & 3486 & -968.37 & -53.20 & 969.83 \\
\hline & & & & & 3495 & 991.16 & 148.46 & 61002.21 \\
\hline
\end{tabular}


$\begin{array}{llll}3476 & -674.93 & 162.21 & 694.15\end{array}$

$\begin{array}{llll}3484 & 0.25 & -0.03 & 0.26\end{array}$

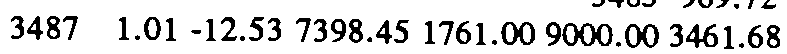

$\begin{array}{llll}3485 & 969.72 & 182.33 & 986.71\end{array}$

$34661323.66 \quad 215.401711 .57$

$\begin{array}{lllll}3488 & 416.97 & 1464.88 & 1523.07\end{array}$

$\begin{array}{llll}3489 & -180.34 & 57.26 & 189.21\end{array}$

$\begin{array}{lllll}3490 & -167.55 & 181.83 & 1051.38\end{array}$

$\begin{array}{lllll}3488 & 0.82 & -15.20 & 763.00 & 778.00\end{array}$

$34951427.04 \quad 373.761475 .18$

$3489 \quad 1.03 \quad 25.522387 .44-175.00$

$$
\begin{array}{lrrr}
3465 & -101.85 & 2.45 & 101.88 \\
3484 & 19.62 & -35.73 & 40.76 \\
3487 & -393.65 & 128.54 & 1237.38 \\
3491 & -82.73 & 24.44 & 86.26 \\
3492 & -264.37 & 51.77 & 269.39 \\
3496 & 30.92 & -25.55 & 40.11
\end{array}
$$

$24768945.97 \quad 134.401335 .48$

$\begin{array}{lllll}3487 & 181.02 & 67.47 & 193.19\end{array}$

$\begin{array}{llll}3490 & 1585.37 & -72.92 & 1587.05\end{array}$

$3491-4356.88 \quad 151.671441 .29$

$\begin{array}{llll}3493 & -29.27 & 22.99 & 37.22\end{array}$

$\begin{array}{llll}3495 & 484.51 & 189.22 & 520.15\end{array}$

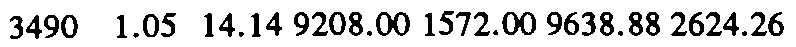

$\begin{array}{llll}3466 & 476.99 & 3.10 & 477.00\end{array}$

$34871047.13 \quad 314.461093 .33$

3489-2365.23 391.491616 .44

$\begin{array}{lllll}3492 & -226.81 & 109.86 & 252.02\end{array}$

$\begin{array}{lllll}3493 & -43.59 & 33.67 & 55.08\end{array}$

$\begin{array}{llll}3495 & 738.91 & 246.78 & 779.03\end{array}$

$\begin{array}{lllllll}3491 & 1.04 & 34.58 & 104.00 & 772.00 & 2200.00 & 1434.04\end{array}$

$\begin{array}{llll}3413 & 7.37 & 8.05 & 10.91\end{array}$

$\begin{array}{llll}3433 & -27.03 & 6.60 & 27.83\end{array}$

$\begin{array}{llll}3436 & -6.42 & 4.90 & 8.08\end{array}$

$\begin{array}{llll}3488 & 91.62 & 60.23 & 109.64\end{array}$

$34891455.76 \quad 76.71 \quad 1457.78$

$\begin{array}{lllll}3492 & 375.79 & 191.95 & 421.97\end{array}$

$\begin{array}{lllll}3496 & 176.03 & 77.30 & 192.25\end{array}$

$\begin{array}{lllll}3492 & 0.97 & 26.70 & 0.00 & 0.00\end{array}$

$$
\begin{array}{cccc}
3416 & -10.45 & 4.26 & 11.28 \\
3433 & -69.11 & -7.14 & 69.47 \\
3436 & -30.38 & -1.43 & 30.42 \\
3465 & 26.70 & -10.15 & 28.57 \\
3467 & -46.32 & 8.89 & 47.16 \\
3488 & 274.51 & 163.76 & 319.65 \\
3490 & 227.37 & -53.67 & 233.62 \\
3491 & -372.04 & -129.42 & 393.91
\end{array}
$$

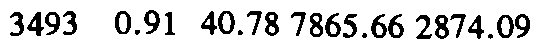


$\begin{array}{llll}3401 & 400.23 & -423.74 & 582.87\end{array}$

$\begin{array}{llll}3489 & 30.41 & -12.84 & 33.01\end{array}$

$\begin{array}{llll}3490 & 47.08 & -9.19 & 47.97\end{array}$

$3494-277.28-943.48 \quad 983.38$

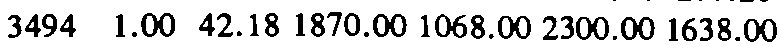

$\begin{array}{llll}3416 & 122.79 & 25.28 & 125.37\end{array}$

$\begin{array}{llll}3417 & 43.14 & -0.86 & 43.15\end{array}$

$\begin{array}{llll}3424 & -17.92 & -20.93 & 27.55\end{array}$

$\begin{array}{lllll}3493 & 279.30 & 1043.86 & 1080.58\end{array}$

$\begin{array}{llll}3498 & 32.48 & 2.62 & 32.59\end{array}$

$\begin{array}{llll}3502 & -22.41 & -141.30 & 143.07\end{array}$

$3495 \quad 1.00-47.278787 .326827 .008799 .438416 .41$

$\begin{array}{llll}3485 & -974.51 & 143.55 & 985.02\end{array}$

$\begin{array}{lllll}3487 & -996.86 & 500.87 & 1460.57\end{array}$

$\begin{array}{llll}3489 & -314.30 & 394.52 & 504.41\end{array}$

$\begin{array}{lllll}3490 & -543.00 & 505.27 & 741.72\end{array}$

$\begin{array}{llll}3496 & -372.14 & 163.53 & 406.48\end{array}$

$\begin{array}{lllll}3496 & 0.92 & -23.89 & 0.00 & 0.00\end{array}$

$\begin{array}{llll}3469 & -146.45 & 17.52 & 147.50\end{array}$

$\begin{array}{llll}3484 & -56.87 & -167.03 & 176.45\end{array}$

$\begin{array}{llll}3488 & -29.81 & 33.42 & 44.78\end{array}$

$\begin{array}{llll}3491 & -138.73 & 96.30 & 168.88\end{array}$

$\begin{array}{llll}3495 & 372.41 & -2.27 & 372.42\end{array}$

$\begin{array}{lllllll}3497 & 1.03 & -0.41 & 310.00 & 210.00 & 394.60 & 199.98\end{array}$

$\begin{array}{llll}3469 & -19.97 & 4.20 & 20.41\end{array}$

$\begin{array}{llll}3473 & -118.62 & 15.17 & 119.59\end{array}$

$\begin{array}{llll}3476 & 160.04 & -32.58 & 163.32\end{array}$

$\begin{array}{llll}3484 & 63.93 & 3.20 & 64.01\end{array}$

$\begin{array}{lllll}3498 & 1.01 & 26.33 & 410.00 & 102.00\end{array}$

$\begin{array}{llll}3419 & -94.52 & -199.38 & 220.65\end{array}$

$\begin{array}{llll}3494 & -31.99 & 6.36 & 32.61\end{array}$

$\begin{array}{llll}3499 & 191.91 & 141.59 & 238.49\end{array}$

$\begin{array}{lllll}3499 & 0.96 & 22.42 & 205.00 & 164.00\end{array}$

$3444-14.41-109.64 \quad 110.58$

$3498-191.53-122.08 \quad 227.13$

$\begin{array}{lllll}3500 & 1.35 & 43.50 & 0.00 & 0.00\end{array}$

$\begin{array}{llll}3408 & 304.30 & 227.86 & 380.15\end{array}$

$\begin{array}{lllll}3501 & 895.84 & 7579.99 & 1344.84\end{array}$

$\begin{array}{llll}3502 & -65.70 & 188.78 & 199.89\end{array}$

$\begin{array}{lllll}3501 & 1.38 & 36.50 & 0.00 & 0.00\end{array}$

$\begin{array}{llll}2499 & 788.62 & -568.13 \quad 971.95\end{array}$

$\begin{array}{llll}3500 & -826.80 & 785.90 & 1140.72\end{array}$

$\begin{array}{lllll}3502 & 1.34 & 43.70 & 367.00 & 247.60\end{array}$

$\begin{array}{lllll}3418 & 417.56 & 352.52 & 546.47\end{array}$

$\begin{array}{lllll}3419 & 105.18 & 46.41 & 114.96\end{array}$

$\begin{array}{lllll}3424 & 27.62 & 342.94 & 344.05\end{array}$

$\begin{array}{lllll}3494 & 24.80 & 188.66 & 190.29\end{array}$

$3500 \quad 65.74-186.77 \quad 198.00$ 
September 4, 1961 Born, Havana, Cuba

1982 B.S. Electrical Engineering Technology Florida International University Miami, Florida

1982 B.S. Computer Science Florida International University Miami, Florida

1982-1984 Engineer Florida Power and Light Miami, Florida

1984-1987 Consultant M.C. Consultants Miami, Florida

1988 M.S. Computer Science Florida International University Miami, Florida

1988-1993 Graduate Assistant in Computer Science Florida International University Miami, Florida

\section{PUBLICATIONS AND PRESENTATION}

D. Tal, J. Comfort, M. Martinez. "The Mapping of the FFT Algorithm on a Systolic Dataflow Machine". Proceedings of the 2nd Symposium on Frontiers of Massively Parallel Computation, GMU, Fairfax, VA, October 10-12, 1988. Ed. R. Milis. IEEE Computer Society Press, 1989.

Maria Cereijo Martinez. "A Parallel Technique for the Solution of the Power System Loadflow Analysis". Interamerican Engineering Conference, Miami, FL, December 1990 .

Maria Cereijo Martinez. "A Parallel Solution to the Power System Load Flow Analysis". Southcon 92, Orlando, Florida, February 1992 .

Referree of the 18th International Conference on Parallel Processing, 1990 . 